\title{
SAMPleD-DATA SUPERVISORY Control
}

\author{
By \\ YU WANG, B.ENG
}

\author{
A Thesis \\ Submitted to the School of Graduate Studies \\ in partial fulfilment of the requirements for the degree of \\ Master of Applied Science \\ Department of Computing and Software \\ McMaster University
}

(C) Copyright by Yu Wang, January 15, 2009 
MASTER OF APPLIED SCIENCE(2006)

(Software Engineering)

TITLE: Sampled-data Supervisory Control

AUTHOR: $\quad$ Yu Wang, B.Eng(McMaster University)

SUPERVISOR: $\quad$ Dr. Ryan Leduc
McMaster University

Hamilton, Ontario

NUMBER OF PAGES: i, 390 


\section{Abstract}

This thesis focuses on issues related to implementing theoretical Discrete-Event Systems (DES) supervisors, and the concurrency and timing delay issues involved.

Sampled-data (SD) supervisory control deals with timed DES (TDES) systems where the supervisors will be implemented as SD controllers. An SD controller is driven by a periodic clock and sees the system as a series of inputs and outputs. On each clock edge (tick event), it samples its inputs, changes states, and updates its outputs.

In this thesis, we identify a set of existing TDES properties that will be useful to our work, but not sufficient. We extend the TDES controllability definition to a new definition, SD controllability, which captures several new properties that will be useful in dealing with concurrency issues, as well as make it easier to translate a TDES supervisor into an SD controller.

We then establish a formal representation of an SD controller as a Moore Finite State Machine (FSM), and describe how to translate a TDES supervisor to a FSM, as well as necessary properties to be able to do so. We discuss how to construct a single centralized controller, as well as a set of modular controllers and show that they will produce equivalent output.

Next, we capture the enablement and forcing action of a translated controller in the form of a TDES supervisory control map, and show that the closed-loop behavior of this map and the plant is the same as that of the plant and the original TDES supervisor. We also show that our method is robust with respect to nonblocking and certain variations in the actual behavior of our physical system.

We also introduce a set of predicate-based algorithms to verify the SD controllability property, as well as certain other conditions that we require. We have created a software tool for verifying these conditions and provide the source code in the appendix. We have implemented these algorithms using binary decision diagrams (BDD).

For illustrative purpose, we have produced a set of examples which fail the key conditions discussed in this thesis, as well as a successful application example based on a Flexible Manufacturing System. We also presented the corresponding FSM, 
iv

translated from the example's supervisors. 


\section{Acknowledgment}

I will definitely first give my thanks to my supervisor, Dr. Ryan Leduc, who I have been working for since I was an undergraduate. I would have never been able to accomplish this task without the great great amount time, constant guidance, and support he has given me. His expertise in the area of discrete event control systems is the most valuable source of help for the whole period of this work.

I'd also like to thank Raoguang Song for his preceding work on the BDD based symbolic verification tool for HISC. His code base saved me a lot of effort in understanding and starting the software implementation.

At last my thanks go to my beloved father Xuhong Wang, and mother Baoxiang Yun for their unlimited support and Wen Xie for her great understanding and confidence in me. This thesis is dedicated to them. 


\section{Contents}

Contents vii

List of Figures $\quad$ xi

$\begin{array}{lll}1 & \text { Introduction } & 1\end{array}$

1.1 Objective . . . . . . . . . . . . . . . . . . . . 4

1.2 Related Work . . . . . . . . . . . . . . . . . . . . . . . . . . 7

\begin{tabular}{|lll}
2 & Discrete-Event Systems Preliminaries & 11
\end{tabular}

$2.1 \quad$ Algebraic Preliminaries . . . . . . . . . . . . . . . . . . . . 11

2.1 .1 Strings . . . . . . . . . . . . . . . . . . . . . . 11

2.1 .2 Languages . . . . . . . . . . . . . . . . . . . . . . . . . . . . . 12

$2.1 .3 \quad$ Nerode Equivalence Relation . . . . . . . . . . . . . . . . . . . 12

2.2 Discrete Event Systems . . . . . . . . . . . . . . . . . . . . . . . . . . 13

2.2 .1 Generator . . . . . . . . . . . . . . . . . . 13

$2.2 .2 \quad$ Synchronization and Product DES . . . . . . . . . . . . . . 17

$2.2 .3 \quad$ Controllability and Supervision . . . . . . . . . . . . . . . . . 19

2.3 Timed Discrete Event Systems . . . . . . . . . . . . . . . . . . . . 21

2.3 .1 Basic Structure . . . . . . . . . . . . . . . . . . . 22

2.3 .2 Controllability and Supervision . . . . . . . . . . . . 22

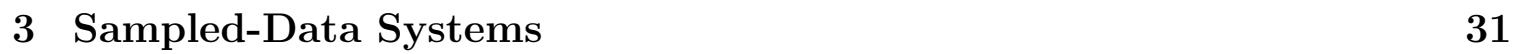

3.1 Sampling Inputs . . . . . . . . . . . . . . . . . . . . . . . . . . . . . 33

3.2 SD Controllable Languages . . . . . . . . . . . . . . . . . . . . . . . . 37

3.3 Future Work . . . . . . . . . . . . . . . . . . . . . . . . . 45

4 Moore Synchronous Finite State Machines 47

4.1 Formal Model . . . . . . . . . . . . . . . . . . . . . . . . . . . . . . . . 48

4.2 Translation Method . . . . . . . . . . . . . . . . . . . . . . . . . . 52

$4.2 .1 \quad$ Event Mapping Functions . . . . . . . . . . . . . . . . . . 53

4.2 .2 Output Equivalence. . . . . . . . . . . . . . . . . . . . . . 54 
4.2 .3 Centralized Controller . . . . . . . . . . . . . . . . 57

4.2 .4 Modular Controllers . . . . . . . . . . . . . . . . . . . 63

\begin{tabular}{|lll}
5 & Control and Nonblocking Verification & 73
\end{tabular}

5.1 Supervisory Control Construction . . . . . . . . . . . . . . . . . 73

$5.2 \quad$ Map V Is Well Defined $\ldots \ldots \ldots \ldots$

5.3 Supervisory Control and SD Supervisors . . . . . . . . . . . . . . 83

5.4 Concurrent Supervisory Control Equivalent. . . . . . . . . . . . . 100

$6 \quad$ Symbolic Verification for SD System 113

6.1 Predicates and Predicate Transformers . . . . . . . . . . . . . . . 113

6.1 .1 State Predicates . . . . . . . . . . . . . . . . . . . . . . . 113

6.1 .2 Predicate Transformers . . . . . . . . . . . . . . . . . . . 115

6.2 Symbolic Representation . . . . . . . . . . . . . . . . . . . . . . . . 116

6.2 .1 State Subsets . . . . . . . . . . . . . . . . . . . 116

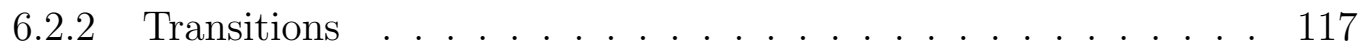

6.3 Symbolic Computation . . . . . . . . . . . . . . . . . . . . . . . . . . . . 119

6.3 .1 Transitions and Inverse Transitions . . . . . . . . . . . . . . 119

6.3 .2 Computation of Predicate Transformers . . . . . . . . . . 121

6.4 Symbolic Verification . . . . . . . . . . . . . . . . . . . . . . . 123

6.4 .1 Untimed Controllability . . . . . . . . . . . . . . . . 124

6.4 .2 Plant Completeness . . . . . . . . . . . . . . . . . . . 126

6.4 .3 Non-blocking . . . . . . . . . . . . . . . . . . . . . . 127

6.4 .4 Activity Loop Free . . . . . . . . . . . . . . . . . 127

6.4 .5 Proper Time Behavior . . . . . . . . . . . . . . . . . . . . 128

$\begin{array}{lll}6.4 .6 & \text { SD Controllability and S-Singular Prohibitable Behavior } \ldots & 129\end{array}$

\begin{tabular}{lll}
\hline 7 & Examples & 143
\end{tabular}

7.1 Examples . . . . . . . . . . . . . . . . . . . . . . . . 144

7.1 .1 Plant Completeness . . . . . . . . . . . . . . . . . . . . . . 144

7.1 .2 Activity Loop Free . . . . . . . . . . . . . . . . . . . . 146

7.1 .3 Proper Time Behavior . . . . . . . . . . . . . . . . . . . . . 147

$7.1 .4 \quad$ SD Controllability . . . . . . . . . . . . . . . . . . . . 148

7.2 SD Controlled Flexible Manufacturing System . . . . . . . . . . . 156

7.2 .1 FMS Plants . . . . . . . . . . . . . . . . . . . 156

7.2 .2 Buffer Supervisors _. . . . . . . . . . . . . . . . . 157

$7.2 .3 \quad$ B4 to Lathe Path . . . . . . . . . . . . . . . . . . . . . . 161

7.2 .4 Moving Parts from B4 to B6/B7 . . . . . . . . . . . . . 164

7.2 .5 AM to Exit Path . . . . . . . . . . . . . . . . . . . . . . . 164

7.2 .6 System Shutdown . . . . . . . . . . . . . . . . . . . . . . . . . 169 
7.2 .7 Algorithm Runtime Statistics . . . . . . . . . . . . . . . . . 171

7.3 Translating FSM Supervisors to Moore FSM . . . . . . . . . . . . . . 174

7.3 .1 Adding More Timing Information . . . . . . . . . . . . . . . . 174

7.3.2 $\quad$ FSM Controllers for Flexible Manufacturing System . . . . . . 176

$\begin{array}{lll}8 & \text { Conclusions } & 183\end{array}$

\begin{tabular}{ll}
\hline Bibliography & 187
\end{tabular}

\begin{tabular}{|ll}
\hline A SD Software Program & 191
\end{tabular}

A.1 FMS Example Input Files . . . . . . . . . . . . . . . . . . . . . . . . 191

A.1.1 FMS Plants . . . . . . . . . . . . . . . . . . . . . . . 191

A.1.2 Helper Plants . . . . . . . . . . . . . . . . . . . . . . 195

A.1.3 Buffer Supervisors . . . . . . . . . . . . . . . . . . . . . . . . 197

A.1.4 Additional Supervisors . . . . . . . . . . . . . . . . . . . . 201

A.2 Source code . . . . . . . . . . . . . . . . . . . . . . . . 208

A.2.1 Main . . . . . . . . . . . . . . . . . . . 209

\begin{tabular}{lll}
\hline A.2.2 Global Functions, Typedefs, Variables, Preprocessors symbols 216 & 235
\end{tabular}

A.2.3 $\quad$ DES Class . . . . . . . . . . . . . . . . . . . . . . . . . . . . 235

A.2.4 Sub Class . . . . . . . . . . . . . . . . . . . . . . . 256

A.2.5 LowSub Class . . . . . . . . . . . . . . . . . . . . . . . . . . . 299 


\section{List of Figures}

1.1 The Occurrences of Two Events . . . . . . . . . . . . . . . . . . 2

$2.1 \quad$ An Example DES $\ldots \ldots \ldots \ldots$

2.2 An Example Failing ALF Property . . . . . . . . . . . . . . . . . 24

$2.3 \quad$ An Example Failing the Proper Time Behavior Property . . . . . . . 27

$3.1 \quad$ Nonminimal Example . . . . . . . . . . . . . . . . . . . . . . . . . . 37

$3.2 \quad$ An Example for Point ii . . . . . . . . . . . . . . . . . . . . . . . 42

3.3 An Example for Point iii.1 . . . . . . . . . . . . . . . . . . . . . 43

3.4 An Example Failing Point iv . . . . . . . . . . . . . . . . . . 43

3.5 SD Controllability and Arbitrary Union. . . . . . . . . . . . . . 44

$4.1 \quad$ FSM Translation Example $\ldots \ldots \ldots \ldots$

4.2 Centralized Control Equivalence Diagram . . . . . . . . . . . . 60

$5.1 \quad$ An Example Failing S-singular Prohibitable Behavior Property . . . . 74

5.2 Part of a TDES plant. . . . . . . . . . . . . . . . 80

5.3 An Example for Concurrent Supervisory Control Equivalence. . . . . . 102

7.1 Legend Used to Display DES . . . . . . . . . . . . . . . . . . 143

7.2 Plant Completeness Example: Plant . . . . . . . . . . . . . . . 145

7.3 Plant Completeness Example: Supervisor. . . . . . . . . . . . . . 145

7.4 Activity Loop Example . . . . . . . . . . . . . . . . . . . . . . . 146

7.5 Proper Time Behavior Example . . . . . . . . . . . . . . . . . 147

$7.6 \quad$ SD Controllability i, ii Example: Plant . . . . . . . . . . . . . . . . 149

7.7 SD Controllability Point i, ii Example: Supervisor . . . . . . . . . . 150

7.8 SD Controllability Point iii.1 Example: Plant . . . . . . . . . . . 152

7.9 SD Controllability Point iii.1 Example: Supervisor . . . . . . . . . . . 152

7.10 SD Controllability Point iii.2 Example: Plant . . . . . . . . . . . 153

7.11 SD Controllability Point iii.2 Example: Supervisor . . . . . . . . . . . 153

7.12 SD Controllability Point iv Example: Plant . . . . . . . . . . . 155 
7.13 SD Controllability Point iv Example: Supervisor . . . . . . . . . . . . 155

7.14 Flexible Manufacturing System Overview . . . . . . . . . . . . . . . . 156

7.15 Conveyor - Con2 . . . . . . . . . . . . . . . . . . . . 157

7.16 Robot . . . . . . . . . . . . . . . . . . . . . . 157

7.17 Lathe . . . . . . . . . . . . . . . . . . . . . . 158

7.18 Finishing Machine - AM . . . . . . . . . . . . . . . . . . . . . . 158

7.19 Conveyor - Con3 . . . . . . . . . . . . . . . . . . . . . . . . 158

7.20 Painting Machine $-\mathbf{P M}$. . . . . . . . . . . . . . . . . . . . 158

7.21 Supervisor B2 $\ldots \ldots \ldots \ldots \ldots$. . . . . . . . . . . . . . . . . . 159

7.22 Supervisor B4 . . . . . . . . . . . . . . . . . . . . . . . . . 160

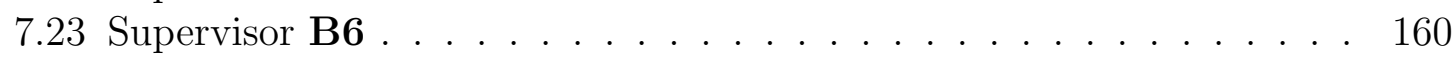

7.24 Supervisor B7 . . . . . . . . . . . . . . . . . . . . . . . . . 160

7.25 Supervisor B8 . . . . . . . . . . . . . . . . . . . . . . . . . 160

7.26 TakeB2 . . . . . . . . . . . . . . . . . . . . . 162

7.27 B4Path . . . . . . . . . . . . . . . . . . . . 162

7.28 LathePick . . . . . . . . . . . . . . . . . . . . . . . 163

7.29 TakeB4PutB6 . . . . . . . . . . . . . . . . . 165

7.30 TakeB4PutB7 . . . . . . . . . . . . . . . . . . . . . 165

7.31 Plant AddNo963 . . . . . . . . . . . . . . . . . . . . 166

7.32 Plant AddNo965 . . . . . . . . . . . . . . . . . . . 166

7.33 Force963 . . . . . . . . . . . . . . . . . . . 167

7.34 Force961 . . . . . . . . . . . . . . . . . . 167

7.35 AMChooser . . . . . . . . . . . . . . . . . . 168

7.36 Force965 . . . . . . . . . . . . . . . . . . . . . 168

7.37 Plant SystDownNup . . . . . . . . . . . . . . . . . . . . . 170

7.38 Supervisor handleSystDown . . . . . . . . . . . . . . . . . . . . . . 170

7.39 Plant AddNo921 . . . . . . . . . . . . . . . . . . . . . . . 170

7.40 Histogram for Memory Usage (Kbytes vs. seconds) . . . . . . . . . . 173

7.41 New B4 . . . . . . . . . . . . . . . . . . . . . . 175

7.42 New B6 . . . . . . . . . . . . . . . . . . . . . 175

7.43 New B7 . . . . . . . . . . . . . . . . . . . . . 175

7.44 New B4Path . . . . . . . . . . . . . . . . . . 175

7.45 FSM B2 . . . . . . . . . . . . . . . . . . . . . . . . 178

7.46 FSM Force963 . . . . . . . . . . . . . . . . . . . . . . . . . . . . . . . 178

7.47 FSM Force965 . . . . . . . . . . . . . . . . . . . . . . . . . . . 178

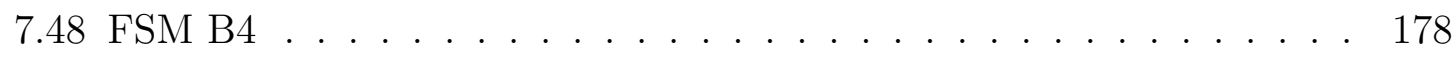

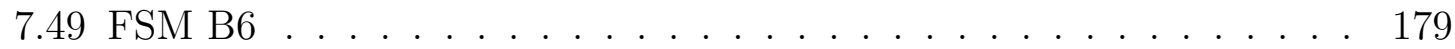

7.50 FSM B7 $\ldots \ldots \ldots \ldots \ldots \ldots$

7.51 FSMB8 $\ldots \ldots \ldots \ldots \ldots \ldots \ldots$

7.52 FSM LathePick . . . . . . . . . . . . . . . . . . . . . . . . . . . 180 
7.53 FSM TakeB2 $\ldots \ldots \ldots \ldots$

7.54 FSM B4Path . . . . . . . . . . . . . . . . . . . . . 180

7.55 FSM Force961 . . . . . . . . . . . . . . . . . . . . . . . . . . . 181

7.56 FSM handleSystDown . . . . . . . . . . . . . . . . . . . . . 181

7.57 FSM TakeB4PutB6 . . . . . . . . . . . . . . . . . . . . . . . . . . . . . . . . . . . . . . . . . .

7.58 FSM TakeB4PutB7 . . . . . . . . . . . . . . . . . . . . . . 181

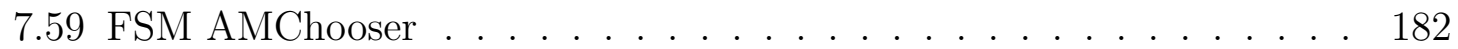




\section{Chapter 1}

\section{Introduction}

In the area of Discrete-Event Systems (DES) [23, [29], [30, a lot of effort has been devoted to studying standard properties such as nonblocking (a form of deadlock detection) and controllability (a check on whether we can actually realize our desired control law) in a theoretical setting. However, limited effort has been made in investigating what an implementation of a DES supervisor would be like, how to do the conversion automatically, whether we can guarantee that it will retain the controllability and nonblocking properties of the theoretical supervisor, and how to handle timing delay and concurrency issues inherent in an implementation. This thesis will be attacking these problems, although issues with respect to timing delay will only be partially dealt with due to time limitations.

A logical implementation method for DES supervisors would be sampled-data (SD) controllers. An SD controller is driven by a periodic clock and sees the system as a series of inputs and outputs. On each clock edge, it samples its inputs, changes state, and updates its outputs. An example of an SD controller might be a programmable logic controller (PLC) [4] or a Moore synchronous finite state machine (FSM) [7. In this thesis, we will focus on FSM SD controllers as they are a complete specification of an SD controller, yet still quite generic allowing an FSM to be implemented in digital logic, or as a computer program. For simplicity, we will assume inputs and outputs of an FSM can take the value of true or false.

When we are using an SD controller to manage a given system, we associate an input with each event, and output with each controllable event. We consider an 
event to have occurred when its corresponding input has gone true during a given clock period. We consider a controllable event to be enabled when its corresponding output has been set true by the controller, disabled otherwise.

As mentioned above, an SD controller samples the value of its inputs on each clock edge, and uses this value to decide what its next internal state will be. This means the SD controller knows nothing about its inputs until the clock edge, and then all it learns is whether a given input is true or false, signifying that the corresponding event has occurred sometime in the clock period that just ended. This means that for the given clock period, all information about event ordering (which event occurred first etc) is lost, as well as how often a given event occurred if it has occurred more than once. The only ordering information that remains is which sampling period (clock period) a given event occurred in.

As an example, consider Figure 1.1. Here we have inputs Event 1 and 2, as well as our sampling clock. The diagram on the left shows when the inputs changed their value, in particular that Event 1 occurred first in the second sampling period. When
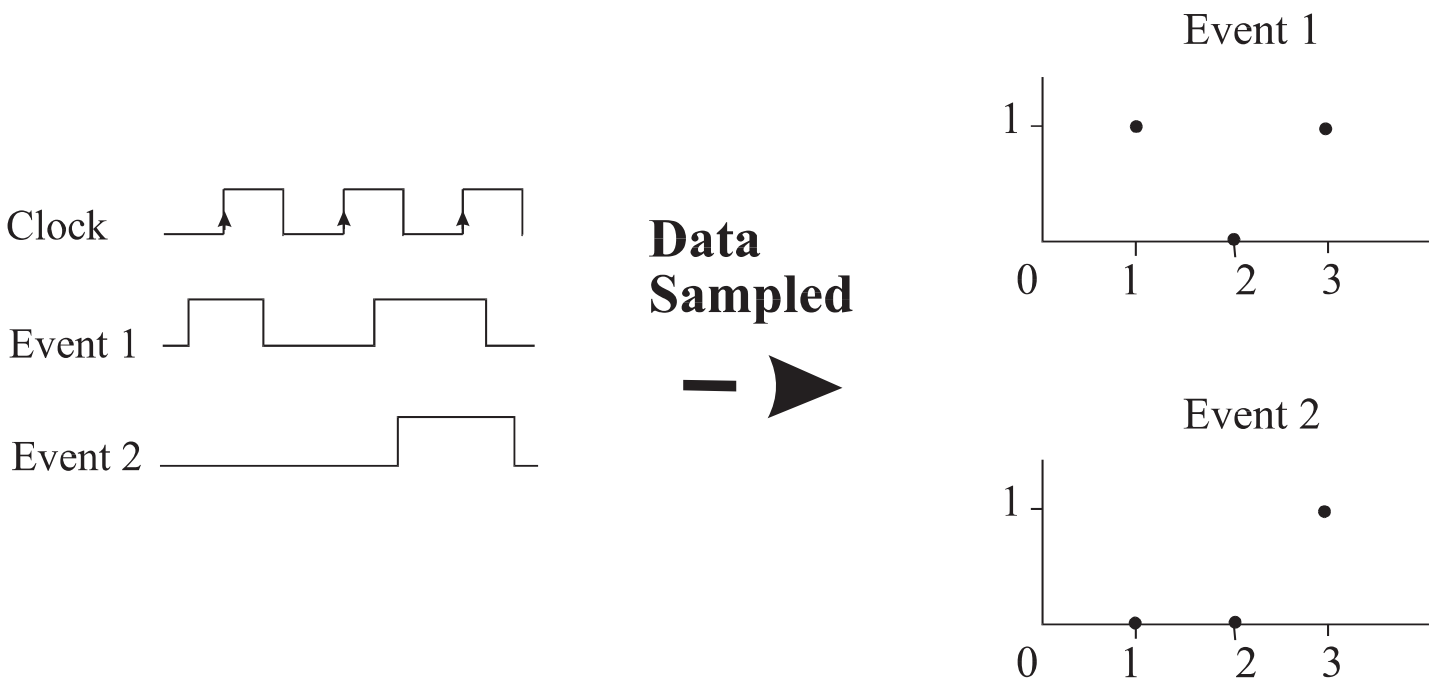

Figure 1.1: The Occurrences of Two Events

the SD controller samples its inputs, it simply gets a true or false value, based on the value of the input at the clock edge ${ }^{1}$ As we can see in the diagram on the right,

\footnotetext{
${ }^{1}$ In our example, we are sampling our inputs when the clock signal rises from low to high (the rising edge of the clock).
} 
the controller simply knows that both Event 1 and 2 occurred in the last sampling period, nothing more.

Another important aspect of an SD controller is that it only changes state on a clock edge, and the value of its outputs are a function of its current state. That means its outputs can only change at a clock edge, and then must stay constant for the rest of the clock period.

For DES supervisors, we generally assume that a supervisor knows immediately when an event occurs, that it can change enablement information right away, and that events occur in an interleaving fashion so the supervisor can always determine the order events occurred in. Based on the above discussion, it is clear that an SD controller implementation violates these assumptions. First, the controller must wait until the next sampling instance (clock edge) before it will know if a given event has occurred. If the control law said something like "once event $\alpha$ occurs, controllable event $\beta$ must not occur." However if $\beta$ can occur in the same sampling period as $\alpha$, $\beta$ may have already occurred before we even know that $\alpha$ has occurred. Of course, even if we did know right away that alpha had occurred, we would not be able to update the enablement information for $\beta$ until the next clock edge anyway, which could be too late. If we wanted to make sure $\beta$ did not occur in this clock period, we would have to disable it at the start of the sampling period. This means that we cannot enforce a policy where an event is initially enabled (disabled) at the start of a clock period, and we then disable (enable) the event somewhere in the middle. Our supervisor must have a policy that is correct and constant for the entire sampling period.

Another important issue is event ordering. If we could get either string ' $\alpha \beta$ ' or ' $\beta \alpha$ ' in the same clock period, our SD controller would only know that at least one $\alpha$ and at least one $\beta$ had occurred. It would not know which of the two had actually occurred. If our DES supervisor enabled event $\gamma$ when string ' $\alpha \beta$ ' occurs, but disables $\gamma$ when string ' $\beta \alpha$ ' occurs, we could not implement this using an SD controller as it would not be able to determine which of the two strings had occurred. This means that a supervisor must always do the same thing for two concurrent strings containing the same individual events, both immediately after the strings have occurred and in the future. Of course, this raises the question of how to determine if two strings are concurrent. 


\section{$1.1 \quad$ Objective}

Clearly, untimed DES does not provide a rich enough modeling method to allow us to work with an SD controller, and its inherent timing information. Therefore, we will base our work on the timed DES (TDES) theory developed by Brandin et al. [5] [6]. TDES extends untimed DES theory by adding a new tick event, corresponding to the tick of a global clock. The event set of a TDES contains the tick event as well as other non-tick events called activity events. The occurrence of a tick event provides us with a concept of time passing, allowing us to model upper and lower time bounds for the occurrence of activity events. It also allows us to introduce a new type of events called forcible events, which we can guarantee to occur and preempt the next clock tick. This means that now we cannot only prevent some events (referred to as prohibitable events in TDES terminology) from occurring by disabling them, but we can also choose to have certain events occur before the next clock tick.

To make the TDES theory work with SD controllers, we identify a tick event occurring with the clock edge that the SD controller uses for sampling and state change. That means that once a tick event occurs, any two strings that are now possible in the system and only contain a single tick at the end of the string, are considered concurrent. We will refer to such strings as concurrent strings. If one of these strings contains at least one different event from the other string, we can distinguish between them. Otherwise, we must treat them the same.

Now that we can force an event to occur in a specific clock period, we have a new concern with respect to nonblocking. The plant model might say that we can do either an ' $\alpha \beta \tau$ ' concurrent string, or a ' $\beta \alpha \tau^{\prime}$ ' string, where $\tau=$ tick. Both might be safe to do, but depending on our implementation, only one of the two might ever occur. Some reasons this could occur are due to time delay, or our implementation might be a sequential program that must choose one version or the other to perform. It might be the case that for some implementations, when two or more concurrent strings are possible and they contain the same events but in a different order or numbers, not all variations might ever actually occur. The problem is that one of the variations that does not occur might have been the only path in the TDES back to a marked state. Basically, if an SD controller cannot tell the difference between concurrent strings, they should have the same marked future. This also means that marked strings can 
only be the empty string (represents the initial state of the system which is always observable), or strings ending in a tick as these are the points in the system's behavior that are observable to an SD controller. We refer to such strings as sampled strings.

The next problem we intend to address is the issue of when a forced event should occur. As noted by Balemi in [2] for untimed systems, controllable events tend to be events fully under the control of our controller implementation.? ${ }^{2}$ They may be a software function we call, an output we set to true, or a message we send. That means that we can make these events occur whenever we want. It is not unusual that a plant might be modeled such that these events are suppose to only occur under certain situations. This might be for flexibility (some implementations have these restrictions, for example) or to make the system easier to model or understand. However, the reality for some controller implementations is that these events could occur even when the plant said they cannot. This also applies to forcible events. When we are forcing an event to occur in a given clock period, we have no information on when it will actually occur. Depending on our implementation, it could occur right away, or in the middle or end of the clock period. We need to make sure that when it finally does occur, it does not contradict the plant model so that our implementation will correspond to the theoretical model in this respect.

The last issue we intend to address is the issue of when a forcible event should actually occur. We want our supervisor specified in such a way that it is straightforward to convert it into an SD controller. Normally for DES systems, we are interested in maximally permissive behavior. We enable all controllable events except for when they must be disabled to enforce our control law, and to ensure the system is nonblocking. However, controller implementations are usually much more procedural. We would disable all controllable events until we want them to occur, and then disable the event again once it has occurred. In our setup, we will be assuming that the set of prohibitable events and forcible events are the same $\mathrm{e}^{3}$ and that we disable the event until we wish to force it, and then disable it once it has occurred. This

\footnotetext{
${ }^{2}$ This is generally a matter of how a system is modeled. We can always model the sending of our enable/disable signal as the controllable event, and the occurrence of the actual action as the uncontrollable event. Of course, the occurrence of the enablement event would toggle the eligibility of the uncontrollable event.

${ }^{3}$ Again, this is a matter of modeling. We can always model our forcing signal as the controllable event, and then model the event corresponding to the actual action as an uncontrollable event that must occur before the next clock tick, once the forcing event has occurred.
} 
requires our supervisor to specify exactly which clock period the event should occur in and this makes it very straight forward to translate to a controller. Currently, a supervisor could say something like controllable event $\alpha$ is now enabled, and will stay enabled for the next three clock cycles, but must occur before the fourth. You could potentially force it sooner, but that might cause blocking. Such an ambiguous supervisor will be a lot harder to translate to an SD controller.

In this thesis, we will develop a new property for TDES systems that will address the above issues, as well as make our TDES supervisor more consistent with SD controllers, making them easy to translate. First, we will provide the preliminaries of untimed and timed DES in Chapter 2, which is required to understand the following chapters.

Then in Chapter 3 we will introduce the sampled-data setting based on timed DES. The sampled-data setting will be formally defined, and we will develop a new property called SD controllability to address the issues we identified above.

In Chapter 4, we will provide the definition of Moore FSM [17] and a method to translate a CS deterministic supervisor (defined in Chapter 3) into a Moore FSM controller. We will present both a centralized translation method and a modular method. We will then show that they will both produce equivalent output information.

Then in Chapter 5 we capture the enablement and forcing action of a translated controller in the form of a TDES supervisory control map, and show that the closed loop behavior of this map and the plant is the same as that of the plant and the original TDES supervisor. We also show that our method is robust with respect to nonblocking and certain variations in the actual behavior of our physical system.

In Chapter 6 we will introduce logic predicates and predicate transformers, as well as symbolic representation and computation based on [26]. Then we will introduce a set of algorithms to verify SD controllability and other properties of interest to us.

Then in Chapter 7 we will present examples which fail the key conditions in this thesis, to help understand the definitions. We will then present a successful application example inspired by the untimed Flexible Manufacturing System from [11], including the Moore FSM controllers translated from the supervisors developed in the example.

We will close the thesis with our conclusions and a brief discussion of future work. Also, in the appendix we will present the input files used for the FSM example 
given in Chapter 7, as well as the source code for our software tool that we have developed that implements the algorithms presented in Chapter 6. The software tool makes use of binary decision diagrams (BDD) 8 .

\subsection{Related Work}

Supervisory control of DES with timing information, known as timed DES (TDES), was firstly introduced in [5], 6], based on the timed transition model from [19], [20], and [21]. The theory added timing information to supervisory control allowing one to specify lower and upper time bounds for events. It also introduced a forcing technology to ensure certain events occur when we desired. We will use this as the basis of our SD supervisory control theory.

Balemi [2] pointed out that typically, controllable events are part of the supervisor implementation, and often can occur whenever we want them to. For simplicity, the plant may be modeled such that these events are assumed to only occur at certain times. Balemi's plant completeness condition helps ensures that the implementation of the supervisor will be consistent with the plant model so that controllable events do not occur when the plant model says that they cannot.

In the sampled-data setting, if the same event occurs once or multiple times in the same sampling period, an SD controller will not be able to detect a difference. In [3], the authors require that the system has the property that an event cannot be generated more than once during a sampling period. The paper also discussed the loss of ordering information when events occur in the same sampling period. To handle these timing related issues, the author adds a dispatcher to the existing supervisor to solve the problems that could occur when event ordering cannot be ignored. The model is implemented based on Petri Nets [16, 33] and an algorithm to translate the Petri Net implementation into computer language is provided.

Translating abstract model into a computer understandable form is an interesting topic for researchers. In [12], Leduc discusses the modeling and implementation of real-life DES problems as well. Theorems for model reduction were created and applied to the DES designed for a programmable logic controller (PLC) based manufacturing testbed. The author investigated implementing DES as Moore finite state machines (FSM) and created an implementation by hand for the testbed. As men- 
tioned earlier, FSM can be converted to other forms of state based logic sequences, such as a relay ladder logic program for the testbed. The idea of implementing SD controllers as FSM is motivated by this thesis.

Similarly, [18 also discusses translating DES into PLC programs. The difference is that they first convert automata into the Grafcet language, which describes the specification of logic controllers. They then translate the Grafcet language into a PLC program. Both [12] and [18] uses automated manufacturing testbeds as examples.

In [9], DES theory is used as a tool to assist programming in the system control area. The authors describe an approach to generate Java code for concurrency control automatically. The approach formalizes each individual code portion without concurrency control into specifications, builds the DES model, and then generates the code with verifications.

A real world application of DES supervisory control is given in [10], where Petri Nets are used to model railway networks and ensure controllability and liveness.

An important tool to allow supervisory control methods to be applied to larger systems, is the use of binary decision diagrams (BDD) [8]. BDD methods have been applied to standard DES [32], [27], state tree structures [14], Hierarchical Interfacebased Supervisory Control [26], and state based control of TDES [24].

When synthesizing controllers there is often a need to consider other components in the system, which lower the flexibility and increase the cost of synthesis in changing environments. With the I/O based hierarchical structure from [22], each controller can be designed independently, and controllability and nonblocking is retained when the controllers are combined.

However, even if the DES supervisor is nonblocking for the DES plant does not mean that the controller implementation is nonblocking as well. To ensure a controller is nonblocking, [15] studied several different systems for implementing controllers. The author suggested conditions to be satisfied for the implemented controllers to be nonblocking.

Another practical issue in implementing controllers based on DES is communication. In [25], the authors study the communication between modular and decentralized supervisors on switch networks. A communication model is then introduced for a large distributed controller network where communication delay and collisions are a concern. In [31], the authors resolve communication issues by introducing an asyn- 


\section{Introduction}

chronous implementation. The work formalizes the delay between the controller and the plant, and defines bounded-delay implementability, in addition to the standard controllability and nonblocking properties. 
1. Introduction 


\section{Chapter 2}

\section{Discrete-Event Systems Preliminaries}

Supervisory control theory provides a framework for the control of discrete-event systems (DES), systems that are discrete in space and time. For a detailed exposition of DES, see [29]. Below, we present a summary of the terminology that we use in this thesis.

\subsection{Algebraic Preliminaries}

\subsubsection{Strings}

An alphabet $\Sigma$ is defined to be a finite set of distinct symbols. A string over $\Sigma$ is a finite sequence of symbols $\sigma_{1} \sigma_{2} . . \sigma_{k}$, where $\sigma_{i} \in \Sigma$ for $i=1,2, . ., k$. Given a string $s=\sigma_{1} \sigma_{2} . . \sigma_{k},|s|=k$ is the length of the string. The string $\epsilon$ is called the empty string with $|\epsilon|=0$. Let $\Sigma^{*}$ be the set of all finite symbol sequences and define $\Sigma^{+}$be

$$
\Sigma^{+}:=\Sigma^{*}-\{\epsilon\}
$$

Definition 2.1.1. Let $s_{1}, s_{2} \in \Sigma^{*}$, where $s_{1}=\sigma_{1} \sigma_{2} . . \sigma_{m}$ and $s_{2}=\tau_{1} \tau_{2} . . \tau_{n}$. The catenation of $s_{1}$ and $s_{2}$ is define to be cat : $\Sigma^{*} \times \Sigma^{*} \rightarrow \Sigma^{*}$ such that

$$
\begin{gathered}
\operatorname{cat}\left(s_{1}, \epsilon\right)=\operatorname{cat}\left(\epsilon, s_{1}\right)=s_{1}=\sigma_{1} \sigma_{2} . . \sigma_{m} \\
\operatorname{cat}\left(s_{1}, s_{2}\right)=s_{1} s_{2}=\sigma_{1} \sigma_{2} . . \sigma_{m} \tau_{1} \tau_{2} . . \tau_{n}
\end{gathered}
$$


As $\left|s_{1}\right|=m$ and $\left|s_{2}\right|=n$, the length of concatenated string is $\left|s_{1} s_{2}\right|=\left|s_{1}\right|+\left|s_{2}\right|=$ $m+n$.

Definition 2.1.2. Let $s, t \in \Sigma^{*}$. We say $\mathrm{s}$ is a prefix of $\mathrm{t}$, denoted as $s \leq t$, if

$$
\left(\exists u \in \Sigma^{*}\right) s u=t
$$

By definition, we can see that a string $s \in \Sigma^{*}$ is a prefix of itself, as $s \leq s$. Also, $\epsilon$ is a prefix of all strings, as $\left(\forall s \in \Sigma^{*}\right) \epsilon \leq s$.

\subsubsection{Languages}

Definition 2.1.3. Let $L \subseteq \Sigma^{*}$. The prefix closure of $L$, denoted as $\bar{L}$, is defined as

$$
\bar{L}=\left\{s \in \Sigma^{*} \mid(\exists t \in L) s \leq t\right\}
$$

By definition, we can see that a language $L$ is a subset of the prefix closure of itself, i.e. $L \subseteq \bar{L}$. We say a language $L \subseteq \Sigma^{*}$ is prefix closed if $L=\bar{L}$. Let $K \subseteq L$. We say $K$ is $L$-closed if $K=\bar{K} \cap L$.

Definition 2.1.4. Let $L \subseteq \Sigma^{*}$. The eligibility operator, $\operatorname{Elig}_{L}: \Sigma^{*} \rightarrow \operatorname{Pwr}(\Sigma)$, is defined for $s \in \Sigma^{*}$ as,

$$
\operatorname{Elig}_{L}(s):=\{\sigma \in \Sigma \mid s \sigma \in L\}
$$

\subsubsection{Nerode Equivalence Relation}

Definition 2.1.5. Let $X$ be a nonempty set. Let $E \subseteq X \times X$ be a binary relation on $X$. The relation $E$ is an equivalence relation on $X$ if

1. $(\forall x \in X) x E x$ (reflexivity)

2. $\left(\forall x, x^{\prime} \in X\right) x E x^{\prime} \Longrightarrow x^{\prime} E x$ (symmetry)

3. $\left(\forall x, x^{\prime}, x^{\prime \prime} \in X\right) x E x^{\prime} \& x^{\prime} E x^{\prime \prime} \Longrightarrow x E x^{\prime \prime}$ (transitivity)

\footnotetext{
${ }^{1}$ We use ' $\&$ ' to stand for logical AND here to avoid confusion with later definitions in this section.
} 
Here we are using standard infix notation, where we use $x E x^{\prime}$ to represent the ordered pair $\left(x, x^{\prime}\right) \in E$. For $x E x^{\prime}$, we may also write $x \equiv x^{\prime}(\bmod E)$.

For $x \in X$, let $[x]_{E} \subseteq X$ represent the subset of elements that are equivalent mod $E$ to $x$. That is

$$
[x]_{E}:=\left\{x^{\prime} \in X \mid x^{\prime} E x\right\}
$$

If relation $E$ is understood by the context, we will just write $[x]$. We will also refer to $[x]$ as the coset or the equivalence class of $x$ with respect to $E$.

Let $s, t \in \Sigma^{*}$, and $L \subseteq \Sigma^{*}$. We say $s$ and $t$ are Nerode equivalent with respect to language $L$, if and only if they can be extended by any string $u \in \Sigma^{*}$ such that the two extended strings are either both in $L$ or neither in $L$. In this case, we write $s \equiv t$ $(\bmod L)$ or $s \equiv_{L} t$. The formal definition is given below.

Definition 2.1.6. Let $L \subseteq \Sigma^{*}$. Let $s, t \in \Sigma^{*}$.

$$
s \equiv{ }_{L} t \text { or } s \equiv t(\bmod L)
$$

iff

$$
\left(\forall u \in \Sigma^{*}\right) s u \in L \quad \Longleftrightarrow t u \in L
$$

Essentially, if strings $s$ and $t$ are equivalent mod $L$, then they can both be extended in the same way by right concatenation.

Example 2.1. Let $\Sigma=\{\alpha, \beta, \gamma\}, L=\left\{\epsilon, \alpha, \beta, \alpha \gamma^{*}, \beta \gamma^{*}\right\}$, then $\alpha \equiv_{L} \beta$.

\subsection{Discrete Event Systems}

\subsubsection{Generator}

We model DES formally as a generator $\mathbf{G}$, which is a five tuple

$$
\mathbf{G}=\left(Q, \Sigma, \delta, q_{0}, Q_{m}\right)
$$

where

$Q$ is the state set. 
$\Sigma$ is the finite set of distinct symbols representing event labels. We partition $\Sigma$ into two parts

$$
\Sigma=\Sigma_{c} \dot{\cup} \Sigma_{u}
$$

where

$\Sigma_{c}$ is the set of controllable events, which can be enabled or disabled by an external agent. A controllable event can only occur when it is enabled.

$\Sigma_{u}$ is the set of uncontrollable events, which cannot be disabled by any external agent. Once the DES has reached a state where an uncontrollable event can occur, the event cannot be prevented.

$\delta: Q \times \Sigma \rightarrow Q$ is the (partial) transition function where each transition is a tuple $\left(q, \sigma, q^{\prime}\right)$, where $\delta(q, \sigma)=q^{\prime}$. We refer to $q$ as the exit (source) state, and $q^{\prime}$ as the entrance (destination) state. We write $\delta(q, \sigma)$ ! if $\delta(q, \sigma)$ is defined.

We can extend the transition function to $\delta: Q \times \Sigma^{*} \rightarrow Q$ as

$$
\begin{aligned}
& \delta(q, \epsilon)=q \quad \text { for } q \in Q . \\
& \delta(q, s \sigma)=\delta(\delta(q, s), \sigma) \quad \text { for } s \in \Sigma^{*}, \sigma \in \Sigma, \text { and } q \in Q .
\end{aligned}
$$

as long as $q^{\prime}=\delta(q, s)$ ! and $\delta\left(q^{\prime}, \sigma\right) !$.

$q_{0} \in Q$ is the initial state.

$Q_{m} \subseteq Q$ is the subset of marked states.

We can extend the transition function to $\delta: Q \times \Sigma^{*} \rightarrow Q$ as

$$
\begin{aligned}
& \delta(q, \epsilon)=q \quad \text { for } q \in Q . \\
& \delta(q, s \sigma)=\delta(\delta(q, s), \sigma) \text { for } s \in \Sigma^{*}, \sigma \in \Sigma, \text { and } q \in Q .
\end{aligned}
$$

as long as $q^{\prime}=\delta(q, s)$ ! and $\delta\left(q^{\prime}, \sigma\right) !$

Example 2.2. Let $\mathbf{G}=\left(Q, \Sigma, \delta, q_{0}, Q_{m}\right)$ be the DES shown in Figure 2.1. By convention, a controllable event is graphically represented by a slash across its transition 
arrow. Marked states are represented by a black dot. The state pointed at by an arrow with no exit state, is the initial state. For the DES shown we have:

$$
\begin{aligned}
& Q=\{I, W, D\} ; \\
& \Sigma=\Sigma_{c} \dot{\cup} \Sigma_{u}, \text { where } \Sigma_{c}=\{\alpha, \mu\} \text { and } \Sigma_{u}=\{\beta, \lambda\} ; \\
& \delta=\{(I, \alpha, W),(W, \beta, I),(W, \lambda, D),(D, \mu, I)\} ; \\
& q_{0}=I ; Q_{m}=\{I\}
\end{aligned}
$$

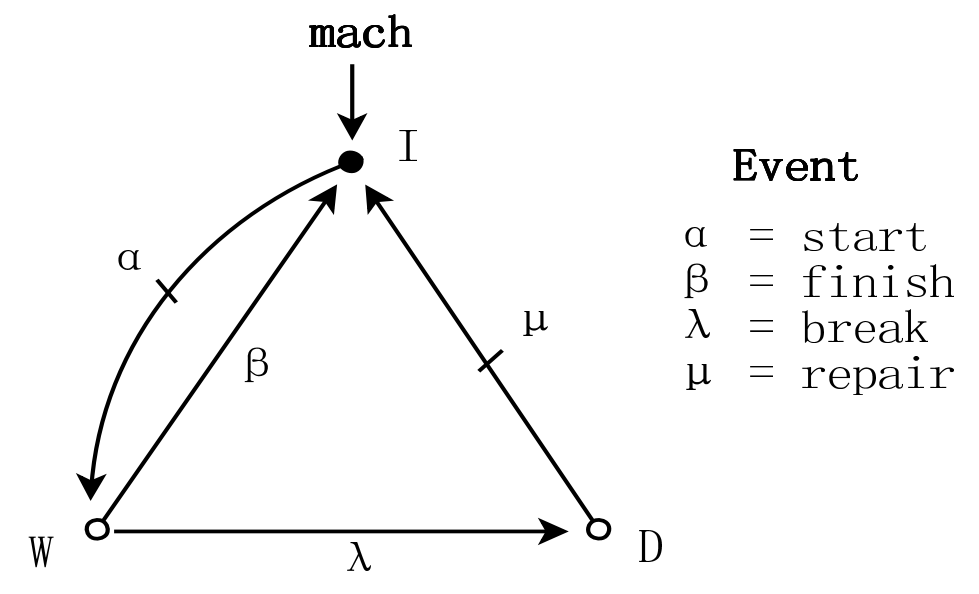

Figure 2.1: An Example DES

Given DES $\mathbf{G}=\left(Q, \Sigma, \delta, q_{0}, Q_{m}\right)$, we have the following definitions.

Definition 2.2.1. A state $q \in Q$ is reachable if

$$
\left(\exists s \in \Sigma^{*}\right) \delta\left(q_{0}, s\right) ! \text { and } q=\delta\left(q_{0}, s\right)
$$

Definition 2.2.2. A state $q \in Q$ is coreachable if

$$
\left(\exists s \in \Sigma^{*}\right) \delta(q, s) ! \text { and } \delta(q, s) \in Q_{m}
$$

To simplify the following discussions, we will always assume a given DES is reachable unless explicitly stated otherwise.

Definition 2.2.3. The closed behavior of DES G is

$$
L(\mathbf{G})=\left\{s \in \Sigma^{*} \mid \delta\left(q_{0}, s\right) !\right\}
$$


Definition 2.2.4. The marked behavior of DES G is

$$
L_{m}(\mathbf{G})=\left\{s \in \Sigma^{*} \mid \delta\left(q_{0}, s\right) ! \& \delta\left(q_{0}, s\right) \in Q_{m}\right\}
$$

Clearly, $L_{m}(\mathbf{G}) \subseteq L(\mathbf{G})$.

Definition 2.2.5. The control action for some $q \in Q$ for DES $\mathbf{G}$ is defined to be a mapping $\zeta: Q \rightarrow \operatorname{Pwr}\left(\Sigma_{c}\right)$ that takes $q$ and returns a set of controllable events enabled at $q$.

Definition 2.2.6. DES $\mathbf{G}$ is said to be nonblocking if every reachable state is also coreachable. This can be expressed as

$$
L(\mathbf{G})=\overline{L_{m}(\mathbf{G})}
$$

Definition 2.2.7. Let $\mathbf{G}=\left(Q, \Sigma, \delta, q_{0}, Q_{m}\right)$ and let $\lambda$ be an equivalence relation on $Q$ such that for $q, q^{\prime} \in Q, q \equiv q^{\prime} \bmod \lambda$ if and only if

1. $\left(\forall s \in \Sigma^{*}\right) \delta(q, s) ! \Longleftrightarrow \delta\left(q^{\prime}, s\right)$ !

2. $\left(\forall s \in \Sigma^{*}\right)\left[\delta(q, s) ! \& \delta(q, s) \in Q_{m}\right] \Longleftrightarrow\left[\delta\left(q^{\prime}, s\right) ! \& \delta\left(q^{\prime}, s\right) \in Q_{m}\right]$

Basically, for states $q$ and $q^{\prime}$ such that $q \equiv q^{\prime} \bmod \lambda$, they have the same future with respect to $L(\mathbf{G})$ and $L_{m}(\mathbf{G})$. Based on this, for string $s \in L(\mathbf{G})$, a state $q=\delta\left(q_{o}, s\right)$ represents all strings in $\Sigma^{*}$ that are equivalent to $s \bmod L(\mathbf{G})$ and $\bmod L_{m}(\mathbf{G})$.

Definition 2.2.8. DES $\mathbf{G}$ is said to be minimal, if

$$
\left(\forall q, q^{\prime} \in Q\right) q \equiv q^{\prime}(\bmod \lambda) \Longleftrightarrow q=q^{\prime}
$$

It says that for all states $q, q^{\prime} \in Q$, if $q$ is equivalent to $q^{\prime} \bmod \lambda$, then $q$ and $q^{\prime}$ are the same state. DES $\mathbf{G}$ is minimal if it does not have two distinct states in $Q$ that are $\lambda$ equivalent. 


\subsubsection{Synchronization and Product DES}

In real world, it is often easier to model a system as several smaller components. For a DES plant, we use the synchronous product operator to combine the individual DES components instead of modeling the whole system at once. We first need to define the natural projection operator and its inverse.

Let $\mathbf{G}=\left(Q, \Sigma, \delta, q_{0}, Q_{m}\right)$ be a DES. Take $\Sigma_{o} \subseteq \Sigma$ to be the set of observable events through some filtering channel of the events generated by $\mathbf{G}$.

Definition 2.2.9. The natural projection $P: \Sigma^{*} \rightarrow \Sigma_{o}^{*}$ is defined as follows. For $s \in \Sigma^{*}, \sigma \in \Sigma$,

$$
\begin{gathered}
P(\epsilon)=\epsilon \\
P(\sigma)= \begin{cases}\epsilon & \text { if } \sigma \notin \Sigma_{o} \\
\sigma & \text { if } \sigma \in \Sigma_{o}\end{cases} \\
P(s \sigma)=P(s) P(\sigma)
\end{gathered}
$$

Example 2.3. For $\Sigma=\{\alpha, \beta, \gamma\}, \Sigma_{o}=\{\alpha, \beta\}$ and $s=\alpha \beta \alpha \gamma \beta \alpha$,

$$
P(s)=P(\alpha) P(\beta) P(\alpha) P(\gamma) P(\beta) P(\alpha)=\alpha \beta \alpha \beta \alpha
$$

Let $L \subseteq \Sigma^{*}$. We define $P(L) \subseteq \Sigma_{o}^{*}$ as an extension of the natural projection as

$$
P(L):=\{P(s) \mid s \in L\}
$$

We also define its inverse image $P^{-1}: \operatorname{Pwr}\left(\Sigma_{o}^{*}\right) \rightarrow \operatorname{Pwr}\left(\Sigma^{*}\right)$ such that, for $H \subseteq \Sigma_{o}^{*}$

$$
P^{-1}(H):=\left\{s \in \Sigma^{*} \mid P(s) \in H\right\}
$$

Example 2.4. For $\Sigma=\{\alpha, \beta, \gamma, \mu\}, \Sigma_{o}=\{\alpha, \beta\}$ and $s_{o}=\alpha \beta \alpha \beta \alpha$, the inverse projection is

$$
P^{-1}\left(\left\{s_{o}\right\}\right):=\{\gamma, \mu\}^{*} \alpha\{\gamma, \mu\}^{*} \beta\{\gamma, \mu\}^{*} \alpha\{\gamma, \mu\}^{*} \beta\{\gamma, \mu\}^{*} \alpha\{\gamma, \mu\}^{*}
$$


Definition 2.2.10. For $i=1,2$, let $L_{i} \subseteq \Sigma_{i}^{*}, \Sigma=\Sigma_{1} \cup \Sigma_{2}$ and $P_{i}: \Sigma^{*} \rightarrow \Sigma_{i}^{*}$ be natural projections. The synchronous product of $L_{1}$ and $L_{2}$ is defined to be

$$
\begin{aligned}
L_{1} \| L_{2} & =P_{1}^{-1}\left(L_{1}\right) \cap P_{2}^{-1}\left(L_{2}\right) \\
& =\left\{s \in \Sigma^{*} \mid P_{1}(s) \in L_{1} \& P_{2}(s) \in L_{2}\right\}
\end{aligned}
$$

Definition 2.2.11. Let $\mathbf{G}_{1}=\left(Q_{1}, \Sigma, \delta_{1}, q_{o, 1}, Q_{m, 1}\right)$ and $\mathbf{G}_{2}=\left(Q_{2}, \Sigma, \delta_{2}, q_{o, 2}, Q_{m, 2}\right)$ be two DES defined over the same event set $\Sigma$. The product of two DES is defined as

$$
\mathbf{G}_{1} \times \mathbf{G}_{2}=\left(Q_{1} \times Q_{2}, \Sigma, \delta_{1} \times \delta_{2},\left(q_{o, 1}, q_{o, 2}\right), Q_{m, 1} \times Q_{m, 2}\right)
$$

where $\delta_{1} \times \delta_{2}: Q_{1} \times Q_{2} \times \Sigma \rightarrow Q_{1} \times Q_{2}$ is defined as

$$
\left(\delta_{1} \times \delta_{2}\right)\left(\left(q_{1}, q_{2}\right), \sigma\right):=\left(\delta_{1}\left(q_{1}, \sigma\right), \delta_{2}\left(q_{2}, \sigma\right)\right)
$$

whenever $\delta_{1}\left(q_{1}, \sigma\right)$ ! and $\delta_{2}\left(q_{2}, \sigma\right)$ !.

By Definition 2.2.11, we have $L\left(\mathbf{G}_{1} \times \mathbf{G}_{2}\right)=L\left(\mathbf{G}_{1}\right) \cap L\left(\mathbf{G}_{2}\right)$ and $L_{m}\left(\mathbf{G}_{1} \times \mathbf{G}_{2}\right)=$ $L_{m}\left(\mathbf{G}_{1}\right) \cap L_{m}\left(\mathbf{G}_{2}\right)$

Definition 2.2.12. The meet of $\mathbf{G}_{1}$ and $\mathbf{G}_{2}$, or meet $\left(\mathbf{G}_{1}, \mathbf{G}_{2}\right)$, is defined to be the reachable subautomaton of the product DES $\mathbf{G}_{1} \times \mathbf{G}_{2}$.

Definition 2.2.13. The synchronous product of DES $\mathbf{G}_{i}=\left(Q_{i}, \Sigma_{i}, \delta_{i}, q_{o_{i}}, Q_{m_{i}}\right)(i=$ $1,2)$, denoted $\mathbf{G}_{1} \| \mathbf{G}_{2}$, is defined to be a reachable DES $\mathbf{G}$ with event set $\Sigma=\Sigma_{1} \cup \Sigma_{2}$ and properties:

$$
L_{m}(\mathbf{G})=L_{m}\left(\mathbf{G}_{1}\right)\left\|L_{m}\left(\mathbf{G}_{2}\right), \quad L(\mathbf{G})=L\left(\mathbf{G}_{1}\right)\right\| L\left(\mathbf{G}_{2}\right)
$$

Definition 2.2.14. Let $\mathbf{G}$ be a DES defined over $\Sigma$ and $\Sigma^{\prime}$ be another set of events such that $\Sigma \cap \Sigma^{\prime}=\emptyset$. The selfloop operation on $\mathbf{G}$ is defined as

$$
\operatorname{selfloop}\left(\mathbf{G}, \Sigma^{\prime}\right)=\left(Q, \Sigma \cup \Sigma^{\prime}, \delta^{\prime}, q_{o}, Q_{m}\right)
$$

where $\delta^{\prime}: Q \times\left(\Sigma \cup \Sigma^{\prime}\right) \rightarrow Q$ is a partial function defined as

$$
\delta^{\prime}(q, \sigma):=\left\{\begin{array}{cc}
\delta(q, \sigma) & \sigma \in \Sigma, \delta(q, \sigma) ! \\
q & \sigma \in \Sigma^{\prime} \\
\text { undefined } & \text { otherwise }
\end{array}\right.
$$


For DES $\mathbf{G}_{i}^{\prime}(i=1,2)$ defined over event set $\Sigma_{i}$, we will always assume that the synchronous product operator is implemented by first extending each DES to be over $\Sigma$ by adding selfloops, and then using the meet operator. More formally, we take $\Sigma=\Sigma_{1} \cup \Sigma_{2}$, and $\mathbf{G}_{i}=\operatorname{selfloop}\left(\mathbf{G}_{i}^{\prime}, \Sigma-\Sigma_{i}\right)$. We then have $G_{1}^{\prime} \| G_{2}^{\prime}=\operatorname{meet}\left(\mathbf{G}_{1}, \mathbf{G}_{2}\right)$.

In the algorithms we develop in this thesis, we will always assume all DES are combined with the product DES operator. If a portion of the system is actually combined together using the synchronous product operator as is commonly done for plant components, we will first add selfloops as above, and then use these new DES from then on in our algorithms.

\subsubsection{Controllability and Supervision}

We will take language $K$ to represent the desired safe behavior of our plant represented by $\mathrm{DES} \mathbf{G}=\left(Q, \Sigma, \delta, q_{0}, Q_{m}\right)$. We want to make sure that the closed loop behavior of the system - that is the behavior of plant $\mathbf{G}$ under control of $K$ - is a subset of $\bar{K}$.

As we mentioned earlier, our system's event set $\Sigma$ is partitioned into controllable and uncontrollable events. If an undesirable controllable event is possible in $\mathbf{G}$ that will cause the system to leave the behavior represented by $\bar{K}$, we disable it and prevent it from occurring. We cannot do this with an uncontrollable event, so we need to make sure the plant never reaches a state where it can leave the desired behavior by an uncontrollable event. We now express this formally below.

Definition 2.2.15. $K$ is said to be controllable with respect to $\mathbf{G}$ if

$$
(\forall s \in \bar{K})\left(\forall \sigma \in \Sigma_{u}\right) s \sigma \in L(\mathbf{G}) \Longrightarrow s \sigma \in \bar{K}
$$

We typically give this definition in the form of $\bar{K} \Sigma_{u} \cap L(\mathbf{G}) \subseteq \bar{K}$ where $\bar{K} \Sigma_{u}$ denotes the string $s \sigma$ for $s \in \bar{K}$ and $\sigma \in \Sigma_{u}$. In other words, if the plant reaches a state where uncontrollable event $\sigma$ is possible, then $\sigma$ must also be accepted by $\bar{K}$. By definition, $\emptyset, L(\mathbf{G})$ and $\Sigma^{*}$ are all controllable with respect to $\mathbf{G}$.

Another way to express this definition is

$$
(\forall s \in \bar{K} \cap L(\mathbf{G})) \operatorname{Elig}_{L(\mathbf{G})}(s) \cap \Sigma_{u} \subseteq \operatorname{Elig}_{\bar{K}}(s)
$$

which is used in Point $\mathbf{i}$ of Definition 3.2.1 in Section 3.2, 
As we prefer to work with finite state automata than typically infinite languages, we want to be able to express $K$ as a DES supervisor.

Definition 2.2.16. Let $\mathbf{G}=\left(Q, \Sigma, \delta, q_{0}, Q_{m}\right)$ be a DES. Let $K \subseteq \Sigma^{*}$. We say $\mathbf{G}$ represents $K$ if

$$
K=L_{m}(\mathbf{G}) \text { and } \bar{K}=L(\mathbf{G})
$$

Definition 2.2.17. Let $\mathbf{S}=\left(X, \Sigma, \xi, x_{o}, X_{m}\right)$ be a DES. Let $K \subseteq \Sigma^{*}$, we say $\mathbf{S}$ implements $K$, if

$$
K=L_{m}(\mathbf{S}) \cap L_{m}(\mathbf{G}) \text { and } \bar{K}=L(\mathbf{S}) \cap L(\mathbf{G})
$$

Recall that $\Sigma=\Sigma_{c} \dot{\cup} \Sigma_{u}$, where $\Sigma_{c}$ is a set of controllable events which can be enabled or disabled by external agents; and $\Sigma_{u}$ is a set of uncontrollable events which cannot be disabled. We refer to such an external agent as a supervisor, which will formally define shortly.

Definition 2.2.18. Let $L(\mathbf{S})$ be the language represented by DES $\mathbf{S}$. We say $\mathbf{S}$ is a supervisor for $\mathbf{G}$, if

1. $L(\mathbf{S})$ is controllable with respect to $\mathbf{G}$, and

2. $\overline{L_{m}(\mathbf{S}) \cap L_{m}(\mathbf{G})}=L(\mathbf{S}) \cap L(\mathbf{G})$

For convenience, we say $\mathbf{S}$ is controllable for $\mathbf{G}$ if $L(\mathbf{S})$ is controllable with respect to $\mathbf{G}$.

We can think of a supervisor $\mathbf{S}=\left(X, \Sigma, \xi, x_{o}, X_{m}\right)$ as a state machine that tracks all the events generated by plant $\mathbf{G}$. Together with current state $x \in X$ as source state, it takes each event as an input to its transition function $\xi$, then moves to the destination state $x^{\prime} \in X$. Events in $\mathbf{G}$ are only allowed to occur when the event is not disabled in $\mathbf{S}$. We refer to the closed loop behavior of the system as the behavior of our plant $\mathbf{G}$ under the control of supervisor $\mathbf{S}$. This is typically represented as the meet of $\mathbf{G}$ and $\mathbf{S}$. If we modeled the system only using the synchronous product, then this would be represented as $\mathbf{G} \| \mathbf{S}$. 
As noted by Balemi in [2], controllable events tend to be events fully under the control of our supervisor's implementation. They may be a software function we call, an output we set to true, or a message we send. That means that we can make these events occur whenever we want. It is not unusual that a plant might be modeled such that these events are suppose to only occur under certain situations. This might be for flexibility (some implementations have these restrictions, for example) or to make the system easier to model or understand. However, the reality for some supervisor implementations is that these events could occur even when the plant said they cannot. We refer to such situations as illegal transitions. The requirement is formally defined in [2] as follows.

Definition 2.2.19. A plant $\mathbf{G}$ is complete for its supervisor $\mathbf{S}$ if

$$
(\forall s \in L(\mathbf{G}) \cap L(\mathbf{S}))\left(\forall \sigma \in \Sigma_{c}\right) s \sigma \in L(\mathbf{S}) \Longrightarrow s \sigma \in L(\mathbf{G})
$$

The definition states that, at each state in plant $\mathbf{G}$, every controllable event enabled by supervisor $\mathbf{S}$ must be accepted by $\mathbf{G}$ as well. This condition can be seen as a dual to the definition of a supervisor $\mathbf{S}$ being controllable for plant $\mathbf{G}$. This definition will be very useful for implementing DES supervisors, as it says that they do not require additional supplementary information from the plant to decide when a controllable event can occur and not violate the plant model.

\subsection{Timed Discrete Event Systems}

So far we have only discussed untimed DES. As we wish to use a richer modeling framework that includes timing requirements of our system, we will now discuss Timed DES (TDES) introduced by Brandin et al [5] [6].

TDES extends untimed DES theory by adding a new tick event, corresponding to the tick of a global clock. The event set of a TDES contains the tick event as well as other non-tick events called activity events $\left(\Sigma_{a c t}\right)$. The occurrence of a tick event provides us with a concept of time passing, allowing us to model upper and lower time bounds for the occurrence of activity events. A lower time bound for a given activity event can be modeled as requiring a certain number of tick events to first occur before the activity event is eligible. Once an activity event is eligible to occur 
in the TDES and the desired number of tick events have occurred, we can model an upper bound for the event by not allowing a tick event to occur until either the event has occurred, or another activity event has occur such that the first event is no longer eligible.

The addition of a tick event also allows us to introduce a new type of events called forcible events $\left(\Sigma_{\text {for }}\right)$, which we guarantee to occur and preempt the next clock tick. This means that now we cannot only prevent some events (referred to as prohibitable events $\left(\Sigma_{h i b}\right)$ in TDES terminology) from occurring by disabling them, but we can also choose to have certain events occur before the next clock tick. As a convention, we sometimes refer to tick as $\tau$ for brevity.

\subsubsection{Basic Structure}

We formally define a TDES as the tuple

$$
\mathbf{G}=\left(Q, \Sigma, \delta, q_{0}, Q_{m}\right)
$$

where,

$Q$ is the state set

$\Sigma=\Sigma_{\text {act }} \dot{\cup}\{\tau\}$ is the set of all events, including activity events and the tick event.

$\delta: Q \times \Sigma \rightarrow Q$ is the (partial) transition function.

$q_{0} \in Q$ is the initial state.

$Q_{m} \subseteq Q$ is the set of marked states.

For convenience, we extend $\delta$ to function $\delta: Q \times \Sigma^{*} \rightarrow Q$ in the same way as we did in the untimed DES definition.

\subsubsection{Controllability and Supervision}

Control action for timed DES is achieved in an analogous fashion as that of untimed DES, by disabling controllable events. As for untimed DES, we also partition our event set $\Sigma$ into controllable and uncontrollable events. The set of controllable events is defined to be 


$$
\Sigma_{c}:=\Sigma_{h i b} \cup\{\tau\}
$$

where $\Sigma_{h i b} \subseteq \Sigma_{a c t}$ the set of activity events that can disabled by an external agents. These event are referred to as prohibitable events to distinguish them from controllable events that include the tick event. As we will see when we define controllability in the TDES setting, we will use disabling the tick event by the supervisor to model forcing an event. A forcible event is an event in the system that we can make occur before the next clock tick, assuming it is not first preempted by another event. The set of uncontrollable events for $\mathbf{G}$ is then defined to be

$$
\Sigma_{u}:=\Sigma-\Sigma_{c}
$$

In Section 2.2.3, we introduced Balemi's concept of completeness of a plant for a given supervisor. Unfortunately, that definition was given in terms of controllable events, which includes the tick event in TDES. As we are only concerned about the occurrence of activity events, we need to define a version of this definition for TDES. When discussing this concept, we will not specify whether or not we mean the timed or untimed version, as this will be clear by the context.

Definition 2.3.1. Let TDES $\mathbf{G}$ be a plant and TDES $\mathbf{S}$ be a supervisor. $\mathbf{G}$ is TDES complete for $\mathbf{S}$ if

$$
(\forall s \in L(\mathbf{G}) \cap L(\mathbf{S}))\left(\forall \sigma \in \Sigma_{h i b}\right) s \sigma \in L(\mathbf{S}) \Longrightarrow s \sigma \in L(\mathbf{G})
$$

We now need to add a technical condition that we most enforce to ensure that our TDES does not allow the physically unrealistic situation where a tick event could be preempted indefinitely by the continued execution of an activity event loop within a given fixed unit time. Formally, a TDES is said to have an activity loop if it satisfies the following definition.

Definition 2.3.2. TDES $\mathbf{G}=\left(Q, \Sigma, \delta, q_{0}, Q_{m}\right)$ has an activity loop if

$$
(\exists q \in Q)\left(\exists s \in \Sigma_{\text {act }}^{+}\right) \delta(q, s)=q
$$

We thus require that a TDES be activity loop free (ALF). We can formalize the ALF concept as defined below. 
Definition 2.3.3. TDES $\mathbf{G}=\left(Q, \Sigma, \delta, q_{0}, Q_{m}\right)$ is activity loop free if

$$
\left(\forall q \in Q_{\text {reach }}\right)\left(\forall s \in \Sigma_{\text {act }}^{+}\right) \delta(q, s) \neq q
$$

We only look at states that are reachable (i.e. in $Q_{\text {reach }}$ ), because we do not care about unreachable states as they do not contribute to the automaton's closed and marked behavior. These unreachable activity loops can be safely ignored. An example that fails the ALF property is shown in Figure 2.2 where the $\alpha \beta$ loop could indefinitely preempt the tick event from occurring.

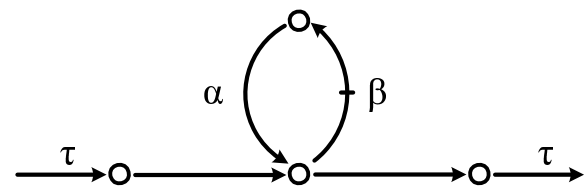

Figure 2.2: An Example Failing ALF Property

We will not require that supervisors be ALF, as they may contain self-loops that are not possible in the plant. We will instead require that the system's closed loop behavior (typically the meet of plant $\mathbf{G}$ and supervisor $\mathbf{S}$ ) be ALF.

For the FSM translation of individual supervisors in Section 4.2, we need a more specific definition as follows.

Definition 2.3.4. Let $\mathbf{G}=\left(Q, \Sigma, \delta, q_{0}, Q_{m}\right)$ be a TDES, and let $\mathbf{G}^{\prime}$ be $\mathbf{G}$ with all activity event selfloops removed. $\mathbf{G}$ is non-selfloop activity loop free if $\mathbf{G}^{\prime}$ is ALF.

Essentially, if we remove the selfloops of any activity events in the TDES, the rest of the TDES must be ALF. This will be a key definition that will allow us to translate the TDES to a Moore finite state machine.

The proposition below states that if individual DES are all ALF, it implies that the synchronous product of these DES is also ALF. This means that we can simply check the individual DES.

Proposition 2.1. For TDES $\mathbf{G}_{1}=\left(Q_{1}, \Sigma_{1}, \delta_{1}, q_{0,1}, Q_{m, 1}\right)$ and $\mathbf{G}_{2}=\left(Q_{2}, \Sigma_{2}, \delta_{2}, q_{0,2}, Q_{m, 2}\right)$, if $\mathbf{G}_{1}$ and $\mathbf{G}_{2}$ are each ALF, then their synchronous product $\mathbf{G}=\mathbf{G}_{1} \| \mathbf{G}_{2}$, is ALF. 
Proof. Let $\mathbf{G}_{1}=\left(Q_{1}, \Sigma_{1}, \delta_{1}, q_{0,1}, Q_{m, 1}\right)$ and $\mathbf{G}_{2}=\left(Q_{2}, \Sigma_{2}, \delta_{2}, q_{0,2}, Q_{m, 2}\right)$ be two TDES and let $P_{1}: \Sigma^{*} \rightarrow \Sigma_{1}^{*}$ and $P_{2}: \Sigma^{*} \rightarrow \Sigma_{2}^{*}$ be natural projections.

Define $\Sigma_{a c t, i}=\Sigma_{a c t} \cap \Sigma_{i}, i=1,2$.

By ALF Definition 2.3.3, for $i=1,2$

$$
\left(\forall q_{i} \in Q_{\text {reach }, i}\right)\left(\forall s_{i} \in \Sigma_{\text {act }, i}^{+}\right) \delta_{i}\left(q_{i}, s_{i}\right) \neq q_{i}
$$

where $Q_{\text {reach, } i}$ is the set of reachable states for $\mathbf{G}_{i}$

Let $\mathbf{G}=\mathbf{G}_{1} \| \mathbf{G}_{2}=\left(Q, \Sigma, \delta, q_{0}, Q_{m}\right)$

Must show

$$
\left(\forall q \in Q_{\text {reach }}\right)\left(\forall s \in \Sigma_{\text {act }}^{+}\right) \delta(q, s) \neq q
$$

We will use proof by contradiction. Assume

$$
\left(\exists q \in Q_{\text {reach }}\right)\left(\exists s^{\prime} \in \Sigma_{\text {act }}^{+}\right) \delta\left(q, s^{\prime}\right)=q
$$

Let $q=\left(q_{1}, q_{2}\right) \in Q_{\text {reach }}$ be this state and let $s^{\prime} \in \Sigma_{\text {act }}^{+}$such that $\delta\left(q, s^{\prime}\right)=q$.

We know that $q$ is a reachable state if and only if $q_{1} \in Q_{1}$ and $q_{2} \in Q_{2}$ are reachable states in $\mathbf{G}_{1}$ and $\mathbf{G}_{2}$, respectively, by Definition of the $\|$ operator. We thus have

$$
\begin{gathered}
\delta\left(q, s^{\prime}\right)=q \Longrightarrow \delta\left(\left(q_{1}, q_{2}\right), s^{\prime}\right)=\left(q_{1}, q_{2}\right) \\
\Longrightarrow \delta\left(\left(q_{1}, q_{2}\right), s^{\prime}\right)=\left(\delta_{1}\left(q_{1}, P_{1}\left(s^{\prime}\right)\right), \delta_{2}\left(q_{2}, P_{2}\left(s^{\prime}\right)\right)\right) \quad \text { by Definition of } \| .
\end{gathered}
$$

This implies

$$
\begin{aligned}
& \delta_{1}\left(q_{1}, P_{1}\left(s^{\prime}\right)\right)=q_{1} \\
& \delta_{2}\left(q_{2}, P_{2}\left(s^{\prime}\right)\right)=q_{2}
\end{aligned}
$$

As $s^{\prime} \in \Sigma_{\text {act }}^{+}$we thus have $s^{\prime} \neq \epsilon$. As $\Sigma=\Sigma_{1} \cup \Sigma_{2}$, it follows that either $P_{1}\left(s^{\prime}\right) \neq \epsilon$ or $P_{2}\left(s^{\prime}\right) \neq \epsilon$ This implies that either $\mathbf{G}_{1}$ or $\mathbf{G}_{2}$ is not ALF, which contradicts $\left(^{*}\right)$.

Therefore it must be that

$$
\left(\forall q \in Q_{\text {reach }}\right)\left(\forall s \in \Sigma_{\text {act }}^{+}\right) \delta(q, s) \neq q
$$


The above proposition can be applied to two TDES combined using the meet operator as meet is a special case of the synchronous product.

We next present a proposition that says that to ensure the synchronous product is ALF, it is sufficient that only one of the two TDES is ALF, as long as the event set of the ALF TDES contains all of the events in the event set of the second TDES. It means that if plant is over $\Sigma$ and the supervisor introduces no new events, then we can just check if the plant is ALF. As indicated by Proposition 2.1, we can check that the plant is ALF by checking if each individual plant component is ALF. Therefore an ALF algorithm does not have to check that the closed loop system is ALF, but can check that the event set of the plant is a superset of the supervisor's event set, then do an ALF check on each individual TDES that makes up the plant. If the check passes, then we are done. Otherwise, we do an ALF check on the entire system.

Proposition 2.2. Let $\mathbf{G}_{1}=\left(Q_{1}, \Sigma_{1}, \delta_{1}, q_{o, 1}, Q_{m, 1}\right)$ and $\mathbf{G}_{2}=\left(Q_{2}, \Sigma_{2}, \delta_{2}, q_{o, 2}, Q_{m, 2}\right)$ be two TDES. If $\mathbf{G}_{1}$ is ALF and $\Sigma_{1} \supseteq \Sigma_{2}$, then $\mathbf{G}_{1} \| \mathbf{G}_{2}$ is also ALF.

Proof. Assume $\mathbf{G}_{1}$ is ALF and $\Sigma_{1} \supseteq \Sigma_{2}$.

Let $\mathbf{G}=\mathbf{G}_{1} \| \mathbf{G}_{2}=\left(Q, \Sigma, \delta, q_{o}, Q_{m}\right)$ with $\Sigma=\Sigma_{1} \cup \Sigma_{2}$ and $P_{i}: \Sigma^{*} \rightarrow \Sigma_{i}^{*}$ for $i=1,2$. Must show $\mathbf{G}$ is ALF.

We will do so by proof of contradiction.

Assume $\mathbf{G}$ is not ALF, then

$$
\left(\exists q \in Q_{\text {reach }}\right)\left(\exists s^{\prime} \in \Sigma^{+} \text {act }\right) \delta\left(q, s^{\prime}\right)=q
$$

Let $q=\left(q_{1}, q_{2}\right) \in Q_{\text {reach }}$, and $s^{\prime} \in \Sigma_{\text {act }}^{+}$such that $\delta\left(q, s^{\prime}\right)=q$.

We first note that $q$ is reachable in $\mathbf{G}$, which implies $q_{1}$ is reachable in $\mathbf{G}_{1}$ and $q_{2}$ is reachable in $\mathbf{G}_{2}$.

We next note that as $\Sigma_{1} \supseteq \Sigma_{2}$, we have $\Sigma=\Sigma_{1} \cup \Sigma_{2}=\Sigma_{1}$. This implies that $P_{1}^{-1} L\left(\mathbf{G}_{1}\right)=L\left(\mathbf{G}_{1}\right)$.

From (2), we have

$$
\begin{array}{ccc} 
& \delta\left(q, s^{\prime}\right)=q \Longrightarrow & \delta\left(\left(q_{1}, q_{2}\right), s^{\prime}\right)=\left(q_{1}, q_{2}\right) \\
\Longrightarrow & \delta_{1}\left(q_{1}, P_{1}\left(s^{\prime}\right)\right)=q_{1} & \\
\Longrightarrow & \delta_{1}\left(q_{1}, s^{\prime}\right)=q_{1} & \text { by }(3)
\end{array}
$$

This contradicts (1) as it implies $\mathbf{G}_{1}$ is not ALF.

We thus conclude that $\mathbf{G}$ must be ALF. 
We are also want to make sure that the plant is not modeled in such a way that our closed loop system could reach a state where no more tick events are possible, as this "stopping the clock" would be physically unrealistic. To help prevent this, we will require that our plant TDES have proper time behavior, as defined by Kai Wong et al. [28].

Definition 2.3.5. TDES $\mathbf{G}$ has a proper time behavior if

$$
(\forall s \in L(\mathbf{G})) \operatorname{Elig}_{L(\mathbf{G})}(s) \cap \Sigma_{u}=\emptyset \Longrightarrow \tau \in \operatorname{Elig}_{L(\mathbf{G})}(s)
$$

This definition can be rewritten as

$$
\left(\forall q \in Q_{\text {reach }}\right)\left(\exists \sigma \in \Sigma_{u} \cup\{\tau\}\right) \delta(q, \sigma) !
$$

In other words, this TDES must guarantee that at all of its reachable states, either a tick event or an uncontrollable event must be possible. This serves two purposes. Combined with TDES G being ALF and having a finite state space, this ensures that we call always reach a state where a tick is possible after at most a finite number of activity events. We prove this shortly in Proposition 2.3. This condition will also ensure we do not stop the clock when we combine our plant with a controllable supervisor. An example that fails the proper time behavior property is shown in Figure 2.3 where after the first tick event, neither an uncontrollable event or a tick are possible, only the prohibitable event $\beta$.

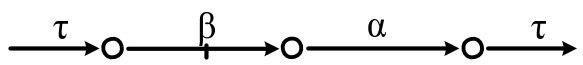

Figure 2.3: An Example Failing the Proper Time Behavior Property

Consider the case where we have a reachable state where tick was ineligible, but only controllable events were possible. If the supervisor disabled these controllable events, there would now be no events possible at all. Proper time behavior ensures that if tick was not possible at this state in the plant, there would be an uncontrollable event possible, even if all the controllable events were disabled. The restriction of proper time behavior applies only to plant TDES. It does not apply to supervisor TDES or the meet of the plant and supervisor (i.e. the closed loop behavior of the system). 
If a TDES G has a finite state space, is activity loop free and has proper time behavior, then we expect that at any reachable state, we can always do a tick event after at most a finite number of activity events. In other words, we will never "stop the clock." The following proposition shows that this is indeed the case.

Proposition 2.3. If a TDES $\mathbf{G}=\left(Q, \Sigma, \delta, q_{0}, Q_{m}\right)$ has a finite statespace, is activity loop free and has proper time behavior, then

$$
\left(\forall q \in Q_{\text {reach }}\right)\left(\exists s \in \Sigma^{*}\right) \delta(q, s \tau) !
$$

where $Q_{\text {reach }}$ is the set of reachable states.

Proof. Assume that $\mathbf{G}$ has a finite statespace, is activity loop free, and has proper time behavior

Let $q \in Q_{\text {reach }}$.

Must show implies $\left(\exists s \in \Sigma^{*}\right) \delta(q, s \tau)$ !

We first note that as $\mathbf{G}$ has a finite statespace and is non-empty, there exists $n \in\{1,2, \ldots\}$ such that $n=|Q|$.

As $\mathbf{G}$ is ALF and has $n$ states, it follows that

$$
\begin{array}{rr}
\left(\exists s \in \Sigma_{\text {act }}^{+}\right)|s| \leq n-1 & \text { and } \\
\left(\exists q^{\prime} \in Q_{\text {reach }}\right) \delta(q, s)=q^{\prime} & \text { and } \\
\left(\forall \sigma \in \Sigma_{\text {act }}\right) \delta\left(q^{\prime}, \sigma\right) y & \text { (1) }
\end{array}
$$

The above follows from the fact that starting at $q$, we can do at most $n-1$ activity event transitions before we have visited all $n$ states. At this point, there must be no activity event transition or we would have to visit a state twice, creating an activity loop and failing the ALF definition.

As $\Sigma_{u} \subseteq \Sigma_{a c t},(1)$ asserts that there are no uncontrollable events at state $q^{\prime}$. It thus follows that $\left.\delta\left(q^{\prime}, \tau\right) !\right)$ as $\mathbf{G}$ has proper time behavior.

We thus have:

$$
\delta(q, s \tau) !
$$

as required. 
We now present the controllability definition for timed DES. Normally, we drop the "TDES" and just say "controllable" as the meaning is clear from the context.

Definition 2.3.6. We define the arbitrary language $K \subseteq L(\mathbf{G})$ to be TDES controllable with respect to $\mathbf{G}$ if,

$$
(\forall s \in \bar{K}) \operatorname{Elig}_{\bar{K}}(s) \supseteq\left\{\begin{array}{cl}
\operatorname{Elig}_{L(\mathbf{G})}(s) \cap\left(\Sigma_{u} \cup\{\tau\}\right) & \text { if } \operatorname{Elig}_{\bar{K}}(s) \cap \Sigma_{\text {for }}=\emptyset \\
\operatorname{Elig}_{L(\mathbf{G})}(s) \cap \Sigma_{u} & \text { if } \operatorname{Elig}_{\bar{K}}(s) \cap \Sigma_{\text {for }} \neq \emptyset
\end{array}\right.
$$

Definition 2.3.6 says that a $\bar{K}$ must accept an uncontrollable event if the event is possible in the plant, and it must accept a tick event if it is possible in the plant, unless there exists an eligible forcible event that can preempt the tick.

Note that the closed and marked behavior of a TDES is defined in the same way as for an untimed DES. A TDES is said to be nonblocking if Definition 2.2.6 is satisfied.

Proposition 2.4. If TDES plant $\mathbf{G}$ and TDES supervisor $\mathbf{S}$ both have finite statespaces, $\mathbf{G}$ has proper time behavior, $\mathbf{G}_{c l}=\operatorname{meet}(\mathbf{G}, \mathbf{S})=\left(Q, \Sigma, \delta, q_{0}, Q_{m}\right)$ is ALF, and $\mathbf{S}$ is controllable for $\mathbf{G}$, then

$$
\left(\forall q \in Q_{\text {reach }}\right)\left(\exists s \in \Sigma^{*}\right) \delta(q, s \tau) !
$$

Proof. Assume:

- $\mathbf{G}$ and $\mathbf{S}$ have finite statespaces

- $\mathrm{G}$ has proper time behavior

- $\mathbf{G}_{c l}$ is ALF

- $\mathbf{S}$ is controllable for $\mathbf{G}$

Let $q \in Q_{\text {reach }}$. Must show $\left(\exists s \in \Sigma^{*}\right) \delta(q, s \tau)$ !

As $\mathbf{G}$ and $\mathbf{S}$ have finite statespaces, it follows from Definition 2.2 .12 of the meet operator, that $\mathbf{G}_{c l}$ has a finite statespace. Let $n=|Q|$. 
As $\mathbf{G}_{c l}$ is ALF and has $n$ states, it follows that

$$
\begin{array}{rr}
\left(\exists s \in \Sigma_{\text {act }}^{+}\right)|s| \leq n-1 & \text { and } \\
\left(\exists q^{\prime} \in Q_{\text {reach }}\right) \delta(q, s)=q^{\prime} & \text { and } \\
\left(\forall \sigma \in \Sigma_{\text {act }}\right) \delta\left(q^{\prime}, \sigma\right) \ngtr & \text { (1) }
\end{array}
$$

The above follows from the fact that starting at $q$, we can do at most $n-1$ activity event transitions before we have visited all $n$ states. At this point, there must be no more activity event transitions or we would have to visit a state twice, creating an activity loop and failing the ALF definition.

We now need to show tick is defined at $q^{\prime}$. From (1), we know that there are no untimed events possible in $\mathbf{G}_{c l}$ at $q^{\prime}$ as $\Sigma_{u} \subseteq \Sigma_{a c t}$. As $\mathbf{S}$ is controllable for $\mathbf{G}$, this implies there are no untimed events possible at the corresponding state in $\mathbf{G}$. As $\mathbf{G}$ has proper time behavior, this implies that $\tau$ is possible at this state in $\mathbf{G}$. As (1) asserts there are no activity event at $q^{\prime}$ and thus no forcible events, $\mathbf{S}$ must accept that tick event as $\mathbf{S}$ is controllable for $\mathbf{G}$.

$$
\begin{array}{ll}
\Longrightarrow & \delta\left(q^{\prime}, \tau\right) ! \\
& \Longrightarrow \quad \delta(q, s \tau) !
\end{array}
$$




\section{Chapter 3}

\section{Sampled-Data Systems}

In this thesis, we will focus on implementing our TDES supervisors as sample-data (SD) controllers. An SD controller is driven by a periodic clock and sees the system as a series of inputs and outputs. On each clock edge, it samples its inputs, changes states, and updates its outputs. For simplicity, we will assume inputs and outputs of an FSM can only take the value of true or false.

When we are using an SD controller to manage a given system, we associate an input with each event, and an output with each controllable event. We consider an event has occurred when its corresponding input has gone true during a given clock period. We consider a controllable event to be enabled when its corresponding output has been set true by the controller, disabled otherwise.

As mentioned above, an SD controller samples the value of its inputs on each clock edge, and uses this value to decide what its next internal state will be. This means the SD controller knows nothing about its inputs until the clock edge, and then all it learns is whether a given input is true or false, signifying that the corresponding event has occurred sometime in the clock period that just ended. This means that for the given clock period, all information about event ordering (which event occurred first etc) is lost, as well as how often a given event occurred if it has occurred more than once. The only ordering information that remains is which sampling period (clock period) a given event occurred in.

Another important aspect of an SD controller is that it only changes state on a clock edge, and the value of its outputs are a function of its current state. That means 
its outputs can only change at a clock edge, and then must stay constant for the rest of the clock period.

In this chapter, we will define the sampled-data setting formally, and develop a new condition to address the issues we identified in Section 1.1 .

We will be making a few assumptions about the systems we work with. They are:

- The set of prohibitable events is exactly equal to the set of forcible events for our system. This is a reasonable assumption that will greatly simplify things. As discussed in the introduction, this is basically a matter of how the system is modeled.

- Our SD controllers will be implemented centrally with a common clock, such that they all sample inputs, and update outputs at the same time. Furthermore, their source of inputs and outputs is common such that their outputs exit to the system at the same place, and their inputs enter from the system at the same place. For their inputs, this means they will always all receive the same results from the sampling inputs. We will never have the case that one controller sees input $\alpha$ go true in a given sampling period, while another does not.

- When a prohibitable event is enabled, we will interpret this to mean we should force the event once in the current clock period. Even if we could cause it to occur twice in one clock period, we will not do that.

- To partially address timing issues, we will assume an event has occurred when its input to the controllers goes true. One exception is if the input goes true so close to a clock edge that it is missed and shows up in the next sampling period. In this case, the event is considered to have occurred at the start of the next sampling period. This should be taken into account in the modeling of the system.

- We are also assuming that when we decide to force an event in a given sampling period, not only will the event physically occur in that sampling period, but it will reach our controller's inputs in time to be detected as occurring in that sampling period, and never in the following one. It is up to the designer and 
user of this theory to make sure that the system they apply it to satisfies these assumptions.

- The input signal should be of an appropriate length so that it will not be missed by the SD controllers (i.e. if its pulse width is shorter than the clock period), nor should it be so long that it is seen at multiple clock edges, unless it is suppose to represent that number of sequential occurrences. For example, if the input is true for two clock edges in a row, it will be considered to have occurred twice, once per clock period. It is the designers responsibility to make sure that the inputs are properly conditioned to ensure this.

\subsection{Sampling Inputs}

To make the TDES theory work with SD controllers, we identify a tick event occurring with the clock edge that the SD controller uses for sampling and state change. This means for a TDES G over event set $\Sigma$, the strings an SD controller can observe from the closed behavior of $\mathbf{G}$ are strings ending with a tick and the empty string, $\epsilon$. We will refer to such strings as sampled strings. The reason the empty string is included is that it represents the initial state of the system, which is usually known. Note also that a non-empty sampled string may contain one or more tick events in addition to the tick event at the end of the string.

Definition 3.1.1. Given a event set $\Sigma$, the set of sampled strings is denoted by $L_{\text {samp }}$ and is define as

$$
L_{\text {samp }}=\Sigma^{*} \cdot \tau \cup\{\epsilon\}
$$

As an SD controller will change from state at each clock edge (tick occurring), the next state of the SD controller will thus be determined by the strings containing a single tick at the end that are possible in the system immediately after the last tick event that brought us to our current state. We will refer to such strings as concurrent strings, defined as below. Essentially, an SD controller starts at its initial, or reset state (corresponding to the empty string), and then transitions from state to state as concurrent strings occur in the corresponding TDES. 
Definition 3.1.2. Given an event set $\Sigma$, we denote the set of concurrent strings as $L_{\text {conc }}$, defined as

$$
L_{\text {conc }}=\Sigma_{\text {act }}^{*} . \text { tick } \subset L_{\text {samp }}
$$

Obviously, $L_{\text {conc }}$ is a strict subset of $L_{\text {samp }}$ since the empty string is not found in $L_{\text {conc }}$.

Next, we want to capture the idea that an SD controller cannot tell the difference between two nonidentical concurrent strings if they contain exactly the same activity events but in a different order, and/or one or more event have a different number of occurrences. For example, strings $\alpha \beta \tau, \beta \alpha \tau$ and $\alpha \beta \alpha \tau$ would all appear the same to an SD controller. We now give the definition of the occurrence operator. It takes a string and returns the set of events (the occurrence image) that make up the string. Essentially, if two concurrent strings have the same occurrence image, they are indistinguishable to an SD controller.

Definition 3.1.3. For $s \in \Sigma^{*}$, the occurrence operator is a function Occu : $\Sigma^{*} \rightarrow$ $\operatorname{Pwr}(\Sigma)$ defined as below

$$
\operatorname{Occu}(s):=\left\{\sigma \in \Sigma \mid s \in \Sigma^{*} \cdot \sigma \cdot \Sigma^{*}\right\}
$$

As an SD controller only gets information about the system it is controlling at sampling instances (ticks), sampled strings represent observable points in the system. Considering a TDES $\mathbf{S}=\left(X, \Sigma, \xi, x_{o}, X_{m}\right)$, states reached by sampling strings represents states in $\mathbf{S}$ that are at least partially observable. We refer to such states as sampling states, and define them formally below.

Definition 3.1.4. A state $x \in X$ from TDES $\mathbf{S}=\left(X, \Sigma, \xi, x_{o}, X_{m}\right)$, is a sampling state for $\mathbf{S}$ if

$$
\left(\exists s \in L(\mathbf{S}) \cap L_{\text {samp }}\right) x=\xi\left(x_{o}, s\right)
$$

We refer to $X_{\text {samp }} \subseteq X$ as the set of sampling states for $\mathbf{S}$. Note that since $\epsilon \in L_{\text {samp }}, x_{o} \in X_{\text {samp }}$ by definition. In other words, the initial state is always observable at least once. It is worth noting that their could exist strings in $L(\mathbf{S})$ that take us to a sampled state $x$, but the strings are not sampled strings. These do not 
represent observable points, and means that a given sampled state may not always be observable relative to $L(\mathbf{S})$. As far as an SD controller is concerned, the system it is observing starts in its initial state, and then goes from sampled state to sampled state via concurrent strings.

If we wished to convert a TDES $\mathbf{S}$ into an SD controller, we make the initial state of $\mathbf{S}$ the start state of the SD controller. We would then determine which concurrent strings are possible from this state. The sampled states of $\mathbf{S}$ reached by these strings will become states of the controller, and the occurrence image of the concurrent strings would define our next state conditions.

Our translation has a problem if we have two concurrent strings with the same occurrence image, but that take us to different states of $\mathbf{S}$. This would mean our SD controller would be nondeterministic. To prevent this, we introduce the concept of CS deterministic, stated formally below. In essence, it requires that if the two concurrent strings possible at a sampled state in $\mathbf{S}$ have the same occurrence image, they take us to the same next state in S. It's possible that the two strings could take us to two different states, but the states are $\lambda$-equivalent. If we determine that the strings satisfy the nerode equivalence portion of the requirement, but do not take us to the same state, we can simply merge these states in $\mathbf{S}$ as they are equivalent. Note that we do not require that $\mathbf{S}$ be minimal, just minimal with respect to the states we care about which is a cheaper condition to check. The CS deterministic definition will also be useful in making sure a given TDES has the correct structure such that we can represent its sampled-data behavior.

Definition 3.1.5. A TDES $\mathbf{S}=\left(X, \Sigma, \xi, x_{o}, X_{m}\right)$ is concurrent string deterministic or $C S$ deterministic, if

$$
\begin{aligned}
\left(\forall s \in L(\mathbf{S}) \cap L_{\text {samp }}\right)\left(\forall s^{\prime}, s^{\prime \prime}\right. & \left.\in L_{\text {conc }}\right) \\
{\left[s s^{\prime}, s s^{\prime \prime}\right.} & \left.\in L(\mathbf{S}) \wedge \operatorname{Occu}\left(s^{\prime}\right)=\operatorname{Occu}\left(s^{\prime \prime}\right)\right] \Longrightarrow \\
& {\left[s s^{\prime} \equiv_{L(\mathbf{S})} s s^{\prime \prime} \wedge s s^{\prime} \equiv_{L_{m}(\mathbf{S})} s s^{\prime \prime} \wedge \xi\left(x_{o}, s s^{\prime}\right)=\xi\left(x_{o}, s s^{\prime \prime}\right)\right] }
\end{aligned}
$$

It is worth noting that SD controllers are concerned with enabling and forcing prohibitable events, and not with marking strings. All an SD controller cares about is that two strings have the same future with respect to the system's closed behavior. 
Following Definition 3.1.5 will ensure our controller is deterministic, but we may end up with some redundant states that we can later minimize using standard digital logic techniques [7] for synchronous finite state machines.

For CS deterministic TDES, we now wish to define some of the tools we will need to express the sampled-data behavior of a TDES. This will be useful when we want to talk about the behavior of a plant under the control of an SD controller, and compare it to the TDES behavior of the plant under the control of its TDES supervisor. The first thing we need to do is define for a given TDES, a next sampling state function. This will represent how a TDES will move from sampling state to sampling state via concurrent strings.

Definition 3.1.6. For the CS deterministic TDES $\mathbf{S}=\left(X, \Sigma, \xi, x_{o}, X_{m}\right)$, we define the partial function, next sampling state function

$$
\Delta: X_{\text {samp }} \times \operatorname{Pwr}\left(\Sigma_{a c t}\right) \rightarrow X_{\text {samp }}
$$

as follows. For $x \in X_{\text {samp }}$ and $\Sigma^{\prime} \subseteq \Sigma_{a c t}$,

$$
\Delta\left(x, \Sigma^{\prime}\right):=\left\{\begin{array}{cc}
\xi(x, s) & \text { if }\left(\exists s \in L_{\text {conc }}\right) \xi(x, s) ! \& \text { Occu }(s) \cap \Sigma_{a c t}=\Sigma^{\prime} \\
\text { undefined } & \text { otherwise }
\end{array}\right.
$$

For the special case $\Sigma^{\prime}=\emptyset, \Delta\left(x, \Sigma^{\prime}\right)$ can still be defined according to the definition. It just returns a sampling state $x^{\prime}=\xi(x, \tau)$, which means that no event except a tick has occurred during the last sampling period. In analogy to the DES transition function, we write $\Delta\left(x, \Sigma^{\prime}\right)$ ! if $\Delta\left(x, \Sigma^{\prime}\right)$ is defined.

As a precondition for the definition of $\Delta$, we require that the TDES be CS deterministic. This means that two concurrent strings with the same occurrence image will take us to exactly the same state in S. For CS deterministic TDES, this means that $\Delta$ is well defined.

To see how a non CS deterministic TDES would cause problems, consider Figure 3.1. For this example, let $\alpha, \beta \in \Sigma_{a c t}$ and $x_{n}, x^{\prime}, x^{\prime \prime} \in X_{\text {samp }}$ for some TDES $\mathbf{S}=$ $\left(X, \Sigma, \xi, x_{o}, X_{m}\right)$. In Figure [3.1, part (a) shows the only portion of $\mathbf{S}$ that is not minimized, such that $s^{\prime}=\alpha \beta \tau$ and $s^{\prime \prime}=\beta \alpha \tau$ end up at two different states, $x^{\prime}$ and $x^{\prime \prime}$ respectively. But (b) shows the minimized version where $x^{\prime}$ and $x^{\prime \prime}$ have been 


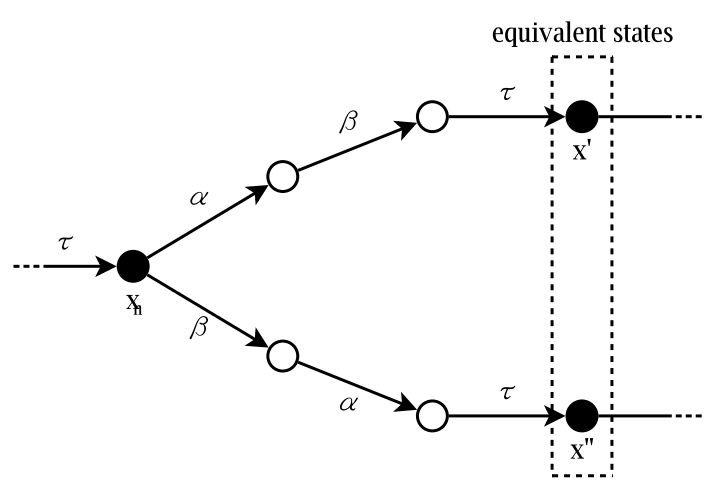

(a) un-minimized supervisor

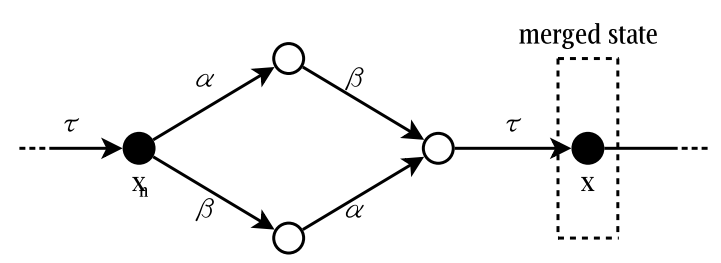

(b) minimized supervisor

Figure 3.1: Nonminimal Example

merge into a single state $x$. Clearly in (a), Occu $\left(s^{\prime}\right) \cap \Sigma_{\text {act }}=\operatorname{Occu}\left(s^{\prime \prime}\right) \cap \Sigma_{\text {act }}$ but $\xi\left(x_{n}, s^{\prime}\right) \neq \xi\left(x_{n}, s^{\prime \prime}\right)$, which would mean that $\delta$ is not well-defined. However in (b), everything is fine. Another problem would be if $x^{\prime}$ and $x^{\prime \prime}$ were not $\lambda$-equivalent. This would mean that we cannot merge the two states, and again $\delta$ would not be well defined.

\subsection{SD Controllable Languages}

So far, we have required that our TDES system have a finite statespace, be ALF and nonblocking, that our plant have proper time behavior and be complete for our supervisor. and that our supervisor be controllable for our plant. However, these conditions are not sufficient to address the concerns that we raised in Section 1.1. In particular, we saw that even though the above conditions are met, our actual system behavior under the control of the corresponding SD controller could block, violate our control law, or even exhibit behavior not contained in our plant model.

To address these issues, we now introduce a new concept called $S D$ controllable languages, defined below. Let $\mathbf{G}=\left(Q, \Sigma, \delta, q_{0}, Q_{m}\right)$ be a TDES where $\Sigma=\Sigma_{c} \dot{\cup} \Sigma_{u}$ for controllable and uncontrollable events. Of course, for a TDES system, $\Sigma_{c}=\Sigma_{h i b} \cup\{\tau\}$. As we will see, this new condition implies TDES controllability, thus we do not have to test for this condition separately. 
It should be noted that the condition we are presenting is a bit conservative. If a system fails it, there may be some situations where things are still fine. Our goal here is to provide a set of conditions that should ensure correct behavior when we implement our TDES supervisors, and be general and flexible enough to apply to a wide range of systems, yet be reasonable conditions to evaluate.

Definition 3.2.1. A language $K \subseteq \Sigma^{*}$ is $S D$ Controllable with respect to $\mathbf{G}=$ $\left(Q, \Sigma, \delta, q_{0}, Q_{m}\right)$ if, $\forall s \in \bar{K} \cap L(\mathbf{G})$, the following statements are satisfied:

i) $\operatorname{Elig}_{L(\mathbf{G})}(s) \cap \Sigma_{u} \subseteq \operatorname{Elig}_{\bar{K}}(s)$

ii) If $\tau \in \operatorname{Elig}_{L(\mathbf{G})}(s)$ then

$$
\tau \in \operatorname{Elig}_{\bar{K}}(s) \Leftrightarrow \operatorname{Elig}_{\bar{K} \cap L(\mathbf{G})}(s) \cap \Sigma_{h i b}=\emptyset
$$

iii) If $s \in L_{\text {samp }}$ then

$$
\begin{gathered}
\text { 1. }\left(\forall s^{\prime} \in \Sigma_{a c t}^{*}\right)\left[s s^{\prime} \in \bar{K} \cap L(\mathbf{G})\right] \Rightarrow \\
{\left[\operatorname{Elig}_{\bar{K} \cap L(\mathbf{G})}\left(s s^{\prime}\right) \cup \operatorname{Occu}\left(s^{\prime}\right)\right] \cap \Sigma_{h i b}=\operatorname{Elig}_{\bar{K} \cap L(\mathbf{G})}(s) \cap \Sigma_{h i b}} \\
\text { 2. }\left(\forall s^{\prime}, s^{\prime \prime} \in L_{c o n c}\right)\left[s s^{\prime}, s s^{\prime \prime} \in \bar{K} \cap L(\mathbf{G}) \wedge \operatorname{Occu}\left(s^{\prime}\right)=\operatorname{Occu}\left(s^{\prime \prime}\right)\right] \Rightarrow \\
s s^{\prime} \equiv \overline{K \cap L(\mathbf{G})} s s^{\prime \prime} \wedge s s^{\prime} \equiv_{K \cap L_{m}(\mathbf{G})} s s^{\prime \prime}
\end{gathered}
$$

iv) $K \cap L_{m}(\mathbf{G}) \subseteq L_{\text {samp }}$

Point $\mathbf{i}$ This is the standard untimed controllability definition and is part of TDES controllability. Intuitively, any uncontrollable events eligible in $\mathbf{G}$ may not be disabled.

Point ii If both a prohibitable event and tick event are enabled and eligible, it will be ambiguous in which clock period the event should occur in. Also, a supervisor must not disable a tick unless there exists a prohibitable (forcible) ${ }^{1}$ event to preempt the tick. The if and only if part only applies if the tick event is eligible in the plant.

The $\Rightarrow$ part states that a tick event must be disabled by $\bar{K}$ if there is an eligible prohibitable event. This is done to ensure that prohibitable events are disabled

\footnotetext{
${ }^{1}$ Remember, we have required that the set of prohibitable events be equal to the set of forcible events.
} 
until they should occur and then they are immediately forced. In other words, it means forcing and enabling are essentially one and the same. This is to make it clear which clock period a prohibitable event should occur in. This in turn will make translating to an SD controller much simpler and straightforward. Part of the goal of this definition is to make the behavior specified by the TDES as close as possible to that which is possible with the actual SD controller. In this case, the SD controller needs to know exactly when to force an event. A range of possible clock periods is no good to it.

The $\Leftarrow$ part states that a tick event cannot be disabled unless there exists an eligible prohibitable event to preempt the tick. Together with Point $\mathbf{i}$, this is equivalent to TDES controllability (Definition 2.3.6).

Point iii The following two points are needed when $s$ is a sampled string.

1) This condition says that the set of prohibitable events eligible in $\bar{K}$ and $L(\mathbf{G})$ after sampled string $s$ (i.e. immediately after a tick occurs (clock edge)) must stay equal to the union of the prohibitable events still eligible, and the prohibitable events that have already occurred since the last tick. In other words, the prohibitable events eligible after the tick must stay eligible until they occur, and no new prohibitable events may become eligible until after the next tick.

This condition is meant to capture two concepts. The first is that since an SD controller only can observe the system at a clock edge (tick event), its enablement and forcing decisions are determined by its current state, and must be constant until the next tick occurs. These cannot change during the current clock cycle in response to events occurring, as it will not know they have occurred until after the next tick, which would be too late.

The second concept is that an SD controller decides to force an event immediately after a tick, based on the information it has at that point (i.e. whether the event is currently enabled and eligible in the plant). Once it decides to force the event, it will occur at some point during the current clock period. So as to not violate the control law or the plant model, this event must stay eligible 
and enabled until it occurs. This is important as we do not know exactly when this event will actually occur, due to the fact that different implementations of our controller could have different timing characteristics. We thus have to ensure that when it does occur, it does not violate our control law, nor exceed the behavior of our plant model.

A side effect of this condition is that it means that we only have to look at the eligibility and enabling information for prohibitable events at the state reached by a tick, and this determines the information for the clock cycle. This makes the conversion to an SD controller easier.

2) This condition says that if sampled string $s$ can be extended by concurrent strings $s^{\prime}$ and $s^{\prime \prime}$ which have the same occurrence image (and thus indistinguishable to an SD controller), then string $s s^{\prime}$ will be Nerode equivalent to string $s s^{\prime \prime}$ with respect to the system's closed and marked behavior. In other words strings $s s^{\prime}$ and $s s^{\prime \prime}$ will have the same closed and marked future. From a TDES perspective, this means that strings $s s^{\prime}$ and $s s^{\prime \prime}$ will go to states that are $\lambda$ equivalent. If the TDES is minimal, this will mean the same state. Otherwise, we may need to check that the two states are $\lambda$-equivalent.

This condition is intended to address two issues. The first is the fact that since the SD controller cannot tell the difference between strings $s^{\prime}$ and $s^{\prime \prime}$, it must take the same control action following either string, both now and in the future. We can capture this by requiring them to have the same future with respect to the system's closed behavior.

The second issue has to do with nonblocking. Depending on the implementation of our SD controller, it maybe the case that we may either always get the string $s^{\prime}$ and never $s^{\prime \prime}$, or vice-versa. If $s^{\prime \prime}$ never actually occurs in the physical system and it is part of the only path back to a marked state, the physical system would block despite the fact the TDES system is nonblocking. By requiring the two strings to have the same marked future, it will not matter which one we actually get, as long as all of the marked strings in the system are also sampled strings (see Point iv for more info on this). In a way, we are ensuring that our system will still be nonblocking for a set of possible closed loop behaviors, that 
differ by which of these concurrent strings can actually happen in the physical system.

Point iv This point says that all marked strings in the closed loop system must be sampled strings. The primary reason is that sampled strings represent observable points in the system. This makes sure that we do not mark a non empty strict substring of a concurrent string accepted by the system. We saw in Point iii.2 that two concurrent strings with the same occurrence image have the same marked future, but the condition says nothing about $\Sigma_{\text {act }}^{+}$substrings of these concurrent strings. Point iii.2 basically says that even if we only get one of the two concurrent strings, we can still get to a new sampled state with an equivalent marked future. i.e. we might lose one of the paths to this sampled state, but we can still get there. However, if we allow marking along the path between sampled states and that is the path we lose, we may no longer be able to reach a marked state. Hence, we require all marked strings to take us to sampled states.

So far, we have only discussed controllable languages. To extend this concept to a TDES supervisor $\mathbf{S}=\left(X, \Sigma, \xi, x_{o}, X_{m}\right)$, we identify $K=L_{m}(\mathbf{S})$ and $\bar{K}=L(\mathbf{S})$ in Definition $3.2 .1{ }^{2}$ This gives us the definition below. Note that the definition is implicitly assuming that $\mathbf{G}$ and $\mathbf{S}$ are combined using the meet operator. If instead we had a plant $\mathbf{G}^{\prime}$ and supervisor $\mathbf{S}^{\prime}$ combined using the synchronous product operator resulting in system event set $\Sigma$, we would first construct plant $\mathbf{G}$ from $\mathbf{G}^{\prime}$ by adding selfloops of any events missing from $\Sigma$, and supervisor $\mathbf{S}$ from $\mathbf{S}^{\prime}$ by again adding needed selfloops. We can then apply the definition below to the these new TDES.

Definition 3.2.2. A supervisor $\mathbf{S}=\left(X, \Sigma, \xi, x_{o}, X_{m}\right)$ is said to be $S D$ controllable with respect to $\mathbf{G}=\left(Q, \Sigma, \delta, q_{0}, Q_{m}\right)$ if, $\forall s \in L(\mathbf{S}) \cap L(\mathbf{G})$, the following statements are satisfied:

i) $\operatorname{Elig}_{L(\mathbf{G})}(s) \cap \Sigma_{u} \subseteq \operatorname{Elig}_{L(\mathbf{S})}(s)$

ii) If $\tau \in \operatorname{Elig}_{L(\mathbf{G})}(s)$ then

$$
\tau \in \operatorname{Elig}_{L(\mathbf{S})}(s) \Leftrightarrow \operatorname{Elig}_{L(\mathbf{S}) \cap L(\mathbf{G})}(s) \cap \Sigma_{h i b}=\emptyset
$$

\footnotetext{
${ }^{2}$ By "identify," we mean make the indicated replacements in the original definition to get the new definition. We do not mean to imply that we require that $\mathbf{S}$ be nonblocking.
} 
iii) If $s \in L_{\text {samp }}$ then

1. $\left(\forall s^{\prime} \in \Sigma_{a c t}^{*}\right)\left[s s^{\prime} \in L(\mathbf{S}) \cap L(\mathbf{G})\right] \Longrightarrow$

$$
\left[\operatorname{Elig}_{L(\mathbf{S}) \cap L(\mathbf{G})}\left(s s^{\prime}\right) \cup \operatorname{Occu}\left(s^{\prime}\right)\right] \cap \Sigma_{h i b}=\operatorname{Elig}_{L(\mathbf{S}) \cap L(\mathbf{G})}(s) \cap \Sigma_{h i b}
$$

2. $\left(\forall s^{\prime}, s^{\prime \prime} \in L_{\text {conc }}\right)\left[s s^{\prime}, s s^{\prime \prime} \in L(\mathbf{S}) \cap L(\mathbf{G}) \wedge \operatorname{Occu}\left(s^{\prime}\right)=\operatorname{Occu}\left(s^{\prime \prime}\right)\right] \Rightarrow$

$$
s s^{\prime} \equiv_{L(\mathbf{S}) \cap L(\mathbf{G})} s s^{\prime \prime} \wedge s s^{\prime} \equiv_{L_{m}(\mathbf{S}) \cap L_{m}(\mathbf{G})} s s^{\prime \prime}
$$

iv) $L_{m}(\mathbf{S}) \cap L_{m}(\mathbf{G}) \subseteq L_{\text {samp }}$

We now discuss a few examples to illustrate the above definition, starting with Point ii. We do not give an example for Point $\mathbf{i}$ or Point iii.2 since the first is essentially untimed controllability, and the second is similar to the CS Deterministic property discussed in Section 3.1.

Figure 3.2 shows an example where prohibitable event $\alpha$ and a tick are both possible at the same state in the plant. When our supervisor decided to enable $\alpha$ here, Point ii required that tick must be disabled. Also, Point ii only allowed us to disable tick here as forcible event $\alpha$ was possible in both the plant and supervisor to preempt the tick.

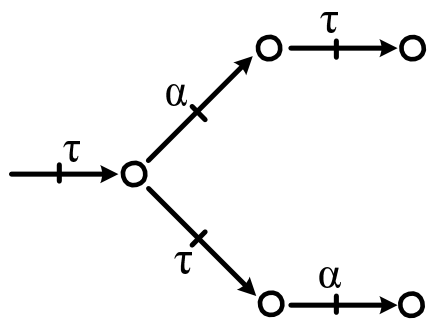

Plant $\mathbf{G}$

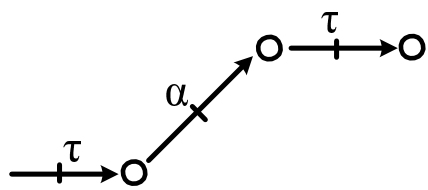

Supervisor $\mathbf{S}$

Figure 3.2: An Example for Point ii

Figure 3.3 shows an example for Point iii.1. In the diagram, we see that the only prohibitable event possible after the tick is $\beta$. We see that $\beta$ stays possible until it occurs on both paths, and no new prohibitable events become eligible before the next tick.

Figure 3.4 shows an example that fails Point iv. Here we see that the state reached by the first tick is marked which is allowed, but then the state reached by $\alpha$ is also marked, which is not. 

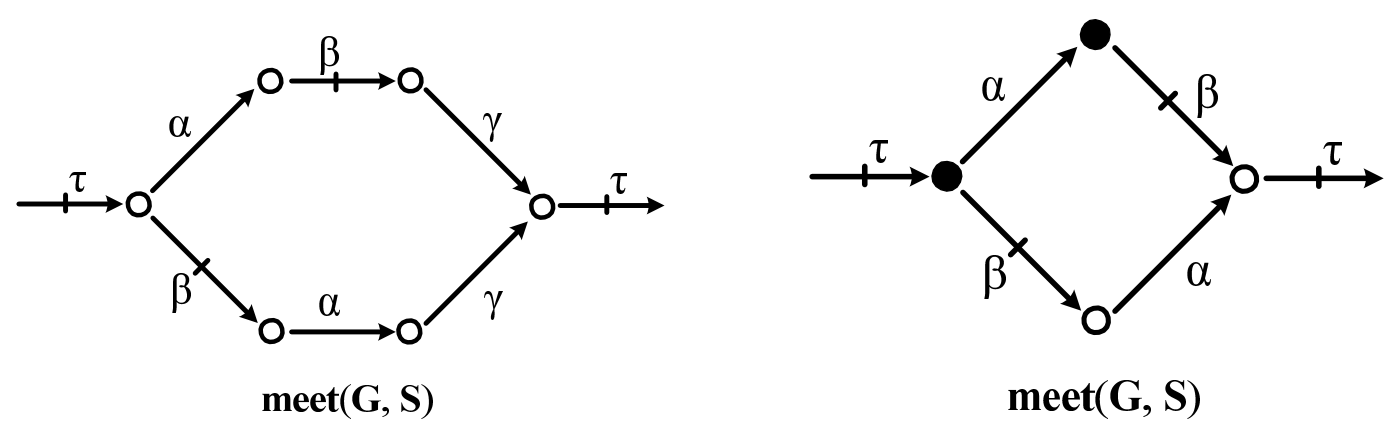

Figure 3.3: An Example for Point iii.1 Figure 3.4: An Example Failing Point iv

Note that Definition 3.2.2 is not closed under arbitrary union. An example is shown in Figure 3.5, where (a) and (b) are two TDES supervisors that enable and force only one event respectively. In (a), $\alpha$ is forced and $\beta$ is disabled. In (b), $\beta$ is forced and $\alpha$ is disabled. It can be shown that both (a) and (b) are SD controllable for our plant shown in (d), but the union of these two languages, shown in (c), is not. The supervisor in (c) fails Point iii.1 as both $\alpha$ and $\beta$ are possible at the initial state, but once one occurs, the other is disabled before the next tick has occurred.

This example suggests that in general, there may not exist a supremal SD controllable sublanguage. For this example, there appears to be two maximal sublanguages but no supremal sublanguage. This likely follows from the fact that in normal TDES controllability, the maximally permissive supervisor might allow several choices as they are each safe, and leave it up to an unmodeled agent to decide which option occurs. As they are all possible, eventually we should get all choices. However for SD controllers, we make the choice with respect to which clock cycle an event gets forced in, meaning that some of these choices might vanish. If two choices are mutual disjoint yet equal in terms of size of behavior we would get, we end up with two or more maximal solutions, and no supremal solution.

We now add another tool that we will need to express the sampled-data behavior of a TDES. We will now define the control action that will take place at a sampling state for our TDES. This is the action the SD controller will take during the corresponding sampling period.

Definition 3.2.3. Let TDES supervisor $\mathbf{S}=\left(X, \Sigma, \xi, x_{o}, X_{m}\right)$ be SD controllable 
with respect to plant $\mathbf{G}=\left(Q, \Sigma, \delta, q_{0}, Q_{m}\right)$. The control action $\zeta: X_{\text {samp }} \rightarrow \operatorname{Pwr}\left(\Sigma_{\text {hib }}\right)$ is defined for $x \in X_{\text {samp }} \subseteq X$ as follows:

$$
\zeta(x):=\left\{\sigma \in \Sigma_{h i b} \mid \xi(x, \sigma) !\right\}
$$

Proposition 3.1. For TDES supervisor $\mathbf{S}=\left(X, \Sigma, \xi, x_{o}, X_{m}\right)$ which is SD controllable with respect to plant $\mathbf{G}=\left(Q, \Sigma, \delta, q_{0}, Q_{m}\right)$, we have

$$
\left(\forall s \in L(\mathbf{S}) \cap L_{\text {samp }}\right) \zeta(x)=\operatorname{Elig}_{L(\mathbf{S})}(s) \cap \Sigma_{h i b}
$$

where $x=\xi\left(x_{o}, s\right)$.

Proof. This follows immediately from the definition of $L(\mathbf{S})$ and the Elig operator.

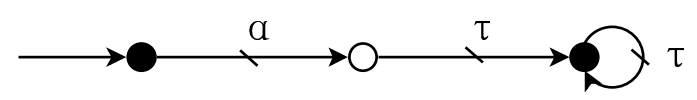

(a)

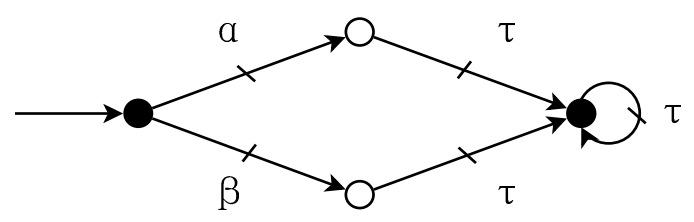

(c)

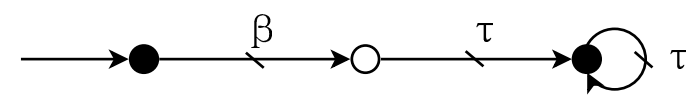

(b)

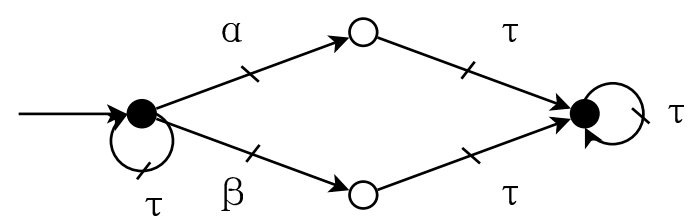

(d)

Figure 3.5: SD Controllability and Arbitrary Union.

We close this chapter with a proposition pointing out the connection of our CS deterministic definition and Point iii.2 of the SD controllability definition.

Proposition 3.2. If TDES supervisor $\mathbf{S}=\left(X, \Sigma, \xi, x_{o}, X_{m}\right)$ is SD controllable for plant $\mathbf{G}=\left(Q, \Sigma, \delta, q_{0}, Q_{m}\right)$, then $\operatorname{meet}(\mathbf{S}, \mathbf{G})$ is $\mathrm{CS}$ deterministic if it is minimal.

Proof. Follows automatically from Point iii.2 in Definition 3.2.2.

However, an SD controllable supervisor $\mathbf{S}$ with respect to plant $\mathbf{G}$ does not imply that $\mathbf{S}$ is CS deterministic by itself, because of the dependency of plant $\mathbf{G}$ in the definition of SD controllability. We use the CS deterministic property when we wish to only discuss the supervisor, instead of the closed loop behavior of the system. 


\subsection{Future Work}

In this thesis, we have presented some new conditions and methods that are intended to address the concurrency and implementation issues raised in Section 1.1. However, we only partly dealt with time delay issues which we have left as future work due to time considerations.

We have tried to mitigate potential time delay problems by the assumptions we have made at the beginning of Chapter 3. Here, we have required that our controllers be implemented on a single machine, that they use a common clock, that they all see the result of a common sampling of the inputs, and that their outputs change at about the same time. These restrictions should protect against time delay issues caused by a distributed implementation of controllers, where they could sample inputs at different times, update enablement information at different times, and this information could reach the plant at different times.

Another potential time delay problem is the difference between when an event physically occurs (say a part arrives at a machine), and when a controller sees that the event has occurred. For instance, the event might physically occur in sampling period $k$, but due to transmission delay, it does not reach the input of the controller until the next clock cycle, so the controller "sees" it one clock cycle late. It is even possible that the signal could reach the input right at the clock edge, and thus is not noticed till the next clock edge. All of these issues could cause the system that the controller "sees" to have slightly different timing information from the formal model.

We have tried to compensate for this by assuming that an event has occurred when its corresponding input goes true at the controller, with one exception. The exception is when the input goes true so close to the clock edge, it does not show up till the next sampling period. In this case, the event is assumed to happen just after the clock edge. We then model the system with this interpretation of what it means for an event to occur, in particular with respect to the timing of the events.

Whereas the steps we have taken to compensate for timing delay are not ideal, they should handle the more pressing issues. However, research needs to be done to identify the existing timing delay issues, and address them directly in a more flexible manner. 


\section{Chapter 4}

\section{Moore Synchronous Finite State Machines}

A Moore state machine is a type of finite state machines introduced by Edward F. Moore in [17]. It chooses its next states based on its current state and inputs. Its outputs are determined by its current state only. We will use Moore state machines with clocked systems whose states change only on a rising or falling edge of the clock. Its current output remains the same until the state is changed again. A Moore state machine used in this way is called a Moore Synchronous Finite State Machine. In the following discussion, we simply use Moore machine or FSM for convenience.

By the properties defined in Chapter 3, an SD Controller can be modeled as a Moore machine. In the following pages, we will first define a formal model for our SD controller in Section 4.1, Then, in Section 4.2 we will introduce translations methods for a centralized controller and for modular controllers. The translation methods require that the given supervisors be CS deterministic and non-selfloop ALF, as defined in Section 3.1 and Section 2.3. Note that we can translate a supervisor as long as its CS deterministic, but it would likely be very hard to evaluate the CS deterministic condition if the TDES is not ALF or non-selfloop ALF, as we would essentially have an infinite number of concurrent strings to evaluate. It is also quite likely such a system would fail the CS deterministic condition. Requiring that the TDES also be ALF or the weaker non-selfloop ALF makes everything easier, and still gives us a general solution as a non ALF system is not physically realistic. 


\subsection{Formal Model}

In this chapter, we will often be discussing vectors of information that will change periodically with respect to some clock. Let $k \in\{0,1,2, .$.$\} . We will say "at time k$ " to indicate the point of time at which $k$ clock ticks have gone by since our starting reference point, which we represent as $k=0$. For any vector $\mathbf{v}=\left[v_{1}, v_{2}, \ldots, v_{n}\right] \in V$ or any of its element $v_{j}$, we write " $\mathbf{v}(k)$ " and " $v_{j}(k)$ " to denote the value of $\mathbf{v}$ and $v_{j}$ at time $k$. Note that $\mathbf{v}(k)$ is not a function of $k$, but a notation to differentiate the value of $\mathbf{v}$ at different points in time. For $k=0, \mathbf{v}(0)$ represents the initial or starting value of $\mathbf{v}$. When we are discussing an SD controller, we can think of $k=0$ as representing the time when the controller has just been turned on.

We can think of when $k$ is incremented as the occurrence of a tick from our clock. With respect to a TDES system, this would correspond to the occurrence of the tick event. As such, $k$ induces a sequence for vector $\mathbf{v}$ with respect to these clock ticks, which we define to be $\{\mathbf{v}(k) \mid k=0,1, \ldots\}$, and is denoted as $\{\mathbf{v}(k)\}$ as a shorthand.

Assumption 4.1. For convenience, we assume every controller is operating based on the same global clock, so that they change state at the same time.

Given a TDES supervisor $\mathbf{S}=\left(X, \Sigma, \xi, x_{o}, X_{m}\right)$, we will refer to the implementation of $\mathbf{S}$ as its corresponding $S D$ controller. We now give a formal definition of SD controllers.

Definition 4.1.1. An SD controller $\mathrm{C}$ is represented by a Moore machine defined as follows.

$$
\mathbf{C}=\left(I, Z, Q, \Omega, \Phi, \mathbf{q}_{\text {res }}\right)
$$

where,

$I$ is the set of possible Boolean vectors that the inputs to our controller can take on. Each vector $\mathbf{i} \in I$ has $v$ input variables, such that

$$
\mathbf{i}=\left[i_{0}, i_{1}, . ., i_{v-1}\right] ; i_{j} \in\{0,1\} ; j=0,1, . ., v-1
$$


Each input vector $\mathbf{i}\left(k^{\prime}\right) \in\{\mathbf{i}(k)\}$ is sampled at the occurrence of a tick event, except for $k=0$ which occurs when the controller is turned on.

Each element of $I$ corresponds to a unique activity event in our system. If that element equals " 1 " at time $k$, then that means the event has occurred at least once since that last clock tick. If it equals zero, then it means the corresponding event has not occurred at all since the last clock tick.

$Z$ is the set of possible Boolean vectors that the controller outputs can take on. Each vector $\mathbf{z} \in Z$ has $r$ output variables, such that

$$
\mathbf{z}=\left[z_{0}, z_{1}, . ., z_{r-1}\right] ; z_{j} \in\{0,1\} ; j=0,1, . ., r-1
$$

Each input vector $\mathbf{z}\left(k^{\prime}\right) \in\{\mathbf{z}(k)\}$ is generated at the occurrence of the tick event, except for $k=0$ which occurs when the controller is turned on. Note that we do not provide separate outputs for forcing, because the forcing of an event is already implied by enabling the event.

The values of vector $Z$ represent enablement information for our prohibitable events. A value of ' 1 ' means the event is enabled, while ' 0 ' means the event is disabled.

$Q$ is the set of possible Boolean vectors that the state of our controller can take on. Each vector $\mathbf{q} \in Q$ has $l$ state variables for state identification, such that

$$
\mathbf{q}=\left[q_{0}, q_{1}, . ., q_{l-1}\right] ; q_{j} \in\{0,1\} ; j=0,1, . ., l-1
$$

Each state $\mathbf{q}\left(k^{\prime}\right) \in\{\mathbf{q}(k)\}$ changes to next state $\mathbf{q}\left(k^{\prime}+1\right) \in\{\mathbf{q}(k)\}$ at the occurrence of the tick event, starting at $k=1$.

$\mathbf{q}_{\text {res }}$ is the default state when the machine is reset or initialized. We take $\mathbf{q}(0)=q_{\text {res }}$. 
$\Omega: Q \times I \rightarrow Q$ is a next state function which takes the current state $\mathbf{q}(k) \in Q$ and an input vector $\mathbf{i}(k+1) \in I$, and returns the next state $\mathbf{q}(k+1) \in Q$.

$$
\mathbf{q}(k+1)=\Omega(\mathbf{q}(k), \mathbf{i}(k+1))
$$

$\Phi: Q \rightarrow Z$ is the state to output map. For state $\mathbf{q} \in Q$, the output $\mathbf{z} \in Z$ at this state is:

$$
\mathbf{z}=\Phi(\mathbf{q})
$$

A few comments are worthwhile here to clarify our notation. We will discuss the notation used for states, but the same applies for input and output variables. If we use $\mathbf{q}$ by itself (i.e. $\mathbf{q} \in Q$ ), then it represents a single instance of $Q$ (i.e. some specific vector of zeros and ones with $j$ elements). When we use $\mathbf{q}\left(k^{\prime}\right)$, then this is the $k^{\prime}$-th element of the sequence $\{\mathbf{q}(k)\}$ where each element of the sequence is some member of $Q$. Obviously, we can construct many different possible $\{\mathbf{q}(k)\}$ sequences. If we wish to label different sequences, we will use different labels for $\mathbf{q}$, such as $\{\mathbf{q}(k)\}$ and $\left\{\mathbf{q}^{\prime}(k)\right\}$.

With respect to our input, a specific sequence $\{\mathbf{i}(k)\}$ would represent a specific pattern of inputs we received for a specific run of the system. If we ran the system again, we could get a completely different sequence. From our definition of $\mathbf{C}$, we see that our state sequence is completely determined by $q_{\text {res }}, \Omega$, and $\{\mathbf{i}(k)\}$. If we get a different input sequence, we could get a different state sequence, depending on how our next state function responds to the input values. As our output is a function of our current state, this means we could also get a different output sequence as well. In other words, input sequence $\{\mathbf{i}(k)\}$ might induce state and output sequences $\{\mathbf{q}(k)\}$ and $\{\mathbf{z}(k)\}$, while input sequence $\left\{\mathbf{i}^{\prime}(k)\right\}$ might induce state and output sequences $\left\{\mathbf{q}^{\prime}(k)\right\}$ and $\left\{\mathbf{z}^{\prime}(k)\right\}$ which may or may not be the same as the other sequences of the same type..

Example 4.1. Inspired by the DES shown in Figure [2.1, we take Figure 4.1] as an example to see how to apply our formal SD controller model.

Figure 4.1(a) shows an example of a TDES and Figure 4.1(b) shows the Moore machine representing this TDES. Our ordering for the input variables is $I=\left[\alpha_{1}, \alpha_{2}, \mu_{1}, \mu_{2}, \beta_{1}, \lambda_{1}\right]$ 


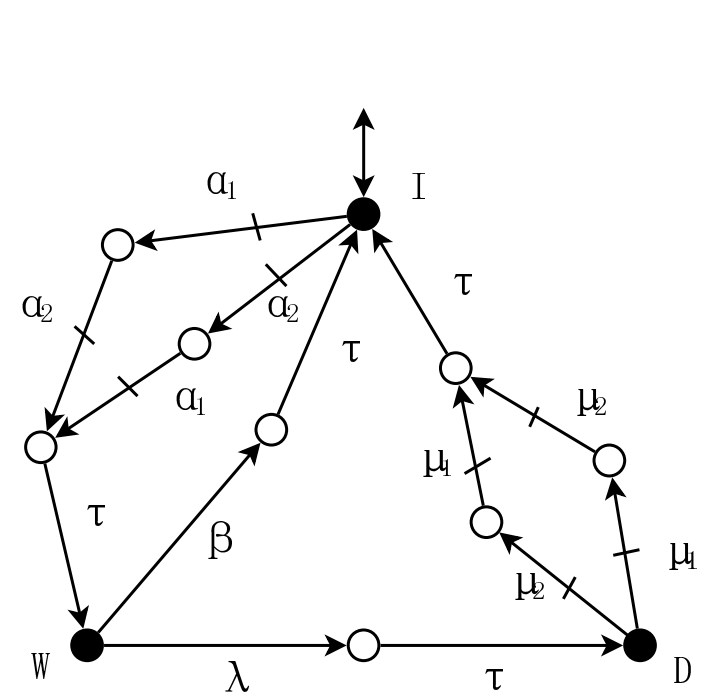

(a) Original TDES

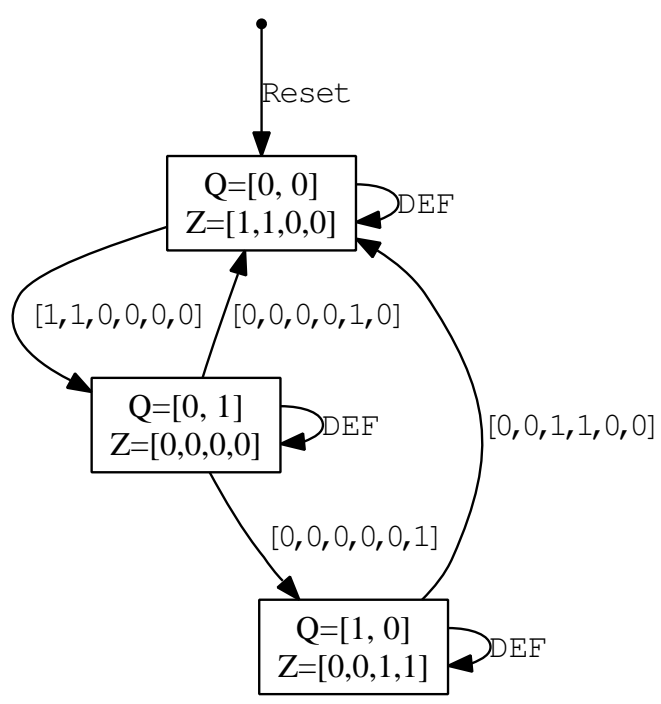

(b) FSM Translation

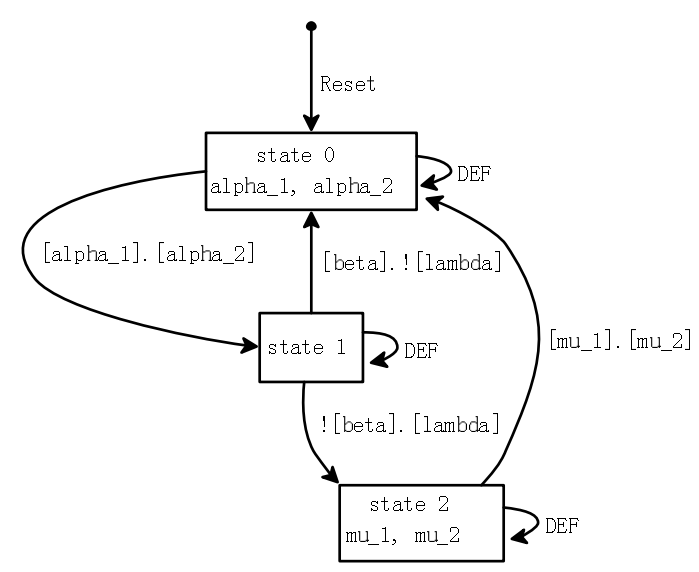

(c) Abbreviated FSM

Figure 4.1: FSM Translation Example

and for our outputs is $Z=\left[\alpha_{1}, \alpha_{2}, \mu_{1}, \mu_{2}\right]$. We have also added a $\boldsymbol{D E F}$ or default transition to cover input combinations that we have not explicitly specified. The reason is that the transition function for a TDES is a partial function, but that of a FSM must be a complete function. The actual translation from the TDES in (a) to the controller in (b) will be presented after the translation method for centralized controllers is introduced in the next section.

In (b), we showed the SD controller for our example in the format of the formal 
SD controller model we just defined. Typically when we give a diagram of an FSM, we use the more compact and readable notation shown in Figure 4.1(c). Here we have given states meaningful names, and we only list at a state those prohibitable events whose outputs are true (1) at that state. Also, rather than listing input vectors on transitions, we use boolean equations that are true for the required input vector. We use '!' as NOT, '+' as OR, and '.' as $A N D^{1}$. We also only use in the equations those events that could occur at a given state, to simplify the equations.

\subsection{Translation Method}

To translate a supervisor to Moore FSM, we require that the supervisor be CS deterministic. CS deterministic is necessary because, for SD systems, we lose the ordering information for the events that occur during a given sampling period. Event sequences that have the same occurrence image must all go to the same next state in the state machine implementation or our controller will be nondeterministic. We can ensure this if we require the supervisors to be CS deterministic before being translated.

We also require that the supervisor be non-selfloop ALF. The reason is to make sure we have a manageable set of next state conditions. If we have activity loops that are not selfloops, then our supervisor does not have enough information for us to determine a reasonable set of concurrent strings to use to define our next state condition. We would thus potentially have a large choice of strings, most of which are not possible in the closed loop system. By requiring that the supervisor be nonselfloop ALF, we should have a reasonable set of possible concurrent strings at a given state. As we discussed earlier, technically the CS deterministic condition is strong enough, however, this condition is hard to evaluate if the system is not ALF or non-selfloop ALF. So, what we would do in practice is first check that our TDES is ALF or non-selfloop ALF, and if so, we will then check if it is CS deterministic.

We note that we require that a supervisor $\mathbf{S}$ be CS deterministic before we can translate it to a controller, but we do not need the supervisor be SD controllable for our plant $\mathbf{G}$ for the conversion process itself. We also note that if we are translating $\mathbf{S}$ to a controller, the fact that $\mathbf{S}$ is $\mathrm{SD}$ controllable for $\mathbf{G}$ is not sufficient to be able to

\footnotetext{
${ }^{1}$ In the following FSM graphs, this operator is represented by '.(period)' instead of '.' due to a technical difficulty.
} 
do the conversion, as it implies that $\mathbf{S} \| \mathbf{G}$ is CS deterministic if $\mathbf{S} \| \mathbf{G}$ is minimal, not $\mathbf{S}$ itself. If $\mathbf{G}$ is not complete for $\mathbf{S}$, we may wish to instead convert $\mathbf{S} \| \mathbf{G}$ instead of $\mathbf{S}$, but typically we prefer to construct modular controllers for the component supervisors that make up $\mathbf{S}$, as they usually are far more compact.

In the following sections, we introduce event mapping functions, and how to translate a CS deterministic TDES supervisor into a centralized controller. We then discuss the translation of modularized CS deterministic supervisors.

\subsubsection{Event Mapping Functions}

As we will often be discussing vectors of boolean values whose elements refer to specific events in $\Sigma_{a c t}$, we will need a way to map events to a vector's elements and vice versa. Let $\mathbf{G}=\left(Y, \Sigma, \delta, y_{o}, Y_{m}\right)$ be the TDES plant to be controlled and let $\mathbf{S}=\left(X, \Sigma_{\mathbf{S}}, \xi, x_{o}, X_{m}\right)$ be an arbitrary CS deterministic TDES supervisor for $\mathbf{G}$. We define $\Sigma_{a c t} \subset \Sigma$ to be the set of all the activity events and $\Sigma_{h i b} \subseteq \Sigma_{a c t}$ to be the set of all prohibitable events. We consider $\Sigma, \Sigma_{a c t}$ and $\Sigma_{h i b}$ to be global event sets that can always be referred to in the following discussion.

We first define a bijective map between an activity event set and an index set we will use for labeling the events.

Definition 4.2.1. Let bijective map $\gamma_{g}: \Sigma_{a c t} \rightarrow\left\{0, . .,\left|\Sigma_{a c t}\right|-1\right\}$ be the canonical event mapping function such that

$$
\left(\forall \sigma_{1}, \sigma_{2} \in \Sigma_{a c t}\right) \sigma_{1}=\sigma_{2} \Longleftrightarrow \gamma_{g}\left(\sigma_{1}\right)=\gamma_{g}\left(\sigma_{2}\right)
$$

For the controller implementation $\mathbf{C}=\left(I, Z, Q, \Omega, \Phi, \mathbf{q}_{\text {res }}\right)$ of $\mathbf{S}$, we include its event mapping information in our translation methods in the following sections, which are the two event mapping functions defined below. The reason we impose the ordering requirement is so that essentially the function $\gamma_{g}$ will induce a single way to define the mapping functions.

Definition 4.2.2. The input event mapping function for $\mathbf{C}$ is defined to be a bijective map $\gamma: \Sigma_{\mathbf{S}} \cap \Sigma_{a c t} \rightarrow\{0,1, . ., v-1\}$ where $v=\left|\Sigma_{\mathbf{S}} \cap \Sigma_{a c t}\right|$. It is defined such that

$$
\left(\forall \sigma_{1}, \sigma_{2} \in \Sigma_{\mathbf{S}} \cap \Sigma_{a c t}\right) \gamma_{g}\left(\sigma_{1}\right)<\gamma_{g}\left(\sigma_{2}\right) \Longrightarrow \gamma\left(\sigma_{1}\right)<\gamma\left(\sigma_{2}\right)
$$


Definition 4.2.3. The output event mapping function for $\mathbf{C}$ is defined to be a bijective map $\eta: \Sigma_{\mathbf{S}} \cap \Sigma_{h i b} \rightarrow\{0,1, . ., r-1\}$ where $r=\left|\Sigma_{\mathbf{S}} \cap \Sigma_{h i b}\right|$. It is defined such that

$$
\left(\forall \sigma_{1}, \sigma_{2} \in \Sigma_{\mathbf{S}} \cap \Sigma_{h i b}\right) \gamma_{g}\left(\sigma_{1}\right)<\gamma_{g}\left(\sigma_{2}\right) \Longrightarrow \eta\left(\sigma_{1}\right)<\eta\left(\sigma_{2}\right)
$$

Since $\gamma_{g}$ is globally available, two input event mapping functions for different controllers will always have the same mapping pairs for the same event domain. In other words, because of the ordering requirement, there is only one way to define the input mapping. Similar logic applies to the output mapping for same event domain. An example is shown below.

Example 4.2. For different controllers $\mathbf{C}_{1}$ and $\mathbf{C}_{2}$ whose supervisors $\mathbf{S}_{1}$ and $\mathbf{S}_{2}$ are defined over $\Sigma=\Sigma_{a c t} \cup\{\tau\}=\{\alpha, \beta, \lambda, \tau\}$. If $\gamma_{g}(\alpha)<\gamma_{g}(\beta)<\gamma_{g}(\lambda)$, then we always have the input event mapping function $\gamma_{1}=\gamma_{2}=\{(\alpha, 0),(\beta, 1),(\lambda, 2)\}$ for $\mathbf{C}_{1}$ and $\mathrm{C}_{2}$.

Sometimes we want to find out which event an index in an input or output vector corresponds to. This can be easily done by applying the inverse event mapping function, since the event mapping functions we have defined are all bijective. i.e. to find the index of event $\alpha$ in the input event index used by the controller, use $\gamma^{-1}(\alpha)$.

For event $\sigma \in \Sigma_{\mathbf{S}} \cap \Sigma_{a c t}$, we can use the inverse event mapping functions to locate the element in a vector that corresponds to $\sigma$. For example, the corresponding element for $\sigma$ in the input vector would be $i_{\gamma^{-1}(\sigma)}$. For convenience, we may write $i_{\sigma}$ instead of $i_{\gamma^{-1}(\sigma)}$ and $z_{\sigma}$ instead of $z_{\eta^{-1}(\sigma)}$.

\subsubsection{Output Equivalence}

If we have two or more controllers for system $\mathbf{G}$, we may wish to determine if they will produce equivalent output (i.e. enablement information) for the same input sequence. The problem is that each controller may care about a slightly different event set, thus we likely cannot use a single $\{\mathbf{i}(k)\}$ input sequence for them. As defined in our formal model, for $n$ controllers $\mathbf{C}_{1}, \mathbf{C}_{2}, \ldots, \mathbf{C}_{n}$, each controller $\mathbf{C}_{j}$ for $1 \leq j \leq n$ has its own input vector $\mathbf{i}_{j} \in I_{j}$ and will generate its own output vector based on the input sequence $\left\{\mathbf{i}_{j}(k)\right\}$ it receives. 
Before we check that their output sequences are equivalent, we need each input sequence $\left\{\mathbf{i}_{j}(k)\right\}$ to contain equivalent input information. However, their input vectors might be incompatible with each other, because their event mapping for the inputs can be different. Therefore, we will provide a single input vector $\mathbf{i}_{g}$ globally available to every controller, and let each controller extract its own input vector $\mathbf{i}_{j}$ from $\mathbf{i}_{g}$. Essentially, $\mathbf{i}_{g}$ represents the inputs the system sees, where each $\mathbf{i}_{j}$ represents the inputs that each controller sees (which may be a strict subset of the system inputs) and is formatted for the input index that controller is using.

Definition 4.2.4. Let $\Sigma_{\text {act }} \subset \Sigma$ be the set of global activity events, we require $\mathbf{i}_{g}=\left[i_{g, 0}, i_{g, 1}, . ., i_{g, v_{g}-1}\right]$ to be defined over $\Sigma_{a c t}$ where $v_{g}=\left|\Sigma_{a c t}\right|$. That is, for any event $\sigma \in \Sigma_{a c t}$, there is an element in $\mathbf{i}_{g}$ corresponds to $\sigma$ and only $\sigma$. We call $\left\{\mathbf{i}_{g}(k)\right\}$ a canonical input sequence and $\mathbf{i}_{g} \in\left\{\mathbf{i}_{g}(k)\right\}$ a canonical input vector ${ }^{2}$.

To extract input vector $\mathbf{i}_{j}=\left[i_{j, 0}, i_{j, 1}, . ., i_{j, v_{j}-1}\right]$ from $\mathbf{i}_{g}$ for controller $\mathbf{C}_{j}$, for $0 \leq$ $l<v_{j}$ we have $i_{j, l}=i_{g, l^{\prime}}$ where $l^{\prime}=\gamma_{g}\left(\left(\gamma_{j}^{-1}(l)\right)\right)$.

Definition 4.2.5. For $j=1,2$, let $\mathbf{C}_{j}=\left(I_{j}, Z_{j}, Q_{j}, \Omega_{j}, \Phi_{j}, \mathbf{q}_{r e s, j}\right)$ be a controller. We say $\mathbf{C}_{1}$ and $\mathbf{C}_{2}$ are output equivalent if for any canonical input sequence $\left\{\mathbf{i}_{g}(k)\right\}$ and induced output $\mathbf{z}_{j}\left(k^{\prime}\right)=\left[z_{j, 1}\left(k^{\prime}\right), z_{j, 2}\left(k^{\prime}\right), \ldots, z_{j, r_{j}}\left(k^{\prime}\right)\right] \in Z_{j}$ at time $k^{\prime}=\{0,1,2, \ldots\}$, the follow conditions are satisfied.

1. $r_{1}=r_{2}$

2. $\left(\forall 0 \leq i<r_{1}\right) \eta_{1}^{-1}(i)=\eta_{2}^{-1}(i)$

3. $\left(\forall k^{\prime} \in\{0,1, .\}.\right) \mathbf{z}_{1}\left(k^{\prime}\right)=\mathbf{z}_{2}\left(k^{\prime}\right)$

In the above definition, by Point 1, 2 we are essentially requiring the outputs of the two controllers be of the same size, and represent the same events in the same order. We could have been more general and only required that they represent the same events but in possibly different order, but this does not gain much and complicates our notation. In Point 3, we are requiring that one controller enables a prohibitable event if and only if the other does, for any value of $k^{\prime}$. In other words, they agree at the reset state, and will continue to agree in the future.

\footnotetext{
${ }^{2}$ Note that our use of "canonical" here refers to the size and ordering of the inputs, not to the actual values of the input sequence or a given vector.
} 
A common situation is that controllers $\mathbf{C}_{1}$ and $\mathbf{C}_{2}$ have been defined relative to a CS deterministic supervisor $\mathbf{S}=\left(X, \Sigma_{\mathbf{S}}, \xi, x_{o}, X_{m}\right)$, and we are only interested that they generate the same output with respect to input sequences that represent valid input strings to the supervisor (i.e. $s \in L(\mathbf{S}) \cap L_{\text {samp }}$ ). We first provide a definition for valid input sequences relative to TDES $\mathbf{S}$, and then a form of output equivalence definition for these sequences.

Definition 4.2.6. For system event set $\Sigma$, with canonical event mapping function $\gamma_{g}$, activity event set $\Sigma_{a c t}$, and CS deterministic TDES supervisor $\mathbf{S}=\left(X, \Sigma_{\mathbf{S}}, \xi, x_{o}, X_{m}\right)$, we say a canonical input sequence $\left\{i_{g}(k)\right\}$ is input valid for $\mathbf{S}$, if

$(\forall k \in\{1,2, \ldots\})\left(\exists s_{1}, s_{2}, \ldots, s_{k} \in L_{\text {conc }}\right)$

$\left[s_{1} s_{2} . . s_{k} \in L(\mathbf{S})\right] \wedge\left[(\forall n \in\{1,2, \ldots, k\})\left(\forall \sigma \in \Sigma_{a c t}\right) i_{g, \gamma_{g}(\sigma)}(n)=1\right.$ iff $\left.\sigma \in \operatorname{Occu}\left(s_{n}\right)\right]$

Essentially in the above definition, we are requiring the sequence $\left\{i_{g}(k)\right\}$ to correspond to a sequence of concurrent strings that supervisor $\mathbf{S}$ will accept. We are specifically excluding input sequences that our supervisor says will never occur. As we will see in the next section, when we translate a CS deterministic supervisor into a controller we will define next state information in an arbitrary manner for invalid input sequences. We will thus not be interested in whether two controllers generate the same output sequences for invalid input sequences.

We now provide a new output equivalence definition that is only concerned about input sequences that are valid for our supervisor.

Definition 4.2.7. For system event set $\Sigma$, with canonical event mapping function $\gamma_{g}$, activity event set $\Sigma_{a c t}$, and CS deterministic TDES supervisor $\mathbf{S}=\left(X, \Sigma_{\mathbf{S}}, \xi, x_{o}, X_{m}\right)$, let $\mathbf{C}_{j}=\left(I_{j}, Z_{j}, Q_{j}, \Omega_{j}, \Phi_{j}, \mathbf{q}_{r e s, j}\right), j=1,2$, be a controller. We say $\mathbf{C}_{1}$ and $\mathbf{C}_{2}$ are output equivalent with respect to $\mathbf{S}$ if for any canonical input sequence $\left\{\mathbf{i}_{g}(k)\right\}$ that is input valid for $\mathbf{S}$, and induced output $\mathbf{z}_{j}\left(k^{\prime}\right)=\left[z_{j, 1}\left(k^{\prime}\right), z_{j, 2}\left(k^{\prime}\right), . ., z_{j, r_{j}}\left(k^{\prime}\right)\right] \in Z_{j}$ at time $k^{\prime}=\{0,1,2, \ldots\}$, the follow conditions are satisfied.

1. $r_{1}=r_{2}$

2. $\left(\forall 0 \leq i<r_{1}\right) \eta_{1}^{-1}(i)=\eta_{2}^{-1}(i)$

3. $\left(\forall k^{\prime} \in\{0,1, .\}.\right) \mathbf{z}_{1}\left(k^{\prime}\right)=\mathbf{z}_{2}\left(k^{\prime}\right)$ 


\subsubsection{Centralized Controller}

We will now discuss how to translate a TDES supervisor into a centralized controller.

Let TDES supervisor $\mathbf{S}=\left(X, \Sigma, \xi, x_{o}, X_{m}\right)$ be CS deterministic and non-selfloop ALF. To translate $\mathbf{S}$ into a controller $\mathbf{C}=\left(I, Z, Q, \Omega, \Phi, \mathbf{q}_{r e s}\right)$, we need to introduce a few definitions.

We start by defining how many state variables are needed for $Q$. Let $X_{\text {samp }} \subseteq X$ be the set of sampling states for $\mathbf{S}$. To map each sampling state to a state in the controller, we define the state size of $Q, l$, to satisfy $2^{l-1}<\left|X_{\text {samp }}\right| \leq 2^{l}$. There are $l$ state variables in vector $\mathbf{q} \in Q$. A state in $\mathbf{S}$ which is not found in $X_{\text {samp }}$, does not correspond to any state variable assignment in $Q$.

We now define a function to map the sampling states of our TDES supervisor, onto states of the controller.

Definition 4.2.8. Let $\mathbf{S}=\left(X, \Sigma, \xi, x_{o}, X_{m}\right)$ be a CS deterministic supervisor. Let $\Lambda: X_{\text {samp }} \rightarrow Q$ be an arbitrary injective map where $X_{\text {samp }} \subseteq X$. We say $\Lambda$ is a state mapping function for controller $\mathbf{C}$ if, for all $x \in X_{\text {samp }}, \Lambda(x)$ returns a vector of state variables $\mathbf{q}=\left[q_{0}, q_{1}, . ., q_{l-1}\right]$ such that,

$$
\left(\forall x_{1}, x_{2} \in X_{\text {samp }}\right) \Lambda\left(x_{1}\right)=\Lambda\left(x_{2}\right) \Longleftrightarrow x_{1}=x_{2}
$$

Recall that the initial state is also a sampling state, and it is mapped to be $\Lambda\left(x_{o}\right)=\mathbf{q}_{\text {res }}=\mathbf{q}(0)$.

We now define a function that will map subsets of $\Sigma_{a c t}$ to a particular assignment of the variables for $I$ (called a valuation of $I$ ) that will represent the events present in the subset, according to the mapping defined by $\gamma$, the controller's input event mapping function. This will be useful for taking the occurrence image of a concurrent string and identifying the corresponding valuation that represents that subset in $I$.

Definition 4.2.9. Let $\mathbf{C}=\left(I, Z, Q, \Omega, \Phi, \mathbf{q}_{\text {res }}\right)$ be the corresponding controller for CS deterministic supervisor $\mathbf{S}=\left(X, \Sigma, \xi, x_{o}, X_{m}\right)$. The size of each input vector $\mathbf{i} \in I$ is defined to be $v=\left|\Sigma_{a c t}\right|$.

Let $\gamma$ be the input event mapping function for controller $\mathbf{C}$. Then we have a bijective map 


$$
\Gamma_{I}: \operatorname{Pwr}\left(\Sigma_{a c t}\right) \rightarrow I
$$

defined as follows. For arbitrary $\Sigma_{I} \subseteq \Sigma_{\text {act }}$, we have $\Gamma_{I}\left(\Sigma_{I}\right)=\left[i_{0}, i_{1}, . ., i_{v-1}\right]$ such that for $j=0,1, . ., v-1$,

$$
i_{j}:=\left\{\begin{array}{cc}
1 & \text { if }\left(\exists \sigma \in \Sigma_{I}\right) \gamma(\sigma)=j \\
0 & \text { otherwise }
\end{array}\right.
$$

We call $\Gamma_{I}$ the input set mapping function for controller $\mathbf{C}$.

The motivation for the above mapping is that at each sampling state, it will be observed which activity events have occurred, and which have not. Since the order of event occurrences is not stored, activity events are observed as if they are concurrent. Thus the occurrence of each event can be represented as a binary value in the corresponding position of the input vector $\mathbf{i}$.

We now define a function that will map subsets of $\Sigma_{h i b}$ to a particular assignment of the variables for $Z$ that will represent the events present in the subset, according to the mapping defined by $\eta$, the controller's output event mapping function. This will be useful for taking the set of prohibitable events eligible at a sampling state of the supervisor, and identifying the corresponding valuation that represents that subset in $Z$.

Definition 4.2.10. Let $\mathbf{C}=\left(I, Z, Q, \Omega, \Phi, \mathbf{q}_{\text {res }}\right)$ be the corresponding controller for CS deterministic supervisor $\mathbf{S}=\left(X, \Sigma, \xi, x_{o}, X_{m}\right)$. The size of each vector in $\mathbf{z} \in Z$ is defined to be $r=\left|\Sigma_{h i b}\right|$. Let $\eta$ be the output event mapping function for controller C. Then we have a bijective map

$$
\Gamma_{Z}: \operatorname{Pwr}\left(\Sigma_{h i b}\right) \rightarrow Z
$$

defined as follows. For arbitrary $\Sigma_{Z} \subseteq \Sigma_{h i b}$, we have $\Gamma_{Z}\left(\Sigma_{Z}\right)=\left[z_{0}, z_{1}, . ., z_{r-1}\right]$ such that for $j=0,1, . ., r-1$,

$$
z_{j}:=\left\{\begin{array}{cc}
1 & \text { if }\left(\exists \sigma \in \Sigma_{Z}\right) \eta(\sigma)=j \\
0 & \text { otherwise }
\end{array}\right.
$$

We call $\Gamma_{Z}$ the output set mapping function for controller $\mathbf{C}$. 
We now discuss how to define the next state function $\Omega$ for our controller, using our CS deterministic supervisor as our starting point. Note that the $\Delta$ function was defined in Section 3.1.

Definition 4.2.11. Let $\mathbf{C}=\left(I, Z, Q, \Omega, \Phi, \mathbf{q}_{\text {res }}\right)$ be the corresponding controller for CS deterministic supervisor $\mathbf{S}=\left(X, \Sigma, \xi, x_{o}, X_{m}\right)$. Let $X_{\text {samp }} \subseteq X$. For state $\mathbf{q} \in Q$ and arbitrary input $\mathbf{i} \in I$, the next state function $\Omega$ is defined to be

$$
\Omega(\mathbf{q}, \mathbf{i})=\Lambda\left(\Delta\left(x, \Gamma_{I}^{-1}(\mathbf{i})\right)\right) \quad \text { if }\left(\exists x \in X_{\text {samp }}\right) \mathbf{q}=\Lambda(x) \& \Delta\left(x, \Gamma_{I}^{-1}(\mathbf{i})\right) !
$$

All remaining values of $\Omega$ are assigned arbitrarily.

Essentially, we define $\Omega$ in terms of $\xi$, the next state function of TDES S. For the given state $\mathbf{q}$ of our controller and input $\mathbf{i}$ which are some valuations of sets $Q$ and $I$, we define the next state of the controller to match that of the supervisor. We define $\Omega(\mathbf{q}, \mathbf{i})$ arbitrarily unless our state $\mathbf{q}$ corresponds to a sampled state $x$ in $\mathbf{S}$, there exists a concurrent string $s$ whose occurrence image matches the set of activity events represented by $\mathbf{i}$, and $\xi(x, s)$ ! in our supervisor. In that case, our new state is $\mathbf{q}^{\prime}=\Lambda(\xi(x, s))$ as per the definition of $\Delta$. If there does not exist such an $x$ and $s$, that means $\mathbf{q}$ and $\mathbf{i}$ do not correspond to possible behavior of our system, so we can define the next state as we like (note $\xi$ is a partial function, but $\Omega$ must be a total function).

In practice, we would not assign the next state randomly. Most likely, we would choose $\mathbf{q}^{\prime}$ to either make our controller simpler, or we would choose $\mathbf{q}^{\prime}$ in a failsafe manner. By failsafe, we mean that we do not believe the combination $\mathbf{q}$ and $\mathbf{i}$ should ever be seen in the physical system, but we will choose our next state in a way to maximize safety should it actually ever occur.

We now discuss how to define the output map $\Phi$ for our controller, using our CS deterministic supervisor as our starting point. Note that the $\zeta$ function was defined in Section 3.2 .

Definition 4.2.12. Let $\mathbf{C}=\left(I, Z, Q, \Omega, \Phi, \mathbf{q}_{\text {res }}\right)$ be the corresponding controller for CS deterministic supervisor $\mathbf{S}=\left(X, \Sigma, \xi, x_{o}, X_{m}\right)$. Let $\zeta(x)$ be the control action for any sampling state $x \in X_{\text {samp }} \subseteq X$ as defined in Definition 3.2.3. For any $\mathbf{q} \in Q$, the output map $\Phi$ is defined to be 


$$
\Phi(\mathbf{q}):=\left\{\begin{array}{cc}
\Gamma_{Z}(\zeta(x)) & \text { if }\left(\exists x \in X_{\text {samp }}\right) \mathbf{q}=\Lambda(x) \\
\Gamma_{Z}(\emptyset) & \text { otherwise }
\end{array}\right.
$$

The definition states that if state $\mathbf{q}$ in controller $\mathbf{C}$ has a corresponding state $x \in X_{\text {samp }}$ in $\mathbf{S}$, then $\Phi(\mathbf{q})$ specifies an output vector based on the control action $\zeta(x)$. $\zeta(x)$ is equal to the set of prohibitable events enabled at state $x$ in $\mathbf{S}$. Otherwise, $\Phi(\mathbf{q})$ leaves all prohibitable events disabled at state $\mathbf{q}$.

Let TDES $\mathbf{S}=\left(X, \Sigma, \xi, x_{o}, X_{m}\right)$ be a CS deterministic supervisor. Then Figure 4.2 shows the control equivalence diagram for $\mathbf{S}$ and its controller $\mathbf{C}=\left(I, Z, Q, \Omega, \Phi, \mathbf{q}_{\text {res }}\right)$, as defined in this section. If, for arbitrary $\Sigma^{\prime} \subseteq \Sigma_{\text {act }}$ and state $x \in X_{\text {samp }}$ of $\mathbf{S}, \Delta\left(x, \Sigma^{\prime}\right)$ is defined, it is easy to see that this diagram commutes.

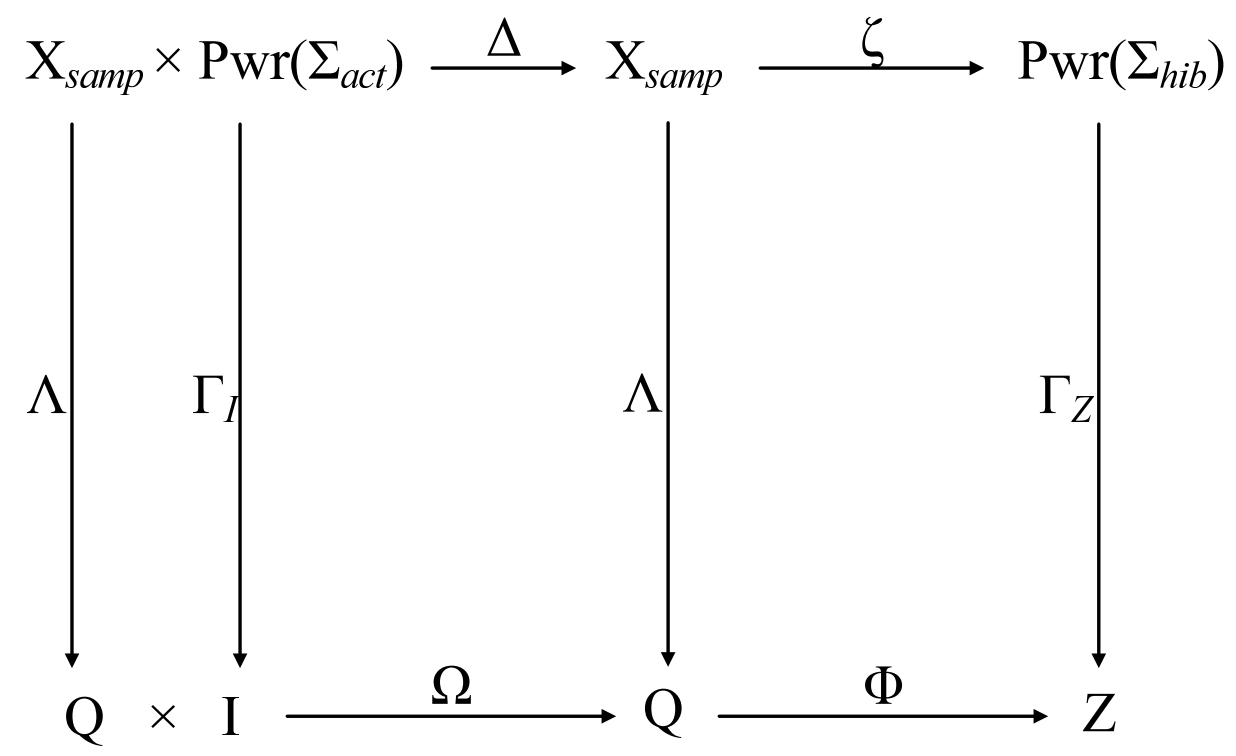

Figure 4.2: Centralized Control Equivalence Diagram

Essentially, the diagram says that as long as $\Delta\left(x, \Sigma^{\prime}\right)$ !, then $\Gamma_{Z}\left(\zeta\left(\Delta\left(x, \Sigma^{\prime}\right)\right)\right)=$ $\Phi\left(\Omega\left(\Lambda(x), \Gamma_{I}\left(\Sigma^{\prime}\right)\right)\right.$ meaning that we can just use the next state function and output map of the controller, and we will produce the correct enablement. Note that the $\Sigma^{\prime}$ represent the occurrence image (minus tick) of the concurrent strings defined at the given sampled state. The figure also says that if $\Delta\left(x, \Sigma^{\prime}\right)$ !, then $\Lambda\left(\left(\Delta\left(x, \Sigma^{\prime}\right)\right)\right)=$ $\Omega\left(\Lambda(x), \Gamma_{I}\left(\Sigma^{\prime}\right)\right.$, meaning that we can simply use the controller's next state function to determine the correct next state. 
Example 4.3. Let $\mathbf{C}=\left(I, Z, Q, \Omega, \Phi, \mathbf{q}_{\text {res }}\right)$ be represented by the Moore machine shown in Figure 4.1(b). We see from Figure 4.1(a), that our set of activity events is $\left\{\alpha_{1}, \alpha_{2}, \beta, \mu_{1}, \mu_{2}, \lambda\right\}$, and our set of prohibitable events are $\left\{\alpha_{1}, \alpha_{2}, \mu_{1}, \mu_{2}\right\}$. We can also see that the TDES is ALF, and CS deterministic.

We have each $\mathbf{i} \in I$ in the form of

$$
\mathbf{i}=\left[i_{0}, i_{1}, i_{2}, i_{3}, i_{4}, i_{5}\right]
$$

For $j=0,1, . ., 5, i_{j}$ corresponds to the occurrence of events $\left[\alpha_{1}, \alpha_{2}, \mu_{1}, \mu_{2}, \beta, \lambda\right]$ respectively, when $i_{j}=1$.

We have each $\mathbf{z} \in Z$ in the form of

$$
\mathbf{z}=\left[z_{0}, z_{1}, z_{2}, z_{3}\right]
$$

For $j=0,1, . ., 3, z_{j}$ corresponds to the enablement of prohibitable events $\left[\alpha_{1}, \alpha_{2}, \mu_{1}, \mu_{2}\right]$, when $z_{j}=1$.

We see from Figure 4.1(a) that our TDES has three sampled states. Our state size, $l$, must thus satisfy $2^{l-1}<3 \leq 2^{l}$. As only $l=2$ satisfies this equation, our state set must have two binary elements. We thus have each $\mathbf{q} \in Q$ in form of

$$
\mathbf{q}=\left[q_{0}, q_{1}\right]
$$

We will let state $\left(q_{0}, q_{1}\right) \in\{(0,0),(0,1),(1,0)\}$ represent states $\{I, W, D\}$ respectively. The fourth state $(1,1)$ is unused and will be unreachable, so we can define transition leaving this state arbitrarily.

Examining Figure 4.1(a), we can determine which concurrent strings are defined at each sampled state. For instance, at state $I$ we could only get strings $\alpha_{1} \alpha_{2} \tau$ or $\alpha_{2} \alpha_{1} \tau$. Both have occurrence image $\left\{\alpha_{1}, \alpha_{2}, \tau\right\}$ and take us to sampled state $W$. As this subset corresponds to $\mathbf{i}=[1,1,0,0,0,0]$, we can see where the transition at state $(0,0)$ in Figure 4.1(b) comes from. Continuing this logic, we can derive the remaining transitions for the SD controller shown in Figure 4.1(b). Note, that we have added the $\boldsymbol{D E F}$ default transitions as we discussed in Section 4.1.

Next,

$$
\mathbf{q}_{\text {res }}=\mathbf{q}(0)=[0,0]
$$


Using the information we have derived for Figure 4.1(b), we can define the next state function, $\Omega$, as below:

$$
\mathbf{q}(k+1)=\Omega(\mathbf{q}(k), \mathbf{i}(k+1))=\Omega\left(\left[q_{0}(k), q_{1}(k)\right],\left[i_{0}(k+1), i_{1}(k+1), . ., i_{5}(k+1)\right]\right)
$$

such that

$$
\begin{aligned}
\Omega([0,0],[1,1,0,0,0,0]) & =[0,1] \\
\Omega([0,0], \mathbf{i}) & =[0,0] \text { for all other } \mathbf{i} \in I \\
\Omega([0,1],[0,0,0,0,1,0]) & =[0,0] \\
\Omega([0,1],[0,0,0,0,0,1]) & =[1,0] \\
\Omega([0,1], \mathbf{i}) & =[0,1] \text { for all other } \mathbf{i} \in I \\
\Omega([1,0],[0,0,1,1,0,0]) & =[0,0] \\
\Omega([1,0], \mathbf{i}) & =[1,0] \text { for all other } \mathbf{i} \in I
\end{aligned}
$$

and $\Omega([1,1], \mathbf{i})$, for any $\mathbf{i} \in I$, can be defined arbitrarily as state $[1,1]$ is unreachable.

Lastly, we define the output function to be

$$
\mathbf{z}=\Phi(\mathbf{q})
$$

such that

$$
\begin{aligned}
& \Phi([0,0])=[1,1,0,0] \\
& \Phi([0,1])=[0,0,0,0] \\
& \Phi([1,0])=[0,0,1,1]
\end{aligned}
$$

We can define $\Phi([1,1])$ arbitrarily, say $\Phi([1,1])=[0,0,0,0]$.

The execution of a centralized controller $\mathbf{C}$ is as follows.

1. Initialize the controller by setting $\mathbf{q}(0)=\mathbf{q}_{\text {res }}, \mathbf{z}=\Phi\left(\mathbf{q}_{\text {res }}\right)$. We have $k=0$.

2. At the next clock pulse

i) sample inputs and set $\mathbf{i}(k+1)$ equal to these values.

ii) calculate our new state and output as follows:

i.e. $\mathbf{q}(k+1)=\Omega(\mathbf{q}(k), \mathbf{i}(k+1))$ and $\mathbf{z}(k+1)=\Phi(\mathbf{q}(k+1))$ 
3. Set $k=k+1$. Go to step 2 .

We say $\mathbf{C}$ acts on $\mathbf{G}$ when controller $\mathbf{C}$ enables or disables events from plant $\mathbf{G}$. Also, since an SD controller forces a prohibitable event as soon as its enabled, the controller is also forcing these events to occur in that clock period. To be consistent, if any controller $\mathbf{C}$ is discussed from now on, we will assume that it has been converted from some CS deterministic supervisor $\mathbf{S}$, using the translation method defined in this section.

Before we close this section, we would like to briefly discuss the case that our TDES supervisor $\mathbf{S}$ is defined over a subset $\Sigma_{S}$ of the system event set, $\Sigma$. This would mean that some activity events would not affect the next state of the controller and could be ignored, thus simplifying the next state logic of the controller. The output for the controller would still cover all events in $\Sigma_{h i b}$. The difference would be that for all $\sigma \in \Sigma_{h i b}-\Sigma_{\mathbf{S}}$, their corresponding output would always be set to 1 .

\subsubsection{Modular Controllers}

For large systems, the centralized supervisor for the system is quite likely large and complex. This would mean that its corresponding controller would also be large and complex, making implementing it directly undesirable. Just as we design modular TDES supervisors for systems to make the design more manageable, we can also implement our controllers by directly translating these modular supervisors into their own controllers. We can then combine the outputs of these controllers together, to create the overall output that would be equivalent to the output provided by a centralized controller.

To implement the composition of modular controllers, we need the following two operations on vectors.

Definition 4.2.13. Let $V$ be the set of Boolean vectors with each vector of size $n$. For $\mathbf{u}=\left[u_{1}, u_{2}, . ., u_{n}\right], \mathbf{v}=\left[v_{1}, v_{2}, . ., v_{n}\right] \in V$, the logical AND operator $\wedge: V \times V \rightarrow V$ is

$$
\mathbf{u} \wedge \mathbf{v}=\left[u_{1} \wedge v_{1}, u_{2} \wedge v_{2}, . ., u_{n} \wedge v_{n}\right]
$$


Definition 4.2.14. Let $\mathbf{u}$ be a Boolean vector of $i$ variables, and $\mathbf{v}$ be another Boolean vector of $j$ variables. The concatenation operator . : $V \times V \rightarrow V$ is defined as follows.

$$
\mathbf{u . v}=\left[u_{1}, u_{2}, . ., u_{i}, v_{1}, v_{2}, . ., v_{j}\right]
$$

For convenience, we will often just write uv instead.

Let the TDES $\mathbf{S}=\mathbf{S}_{1}\left\|\mathbf{S}_{2}\right\| . .|| \mathbf{S}_{n}$ be a supervisor where each modular supervisor $\mathbf{S}_{i}$, for $1 \leq i \leq n$, is CS deterministic.

To avoid implementing the likely large $\mathbf{S}$ directly, we wish to implement each supervisor $\mathbf{S}_{i}$ as controller $\mathbf{C}_{i}$, then combine the controllers $\mathbf{C}_{1}, \mathbf{C}_{2}, . ., \mathbf{C}_{n}$ (referred to as the composite controller) to generate the actual final output. We call each $\mathbf{C}_{i}$ the modular controller for supervisor $\mathbf{S}_{i}$. To be able to reuse the implementation technique discussed in the previous section, we assume each supervisor $\mathbf{S}_{i}$ is CS deterministic.

When comparing a centralized controller implementation to a modular controller implementation, all we care about is the output equivalence of the centralized controller and the composite controller created from $\mathbf{C}_{1}, \mathbf{C}_{2}, \ldots, \mathbf{C}_{n}$. If we take $\mathbf{S}$ and implement it directly as a controller $\mathbf{C}$, we want the composition of the outputs from $\mathbf{C}_{1}, \mathbf{C}_{2}, . ., \mathbf{C}_{n}$ to be equivalent to the output from $\mathbf{C}$.

We will now discuss how to implement the modular supervisors as individual controllers, and then combine them into a composite controller to handle the system. It is key to note that the modular supervisors may be defined over strict subsets of the system event set, $\Sigma$. Essentially, supervisor $\mathbf{S}_{j}$ will have activity event set $\Sigma_{a c t, j} \subseteq \Sigma_{a c t}$ and prohibitable event set $\Sigma_{h i b, j} \subseteq \Sigma_{h i b}$. To translate the CS deterministic supervisor $\mathbf{S}_{j}$ to a controller, we will use the method defined in Section 4.2.3, but the key difference is that we replace every $\Sigma_{a c t}$ in the definitions with $\Sigma_{a c t, j}$, and each $\Sigma_{h i b}$ with $\Sigma_{h i b, j}$. This means that the input and the output sets for the controller may only represent a subset of $\Sigma_{a c t}$ and $\Sigma_{h i b}$, respectively.

Definition 4.2.15. Let $\mathbf{G}$ be the plant to be controlled, $\gamma_{g}$ be the canonical event mapping function, $\Sigma$ be the system event set, $\Sigma_{a c t}$ the system activity event set, and $\Sigma_{h i b}$ the prohibitable event set. For $j=1,2, . ., n$, let $\mathbf{S}_{j}=\left(X_{j}, \Sigma_{j}, \xi_{j}, x_{o, j}, X_{m, j}\right)$ be the $j$-th CS deterministic supervisor, where $\Sigma_{j}=\Sigma_{a c t, j} \cup\{\tau\} \subseteq \Sigma$. Here we have $\Sigma_{a c t, j} \subseteq$ 
$\Sigma_{a c t}$ the activity event set for supervisor $\mathbf{S}_{j}, \Sigma_{h i b, j} \subseteq \Sigma_{h i b}$ the prohibitable event set for $\mathbf{S}_{j}$. We also require that $\Sigma=\bigcup_{j \in\{1,2, . ., n\}} \Sigma_{j}$. Then we define the composition of modular controllers as follows.

Let $\mathbf{C}_{j}=\left(I_{j}, Z_{j}, Q_{j}, \Omega_{j}, \Phi_{j}, q_{r e s, j}\right)$ be the controller for $\mathbf{S}_{j}$ with the following configuration:

- $l_{j}$ is the number of state variables for each $\mathbf{q}_{j}=\left[q_{j, 0}, q_{j, 1}, . ., q_{j, l_{j}-1}\right] \in Q_{j}$

- $v_{j}=\left|\Sigma_{a c t, j}\right|$ is number of input variables for each $\mathbf{i}_{j}=\left[i_{j, 0}, i_{j, 1}, . ., i_{j, v_{j}-1}\right] \in I_{j}$

- $r_{j}=\left|\Sigma_{h i b, j}\right|$ is number of output variables for each $\mathbf{z}_{j}=\left[z_{j, 0}, z_{j, 1}, . ., z_{j, r_{j}-1}\right] \in Z_{j}$ The composition of $\mathbf{C}_{1}, \mathbf{C}_{2}, . ., \mathbf{C}_{n}$,

$$
\mathbf{C}=\left(I, Z, Q, \Omega, \Phi, q_{\text {res }}\right)=\operatorname{comp}\left(\mathbf{C}_{1}, \mathbf{C}_{2}, . ., \mathbf{C}_{n}\right)
$$

is defined as follows.

1. $\Sigma_{a c t}=\bigcup_{j=1,2, . ., n} \Sigma_{a c t, j}$ and $\Sigma_{h i b}=\bigcup_{j=1,2, . ., n} \Sigma_{h i b, j}$, thus $\Sigma_{h i b} \subseteq \Sigma_{a c t} \subset \Sigma$ is guaranteed.

2. The number of state variables for vectors $\mathbf{q} \in Q$ is defined to be $l=\sum_{j=1}^{n} l_{j}$. The state vector $\mathbf{q}$ is defined to be

$$
\begin{aligned}
\mathbf{q} & =\mathbf{q}_{1} \mathbf{q}_{2} . . \mathbf{q}_{n} \\
& =\left[q_{1,0}, q_{1,1}, . ., q_{1, l_{1}-1}\right]\left[q_{2,0}, q_{2,1}, . ., q_{2, l_{2}-1}\right] . .\left[q_{n, 0}, q_{n, 1}, . ., q_{n, l_{n}-1}\right] \\
& =\left[q_{1,0}, q_{1,1}, . ., q_{1, l_{1}-1}, q_{2,0}, q_{2,1}, . ., q_{2, l_{2}-1}, . ., q_{n, 0}, q_{n, 1}, . ., q_{n, l_{n}-1}\right]
\end{aligned}
$$

3. The size of each input vector $\mathbf{i} \in I$ is defined to be $v=\left|\Sigma_{\text {act }}\right|$.

Then we define $\gamma: \Sigma_{a c t} \rightarrow\{0,1, . ., v-1\}$ to be the input event mapping function for $\mathbf{C}$ such that

$$
\left(\forall \sigma_{1}, \sigma_{2} \in \Sigma_{a c t}\right) \gamma_{g}\left(\sigma_{1}\right)<\gamma_{g}\left(\sigma_{2}\right) \Longrightarrow \gamma\left(\sigma_{1}\right)<\gamma\left(\sigma_{2}\right)
$$

4. The size of each output vector $\mathbf{z} \in Z$ is defined to be $r=\left|\Sigma_{h i b}\right|$.

Then we define $\eta: \Sigma_{h i b} \rightarrow\{0,1, . ., r-1\}$ to be the output event mapping function for $\mathbf{C}$ such that

$$
\left(\forall \sigma_{1}, \sigma_{2} \in \Sigma_{h i b}\right) \gamma_{g}\left(\sigma_{1}\right)<\gamma_{g}\left(\sigma_{2}\right) \Longrightarrow \eta\left(\sigma_{1}\right)<\eta\left(\sigma_{2}\right)
$$


5. The next state function $\Omega: Q \times I \rightarrow Q$ is defined such that, for $\mathbf{q}(k)=$ $\mathbf{q}_{1}(k) \mathbf{q}_{2}(k) . . \mathbf{q}_{n}(k) \in Q$ and $\mathbf{i}(k+1) \in I$,

$$
\begin{aligned}
\mathbf{q}(k+1) & =\Omega(\mathbf{q}(k), \mathbf{i}(k+1)) \\
& =\Omega_{1}\left(\mathbf{q}_{1}(k), \mathbf{i}_{1}(k+1)\right) \Omega_{2}\left(\mathbf{q}_{2}(k), \mathbf{i}_{2}(k+1)\right) \ldots \Omega_{n}\left(\mathbf{q}_{n}(k), \mathbf{i}_{n}(k+1)\right)
\end{aligned}
$$

For above, the input vector $\mathbf{i}(k+1)$ is in canonical form with respect to $\gamma_{g}$. To use it as an input to each controller $\mathbf{C}_{j}$, we need to map it to input vector $\mathbf{i}_{j}(k+1)$ using $\gamma_{j}$, the input event mapping function for Controller $\mathbf{C}_{j}$. To do this, we need to map input vector

$$
\mathbf{i}(k+1)=\left[i_{0}(k+1), i_{1}(k+1), . ., i_{v-1}(k+1)\right]
$$

onto input vectors $\mathbf{i}_{j}(k+1)=\left[i_{j, 0}(k+1), i_{j, 1}(k+1), . ., i_{j, v_{j}-1}(k+1)\right]$ for modular controller $\mathbf{C}_{j}$, as follows

$$
\left(\forall \sigma \in \Sigma_{a c t, j}\right) i_{j, \gamma_{j}(\sigma)}(k+1)=i_{\gamma_{g}(\sigma)}(k+1)
$$

6. The output map $\Phi: Q \rightarrow Z$ is defined as follows.

Given $\mathbf{q}=\mathbf{q}_{1} \mathbf{q}_{2} . . \mathbf{q}_{n} \in Q$, let

$$
\mathbf{z}_{j}=\Phi_{j}\left(\mathbf{q}_{j}\right)=\left[z_{j, 0}, z_{j, 1}, . ., z_{j, r_{j}-1}\right] \in Z_{j}
$$

For each $\mathbf{z}_{j}$ we expand it to

$$
\mathbf{z}_{j}^{\prime}=\left[z_{j, 0}^{\prime}, z_{j, 1}^{\prime}, . ., z_{j, r-1}^{\prime}\right] \in Z
$$

such that,

$$
\left(\forall \sigma \in \Sigma_{h i b}\right) z_{j, \eta(\sigma)}^{\prime}=\left\{\begin{array}{cc}
z_{j, \eta_{j}(\sigma)} & \text { if } \sigma \in \Sigma_{h i b, j} \\
1 & \text { otherwise }
\end{array}\right.
$$

In above, $\eta_{j}$ is the output event mapping for controller $\mathbf{C}_{j}$. Essentially, what we are doing is mapping the output value for $\mathbf{C}_{j}$ to the corresponding position in $\mathbf{z}_{j}^{\prime}$ if $\sigma \in \Sigma_{h i b, j}$, else we always set the value equal to 1 . 
With expanded output vectors $\mathbf{z}_{1}^{\prime}, \mathbf{z}_{2}^{\prime}, . ., \mathbf{z}_{n}^{\prime} \in Z$ defined, the next state function is then defined to be

$$
\Phi(\mathbf{q})=\bigwedge_{j \in\{1,2, . ., n\}} \mathbf{z}_{j}^{\prime}
$$

We simply logically AND each $\mathbf{z}_{j}^{\prime}$ together to obtain the output vector.

In Definition 4.2.15, we assumed that when the supervisors are combined together, they are defined over $\Sigma$, the systems event set (i.e. $\bigcup_{j=1,2, . ., n} \Sigma_{j}$ ). As for the centralized supervisor, it may be the case that the supervisors only care about a subset of $\Sigma$. This would mean that some activity events would not affect the next state of the controller and could be ignored, thus simplifying the next state logic of the controller. The output for the composite controller would still cover all events in $\Sigma_{h i b}$. The difference would be that for all $\sigma \in \Sigma_{\text {hib }}$ but not covered by any modular supervisor, their corresponding output would always be set to 1 .

We now present a theorem that shows that we can either implement our supervisor centrally or modularly, and we will get the same enablement information for valid input sequences.

Theorem 4.1. Let $\mathbf{G}$ be the plant to be controlled, $\gamma_{g}$ be the canonical event mapping function, $\Sigma$ be the system event set, $\Sigma_{a c t}$ the system activity event set, and $\Sigma_{h i b}$ the prohibitable event set. Also, let CS deterministic supervisor $\mathbf{S}=\left(X, \Sigma, \xi, x_{o}, X_{m}\right)$ be composed of $n$ component CS deterministic supervisor $\mathbf{S}_{j}=\left(X_{j}, \Sigma_{j}, \xi_{j}, x_{o, j}, X_{m, j}\right)$ for $j=1,2, . ., n$, such that $\mathbf{S}=\mathbf{S}_{1}\left\|\mathbf{S}_{2}\right\| . . \| \mathbf{S}_{n}$. Let $\Sigma_{a c t, j} \subseteq \Sigma_{a c t}$ and $\Sigma_{h i b, j} \subseteq \Sigma_{h i b}$ be the activity event set and prohibitable event set for $\mathbf{S}_{j}$.

For $j=1,2, . ., n$, let $\mathbf{C}_{j}=\left(I_{j}, Z_{j}, Q_{j}, \Omega_{j}, \Phi_{j}, \mathbf{q}_{r e s, j}\right)$ be the controller translated from $\mathbf{S}_{j}$ using translation method defined in Section 4.2.3 but replacing every $\Sigma_{a c t}$ in the definitions with $\Sigma_{a c t, j}$, and each $\Sigma_{h i b}$ with $\Sigma_{h i b, j}$. Let $\mathbf{C}^{\prime}=\operatorname{comp}\left(\mathbf{C}_{1}, \mathbf{C}_{2}, . ., \mathbf{C}_{n}\right)$ be the composed controller of $\mathbf{C}_{1}, \mathbf{C}_{2}, . ., \mathbf{C}_{n}$. Let $\mathbf{C}=\left(I, Z, Q, \Omega, \Phi, \mathbf{q}_{\text {res }}\right)$ be the controller translated from $\mathbf{S}$ using the translation method defined in Section 4.2.3.

Then $\mathbf{C}$ and $\mathbf{C}^{\prime}$ are output equivalent with respect to $\mathbf{S}$.

Proof. Assume the required initial conditions for the proof. 
Next, we need to define the following items for our proof, to ensure clarity.

Let $X_{\text {samp }} \subseteq X$ and $X_{s a m p, j} \subseteq X_{j}$ be the sets of sampling states for $\mathbf{S}$ and $\mathbf{S}_{j}$, respectively.

Let $\Delta: X_{\text {samp }} \times \operatorname{Pwr}\left(\Sigma_{a c t}\right) \rightarrow X_{\text {samp }}$ and $\Delta_{j}: X_{\text {samp }, j} \times \operatorname{Pwr}\left(\Sigma_{a c t, j}\right) \rightarrow X_{\text {samp }, j}$ be the next sampling state functions for $\mathbf{S}$ and $\mathbf{S}_{j}$, respectively.

Let $\Lambda: X_{\text {samp }} \rightarrow Q$ and $\Lambda_{j}: X_{\text {samp }, j} \rightarrow Q_{j}$ be the state mapping functions for $\mathbf{C}$ and $\mathbf{C}_{j}$, respectively.

Let $\Gamma_{I}: \operatorname{Pwr}\left(\Sigma_{a c t}\right) \rightarrow I$ and $\Gamma_{I, j}: \operatorname{Pwr}\left(\Sigma_{a c t, j}\right) \rightarrow I_{j}$ be the input set mapping functions for $\mathbf{C}$ and $\mathbf{C}_{j}$, respectively.

Let $\Gamma_{Z}: \operatorname{Pwr}\left(\Sigma_{h i b}\right) \rightarrow Z$ and $\Gamma_{Z, j}: \operatorname{Pwr}\left(\Sigma_{h i b, j}\right) \rightarrow Z_{j}$ be the output set mapping functions for $\mathbf{C}$ and $\mathbf{C}_{j}$, respectively.

Let $\gamma: \Sigma_{a c t} \rightarrow\{0,1, . ., v-1\}, \gamma^{\prime}: \Sigma_{a c t} \rightarrow\left\{0,1, . ., v^{\prime}-1\right\}, \gamma_{j}: \Sigma_{a c t, j} \rightarrow\left\{0,1, . . v_{j}-1\right\}$ be the input event mapping functions for $\mathbf{C}, \mathbf{C}^{\prime}$, and $\mathbf{C}_{j}$, respectively. We note that since $\gamma$ and $\gamma^{\prime}$ have domain $\Sigma_{a c t}$, they must both equal $\gamma_{g}$ due to how they are defined i.e. given a specific $\gamma_{g}$, there is only one way to define the other two functions and it must be the same as $\gamma_{g}$, if Definition 4.2 .2 is to be satisfied.

Let $\eta: \Sigma_{h i b} \rightarrow\{0,1, . ., r-1\}, \eta^{\prime}: \Sigma_{h i b} \rightarrow\left\{0,1, . . r^{\prime}-1\right\}, \eta_{j}: \Sigma_{h i b, j} \rightarrow\left\{0,1, . . r_{j}-1\right\}$ be the output event mapping functions for $C, C^{\prime}$, and $C_{j}$, respectively. We note that since $\eta$ and $\eta^{\prime}$ have domain $\Sigma_{h i b}$, they must be equal due to how they are defined (see Definition 4.2.3). This means that $Z$ and $Z^{\prime}$ represent the same prohibitable events, in the same order, and can be directly compared.

Given the above setting, we will now show that $\mathbf{C}$ and $\mathbf{C}^{\prime}$ are output equivalent with respect to $\mathbf{S}$.

Let $\left\{\mathbf{i}\left(k^{\prime \prime}\right)\right\}$ be a canonical input sequence with respect to $\gamma_{g}$, the canonical event mapping function for the system, and let the sequence be input valid with respect to S. From (1), we have $\gamma=\gamma^{\prime}=\gamma_{g}$. This means that $\left\{\mathbf{i}\left(k^{\prime \prime}\right)\right\}$ can be used as an input for both $\mathbf{C}$ and $\mathbf{C}^{\prime}$ directly, without any mapping required.

Let $\mathbf{z}^{\prime}(k)=\left[z_{1}^{\prime}(k), z_{2}^{\prime}(k), . ., z_{r^{\prime}}^{\prime}(k)\right] \in Z^{\prime}$ be the induced output vector in $\mathbf{C}^{\prime}$ at time $k$, from input sequence $\left\{\mathbf{i}\left(k^{\prime \prime}\right)\right\}$.

Let $\mathbf{z}(k)=\left[z_{1}(k), z_{2}(k), . ., z_{r}(k)\right] \in Z$ be the induced output vector in $\mathbf{C}$ at time $k$, from input sequence $\left\{\mathbf{i}\left(k^{\prime \prime}\right)\right\}$. 
We now need to show the following three points from Definition 4.2.7.

1. Show $r^{\prime}=r$

As both $\mathbf{C}$ and $\mathbf{C}^{\prime}$ are defined relative to $\Sigma$, their outputs are both defined relative to $\Sigma_{h i b}$. It follows immediately from the definition of $r^{\prime}$ and $r$ in Definition 4.1 .1 that $r^{\prime}=r$.

2. Show $(\forall 0 \leq i<r) \eta(i)=\eta^{\prime}(i)$

Let $i \in\{0,1, . ., r-1\}$, show $\eta(i)=\eta^{\prime}(i)$.

This follows immediately from (2).

3. Show $(\forall k \in\{0,1, .\}.) \mathbf{z}(k)=\mathbf{z}^{\prime}(k)$

(A) First we will show that if $\mathbf{C}$ is in state $\mathbf{q}=\Lambda(x)$ for some $x \in X_{\text {samp }}$, and each $\mathbf{C}_{j}$ is in state $\mathbf{q}_{j}=\Lambda_{j}\left(x_{j}\right)$ for some $x_{j} \in X_{\text {samp }}$ such that $x=$ $\left(x_{1}, x_{2}, . ., x_{n}\right)$, then $\mathbf{C}$ at state $\mathbf{q}$ and $\mathbf{C}^{\prime}$ at state $\mathbf{q}_{1} \mathbf{q}_{2} . . \mathbf{q}_{n}$ will have the same output.

(B) Then we will show for all $k \in\{0,1, .$.$\} that after inputs \mathbf{i}(1), \mathbf{i}(2), . ., \mathbf{i}(k)$ from our input sequence $\left\{\mathbf{i}\left(k^{\prime \prime}\right)\right\}, \mathbf{C}$ will be in state $\mathbf{q}(k)=\Lambda(x(k))$ for some $x(k) \in X_{\text {samp }}$, and $\mathbf{C}_{j}$ will be in state $\mathbf{q}_{j}(k)=\Lambda_{j}\left(x_{j}(k)\right)$ for some $x_{j}(k) \in X_{\text {samp }, j}$ such that $x(k)=\left(x_{1}(k), x_{2}(k), . ., x_{n}(k)\right)$.

Combining the two points will give the desired result.

Claim A: We will now prove point (A).

Let $\mathbf{C}$ be in state $\mathbf{q}=\Lambda(x)$ for some $x \in X_{\text {samp }}$.

Let each $\mathbf{C}_{j}$ be in state $\mathbf{q}_{j}=\Lambda_{j}\left(x_{j}\right)$ for some $x_{j} \in X_{s a m p, j}$.

Assume $x=\left(x_{1}, x_{2}, . ., x_{n}\right)$.

We thus have $\mathbf{C}^{\prime}$ at state $\mathbf{q}^{\prime}=\mathbf{q}_{1} \mathbf{q}_{2} . . \mathbf{q}_{n}$.

Let $\mathbf{z}=\Phi(\mathbf{q})$ and $\mathbf{z}^{\prime}=\Phi^{\prime}\left(\mathbf{q}^{\prime}\right)$. Must show $\mathbf{z}^{\prime}=\mathbf{z}$.

By Definition 4.2 .12 of the output map, we have for $\mathbf{C}$ that $\Phi(\mathbf{q})=\Gamma_{Z}(\zeta(x))$ 
The set of prohibitable events enabled at $\mathbf{q}$ can be represented as

$$
\begin{array}{rlr}
\Sigma_{Z} & =\zeta(x) & \\
& =\left\{\sigma \in \Sigma_{h i b} \mid \xi(x, \sigma) !\right\} & \text { by definition of } \zeta(x) \\
& =\bigcap_{j \in\{1,2, . ., n\}}\left\{\sigma \in \Sigma_{h i b} \mid\left(\sigma \notin \Sigma_{j}\right) \vee\left(\xi_{j}\left(x_{j}, \sigma\right) !\right)\right\} &
\end{array}
$$

by definition of synchronous product

We next note that by point (1) and (2), $\mathbf{C}$ and $\mathbf{C}^{\prime}$ represent exact the same events in $\Sigma_{h i b}$ in exactly the same order. It is sufficient to show that $\mathbf{C}^{\prime}$ enables the same event as $\mathbf{C}$.

By Definition 4.2.15, we have

$$
\mathbf{z}^{\prime}=\Phi^{\prime}\left(\mathbf{q}^{\prime}\right)=\bigwedge_{i \in\{1,2, . ., n\}} \mathbf{z}_{j}^{\prime}
$$

where $\mathbf{z}_{j}^{\prime}$ is the expanded output from controller $\mathbf{C}_{j}$.

As defined, an event is enabled in $\mathbf{z}_{j}^{\prime}$ if the event is enabled in $\mathbf{z}_{j}=\Phi_{j}\left(\mathbf{q}_{j}\right)$, or the event is not in $\Sigma_{h i b, j}$ and thus not in $\Sigma_{j}$. Otherwise, the event is disabled.

Therefore, the set of events enabled by $\mathbf{z}_{j}^{\prime}$ can be represented as

$$
\begin{aligned}
\Sigma_{Z, j}^{\prime} & =\zeta_{j}\left(x_{j}\right) \cup\left\{\Sigma_{h i b}-\Sigma_{h i b, j}\right\} \\
& =\left\{\sigma \in \Sigma_{h i b} \mid\left(\sigma \notin \Sigma_{j}\right) \vee\left(\xi_{j}^{\prime}\left(x_{j}, \sigma\right) !\right)\right\} \quad \text { by definition of } \zeta_{j}\left(x_{j}\right)
\end{aligned}
$$

The set of events enabled by $\mathbf{z}^{\prime}$ and thus $\mathbf{C}^{\prime}$ can be represented as

$$
\begin{aligned}
\Sigma_{Z}^{\prime} & =\bigcap_{j \in\{1,2, . ., n\}} \Sigma_{Z, j}^{\prime} \\
& =\bigcap_{j \in\{1,2, . ., n\}}\left\{\sigma \in \Sigma_{h i b} \mid\left(\sigma \notin \Sigma_{j}\right) \vee\left(\xi_{j}\left(x_{j}, \sigma\right) !\right)\right\} \\
& =\Sigma_{Z}
\end{aligned}
$$

Claim A proven.

Claim B: We will now prove point (B). 
We first consider $k=0$

By definition, $\mathbf{q}(0)=\mathbf{q}_{r e s}=\Lambda\left(x_{o}\right)$ and for each $\mathbf{C}_{j}, \mathbf{q}_{j}(0)=\mathbf{q}_{r e s, j}=\Lambda_{j}\left(x_{o, j}\right)$

We next note that initial states are always sampled states, so we have $x(0)=x_{o}$ and $x_{j}(0)=x_{o, j}$. Also, $x_{o}=\left(x_{o, 1}, x_{o, 2}, . ., x_{o, n}\right)$ by definition of the synchronous product. We note that input $\mathbf{i}(0)$ is ignored as the controller always starts at its reset state.

We now consider $k \in\{1,2, .$.

As $\left\{\mathbf{i}\left(k^{\prime \prime}\right)\right\}$ is input valid for $\mathbf{S}$, we know by definition that:

$(\forall k \in\{1,2, \ldots\})\left(\exists s_{1}, s_{2}, \ldots, s_{k} \in L_{\text {conc }}\right)\left[s_{1} s_{2} . . s_{k} \in L(\mathbf{S})\right] \wedge$

$$
\left[(\forall t \in\{1,2, \ldots, k\})\left(\forall \sigma \in \Sigma_{a c t}\right) i_{g, \gamma_{g}(\sigma)}(t)=1 \Leftrightarrow \sigma \in \operatorname{Occu}\left(s_{t}\right)\right]
$$

This implies that for $t \in\{1,2, . ., k\}, \operatorname{Occu}\left(s_{t}\right)=\Gamma_{I}^{-1}(\mathbf{i}(t))$

We thus have $\Delta\left(x(0), \Gamma_{I}^{-1}(\mathbf{i}(1))\right)=x(1) \in X_{\text {samp }}$.

We note that as $\mathbf{S}$ is CS deterministic, $x(1)=\xi\left(x_{o}, s_{1}\right)$ as any concurrent string with same occurrence image would come to the same state. We thus have $x(2)=\Delta\left(x(1), \Gamma_{I}^{-1}(\mathbf{i}(2))\right)$ with $x(2)=\xi\left(x_{o}, s_{1} s_{2}\right) \in X_{\text {samp }}$ and so on, until we have $x(k)=\Delta\left(x(k-1), \Gamma_{I}^{-1}(\mathbf{i}(k))\right)$ with $x(k)=\xi\left(x_{o}, s_{1} s_{2} . . s_{k}\right) \in X_{\text {samp }}$.

Let $P_{j}: \Sigma^{*} \rightarrow \Sigma_{j}^{*}$, where $j=1,2, . ., n$, be a natural projection.

By (3) and definition of the synchronous product, it follows that for $t \in\{1,2, . ., k\}$, $\operatorname{Occu}\left(P_{j}\left(s_{t}\right)\right)=\Gamma_{I, j}^{-1}\left(\mathbf{i}_{j}(t)\right)$ and $P_{j}\left(s_{1}\right) P_{j}\left(s_{2}\right) . . P_{j}\left(s_{t}\right) \in L\left(\mathbf{S}_{j}\right)$

By a similar logic as above, we have

$$
\begin{aligned}
& \Delta_{j}\left(x(0), \Gamma_{I, j}^{-1}\left(\mathbf{i}_{j}(1)\right)\right) \\
= & x_{j}(1) \\
= & \xi_{j}\left(x_{o, j}, P_{j}\left(s_{1}\right)\right) \in X_{s a m p, j}
\end{aligned}
$$

until we get

$$
x_{j}(k)=\Delta_{j}\left(x(k-1), \Gamma_{I, j}^{-1}\left(\mathbf{i}_{j}(k)\right)\right)
$$

with $x_{j}(k)=\xi_{j}\left(x_{o, j}, P_{j}\left(s_{1}\right) P_{j}\left(s_{2}\right) . . P_{j}\left(s_{k}\right)\right) \in X_{s a m p, j}$ By Definition 4.2.11 for $\Omega$, it is easy to see that $(\forall t \in\{1,2, . ., k\}) \mathbf{q}(t)=\Lambda(x(t))$ and $\mathbf{q}_{j}(t)=\Lambda_{j}\left(x_{j}(t)\right)$. 
By definition of the synchronous product

$$
\begin{aligned}
x(k)= & \xi\left(x_{o}, s_{1} s_{2} . . s_{k}\right) \\
= & \left(\xi_{1}\left(x_{o, 1}, P_{1}\left(s_{1}\right) P_{1}\left(s_{2}\right) . . P_{1}\left(s_{k}\right)\right),\right. \\
& \xi_{2}\left(x_{o, 2}, P_{2}\left(s_{1}\right) P_{2}\left(s_{2}\right) . . P_{2}\left(s_{k}\right)\right), . ., \\
& \left.\xi_{n}\left(x_{o, n}, P_{n}\left(s_{1}\right) P_{n}\left(s_{2}\right) . . P_{n}\left(s_{k}\right)\right)\right) \\
= & \left(x_{1}(k), x_{2}(k), . ., x_{n}(k)\right)
\end{aligned}
$$

Claim B proven.

Let $k \in\{0,1, .$.

We are now ready to show that $\mathbf{z}(k)=\mathbf{z}^{\prime}(k)$

We next note that by Claim $\mathrm{B}$, that after inputs $\mathbf{i}(0), \mathbf{i}(1), \ldots \mathbf{i}(k)$ from $\left\{\mathbf{i}\left(k^{\prime \prime}\right)\right\}$, controller $\mathbf{C}$ is in state $\mathbf{q}(k)=\Lambda(x(k))$ for some $x(k) \in X_{\text {samp }}$ and each $\mathbf{C}_{j}$ is in state $\mathbf{q}_{j}(k)=\Lambda_{j}\left(x_{j}(k)\right)$ for some $x_{j}(k) \in X_{\text {samp }, j}$ and $x(k)=\left(x_{1}(k), x_{2}(k), . ., x_{n}(k)\right)$.

We can now apply Claim A with $\mathbf{q}=\mathbf{q}(k)$ and each $\mathbf{q}_{j}=\mathbf{q}_{j}(k)$ for $j=1,2, \ldots, n$, and conclude that for $\mathbf{C}$ at state $\mathbf{q}(k)$ and $\mathbf{C}^{\prime}$ at state $\mathbf{q}_{1}(k) \mathbf{q}_{2}(k) . . \mathbf{q}_{n}(k)$, they will produce the same output. In other words, $\mathbf{z}(k)=\mathbf{z}^{\prime}(k)$, as required.

By steps 1., 2., and 3., we can thus conclude that $\mathbf{C}$ and $\mathbf{C}^{\prime}$ are output equivalent with respect to $\mathbf{S}$. 


\section{Chapter 5}

\section{Control and Nonblocking Verification}

A controller is more constrained than a supervisor. Every time an event occurs, the supervisor changes its state, but a controller reacts only on sampling instances (tick event). This means it is possible that the enablement information from the controller may not always be exactly the same as that of the supervisor's, as a supervisor can be more expressive in this regard. We want to make sure that the corresponding enablement information that the controller applies to the plant is such that the system's closed loop behavior (the actual behavior of the plant reacting to the controller's enablement information and the event forcing initiated by the controller) stays a subset of the desired behavior specified by the supervisor.

\subsection{Supervisory Control Construction}

First we have the following definition from [6].

Definition 5.1.1. A TDES supervisory control for $\mathbf{G}=\left(Y, \Sigma, \delta, y_{o}, Y_{m}\right)$ is any map $V: L(\mathbf{G}) \rightarrow \operatorname{Pwr}(\Sigma)$, such that,

$$
(\forall s \in L(\mathbf{G})) V(s) \supseteq\left\{\begin{array}{cl}
\Sigma_{u} \cup\left(\{\tau\} \cap \operatorname{Elig}_{L(\mathbf{G})}(s)\right) & \text { if } V(s) \cap \operatorname{Elig}_{L(\mathbf{G})}(s) \cap \Sigma_{h i b}=\emptyset \\
\Sigma_{u} & \text { if } V(s) \cap \operatorname{Elig}_{L(\mathbf{G})}(s) \cap \Sigma_{h i b} \neq \emptyset
\end{array}\right.
$$

From now on, we will just use the term supervisory control when it is clear by our context that we are referring to TDES. 
We will be requiring that prohibitable events can only occur at most once per sampling period. This is to simplify things a bit, but is primarily as we only decide to force an event once per clock cycle, it makes sense that the event only occurs once per clock cycle. If the controller has full control over when the event occurs, this is what will happen so the TDES behavior should reflect this. It makes it easier to keep track of things. Also, Point iii.1 of the SD controllability definition does not say anything about eligibility of $\Sigma_{h i b}$ events after they have occurred once. As we will see in the proofs in this section, this assumption will be a key part in making the proofs work.

Definition 5.1.2. For TDES $\mathbf{G}=\left(Y, \Sigma, \delta, y_{o}, Y_{m}\right)$, we say that $\mathbf{G}$ has singular prohibitable behavior if,

$$
\begin{aligned}
&\left(\forall s \in L(\mathbf{G}) \cap L_{\text {samp }}\right)\left(\forall s^{\prime} \in L_{\text {conc }}\right) s s^{\prime} \in L(\mathbf{G}) \\
& \Longrightarrow\left(\forall \sigma \in \operatorname{Occu}\left(s^{\prime}\right) \cap \Sigma_{\text {hib }}\right)\left(\exists s_{1}, s_{2} \in\left(\Sigma_{a c t}-\{\sigma\}\right)^{*}\right) s^{\prime}=s_{1} \sigma s_{2} \tau
\end{aligned}
$$

In other words, the above condition says that for TDES G, a prohibitable event is allowed to occur at most once per sampling period.

If TDES $\mathbf{G}$ is our plant and TDES $\mathbf{S}$ is our supervisor, we likely only care about checking this condition for strings in $L(\mathbf{S}) \cap L(\mathbf{G})$. We thus introduce the definition below. An example that fails the $\mathbf{S}$-singular prohibitable behavior property is shown in Figure 5.1. Here we see the prohibitable event $\alpha$ occurring twice in a sampling period.

Definition 5.1.3. For TDES $\mathbf{G}=\left(Y, \Sigma, \delta, y_{o}, Y_{m}\right)$ and $\operatorname{TDES} \mathbf{S}=\left(X, \Sigma, \xi, x_{o}, X_{m}\right)$, we say that $\mathbf{G}$ has $\mathbf{S}$-singular prohibitable behavior if

$$
\begin{aligned}
& \left(\forall s \in L(\mathbf{S}) \cap L(\mathbf{G}) \cap L_{\text {samp }}\right)\left(\forall s^{\prime} \in \Sigma_{\text {act }}^{*}\right) s s^{\prime} \in L(\mathbf{S}) \cap L(\mathbf{G}) \\
& \Longrightarrow\left(\forall \sigma \in \operatorname{Occu}\left(s^{\prime}\right) \cap \Sigma_{h i b}\right) \sigma \notin \operatorname{Elig}_{L(\mathbf{G})}\left(s s^{\prime}\right) \\
& \stackrel{\tau}{\longrightarrow} \mathrm{O} \stackrel{\alpha}{\longrightarrow} \mathrm{P} \stackrel{\beta}{\longrightarrow} \mathrm{O} \stackrel{\alpha}{\stackrel{\alpha}{\longrightarrow}} \mathrm{O} \stackrel{\tau}{\longrightarrow} \mathrm{O}
\end{aligned}
$$

Figure 5.1: An Example Failing S-singular Prohibitable Behavior Property

Let $\mathbf{G}=\left(Y, \Sigma, \delta, y_{o}, Y_{m}\right)$ be a TDES plant. For the rest of this chapter, we will require plant $\mathbf{G}$ to be complete for our supervisor $\mathbf{S}$, have proper time behavior and 
S-singular prohibitable behavior, and that $\operatorname{meet}(\mathbf{G}, \mathbf{S})$ be ALF. This will ensure that for any string $s \in L(\mathbf{G})$ (or $L(V / \mathbf{G})$ if $\mathbf{G}$ is not ALF on its own), we will always be able to reach a state where tick is possible after at most a finite number of activity events. In other words, we will not "stop the clock." This is important as it ensures that after every sampled string in our system has occurred, all new behavior can be represented as a series of concurrent strings.

Definition 5.1.4. We write $V / \mathbf{G}$ to represent $\mathbf{G}=\left(Y, \Sigma, \delta, y_{o}, Y_{m}\right)$ under the supervision of $V$. The closed behavior of $V / \mathbf{G}$ is defined to be $L(V / \mathbf{G}) \subseteq L(\mathbf{G})$ such that

1. $\epsilon \in L(V / \mathbf{G})$;

2. if $s \in L(V / \mathbf{G}), \sigma \in V(s)$ and $s \sigma \in L(\mathbf{G})$, then $s \sigma \in L(V / \mathbf{G})$;

3. no other strings are in $L(V / \mathbf{G})$.

It follows from the above definition, that $L(V / \mathbf{G})$ is prefix closed.

Let supervisor $\mathbf{S}=\left(X, \Sigma, \xi, x_{o}, X_{m}\right)$ be CS deterministic and SD controllable with respect to our plant $\mathbf{G}$. Let $\mathbf{C}=\left(I, Z, Q, \Omega, \Phi, \mathbf{q}_{\text {res }}\right)$ be a centralized controller translated from $\mathbf{S}$ using the method described in Section 4.2.3, with input and output event mapping functions $\gamma$ (see Definition 4.2.2) and $\eta$ (see Definition 4.2.3), and input and output set mapping functions $\Gamma_{I}$ (see Definition 4.2.9) and $\Gamma_{Z}$ (see Definition 4.2.10).

To verify that our controller $\mathbf{C}$ will generate the correct enablement information for our plant, we construct the corresponding supervisory control $V$ for $\mathbf{G}$. The idea is to express the enablement information that the controller would provide to the plant as a supervisory control. In particular, we wish to capture the idea that enablement information only changes after a tick, and then stays constant till the next tick. We also want to express the forcing information the controller provides to the plant, in particular the fact that as soon as a prohibitable event is enabled, the controller will force the event to occur within the current sampling period.

The construction of our supervisory control $V$ will be presented as an algorithm. We will use the logic in the algorithm to do the verification. First, we need to have the following definition. An important aspect of sampled strings is that they delineate 
the concurrent behavior of $\mathbf{G}$, which interprets how $\mathbf{G}$ moves from one sampling state to another.

Definition 5.1.5. For TDES $\mathbf{G}=\left(Y, \Sigma, \delta, y_{o}, Y_{m}\right)$, the concurrent behavior of $\mathbf{G}$ is defined to be a map $C B_{\mathbf{G}}: L(\mathbf{G}) \cap L_{\text {samp }} \rightarrow L_{\text {conc }}$, such that for $s \in L(\mathbf{G}) \cap L_{\text {samp }}$,

$$
C B_{\mathbf{G}}(s):=\left\{s^{\prime} \in L_{\text {conc }} \mid s s^{\prime} \in L(\mathbf{G})\right\}
$$

It states that the possible concurrent behavior for a TDES $\mathbf{G}$ after sampled string $s \in L(\mathbf{G}) \cap L_{\text {samp }}$, is the set of concurrent strings that can extend $s$ to a string in the closed behavior of $\mathbf{G}$.

We now discuss our conversion algorithm, labeled Algorithm 5.1. Given G and the controller $\mathbf{C}$ acting on $\mathbf{G}$, our algorithm constructs our supervisory control map $V$, by keeping track of how our controller changes state in response to strings generated by our plant. In our next section, we will show the map $V$ is well defined. We first describe some variables that we will use in our algorithm.

Pend $\subseteq L_{\text {samp }} \times Q$ is the set of pending $(s, \mathbf{q})$ pairs to be analyzed, where $s$ is a sampled string in $L(\mathbf{G})$, and $\mathbf{q}$ is the corresponding state in the controller that the sequence of inputs that would match the concurrent strings that make up $s$, unless of course $s=\epsilon$. If $s=\epsilon$, then $\mathbf{q}$ would be our reset state.

$\Sigma_{V}$ is the set of prohibitable events enabled by $V(s)$, for current sampled string $s$ that we are processing.

$\Sigma_{\text {temp }}$ is a copy of $\Sigma_{V}$ that we make when we are processing a concurrent string that extends the sampled string, $s$, that we are currently processing. This will be used to keep track of which prohibitable events in $\Sigma_{V}$ have not yet occurred in substrings of the concurrent strings that extend $s$ in $L(\mathbf{G})$.

Next, we will explain the statements in Algorithm 5.1 in detail. Note that Algorithm 5.1 may never terminate as the language $L(\mathbf{G})$ may not be finite, thus giving us a non-finite number of string-state pairs to evaluate. The algorithm merely describes abstractly how map $V$ is related to controller $\mathbf{C}$. We will then use this to compare the control behavior of $V$ to that of our supervisor, $\mathbf{S}$, that $\mathbf{C}$ was translated from. 


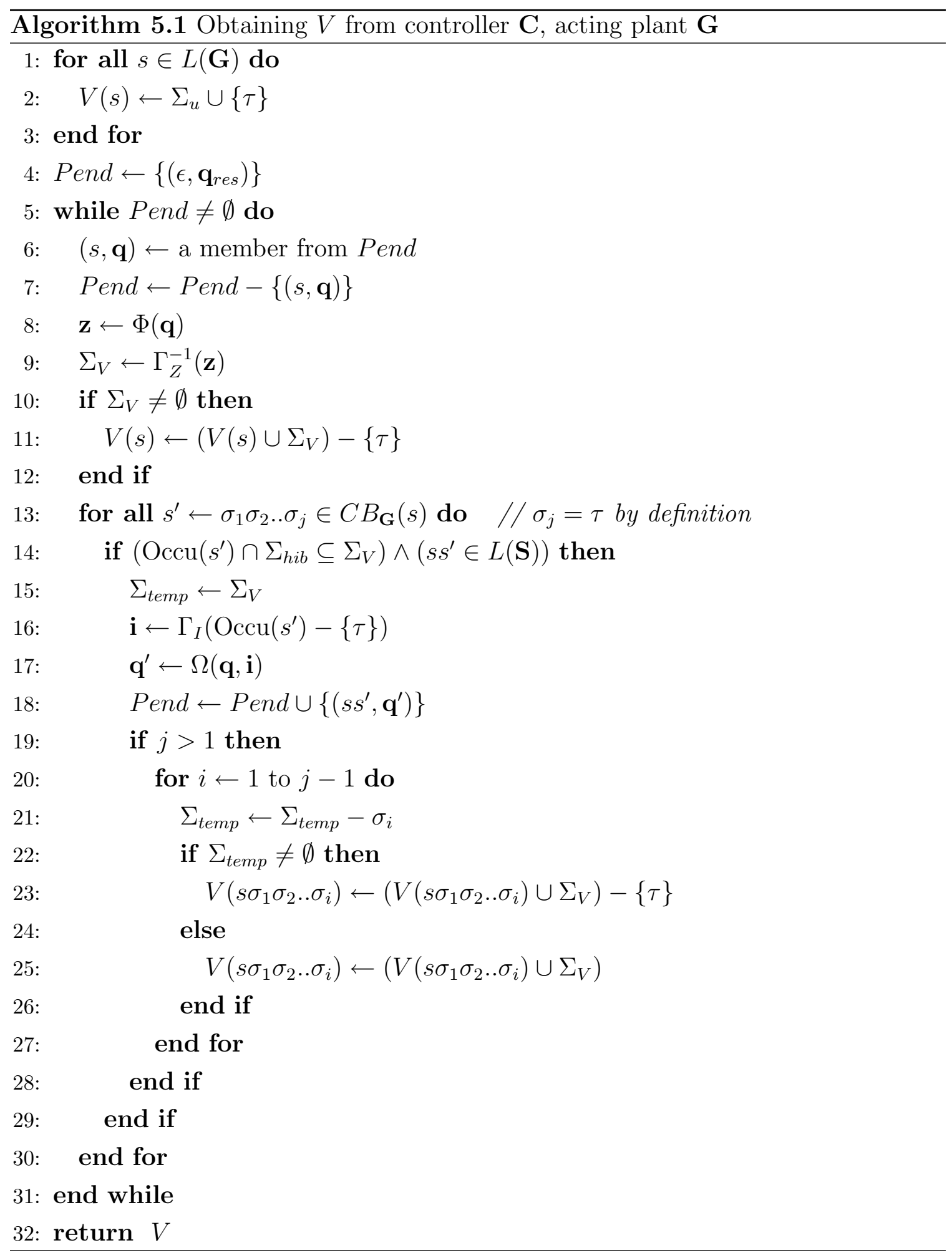


Initially, the for-loop from line 1 to line 3 includes all events $\sigma \in \Sigma_{u} \cup\{\tau\}$ in $V(s)$ for all $s \in L(\mathbf{G})$, to ensure all uncontrollable events are eligible in $V(s)$. This is needed to satisfy the controllability definition. This is the default setting for each possible string $s$. The tick event will be removed later, if we are suppose to be forcing an event.

A controller always starts operating at its reset state, so this will be the first state we will examine. As this corresponds to the empty string, our starting place is thus the tuple $(\epsilon, \mathbf{q})$. On line $\mathbf{4}$, we thus initialize our set of pending tuples to $(\epsilon, \mathbf{q})$.

The set Pend contains all the state-string pairs that have not been analyzed, and its members will be extracted one by one in the while-loop running from line 5 to line 31. There are two parts in the while-loop, where we process $V(s)$ and then $V\left(s \sigma_{1} \sigma_{2} . . \sigma_{i}\right)$ for $i<\left|s^{\prime}\right|, s^{\prime} \in C B_{\mathbf{G}}(s)$.

At line $\mathbf{6}$ in the while-loop, a member $(s, \mathbf{q})$ is extracted from the set Pend. This is the next tuple to be analyzed.

At line $\mathbf{8}$ output vector $\mathbf{z}$ is obtained from the current controller state $\mathbf{q}$ by applying output function $\Phi$. Vector $\mathbf{z}$ represents all the prohibitable events that the controller enables while it is at state $Q$. Then at line $\mathbf{9}$, all the prohibitable events enabled by the controller at current state $\mathbf{q}$ are included in $\Sigma_{V}$. This is done by using the inverse of the output set mapping function $\Gamma_{Z}^{-1}(\mathbf{q})$ from Definition 4.2.10,

At line 11 the enablement information $\Sigma_{V}$ is included in $V(s)$ for current sampled string $s$. As mentioned, the tick event included at line $\mathbf{2}$ is removed here in accordance to Point ii of the SD controllability definition (Definition 3.2.2). Basically, it says if we have eligible prohibitable events enabled, we must disable a tick and force the event. Of course, when we later show that the map $V$ we have defined is indeed a TDES supervisor control, we will have to show that these prohibitable events were eligible in $L(\mathbf{G})$ at this point.

The for-loop from line $\mathbf{1 3}$ to line $\mathbf{3 0}$ loops through all possible concurrent strings $s^{\prime}=\sigma_{1} \sigma_{2} . . \sigma_{j} \in C B_{\mathbf{G}}(s)$ (i.e. those that can extend $s$ in $L(\mathbf{G})$ ). First, it calculates the input vector, $\mathbf{i}$, that would correspond to $s^{\prime}$ occurring. This is done by using the controller's input event mapping function, $\Gamma_{I}$. We then use the controller's next state function, $\Omega$, to calculate $\mathbf{q}^{\prime}$, the state reached from $\mathbf{q}$ by input vector $\mathbf{i}$. Recall that $C B_{\mathbf{G}}(s)$ from Definition 5.1.5 is the concurrent behavior at state $\delta\left(y_{o}, s\right)$ in $\mathbf{G}$.

At line 14, we ignore concurrent strings whose occurrence images contain pro- 
hibitable events that are not in $\Sigma_{V}$. The reason is that these events have been disabled by the controller, so this represents behavior that will not occur in the closed loop system, so we just leave it at the default enablement information specified at line 2 .

We also ignore concurrent strings that do not represent behavior in $L(\mathbf{S})$, thus restricting the strings we can change from their line 2 defaults, to strings in $L(\mathbf{S}) \cap$ $L(\mathbf{G})$. The reason is that we later need to prove that our $V$ satisfies Definition 5.1.1, We will do this later by first showing that $L(V / \mathbf{G})=L(\mathbf{S}) \cap L(\mathbf{G})$, and then use the fact that $\mathbf{S}$ is $\mathrm{SD}$ controllable for $\mathbf{G}$.

At line 15, all prohibitable events in $\Sigma_{V}$ are copied to $\Sigma_{t e m p}$, which stores prohibitable events in $\Sigma_{V}$ that have not yet occurred in this sampling period. At line 18, the new string-state pair $\left(s s^{\prime}, \mathbf{q}^{\prime}\right)$ is added to set Pend.

At line 19, the if statement checks if $s^{\prime}$ contains events other than tick. Since the only tick event in a concurrent string is the ending event, it only checks if $j>1$ for $j=\left|s^{\prime}\right|$. If so, we execute lines 20 to 27 .

In the inner most loop from line 20 to line 27, we analyze each substring $\sigma_{1} \sigma_{2} . . \sigma_{i}$, $i<j$.

For lines 22 to line 26, if there are still prohibitable events in $\Sigma_{t e m p}$ that have not yet occurred, the map $V\left(s \sigma_{1} \sigma_{2} . . \sigma_{i}\right)$ has to remove the tick event since in our setting, enabling a prohibitable event also means we want to force it. Otherwise we leave the tick event in $V\left(s \sigma_{1} \sigma_{2} . . \sigma_{i}\right)$. In either case, we add $\Sigma_{V}$ to $V\left(s \sigma_{1} \sigma_{2} . . \sigma_{i}\right)$ since the enablement information of a controller is constant until the next tick event.

In the rest of the chapter, when we are discussing a system with plant $\mathbf{G}$, and $\mathrm{CS}$ deterministic TDES supervisor $\mathbf{S}$ that is SD controllable for $\mathbf{G}$, we will be concerned about the SD controller $\mathbf{C}$ that is constructed from $\mathbf{S}$ using the translation method described in Section 4.2, and TDES supervisory control $V^{1}$ that is constructed from C using Algorithm 5.1.

Definition 5.1.6. For plant $\mathbf{G}$, and CS deterministic supervisor $\mathbf{S}$ that is SD controllable for $\mathbf{G}$, let $\mathbf{C}$ be the SD controller that is constructed from $\mathbf{S}$ using the translation method described in Section 4.2, and $V$ be the map that is constructed from $\mathbf{C}$ using

\footnotetext{
${ }^{1}$ We still need to prove that our map $V$ is indeed a TDES supervisory control, and that the map is well defined. We will prove this in the following sections.
} 
Algorithm 5.1. The marked behavior of $V / \mathbf{G}$ is defined to be

$$
L_{m}(V / \mathbf{G}):=L(V / \mathbf{G}) \cap L_{m}(\mathbf{S}) \cap L_{m}(\mathbf{G})
$$

We say $V$ is nonblocking for $\mathbf{G}$ if

$$
\overline{L_{m}(V / \mathbf{G})}=L(V / \mathbf{G})
$$

That is, a nonblocking supervisory control $V$ for $\mathbf{G}$ can always reach a marked state in both $\mathbf{G}$ and $\mathbf{S}$ by extending the current string $s \in L(V / \mathbf{G})$.

\subsection{Map V Is Well Defined}

We want to show that the map $V$ constructed using Algorithm 5.1 is well defined for any possible string $s \in L(\mathbf{G})$ so that it can be considered as a possible supervisory control.

For example, Let TDES $\mathbf{G}$ be a plant defined over $\Sigma=\{\alpha, \beta, \gamma, \omega, \tau\}$ where $\tau=$ tick. Let $\Sigma_{h i b}=\{\alpha, \beta, \gamma\}$. Let $\mathbf{C}$ be the controller acting on $\mathbf{G}$. Imagine a part of $\mathbf{G}$ as shown in Figure 5.2 .

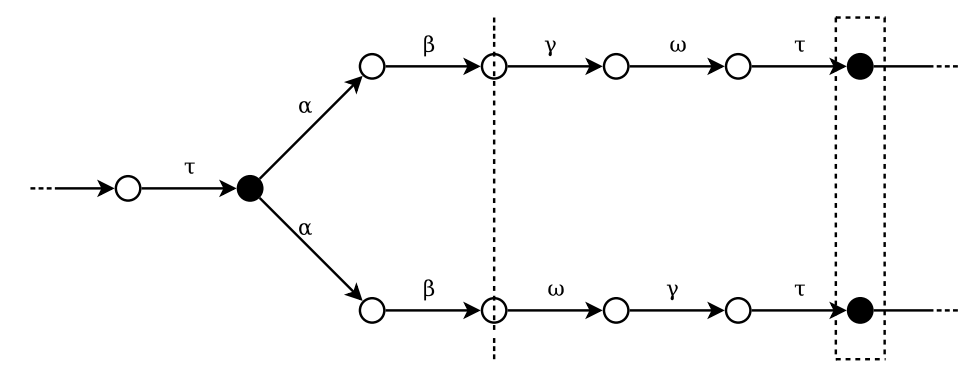

Figure 5.2: Part of a TDES plant

In the figure, let $s \in L(\mathbf{G})$ be the string taking us to the left most sampling state. We see there are two concurrent strings $s_{1}^{\prime}=\alpha \beta \gamma \omega \tau$ and $s_{1}^{\prime}=\alpha \beta \omega \gamma \tau$ extending $s$ in different paths so that $s s_{1}^{\prime}, s s_{2}^{\prime} \in L(\mathbf{G})$. Let $\hat{s}=\alpha \beta$ to be the prefix of both $s_{1}^{\prime}$ and $s_{2}^{\prime}$. Since Algorithm 5.1 will evaluate $V(s \hat{s})$ twice (lines 22 to 26), we want to make sure each time $V(s \hat{s})$ is assigned the same control action for both paths in the figure. We also need to make sure that every string $s \in L_{\text {samp }} \cap L(\mathbf{G})$ is either evaluated once, or is always associated with the same state $\mathbf{q}$ of the controller. We then have the following proposition to be proven. 
Proposition 5.1. For plant $\mathbf{G}=\left(Y, \Sigma, \delta, y_{o}, Y_{m}\right)$, and CS deterministic supervisor $\mathbf{S}=\left(X, \Sigma, \xi, x_{o}, X_{m}\right)$ that is SD controllable for $\mathbf{G}$, let $\mathbf{C}$ be the SD controller that is constructed from $\mathbf{S}$ using the translation method described in Section 4.2.3, and $V$ be map that is constructed from $\mathbf{C}$ using Algorithm 5.1. Then, map $V$ is well defined.

Proof. Assume initial conditions for proposition.

To show that $V$ is well defined, we need to show that for every $s \in L(\mathbf{G})$, our algorithm will define $V(s)$ in only one way.

From the definition of Algorithm 5.1 , it is clear that for all $s \notin \overline{L(\mathbf{S}) \cap L(\mathbf{G}) \cap L_{\text {samp }}}$, the algorithm only defines $V(s)$ exactly once on line $\mathbf{2}$.

This means we only have to examine strings $s \in \overline{L(\mathbf{S}) \cap L(\mathbf{G}) \cap L_{\text {samp }}}$.

Let $s \in \overline{L(\mathbf{S}) \cap L(\mathbf{G}) \cap L_{\text {samp }}}$.

Further examination of Algorithm 5.1 shows that $V(s)$ is only updated on line 11, line 23 and line 25, if at all.

Examining these cases, we see that if $s \in L_{\text {samp }}$, it will only be updated at line 11. Otherwise, it could be updated once or more at line 23 or line $\mathbf{2 5 .}$

Case A) $s \in L_{\text {samp }}$

We first note that we only care about sampled strings that are added to Pend. If $s$ is never added to Pend, it is only defined at line $\mathbf{2}$ and is never updated, thus is uniquely defined. We can thus assume that $\mathrm{s}$ is added at some point to Pend, without loss of generality.

To show that there is only one way to define $V(s)$, it is sufficient to show that whenever line 11 was executed for $s, \Sigma_{V}$ was always the same. Clearly, as long as $\Sigma_{V}$ is the same, then executing line $\mathbf{1 1}$ again will produce the same result as the first time. As $\Sigma_{V}$ is uniquely defined by state $\mathbf{q}$ of controller $\mathbf{C}$, it is thus sufficient to show that string $s$ will always be paired with state $\mathbf{q}$.

If $s=\epsilon$, then by definition this is always paired with state $\mathbf{q}_{r e s}$. Studying the algorithm, it is easy to see this is the case. We thus need only consider the case of $s \in \Sigma^{*} \cdot \tau$.

Examining Algorithm 5.1, we can see that every such string in Pend, is constructed by concatenating one or more concurrent strings together. 
For $s$, we thus have:

$$
(\exists n \in\{1,2, . .\})\left(\exists s_{1}, s_{2}, . ., s_{n} \in L_{\text {conc }}\right) s_{1} s_{2} . . s_{n}=s
$$

As $L_{\text {conc }}=\Sigma_{\text {act }}^{+} \tau$, there is only way to define strings $s_{1}$ to $s_{n}$.

Examining line 16 and line 17 of the algorithm, we see how starting with $\mathbf{q}_{r e s}$, each new state would be calculated using the next concurrent string in the list. Examining the definition of $\Gamma_{I}$ and $\Omega$ from Section 4.2.3, and $\Delta$ from Section 3.1 , we can see that since supervisor $\mathbf{S}$ is CS deterministic and $s \in L(\mathbf{S}) \cap L_{\text {samp }}$, this sequence of states is unique, meaning the final state $\mathbf{q}$ associated with $s$ is unique for controller C.

We thus conclude that we will always associate the same state $\mathbf{q}$ with $s$, thus the same set $\Sigma_{V}$.

Case B) $s \notin L_{\text {samp }}$

This implies $\left(\exists t \in L_{\text {samp }}\right)\left(\exists \hat{t} \in L_{\text {conc }}\right) t<s<t \hat{t}$

We note that this implies $(\exists j>1)\left(\exists \sigma_{1}, \sigma_{2}, . ., \sigma_{j} \in \Sigma\right) \hat{t}=\sigma_{1} \sigma_{2} . . \sigma_{j}$

We thus have $(\exists i \in\{1,2, . ., j-1\}) t \sigma_{1}, \sigma_{2}, . ., \sigma_{i}=s$

Note that in above, we have $j>1$ since as $t<s<t \hat{t}, j=0,1$ would cause a contradiction. Basically, $j=0$ would mean $\hat{t}=\epsilon$, thus $t \hat{t}=t$ and we could not have $t<s<t$. If we had $j=1$, we would have $\hat{t}=\tau$ as $\hat{t} \in L_{\text {conc }}$. As we require $t<s, s$ must contain at least one event more than $t$, but that would not also allow $s<t \hat{t}$ as $\hat{t}$ only contains one event. We thus must have $j>1$.

We note if for all such $\hat{t}$ they fail the condition on line 14, or if $\mathrm{t}$ was never added to Pend, then $V(s)$ will never be updated again, and will retain the value it was assigned on line $\mathbf{2}$. Thus, with no loss of generality, we can assume that t was added to Pend and our $\hat{t}$ passes the condition on line 14. We thus have $t, t \hat{t} \in L(\mathbf{S}) \cap L(\mathbf{G})$.

Given the definition of $L_{\text {conc }}$ and sampled strings, it is easy to see that there is only one way to define $\sigma_{1}, \sigma_{2}, . ., \sigma_{i}$, and thus sampled string $t$.

From Part A, we saw that for a given sampled string, there is only one way to define the corresponding $\Sigma_{V}$ set. Of course, it is possible that there are multiple ways to define $\sigma_{i+1} . . \sigma_{j}$.

Examining Algorithm 5.1, we see that the portion that we are concerned with corresponds to line 19 to line 28. Examining these lines, we see that the definition of $V(s)$ is determined only by $\Sigma_{V}$ and $t \sigma_{1}, \sigma_{2}, . ., \sigma_{i}$, which are unique for $s$. 
It thus follows that $V(s)$ is unique defined for our $s$.

By Case A and Case B, we have shown that $V$ is well defined.

\subsection{Supervisory Control and SD Supervisors}

Given the map $V$ constructed from $\mathbf{C}$ by Algorithm 5.1, we want show that the closed loop behavior $L(V / \mathbf{G})$ equals the behavior of $\operatorname{meet}(\mathbf{G}, \mathbf{S})$, i.e.

$$
L(V / \mathbf{G})=L(\mathbf{S}) \cap L(\mathbf{G})
$$

By Definition 5.1.4 for $L(V / \mathbf{G})$, we find that $\{\epsilon\} \subseteq L(V / \mathbf{G}) \subseteq L(\mathbf{G})$. We thus need to make sure that $L(\mathbf{G}) \neq \emptyset$. This is automatic as long as $\mathbf{G}$ has an initial state.

Theorem 5.1. For plant $\mathbf{G}=\left(Y, \Sigma, \delta, y_{o}, Y_{m}\right)$, and CS deterministic supervisor $\mathbf{S}=$ $\left(X, \Sigma, \delta, x_{o}, X_{m}\right)$ that is SD controllable for $\mathbf{G}$, let both TDES have finite statespaces, let $\mathbf{G}$ be complete for $\mathbf{S}$, have proper time and $\mathbf{S}$-singular prohibitable behavior, let $\operatorname{meet}(\mathbf{G}, \mathbf{S})$ be $\mathrm{ALF}$, let $\mathbf{C}=\left(I, Z, Q, \Omega, \Phi, \mathbf{q}_{\text {res }}\right)$ be the $\mathrm{SD}$ controller that is constructed from $\mathbf{S}$ using the translation method described in Section 4.2.3, and let $V$ be the map that is constructed from $\mathbf{C}$ using Algorithm 5.1. Then,

$$
L(V / \mathbf{G})=L(\mathbf{S}) \cap L(\mathbf{G})
$$

Proof. Assume assumptions in proposition setup.

To show $L(V / \mathbf{G})=L(\mathbf{S}) \cap L(\mathbf{G})$, we must

1. show $L(V / \mathbf{G}) \subseteq L(\mathbf{S}) \cap L(\mathbf{G})$

2. show $L(V / \mathbf{G}) \supseteq L(\mathbf{S}) \cap L(\mathbf{G})$

To show $\mathbf{1}$, must show $\mathbf{1 . 1}$ and $\mathbf{1 . 2}$ as follows.

1.1 show $L(V / \mathbf{G}) \subseteq L(\mathbf{G})$

This is automatic by Definition of $L(V / \mathbf{G})$ and the fact $\mathbf{G}$ contains an initial state. 
1.2 show $L(V / \mathbf{G}) \subseteq L(\mathbf{S})$

To show this, we must show

$$
(\forall s \in L(V / \mathbf{G})) s \in L(\mathbf{S})
$$

Let $s \in L(V / \mathbf{G})$. We can show it by induction as follows.

base case $s=\epsilon$.

As $\mathbf{S}$ contains an initial state, it follows that $\epsilon \in L(\mathbf{S})$.

inductive step We assume that $s=\sigma_{1} . . \sigma_{k} \in L(V / \mathbf{G}) \cap L(\mathbf{S})$ and $s \sigma_{k+1} \in$ $L(V / \mathbf{G})$ for some $k \geq 0$. We will now show this implies that

$$
s \sigma_{k+1} \in L(\mathbf{S})
$$

Since $\Sigma=\Sigma_{u} \dot{\cup} \Sigma_{c}=\Sigma_{u} \dot{\cup} \Sigma_{h i b} \dot{\cup}\{\tau\}$ by definition of TDES, we have 3 cases for $\sigma_{k+1} \in \Sigma$

(i) $\sigma_{k+1} \in \Sigma_{u}$

As $s \sigma_{k+1} \in L(V / \mathbf{G})$, it follows that $\sigma_{k+1} \in \operatorname{Elig}_{L(\mathbf{G})}(s) \cap \Sigma_{u}$ As $\mathbf{S}$ is

SD controllable for $\mathbf{G}$, it follows that $\sigma_{k+1} \in \operatorname{Elig}_{L(\mathbf{S})}(s)$, thus $s \sigma_{k+1} \in$ $L(\mathbf{S})$

(ii) $\sigma_{k+1}=\tau$

To show $\tau \in \operatorname{Elig}_{L(\mathbf{S})}(s)$, by Point ii in Definition 3.2.2 of SD controllability (since $\mathbf{S}$ is $\mathrm{SD}$ controllable for $\mathbf{G}$ ) we need to show

$$
\left(\tau \in \operatorname{Elig}_{L(\mathbf{G})}(s)\right) \wedge\left(\operatorname{Elig}_{L(\mathbf{S}) \cap L(\mathbf{G})}(s) \cap \Sigma_{h i b}=\emptyset\right)
$$

Since $L(V / \mathbf{G}) \subseteq L(\mathbf{G})$ as shown in $\mathbf{1 . 1}$, we have

$$
\begin{aligned}
\tau & \in \operatorname{Elig}_{L(V / \mathbf{G})}(s) \\
\Longrightarrow \tau & \in \operatorname{Elig}_{L(\mathbf{G})}(s)
\end{aligned}
$$

Now we need to show

$$
\operatorname{Elig}_{L(\mathbf{S}) \cap L(\mathbf{G})}(s) \cap \Sigma_{h i b}=\emptyset
$$


By default, the tick event is included in $V(s)$ at line 2. In the algorithm, tick is only removed if $\Sigma_{V} \neq \emptyset$ at line 11 or $\Sigma_{\text {temp }} \neq \emptyset$ at line 23.

We thus have four possibilities: a) $s \in L_{\text {samp }}, \Sigma_{V}=\emptyset$ and $s$ was added to Pend, b) $s \notin L_{\text {samp }}, \Sigma_{\text {temp }}=\emptyset$, and $V(s)$ is re-evaluated $\mathbf{c}$ ) $s \in L_{\text {samp }}$ and $s$ was not added to Pend, or d) $s \notin L_{\text {samp }}$ and $V(s)$ is not re-evaluated. We now examine these cases.

(ii.a) $s \in L_{\text {samp }}, \Sigma_{V}=\emptyset$, and $s$ was added to Pend.

As $s \in L_{\text {samp }}$, either it is the empty string, or $s \in \Sigma^{*} . \tau$. For the case $s=\epsilon$, we have $\Lambda\left(x_{o}\right)=\mathbf{q}_{\text {res }}$ (see Definition 4.2.8), which matches the state-string association that Algorithm 5.1 makes.

Otherwise, $s$ is composed of one or more concurrent strings. We thus have

$$
(\exists n \in 1,2, . .)\left(\exists s_{1}, s_{2}, . ., s_{n} \in L_{\text {conc }}\right) s_{1} s_{2} . . s_{n}=s
$$

As $L_{\text {conc }}=\Sigma_{\text {act }}^{+} \tau$, there is only way to define strings $s_{1}$ to $s_{n}$.

Based on the definitions from Section 4.2.3, we can determine the state in $\mathbf{C}$ that will correspond to string $s$, by starting with $\mathbf{q}_{r e s}$, and evaluating

$$
\Omega\left(\mathbf{q}_{r e s}, \Gamma_{I}\left(\operatorname{Occu}\left(s_{1}\right)-\{\tau\}\right)\right)=\mathbf{q}_{1}
$$

As $\mathbf{S}$ is CS deterministic, it follows from the definitions of $\Gamma_{I}$ and $\Omega$ that $\mathbf{q}_{1}=\Lambda\left(x_{1}\right)$, where $x_{1}=\xi\left(x_{o}, s_{1}\right)$. Note that we have $s_{1} \in L(\mathbf{S})$ as the language is closed.

By the same logic we have

$$
\Omega\left(\mathbf{q}_{1}, \Gamma_{I}\left(\operatorname{Occu}\left(s_{2}\right)-\{\tau\}\right)\right)=\mathbf{q}_{2}
$$

and $\mathbf{q}_{2}=\Lambda\left(x_{2}\right)$, where $x_{2}=\xi\left(x_{o}, s_{1} s_{2}\right)$. 
Extending this logic to the end, we have

$$
\Omega\left(\mathbf{q}_{n-1}, \Gamma_{I}\left(\operatorname{Occu}\left(s_{n}\right)-\{\tau\}\right)\right)=\mathbf{q}_{n}
$$

and $\mathbf{q}_{n}=\Lambda\left(x_{n}\right)$, where $x_{n}=\xi\left(x_{o}, s_{1} s_{2} . . s_{n}\right)$. To simplify the notation, we will take $\mathbf{q}=\mathbf{q}_{n}$ and $x=x_{n}$.

We thus have $\mathbf{q}$ the state the controller $\mathbf{C}$ will be in after string $s$, and $x \in X_{\text {samp }}$ the state that $\mathbf{S}$ will be in, while $\mathbf{q}=\Lambda(x)$. It is easy to see by the logic of Algorithm 5.1, that string $s$ will be paired with state q. See proof of Proposition 5.1 for more details.

We next note that the outputs at state $\mathbf{q}$ are $\mathbf{z}=\Phi(\mathbf{q})$. We thus have by Definition 4.2.12 that $\mathbf{z}=\Gamma_{Z}(\zeta(x))$.

By the definition of control action given in Definition 3.2.3, it follows that $\zeta(x)=\left\{\sigma \in \Sigma_{h i b} \mid \xi(x, \sigma) !\right\}$. As $\Sigma_{V}=\Gamma_{Z}^{-1}(\mathbf{z})$ as per line 9 of Algorithm 5.1, we thus have $\Sigma_{V}=\left\{\sigma \in \Sigma_{\text {hib }} \mid \xi(x, \sigma) !\right\}$. As we have $\Sigma_{V}=\emptyset$ by assumption, we thus have

$$
\left\{\sigma \in \Sigma_{h i b} \mid \xi(x, \sigma) !\right\}=\emptyset
$$

which implies

$$
\begin{aligned}
& \operatorname{Elig}_{L(\mathbf{S})}(s) \cap \Sigma_{h i b}=\emptyset \\
\Longrightarrow & \operatorname{Elig}_{L(\mathbf{S}) \cap L(\mathbf{G})}(s) \cap \Sigma_{h i b}=\emptyset
\end{aligned}
$$

as required.

Part (ii.a) complete.

(ii.b) $s \notin L_{\text {samp }}, \Sigma_{\text {temp }}=\emptyset$ and $V(s)$ is re-evaluated.

As $V(s)$ is re-evaluated and $s \notin L_{\text {samp }}$, then it follows from the logic of Algorithm 5.1 that

$$
\begin{array}{r}
\left(\exists t \in L_{\text {samp }} \cap L(\mathbf{S}) \cap L(\mathbf{G})\right)\left(\exists \hat{t} \in L_{\text {conc }}\right) \\
(t<s<t \hat{t}) \wedge(t \hat{t} \in L(\mathbf{S}) \cap L(\mathbf{G}))
\end{array}
$$


It also follows that:

$(\exists l \in\{1,2, .\}).\left(\exists \sigma_{1}, \sigma_{2}, . ., \sigma_{l} \in \Sigma_{a c t} \subset \Sigma\right) t \sigma_{1} \sigma_{2} . . \sigma_{l}=s$

Now, from the logic of part (ii.a), we know that string $t$ will be paired in Pend with a state $\mathbf{q}$, such that $\mathbf{q}=\Lambda(x)$, where $x=\xi\left(x_{o}, t\right)$. We thus have

$$
\begin{aligned}
& \Sigma_{V}=\operatorname{Elig}_{L(\mathbf{S})}(t) \cap \Sigma_{h i b} \\
\Longrightarrow & \operatorname{Elig}_{L(\mathbf{S}) \cap L(\mathbf{G})}(t) \cap \Sigma_{h i b} \subseteq \Sigma_{V}
\end{aligned}
$$

We now note that as $\mathbf{S}$ is SD controllable for $\mathbf{G}$, we have by Point iii.1 of Definition 3.2.2,

$$
\begin{aligned}
& {\left[\operatorname{Elig}_{L(\mathbf{S}) \cap L(\mathbf{G})}(s) \cup \operatorname{Occu}\left(\sigma_{1} \sigma_{2} . . \sigma_{l}\right)\right] \cap \Sigma_{h i b} } \\
= & \operatorname{Elig}_{L(\mathbf{S}) \cap L(\mathbf{G})}(t) \cap \Sigma_{h i b} \subseteq \Sigma_{V} \\
\Rightarrow & \left(\operatorname{Elig}_{L(\mathbf{S}) \cap L(\mathbf{G})}(s) \cup \operatorname{Occu}\left(\sigma_{1} \sigma_{2} . . \sigma_{l}\right)\right) \cap \Sigma_{h i b} \subseteq \Sigma_{V} \\
\Rightarrow & \operatorname{Elig}_{L(\mathbf{S}) \cap L(\mathbf{G})}(s) \cap \Sigma_{h i b} \subseteq \Sigma_{V}
\end{aligned}
$$

It follows from the logic of Algorithm 5.1 and fact that $\Sigma_{t e m p}=\emptyset$, that

$$
\operatorname{Occu}\left(\sigma_{1} \sigma_{2} . . \sigma_{l}\right) \cap \Sigma_{h i b} \supseteq \Sigma_{V}
$$

In other words, every prohibitable event in $\Sigma_{V}$ has occurred at least once since $t$ (i.e. this sampling period).

As $t \in L(\mathbf{S}) \cap L(\mathbf{G})$ by (1), and $\mathbf{G}$ has $\mathbf{S}$-singular prohibitable behavior by our initial assumptions, it follows that the prohibitable events in $\Sigma_{V}$ cannot occur again in $\hat{t}$ (i.e. not until after next tick). This implies

$$
\operatorname{Elig}_{L(\mathbf{G})}(s) \cap \Sigma_{V}=\emptyset
$$

Since $\operatorname{Elig}_{L(\mathbf{S}) \cap L(\mathbf{G})}(s) \cap \Sigma_{h i b} \subseteq \Sigma_{V}$ by (2), it follows that

$$
\operatorname{Elig}_{L(\mathbf{S}) \cap L(\mathbf{G})}(s) \cap \Sigma_{h i b}=\emptyset
$$

as required.

Part (ii.b) complete. 
(ii.c) $s \in L_{\text {samp }}$ and $s$ not added to Pend

We will show that $s \in L_{\text {samp }}$ causes a contradiction and thus $s$ must be added to Pend. This means that case (ii.a) represents the only valid possibility, if $s \in L_{\text {samp }}$

We note that $L_{\text {samp }}=\Sigma^{*}$.tick $\cup\{\epsilon\}$. If $s=\epsilon$, then we know $\epsilon$ is always added to Pend (line 4 of Algorithm 5.1), so this section does not apply. We can thus assume $s \neq \epsilon$, and thus $s \in \Sigma^{*} . \tau$

Our goal is to show that $s$ will always be added to Pend, thus (ii.c) never applies.

As $s \in \Sigma^{*} . \tau$, if follows

$$
(\exists n \in\{1,2, . .\})\left(\exists s_{1}, s_{2}, . ., s_{n} \in L_{\text {conc }}\right) s_{1} s_{2} . . s_{n}=s
$$

To show that $s$ must be added to Pend, we need to show:

$$
\begin{aligned}
& (\forall l \in\{1,2, \ldots, n\}) \\
& \quad\left(s_{1} s_{2} . . s_{l} \in L(\mathbf{S}) \cap L(\mathbf{G})\right) \wedge\left(\operatorname{Occu}\left(s_{l}\right) \cap \Sigma_{h i b} \subseteq \Sigma_{V}\left(s_{1} s_{2} . . s_{l-1}\right)\right)
\end{aligned}
$$

where $\Sigma_{V}\left(s_{1} s_{2} . . s_{l-1}\right)$ is the value of $\Sigma_{V}$ at line 14 in the algorithm when sampled string $s_{1} s_{2} . . s_{l-1}$ is being evaluated.

Let $l \in\{1,2, \ldots, n\}$.

As $s \in L(\mathbf{S}) \cap L(\mathbf{G})$ by assumption, and $L(\mathbf{S})$ and $L(\mathbf{G})$ are closed languages, $s_{1} s_{2} . . s_{l} \in L(\mathbf{S}) \cap L(\mathbf{G})$ is automatic.

All that remains is showing

$$
\operatorname{Occu}\left(s_{l}\right) \cap \Sigma_{h i b} \subseteq \Sigma_{V}\left(s_{1} s_{2} . . s_{l-1}\right)
$$

We know from part (ii.a) that sampled string $s_{1} s_{2} . . s_{l-1}$ will always be paired with state $\mathbf{q}$ of the controller, with $\mathbf{q}=\Lambda(x)$, where $x=$ $\xi\left(x_{o}, s_{1} s_{2} . . s_{l-1}\right)$. This state $\mathbf{q}$ is the state the controller will be in after this string, thus $\Sigma_{V}\left(s_{1} s_{2} . . s_{l-1}\right)$ will be the enablement output (as per definition of Algorithm 5.1) of the controller until after the 
next tick occurs. That means for all $\sigma \in \Sigma_{h i b}-\Sigma_{V}\left(s_{1} s_{2} . . s_{l-1}\right), \sigma$ will be disabled until after concurrent string $s_{l}$ has occurred. As $s \in L(V / \mathbf{G})$ by assumption, we also have $s_{1} s_{2} . . s_{l} \in L(V / \mathbf{G})$ as $L(V / \mathbf{G})$ is closed and $s_{1} s_{2} . . s_{l} \leq s$. This means $s_{l}$ cannot contain any events in $\Sigma_{h i b}-\Sigma_{V}\left(s_{1} s_{2} . . s_{l-1}\right)$, thus

$$
\operatorname{Occu}\left(s_{l}\right) \cap \Sigma_{h i b} \subseteq \Sigma_{V}\left(s_{1} s_{2} . . s_{l-1}\right)
$$

We have thus shown that for $s=\epsilon$ or $s \in \Sigma^{*} . \tau$, it must have been added to Pend. This means that (ii.c) does not apply to $s$ at all, so string $s$ must be covered by case (ii.a).

Part (ii.c) complete.

(ii.d) $s \notin L_{\text {samp }}$ and $V(s)$ is not re-evaluated.

We now examine case of $s \notin L_{\text {samp }}$ and show that it must have been re-evaluated at line $\mathbf{2 3}$ or line $\mathbf{2 5}$, thus case (ii.b) is the only valid possibility for $s \notin L_{\text {samp }}$.

As $s \notin L_{\text {samp }}$, it follows that: $\left(\exists t \in L_{\text {samp }}\right)\left(\exists \hat{t} \in L_{\text {conc }}\right) t<s<t \hat{t}$

It also follows that

$$
(\exists l \in\{1,2, . .\})\left(\exists \sigma_{1}, \sigma_{2}, . ., \sigma_{l} \in \Sigma_{a c t} \subset \Sigma\right) t \sigma_{1} \sigma_{2} . . \sigma_{l}=s
$$

To show that $V(s)$ must have been re-evaluated at line $\mathbf{2 3}$ or line $\mathbf{2 5}$, it is sufficient to show that $t$ must be added to Pend, and that there exist a $\hat{t}$ that will pass the condition on line $\mathbf{1 4}$.

We first note that as $s \in L(\mathbf{S}) \cap L(\mathbf{G})$, it follows that $t \in L(\mathbf{S}) \cap$ $L(\mathbf{G})$ as $L(\mathbf{G})$ and $L(\mathbf{S})$ are closed languages. Similarly, as $s \in$ $L(V / \mathbf{G})$, we also have $t \in L(V / \mathbf{G})$.

We can thus apply the logic from (ii.c), and conclude that $t$ must be added to Pend, and it will be paired with state $\mathbf{q}$ of the controller with $\mathbf{q}=\Lambda(x)$ where $x=\xi\left(x_{o}, t\right)$. State $\mathbf{q}$ is the state the 
controller will be in after string $t$, thus $\Sigma_{V}$ will be the enablement output of the controller, where

$$
\Sigma_{V}=\operatorname{Elig}_{L(\mathbf{S})}(t) \cap \Sigma_{h i b}
$$

We now need to show:

$\left(\exists \hat{t} \in L_{\text {conc }}\right)$

$$
(s<t \hat{t}) \wedge(t \hat{t} \cap L(\mathbf{S}) \cap L(\mathbf{G})) \wedge \operatorname{Occu}(\hat{t}) \cap \Sigma_{h i b} \subseteq \Sigma_{V}
$$

We start by constructing a string $\hat{t} \in L_{\text {conc }}$ that satisfies the first two conditions.

We note that by assumption, $\mathbf{G}$ and $\mathbf{S}$ have finite statespaces, $\mathbf{G}$ has proper time behavior, $\operatorname{meet}(\mathbf{G}, \mathbf{S})$ is $\mathrm{ALF}$, and that $\mathbf{S}$ is controllable for $\mathbf{G}$ (this is implied by fact $\mathbf{S}$ is $\mathrm{SD}$ controllable for G). We can thus apply Proposition 2.4 and conclude

$$
\left(\exists s^{\prime} \in \Sigma^{*}\right) s s^{\prime} \tau \in L(\mathbf{S}) \cap L(\mathbf{G})
$$

We can thus take $\hat{t}=\sigma_{1} \sigma_{2} . . \sigma_{l} s^{\prime} \tau$ and we have $s<t \hat{t}$ (by (3)) and $t \hat{t} \in L(\mathbf{S}) \cap L(\mathbf{G})$.

All that remains is to show

$$
\operatorname{Occu}(\hat{t}) \cap \Sigma_{h i b} \subseteq \Sigma_{V}
$$

From (4), we have

$$
\begin{aligned}
& \Sigma_{V}=\operatorname{Elig}_{L(\mathbf{S})}(t) \cap \Sigma_{h i b} \\
\Longrightarrow & \operatorname{Elig}_{L(\mathbf{S}) \cap L(\mathbf{G})}(t) \subseteq \Sigma_{V}
\end{aligned}
$$

We now note that as $\mathbf{S}$ is $\mathrm{SD}$ controllable for $\mathbf{G}$, we have by Point iii.1 of Definition 3.2 .2

$$
\begin{aligned}
& \left(\forall t^{\prime} \in \Sigma_{a c t}^{*}\right)\left(t^{\prime}<\hat{t}\right) \Longrightarrow \\
& {\left[\operatorname{Elig}_{L(\mathbf{S}) \cap L(\mathbf{G})}\left(t t^{\prime}\right) \cup \operatorname{Occu}\left(t^{\prime}\right)\right] \cap \Sigma_{h i b}=\operatorname{Elig}_{L(\mathbf{S}) \cap L(\mathbf{G})}(t) \cap \Sigma_{h i b} \subseteq \Sigma_{V}}
\end{aligned}
$$


If we take $t^{\prime}=\sigma_{1} \sigma_{2} . . \sigma_{l} s^{\prime}<t$ we have

$$
\begin{aligned}
& {\left[\operatorname{Elig}_{L(\mathbf{S}) \cap L(\mathbf{G})}\left(t t^{\prime}\right) \cup \operatorname{Occu}\left(t^{\prime}\right)\right] \cap \Sigma_{h i b} \subseteq \Sigma_{V} } \\
\Longrightarrow & \operatorname{Occu}\left(t^{\prime}\right) \cap \Sigma_{h i b} \subseteq \Sigma_{V}
\end{aligned}
$$

As $t^{\prime} \tau=\hat{t}$, we thus have $\operatorname{Occu}(\hat{t}) \cap \Sigma_{h i b} \subseteq \Sigma_{V}$

We have now shown, that for $s \notin L_{\text {samp }}$, we must have re-evaluated $V(s)$ at line $\mathbf{2 3}$ or line $\mathbf{2 5}$, so (ii.d) does not apply. This means that string $s$ must be covered under (ii.b).

Part (ii.d) complete.

We thus have shown by (ii.a-d), that

$$
\begin{aligned}
& \operatorname{Elig}_{L(\mathbf{S}) \cap L(\mathbf{G})}(s) \cap \Sigma_{h i b}=\emptyset \wedge \tau \in \operatorname{Elig}_{L(\mathbf{G})}(s) \\
\Longrightarrow & \tau \in \operatorname{Elig}_{L(\mathbf{S})}(s) \quad \text { by Point ii of Definition 3.2.2 } \\
\Longrightarrow & s \sigma_{k+1} \in L(\mathbf{S})
\end{aligned}
$$

(iii) $\sigma_{k+1} \in \Sigma_{h i b}$

For $s \in L(\mathbf{S}) \cap L(\mathbf{G})$ and $s \in L(V / \mathbf{G})$, we know there exists $t \in L_{\text {samp }}$ and $t^{\prime} \in \Sigma_{a c t}^{*}$ such that $s=t t^{\prime}$.

From (ii.a), we know that Algorithm 5.1 will pair sampled string $t$ with state $\mathbf{q}$ in the controller, with $\mathbf{q}=\Lambda(x)$, where $x=\xi\left(x_{o}, t\right)$.

Also, we have

$$
\begin{gathered}
\Sigma_{V}=\operatorname{Elig}_{L(\mathbf{S})}(t) \cap \Sigma_{h i b} \\
\Rightarrow \operatorname{Elig}_{L(\mathbf{S}) \cap L(\mathbf{G})}(t) \cap \Sigma_{h i b} \subseteq \Sigma_{V}
\end{gathered}
$$

We now will show that $\sigma_{k+1} \in \Sigma_{V}$.

We note that as $s \sigma_{k+1} \in L(V / \mathbf{G})$, we have $\sigma_{k+1} \in V(s)$.

From line 2, we see $V(s)$ is initially set to $\Sigma_{u} \cup\{\tau\}$. This means that $\sigma_{k+1}$ must have been added at line $\mathbf{1 1}$ if $t^{\prime}=\epsilon$ and $t=s$, or at line 
23 or line 25. In either case it implies our prohibitable event is in $\Sigma_{V}$

We thus have

$$
\begin{aligned}
\sigma_{k+1} \in \Sigma_{V} & \Longrightarrow \sigma_{k+1} \in \operatorname{Elig}_{L(\mathbf{S})}(t) \\
& \Longrightarrow \sigma_{k+1} \in \operatorname{Elig}_{L(\mathbf{G})}(t) \quad \text { as } \mathbf{G} \text { is complete for } \mathbf{S} .
\end{aligned}
$$

As $\mathbf{S}$ is SD controllable for $\mathbf{G}$, we have from Point iii.1 of Definition 3.2 .2 that

$$
\left[\operatorname{Elig}_{L(\mathbf{S}) \cap L(\mathbf{G})}\left(t t^{\prime}\right) \cup \operatorname{Occu}\left(t^{\prime}\right)\right] \cap \Sigma_{h i b}=\operatorname{Elig}_{L(\mathbf{S}) \cap L(\mathbf{G})}(t) \cap \Sigma_{h i b}
$$

We note that as $s \sigma_{k+1} \in L(V / \mathbf{G})$, we have $s \sigma_{k+1} \in L(\mathbf{G})$. As $\mathbf{G}$ has $\mathbf{S}$ singular prohibitable behavior, this implies $\sigma_{k+1}$ has not yet occurred in this sampling period. Thus

$$
\sigma_{k+1} \notin \operatorname{Occu}\left(t^{\prime}\right)
$$

From (5), we have $\sigma_{k+1} \in \operatorname{Elig}_{L(\mathbf{S}) \cap L(\mathbf{G})}(t)$.

From (6), we thus have

$$
\sigma_{k+1} \in\left[\operatorname{Elig}_{L(\mathbf{S}) \cap L(\mathbf{G})}\left(t t^{\prime}\right) \cup \operatorname{Occu}\left(t^{\prime}\right)\right]
$$

As $\sigma_{k+1} \notin \operatorname{Occu}\left(t^{\prime}\right)$ from $(7)$, it follows that

$$
\begin{aligned}
& \sigma_{k+1} \in \operatorname{Elig}_{L(\mathbf{S}) \cap L(\mathbf{G})}(s) \quad\left(\text { as } t t^{\prime}=s\right) \\
& \Longrightarrow s \sigma_{k+1} \in L(\mathbf{S}) \quad \text { as required }
\end{aligned}
$$

By (i), (ii) and (iii), we have shown $s \sigma_{k+1} \in L(\mathbf{S})$ for any $\sigma_{k+1} \in \Sigma$, thus our inductive step is complete.

Thus by our base case and our inductive step, we have $s \in L(\mathbf{S})$ for arbitrary $s \in L(V / \mathbf{G})$. Therefore, $\mathbf{1 . 2}$ is complete.

By step $\mathbf{1 . 1}$ and $\mathbf{1 . 2}$, we have shown $L(V / \mathbf{G}) \subseteq L(\mathbf{S}) \cap L(\mathbf{G})$. 
2. Show $L(V / \mathbf{G}) \supseteq L(\mathbf{S}) \cap L(\mathbf{G})$

Let $s \in L(\mathbf{S}) \cap L(\mathbf{G})$. We need to show this implies

$$
s \in L(V / \mathbf{G})
$$

We will show this using proof by induction.

base case $s=\epsilon$

Automatic that $s \in L(V / \mathbf{G})$, by Definition 5.1 .4 for $L(V / \mathbf{G})$.

inductive step We assume that $s=\sigma_{1} \sigma_{2} . . \sigma_{k} \in L(V / \mathbf{G}) \cap L(\mathbf{S}) \cap L(\mathbf{G})$ and $s \sigma_{k+1} \in$ $L(\mathbf{S}) \cap L(\mathbf{G})$ for some $k \geq 0$.

We will now show this implies $s \sigma_{k+1} \in L(V / \mathbf{G})$.

Sufficient to show $\sigma_{k+1} \in V(s)$ by Definition of $L(V / \mathbf{G})$, and fact we already have $s \sigma_{k+1} \in L(\mathbf{G})$

Again, since $\Sigma=\Sigma_{u} \dot{\cup} \Sigma_{h i b} \dot{U}\{\tau\}$ by definition of TDES, we have 3 cases for $\sigma_{k+1} \in \Sigma$.

(i) $\sigma_{k+1} \in \Sigma_{u}$

This is automatic by line 2 in Algorithm 5.1, where all uncontrollable events are included in $V(s)$ for each possible string $s$ by default. Examining the algorithm, it is clear that uncontrollable events are never later removed.

(ii) $\sigma_{k+1}=\tau$

As we have $s \tau \in L(\mathbf{G})$ and $\mathbf{S}$ is SD controllable for $\mathbf{G}$ by assumption, we can conclude by Point ii in Definition 3.2.2 that

$$
\tau \in \operatorname{Elig}_{L(\mathbf{S})}(s) \Longleftrightarrow \operatorname{Elig}_{L(\mathbf{S}) \cap L(\mathbf{G})}(s) \cap \Sigma_{h i b}=\emptyset
$$

As we have $s \tau \in L(\mathbf{S})$ by assumption, we thus have

$$
\begin{aligned}
& \operatorname{Elig}_{L(\mathbf{S}) \cap L(\mathbf{G})}(s) \cap \Sigma_{h i b}=\emptyset \\
\Longrightarrow & \operatorname{Elig}_{L(\mathbf{S})}(s) \cap \Sigma_{h i b}=\emptyset
\end{aligned}
$$

as $\mathbf{G}$ is complete for $\mathbf{S}(9)$ 
Essentially, $\mathbf{G}$ complete for $\mathbf{S}$ means that if a prohibitable event was accepted by $\mathbf{S}$, it must also be accepted by $\mathbf{G}$, thus in $L(\mathbf{S}) \cap L(\mathbf{G})$. Thus, the only way there could be no eligible prohibitable events in both, is if there are none in $L(\mathbf{S})$, otherwise we would have a contradiction.

We next note that $\tau$ is initially added to $V(\mathbf{S})$ at line 2 of Algorithm 5.1 , thus we would have $\tau \in V(s)$ unless it is removed at line $\mathbf{1 1}$ or line 23 . Now, it is possible that $s$ will never be added to Pend if $s$ is a sampled string, or that it will never be processed in the for-loop from line 20 to line 27 if $s$ is not a sampled string. If that was the case, $V(s)$ would have the default value and we have $\tau \in V(s)$ as required. We can thus, without any loss of generality, assume that $s$ is added to Pend if $s \in L_{s a m p}$, or $s$ is processed by the for-loop from line 20 to line 27 if $s \notin L_{\text {samp }}$.

It is thus sufficient to show that the tick event is not removed at line $\mathbf{1 1}$ when $s \in L_{\text {samp }}$ or at line 23 when $s \notin L_{\text {samp. }}$. The two situations are discussed individually below.

(ii.a) $s \in L_{\text {samp }}$

If $s \in L_{\text {samp }}, \tau$ could only be removed at line 11 . To show that it is not, it is sufficient to show that $\Sigma_{V}=\emptyset$.

As we know from (ii.a) in the proof of part 1, Algorithm5.1 will always associate with $s$ in Pend, the state $\mathbf{q}$ in the controller with $\mathbf{q}=\Lambda(x)$ where $x=\xi\left(x_{o}, s\right) \in X_{\text {samp }}$. Also, we will have $\Sigma_{V}=\operatorname{Elig}_{L(\mathbf{S})}(s) \cap \Sigma_{h i b}$ From (9) we know $\operatorname{Elig}_{L(\mathbf{S})}(s) \cap \Sigma_{h i b}=\emptyset$, thus $\Sigma_{V}=\emptyset$, as required.

(ii.b) $s \notin L_{\text {samp }}$

As $s \notin L_{\text {samp }}$, we know: $\left(\exists t \in L_{\text {samp }}\right)\left(\exists \hat{t} \in L_{\text {conc }}\right) t<s<t \hat{t}$.

Also, we know: $(\exists i \in\{1,2, .\}).\left(\exists \sigma_{1}, \sigma_{2}, . ., \sigma_{i} \in \Sigma_{a c t} \subset \Sigma\right) t \sigma_{1} \sigma_{2} . . \sigma_{i}=s$

As $s$ is being processed by the for-loop from line 20 to line 27 , by assumption we have $t \hat{t} \in L(\mathbf{S}) \cap L(\mathbf{G})$. 
Examining from line 22 to line 26 of Algorithm 5.1, we see that to show $\tau$ is not removed, it is sufficient to show that when $s$ is processed, $\Sigma_{\text {temp }}=\emptyset$

From the logic of Algorithm [5.1, we see that initially $\Sigma_{\text {temp }}=\Sigma_{V}$, and $\Sigma_{\text {temp }}=\Sigma_{V}-\left\{\sigma_{1}, \sigma_{2}, . ., \sigma_{i}\right\}$ by the time $s$ is evaluated.

As we know from the logic of (ii.a) in part 1, Algorithm 5.1 will pair string $t$ in Pend with state $\mathbf{q}$ from the controller, where $\mathbf{q}=\Lambda(x)$ and $x=\xi\left(x_{o}, t\right) \in X_{\text {samp. }}$. Also,

$$
\begin{aligned}
& \Sigma_{V}=\operatorname{Elig}_{L(\mathbf{S})}(t) \cap \Sigma_{h i b} \\
\Longrightarrow & \operatorname{Elig}_{L(\mathbf{S}) \cap L(\mathbf{G})}(t) \cap \Sigma_{h i b} \subseteq \Sigma_{V}
\end{aligned}
$$

We will now show that $\Sigma_{V} \subseteq \operatorname{Elig}_{L(\mathbf{S}) \cap L(\mathbf{G})}(t) \cap \Sigma_{h i b}$, and thus $\Sigma_{V}=$ $\operatorname{Elig}_{L(\mathbf{S}) \cap L(\mathbf{G})}(t) \cap \Sigma_{h i b}$

Let $\sigma \in \Sigma_{V}=\operatorname{Elig}_{L(\mathbf{S})}(t) \cap \Sigma_{h i b}$

As $\sigma$ is prohibitable, we immediately know $\sigma \in \operatorname{Elig}_{L(\mathbf{G})}(t)$ as $\mathbf{G}$ is complete for $\mathbf{S}$, which implies $\sigma \in \operatorname{Elig}_{L(\mathbf{S}) \cap L(\mathbf{G})}(t) \cap \Sigma_{h i b}$.

$\Rightarrow \Sigma_{V}=\operatorname{Elig}_{L(\mathbf{S}) \cap L(\mathbf{G})}(t) \cap \Sigma_{h i b}$

As $t<s<t \hat{t}$, and $\operatorname{Elig}_{L(\mathbf{S}) \cap L(\mathbf{G})}(s) \cap \Sigma_{h i b}=\emptyset$ by (8), it thus follows that all prohibitable events that were possible at $t$, are no longer possible at $s$ in $\operatorname{meet}(\mathbf{G}, \mathbf{S})$.

As $\mathbf{S}$ is SD controllable for $\mathbf{G}$, we can apply Point iii.1 of Definition 3.2.2, and conclude

$$
\begin{aligned}
& \left(\operatorname{Elig}_{L(\mathbf{S}) \cap L(\mathbf{G})}(s) \cup \operatorname{Occu}\left(\sigma_{1} \sigma_{2} . . \sigma_{i}\right)\right) \cap \Sigma_{h i b}=\operatorname{Elig}_{L(\mathbf{S}) \cap L(\mathbf{G})}(t) \cap \Sigma_{h i b} \\
\Rightarrow & \left(\operatorname{Elig}_{L(\mathbf{S}) \cap L(\mathbf{G})}(s) \cup \operatorname{Occu}\left(\sigma_{1} \sigma_{2} . . \sigma_{i}\right)\right) \cap \Sigma_{h i b}=\Sigma_{V} \text { by }(10) .
\end{aligned}
$$

As $\operatorname{Elig}_{L(\mathbf{S}) \cap L(\mathbf{G})}(s)=\emptyset$, by (8) we have

$$
\operatorname{Occu}\left(\sigma_{1} \sigma_{2} . . \sigma_{i}\right) \cap \Sigma_{h i b}=\Sigma_{v}
$$


This means, after string $t \sigma_{1} \sigma_{2} . . \sigma_{i}$ has occurred, every event in $\Sigma_{V}$ has occurred at least once in $\sigma_{1} \sigma_{2} . . \sigma_{i}$. Thus, by the time $s$ is evaluated,

$$
\Sigma_{\text {temp }}=\Sigma_{V}-\left\{\sigma_{1}, \sigma_{2}, . ., \sigma_{i}\right\}=\emptyset
$$

This means that for $s$, line 25 is executed in Algorithm 5.1, not line 23, so tick is not removed from $V(s)$. Thus $\tau \in V(s)$, as required.

Part (ii.b) complete.

By (ii.a) and (ii.b) we have shown $s \sigma_{k+1} \in V(s)$ and thus

$$
s \sigma_{k+1} \in L(V / \mathbf{G})
$$

(iii) $\sigma_{k+1} \in \Sigma_{h i b}$

We thus have by assumption

$$
\sigma_{k+1} \in \operatorname{Elig}_{L(\mathbf{S}) \cap L(\mathbf{G})}(s) \cap \Sigma_{h i b}
$$

Examining Algorithm 5.1, we see no prohibitable event is added to $V(s)$ at line 2. This means, $\sigma_{k+1}$ could only be added at line 11 if $s \in L_{\text {samp }}$,

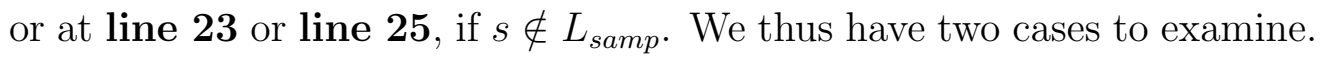
(iii.a) $s \in L_{\text {samp }}$

First, we have to show that $s$ will be added to Pend, or it will never get to line 11 .

As we have $s \in L(\mathbf{S}) \cap L(\mathbf{G}) \cap L(V / \mathbf{G})$ by assumption, we can apply the same logic that we used in (ii.c) in part 1, to show that $s$ will always be added to Pend.

We next note that from the logic of (ii.a) in part 1, Algorithm 5.1 will always associate with $s$ in Pend the state $\mathbf{q}$ in the controller with $\mathbf{q}=\Lambda(x)$, where $x=\xi\left(x_{o}, s\right) \in X_{\text {samp. }}$. Also we will have

$$
\Sigma_{V}=\operatorname{Elig}_{L(\mathbf{S})}(s) \cap \Sigma_{h i b}
$$

As $\sigma_{k+1} \in \operatorname{Elig}_{L(\mathbf{S}) \cap L(\mathbf{G})}(s) \cap \Sigma_{h i b}$, we thus have $\sigma_{k+1} \in \Sigma_{V}$. This means that the condition at line $\mathbf{1 0}$ of Algorithm 5.1 is satisfied, and thus $V(s) \leftarrow\left(V(s) \cup \Sigma_{V}\right)-\{\tau\}$.

Therefore, $\sigma_{k+1} \in V(s)$ as required. 
(iii.b) $s \notin L_{\text {samp }}$

First, we need to show that we will reach line $\mathbf{2 3}$ or line $\mathbf{2 5}$ for $s$, or $s$ could only be assigned the default value at line $\mathbf{2}$.

As we have $s \in L(\mathbf{S}) \cap L(\mathbf{G}) \cap L(V / \mathbf{G})$ by assumption, we can apply the logic of (ii.d) in part 1, and conclude

$$
\left(\exists t \in L_{\text {samp }}\right)\left(\exists \hat{t} \in L_{\text {conc }}\right) t<s<t \hat{t}
$$

such that $t$ will be added to Pend and associated with state $\mathbf{q}$ in the controller with $\mathbf{q}=\Lambda(x)$, where $x=\xi\left(x_{o}, t\right)$. Also, $\Sigma_{V}=\operatorname{Elig}_{L(\mathbf{S})}(t) \cap$ $\Sigma_{h i b}$.

Also, $\hat{t}$ is such that the condition at line $\mathbf{1 4}$ will be satisfied, and thus line 23 or line 25 will be reached.

We also note

$$
(\exists i \in\{1,2, . .\})\left(\exists \sigma_{1}, \sigma_{2}, . ., \sigma_{i} \in \Sigma_{a c t} \subset \Sigma\right) t \sigma_{1} \sigma_{2} . . \sigma_{i}=s
$$

Now, since either line $\mathbf{2 3}$ or line $\mathbf{2 5}$ will be executed, $\Sigma_{V}$ will be added to $V(s)$. It is thus sufficient to show $\sigma_{k+1} \in \Sigma_{V}$.

Since by assumption $\sigma_{k+1} \in \Sigma_{h i b}$, and $\sigma_{k+1} \in \operatorname{Elig}_{L(\mathbf{S}) \cap L(\mathbf{G})}(s)$, it follows that

$$
\sigma_{k+1} \in \operatorname{Elig}_{L(\mathbf{S}) \cap L(\mathbf{G})}(s) \cap \Sigma_{h i b}
$$

As $\mathbf{S}$ is SD controllable for $\mathbf{G}$, we can apply Point iii.1 of Definition 3.2.2, and conclude

$$
\begin{aligned}
& \left(\operatorname{Elig}_{L(\mathbf{S}) \cap L(\mathbf{G})}(s) \cup \operatorname{Occu}\left(\sigma_{1} \sigma_{2} . . \sigma_{i}\right)\right) \cap \Sigma_{h i b}=\operatorname{Elig}_{L(\mathbf{S}) \cap L(\mathbf{G})}(t) \cap \Sigma_{h i b} \\
\Rightarrow & \sigma_{k+1} \in\left(\operatorname{Elig}_{L(\mathbf{S}) \cap L(\mathbf{G})}(s) \cup \operatorname{Occu}\left(\sigma_{1} \sigma_{2} . . \sigma_{i}\right)\right) \cap \Sigma_{h i b}, \text { by }(12) . \\
\Rightarrow & \sigma_{k+1} \in \operatorname{Elig}_{L(\mathbf{S}) \cap L(\mathbf{G})}(t) \cap \Sigma_{h i b} \\
\Rightarrow & \sigma_{k+1} \in \operatorname{Elig}_{L(\mathbf{S})}(t) \cap \Sigma_{h i b}=\Sigma_{V}, \text { by }(11) \\
& \text { Thus } \sigma_{k+1} \in V(s), \text { as required. }
\end{aligned}
$$


By part (iii.a) and (iii.b), we have $\sigma_{k+1} \in V(s)$, as required.

Part iii complete.

By cases i-iii, we have $\sigma_{k+1} \in V(s)$. We thus have $s \sigma_{k+1} \in L(V / \mathbf{G})$, thus our inductive step is complete.

Thus by our base case and our inductive step, we have shown $s \in L(V / \mathbf{G})$ for arbitrary $s \in L(S) \cap L(G)$.

Part $\mathbf{2}$ is complete.

We have shown $\mathbf{1}$ and $\mathbf{2}$, thus we have shown $L(V / \mathbf{G})=L(\mathbf{S}) \cap L(\mathbf{G})$.

We are now ready to show that the $V$ we constructed in Algorithm 5.1 with the given system requirements, is indeed a TDES supervisory control.

Proposition 5.2. For plant $\mathbf{G}=\left(Y, \Sigma, \delta, y_{o}, Y_{m}\right)$, and CS deterministic supervisor $\mathbf{S}=\left(X, \Sigma, \delta, x_{o}, X_{m}\right)$ that is SD controllable for $\mathbf{G}$, let both TDES have finite statespaces, let $\mathbf{G}$ be complete for $\mathbf{S}$, have proper time and $\mathbf{S}$-singular prohibitable behavior, let $\operatorname{meet}(\mathbf{G}, \mathbf{S})$ be $\mathrm{ALF}$, let $\mathbf{C}=\left(I, Z, Q, \Omega, \Phi, \mathbf{q}_{\text {res }}\right)$ be the SD controller that is constructed from $\mathbf{S}$ using the translation method described in Section 4.2.3, and let $V$ be the map that is constructed from $\mathbf{C}$ using Algorithm 5.1. Then map $V$ is a TDES supervisory control for $\mathbf{G}$.

Proof. Let $s \in L(\mathbf{G})$.

To show that $V$ satisfies Definition 5.1.1, we need to show 1) $V(s) \supseteq \Sigma_{u}$ and 2) $\left[\left(\tau \in \operatorname{Elig}_{L(\mathbf{G})}(s)\right) \wedge\left(V(s) \cap \operatorname{Elig}_{L(\mathbf{G})}(s) \cap \Sigma_{h i b}=\emptyset\right)\right] \Longrightarrow \tau \in V(s)$.

1) Show $V(s) \supseteq \Sigma_{u}$

This is automatic as $V(s)=\Sigma_{u} \cup\{\tau\}$ is set at line 2 of Algorithm 5.1, and as $\tau \notin \Sigma_{u}$, no $\sigma \in \Sigma_{u}$ is ever removed from $V(s)$.

2) Show $\left[\left(\tau \in \operatorname{Elig}_{L(\mathbf{G})}(s)\right) \wedge\left(V(s) \cap \operatorname{Elig}_{L(\mathbf{G})}(s) \cap \Sigma_{h i b}=\emptyset\right)\right] \Longrightarrow \tau \in V(s)$ Assume $\tau \in \operatorname{Elig}_{L(\mathbf{G})}(s)$ and $V(s) \cap \operatorname{Elig}_{L(\mathbf{G})}(s) \cap \Sigma_{h i b}=\emptyset$ 
We will now show this implies $\tau \in V(s)$

We first note that as the assumptions of Theorem 5.1 are satisfied, we can conclude $L(V / \mathbf{G})=L(\mathbf{S}) \cap L(\mathbf{G})$

We next note that $\tau$ is initially added to $V(s)$ at line 2 of Algorithm 5.1. If $s$ is not processed again at line $\mathbf{1 1}$, line 23 and line 25, we have $\tau \in V(s)$

As we initializes Pend to $\left(\epsilon, \mathbf{q}_{\text {res }}\right)$, and we see that only strings in $L(\mathbf{S}) \cap L(\mathbf{G})$ will ever be added to Pend or processed at line 23 or line 25 (this can be seen by line 13 and line 14). This means if $s \notin L(\mathbf{S}) \cap L(\mathbf{G})$, we get the default value from line 2 and thus have $\tau \in V(s)$.

We only need to still consider $s \in L(\mathbf{S}) \cap L(\mathbf{G})$.

We will now show that $\operatorname{Elig}_{L(\mathbf{G})}(s) \cap \Sigma_{h i b}=\emptyset$.

By definition of $L(V / \mathbf{G})$ (Definition 5.1.4), for $\sigma \in \Sigma_{h i b}$, we would only have $s \sigma \in L(V / \mathbf{G})$ if $s \in L(V / \mathbf{G}), \sigma \in V(s)$ and $s \sigma \in L(\mathbf{G})$.

We have $s \in L(V / \mathbf{G})=L(\mathbf{S}) \cap L(\mathbf{G})$ from $(1)$, so for $s \sigma \in L(V / \mathbf{G})$, we would need $\sigma \in V(s) \cap \operatorname{Elig}_{L(\mathbf{G})}(s)$. However, we have $V(s) \cap \operatorname{Elig}_{L(\mathbf{G})}(s) \cap \Sigma_{h i b}=\emptyset$ by assumption, thus

$$
\begin{aligned}
& \left(\forall \sigma \in \Sigma_{h i b}\right) s \sigma \notin L(V / \mathbf{G})=L(\mathbf{S}) \cap L(\mathbf{G}) \\
\Longrightarrow & \operatorname{Elig}_{L(\mathbf{S}) \cap L(\mathbf{G})}(s) \cap \Sigma_{h i b}=\emptyset
\end{aligned}
$$

As $\mathbf{S}$ is SD controllable for $\mathbf{G}$, we can conclude by Point ii of Definition 3.2 .2 that, $\tau \in \operatorname{Elig}_{L(\mathbf{S})}(s)$. Since by assumption, we have $\tau \in \operatorname{Elig}_{L(\mathbf{G})}(s)$, we have

$$
\begin{aligned}
& \tau \in \operatorname{Elig}_{L(\mathbf{S}) \cap L(\mathbf{G})}(s)=\operatorname{Elig}_{L(V / \mathbf{G})}(s) \\
\Longrightarrow & s \tau \in L(V / \mathbf{G})
\end{aligned}
$$

As $s \in L(V / \mathbf{G}), s \tau \in L(\mathbf{G})$ and $s \tau \in L(V / \mathbf{G})$, it follows from Definition 5.1.4 that $\tau \in V(s)$, as required.

From points 1) and 2), we thus conclude that $V$ is a TDES supervisory control. 
We have now captured the enablement and forcing behavior of our controller as a map $V$, and shown that if $\mathbf{G}$ and $\mathbf{S}$ have the appropriate properties, $V$ is indeed a TDES supervisory control. We have also shown that the closed behavior of $\mathbf{G}$ under the control of $V, L(V / \mathbf{G})$, is exactly that of the closed behavior of the $\operatorname{meet}(\mathbf{G}, \mathbf{S})$, namely $L(\mathbf{S}) \cap L(\mathbf{G})$. This means that the behavior we get when our controller acts on our plant is what we expect, at least with respect to enablement and forcing behavior. Of course, this is assuming that none of the time delay issues we discussed in Section 3.3 occur.

We will now show that $V$ is nonblocking for $\mathbf{G}$ if and only if the meet of $\mathbf{G}$ and $\mathbf{S}$ are nonblocking.

Proposition 5.3. For plant $\mathbf{G}=\left(Y, \Sigma, \delta, y_{o}, Y_{m}\right)$, and CS deterministic supervisor $\mathbf{S}=\left(X, \Sigma, \delta, x_{o}, X_{m}\right)$ that is SD controllable for $\mathbf{G}$, let both TDES have finite statespaces, let $\mathbf{G}$ be complete for $\mathbf{S}$, have proper time and $\mathbf{S}$-singular prohibitable behavior, let $\operatorname{meet}(\mathbf{G}, \mathbf{S})$ be $\mathrm{ALF}$, let $\mathbf{C}=\left(I, Z, Q, \Omega, \Phi, \mathbf{q}_{\text {res }}\right)$ be the SD controller that is constructed from $\mathbf{S}$ using the translation method described in Section 4.2.3, and let $V$ be the map that is constructed from $\mathbf{C}$ using Algorithm 5.1. Then $V$ is non-blocking for $\mathbf{G}$ if and only if $\operatorname{meet}(\mathbf{G}, \mathbf{S})$ is non-blocking.

Proof. To show this, it is sufficient to show that $L(V / \mathbf{G})=L(\mathbf{S}) \cap L(\mathbf{G})$, and $L_{m}(V / \mathbf{G})=L_{m}(\mathbf{S}) \cap L_{m}(\mathbf{G})$.

As the assumptions of Theorem 5.1 are satisfied, we can conclude $L(V / \mathbf{G})=$ $L(\mathbf{S}) \cap L(\mathbf{G})$.

We next note that by Definition 5.1.6, we have

$$
\begin{aligned}
L_{m}(V / \mathbf{G}) & =L(V / \mathbf{G}) \cap L_{m}(\mathbf{S}) \cap L_{m}(\mathbf{G}) \\
& =L(\mathbf{S}) \cap L(\mathbf{G}) \cap L_{m}(\mathbf{S}) \cap L_{m}(\mathbf{G}) \quad \text { after substitution for } L(V / \mathbf{G}) \\
& =L_{m}(\mathbf{S}) \cap L_{m}(\mathbf{G}) \quad \text { as } L_{m}(\mathbf{G}) \subseteq L(\mathbf{G}) \text { and } L_{m}(\mathbf{S}) \subseteq L(\mathbf{S})
\end{aligned}
$$

as required.

\subsection{Concurrent Supervisory Control Equivalent}

In general, the order that events occur in the physical plant during a given sampling period, are that dictated by the plant model, and are allowed by the enablement 
and forcing behavior of the plant's SD controller. However, in practice time delay restrictions and the particular implementation of our controller might mean that all concurrent strings that should be possible in a given sampling period according to our plant model, may not actually be possible in practice.

For instance, we may be expecting that we could either get string $\alpha \beta \tau$ or $\beta \alpha \tau$ $\left(\alpha, \beta \in \Sigma_{\text {hib }}\right)$, yet it may be that only string $\alpha \beta \tau$ will ever occur due to time delay or the specific implementation of our controller. With respect to time delay, it could be possible, for example, that $\alpha$ always reaches our controller's inputs first. With respect to implementation, our controller might have to execute the events sequentially and always chooses to first do an $\alpha$ then a $\beta$ as it must choose an execution order (people typically would not design a controller that randomly chooses an execution order each time). Another possibility is that the controller could start $\alpha$ and $\beta$ tasks at about the same time, but $\beta$ always takes longer (in this particular implementation of our controller) to occur.

This could be a problem if, for example, only string $\beta \alpha \tau$ lead back to a marked state. In such a case, our TDES system would be nonblocking and controllable, but our implementation would block. We want to ensure that if our TDES system is nonblocking, and in our actual controlled system where we have a set of possible concurrent strings with the same occurrence image that could occur (according to our TDES model) in a given sampling period, if at least one of these strings can actually occur, our implementation would still be nonblocking. In other words, we wish our system to be robust with respect to nonblocking and this uncertainty.

We will now show that the conditions that we have developed will in fact guarantee this. We will frame our argument in terms of supervisory controls. Given a TDES $\mathbf{G}=\left(Q, \Sigma, \delta, q_{o}, Q_{m}\right)$ and supervisor control $V$ for $\mathbf{G}$, we want to be able define a supervisor control $V^{\prime}$ such that if $V$ allows a set of concurrent strings with the same occurrence image to occur in $\mathbf{G}$ in a given sampling instance, $V^{\prime}$ will always allow at least one of them to occur, but not necessarily all of them. We want to make sure that as long as our actual controlled system exhibits the behavior of at least one of these $V^{\prime}$, it will still be nonblocking. Note that we are only modeling variations in which prohibitable events are enabled and possibly forced. We capture this notation in the following definition.

Definition 5.4.1. Let $\mathbf{G}=\left(Q, \Sigma, \delta, q_{o}, Q_{m}\right)$ be a TDES plant and let $V$ and $V^{\prime}$ be 
supervisory controls for $\mathbf{G}$. We say $V^{\prime}$ is concurrent supervisory control equivalent to $V$ if

1. $(\forall s \in L(\mathbf{G})) V^{\prime}(s) \subseteq V(s)$

2. $\left(\forall s \in L\left(V^{\prime} / \mathbf{G}\right) \cap L_{\text {samp }}\right)\left(\forall s^{\prime} \in L_{\text {conc }}\right) s s^{\prime} \in L(V / \mathbf{G})$

$\Longrightarrow\left(\exists s^{\prime \prime} \in L_{\text {conc }}\right) s s^{\prime \prime} \in L\left(V^{\prime} / \mathbf{G}\right) \cap \operatorname{Occu}\left(s^{\prime}\right)=\operatorname{Occu}\left(s^{\prime \prime}\right)$

By point 1 in the definition above, we require each event that $V^{\prime}(s)$ allows is also allowed by $V(s)$, so that $L\left(V^{\prime} / \mathbf{G}\right)$ does not include unwanted behavior.

By point 2, we require that if $V^{\prime} / \mathbf{G}$ accepts sampled string $s$, and $V / \mathbf{G}$ accepts concurrent string $s^{\prime}$ after it accepts string $s$, then $V^{\prime} / \mathbf{G}$ must accept a concurrent string $s^{\prime \prime}$ that has the same occurrence image as $s^{\prime}$. We use the the term concurrent equivalent because strings $s^{\prime}$ and $s^{\prime \prime}$ in the definition could both occur in the same sampling period and would be indistinguishable to an SD controller.

Figure 5.3 shows an example for the concurrent supervisory control equivalence definition. Here we see that for $V / \mathbf{G}$, we have two paths with the same occurrence image. For $V^{\prime} / \mathbf{G}$, only one of the two paths are still possible, but that is enough to satisfy the definition.

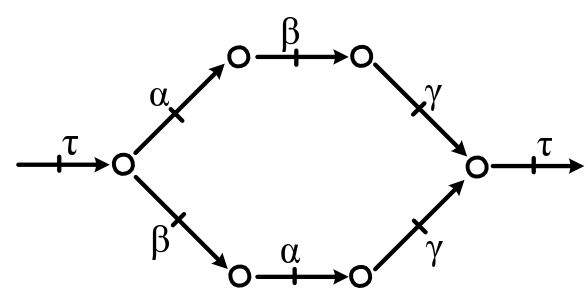

V/G

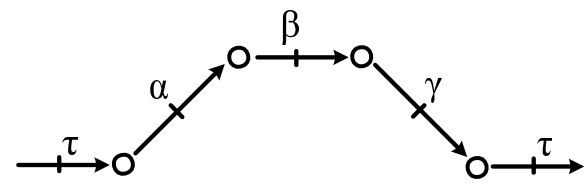

$\mathbf{V}^{\prime} / \mathbf{G}$

Figure 5.3: An Example for Concurrent Supervisory Control Equivalence 
Proposition 5.4. For TDES plant $\mathbf{G}=\left(Q, \Sigma, \delta, q_{o}, Q_{m}\right)$, let $V$ and $V^{\prime}$ be supervisory controls for G. If $V^{\prime}$ is concurrent supervisory control equivalent to $V$, then

$$
L\left(V^{\prime} / \mathbf{G}\right) \subseteq L(V / \mathbf{G})
$$

Proof. Let $V$ and $V^{\prime}$ be supervisory controls for $\mathbf{G}$.

Assume $V^{\prime}$ is concurrent supervisory control equivalent to $V$.

Must show

$$
\left(\forall s \in L\left(V^{\prime} / \mathbf{G}\right)\right) s \in L(V / \mathbf{G})
$$

We will show this using proof by induction.

base case Let $s=\epsilon$

This automatically implies $\epsilon \in L(V / \mathbf{G})$ by definition of $L(V / \mathbf{G})$.

inductive step Let $s \in L\left(V^{\prime} / \mathbf{G}\right) \cap L(V / \mathbf{G})$. Let $\sigma \in \Sigma$ such that $s \sigma \in L\left(V^{\prime} / \mathbf{G}\right)$.

Need to show implies $s \sigma \in L(V / \mathbf{G})$.

As we already have $s \in L(V / \mathbf{G})$ by assumption, it is sufficient to show

1. $\sigma \in V(s)$

As $s \sigma \in L\left(V^{\prime} / \mathbf{G}\right)$, we have by definition of $L\left(V^{\prime} / \mathbf{G}\right)$ that $\sigma \in V^{\prime}(s)$ and $s \in L(\mathbf{G})$. This implies $\sigma \in V(s)$ by point 1 of Definition 5.4.1.

2. $s \sigma \in L(\mathbf{G})$

By assumption, we have $s \sigma \in L\left(V^{\prime} / \mathbf{G}\right)$, which implies $s \sigma \in L(\mathbf{G})$ by definition of $L\left(V^{\prime} / \mathbf{G}\right)$.

Thus by definition 5.1.4 of supervisory control, we have $s \sigma \in L(V / \mathbf{G})$.

Thus by our base case and inductive step, we conclude

$$
\left(\forall s \in L\left(V^{\prime} / \mathbf{G}\right)\right) s \in L(V / \mathbf{G})
$$

which implies

$$
L\left(V^{\prime} / \mathbf{G}\right) \subseteq L(V / \mathbf{G})
$$


We will now show that if $V$ is the TDES supervisor control we constructed with Algorithm 5.1 and $V^{\prime}$ is a TDES supervisor control that is concurrent control equivalent to $V$, then $V$ nonblocking for $\mathbf{G}$ implies that $V^{\prime}$ is also nonblocking for $\mathbf{G}$ (per Definition 5.1.6).

Theorem 5.2. For plant $\mathbf{G}=\left(Q, \Sigma, \delta, q_{o}, Q_{m}\right)$, and CS deterministic supervisor $\mathbf{S}=\left(X, \Sigma, \xi, x_{o}, X_{m}\right)$ that is SD controllable for $\mathbf{G}$, let both TDES have finite state spaces, let $\mathbf{G}$ be complete for $\mathbf{S}$, and have proper time and $\mathbf{S}$-singular prohibitable behavior, let meet $(\mathbf{G}, \mathbf{S})$ be ALF, let $\mathbf{C}$ be the SD controller that is constructed from $\mathrm{S}$ using the translation method described in Section 4.2.3, and let $V$ be the map that is constructed from $\mathbf{C}$ using Algorithm 5.1, Let $V^{\prime}$ be a supervisor control for G. If $V$ is nonblocking for $\mathbf{G}$ and $V^{\prime}$ is concurrent supervisory control equivalent to $V$, then $V^{\prime}$ is also nonblocking for $\mathbf{G}$.

Proof. Assume the initial conditions for the proposition, including that $V$ is nonblocking and $V^{\prime}$ is concurrent supervisory control equivalent to $V$.

We must show this implies

$$
L\left(V^{\prime} / \mathbf{G}\right)=\overline{L\left(V^{\prime} / \mathbf{G}\right) \cap L_{m}(\mathbf{S}) \cap L_{m}(\mathbf{G})}
$$

It is sufficient to show points 1 and 2 as follows.

1. $L\left(V^{\prime} / \mathbf{G}\right) \supseteq \overline{L\left(V^{\prime} / \mathbf{G}\right) \cap L_{m}(\mathbf{S}) \cap L_{m}(\mathbf{G})}$

Let $s \in \overline{L\left(V^{\prime} / \mathbf{G}\right) \cap L_{m}(\mathbf{S}) \cap L_{m}(\mathbf{G})}$.

Must show implies $s \in L\left(V^{\prime} / \mathbf{G}\right)$.

Since $s \in \overline{L\left(V^{\prime} / \mathbf{G}\right) \cap L_{m}(\mathbf{S}) \cap L_{m}(\mathbf{G})}$, there exists $s^{\prime \prime} \in \Sigma^{*}$ such that

$$
\begin{aligned}
& s s^{\prime \prime} \in L\left(V^{\prime} / \mathbf{G}\right) \cap L_{m}(\mathbf{S}) \cap L_{m}(\mathbf{G}) \\
\Longrightarrow & s s^{\prime \prime} \in L\left(V^{\prime} / \mathbf{G}\right) \\
\Longrightarrow & s \in \overline{L\left(V^{\prime} / \mathbf{G}\right)} \\
\Longrightarrow & s \in L\left(V^{\prime} / \mathbf{G}\right) \quad \text { as } L\left(V^{\prime} / \mathbf{G}\right) \text { is prefix closed, by Definition } 5.1 .4
\end{aligned}
$$

2. $L\left(V^{\prime} / \mathbf{G}\right) \subseteq \overline{L\left(V^{\prime} / \mathbf{G}\right) \cap L_{m}(\mathbf{S}) \cap L_{m}(\mathbf{G})}$ 
Let $s \in L\left(V^{\prime} / \mathbf{G}\right)$.

Must show $s \in \overline{L\left(V^{\prime} / \mathbf{G}\right) \cap L_{m}(\mathbf{S}) \cap L_{m}(\mathbf{G})}$.

Sufficient to show

$$
\left(\exists s^{\prime \prime} \in \Sigma^{*}\right) s s^{\prime \prime} \in L\left(V^{\prime} / \mathbf{G}\right) \cap L_{m}(\mathbf{S}) \cap L_{m}(\mathbf{G})
$$

For the case that

$$
s \in L_{m}(\mathbf{S}) \cap L_{m}(\mathbf{G})
$$

We have $s s^{\prime \prime} \in L\left(V^{\prime} / \mathbf{G}\right) \cap L_{m}(\mathbf{S}) \cap L_{m}(\mathbf{G})$, with $s^{\prime \prime}=\epsilon$.

We now examine the case

$$
s \notin L_{m}(\mathbf{S}) \cap L_{m}(\mathbf{G})
$$

We first note that the assumptions of Theorem 5.1 have been met, so we can conclude that

$$
L(V / \mathbf{G})=L(\mathbf{S}) \cap L(\mathbf{G})
$$

Also, as $L_{m}(\mathbf{G}) \subseteq L(\mathbf{G})$ and $L_{m}(\mathbf{S}) \subseteq L(\mathbf{S})$, it follows that

$$
L_{m}(\mathbf{G}) \cap L_{m}(\mathbf{S}) \subseteq L(V / \mathbf{G})
$$

We have two sub-cases for $s:$ 1) $s \in L_{\text {samp }}$ and 2) $s \notin L_{\text {samp }}$.

$2.1 s \in L_{\text {samp }}$

By Proposition 5.4 we have $L\left(V^{\prime} / \mathbf{G}\right) \subseteq L(V / \mathbf{G})$, we thus have

$$
s \in L(V / \mathbf{G})
$$

Since $V$ is nonblocking for $\mathbf{G}$, we have

$$
\begin{aligned}
s \in L(V / \mathbf{G}) & \Longrightarrow\left(\exists s^{\prime} \in \Sigma^{*}\right) s s^{\prime} \in L(V / \mathbf{G}) \cap L_{m}(\mathbf{S}) \cap L_{m}(\mathbf{G}) \\
& \Longrightarrow\left(\exists s^{\prime} \in \Sigma^{*}\right)\left(s s^{\prime} \in L(V / \mathbf{G})\right) \wedge\left(s s^{\prime} \in L_{m}(\mathbf{S}) \cap L_{m}(\mathbf{G})\right)
\end{aligned}
$$


Let $s^{\prime} \in \Sigma^{*}$ such that

$$
\left(s s^{\prime} \in L(V / \mathbf{G})\right) \wedge\left(s s^{\prime} \in L_{m}(\mathbf{S}) \cap L_{m}(\mathbf{G})\right)
$$

As $\mathbf{S}$ is $\mathbf{S D}$ controllable for $\mathbf{G}$, we have by point iv in Definition 3.2 .2 that

$$
L_{m}(\mathbf{S}) \cap L_{m}(\mathbf{G}) \subseteq L_{\text {samp }}
$$

We can thus divide $s^{\prime}$ into consecutive strings $s_{1}^{\prime}, s_{2}^{\prime}, . ., s_{n}^{\prime} \in L_{\text {conc }}$ such that

$$
s^{\prime}:=s_{1}^{\prime} s_{2}^{\prime} . . s_{n}^{\prime}
$$

We note that $n \geq 1$ as $s \notin L_{m}(\mathbf{S}) \cap L_{m}(\mathbf{G})$, by (2). We thus have

$$
s s_{1}^{\prime} s_{2}^{\prime} . . s_{n}^{\prime} \in L(V / \mathbf{G})
$$

We will now use $s_{1}^{\prime}, s_{2}^{\prime}, . ., s_{n}^{\prime}$ to construct $s_{1}^{\prime \prime}, s_{2}^{\prime \prime}, . ., s_{n}^{\prime \prime} \in L_{\text {conc }}$ such that $s s_{1}^{\prime \prime} s_{2}^{\prime \prime} . . s_{n}^{\prime \prime} \in L\left(V^{\prime} / \mathbf{G}\right) \cap L_{m}(\mathbf{S}) \cap L_{m}(\mathbf{G})$

We will use a proof by induction to show that, for all $k \in\{2,3, . ., n\}$

$$
\begin{aligned}
& \left(\exists s_{1}^{\prime \prime}, s_{2}^{\prime \prime}, . ., s_{k-1}^{\prime \prime} \in L_{\text {conc }}\right) \\
& \quad\left[s s_{1}^{\prime \prime} s_{2}^{\prime \prime} . . s_{k-1}^{\prime \prime} s_{k}^{\prime} . . s_{n}^{\prime} \in L_{m}(\mathbf{S}) \cap L_{m}(\mathbf{G})\right] \wedge\left[s s_{1}^{\prime \prime} s_{2}^{\prime \prime} . . s_{k-1}^{\prime \prime} \in L\left(V^{\prime} / \mathbf{G}\right)\right] \\
& \Longrightarrow \\
& \left(\exists s_{k}^{\prime \prime} \in L_{\text {conc }}\right) \\
& \quad\left[s s_{1}^{\prime \prime} s_{2}^{\prime \prime} . . s_{k}^{\prime \prime} s_{k+1}^{\prime} . . s_{n}^{\prime} \in L_{m}(\mathbf{S}) \cap L_{m}(\mathbf{G})\right] \wedge\left[s s_{1}^{\prime \prime} s_{2}^{\prime \prime} . . s_{k}^{\prime \prime} \in L\left(V^{\prime} / \mathbf{G}\right)\right]
\end{aligned}
$$

base case Show: $\left(\exists s_{1}^{\prime \prime} \in L_{\text {conc }}\right)$

$$
\left[s s_{1}^{\prime \prime} s_{2}^{\prime} . . s_{n}^{\prime} \in L_{m}(\mathbf{S}) \cap L_{m}(\mathbf{G})\right] \wedge\left[s s_{1}^{\prime \prime} \in L\left(V^{\prime} / \mathbf{G}\right)\right]
$$

From (6), (7) and (8) we have $s_{1}^{\prime}, s_{2}^{\prime}, . ., s_{n}^{\prime} \in L_{\text {conc }}$ and

$$
\begin{aligned}
& s s_{1}^{\prime} s_{2}^{\prime} . . s_{n}^{\prime} \in L(V / \mathbf{G}) \cap L_{m}(\mathbf{S}) \cap L_{m}(\mathbf{G}) \\
\Longrightarrow & s s_{1}^{\prime} \in L(V / \mathbf{G}) \quad \text { as } L(V / \mathbf{G}) \text { is prefix closed }
\end{aligned}
$$

By (1) and (5), we have $s \in L\left(V^{\prime} / \mathbf{G}\right) \cap L_{\text {samp }}$

Putting this together we see we have

$$
\left(s \in L\left(V^{\prime} / \mathbf{G}\right) \cap L_{\text {samp }}\right) \wedge\left(s_{1}^{\prime} \in L_{\text {conc }}\right) \wedge\left(s s_{1}^{\prime} \in L(V / \mathbf{G})\right)
$$


As $V^{\prime}$ is concurrent supervisory control equivalent to $V$, we can apply Definition 5.4.1 and conclude

$$
\left(\exists s_{1}^{\prime \prime} \in L_{\text {conc }}\right)\left(s s_{1}^{\prime \prime} \in L\left(V^{\prime} / \mathbf{G}\right)\right) \wedge\left(\mathrm{Occu}\left(s_{1}^{\prime}\right)=\operatorname{Occu}\left(s_{1}^{\prime \prime}\right)\right)
$$

As $L\left(V^{\prime} / \mathbf{G}\right) \subseteq L(V / \mathbf{G})$ by Proposition 5.4, we have $s s_{1}^{\prime \prime} \in L(V / \mathbf{G})$

As $s, s s_{1}^{\prime}, s s_{1}^{\prime \prime} \in L(V / \mathbf{G})$, we have

$$
s, s s_{1}^{\prime}, s s_{1}^{\prime \prime} \in L(\mathbf{S}) \cap L(\mathbf{G})
$$

As $s \in L_{\text {samp }}$ and $\mathbf{S}$ is SD controllable for $\mathbf{G}$, we can apply Point iii.2 and conclude: $s s_{1}^{\prime} \equiv_{L_{m}(\mathbf{S}) \cap L_{m}(\mathbf{G})} s s_{1}^{\prime \prime}$.

As $s s_{1}^{\prime} s_{2}^{\prime} . . s_{n}^{\prime} \in L_{m}(\mathbf{S}) \cap L_{m}(\mathbf{G})$ from (6) and (7), we have $s s_{1}^{\prime \prime} s_{2}^{\prime} . . s_{n}^{\prime} \in$ $L_{m}(\mathbf{S}) \cap L_{m}(\mathbf{G})$ as $s s_{1}^{\prime}$ and $s s_{1}^{\prime \prime}$ are Nerode equivalent $\bmod L_{m}(\mathbf{S}) \cap$ $L_{m}(\mathbf{G})$.

We have thus shown

$$
\left(\exists s_{1}^{\prime \prime} \in L_{\text {conc }}\right)\left[s s_{1}^{\prime \prime} s_{2}^{\prime} . . s_{n}^{\prime} \in L_{m}(\mathbf{S}) \cap L_{m}(\mathbf{G})\right] \wedge\left[s s_{1}^{\prime \prime} \in L\left(V^{\prime} / \mathbf{G}\right)\right]
$$

Base case complete.

inductive step Let $k \in\{2,3, . ., n\}$.

Assume:

$$
\begin{aligned}
& \left(\exists s_{1}^{\prime \prime}, s_{2}^{\prime \prime}, . ., s_{k-1}^{\prime \prime} \in L_{\text {conc }}\right) \\
& \quad\left[s s_{1}^{\prime \prime} s_{2}^{\prime \prime} . . s_{k-1}^{\prime \prime} s_{k}^{\prime} . . s_{n}^{\prime} \in L_{m}(\mathbf{S}) \cap L_{m}(\mathbf{G})\right] \wedge\left[s s_{1}^{\prime \prime} s_{2}^{\prime \prime} . . s_{k-1}^{\prime \prime} \in L\left(V^{\prime} / \mathbf{G}\right)\right]
\end{aligned}
$$

We will show this implies condition (9) is satisfied for this $k$.

We first note that as $s_{1}^{\prime \prime}, s_{2}^{\prime \prime}, . ., s_{k-1}^{\prime \prime} \in L_{\text {conc }}, s s_{1}^{\prime \prime} s_{2}^{\prime \prime} . s_{k-1}^{\prime \prime} \in L_{s a m p}$.

As $L_{m}(\mathbf{S}) \cap L_{m}(\mathbf{G}) \subseteq L(V / \mathbf{G})$ by $(4)$, we have $s s_{1}^{\prime \prime} s_{2}^{\prime \prime} . s_{k-1}^{\prime \prime} s_{k}^{\prime} . . s_{n}^{\prime} \in$ $L(V / \mathbf{G})$.

As $L(V / \mathbf{G})$ is prefix closed, we have $s s_{1}^{\prime \prime} . s_{k-1}^{\prime \prime} s_{k}^{\prime} \in L(V / \mathbf{G})$. Also, $s_{k}^{\prime} \in L_{\text {conc }}$ by $(7)$. 
We thus have:

$$
\begin{aligned}
& \left(s s_{1}^{\prime \prime} s_{2}^{\prime \prime} . . s_{k-1}^{\prime \prime} \in L\left(V^{\prime} / \mathbf{G}\right) \cap L_{\text {samp }}\right) \\
\wedge & \left(s_{k}^{\prime} \in L_{\text {conc }}\right) \\
\wedge & \left(s s_{1}^{\prime \prime} s_{2}^{\prime \prime} . . s_{k-1}^{\prime \prime} s_{k}^{\prime} \in L(V / \mathbf{G})\right)
\end{aligned}
$$

As $V^{\prime}$ is concurrent supervisory control equivalent to $V$, we can thus apply point 2 of Definition 5.4 .1 and conclude

$$
\left(\exists s_{k}^{\prime \prime} \in L_{\text {conc }}\right) s s_{1}^{\prime \prime} s_{2}^{\prime \prime} . . s_{k}^{\prime \prime} \in L\left(V^{\prime} / \mathbf{G}\right) \text { and } \operatorname{Occu}\left(s_{k}^{\prime}\right)=\operatorname{Occu}\left(s_{k}^{\prime \prime}\right)
$$

As $L\left(V^{\prime} / \mathbf{G}\right) \subseteq L(V / \mathbf{G})$, we have $s s_{1}^{\prime \prime} s_{2}^{\prime \prime} . s_{k}^{\prime \prime} \in L(V / \mathbf{G})$

We thus have

$$
\begin{gathered}
s s_{1}^{\prime \prime} s_{2}^{\prime \prime} . . s_{k-1}^{\prime \prime} \in L(V / \mathbf{G}) \cap L_{s a m p} \\
\Longrightarrow s s_{1}^{\prime \prime} s_{2}^{\prime \prime} . . s_{k-1}^{\prime \prime} \in L(\mathbf{S}) \cap L(\mathbf{G}) \cap L_{s a m p}
\end{gathered}
$$

and

$$
s s_{1}^{\prime \prime} s_{2}^{\prime \prime} . . s_{k-1}^{\prime \prime} s_{k}^{\prime}, s s_{1}^{\prime \prime} s_{2}^{\prime \prime} . . s_{k}^{\prime \prime} \in L(V / \mathbf{G})=L(\mathbf{S}) \cap L(\mathbf{G})
$$

As $\mathbf{S}$ is SD controllable for $\mathbf{G}$, we can apply Point iii.2 of Definition 3.2 .2 and conclude

$$
s s_{1}^{\prime \prime} s_{2}^{\prime \prime} . . s_{k-1}^{\prime \prime} s_{k}^{\prime} \equiv_{L_{m}(\mathbf{S}) \cap L_{m}(\mathbf{G})} s s_{1}^{\prime \prime} s_{2}^{\prime \prime} . . s_{k}^{\prime \prime}
$$

As $s s_{1}^{\prime \prime} s_{2}^{\prime \prime} . . s_{k-1}^{\prime \prime} s_{k}^{\prime} . . s_{n}^{\prime} \in L_{m}(\mathbf{S}) \cap L_{m}(\mathbf{G})$ by assumption, we thus have $s s_{1}^{\prime \prime} s_{2}^{\prime \prime} . . s_{k}^{\prime \prime} s_{k+1}^{\prime} . . s_{n}^{\prime} \in L_{m}(\mathbf{S}) \cap L_{m}(\mathbf{G})$ as $s s_{1}^{\prime \prime} s_{2}^{\prime \prime} . . s_{k-1}^{\prime \prime} s_{k}^{\prime}$ and $s s_{1}^{\prime \prime} s_{2}^{\prime \prime} . . s_{k}^{\prime \prime}$ are Nerode equivalent $\bmod L_{m}(\mathbf{S}) \cap L_{m}(\mathbf{G})$.

We have thus shown

$$
\begin{aligned}
& \left(\exists s_{k}^{\prime \prime} \in L_{\text {conc }}\right) \\
& \quad\left[s s_{1}^{\prime \prime} s_{2}^{\prime \prime} . . s_{k}^{\prime \prime} s_{k+1}^{\prime} . . s_{n}^{\prime} \in L_{m}(\mathbf{S}) \cap L_{m}(\mathbf{G})\right] \text { and }\left[s s_{1}^{\prime \prime} s_{2}^{\prime \prime} . . s_{k}^{\prime \prime} \in L\left(V^{\prime} / \mathbf{G}\right)\right]
\end{aligned}
$$

Inductive step complete. 
Combining our base case and inductive step, we can take $k=n$, and conclude

$$
\left(\exists s_{1}^{\prime \prime}, s_{2}^{\prime \prime}, . ., s_{n}^{\prime \prime} \in L_{\text {conc }}\right) s s_{1}^{\prime \prime} s_{2}^{\prime \prime} . . s_{n}^{\prime \prime} \in L_{m}(\mathbf{S}) \cap L_{m}(\mathbf{G}) \cap L\left(V^{\prime} / \mathbf{G}\right)
$$

We thus take $s^{\prime \prime}=s_{1}^{\prime \prime} s_{2}^{\prime \prime} . . s_{n}^{\prime \prime}$ and Case 2.1 is complete.

\section{$2.2 s \notin L_{s a m p}$}

As we want to reuse the result from 2.1 for this part, we first need to extend $s$ to a string in $L\left(V^{\prime} / \mathbf{G}\right) \cap L_{\text {samp }}$.

As $\mathbf{G}$ and $\mathbf{S}$ have finite statespaces, and $\operatorname{meet}(\mathbf{G}, \mathbf{S})$ is activity loop free, it follows that $\operatorname{meet}(\mathbf{G}, \mathbf{S})$ will accept at most a finite number of nontick events, before no more non-tick events can occur. Note $L(V / \mathbf{G})=$ $L(\mathbf{S}) \cap L(\mathbf{G})$ by $(3)$.

This means that at the state reached by $s$ in $\operatorname{meet}(\mathbf{G}, \mathbf{S})$, either there are no activity events possible, or after at most a finite number of activity events occur, we will be in a state where no activity events are possible.

The reason is that we have a finite number of states in $\operatorname{meet}(\mathbf{G}, \mathbf{S})$, thus after at most a finite number of non-tick transitions, we will have either reached a state where no activity events are possible, or we will have visited each state once as meet $(\mathbf{G}, \mathbf{S})$ is ALF. If we have visited each state once, we can't have another activity event possible, or it would create a loop, violating the assumption that $\operatorname{meet}(\mathbf{G}, \mathbf{S})$ is $A L F$.

As $L\left(V^{\prime} / \mathbf{G}\right) \subseteq L(V / \mathbf{G})$ by Proposition 5.4, it thus follows

$$
\left(\exists \hat{t} \in \Sigma_{a c t}^{*}\right)\left(s \hat{t} \in L\left(V^{\prime} / \mathbf{G}\right)\right) \wedge\left(\operatorname{Elig}_{L\left(V^{\prime} / \mathbf{G}\right)}(s \hat{t}) \cap \Sigma_{a c t}=\emptyset\right)
$$

We will now show that: $s \hat{t} \tau \in L\left(V^{\prime} / \mathbf{G}\right) \cap L_{\text {samp }}$.

We first note that by definition of $L\left(V^{\prime} / \mathbf{G}\right)$,

$$
\operatorname{Elig}_{L\left(V^{\prime} / \mathbf{G}\right)}(s \hat{t})=V^{\prime}(s \hat{t}) \cap \operatorname{Elig}_{L(\mathbf{G})}(s \hat{t})
$$


As $V^{\prime}$ is a TDES supervisory control, we have $V^{\prime}(s \hat{t}) \supseteq \Sigma_{u}$. Thus

$$
\begin{aligned}
& V^{\prime}(s \hat{t}) \cap \operatorname{Elig}_{L(\mathbf{G})}(s \hat{t}) \cap \Sigma_{a c t}=\emptyset \\
\Longrightarrow & \operatorname{Elig}_{L(\mathbf{G})}(s \hat{t}) \cap \Sigma_{u}=\emptyset \\
\Longrightarrow & \tau \in \operatorname{Elig}_{L(\mathbf{G})}(s \hat{t}) \quad \text { as } \mathbf{G} \text { has proper time behavior }
\end{aligned}
$$

We next note

$$
\begin{aligned}
& V^{\prime}(s \hat{t}) \cap \operatorname{Elig}_{L(\mathbf{G})}(s \hat{t}) \cap \Sigma_{a c t}=\emptyset \\
\Longrightarrow & V^{\prime}(s \hat{t}) \cap \operatorname{Elig}_{L(\mathbf{G})}(s \hat{t}) \cap \Sigma_{h i b}=\emptyset \\
\Longrightarrow & \tau \in V^{\prime}(s \hat{t}) \quad \text { as } V^{\prime} \text { is a TDES supervisory control }
\end{aligned}
$$

Combining the two results, we have $s \hat{t} \tau \in L\left(V^{\prime} / \mathbf{G}\right)$.

Taking $t=s \hat{t} \tau$, we first note that if $t \in L_{m}(\mathbf{S}) \cap L_{m}(\mathbf{G})$ we can take $s^{\prime \prime}=\hat{t} \tau$ and we have

$$
s s^{\prime \prime} \in L\left(V^{\prime} / \mathbf{G}\right) \cap L_{m}(\mathbf{S}) \cap L_{m}(\mathbf{G})
$$

and we are done.

We then consider the case $t \notin L_{m}(\mathbf{S}) \cap L_{m}(\mathbf{G})$.

As $t=s \hat{t} \tau$, we thus have $t \in L_{\text {samp }} \cap L\left(V^{\prime} / \mathbf{G}\right)$

We can now apply the logic of part 2.1, but use $t$ instead of $s$ as our starting place.

We can thus conclude

$$
\left(\exists s_{1}^{\prime \prime}, s_{2}^{\prime \prime}, . ., s_{n}^{\prime \prime} \in L_{\text {conc }}\right) t s_{1}^{\prime \prime} s_{2}^{\prime \prime} . . s_{n}^{\prime \prime} \in L_{m}(\mathbf{S}) \cap L_{m}(\mathbf{G}) \cap L\left(V^{\prime} / \mathbf{G}\right)
$$

We thus take $s^{\prime \prime}=\hat{t} \tau s_{1}^{\prime \prime} s_{2}^{\prime \prime} . . s_{n}^{\prime \prime}$ and part 2.2 is complete.

By both part 2.1 and 2.2, we have constructed a string $s^{\prime \prime} \in \Sigma^{*}$, where

$$
\begin{aligned}
& s s^{\prime \prime} \in L_{m}(\mathbf{S}) \cap L_{m}(\mathbf{G}) \cap L\left(V^{\prime} / \mathbf{G}\right) \\
\Longrightarrow & s \in \overline{L_{m}(\mathbf{S}) \cap L_{m}(\mathbf{G}) \cap L\left(V^{\prime} / \mathbf{G}\right)}
\end{aligned}
$$

as required.

Part 2 is complete. 
By part 1 and 2, we thus have

$$
L\left(V^{\prime} / \mathbf{G}\right)=\overline{L\left(V^{\prime} / \mathbf{G}\right) \cap L_{m}(\mathbf{S}) \cap L_{m}(\mathbf{G})}
$$

i.e. $V^{\prime}$ is non-blocking for $\mathbf{G}$. 


\section{Chapter 6}

\section{Symbolic Verification for SD System}

In this section, we will present algorithms to verify nonblocking, untimed controllability, ALF, proper time behavior, plant completeness, $\mathbf{S}$-singular prohibitable behavior, and SD controllability. To ensure scalability, we will develop predicate based algorithms that are built upon the work of Song [26]. We will first introduce predicates, and then discuss how we can use them to verify properties of interest. We then present our new algorithms, as well as a few that we will re-use from [26].

All the data representations, computations and verifications are based on ordered binary decision diagram [8]. For simplicity, we will just use the term BDD. In the appendix, you will find the source code for the software tool we developed to implement our algorithms. The code is based on the software developed by Song [26], and uses his BDD variable ordering algorithm. The code also uses the BuDDy library [13] which is a $\mathrm{C}++$ library that implements standard BDD structures and operations.

\subsection{Predicates and Predicate Transformers}

\subsubsection{State Predicates}

From now on, we will use ' $\equiv$ ' to mean logical equivalence between state predicates. We will also use ' $T$ ' and ' $F$ ' for logical true and false. 
Let $\mathbf{G}=\left(Q, \Sigma, \delta, q_{0}, Q_{m}\right)$ be a TDES.

Definition 6.1.1. A predicate $P$ defined on state set $Q$ is a function

$$
P: Q \rightarrow\{T, F\}
$$

identified by the corresponding state subset

$$
Q_{P}:=\{q \in Q \mid P(q)=T\} \subseteq Q
$$

We identify state predicate true by $Q$, state predicate false by $\emptyset$, and state predicate $P_{m}$ by $Q_{m}$.

We write $q \models P$ if $q \in Q_{P}$ and say " $q$ satisfies $P$ " or " $P$ includes $q$ ". Thus we have

$$
q \models P \Longleftrightarrow P(q)=T
$$

We write $\operatorname{Pred}(Q)$ for the set of all predicates defined on $Q$; thus $\operatorname{Pred}(Q)$ is identified by $\operatorname{Pwr}(Q)$. For $P \in \operatorname{Pred}(Q)$, we write $s t(P)$ for the corresponding state subset $Q_{P} \subseteq Q$ which identifies $P$. We write $\operatorname{pr}(Q)$ to represent the predicate that is identified by $Q$.

Definition 6.1.2. For $P, P_{1}, P_{2} \in \operatorname{Pred}(Q)$ and $q \in Q$, we can build boolean expressions by using the following predicate operations.

$$
\begin{aligned}
(\neg P)(q)=T & \Longleftrightarrow \quad P(q)=F \\
\left(P_{1} \wedge P_{2}\right)(q)=T \quad & \Longleftrightarrow \quad P_{1}(q)=T \text { and } P_{2}(q)=T \\
\left(P_{1} \vee P_{2}\right)(q)=T & \Longleftrightarrow \quad P_{1}(q)=T \text { or } P_{2}(q)=T \\
\left(P_{1}-P_{2}\right)(q)=T & \Longleftrightarrow \quad P_{1}(q)=T \text { and } P_{2}(q)=F
\end{aligned}
$$

Definition 6.1.3. The partial order relation $\preceq$ over $\operatorname{Pred}(Q)$ is defined as

$$
\left(\forall P_{1}, P_{2} \in \operatorname{Pred}(Q)\right) P_{1} \preceq P_{2} \Longleftrightarrow\left(P_{1} \wedge P_{2}\right) \equiv P_{1}
$$

It is obvious that $Q_{P_{1}} \subseteq Q_{P_{2}} \Longleftrightarrow P_{1} \preceq P_{2}$. In this case,

$$
(\forall q \in Q) q \models P_{1} \Longrightarrow q \models P_{2}
$$

Definition 6.1.4. Let $P_{1}, P_{2} \in \operatorname{Pred}(Q)$ for some state set $Q . P_{1}$ is a subpredicate of $P_{2}$ if $P_{1} \preceq P_{2}$. We say $P_{1}$ is stronger than $P_{2}$ and $P_{2}$ is weaker than $P_{1}$.

We write $\operatorname{Sub}(P)$ to be the set of all the subpredicates of $P \in \operatorname{Pred}(Q)$ such that $\operatorname{Sub}(P)$ is identified by $\operatorname{Pwr}\left(Q_{P}\right)$. 


\subsubsection{Predicate Transformers}

Let $\mathbf{G}=\left(Q, \Sigma, \delta, q_{0}, Q_{m}\right)$ be a TDES and $P \in \operatorname{Pred}(Q)$. A predicate transformer is a function $f: \operatorname{Pred}(Q) \rightarrow \operatorname{Pred}(Q)$. Here we introduce several basic predicate transformers from [26] which are required by the following sections.

- $R(\mathbf{G}, P)$

The reachability predicate $R(\mathbf{G}, P)$ is true for exactly the states in $\mathbf{G}$ that can be reached from $q_{o}$ by states satisfying $P$. It is inductively defined as follows.

1. $q_{o} \models P \Longrightarrow q_{o} \models R(\mathbf{G}, P)$

2. $q \models R(\mathbf{G}, P) \& \sigma \in \Sigma \& \delta(q, \sigma)$ ! \& $\delta(q, \sigma) \models P \Longrightarrow \delta(q, \sigma) \models R(\mathbf{G}, P)$

3. No other states satisfy $R(\mathbf{G}, P)$.

It says that a state $q \models R(\mathbf{G}, P)$ if and only if there exists a path from $q_{o}$ to $q$ in $\mathbf{G}$ and each state in that path satisfies $P$. To represent the set of all reachable states in $Q$, we use $R(\mathbf{G}$, true $)$.

- $C R(\mathbf{G}, P)$

The coreachability predicate $C R(\mathbf{G}, P)$ is true for exactly the states in $\mathbf{G}$ that can reach a marked state by states satisfying $P$. It is inductively defined as follows.

1. $P_{m} \wedge P \equiv$ false $\Longrightarrow C R(\mathbf{G}, P) \equiv$ false

2. $q \models P_{m} \wedge P \Longrightarrow q \models C R(\mathbf{G}, P)$

3. $q \models C R(\mathbf{G}, P) \& q^{\prime} \models P \& \sigma \in \Sigma \& \delta\left(q^{\prime}, \sigma\right) ! \& \delta\left(q^{\prime}, \sigma\right)=q \Longrightarrow q^{\prime} \models$ $C R(\mathbf{G}, P)$

4. No other states satisfy $C R(\mathbf{G}, P)$.

It says that a state $q \models C R(\mathbf{G}, P)$ if and only if there exists a path from $q$ to some marked state in $\mathbf{G}$ and each state in that path satisfies $P$. To represent the set of all coreachable states in $Q$, we use $C R(\mathbf{G}$, true $)$. 
- $\mathcal{C R}\left(\mathbf{G}, P^{\prime}, \Sigma^{\prime}, P\right)$

Let $P^{\prime} \in \operatorname{Pred}(Q)$ and $\Sigma^{\prime} \subseteq \Sigma$. Once we fix $\mathbf{G}, P^{\prime}$ and $\Sigma^{\prime}, \mathcal{C R}\left(\mathbf{G}, P^{\prime}, \Sigma^{\prime}, P\right)$ is then a predicate transformer. The predicate $\mathcal{C R}\left(\mathbf{G}, P^{\prime}, \Sigma^{\prime}, P\right)$ is true for exactly the states in $\mathbf{G}$ that can reach a state in $\mathbf{G}$ satisfying $P^{\prime}$, by states that satisfy $P$ and by transition with events in $\Sigma^{\prime}$. It is inductively defined as follows.

1. $P^{\prime} \wedge P \equiv$ false $\Longrightarrow \mathcal{C} \mathcal{R}\left(\mathbf{G}, P^{\prime}, \Sigma^{\prime}, P\right) \equiv$ false

2. $q \models P^{\prime} \wedge P \Longrightarrow q \models \mathcal{C} \mathcal{R}\left(\mathbf{G}, P^{\prime}, \Sigma^{\prime}, P\right)$

3. $q \models \mathcal{C R}\left(\mathbf{G}, P^{\prime}, \Sigma^{\prime}, P\right) \& q^{\prime} \models P \& \sigma \in \Sigma^{\prime} \& \delta\left(q^{\prime}, \sigma\right)$ ! \& $\delta\left(q^{\prime}, \sigma\right)=q$ $\Longrightarrow q^{\prime} \models \mathcal{C} \mathcal{R}\left(\mathbf{G}, P^{\prime}, \Sigma^{\prime}, P\right)$

4. No other states satisfy $\mathcal{C R}\left(\mathbf{G}, P^{\prime}, \Sigma^{\prime}, P\right)$.

By comparing with definition of coreachablity predicate $C R$, we have

$$
\mathcal{C R}\left(\mathbf{G}, P_{m}, \Sigma, P\right) \equiv C R(\mathbf{G}, P)
$$

\subsection{Symbolic Representation}

For symbolic verification of SD systems, we need to have a representation for states and transitions. We will use the symbolic representation from Song [26], who in turn based his work on Ma [14]. In this section, we only introduce the necessary definitions from this representation that are needed for the computation and verification in the following sections.

\subsubsection{State Subsets}

Let $\mathbf{G}=\left(Q, \Sigma, \delta, q_{o}, Q_{m}\right)=\mathbf{G}_{1} \times \mathbf{G}_{2} \times . . \times \mathbf{G}_{n}$ be the product TDES of component TDES $\mathbf{G}_{i}$ where $\mathbf{G}_{i}=\left(Q_{i}, \Sigma_{i}, \delta_{i}, q_{o, i}, Q_{m, i}\right)$ for $i=1,2, . ., n$. For any state $q \in Q$, we have $q=\left(q_{1}, q_{2}, . ., q_{n}\right)$ where $q_{i} \in Q_{i}$.

In later sections we will be evaluating the meet of component TDES for some of the verifications. The only difference between the meet and the product of these TDES is that, the product might contain unreachable states but the meet does not. However, the checking of unreachable states is expensive and therefore the reachability check is 
performed over the entire system at the end. In addition, since including unreachable states does not effect the closed loop behavior, using the product TDES will not introduce any error.

Definition 6.2.1. For $\mathbf{G}=\mathbf{G}_{1} \times \mathbf{G}_{2} \times \ldots \times \mathbf{G}_{n}$, let $i=1,2, . ., n$ and $q_{i} \in Q_{i}$. The state variable $v_{i}$ for the $i$-th component TDES $\mathbf{G}_{i}$ is a variable of domain $Q_{i}$. If $v_{i}$ has assigned value $q_{i}$, then $v_{i}=q_{i}$ returns $T$; otherwise it returns $F$.

Here we use ' $=$ ' to if $v_{i}$ has been assigned value $q_{i}$, because ' $\equiv$ ' has been used for logical equivalence between state predicates.

Definition 6.2.2. For $\mathbf{G}=\mathbf{G}_{1} \times \mathbf{G}_{2} \times \ldots \times \mathbf{G}_{n}$, the state variable vector $\mathbf{v}$ is a vector $\left[v_{1}, v_{2}, . ., v_{n}\right]$ of state variables $v_{i}$ from each component TDES $\mathbf{G}_{i}$. For state subset $A \subseteq Q$, we write predicate

$$
P_{A}(\mathbf{v}):=\bigvee_{q \in A}\left(v_{1}=q_{1} \wedge v_{2}=q_{2} \wedge . . \wedge v_{n}=q_{n}\right)
$$

or $P_{A}$ if $\mathbf{v}$ is understood.

\subsubsection{Transitions}

Let $\mathbf{G}=\left(Q, \Sigma, \delta, q_{o}, Q_{m}\right)=\mathbf{G}_{1} \times \mathbf{G}_{2} \times . . \times \mathbf{G}_{n}$ be the product TDES of component TDES $\mathbf{G}_{i}=\left(Q_{i}, \Sigma_{i}, \delta_{i}, q_{o, i}, Q_{m, i}\right)$ for $i=1,2, . ., n$ as defined in previous section.

Definition 6.2.3. For $\mathbf{G}=\mathbf{G}_{1} \times \mathbf{G}_{2} \times \ldots \times \mathbf{G}_{n}$, let $\sigma \in \Sigma$. A transition predicate $N_{\sigma}: Q \times Q \rightarrow\{T, F\}$ identifies all the transitions for $\sigma$ in $\mathbf{G}$ and is defined as follows.

$$
\left(\forall q, q^{\prime} \in Q\right) N_{\sigma}\left(q, q^{\prime}\right):=\left\{\begin{array}{lc}
T, & \text { if } \delta(q, \sigma) ! \& \delta(q, \sigma)=q^{\prime} \\
F, & \text { otherwise. }
\end{array}\right.
$$

To distinguish between source states and destination states, we need to have two different vectors of state variables, as defined below.

Definition 6.2.4. For $\mathbf{G}=\mathbf{G}_{1} \times \mathbf{G}_{2} \times \ldots \times \mathbf{G}_{n}$, let $i=1,2, . ., n$. For each $\mathbf{G}_{i}$, we have the normal state variable $v_{i}$ (source state) and the prime state variable $v_{i}^{\prime}$ (destination state), both with domain $Q_{i}$. For $\mathbf{G}$, we have the normal state variable vector $\mathbf{v}=\left[v_{1}, v_{2}, . ., v_{n}\right]$ and the prime state variable vector $\mathbf{v}^{\prime}=\left[v_{1}^{\prime}, v_{2}^{\prime}, . ., v_{n}^{\prime}\right]$. 
For each $\sigma \in \Sigma$, we can write the transition predicate for $\sigma, N_{\sigma}$, as below. Essentially, if we set $\mathbf{v}=q$ and $\mathbf{v}^{\prime}=q^{\prime}$ such that $\delta(q, \sigma)=q^{\prime}$, then $N_{\sigma}\left(\mathbf{v}, \mathbf{v}^{\prime}\right)$ will return $T$.

$$
N_{\sigma}\left(\mathbf{v}, \mathbf{v}^{\prime}\right):=\bigwedge_{\{1 \leq i \leq n\}}\left(\bigvee_{\left\{q_{i}, q_{i}^{\prime} \in Q_{i} \mid \delta_{i}\left(q_{i}, \sigma\right)=q_{i}^{\prime}\right\}}\left(v_{i}=q_{i}\right) \wedge\left(v_{i}^{\prime}=q_{i}^{\prime}\right)\right)
$$

However, when designing a system with multiple component TDES defined over different event set, such as when we use the synchronous product operator, each component TDES must be selflooped at each state with events that are not in its own event set. This of course makes the transition predicate much more complicated. A new representation to avoid this issue is defined as below. Note that the size of $v_{\sigma}$ and $v_{\sigma}^{\prime}$ will always be the same.

Definition 6.2.5. We use the transition tuple $\left(\mathbf{v}_{\sigma}, \mathbf{v}_{\sigma}^{\prime}, N_{\sigma}\right)$ to represent the transition on $\sigma$, where $\mathbf{v}_{\sigma}=\left\{v_{i} \in \mathbf{v} \mid \sigma \in \Sigma_{i}\right\}, \mathbf{v}_{\sigma}^{\prime}=\left\{v_{i}^{\prime} \in \mathbf{v}^{\prime} \mid \sigma \in \Sigma_{i}\right\}$ and

$$
N_{\sigma}\left(\mathbf{v}, \mathbf{v}^{\prime}\right):=\bigwedge_{\left\{1 \leq i \leq n \mid \sigma \in \Sigma_{i}\right\}}\left(\bigvee_{\left\{q_{i}, q_{i}^{\prime} \in Q_{i} \mid \delta_{i}\left(q_{i}, \sigma\right)=q_{i}^{\prime}\right\}}\left(v_{i}=q_{i}\right) \wedge\left(v_{i}^{\prime}=q_{i}^{\prime}\right)\right)
$$

Although selflooped transitions are not specified in the definition, the selfloop information is still expressed. For those state variables that are not in $\mathbf{v}_{\sigma}$, we know that the corresponding component TDES must be selflooped with event $\sigma$ on each state, so we do not need to express this explicitly. Definition 6.2 .5 will work fine with systems where these self-loops have already been added.

Since BDD [8] does not support first order logic by itself, to compute state transitions we will need the following definition taken from the existential quantifier elimination method for finite domain [1].

Definition 6.2.6. For $\mathbf{G}=\mathbf{G}_{1} \times \mathbf{G}_{2} \times . . \times \mathbf{G}_{n}$, let $\sigma \in \Sigma$ and $\left(\mathbf{v}_{\sigma}, \mathbf{v}_{\sigma}^{\prime}, N_{\sigma}\right)$ be the transition tuple for $\sigma$ in $\mathbf{G}$. For $i=1,2, . ., n$, if $v_{i} \in \mathbf{v}_{\sigma}$ and $v_{i}^{\prime} \in \mathbf{v}_{\sigma}^{\prime}$, then define

$$
\exists v_{i} N_{\sigma}:=\bigvee_{q_{i} \in Q_{i}} N_{\sigma}\left[q_{i} / v_{i}\right] \quad \exists v_{i}^{\prime} N_{\sigma}:=\bigvee_{q_{i} \in Q_{i}} N_{\sigma}\left[q_{i} / v_{i}^{\prime}\right]
$$

where $N_{\sigma}\left[q_{i} / v_{i}\right]$ is the predicate $N_{\sigma}$ with each term $v_{i}$ substituted by $q_{i}$, and $N_{\sigma}\left[q_{i} / v_{i}^{\prime}\right]$ is defined analogously. 
We use the above method to eliminate either the normal or prime variable, so that we can express the statement using propositional logic that we can represent as a BDD.

Let $\mathbf{v}_{\sigma}=\left\{v_{1}, v_{2}, . ., v_{m}\right\}$ for $m>0$. For convenience, we write $\exists \mathbf{v}_{\sigma} N_{\sigma}$ to represent $\exists v_{1}\left(\exists v_{2} . .\left(\exists v_{m} N_{\sigma}\right) ..\right)$ and the resulting predicate should contain only prime variables in $\mathbf{v}_{\sigma}^{\prime}$. For any computation of state predicates, we need all input variables to be consistent. That is, either all predicates in the computation have to be expressed as normal variables or prime variables. We thus need to substitute all the prime variables by normal variables, denoted as $\exists \mathbf{v}_{\sigma} N_{\sigma}\left[\mathbf{v}_{\sigma}^{\prime} \rightarrow \mathbf{v}_{\sigma}\right]$. The substitution should return the predicate for the set of target states for $\sigma$ transitions in G. This means that each state in this set has a $\sigma$ transition entering it.

Let $\mathbf{v}_{\sigma}^{\prime}=\left\{v_{1}^{\prime}, v_{2}^{\prime}, . ., v_{m}^{\prime}\right\}$. For convenience, we also write $\exists \mathbf{v}_{\sigma}^{\prime} N_{\sigma}$ to represent $\exists v_{1}^{\prime}\left(\exists v_{2}^{\prime} . .\left(\exists v_{m}^{\prime} N_{\sigma}\right) ..\right)$ and the resulting predicate should contain only normal variables in $\mathbf{v}_{\sigma}$, which represents the set of source states for $\sigma$ transitions in G. This means that each state in this set has a $\sigma$ transition leaving it.

\subsection{Symbolic Computation}

We will now discuss symbolic computation based on the symbolic representation we just introduced. This work is based on the work of Song [26] who in turn based his work on Ma [14].

\subsubsection{Transitions and Inverse Transitions}

Let $\mathbf{G}=\left(Q, \Sigma, \delta, q_{o}, Q_{m}\right)=\mathbf{G}_{1} \times \mathbf{G}_{2} \times \ldots \times \mathbf{G}_{n}$ be a TDES plant. For a state $q \in Q$ and a event $\sigma \in \Sigma$, we want to compute the transition $\delta(q, \sigma)$ using the symbolic representation introduced previously. To do this, for $Q_{P} \subseteq Q$, where $P \in \operatorname{Pred}(Q)$, we can compute

$$
Q_{P}^{\prime}=\bigcup_{q \in Q_{p}}\{\delta(q, \sigma)\}
$$

and then find $P^{\prime}:=\operatorname{pr}\left(Q_{P}^{\prime}\right)$. However, computing $q^{\prime}$ one by one is time consuming for systems with large statespaces. Instead, we can directly compute the predicate of the set of next states from the predicate of the set of current states. 
The computation is based on a function $\hat{\delta}: \operatorname{Pred}(Q) \times \Sigma \rightarrow \operatorname{Pred}(Q)$ defined to be

$$
(\forall P \in \operatorname{Pred}(Q))(\forall \sigma \in \Sigma) \hat{\delta}(P, \sigma):=\operatorname{pr}\left(\left\{q^{\prime} \in Q \mid(\exists q \models P) \delta(q, \sigma)=q^{\prime}\right\}\right)
$$

As discussed in previous section, the formula $\exists \mathbf{v}_{\sigma} N_{\sigma}\left[\mathbf{v}_{\sigma}^{\prime} \rightarrow \mathbf{v}_{\sigma}\right]$ returns a predicate representing the set of target states $\left\{q^{\prime} \in Q \mid(\exists q \in Q) \delta(q, \sigma)=q^{\prime}\right\}$. We thus have the following definition.

Definition 6.3.1. Let $\sigma \in \Sigma$ and $\left(\mathbf{v}_{\sigma}, \mathbf{v}_{\sigma}^{\prime}, N_{\sigma}\right)$ be the transition tuple for $\sigma$ in G. For $P \in \operatorname{Pred}(Q)$,

$$
\hat{\delta}(P, \sigma):=\left(\exists \mathbf{v}_{\sigma}\left(N_{\sigma} \wedge P\right)\right)\left[\mathbf{v}_{\sigma}^{\prime} \rightarrow \mathbf{v}_{\sigma}\right]
$$

By first computing $N_{\sigma} \wedge P$ in the above definition, we are restricting the source states to those satisfying $P$.

We also need an inverse function $\hat{\delta}^{-1}: \operatorname{Pred}(Q) \times \Sigma \rightarrow \operatorname{Pred}(Q)$ to compute the predicate of the set of source states from the predicate representing the set of target states, where $\hat{\delta}^{-1}$ is defined to be

$$
(\forall P \in \operatorname{Pred}(Q))(\forall \sigma \in \Sigma) \hat{\delta}^{-1}(P, \sigma):=\operatorname{pr}(\{q \in Q \mid \delta(q, \sigma) \models P\})
$$

Since the formula $\exists \mathbf{v}_{\sigma}^{\prime} N_{\sigma}$ returns a predicate representing the set of source states $\{q \in Q \mid \delta(q, \sigma) !\}$, we have the following definition.

Definition 6.3.2. Let $\sigma \in \Sigma$ and $\left(\mathbf{v}_{\sigma}, \mathbf{v}_{\sigma}^{\prime}, N_{\sigma}\right)$ be the transition tuple for $\sigma$ in $\mathbf{G}$. For $P \in \operatorname{Pred}(Q)$,

$$
\hat{\delta}^{-1}(P, \sigma):=\exists \mathbf{v}_{\sigma}^{\prime}\left(N_{\sigma} \wedge\left(P\left[\mathbf{v}_{\sigma} \rightarrow \mathbf{v}_{\sigma}^{\prime}\right]\right)\right)
$$

In the definition, $P\left[\mathbf{v}_{\sigma} \rightarrow \mathbf{v}_{\sigma}^{\prime}\right]$ returns predicate $P$ with its normal variables substituted by prime variables. As prime variables represent target states, this has the effect of restricting the target states to those satisfying $P$. 


\subsubsection{Computation of Predicate Transformers}

Let $\mathbf{G}=\left(Q, \Sigma, \delta, q_{o}, Q_{m}\right)=\mathbf{G}_{1} \times \mathbf{G}_{2} \times . . \times \mathbf{G}_{n}$ be the cross product TDES of component TDES $\mathbf{G}_{i}$ for $i=1,2, . ., n$. Let $P \in \operatorname{Pred}(Q)$. To compute the predicate transformers $R$ and $C R$ introduced in Section 6.1.2, we have the following algorithms which are taken from [26].

\section{Reachability Check}

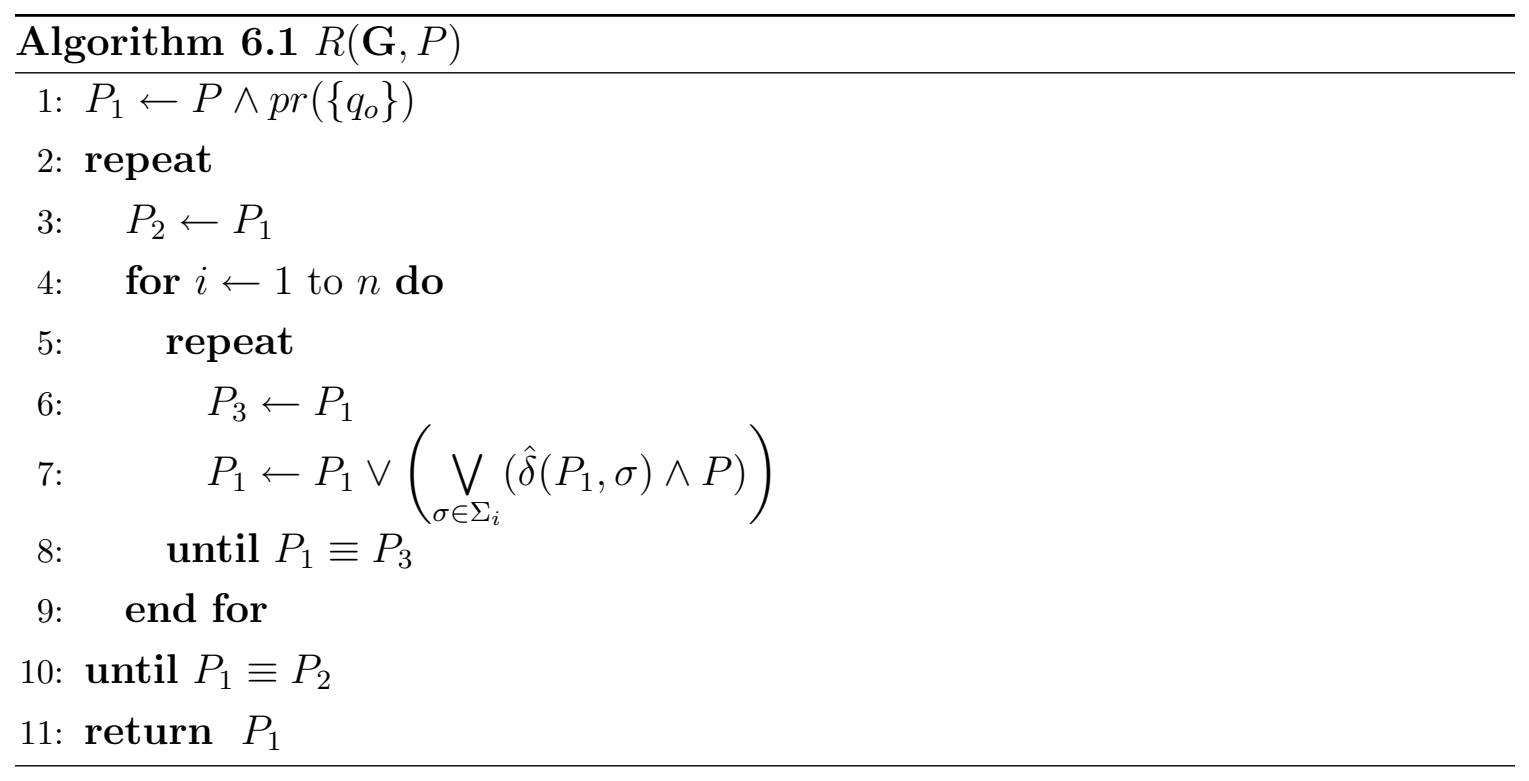

In Algorithm 6.1, procedure $R(\mathbf{G}, P)$ takes a TDES $\mathbf{G}$ and a predicate $P$, then returns a predicate which holds a set of states in $\mathbf{G}$ that can be reached from $q_{o}$ by states satisfying $P$.

At line $1, P_{1}$ is initialized to be the predicate which represents the initial state $q_{o}$ or $\emptyset$ if $q_{o} \not \models P$.

From line 2 to line 10, for $i \in 1, . ., n$, we loop over $\sigma \in \Sigma_{i}$ and determine states that satisfy $P$, and are reachable from a state that satisfies $P_{1}$ by a $\sigma$ transition.

Due to the intermediate logic formula expansion problem described in [26], that intermediate logic formula can become large and complicated even though the final predicate might be relatively small, the for loop on line 4 to line 9 repeatedly modifies $P_{1}$ on a component TDES basis. We start with a specific TDES, $\mathbf{G}_{i}$, and 
determine next states using only events from $\Sigma_{i}$ until no more changes. Then move onto next TDES. For each component TDES $\mathbf{G}_{i}, P_{1}$ is modified until it is logical equivalent to its previous value, $P_{3}$. We cycle through all the TDES until no further changes.

\section{Coreachability Check}

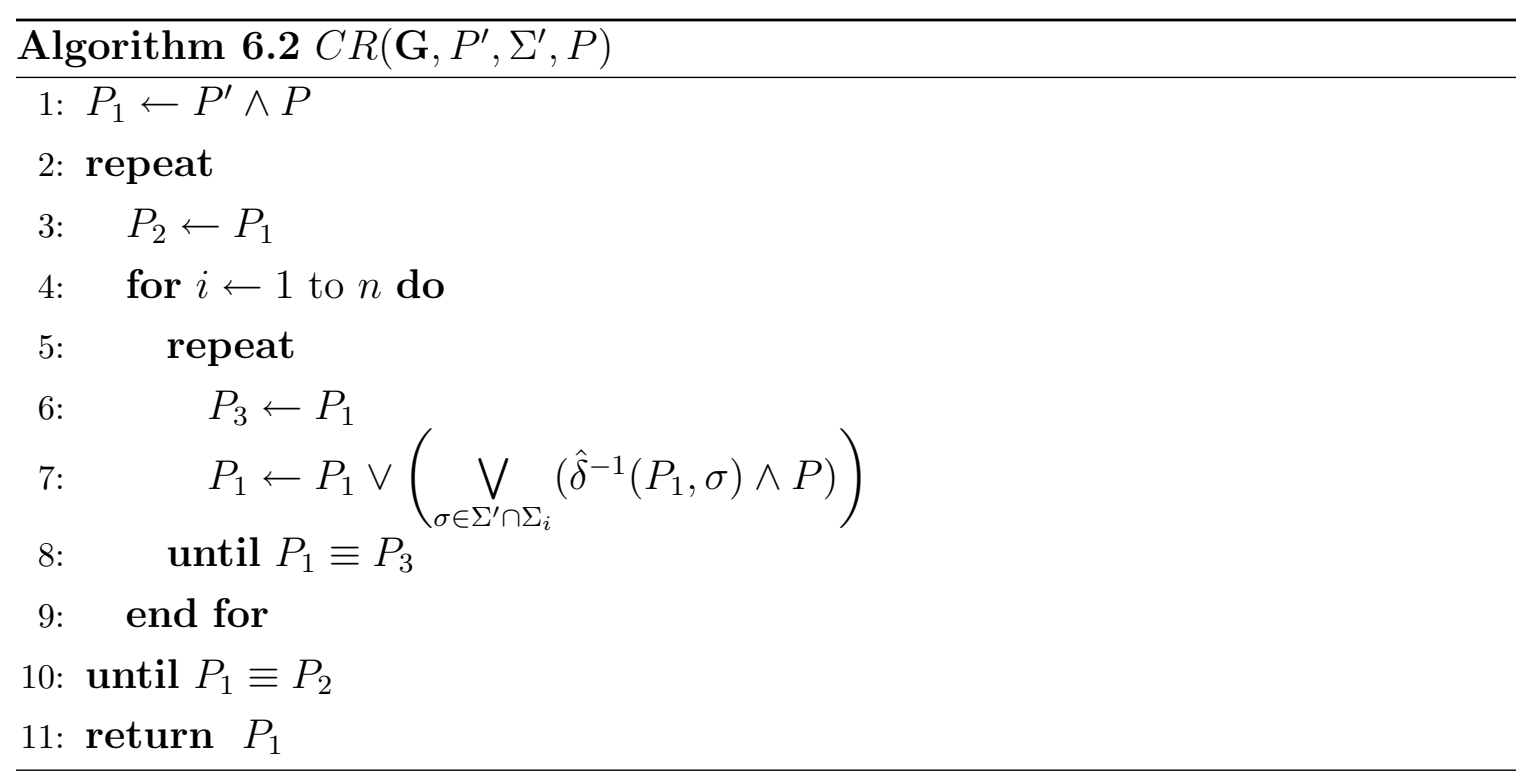

In Algorithm 6.2. procedure $\mathcal{C R}\left(\mathbf{G}, P^{\prime}, \Sigma^{\prime}, P\right)$ takes a TDES $\mathbf{G}$, a predicate $P^{\prime}$, an event set $\Sigma^{\prime}$ and a predicate $P$, then returns a predicate which represents a set of states in $\mathbf{G}$ that can reach a state in $\mathbf{G}$ satisfying $P^{\prime}$ by states that satisfy $P$ and by transition with events in $\Sigma^{\prime}$. We do not present an algorithm for $C R(Q, P)$ as it is a special case which is equivalent to $\mathcal{C} \mathcal{R}\left(\mathbf{G}, P_{m}, \Sigma, P\right)$.

At line $1, P_{1}$ is initialized to be the predicate which represents the set of states in $Q_{P^{\prime}}$ which satisfies predicate $P$ as well.

Like in Algorithm 6.1, line 4 to line 9 focus on one TDES event set at a time to reduce the complexity of intermediate logic formulas. In line 7 , we are adding to $P_{1}$ the states in $P$ that can be reached by a state in $P_{1}$ via an event in $\Sigma^{\prime} \cap \Sigma_{i}$.

We iterate until there are no more changes. 


\subsection{Symbolic Verification}

The TDES systems we are interested in are composed of a plant $\mathbf{G}$ and a supervisor S, with system event set $\Sigma$.

Given $\mathbf{G}_{i}^{\prime}=\left(Y_{i}, \Sigma_{i}, \delta_{i}, y_{o, i}, Y_{m, i}\right)$ and $\mathbf{G}^{\prime}=\mathbf{G}_{1}^{\prime}\left\|\mathbf{G}_{2}^{\prime}\right\| . . \| \mathbf{G}_{n}^{\prime}$, for $i=1,2, . ., n$, let $\mathbf{G}_{i}=\operatorname{selfloop}\left(\mathbf{G}_{i}^{\prime}, \Sigma-\Sigma_{i}\right)$. The plant is defined as:

$$
\mathbf{G}=\mathbf{G}_{1} \times \mathbf{G}_{2} \times \ldots \times \mathbf{G}_{n}:=\left(Y, \Sigma, \delta, y_{o}, Y_{m}\right)
$$

Given $\mathbf{S}_{i}=\left(X_{i}, \Sigma, \xi_{i}, x_{o, i}, X_{m, i}\right)$, the supervisor is defined as

$$
\mathbf{S}=\mathbf{S}_{1} \times \mathbf{S}_{2} \times \ldots \times \mathbf{S}_{m}:=\left(X, \Sigma, \xi, x_{o}, X_{m}\right)
$$

Therefore both $\mathbf{G}$ and $\mathbf{S}$ are defined over the global event set $\Sigma$. If our component supervisors were defined over subsets of $\Sigma$ and combined together using the synchronous product, we would add selfloops of the missing events as we did for the plant components, and then use these new DES from then on.

The closed-loop system, $\mathbf{G}_{c l}$, is the product of the plant and supervisor

$$
\mathbf{G}_{c l}=\mathbf{G} \times \mathbf{S}:=\left(Q, \Sigma, \eta, q_{o}, Q_{m}\right)
$$

where $Q=Y \times X=Y_{1} \times Y_{2} \times \ldots \times Y_{n} \times X_{1} \times X_{2} \times \ldots \times X_{m}, \Sigma=\Sigma_{c} \dot{\cup} \Sigma_{u}, \eta=\delta \times \xi$, $q_{o}=\left(y_{o}, x_{o}\right)$ and $Q_{m}=Y_{m} \times X_{m}$. See Definition 2.2.11 for more details.

Note that we cannot use $\mathbf{G}_{c l}=\operatorname{meet}(\mathbf{G}, \mathbf{S})$ as meet by definition only contains reachable states, which is too restrictive. The product DES is the same as meet, but it can include unreachable states.

Definition 6.4.1. Let $\mathbf{G}_{c l}=\mathbf{G} \times \mathbf{S}:=\left(Q, \Sigma, \eta, q_{o}, Q_{m}\right)$ where $\mathbf{G}=\mathbf{G}_{1} \times \mathbf{G}_{2} \times . . \times \mathbf{G}_{n}=$ $\left(Y, \Sigma, \delta, y_{o}, Y_{m}\right)$ and $\mathbf{S}=\mathbf{S}_{1} \times \mathbf{S}_{2} \times . . \times \mathbf{S}_{m}=\left(X, \Sigma, \xi, x_{o}, X_{m}\right)$. For a given event $\sigma \in \Sigma$, the $\sigma$ plant transition predicate $N_{\mathbf{G}, \sigma}: Q \times Q \rightarrow\{T, F\}$ can be written as

$$
N_{\mathbf{G}, \sigma}\left(\mathbf{v}, \mathbf{v}^{\prime}\right):=\bigwedge_{\{1 \leq i \leq n\}}\left(\bigvee_{\left\{y_{i}, y_{i}^{\prime} \in Y_{i} \mid \delta_{i}\left(y_{i}, \sigma\right)=y_{i}^{\prime}\right\}}\left(v_{i}=y_{i}\right) \wedge\left(v_{i}^{\prime}=y_{i}^{\prime}\right)\right)
$$

and the $\sigma$ supervisor transition predicate $N_{\mathbf{S}, \sigma}: Q \times Q \rightarrow\{T, F\}$ can be written as

$$
N_{\mathbf{S}, \sigma}\left(\mathbf{v}, \mathbf{v}^{\prime}\right):=\bigwedge_{\{1 \leq i \leq m\}}\left(\bigvee_{\left\{x_{i}, x_{i}^{\prime} \in X_{i} \mid \xi_{i}\left(x_{i}, \sigma\right)=x_{i}^{\prime}\right\}}\left(v_{i+n}=x_{i}\right) \wedge\left(v_{i+n}^{\prime}=x_{i}^{\prime}\right)\right)
$$


$N_{\mathbf{G}, \sigma}$ and $N_{\mathbf{S}, \sigma}$ are state predicates defined on $Q \times Q$ and use the $\mathbf{v}$ and $\mathbf{v}^{\prime}$ variables like $N_{\sigma}$. We use $N_{\mathbf{G}, \sigma}$ when we wish to determine if there is a $\sigma$ defined at the plant portion of the indicated states, say for when we are checking controllability. Similarly, we use $N_{\mathbf{S}, \sigma}$ when we wish to determine if there is a $\sigma$ defined at the supervisor portion of the indicated states. They must be defined over $Q \times Q$ so the results of each can be compared and combined with other state predicates on $Q$.

Definition 6.4.2. Let $\sigma \in \Sigma$ and $N_{\mathbf{G}, \sigma}$ be the $\sigma$ transition predicate for plant $\mathbf{G}=$ $\left(Y, \Sigma, \delta, y_{o}, Y_{m}\right)$. We define $\hat{\delta}_{\mathrm{G}}: \operatorname{Pred}(Q) \times \Sigma \rightarrow \operatorname{Pred}(Q)$, for $P \in \operatorname{Pred}(Q)$, to be

$$
\hat{\delta}_{\mathbf{G}}(P, \sigma):=\left(\exists \mathbf{v}\left(N_{\mathbf{G}, \sigma} \wedge P\right)\right)\left[\mathbf{v}^{\prime} \rightarrow \mathbf{v}\right]
$$

and we also define $\hat{\delta}_{\mathbf{G}}^{-1}: \operatorname{Pred}(Q) \times \Sigma \rightarrow \operatorname{Pred}(Q)$ to be

$$
\hat{\delta}_{\mathbf{G}}^{-1}(P, \sigma):=\exists \mathbf{v}^{\prime}\left(N_{\mathbf{G}, \sigma} \wedge\left(P\left[\mathbf{v} \rightarrow \mathbf{v}^{\prime}\right]\right)\right)
$$

Definition 6.4.3. Let $\sigma \in \Sigma$ and $N_{\mathbf{S}, \sigma}$ be the $\sigma$ transition predicate for supervisor $\mathbf{S}=\left(X, \Sigma, \xi, x_{o}, X_{m}\right)$. We define $\hat{\xi}: \operatorname{Pred}(Q) \times \Sigma \rightarrow \operatorname{Pred}(Q)$, for $P \in \operatorname{Pred}(Q)$, to be

$$
\hat{\xi}(P, \sigma):=\left(\exists \mathbf{v}\left(N_{\mathbf{S}, \sigma} \wedge P\right)\right)\left[\mathbf{v}^{\prime} \rightarrow \mathbf{v}\right]
$$

and we also define $\hat{\xi}^{-1}: \operatorname{Pred}(Q) \times \Sigma \rightarrow \operatorname{Pred}(Q)$ to be

$$
\hat{\xi}^{-1}(P, \sigma):=\exists \mathbf{v}^{\prime}\left(N_{\mathbf{S}, \sigma} \wedge\left(P\left[\mathbf{v} \rightarrow \mathbf{v}^{\prime}\right]\right)\right)
$$

\subsubsection{Untimed Controllability}

To verify that a supervisor $\mathbf{S}=\left(X, \Sigma, \xi, x_{o}, X_{m}\right)$ is controllable with respect to plant $\mathbf{G}=\left(Y, \Sigma, \delta, y_{o}, Y_{m}\right)$, we need the closed loop system $\mathbf{G}_{c l}=\left(Q, \Sigma, \eta, q_{o}, Q_{m}\right)$ as defined in Section 6.4. For $q \in Q$, there must exist a state $x \in X$ and $y \in Y$ such that $q=(y, x)$.

According to Definition 2.2.15 for untimed controllability, we can express the states that could cause $\mathbf{S}$ to be uncontrollable for $\mathbf{G}$ (if they are reachable), as follows:

Definition 6.4.4. Let $\mathbf{S}=\left(X, \Sigma, \xi, x_{o}, X_{m}\right)$ be a supervisor. Let $\mathbf{G}=\left(Y, \Sigma, \delta, y_{o}, Y_{m}\right)$ be a plant, then

$$
Q_{\text {bad }}=\left\{q=(y, x) \in Q \mid\left(\exists \sigma_{u} \in \Sigma_{u}\right) \delta\left(y, \sigma_{u}\right) ! \& \xi\left(x, \sigma_{u}\right) \not y\right\}
$$


By this definition, the state set $Q_{b a d}$ includes all states $q \in Q$ in system $\mathbf{G}_{c l}$ that an uncontrollable event is eligible at the corresponding state in plant $\mathbf{G}$ but not eligible in the corresponding state in supervisor $\mathbf{S}$. We consider such states bad. Of course, not all states in $Q_{b a d}$ are necessarily reachable. Therefore $\mathbf{S}$ is controllable with to respect to $\mathbf{G}$ if $Q_{\text {bad }} \cap Q_{\text {reach }}=\emptyset$ where $Q_{\text {reach }}$ is the set of reachable states.

The corresponding predicate $P_{b a d}:=\operatorname{pr}\left(Q_{b a d}\right)$ is defined to be

$$
P_{\text {bad }}=\bigvee_{\sigma_{u} \in \Sigma_{u}}\left(\hat{\delta}_{\mathbf{G}}^{-1}\left(\text { true }, \sigma_{u}\right) \wedge \neg \hat{\xi}^{-1}\left(\text { true }, \sigma_{u}\right)\right)
$$

where $\hat{\delta}_{\mathbf{G}}^{-1}$ and $\hat{\xi}^{-1}$ are the inverse transition predicate functions for $\mathbf{G}$ and $\mathbf{S}$ respectively. We thus have $\mathbf{S}$ is controllable with respect to $\mathbf{G}$ if $P_{b a d} \wedge P_{\text {reach }} \equiv$ false where $P_{\text {reach }}:=\operatorname{pr}\left(Q_{\text {reach }}\right)$ holds the set of reachable states. Otherwise, $P_{\text {bad }} \wedge P_{\text {reach }}$ represents the set of bad states where supervisor $\mathrm{S}$ has disabled an uncontrollable event.

Algorithm [6.3, from [26], checks untimed controllability. For each uncontrollable event $\sigma_{u}$, it looks for the reachable composite state at which $\sigma_{u}$ is eligible in $\mathbf{G}$ but not eligible in $\mathbf{S}$. If such a state exists, then $\mathbf{S}$ is not controllable with respect to $\mathbf{G}$. The algorithm returns $\operatorname{Tru} e^{\square}$ if the supervisor $\mathbf{S}$ is controllable with respect to $\mathbf{G}$ and False otherwise.

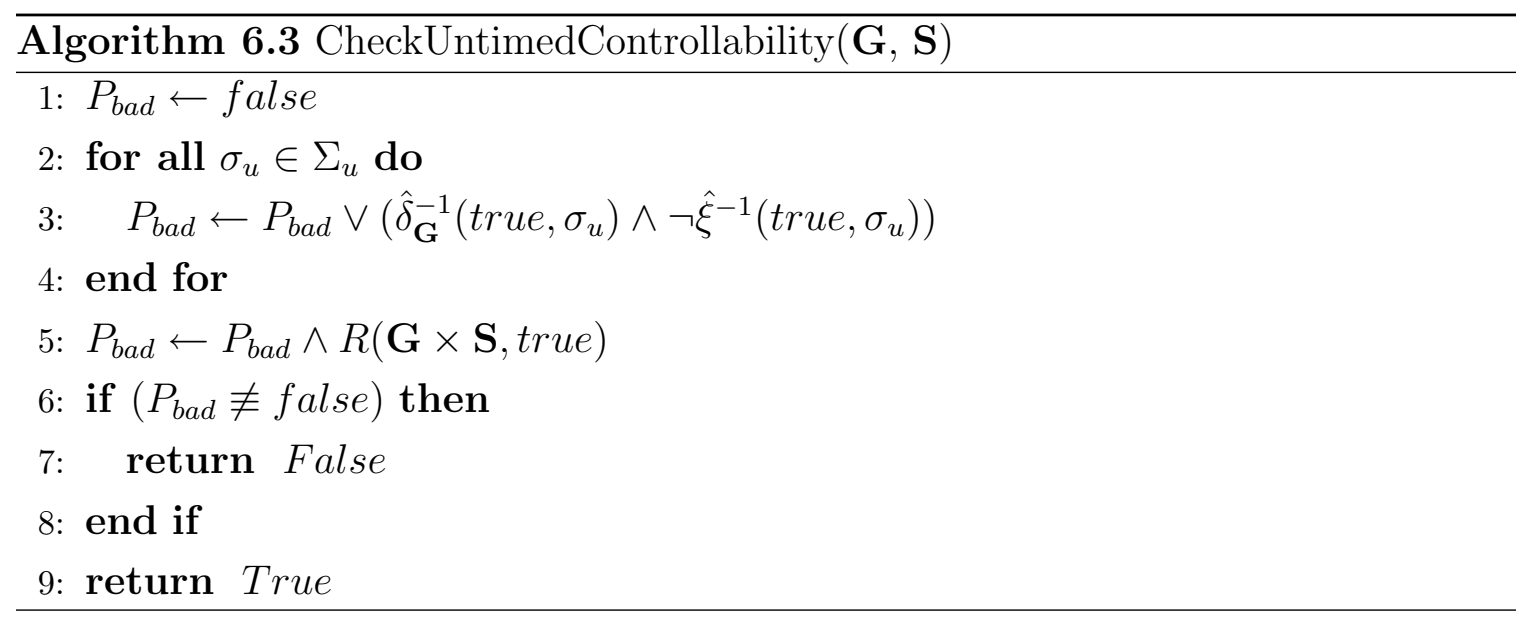

\footnotetext{
${ }^{1}$ We use True and False here because it is a boolean returned by the algorithm, instead of a state predicate.
} 


\subsubsection{Plant Completeness}

Similar to checking untimed controllability, we have the following definition for plant completeness.

Definition 6.4.5. Let $\mathbf{S}=\left(X, \Sigma, \xi, x_{o}, X_{m}\right)$ be a DES supervisor. Let $\mathbf{G}=\left(Y, \Sigma, \delta, y_{o}, Y_{m}\right)$ be a DES plant, then

$$
Q_{\text {incomplete }}=\left\{q=(y, x) \in Q \mid\left(\exists \sigma \in \Sigma_{h i b}\right) \xi(x, \sigma) ! \& \delta(y, \sigma) \not h\right\}
$$

By this definition, the state set $Q_{\text {incomplete }}$ includes all states $q$ in system $\mathbf{G}_{c l}$ that a prohibitable event is eligible at the corresponding state in supervisor $\mathbf{S}$ but not eligible in the corresponding state in plant $\mathbf{G}$. Plant $\mathbf{G}$ is complete for its supervisor $\mathbf{S}$ only if $Q_{\text {incomplete }} \cap Q_{\text {reach }}=\emptyset$. We only care about states in $Q_{\text {incomplete }}$ that are reachable.

The corresponding predicate $P_{\text {incomplete }}:=\operatorname{pr}\left(Q_{\text {incomplete }}\right)$ is defined to be

$$
P_{\text {incomplete }}=\bigvee_{\sigma \in \Sigma_{\text {hib }}}\left(\hat{\xi}^{-1}(\text { true }, \sigma) \wedge \neg \hat{\delta}_{\mathbf{G}}^{-1}(\text { true }, \sigma)\right)
$$

where $\hat{\delta}_{\mathbf{G}}^{-1}$ and $\hat{\xi}^{-1}$ are the inverse transition predicate functions for $\mathbf{G}$ and $\mathbf{S}$ respectively. Therefore the plant $\mathbf{G}$ is complete for its supervisor $\mathbf{S}$ only if $P_{\text {incomplete }} \wedge$ $P_{\text {reach }} \equiv$ false. Otherwise, $P_{\text {incomplete }} \wedge P_{\text {reach }}$ represents the set of states which fail the condition.

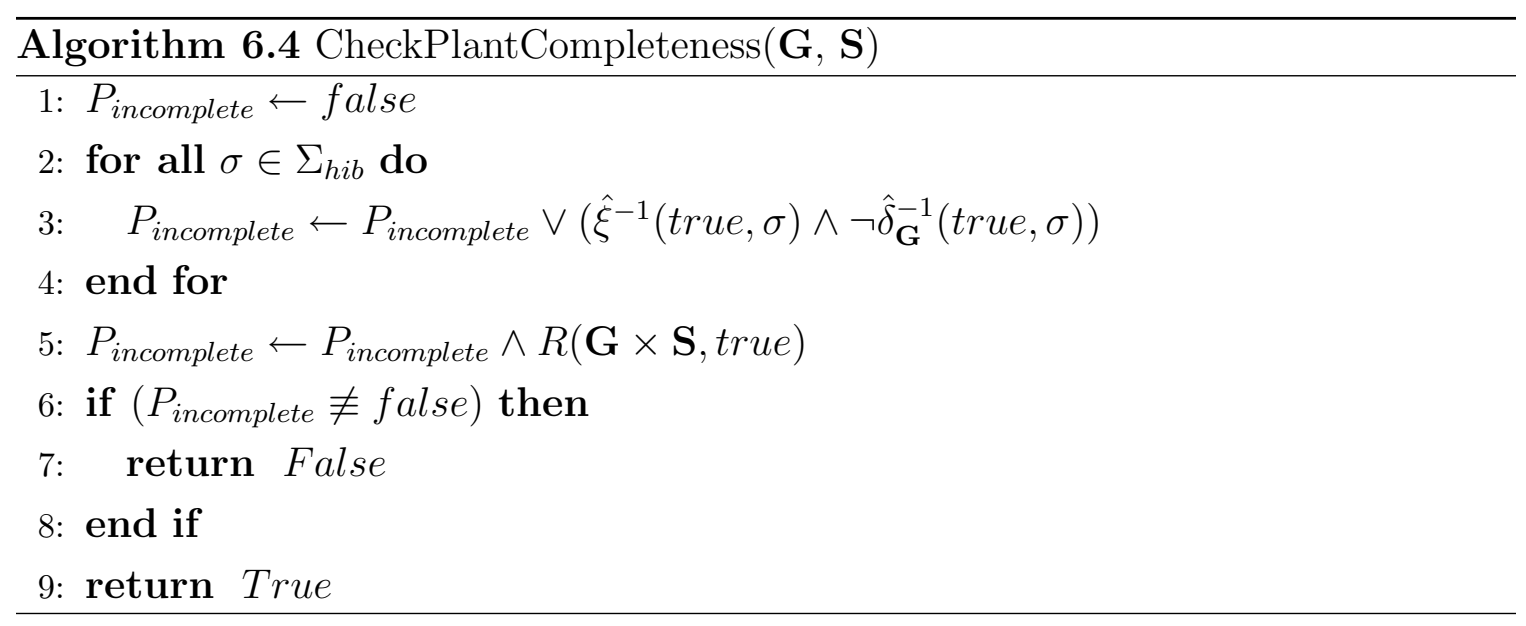

Algorithm 6.4 checks for plant completeness. For each prohibitable event $\sigma$, it looks for reachable composite states at which $\sigma$ is eligible in $\mathbf{S}$ but not eligible in 
G. If such a state exists, then plant $\mathbf{G}$ fails to be complete for supervisor $\mathbf{S}$ and the algorithm returns False. Otherwise it returns True.

\subsubsection{Non-blocking}

Algorithm 6.5 checks for non-blocking as defined in Definition 2.2.6. It compares the set of reachable states with the set of coreachable states, then returns True if there is no reachable state that is not coreachable and False otherwise.

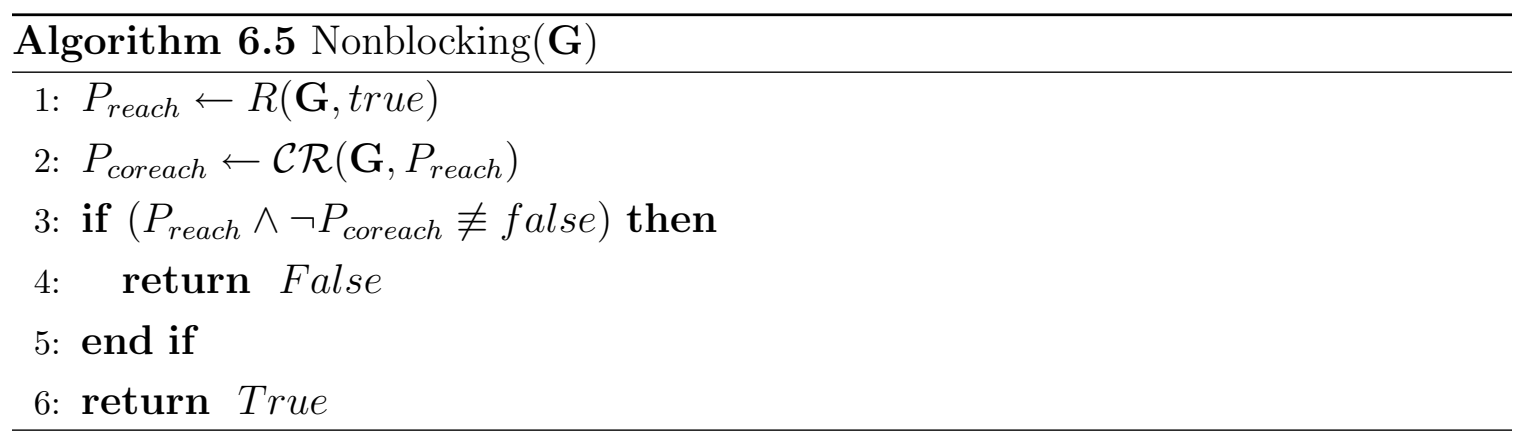

\subsubsection{Activity Loop Free}

By Definition 2.3.3 of Activity Loop Free (ALF), we require that for each reachable state in a TDES there will not be a non-empty string of activity events leaving from that state and back to itself. This is to prevent the TDES from "stopping the clock". Algorithm 6.6 checks the given TDES $\mathbf{G}$ and returns True if it is ALF and False otherwise.

At line 1, Algorithm 6.6 first calculates all the reachable states. Then for each state $q$ in $P_{c h k}$, it starts from any states $P_{v i s i t}$ reached via activity events from $q$ at line 4. From there, in the following loop from line $\mathbf{7}$ to line $\mathbf{1 7}$ it traverses to next states $P_{\text {next }}$ until no more state can be reached by activity events.

At each iteration of the loop, the algorithm first checks if there is an overlap between $P_{\text {visit }}$ and $P_{n e x t}$. Then it checks if state $q$ has been reached again. If state $q$ has been reached again, then the system is not ALF. Otherwise, the loop continues.

Once the check is done for state $q$, this state is removed from $P_{c h k}$. If there is no overlap found in the loop, all the visited states are removed from $P_{c h k}$. After that, 


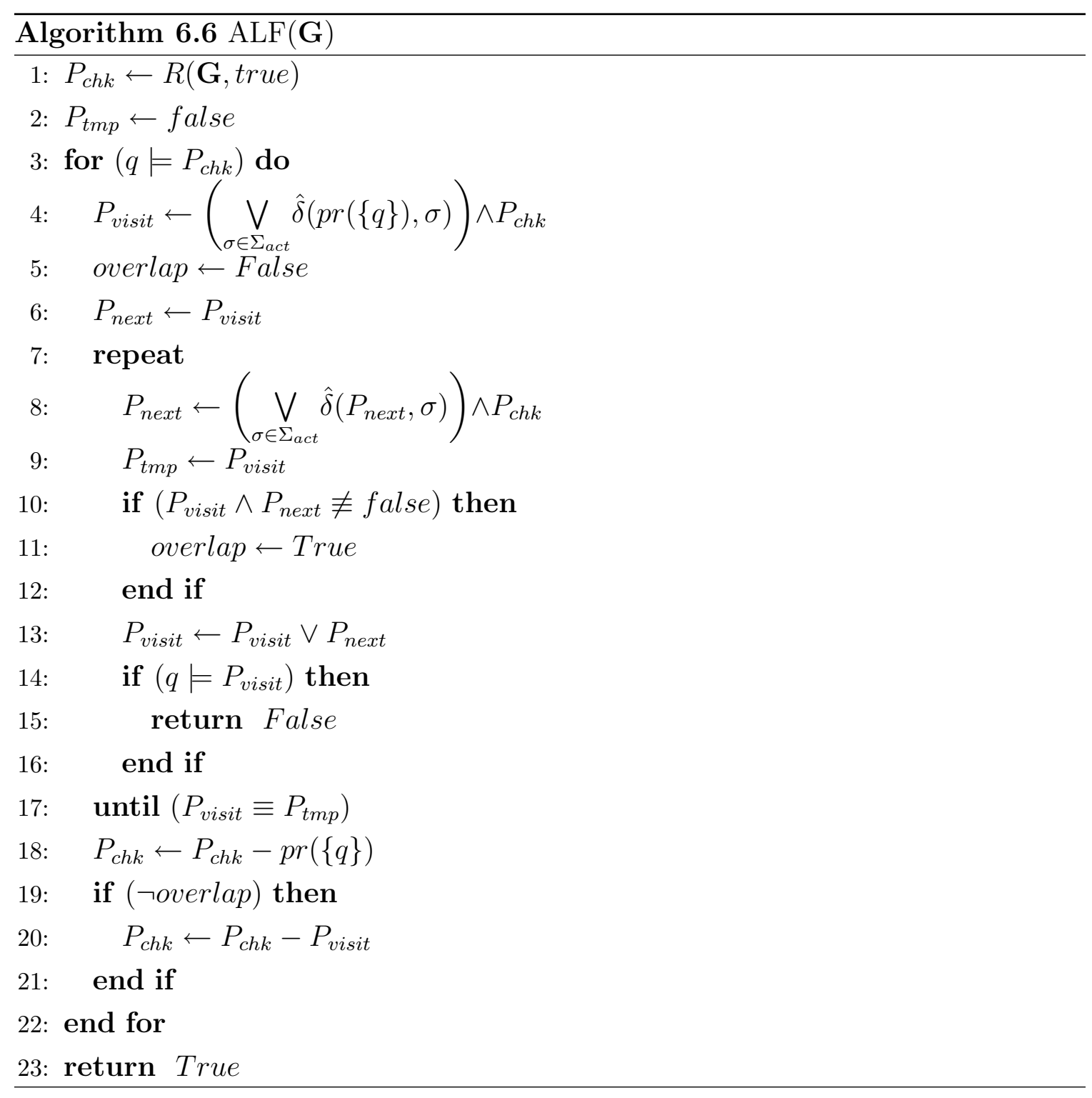

the algorithm moves to next state in $P_{c h k}$. If there was no False returned during the loop, the algorithm will consider it to be ALF and returns True.

\subsubsection{Proper Time Behavior}

By Definition 2.3.5 for Proper Time Behavior, we require that at each reachable state in a TDES plant, either an uncontrollable event or a tick event is eligible. Algorithm 
6.7 checks the given TDES plant $\mathbf{G}$ and returns True if it has a proper time behavior and False otherwise.

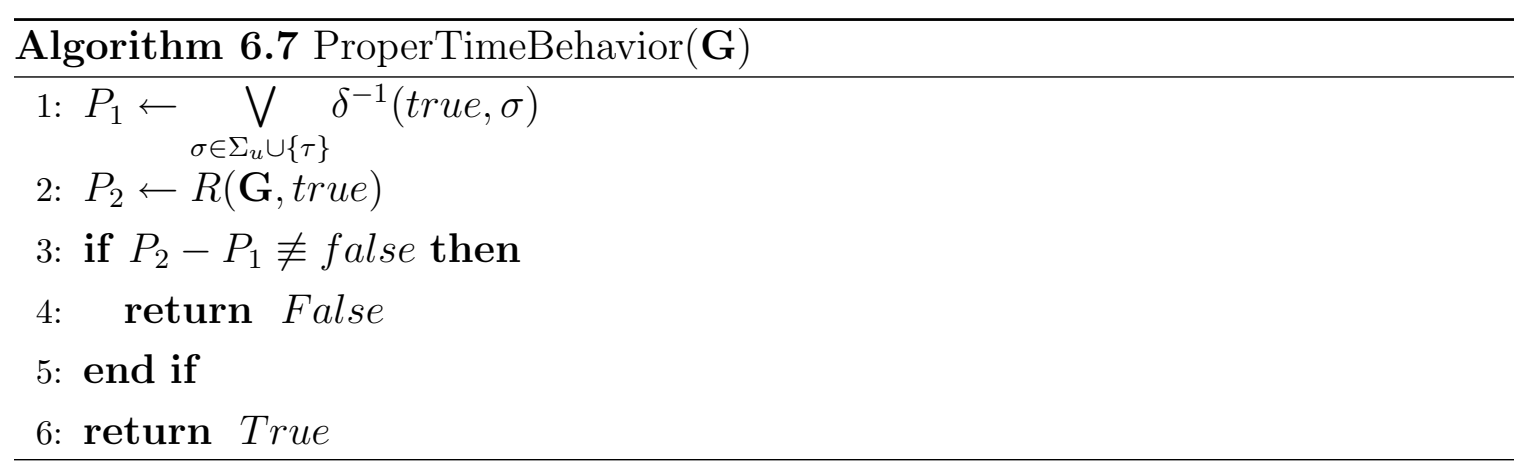

Algorithm 6.7first calculates $P_{1}$, the set of all states that have a $\Sigma_{u} \cup\{\tau\}$ transition leaving it. It then compares $P_{1}$ to the set $P_{2}$ of reachable states. When there is a state in $P_{2}$ but not in $P_{1}$, it implies that the state is reachable and neither a tick or an uncontrollable event is eligible at this state.

\subsubsection{SD Controllability and S-Singular Prohibitable Behav- ior}

Algorithm 6.8 evaluates SD controllability for supervisor $\mathbf{S}=\left(X, \Sigma, \xi, x_{o}, X_{m}\right)$ with respect to plant $\mathbf{G}=\left(Y, \Sigma, \delta, y_{o}, Y_{m}\right)$, where $\mathbf{G}, \mathbf{S}$, and the closed loop system, $\mathbf{G}_{c l}=$ $\mathbf{G} \times \mathbf{S}$ are as defined in Section 6.4. In addition, the algorithm's subroutine, Algorithm 6.11, also checks that $\mathbf{G}$ has $\mathbf{S}$-singular prohibitable behavior. As checking Point $\mathbf{i}$ of the SD controllability definition is the same as checking untimed controllability (Algorithm 6.3), we will not mention it explicitly here.

$\Sigma$ is defined to be $\Sigma=\Sigma_{h i b} \cup \dot{U} \Sigma_{u} \dot{\cup}\{\tau\}$, where $\Sigma_{h i b}$ is the set of prohibitable events in $\mathbf{G}$ and $\Sigma_{u}$ is the set of uncontrollable events in $\mathbf{G}$. The set of controllable events is $\Sigma_{c}=\Sigma_{h i b} \cup\{\tau\}$, and the set of activity events is $\Sigma_{a c t}=\Sigma_{h i b} \cup \Sigma_{u}$.

The algorithm makes the following assumptions:

- The set $\Sigma_{h i b}$ of prohibitable events equals the set $\Sigma_{\text {for }}$ of forcible events

- The plant has proper time behavior (checked by Algorithm 6.7)

- All TDES are finite and deterministic 
- The closed loop system, $\mathbf{G}_{c l}$, is activity loop free (ALF) (checked by Algorithm 6.6)

The algorithm uses certain variables as it executes.

$P_{\text {reach }}$ : The predicate of the set of reachable states of $\mathbf{G}_{c l}$.

$P_{S F}$ : The predicate of the set that contains sampling states of $\mathbf{G}_{c l}$ found by the algorithm.

$Z_{S P}$ : This set contains the predicates of sampling states in $\mathbf{G}_{c l}$ found and not yet analyzed by the algorithm.

$N_{\mathbf{G}, \sigma}, N_{\mathbf{S}, \sigma}$ : Transition predicates for $\sigma$ for $\mathbf{G}$ and $\mathbf{S}$ as in Definition 6.4.1.

$N_{\sigma}$ : Transition predicate for $\sigma$ for $\mathbf{G}_{c l}$ as in Definition 6.2.5.

$\hat{\delta}$ : Transition function for state predicates for $\mathbf{G}_{c l}$ as in Definition 6.3.1.

$\hat{\delta}_{\mathbf{G}}$ : Transition function for state predicates for $\mathbf{G}$ only as in Definition 6.4 .2 .

$\hat{\xi}$ : Transition function for state predicates for $\mathbf{S}$ only as in Definition 6.4.3.

pNerFail: This set $p N e r F a i l \subseteq \operatorname{Pwr}(\operatorname{Pred}(Q))$ is a set of sets of predicates that stores information where Point iii.2 in Definition 3.2 .2 of SD controllability may have failed.

SDControllable: This flag asserts if $\mathbf{S}$ is $\mathrm{SD}$ controllable with respect to $\mathbf{G}$.

Algorithm 6.8] starts at the initial state, which is always a sampling state. Then it analyzes the concurrent behavior of this state by creating a reachability tree with the initial state as a node. It expands the tree until all paths terminate at a tick event. Since we first check that the closed loop system is activity loop free, the system has a finite state space and that the plant has proper time behavior, we are either guaranteed that we will reach a tick after a finite number of events, or the system will fail Point ii of the SD controllability definition. Any new sampling states found are then analyzed as above, until all reachable sampling states have been analyzed.

As the reachability tree for a given sampling period is created, conformance to Definition 3.2.2 of SD controllability is tested. We also test here that $\mathbf{G}$ has $\mathbf{S}$-singular 


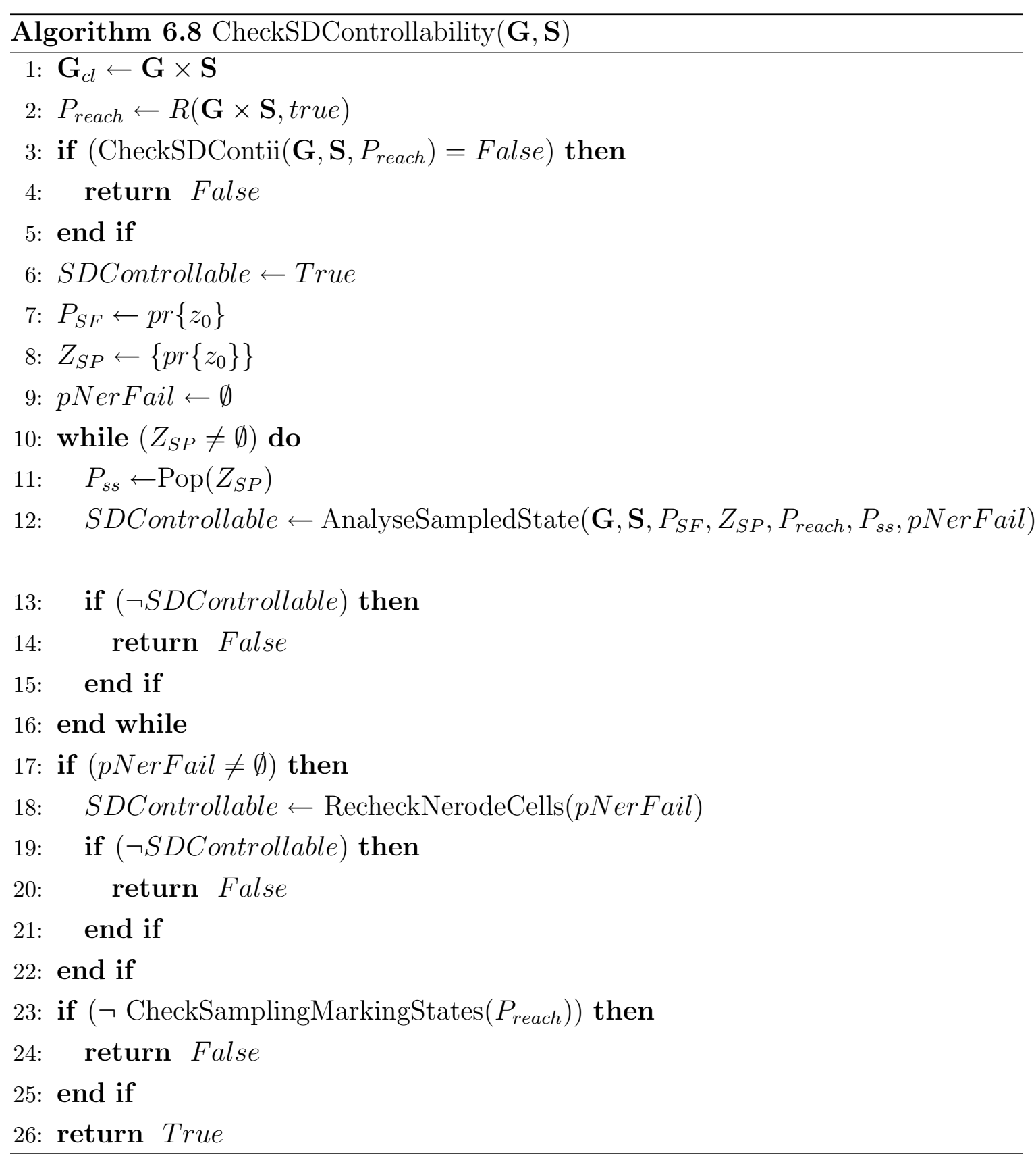

prohibitable behavior. With the exception of Point iii.2, evaluation stops if the test for any of the other points fail. If the test for Point iii.2 fails, the problem area is noted and the algorithm continues until all reachable sampling states have been analyzed. Nerode cells will be rechecked and then Point iii.2 is tested again. 
In the algorithm, pNerFail represents states reached by concurrent strings with the same occurrence image, thus should belong to the same equivalence classes for

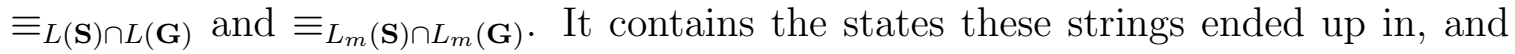
we will now check to see if these states actually represent the same equivalence cells. i.e. they are equivalent $\bmod \lambda$ (Definition 2.2.7).

Finally, the algorithm checks Point iv in Definition 3.2 .2 of SD controllability by comparing the set of marked states, implied by $P_{m}$, with the set of states reached by a tick event. If not all states implied by $P_{m}$ are reached by a tick and if that state not reached by a tick is not the initial state $z_{o}$, then it returns False.

If all tests pass, the algorithm returns True at the end.

See following sections for subroutines in Algorithm 6.8. The subroutine CheckSDContii is defined in Algorithm 6.9. The subroutine AnalyseSampledState is defined in Algorithm 6.10. The subroutine RecheckNerodeCells is defined in Algorithm 6.13. The subroutine CheckSamplingMarkingStates is defined in Algorithm 6.15.

\section{Point ii of SD Controllability}

Algorithm 6.9 checks Point ii of the SD Controllability definition. The algorithm takes the following three parameters: a plant $\mathbf{G}$, a supervisor $\mathbf{S}$ and a predicate $P_{\text {reach }}$ of all reachable states in $\mathbf{G}_{c l}$.

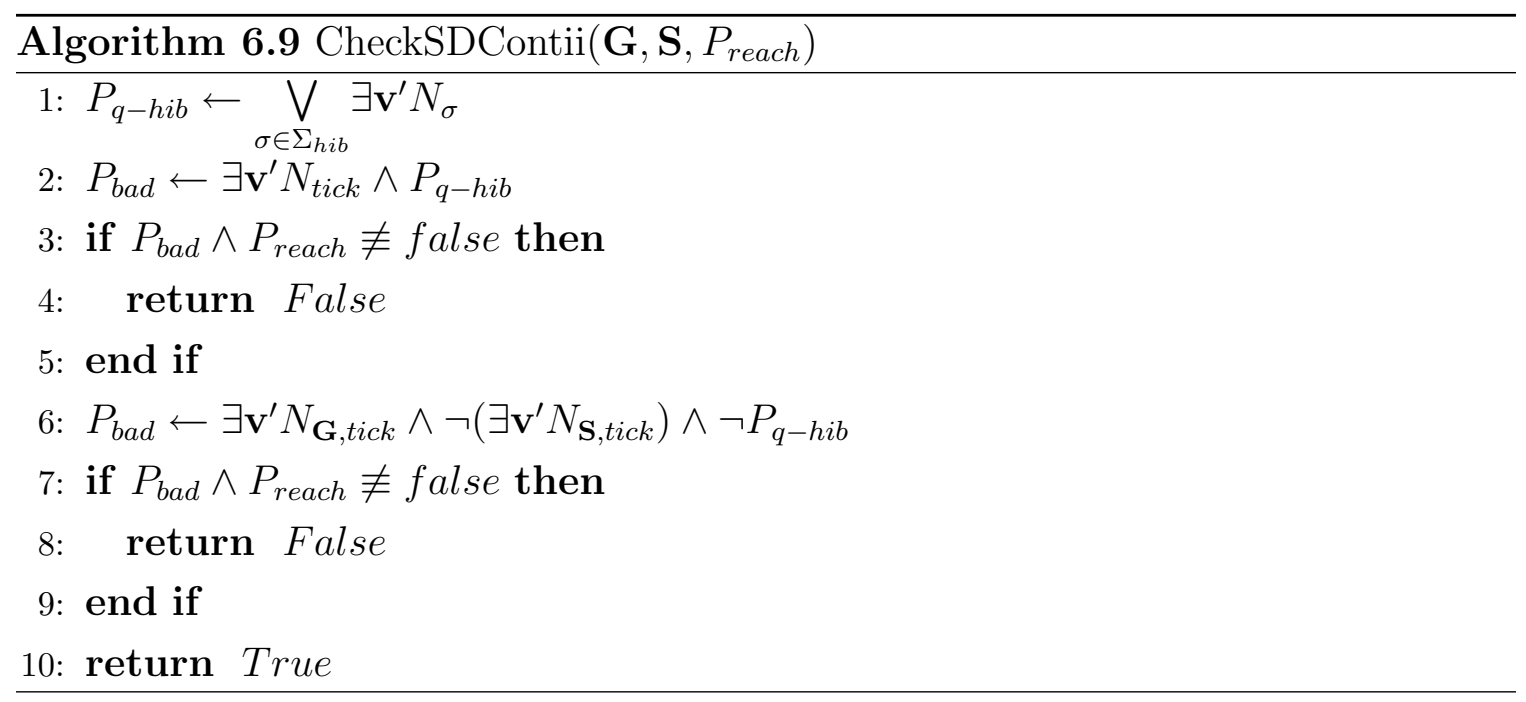

From line 1 to line 5 the algorithm checks the " $\Rightarrow$ " part of Point ii. It checks 
for any reachable states in $\mathbf{G}_{c l}$ that has both a prohibitable event and tick event enabled. If such a state exists, then it returns False.

Then from line 6 to line $\mathbf{9}$, the algorithm checks " $\Leftarrow$ " part of Point ii. It checks to see if a reachable state exists in $\mathbf{G}_{c l}$ where no prohibitable events are eligible, but a tick is eligible in $\mathbf{G}$ but not in $\mathbf{S}$. If such a state exists, then it returns False.

\section{AnalyzeSampledState}

Algorithm 6.10 analyzes the concurrent behavior for sampling state $q_{s s}$, represented by predicate $P_{s s}$. The algorithm takes seven parameters. See Algorithm 6.8 for their definitions.

During the execution, the algorithm uses the following variables:

$\Sigma_{\text {Elig }}$ : The set of prohibitable events eligible in both $\mathbf{G}$ and $\mathbf{S}$ at $q_{s s}$, the sampling state in $\mathbf{G}_{c l}$ that we are processing.

$P_{q}$ : The predicate of current state in $\mathbf{G}_{c l}$.

$\Sigma_{\text {poss }}$ : The set of events eligible in both $\mathbf{G}$ and $\mathbf{S}$ at predicate $P_{q}$ of current state in $\mathbf{G}_{c l}$.

$\Sigma_{\mathbf{G} p o s s}$ : The set of prohibitable events eligible in $\mathbf{G}$ at predicate $P_{q}$ of current state in $\mathbf{G}_{c l}$.

nextLabel: This number represents the next unused node in $B_{\text {map }}$. It is used to name newly discovered nodes of the reachability tree.

$B_{\text {map }}$ : This partial function $B_{\text {map }}: \mathcal{N} \rightarrow \operatorname{Pred}(Q)$ maps the nodes of the reachability tree to the predicates of the states of $\mathbf{G}_{c l}$ which the nodes represent. This function will sometimes be treated like the set $B_{\text {map }} \subseteq \mathcal{N} \times \operatorname{Pred}(Q)$. Note, $\mathcal{N}=\{0,1,2, \ldots\}$ is the set of natural numbers.

$B_{p}$ : This is the set of nodes pending to be expanded in the reachability tree.

$B_{\text {conc }}$ : The set $B_{\text {conc }} \subseteq \mathcal{N} \times \operatorname{Pred}(Q)$ contains nodes that represent concurrent strings and the sampled states the strings lead to. For $(b, q) \in B_{\text {conc }}$, the node $b$ is a node at which tick is eligible in $\mathbf{G}$ and $\mathbf{S}$, and $q$ is the sampling state of $\mathbf{G}_{c l}$ that the tick leads to. 


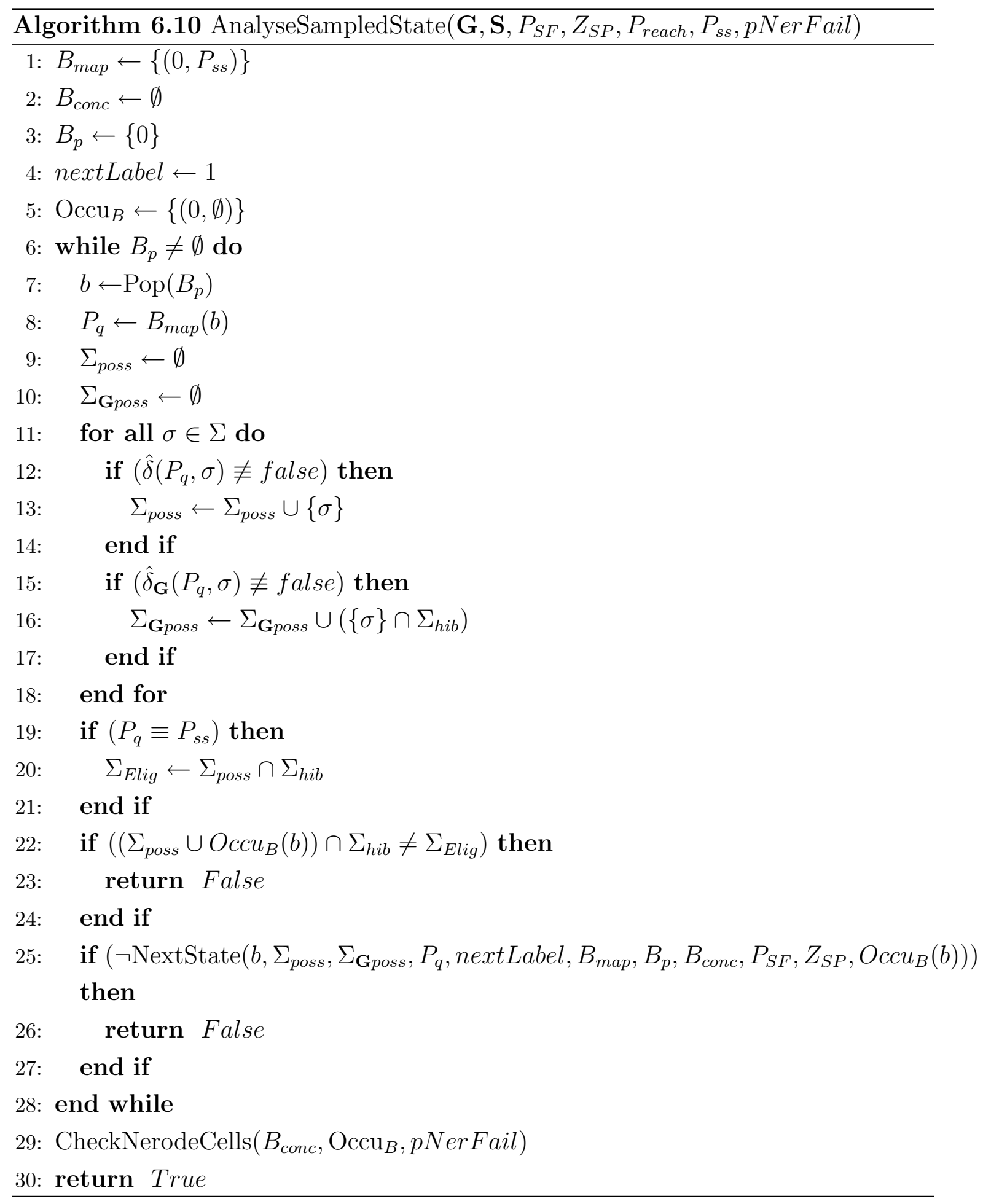


$\mathrm{Occu}_{B}$ : The partial function $\mathrm{Occu}_{B}: \mathcal{N} \rightarrow \operatorname{Pwr}(\Sigma)$ maps the nodes of the reachability tree to the occurrence image of the string that they represent. This function will sometimes be treated like the set $\operatorname{Occu}_{B} \subseteq \mathcal{N} \times \operatorname{Pwr}(\Sigma)$.

The algorithm builds the reachability tree, starting at $q_{s s}$, until all nodes terminates at a tick event or one of our checks fail. As we need to evaluate the strings taking us from the sampled state, we need to know how we got to a given state. So we introduce nodes for the states we reach, and associate with the node the occurrence image of the string that brought us to that node. We use map $\mathrm{Occu}_{B}$ to do this. The function $B_{\text {map }}$ maps the nodes back to the states in $\mathbf{G}_{c l}$ that they represent. The information is stored per node, not per state of $\mathbf{G}_{c l}$. It means there could be two or more nodes that corresponds to the same state, but have possibly different occurrence images, as they were reached by different strings.

When the algorithm starts, we store the set of prohibitable events that are eligible at our starting sampling state. Point iii.1 in Definition 3.2.2 for SD controllability is analyzed as the tree is built. In the algorithm, a concurrent string is represented by the label $b$ of the node it is associated with, and a sampled string is represented by the sampling state $q_{s s}$. From line $\mathbf{2 2}$ to line 24, the algorithm checks this condition. If the test fails, the algorithm returns False.

After the reachability tree is complete, $B_{\text {conc }}$ will represent the concurrent strings leaving the sampling state implied by predicate $P_{s s}$, and the sampling state each string leads to. We then call CheckNerodeCells which will indicate via $p N e r F a i l$ what further checks are needed. This is how Point iii.2 is checked.

In next section we will discuss subroutine NextState (Algorithm 6.11) and subroutine CheckNerodeCells (Algorithm 6.12), as both algorithms are called from AnalyseSampledState.

\section{NextState}

Algorithm 6.11]determines the next states to be processed for Algorithm 6.10. Subroutine NextState takes parameters $b, \Sigma_{\text {poss }}, \Sigma_{\mathbf{G} \text { poss }}, P_{q}$, nextLabel, $B_{\text {map }}, B_{p}, B_{\text {conc }}, P_{S F}, Z_{S P}$, and $\operatorname{Occu}_{B}(b)$. See Algorithms 6.8 and 6.10 for their definitions.

The algorithm returns if the set of eligible events, $\Sigma_{\text {poss }}$, at state $q$ (implied by $P_{q}$ ) of $\mathbf{G}_{c l}$, is empty. If tick is possible at state $q$, we determine the new sampling 


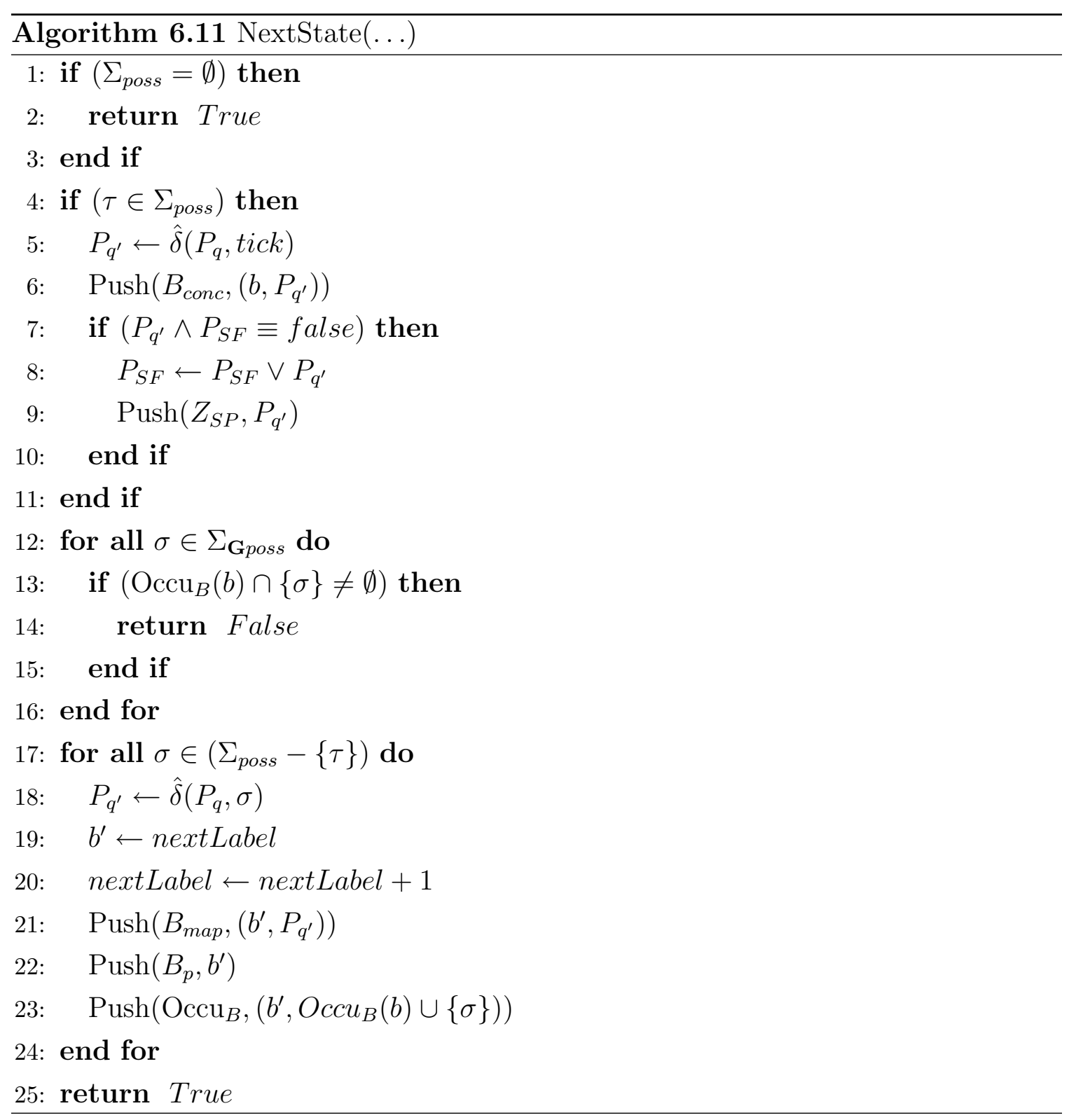

state that tick takes us to, and then add $\mathrm{b}$ and the state to $B_{\text {conc }}$. If we have not yet encountered this state, it is added to $P_{S F}$ and $Z_{S P}$.

In lines 12 to $\mathbf{1 6}$, we check that no prohibitable event is currently eligible in $\mathbf{G}$ if it has already occurred this sampling period. This is part of checking if $\mathbf{G}$ has S-singular prohibitable behavior.

Then for each non-tick event $\sigma$, it finds the next state implied by $P_{q^{\prime}}$, assigns a 
new node $b^{\prime}$ to it and pushes $\left(b^{\prime}, q^{\prime}\right)$ onto $B_{\text {map }}$, and $b^{\prime}$ onto the set of pending nodes, $B_{p}$. It also associates the occurrence image of the strings that took us to $b^{\prime}$ with node $b^{\prime}$, via $\mathrm{Occu}_{B}$.

\section{CheckNerodeCells}

Algorithm6.12 is used to determine if we have possible violations of Point iii.2 of the SD controllability definition. Subroutine CheckNerodeCells is passed a set of sampled states reached in the recent search, plus information on the occurrence images of the concurrent strings that took us to that state. For more details on these parameters, see Algorithm 6.10.

Point iii.2 of the SD Controllability definition requires that if two concurrent strings have the same occurrence image, they must take us to states representing the

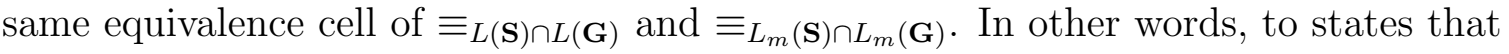
are $\lambda$-equivalent (see Definition 2.2.7). If $\mathbf{G}_{c l}$ is minimal, they must go to the same state. If they do not, we add each set of non-equal states, represented by variable $Z_{\text {eqv }} \subseteq \operatorname{Pred}(Q)$, to $p N e r F$ ail, and we will later check to see if they are indeed $\lambda$-equivalent. Note that every state predicate in $Z_{\text {eqv }}$ represents a single state.

\section{RecheckNerodeCells}

Algorithm 6.13 checks state subsets of $\mathbf{G}_{c l}$ stored in $p N e r F$ ail to see if the states in a given subset actually are equivalent mod $\lambda$ (see Definition 2.2.7) to each other. Subroutine RecheckNerodeCells is passed parameter $p N e r F a i l$. See Algorithm 6.8 for the definition of $p N e r F a i l$.

At a given sampling state, if we found two or more concurrent strings that had the same occurrence image but terminated in different states, we stored the predicates that identified the states these strings led us to, in $p N e r F a i l$. Variable $p N e r F a i l$ contains all such sets found by Algorithm 6.8 as it processed all the reachable sampling states of $\mathbf{G}_{c l}$. For the system to pass Point iii.2 of Definition 3.2 .2 , the states in a given state predicate in $p N e r F$ ail must all be $\lambda$-equivalent to each other. If a single set fails this test, the system fails Point iii.2 of Definition 3.2.2.

From line 1 to line 3, the algorithm first sees if there is actually any state sets in $p$ Ner Fail to be checked. If it is empty, it returns True. 


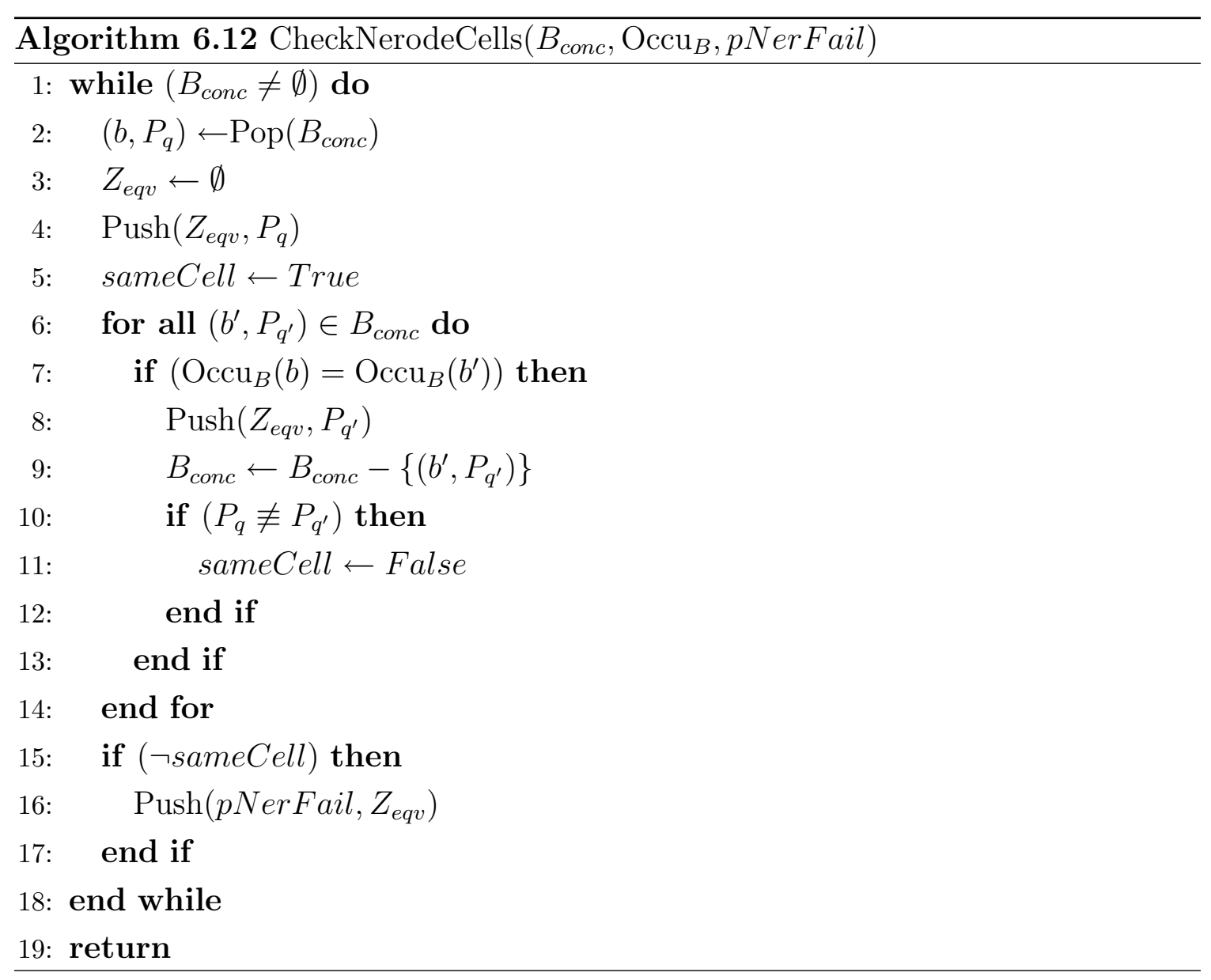

At line 4, variable Visited $\subseteq \operatorname{Pred}(Q) \times \operatorname{Pred}(Q)$ is initialized to the empty set. After each call to RecheckNerodeCell (Algorithm 6.14) that returns True, Visited will contain tuples of state predicates, where each predicate in the tuple represents a single state in $Q$. Essentially, a tuple belonging to Visited means that RecheckNerodeCell has determined that those two states are $\lambda$-equivalent. We pass it back into RecheckNerodeCell so that this information can be reused in future checks.

During the while loop from lines 5 to line 10, we call RecheckNerodeCell for each element $Z_{\text {eqv }} \subseteq \operatorname{Pred}(Q)$ in $p N e r F a i l$. If RecheckNerodeCell returns False, then the system fails Point iii.2 of Definition 3.2.2. 


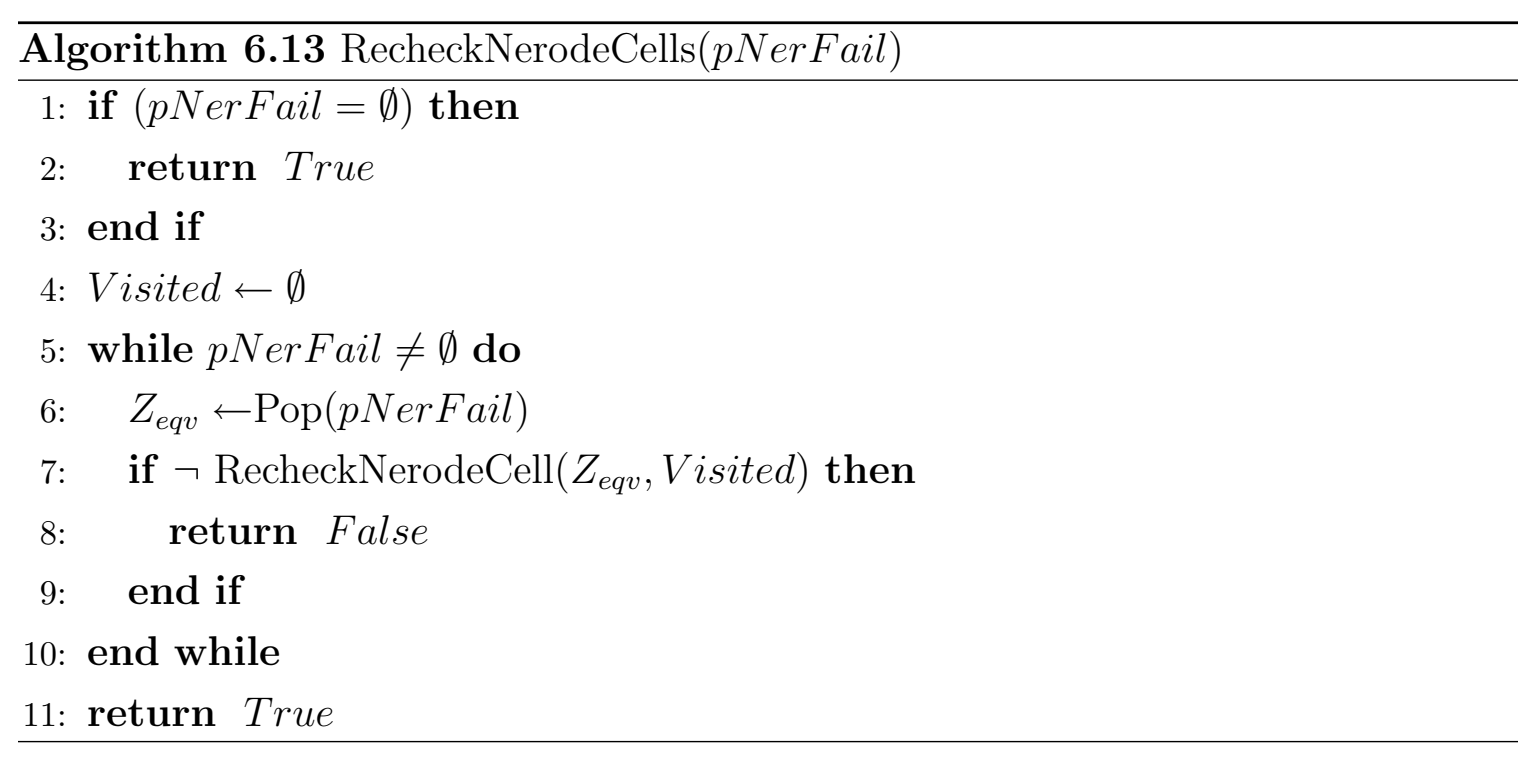

\section{RecheckNerodeCell}

For each set of state predicates $Z_{\text {eqv }} \subseteq \operatorname{Pred}(Q)$ that Algorithm 6.14 is called with, we will check that these states identified by the predicates are $\lambda$-equivalent to each other, and return False if they are not. When Subroutine RecheckNerodeCell is called, parameter Visited $\subseteq \operatorname{Pred}(Q) \times \operatorname{Pred}(Q)$ represents tuples of states that are known to be $\lambda$-equivalent. See Algorithm 6.13 for further details about these parameters.

At line 1, a state predicate is popped out of $Z_{\text {eqv }}$ and labeled as $P_{q_{1}}$.

From line 2 to line $\mathbf{6}$, the algorithm populates the Pending set with all pairs of $P_{q_{1}}$ and $P_{q_{2}}$, where $P_{q_{2}}$ is also popped from $Z_{e q v}$. Note that state predicates $P_{q_{1}}$ and $P_{q_{2}}$ each represent a single state in $Q$. Set Pending represents all the state pairs that we wish to show to be $\lambda$-equivalent. Of course, we will likely finding new state pairs that we will also need to test, as our algorithm progresses.

Two states $q_{1}, q_{2} \in Q$ are $\lambda$-equivalent if they have the same future with respect to the marked and closed behavior of $\mathbf{G}_{c l}$. That means that both states are either marked, or neither is marked (lines 10-12). It also means that for each $\sigma \in \Sigma$ (lines 13-28), there is a $\sigma$ transition at one state if and only if there is a $\sigma$ transition at the other (line 17-18). Also, if there is a $\sigma$ transition leaving each state, the two new states reached must be $\lambda$-equivalent. Obviously if $q_{1}=q_{2}$ (line 19), then the two 


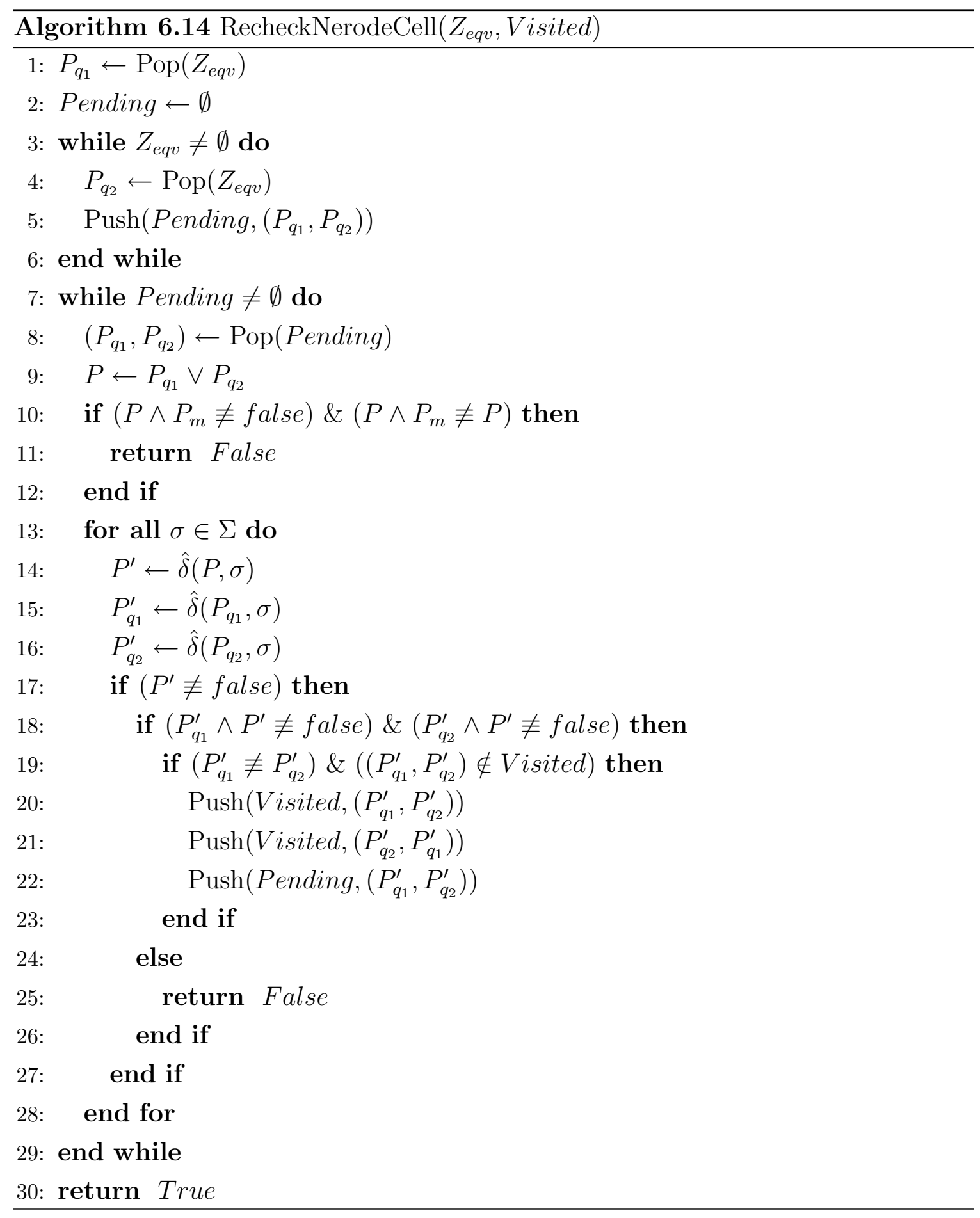


states are $\lambda$-equivalent.

Our approach to prove that $q_{1}, q_{2} \in Q$ are $\lambda$-equivalent will be to attempt to prove they are not. We will check the per state conditions (lines 10-12 and lines 17-27), and then if the states take us to two different states for a common $\sigma$ transition (line 19), we check to see if the new states already have a tuple in Visited (line 19). If they do, either they are known to be equivalent or we have already processed the pair and added their requirements to Pending. If they do not, we add the pair to Pending and Visited (lines 20-22). This ensures that a state pair is added to pending at most once, so we will terminate after a finite number of iterations as $\mathbf{G}_{c l}$ has a finite statespace. There is no sense in adding the pair to Pending twice as processing the pair twice would not provide new information to check.

The idea is that if the state pair are not equivalent, then we must eventually reach a state pair that we need to be equivalent, but the states do not have the same marking information and/or the same possible outgoing event transitions. If we never reach such a pair (and we have a finite number of possible state pairs to check), then the original state pairs must be equivalent. Not only that, then every state pair that we encountered to check, must also be equivalent to each other, or they would have caused the test to fail. This is why all state pairs in Visited are known to be equivalent if the algorithm returns true.

As we expect that our plant and supervisor TDES components are typically minimal or close to it, we also expect that $\mathbf{G}_{c l}$ is likely minimal or close to it. As such, we believe that when we start to check that a state pair is equivalent, we expect to either quickly find out it is not, or have the test terminate successfully as the new state pairs we encounter to test are actually the same state.

We now make a few additional comments to clarify a few steps of the algorithm. For lines 14-16, predicate $P^{\prime}$ represents states reached via $\sigma$ from either state $q_{1}$ or state $q_{2}$, while $P_{q_{1}}^{\prime}$ and $P_{q_{1}}^{\prime}$ represents states reached via $\sigma$ only from the indicated state. The condition on line $\mathbf{1 7}$ will be satisfied if either state $q_{1}$ or state $q_{2}$ has a $\sigma$ transition leaving that state. The condition on line 18 will fail if only one of the two states has a $\sigma$ transition leaving that state. 


\section{Checking Point iv of SD Controllability}

Point iv in Definition 3.2.2 for SD Controllability is checked by Algorithm 6.15, Subroutine CheckSamplingMarkingStates is passed the state predicate $P_{\text {reach }}$, which represents the set of reachable states of $\mathbf{G}_{c l}$, when it is called by Algorithm 6.8.

Point iv of SD Controllability states that only sampled strings can be marked strings. This implies that every reachable marked state of $\mathbf{G}_{c l}$ can only have at most incoming tick transitions from other reachable states.

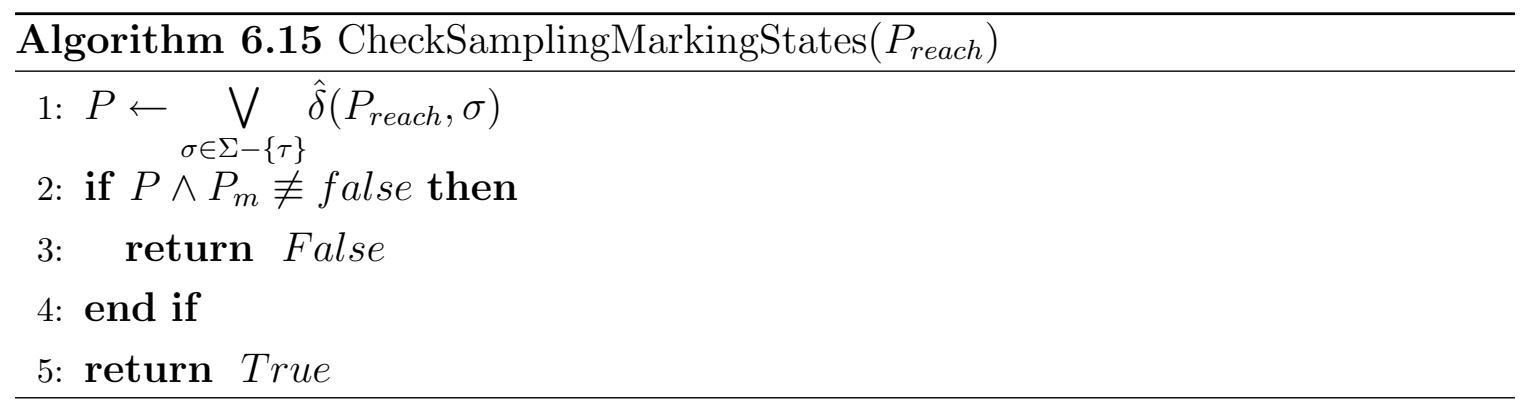

At line 1, we first identify all states with an incoming non-tick transition from a reachable state. This implies that all of these states are also reachable. At line 2, we check to see if any of these states are also marked. If one of them is marked, then $\mathbf{G}_{c l}$ fails this condition and we return False. 


\section{Chapter 7}

\section{Examples}

In this chapter we provide illustrative examples for key required conditions we have defined for an SD system (see Section 7.1), as well as a successful example based on Hill's Flexible Manufacturing System (FMS) from [11] (see Section 7.2). Then in Section 7.3, we translate the FMS example into Moore FSM, using the approach we discussed in Chapter 4

All the DES examples have been verified to be either passing or failing using the software tool we implemented, based on the algorithms from Chapter 6. The examples are illustrated as per the legend shown in Figure 7.1.

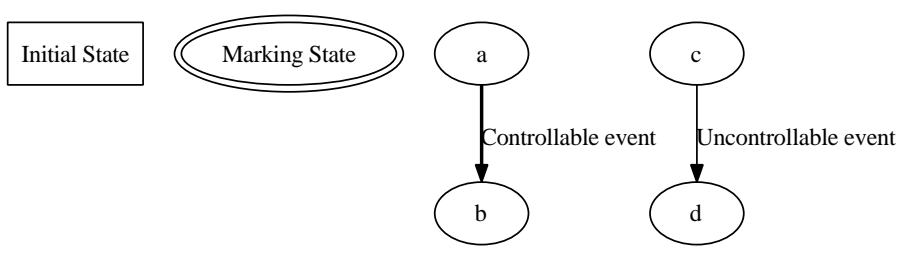

Figure 7.1: Legend Used to Display DES

As shown in Figure 7.1,

- An initial state is a box shape with its border single lined.

- A marked state is a ellipse shape with its border doubled lined.

- By default, a regular state is a ellipse shape with its border single lined.

- A controllable event transition is shown as a bold arrow. 
- An uncontrollable event transition is shown as a thin arrow.

\subsection{Examples}

In this section we provide some examples which fail key conditions that we require, in order to provide a better understanding of these conditions. The conditions we cover include plant completeness, activity loop free, proper time behavior, and SD controllability. We have not included examples for untimed controllability and nonblocking conditions since these two conditions are already well studied.

\subsubsection{Plant Completeness}

Figures 7.2 and 7.3 show a plant and a supervisor such that the plant fails to be complete for the supervisor, as per Definition 2.3.1. This is because event repair.2 is not eligible at state down in the plant, while this event is eligible at state down in the supervisor. This could be a problem if event repair. 2 is being generated by the controller, and can occur whenever it is enabled. This would mean that the event could potentially occur when the plant model says it can't, resulting in unmodeled behavior.

\section{Listing 7.1: Output}

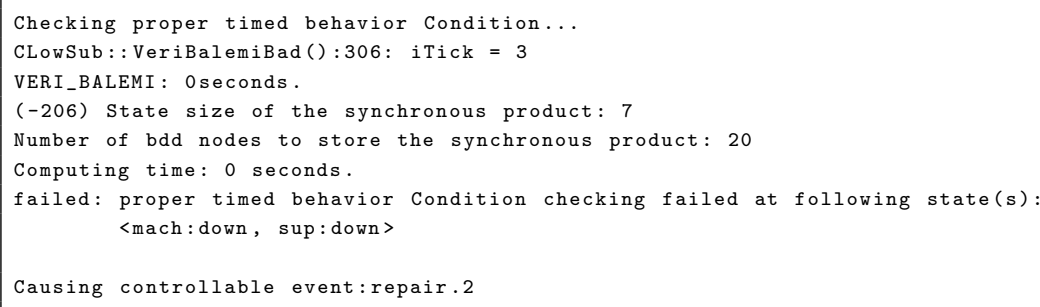



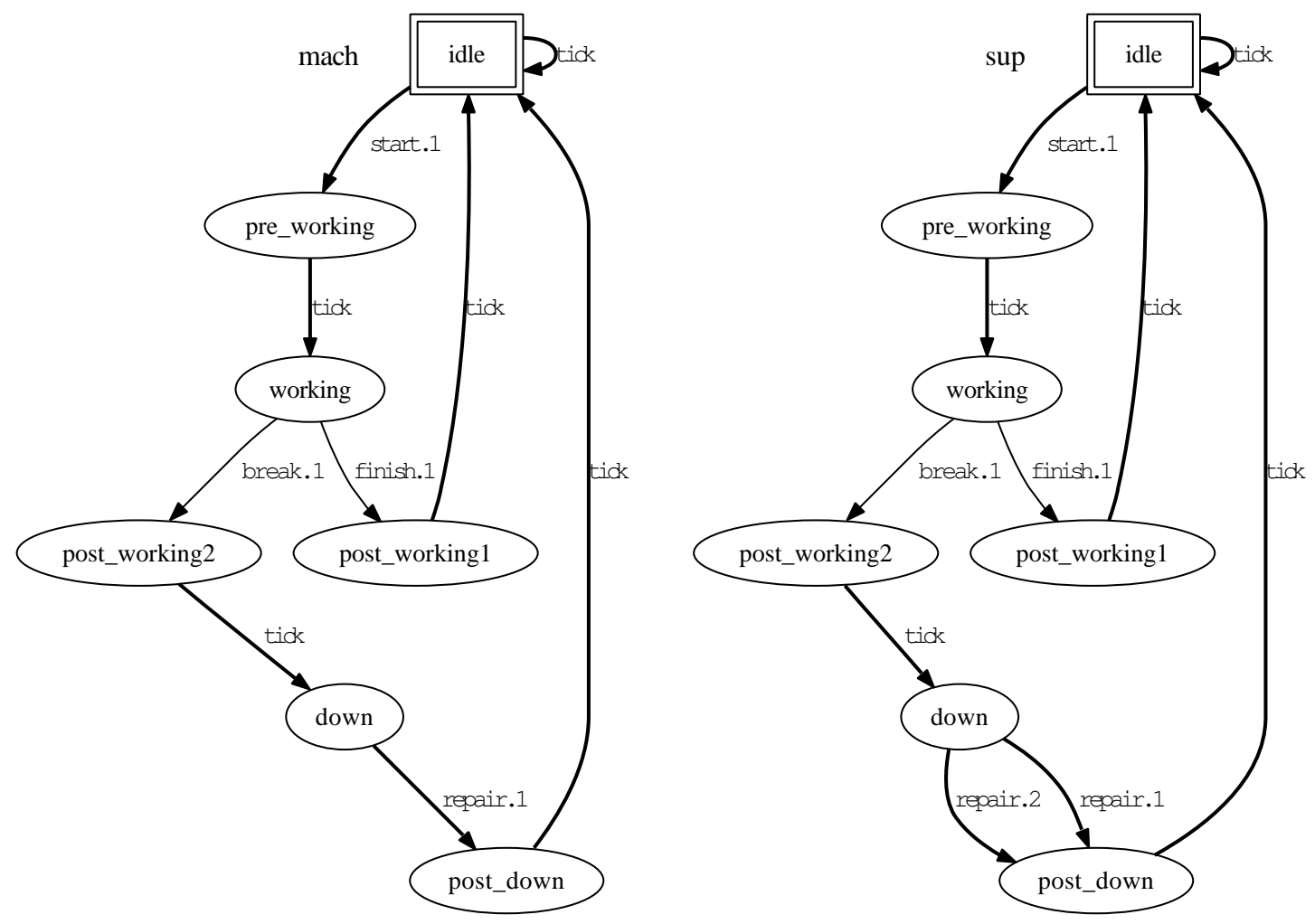

Figure 7.2: Plant Completeness Example: Figure 7.3: Plant Completeness Example: Plant Supervisor 


\subsubsection{Activity Loop Free}

Figure 7.4 shows a TDES which is not activity loop free, as per Definition 2.3.3. This is because at state (b) the event down.1 is able to preempt the tick event and proceed to state (c) and after that to state (a). This creates a tick-less cycle. This cycle of 'start.1-down.1-repair.1' can occur an unlimited number of times. This implies the physically unrealistic situation that we can have an infinite number of these events occur in a finite time period, and thus must not be allowed.

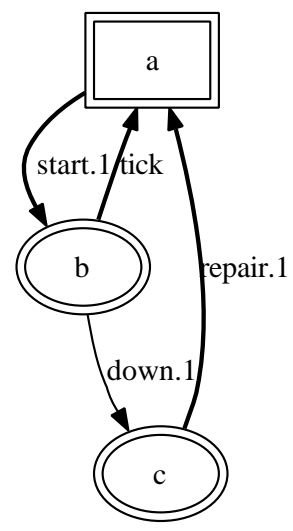

Figure 7.4: Activity Loop Example 


\subsubsection{Proper Time Behavior}

Figure 7.5 shows a plant which fails to satisfy proper time behavior as per Definition 2.3.5. At state down, neither a tick event nor an uncontrollable event is eligible, just the controllable event repair.1. This causes two problems: First, it implies that the controllable event must occur in a particular time frame, yet the event can be disabled forever by a supervisor, and thus never occur. Second, because its controllable, it can be disabled by a supervisor. Since no other events are possible, if this event is disabled, we effectively "stop the clock", which is physically unrealistic. Note that supervisor could disable repair.1 here and still be TDES controllable. i.e. this problem is not caught by the TDES controllability definition.

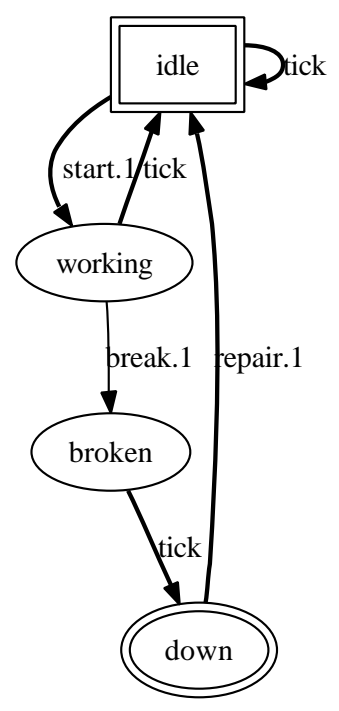

Figure 7.5: Proper Time Behavior Example 


\subsubsection{SD Controllability}

We now examine the the various points of the SD controllability condition from Definition 3.2 .2 .

\section{Point i and Point ii}

As Point $\mathbf{i}$ and the ' $\Leftarrow$ ' part of Point ii are essentially equivalent to the standard TDES controllability condition, we will not provide an example here for them. We will instead focus on the ' $\Rightarrow$ ' part of Point ii as this is a new condition introduced bu SD Controllability.

Figure 7.6 and Figure 7.7 show a plant and a supervisor such that Supervisor fails to satisfy the ' $\Rightarrow$ ' part of Definition 3.2 .2 , with respect to Plant. The prohibitable event is job and the uncontrollable events are verified and done. We first note that a tick event is eligible at state 3 in the Plant. Since the prohibitable event job is eligible at state (Plant:3, Supervisor:3) in the synchronous product, the supervisor should disable tick at its state 3 since a prohibitable event should only be enabled when it is to be forced. Alternately, if we do not yet wish event job to occur, it should be disabled until we are ready for it.

Listing 7.2: Output

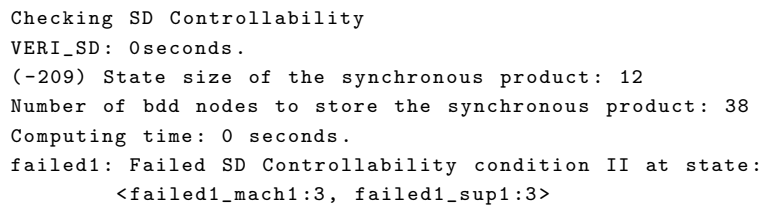

\section{Point iii.1}

Figure 7.8 and Figure 7.9 show a plant and a supervisor such that Supervisor fail to satisfy Point iii.1 of Definition 3.2.2 with respect to Plant. The only prohibitable event is job. The uncontrollable events are $\{$ verified1, verified 2, done $\}$.

In the system, prohibitable event job is eligible at sampling state 1 in the Plant, so the eligible prohibitable event set for this sampling period is $\{j o b\}$. However when we reach state 3 , event job has not yet occurred, but is no longer eligible, violating Point iii.1. 


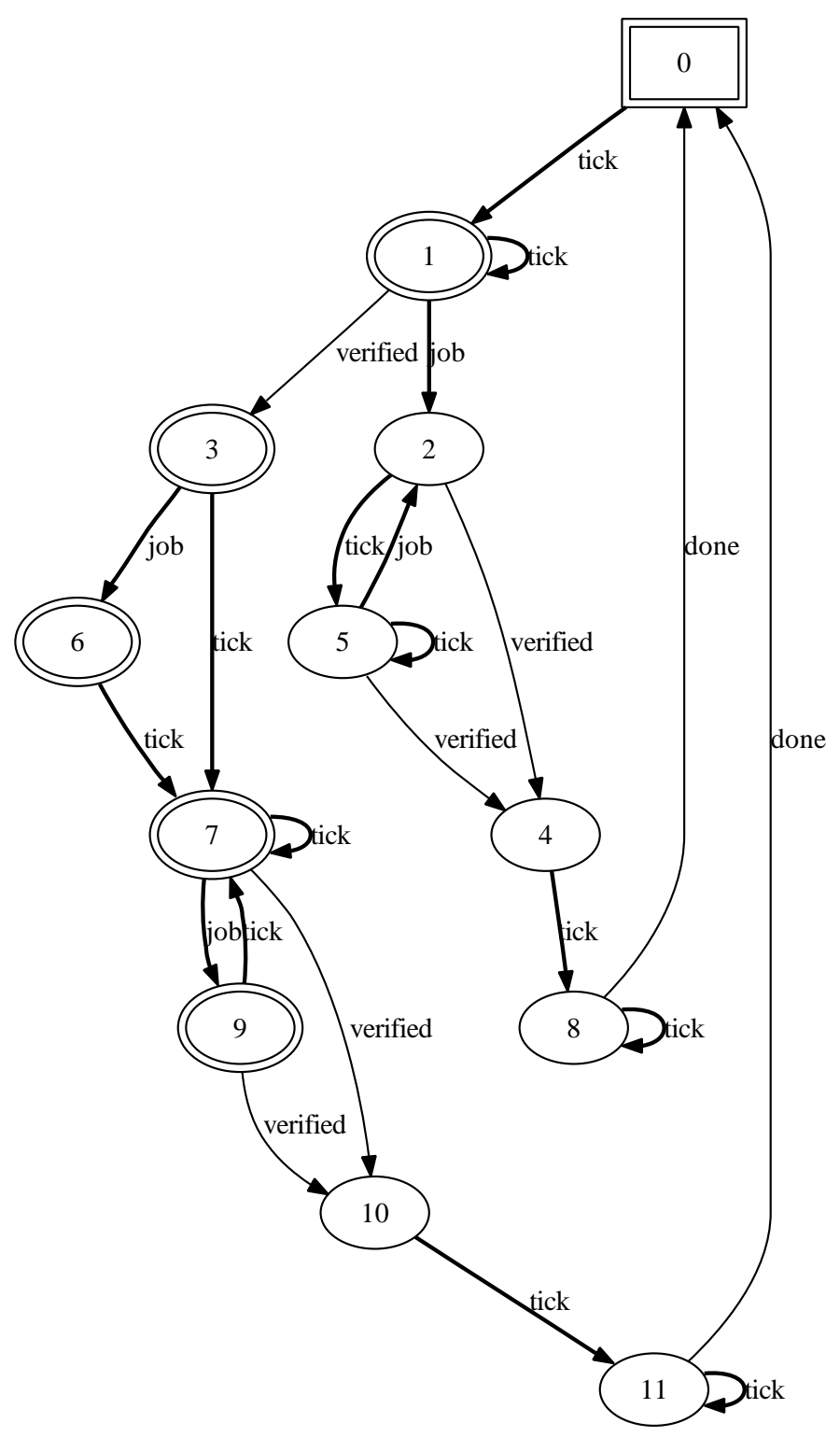

Figure 7.6: SD Controllability i, ii Example: Plant

This is a problem as often when a prohibitable event occurs is completely under the control of the implementation (as discussed before, this is a modeling issue). Also, this event may occur at different times during a sampling period, depending on the implementation used. As an SD controller makes its forcing decisions immediately after a tick, it will cause event job to occur at state 1 in the physical system. If the 


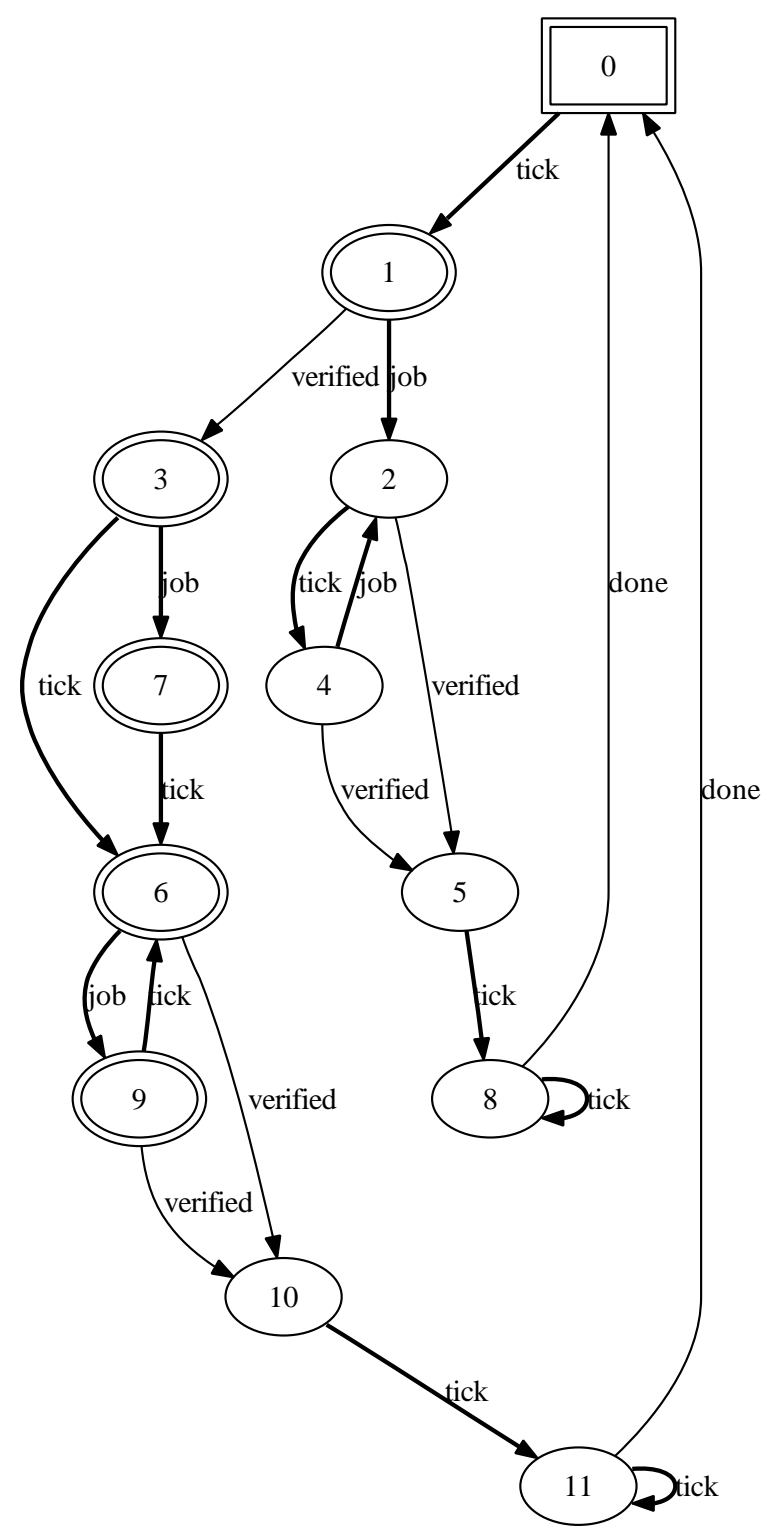

Figure 7.7: SD Controllability Point i, ii Example: Supervisor

implementation is such that event job is delayed and event verified 1 occurs first, we could get event job after event verified1 in the physical system, which does not match our plant model.

In this example, it was the plant model that made event job become ineligible. A related issue would have been if event job was possible at state 3 in the plant, but not 
in the supervisor. This would imply that the SD controller must detect that event verified 1 has occurred in the current sampling period, and disable event job in time to prevent it from occurring. This of course cannot be done as the event has already been initiated after the tick occurred and even if could be stopped, the SD controller will not even see that event verified1 has occurred until after the next tick, at which point it would be too late. If the implementation is such that event verified1 occurs before event job, we would still get a job transition in the current sampling period in the physical system, violating our control law. For example, if event job was "walk through doorway", and event verified1 was "door closes", this would mean we would walk into a closed door.

A second related problem this condition can catch is when a prohibitable event is not eligible at state 1 , but becomes eligible at state 3 . The supervisor is trying to express that the event should occur this sampling period, but not until after event verified1 has occurred. This cannot be implemented as the SD controller would not know event verified1 had occurred until after the next tick, thus too late to force a new event. If we tried to simply force the prohibitable event at state one in the controller, we might get the situation that the event occurs before event verified1 (depending on our implementation). Again, this would violate our control law.

\section{Point iii.2}

Figure 7.10 and Figure 7.11 show a plant and a supervisor such that Supervisor fails to satisfy Point iii.2 of Definition 3.2 .2 , with respect to Plant. The prohibitable events are $\{j o b 1, j o b 2\}$. The uncontrollable events are $\{$ done 1, done 2$\}$.

In the system, states 6 and 7 are reached from sampled state 1 by concurrent strings $j o b 1-j o b 2-t i c k$ and $j o b 2-j o b 1-\tau$, respectively. As these strings have the same occurrence image, Point iii. 2 requires that states 6 and 7 represent the same Nerode equivalence cells of the closed loop system's closed and marked language's. However, as strings reaching state 6 can be extended by a done 1 event, while strings reaching state 7 can be extended by a done2 event, the states clearly do not represent the same Nerode equivalence cell of the system's closed behavior. Similarly, as strings reaching state 6 can be extended by a done1 event to a marked string while strings reaching state 7 can be extended by a done2 event to a marked string, they do not represent the same Nerode equivalence cell of the system's marked language either. 


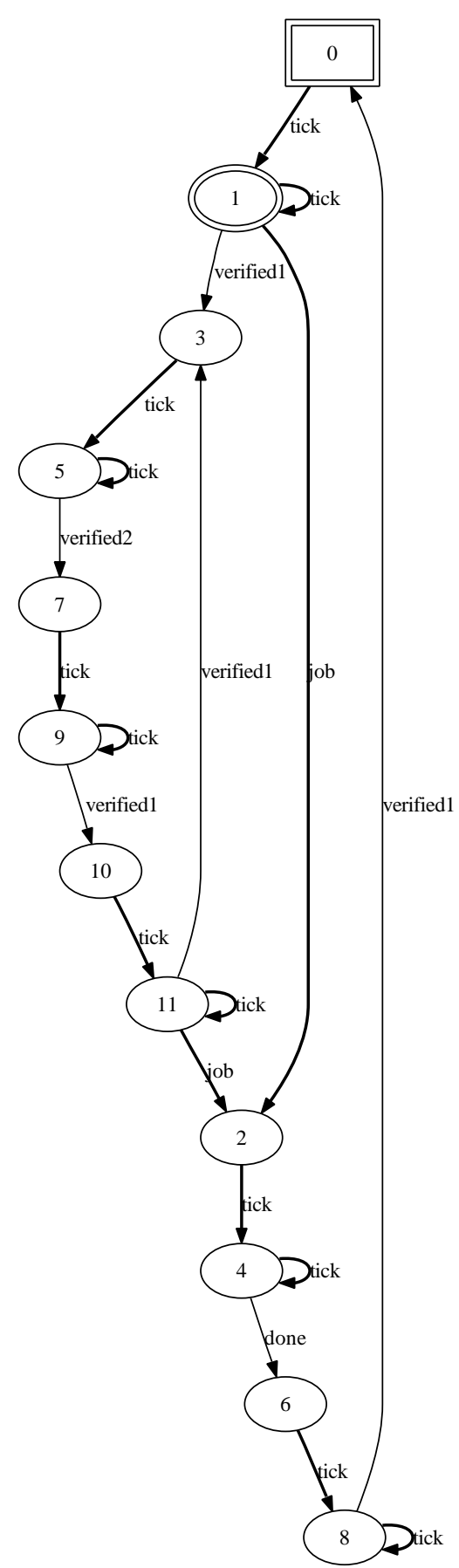

Figure 7.8: SD Controllability Point iii.1 Figure 7.9: SD Controllability Point iii.1 Example: Plant

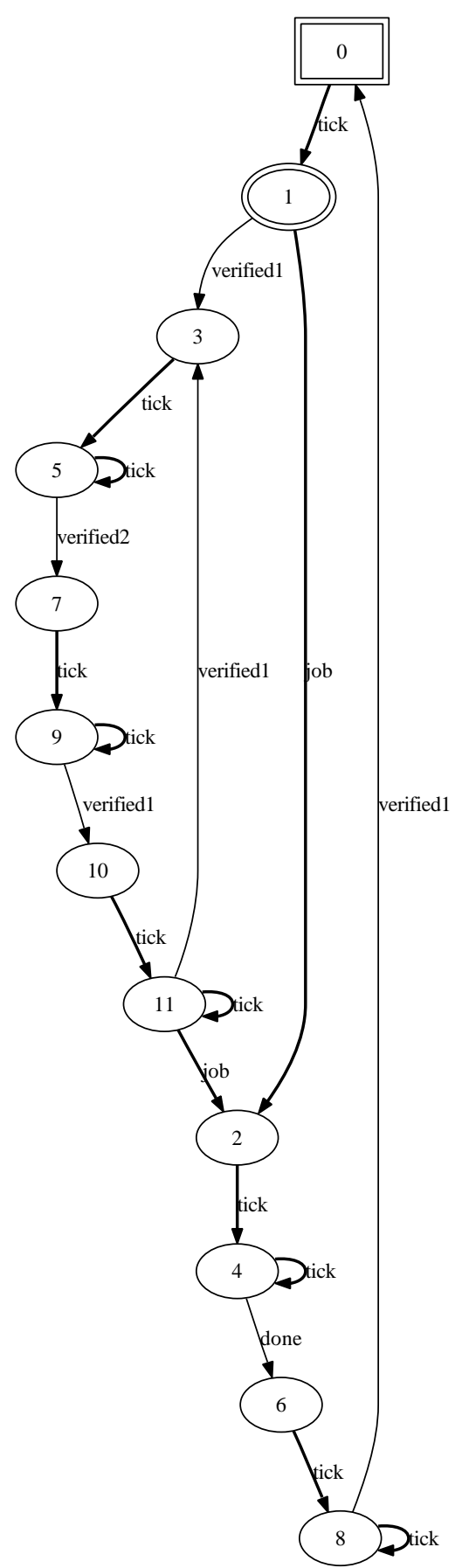

Example: Supervisor 

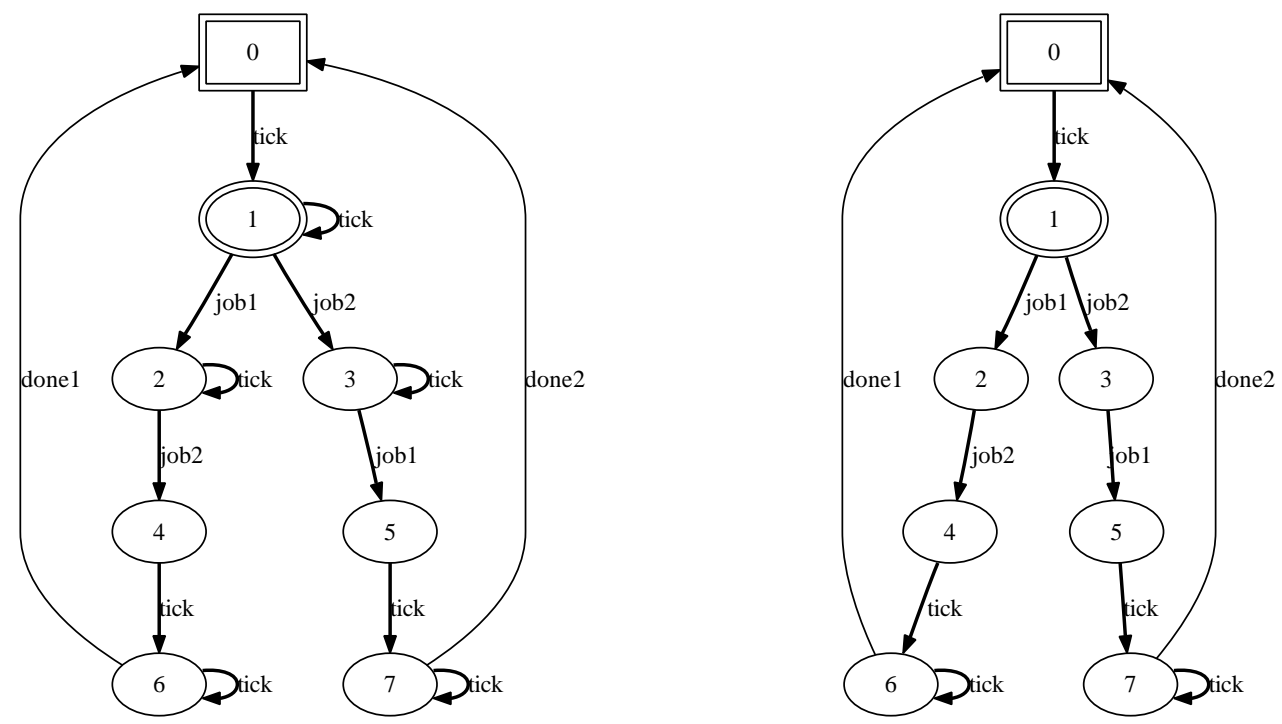

Figure 7.10: SD Controllability Point iii.2 Figure 7.11: SD Controllability Point iii.2 Example: Plant

Example: Supervisor

This condition is important for controllability and nonblocking. The reason is that an SD controller cannot tell the difference between the two concurrent strings, so it does not know whether it should be in state 6 or state 7 . If events done1 and done2 were controllable, it would not know if it should be enabling event done1 or event done2. Clearly, we could not enforce such a control law.

The reason this is important for nonblocking is also that we cannot tell the difference between the two strings. If we had a sequence of possible concurrent strings such that each pair had the same occurrence image and only one path of the pair ever reached a marked state, we would never be able to determine if our system reached a marked state.

A related issue is how our controller is implemented. The control law says that either sequence $j o b 1-j o b 2$ or sequence $j o b 2-j o b 1$ is fine, but not which one will actually occur. It might be that we will get a bit of both, but we might always get only one due to timing issues; or perhaps we have a sequential implementation that knows that job1 and job2 must occur, so its designers choose the order job1 - job2, and the implementation always executes these events in this order. If the sequence job2-job1 was the only path back to a marked state, the implementation would block despite the fact the TDES system was nonblocking. This condition, in conjunction 
with Point iv of the SD controllability definition, helps make sure nonblocking does not depend on the order of the events and allows things to function if we only get one of the variations of the possible concurrent strings with the same occurrence image. One can image that we have a family of possible physical systems that we could get based on how we implement our controllers, each differing based on which of the possible variations of the concurrent strings can actually occur. We are assuming we will see at least one variation, possibly more. These conditions are intended to ensure that whichever system we get, it will still be nonblocking if the TDES system was nonblocking.

Listing 7.3: Output

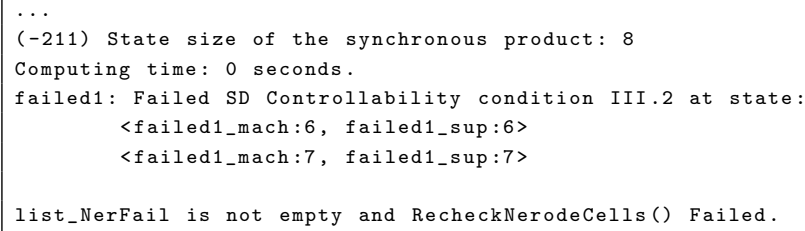

\section{Point iv}

Figure 7.12 and Figure 7.13 show a plant and a supervisor such that Supervisor fails to satisfy Point iv in Definition 3.2.2 with respect to Plant. Since state $\mathbf{0}$ is a marked state and is reached from state 6 by activity event done, the system does not satisfy the condition as clearly its marked language is not a subset of the sampled strings (empty string and strings ending in a tick).

If a marked state is reachable by a non-tick event, it means the system can reach a marked state in a way that is invisible to the SD controller as it can only observe sampled strings. This by itself is undesirable, as we could have a system that can only reach marked states by non-tick events and we would never be able to tell if we had actually reached a marked state. Also, if we have multiple concurrent strings with the same concurrence image, we could have the situation that only some of them pass through a marked state in that sampling period. Worse, our implementation might be such that we only get the variations that do not pass through a marked state! Note also, that Point iii.2 of the SD controllability definition only says that concurrent strings with same occurrence image must have same marked future. it does not say 

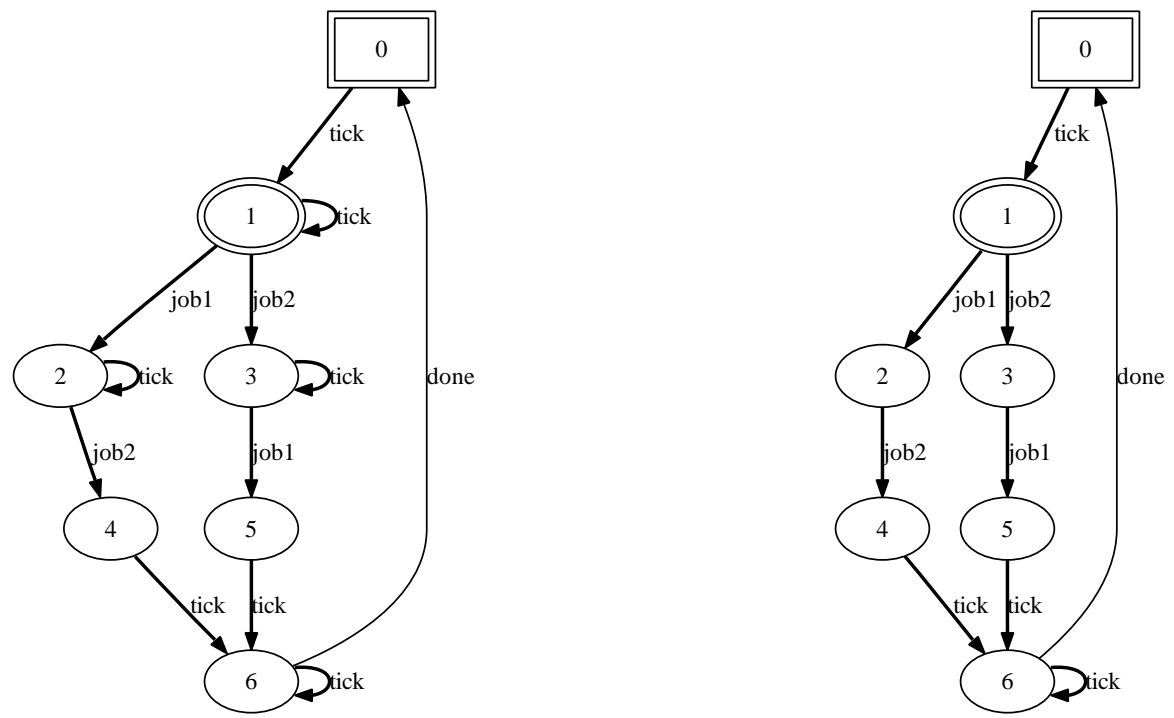

Figure 7.12: SD Controllability Point iv Figure 7.13: SD Controllability Point iv Example: Plant

Example: Supervisor

much about the prefixes of these concurrent strings. That is where Point iv comes in, making sure the $\Sigma_{\text {act }}^{+}$prefixes are not marked.

Listing 7.4: Output

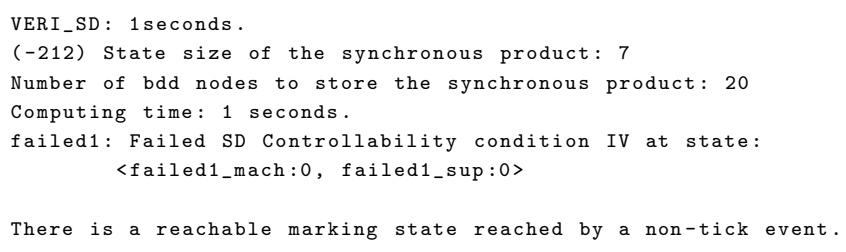




\subsection{SD Controlled Flexible Manufacturing System}

In this section we present a working example based on the untimed Flexible Manufacturing System (FMS) from [11]. The system, shown in Figure 7.14, is composed of six plant components and five one slot buffers. We will treat the buffers as specifications, requiring that they do not overflow or underflow. Table 7.1 below shows a mapping from the event labels used in the diagrams to their meaning. The events labeled as numbers are directly from the Hill untimed example. We kept the same labeling to make it easy to see the correspondence.

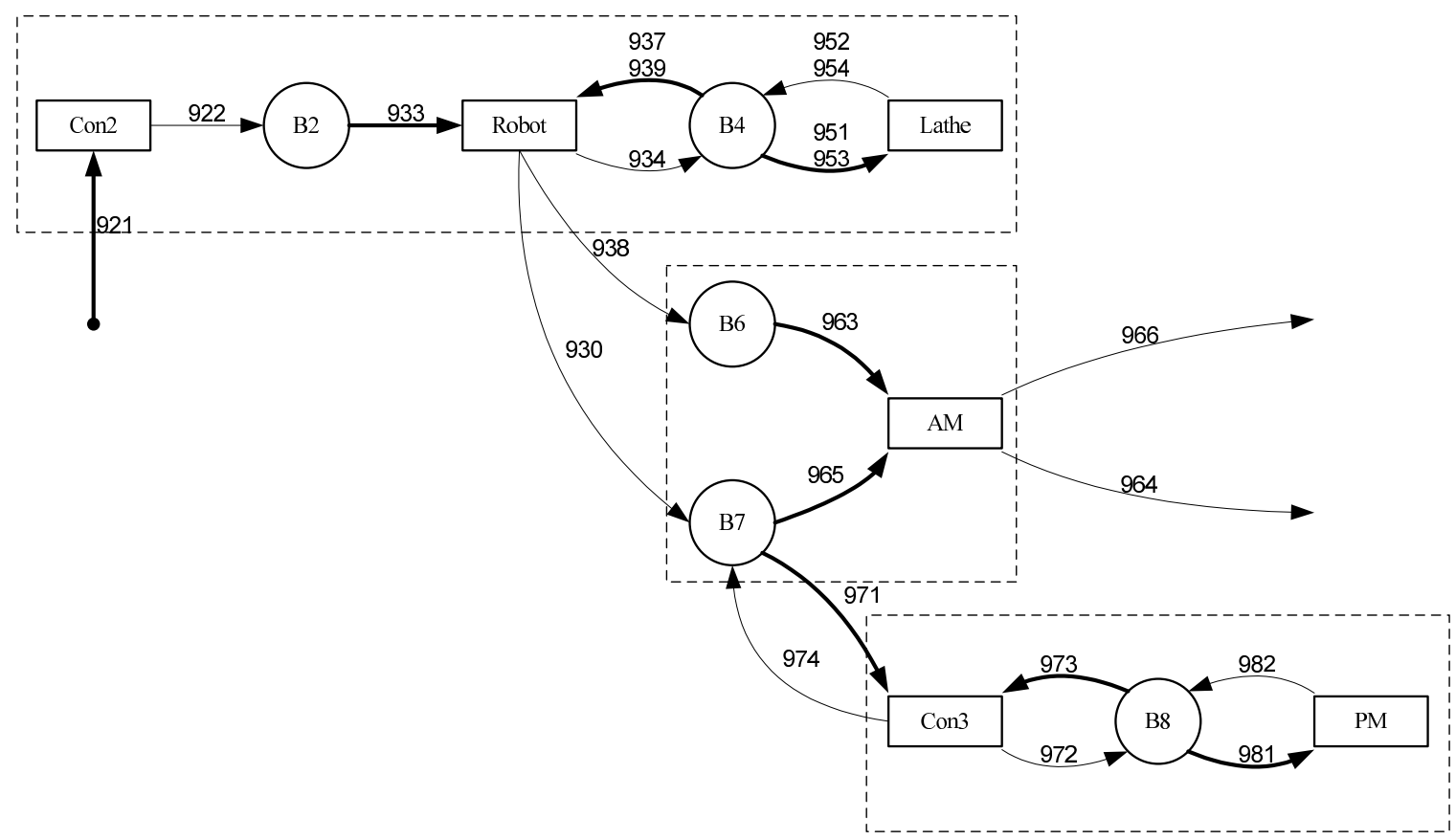

Figure 7.14: Flexible Manufacturing System Overview

\subsubsection{FMS Plants}

The plant components consist of two conveyors (Con2 and Con3), a handling robot (Robot), a lathe that can produce two different parts, a painting machine (PM), and a finishing machine (AM). The flow of material is illustrated in Figure 7.14, See Figures 7.15-7.20 for the TDES models of the components. 
Table 7.1: Explanation of Event Labels

\begin{tabular}{|l|l||l|l||l|l|}
\hline Label & Meaning & Label & Meaning & Label & Meaning \\
\hline \hline 921 & Part enters system & 922 & Part enters B2 & 933 & Robot takes from B2 \\
\hline 934 & Robot to B4 & 937 & B4 to Robot for B6 & 939 & B4 to Robot for B7 \\
\hline 938 & Robot to B6 & 930 & Robot to B7 & 951 & B4 to Lathe (A) \\
\hline 953 & B4 to Lathe (B) & 952 & Lathe to B4 (A) & 954 & Lathe to B4 (B) \\
\hline 971 & B7 to Con3 & 974 & Con3 to B7 & 972 & Con3 to B8 \\
\hline 973 & B8 to Con3 & 981 & B8 to PM & 982 & PM to B8 \\
\hline 961 & Initialize AM & 963 & B6 to AM & 965 & B7 to AM \\
\hline 966 & Finished from B7 & 964 & Finished from B6 & & \\
\hline
\end{tabular}
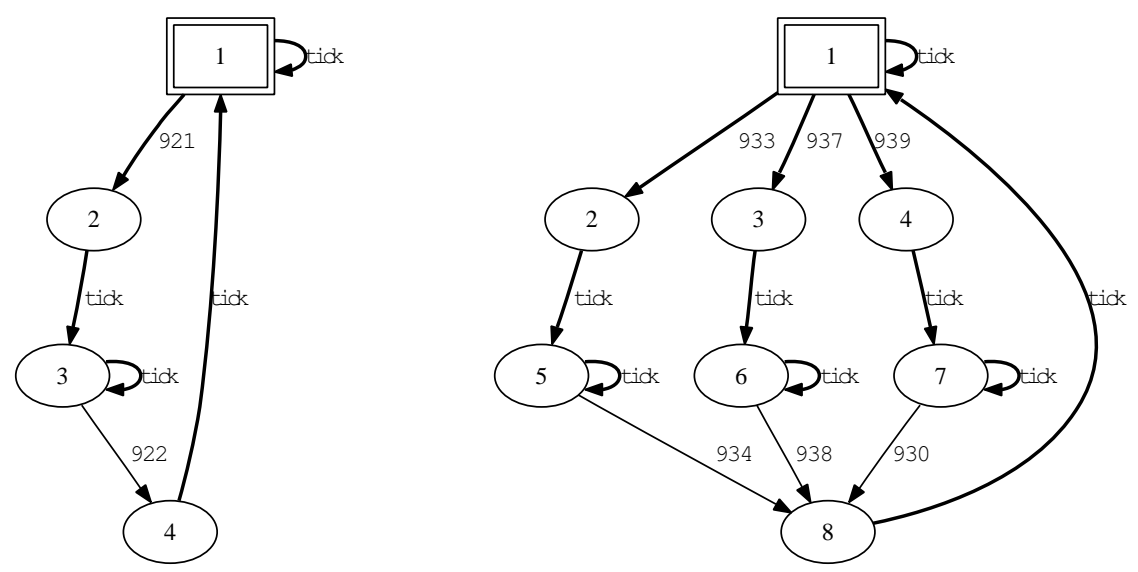

Figure 7.15: Conveyor - Con2

Figure 7.16: Robot

\subsubsection{Buffer Supervisors}

We now discuss the TDES supervisors, shown in Figures 7.21- 7.25, that control the flow of parts in and out of the buffers. Their goal is to make sure the buffers do not overflow or underflow. They are based on the original untimed buffer specification of [11, but extended to the SD controllable setting. In some of the supervisors in this section such as B4 in Figure 7.22, we have activity events selflooped (i.e. event 933 at state 0 of B4). This will not cause the system to have an activity loop, as it will be combined with the plant TDES which only allow these events to occur once per clock cycle. 


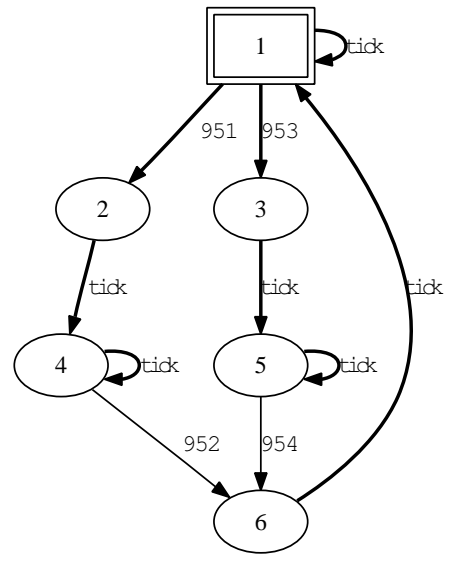

Figure 7.17: Lathe

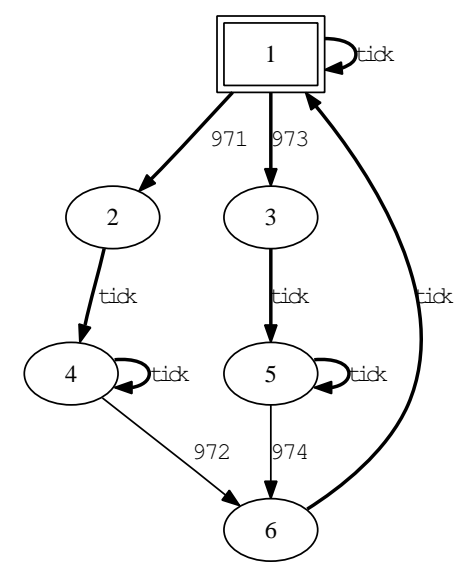

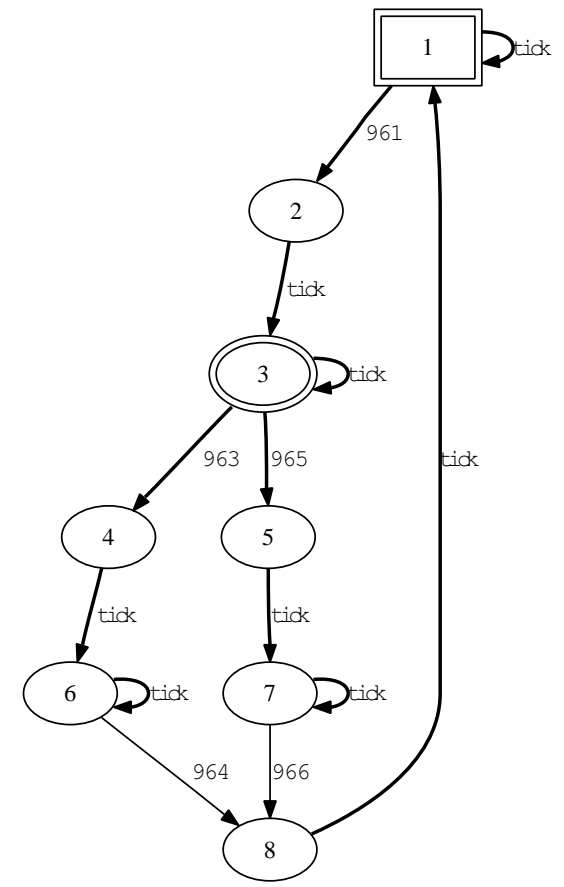

Figure 7.18: Finishing Machine - AM

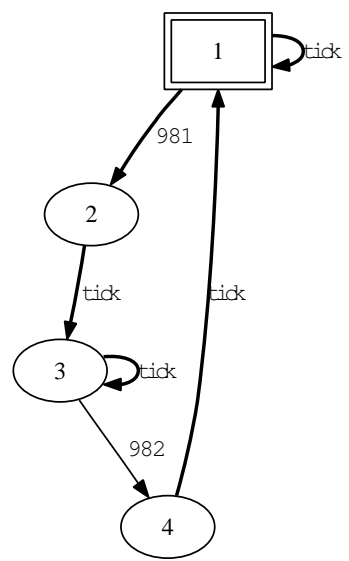

Figure 7.19: Conveyor - Con3 Figure 7.20: Painting Machine PM

Supervisor B2 not only prevents overflow and underflow of buffer B2, it also decides when event 921 should occur. As soon as the system is turned on, it immediately enables and forces 921, causing Con2 to accept a new piece into the system. It then waits for the piece to enter B2, before it enables event 933, allowing the 


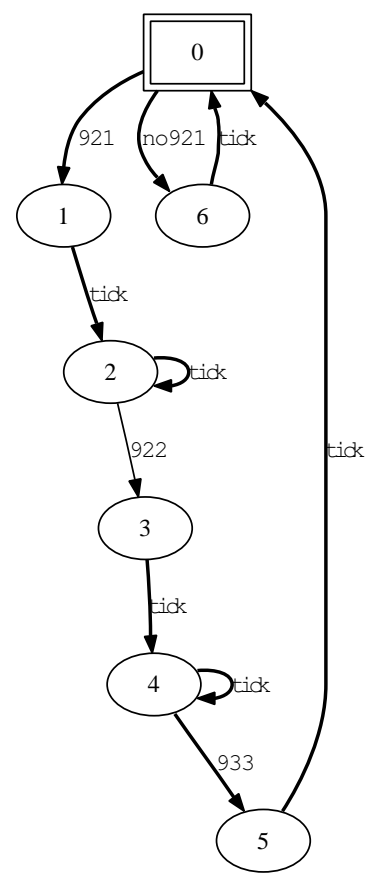

Figure 7.21: Supervisor B2

Robot to remove the part. It does not cause another 921 to occur until 933 does, ensuring that the buffer is empty. A few things are worth noting. First, B2 enables prohibitable event 933, but does not disable the tick at state 4 . This tells us that it wants to prevent the event from occurring too soon, but does not decide when the event will actually occur. This is controlled by another supervisor. Second, B2 makes sure there is a tick between 933 occurring, and enabling and forcing event 921. This is to satisfy Point iii.1 of the SD controllability definition. Third, Supervisor B2 contains a special event, no921, which we will discuss in a later section. This is a "virtual event" that was not part of the original plant, but that we added to aid in communication between supervisors.

Supervisors B4, B6, and B7 manage their respective buffers. They strictly disable and enable events to prevent buffer overflow and underflow. They do not force any events, telling us that other supervisors make these decisions. This is because the decision of when these events should occur requires more than just a local view of whether a buffer is empty or not. We will discuss these other supervisors in later sections. 


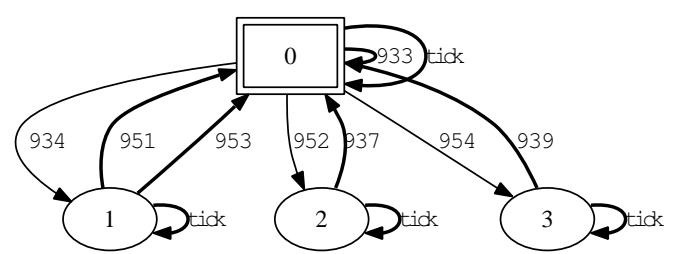

Figure 7.22: Supervisor B4

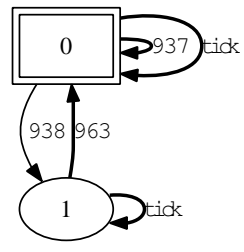

Figure 7.23: Supervisor B6

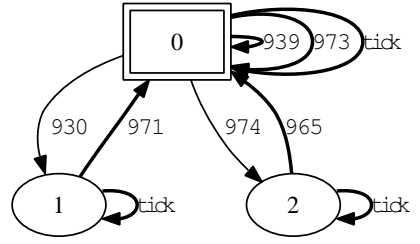

Figure 7.24: Supervisor B7

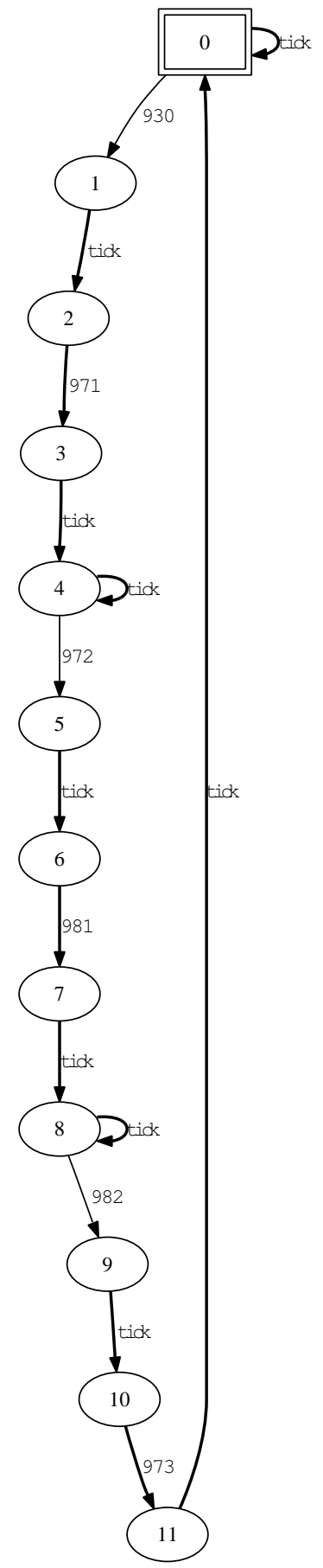

Figure 7.25: Supervisor B8 
Supervisor B8 not only prevents overflow and underflow of buffer B8, it also controls the flow of pieces once a part enters buffer B7 (event 930), flows to TDES $\mathbf{P M}$, and then back to buffer B7. It does this by watching the parts progress, and then forcing events 971, 981, and 973 as needed. As B8 determines when these events occur, it disables tick as soon as it enables these events to comply with Point ii of the SD controllability definition. In other words, once the event is enabled by all the supervisors and possible in the plant, the event is also forced.

The fact that the supervisor must not only decide when to enable an event, but also when to force the event, makes things more complicated. It must not only decide when to enable the event, but also must know that the event is not disabled by another supervisor and that it is eligible in the plant. Otherwise, it could disable a tick when the desired event cannot occur, either forcing the wrong event or becoming uncontrollable.

\subsubsection{B4 to Lathe Path}

In addition to the buffer supervisors we represented in Section 7.2.2, we need to add the following supervisors to resolve some nonblocking and concurrency issues on the B4 to lathe part path of Figure 7.14.

We first need to address a nonblocking issue with respect to buffer B4 and B2. We see from Figure 7.14 and Figure 7.16, that Robot takes a piece from buffer B2 (event 933), and places it in B4. The piece then goes to the Lathe, and then back to buffer B4. The robot will then take the piece from B4, and put it in either buffer B7 (event 930), or buffer B6 (event 938).

There are two issues here. The first issue is how to decide which action the Robot should take if both buffer B2 and buffer B4 have a part waiting. In normal supervisory control theory, we can just enable the safe choices, and allow the plant to somehow make the decision. However, we want to be able to convert from a TDES supervisor to an SD controller in an easy, deterministic fashion. This means we must dictate which prohibitable events occur, and in which sampling period they occur in. We thus have to choose to service either buffer $\mathbf{B 2}$ or $\mathbf{B} 4$, as we cannot do both at the same time.

This issue is handled by supervisor TakeB2, shown in Figure 7.26. It forces 
Robot to first service buffer B2, then buffer B4, then back to buffer B2. It waits until there is a piece in $\mathbf{B 2}$ (event 922), then it immediately enables and forces event 933 to move the piece to buffer B4. It then waits until the piece goes to the Lathe, returns to $\mathbf{B} 4$, and then moved to either $\mathbf{B} 6$ or $\mathbf{B} 7$, before it allows the Robot to service $\mathbf{B 2}$ again.

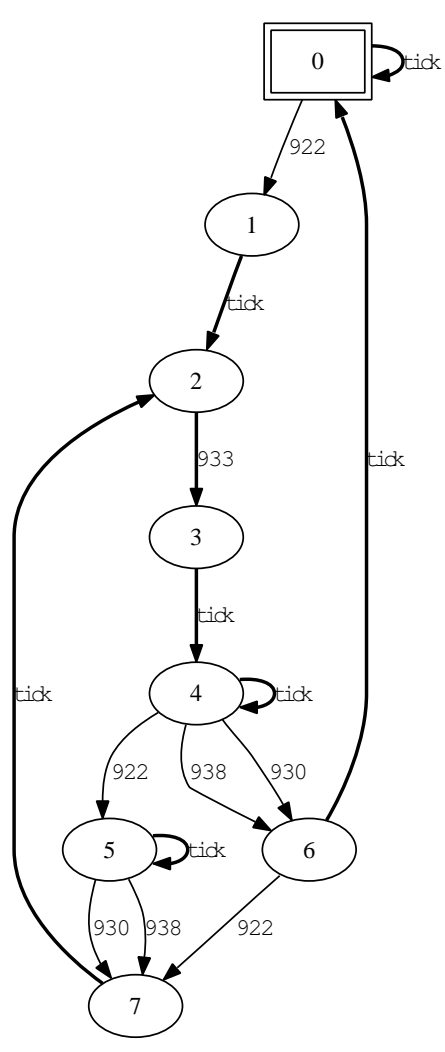

Figure 7.26: TakeB2

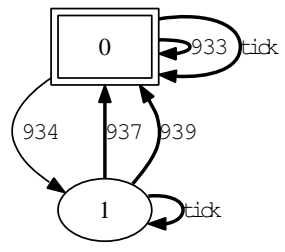

Figure 7.27: B4Path

The second issue is to prevent a conflict with respect to buffer $\mathbf{B} 4$. Once the Robot puts a piece in B4 and the piece is taken by the Lathe, the Robot could put a second piece in B4. This would mean the Lathe has no place to return its part, and the system blocks. TakeB2 prevents this by disabling event 933 until the current part has returned to $\mathbf{B} 4$, and then removed to either $\mathbf{B 6}$ or B7.

We now discuss supervisor B4Path. It works with buffer supervisor B4 to ensure the proper behavior of the B4 to lathe part path. Supervisor B4 primarily ensures that buffer B4 does not overflow or underflow. It serves an additional role in making 
sure that once a piece is put in $\mathbf{B} 4$, the correct action is taken when it is taken out. When the robot initially puts a piece in B4 (event 934), it makes sure that only events 951 and 953 can be used to take the piece out. This ensures the part goes to the Lathe for processing. The Lathe can process the piece as type A (event 951) or type B (event 953), producing different results. The Lathe then returns the part to B4 using events 952 (part is type A) or event 954 (part is type B). Since type A parts go to buffer B6 (events 937 then 938), and type B parts (event 939 then 930 ) must go to buffer B7, supervisor B4 ensures only the correct follow up event is possible. B4Path contributes to the proper behavior of the $\mathbf{B} 4$ to lathe path, by disabling event 933 once a part is put into B4 from B2, and disabling events 937 and 939 until a part is placed into B4 from B2.

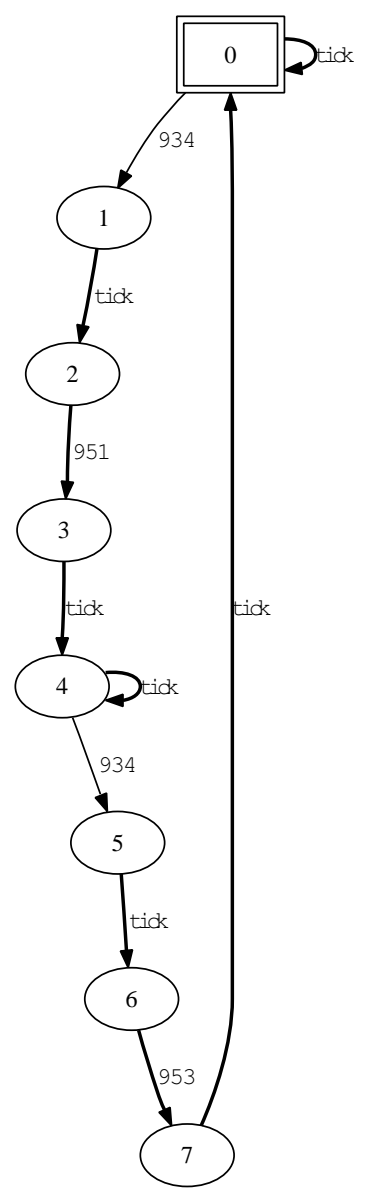

Figure 7.28: LathePick 
Supervisor LathePick, shown in Figure 7.28, also contributes to control of the B4 to lathe part path. To satisfy Point ii of SD controllability, we cannot just enable both event 951 and 953 and let the system "decide." We have to dictate when these events are to occur. That means we have to make a choice. In LathePick, we have required that the Lathe first produce a type A part, then a type B part, and then alternate. Note that the supervisor has enough information to know when the events are possible in the plant, so it does not try to force them at the wrong time, possibly "stopping the clock."

\subsubsection{Moving Parts from B4 to B6/B7}

We now discuss some concurrency and blocking issues involved with moving pieces from buffer $\mathbf{B} 4$, to either buffer $\mathbf{B} 6$ or $\mathbf{B 7}$. To move a part from buffer $\mathbf{B} 4$ to $\mathbf{B 6}$, we use event 93\%. To satisfy Point ii of SD controllability, we need to decide when to enable and force this event. This is handled by supervisor TakeB4PutB6, shown in Figure 7.29, It waits for event 952 to occur, which signifies a piece of type $\mathrm{A}$ is ready to be transferred to buffer B6. It forces event 937 and then waits for event 963 to occur, signifying that the piece has been taken by $\mathbf{A M}$ and that $\mathbf{B} 6$ is ready for a new part.

We now consider moving a part from B4 to B7. We do this using event 939. We have to decide when to force 939 in order to satisfy Point ii of SD controllability, but we also have to deal with a potential blocking situation. Because a part placed in B7 first goes to $\mathbf{P M}$ for processing, it is possible that the robot could put a part in the now empty buffer B7, leaving no place for the first part to return to. Supervisor TakeB4PutB7, shown in Figure 7.30, handles both issues. It watches for event 954 to occur, signaling that a part of type B has been placed in $\mathbf{B} 4$, and is ready to be transferred to buffer B7. TakeB4PutB7 forces event 939 to make the transfer. It then waits for event 965 to occur signaling that AM has removed the part from B7, before allowing another 939 to occur, thus preventing blocking.

\subsubsection{AM to Exit Path}

We now discuss the paths from $\mathbf{B} \mathbf{6}$ and $\mathbf{B 7}$, leading through machine $\mathbf{A M}$ and then to where the parts exit the system. We have several concurrency issues to deal with here. 


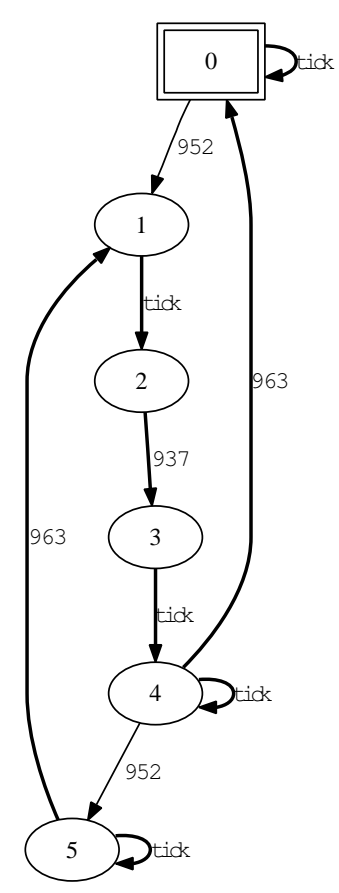

Figure 7.29: TakeB4PutB6

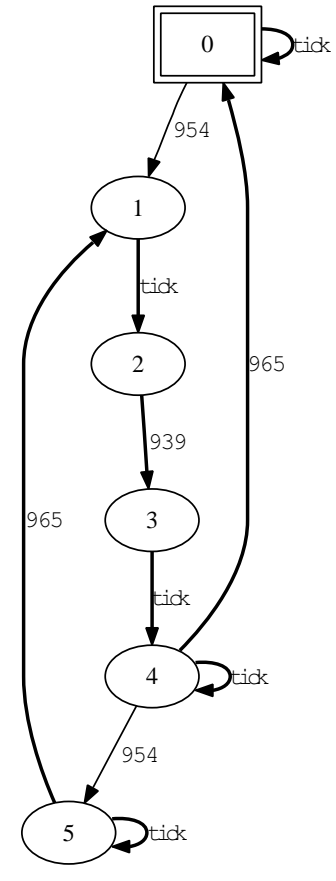

Figure 7.30: TakeB4PutB7

First, we have to specify when prohibitable events 961, 963, and 965 are suppose to occur in order to satisfy Point ii of SD controllability. This is complicated by the fact that a piece could be waiting for $\mathbf{A M}$ in both $\mathbf{B} 6$ and $\mathbf{B 7}$, so we need to specify how to choose which buffer to service first.

The problem is that these three events are linked and we have to keep track of several issues in order to decide when to force which event. We could create a single supervisor to do this, but it would be quite large and complicated, thus difficult to design correctly. It would be nice to be able to design several modular supervisors. If we were only enabling and disabling events, this would not be that hard. However, since we must decide when to force the events, we have to make sure we do not try to force an event when it is not possible in the plant, or disabled by another supervisor. It was very non-obvious how to do this modularly, without significant reuse of logic from other supervisors.

We then came up with the solution of using prohibitable "virtual events" no963a, no963b, no965a, and no965b. We introduced these new events to the system by adding plants AddNo963 and AddNo965, shown in Figures 7.31 and 7.32, Note that we 
made sure the plants specify that these events can only occur once per sampling period, so that we do not have to specify this in our supervisors.

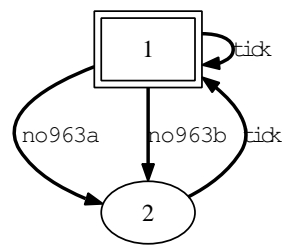

Figure 7.31: Plant AddNo963

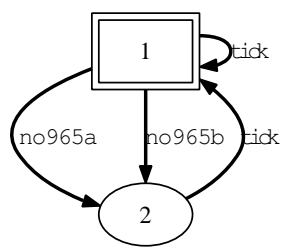

Figure 7.32: Plant AddNo965

Let's first discuss how to handle event 963. The idea is that when we want to disable the tick to force event 963 in one supervisor, events no963a/b can be used as an alternate event to force if event 963 is disabled by another supervisor, or not possible yet in the plant. The other supervisors will only enable event no963a or no963b when they know 963 is not possible, and they will make sure only one of the three events are possible at a given time. The reason there is an 'a' and 'b' event is that there are three supervisors with which we need to coordinate enablement information. This will become clear later.

The primary supervisor for event 963 is Force963, shown in Figure [7.33. It watches for event 938 to occur, signifying that there is a part in $\mathbf{B} 6$ waiting to go to AM. The supervisor then disables the tick to force 963. Note, that it is the only supervisor that tries to force this event. However, event 963 could be ineligible in plant component AM, or disabled by supervisors Force961 or AMChooser, the latter two TDES shown in Figures 7.34 and 17.35. Force963 has no way of knowing this. It handles this by adding the no963a/b-tick loop at state 2 . Supervisors Force961 and AMChooser will ensure that out of events 963, no963a, and no963b, one and only event will be eligible and enabled immediately after a tick. If 963 is ineligible or disabled, then no963a or no963b gets forced instead, and then we try again after the tick. This way we signal we want 963 to occur as soon as it can, but do not stop the clock. We also do not need to repeat information from the plant and other supervisors about when these events are eligible/enabled.

The reason that only one of the three events are ever allowed to be eligible/enabled at the start of a tick, is to avoid violating Point iii.1 of the SD controllability definition. Examining state 2 of Force963, we see that once one of the three events 
occurs, the others are disabled. If more than one was enabled and eligible at state 2 , this would cause one of them to change eligibility status between ticks, violating Point iii.1 of the SD controllability definition.

For event 965, we have similar behavior represented by supervisor Force965, shown in Figure 7.36. It interacts in a similar way with plant component AM, and supervisors Force961 and AMChooser.
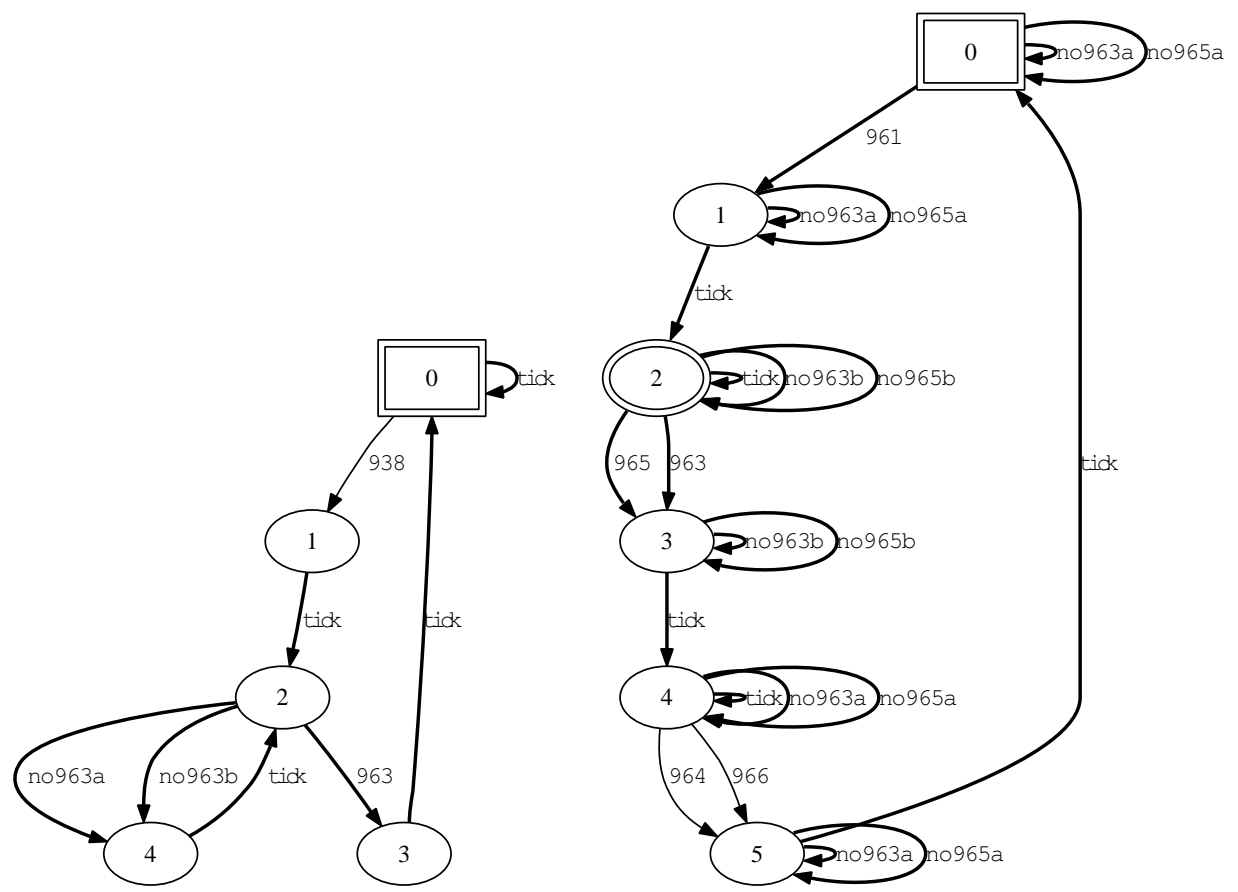

Figure 7.33: Force963

Figure 7.34: Force961

We now discuss supervisor Force961, shown in Figure 7.34, Its primary task is to determine when to force event 961 which readies AM to process parts. Force961 forces 961 right away, and then waits for events 964 or 966 (signifies AM has finished processing the part) to occur, before forcing event 961 again.

The secondary task of Force961 is to only enable events no963a and no965a when events 963 and 965 are not possible in the plant component AM. When they are possible in the plant, Force961 enables no963b and no965b instead. This insures that events no963a and no965a will always be possible after a tick when events 963 and 965 are ineligible in the plant. It also ensures that the ' $a$ ' and 'b' events are never 


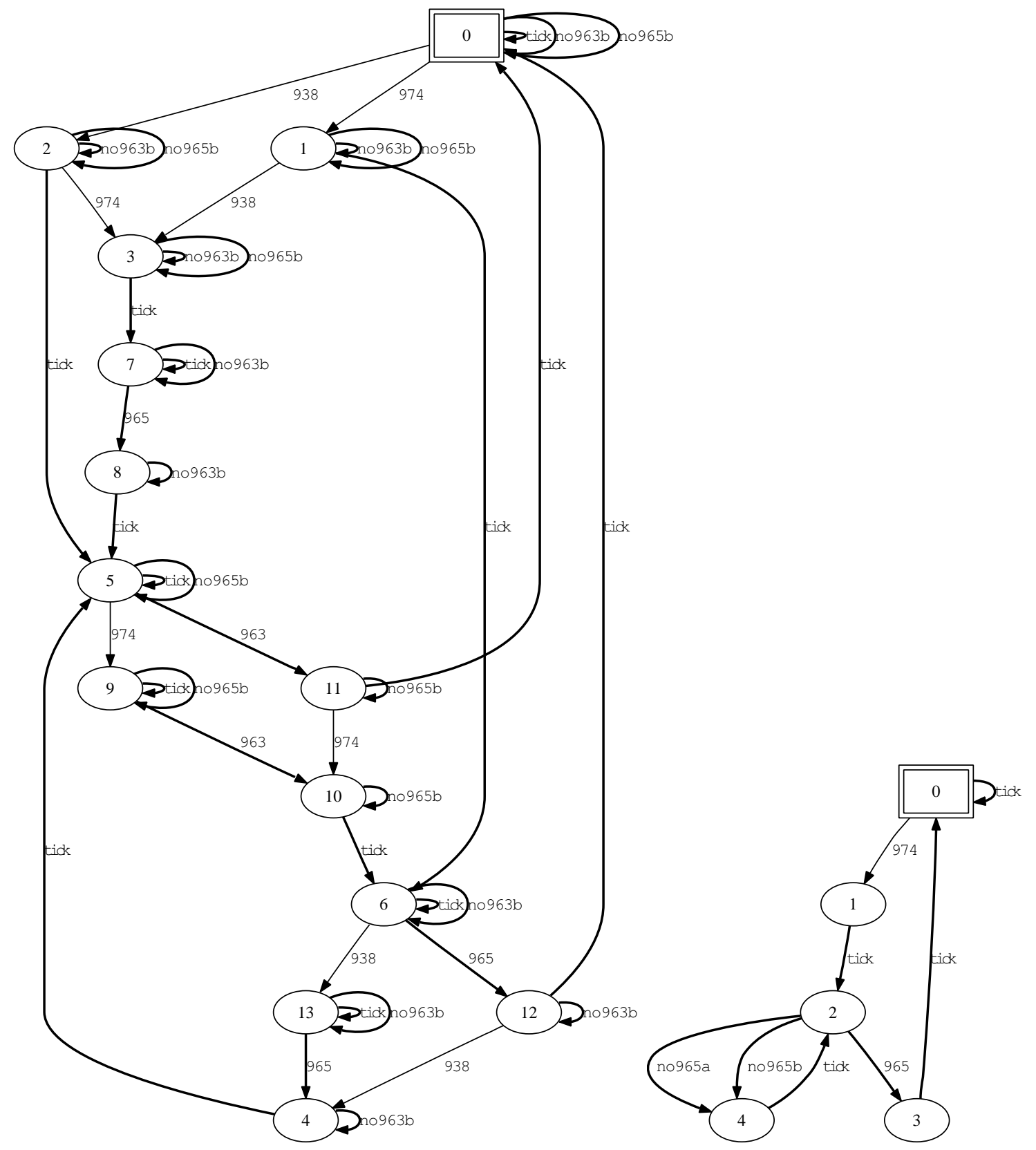

Figure 7.36: Force965

Figure 7.35: AMChooser

eligible at the same time. Also, as supervisor AMChooser ignores the 'a' events, they will never be disabled when Force961 needs them. As Force961 never disables 
the 'b' events when 963 and 965 are possible in the plant, this ensures that they will not be disabled when AMChooser needs them. This means the two supervisors do not interfere with each other with respect to these events.

Finally, please note that when we switch from the 'a' to the 'b' events in Force961, we only do so immediately after a tick (consider states 1 to state 4 as an example). This is to not violate Point iii.1 of the SD controllability definition.

We now consider our last supervisor for this section, AMChooser, shown in Figure 7.35. The role of this supervisor is to choose between taking a piece from buffer B7 (event 965) or buffer B6 (event 963), when both have a waiting part. If both receive a part in the same sampling period, we take the piece from buffer B7 first as there are other machines to keep busy along the B7 to PM path. We then take a piece from B6. If there is already a new piece from B7 waiting, we continue in an alternating fashion. If there is only one piece waiting in a given sampling period, then we handle that piece. Because AMChooser sometimes disables event 963 or 965 in order to enforce this order, it enables the appropriate no963b or no965b event as a forcing substitute. It also ensures that event 963 and no963b are never enabled at the same time. It behaves similarly for events 965 and no965b.

\subsubsection{System Shutdown}

When we tested the previous supervisors (excepting supervisor B2 originally did not have its state 6, plant component $\mathbf{A} \mathbf{M}$ was not marked at its state 3, and supervisor Force961 was not marked at its state 2) we found that the system was blocking. It was not that the system was deadlocking or not completing its tasks, it was simply the fact that, due to forcing events as soon as they were ready, the entire system was never in a marked state at the same time. We could have delayed some events to achieve this, but that would have been less efficient.

The real cause was the fact that the system did not have a shutdown mechanism. Once started, it just kept running. A shutdown mechanism would cause the system to empty out, allowing a non-deadlocked/livelocked system to return to its idle state. The easiest way to cause the system to go idle, is to prevent plant component Con2 from taking new parts (event 921). Once new parts stopped coming in, the system would process the existing ones, allow them to leave, and then the TDES should 
return to their idle states which are marked.

To achieve this, we added a new plant component SystDownNup, shown in Figure 7.37, It contains an event shutdown to empty the system, and an event restart to bring the system back up. This could correspond to a physical switch an operator could throw to control this behavior.
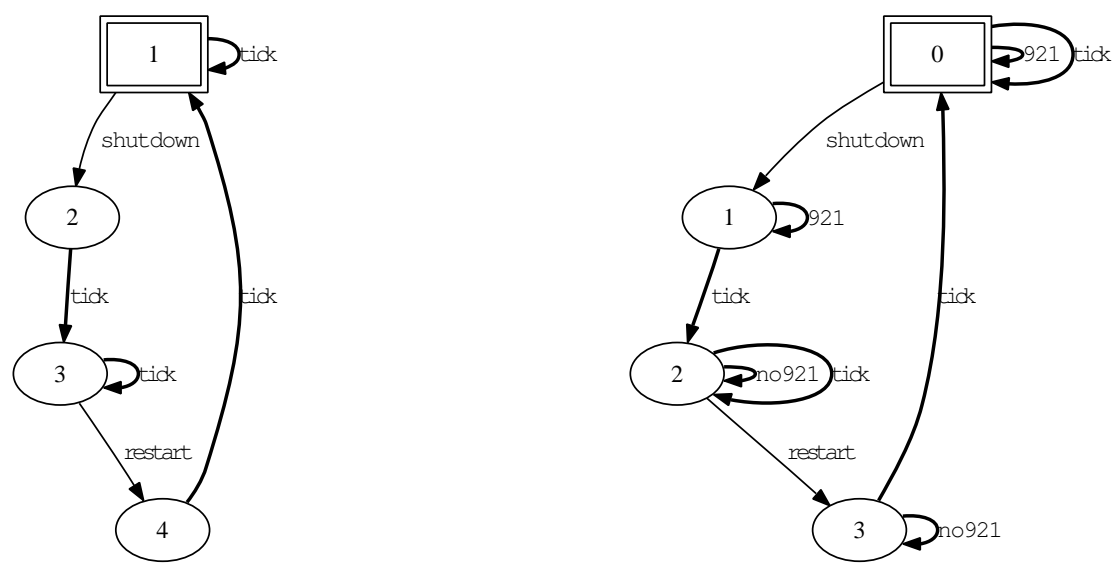

Figure 7.37: Plant SystDownNup Figure 7.38: Supervisor handleSystDown

Our next task was to stop new pieces from entering the system. The problem was that supervisor B2 forced event 921, causing Con2 to take a new part, as soon as buffer B2 was empty. As we wanted to keep supervisor B2 simple, we added a new prohibitable "virtual event," no921. This was introduced by adding plant AddNo921, shown in Figure 7.39. We then added the no921-tick loop at state 0 of supervisor B2. We would use event no921 as an alternate event to force, when we disabled event 921.

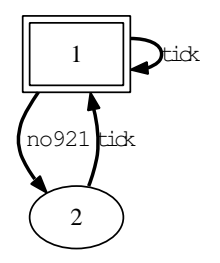

Figure 7.39: Plant AddNo921

Finally, we added supervisor handleSystDown, shown in Figure 7.38, Its job was to enable event 921 and disable no921 initially, and then disable 921 and enable 
no921 once the shutdown event occurs. When the restart event occurs, the process is reversed. We also make sure events 921 and no921 are never enabled at the same time, and that one of the two are always eligible and enabled immediately after a tick.

However, after the above, we were still nonblocking. The culprit was supervisor Force961. As soon as event 961 was eligible, it was forced so that AM was ready to process a part. We could have created a no961 event like we did for B2, but this would have been trickier as we needed to allow enough 961 events to occur to allow the existing pieces to leave. Rather than do this, we decided that for AM, state 3 was a rest state, and it was fine to leave it there. So, we marked state 3 of $\mathbf{A M}$, and state 2 of Force961, and the system was nonblocking. Note that we could have marked state 2 of TDES AM, and state 1 of TDES Force961, but that would have caused Point iv of the SD controllability definition to fail.

\subsubsection{Algorithm Runtime Statistics}

To test the performance of the algorithm on this example, the following machine configuration was used:

- $1.8 \mathrm{GHz}$ Dual core AMD processor

- 4GB of Dual channel DDR2 RAM

- Cygwin 1.5.25-15 with gcc version 4.3.2

For testing purpose, the source code is compiled with -03 optimization ${ }^{1}$.

As we can see from the log output for the FMS example, shown in Listing 7.5, our supervisor $\mathbf{S}$ is SD controllable for our plant. We also see that our plant has proper time behavior, is complete for our supervisor, and has $\mathbf{S}$-singular prohibitable behavior. Finally, we see that our closed loop system is ALF and nonblocking. From the $\log$, the total number of states of the synchronous product is 82,608 . The verifications take about 2 minutes and 51 seconds. The memory usage is around 183 megabytes at the highest point, as shown in Figure 7.40, For the input files of all the DES in this example, please see the appendix.

\footnotetext{
${ }^{1}$ More information can be found by running man gcc.
} 
Listing 7.5: Output

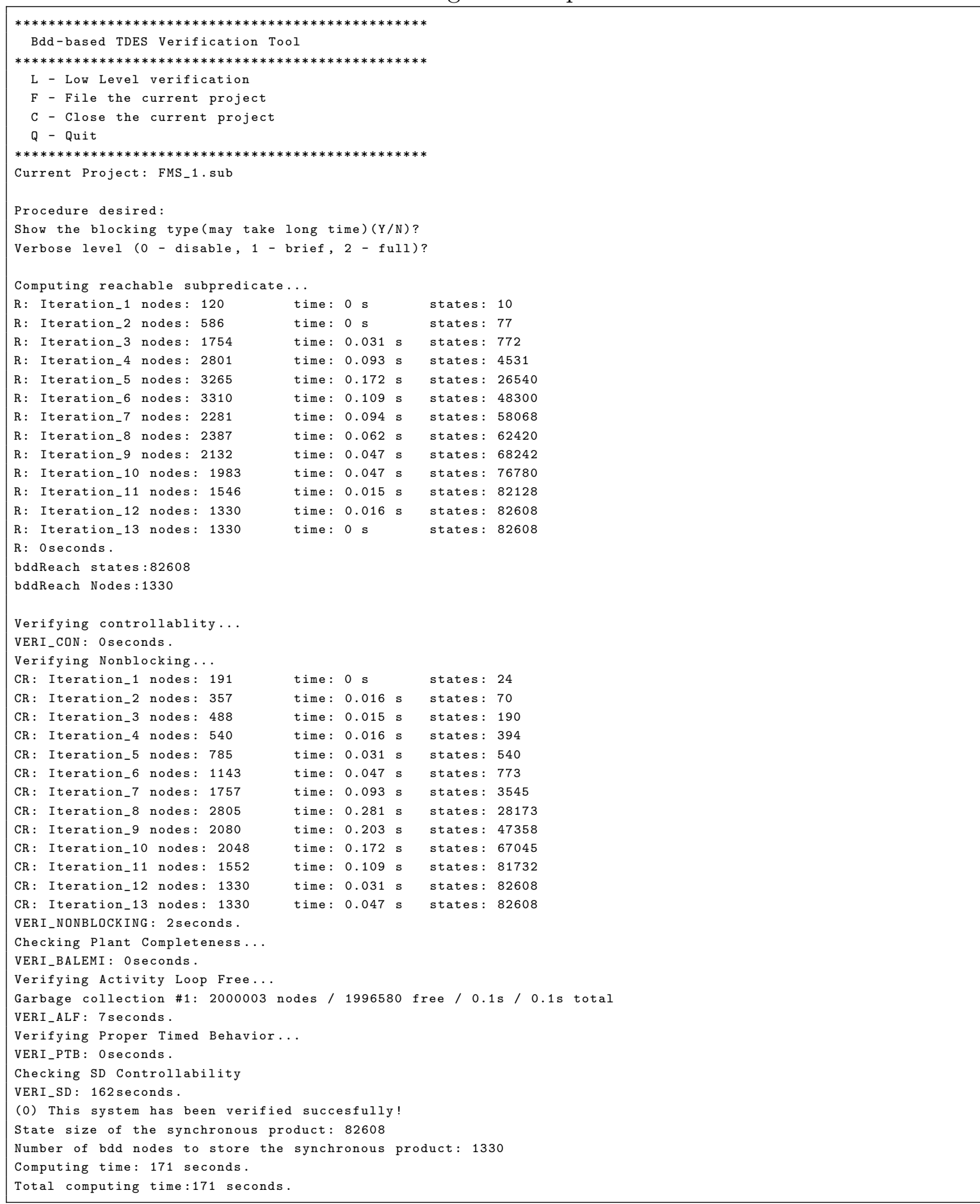




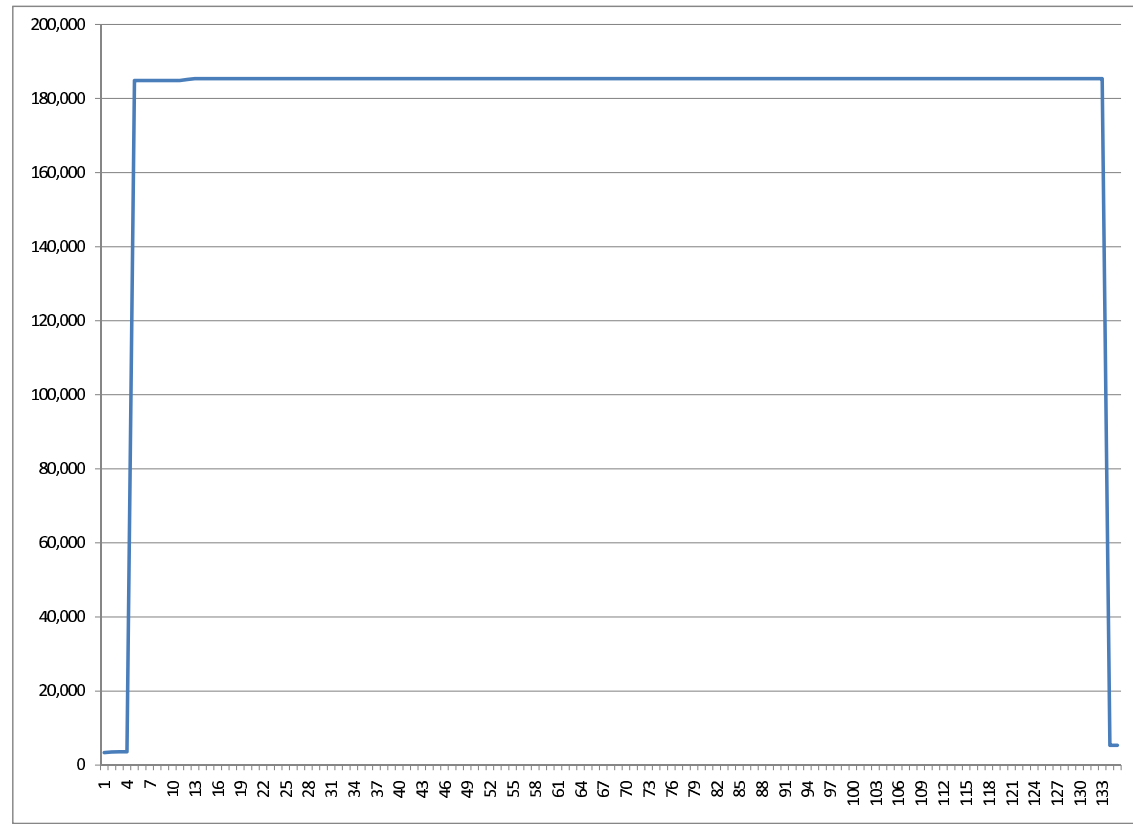

Figure 7.40: Histogram for Memory Usage (Kbytes vs. seconds) 


\subsection{Translating FSM Supervisors to Moore FSM}

In Section 7.2, we presented an example of a Flexible Manufacturing System with SD controllable TDES supervisors. In this section, we apply the method in Section 4.2 to translate individual FMS supervisors into Moore finite state machines (FSM) (see Section 4.1). This is possible because our supervisor is SD controllable, and our plant is complete for our supervisor. If the plant was not complete, we would have had to use additional information from the plant components to determine when the problematic prohibitable events were not possible in the plant. This can be accomplished by converting the plant components that contain the needed information into FSM as well, and combining them with the FSM for the supervisors as modular controllers.

\subsubsection{Adding More Timing Information}

Before we can translate the individual TDES supervisors into FSM, they must be CS deterministic as in Definition 3.1.5 and non-selfloop ALF. A TDES is non-selfloop ALF if once any activity selfloops are removed, the resulting TDES is ALF. For example, supervisor B4 in Figure 7.22 is neither CS deterministic or non-selfloop ALF. This is a problem as the possible next state transitions of the FSM are too numerous, and many of them are not actually possible in the plant. For example, we could have according to the TDES a 934-tick sequence, a 934-951-tick, or even a \{934-951 $\}^{*}$-tick sequence. We simply have too many choices, and this would result in an overly complex FSM. Also, concurrent strings 934-951-tick and 934-951-934tick have the same occurrence image but lead to different states, which would result in a nondeterministic controller. Examining the plant and other supervisors, we see that there will always be a tick between events 934 and 951, so we can add this to TDES B4, as we have done in Figure 7.41.

Making similar observations for the other non-selfloop activity loops, we get the supervisor in Figure 7.41 which should provide us with the same over all closed loop behavior as the original B4 supervisor. However, we note that prohibitable event 933 is still selflooped at state 0 , so the TDES is not ALF. We could modify the supervisor to remove this loop, but we do not need to as the selfloop provides enablement information, but does not affect the next state information. As such, it does not impede our translation. i.e. our next state information is $\{933\}^{*}-934$-tick to state 
1 of B4. Essentially, as long as the supervisor is CS deterministic and non-selfloop ALF, we can do the translation. As was discussed in Chapter 4 , all we require is that the TDES be CS deterministic, but typically if the TDES is not non-selfloop ALF it will also not be CS deterministic. Also, it is often difficult to even check the CS deterministic condition if the TDES is not non-selfloop ALF.

We then made similar changes to supervisors B6, B7, and B4Path. The new supervisors are shown in Figures 7.42-7.44. All remaining supervisors can be converted directly. We reran our software on the FMS system with these new supervisors, and all conditions still passed.

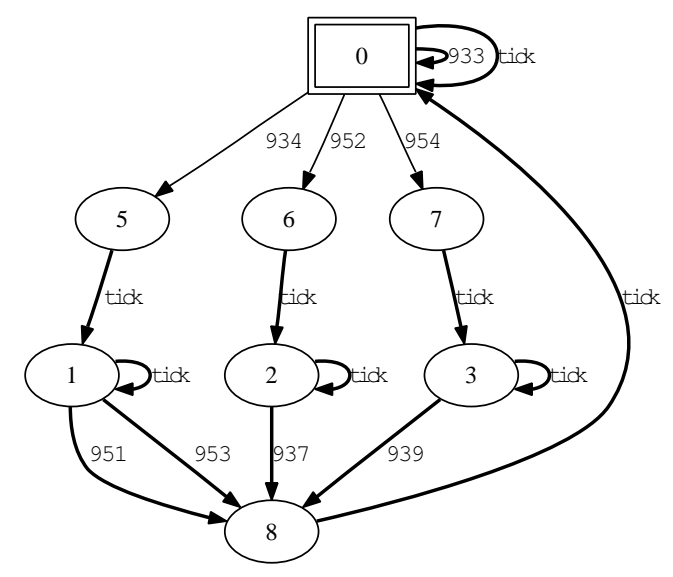

Figure 7.41: New B4

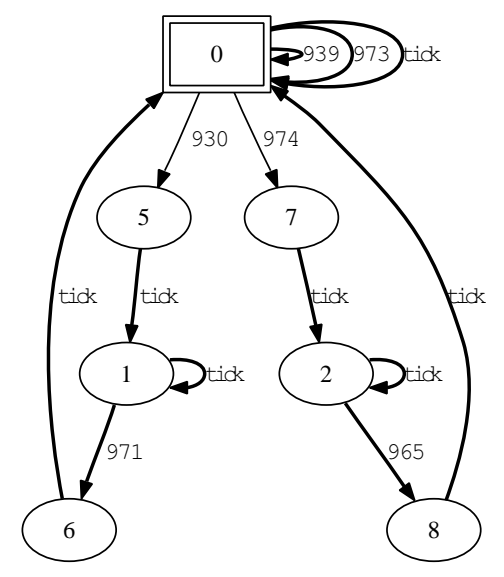

Figure 7.43: New B7

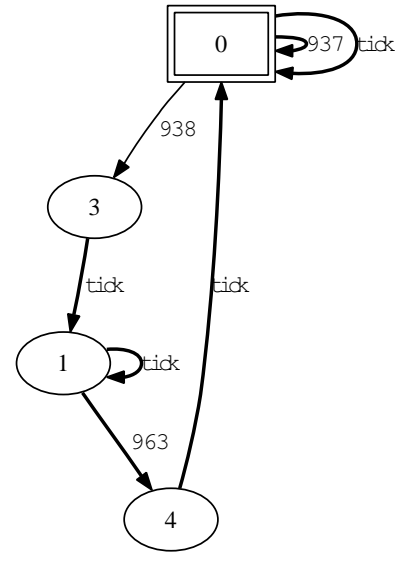

Figure 7.42: New B6

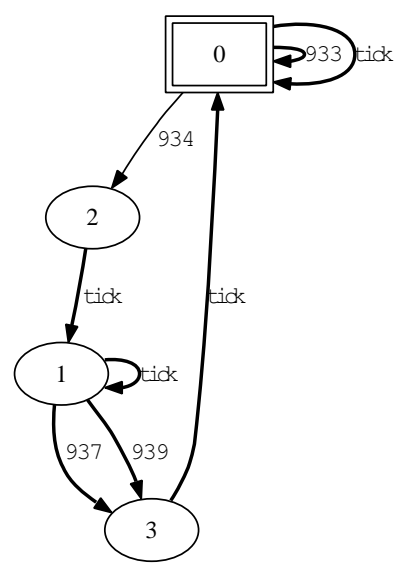

Figure 7.44: New B4Path 


\subsubsection{FSM Controllers for Flexible Manufacturing System}

This section lists all the FSM Controllers for the Flexible Manufacturing System we presented in Section 7.2 and 7.3.1, using the method developed in Section 4.2. We first briefly discuss some implementation and modeling details, as well as introduce some notation that we will use.

Each FSM samples its inputs on the clock edge when tick occurs, and then changes state based on its current state, the value of each relevant input, and the next state arcs for that state. The timing info is implicit as it only changes state on a clock edge. If an input for an event is true when sampled on the clock edge, then it is considered to have occurred during the last clock period. The designer must make sure that the input for a given value has a pulse length equal to the period of our clock so that the input will not be lost. If an input is seen at two clock edges in a row, it is considered to have occurred twice. As such, the designer must make sure an input does not have an overly long pulse length. Remember, except for one exception, an event is considered to occur when its input goes true at the controller. The exception is when the input goes true so close to a sampling edge it is detected in the next sampling period, then it is considered to have occurred in the next clock period. This should be taken in to account in modeling the system.

To represent the FSM visually, the following notations are defined. For the given FSM,

- At each state in the FSM, a prohibitable event is listed if its corresponding output is true at that state, which means the controller enables this event at this state. An event is not listed if its output is false.

- At each transition, we use logical operators to represent the sampled input. We use '!' as NOT, '+' as OR, '.' as AND.

- To distinguish from a DES event label and the event input being true at the clock edge, the event name is surrounded by '[ ]' to indicate that the input was true at the clock edge.

If the controller is following a concurrent string, for example $\alpha-\beta-\tau$ from one sampling state to the next, we add a transition arc with ' $'$ (AND) between the non- 
tick events. For example ' $[\alpha] \cdot[\beta]^{\prime}$ ? 2 This would be interpreted as events $\alpha$ and $\beta$ occurred in the last sampling period, and no other activity events. Of course, there is no implied ordering of the two events, nor do we know how many times each event actually occurred during the last clock period.

Technically, if a supervisor has event set $\Sigma=\alpha, \beta, \gamma$, tick, the next state condition for a given concurrent string should include a term for each activity event in the event set. When the event is missing, it is negated. For string $\alpha-\beta-\tau$, this would be ' $[\alpha] \cdot[\beta] \cdot ! \gamma]$ '. However, we can often simplify these equations using Boolean logic. For instance, if none of the possible strings at the current sampled state contain $\gamma$, we can leave it out of the equations.

If the controller is getting to the same state by different strings which are not occurrence equivalent, then we can use ' + '(OR) to combine the conditions together. For example ' $[\alpha]+[\beta]$ '. This would be interpreted as event $\alpha$ or event $\beta$ occurred in the last sampling period, but no others.

If at a given state in the controller we can do concurrent string $\alpha-\tau$ and $\alpha-\beta-\tau$, we need to make sure their next state equations do not overlap. Using conditions ' $[\alpha]$ ' and ' $[\alpha] \cdot[\beta]$ ' is not enough as first condition is true as long as $\alpha$ occurred, irrespective of $\beta$. Instead, the condition for $\alpha-\tau$ should be ' $[\alpha] \cdot ![\beta]$ '.

For each FSM, the initial state is identified by a Reset signal. This Reset signal represents the "power on" behavior or a restart of the controller. This state is equivalent to the initial state of each TDES. It also explains why the initial state of a timed DES is a sampling state that does not need to be reached by a tick, since the FSM always starts at this state.

For each FSM state, we typically define a default transition DEF. This is because a TDES transition function is a partial function and an FSM next state function is a total function. Basically, it is a shorthand for all the next state equations that we have not explicitly specified. It is equivalent to taking the logical OR of all existing outgoing next state conditions from that state, and then negating the result. Sometimes, when we are translating a supervisor, we end up with a specified next state equation going to the same place as our DEF transition. That means this transition can be removed as it will be covered by the DEF condition.

\footnotetext{
${ }^{2}$ In the following FSM graphs, this operator is represented by '.'(period) instead of '.' due to a technical difficulty.
} 
Our first FSM is for supervisor B2, and is shown in Figure 7.45. At state 0, we have merged selfloop transition '![921] • [no921]' with the DEF transition. It is worth noting how much simpler the FSM tends to be than the corresponding supervisor. For B2, we went from a 7 state supervisor to a 3 state FSM.

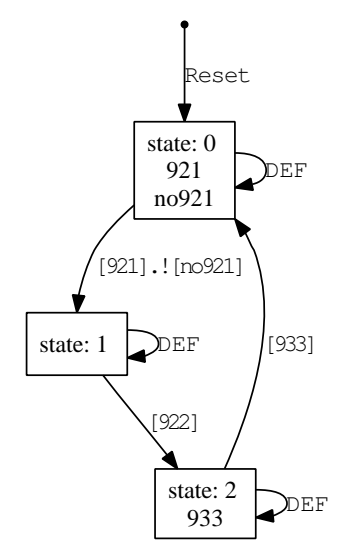

Figure 7.45: FSM B2

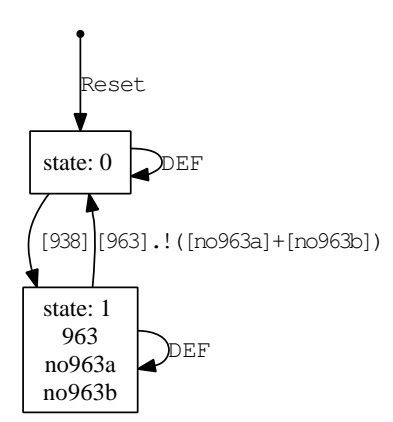

Figure 7.46: FSM Force963

We do a similar simplification for supervisors Force963 and Force965. The translated FSM are shown in Figures 7.46 and 7.47. For Force963, we should have a ! [963] $\cdot([n o 963 a]+[$ no963b] $)$ selfloop at state 1 , but we have absorbed this into the DEF transition. For Force965, we have absorbed the ![965] $([$ no965a $]+[n o 965 b])$ transition at state 1 , into the DEF transition.

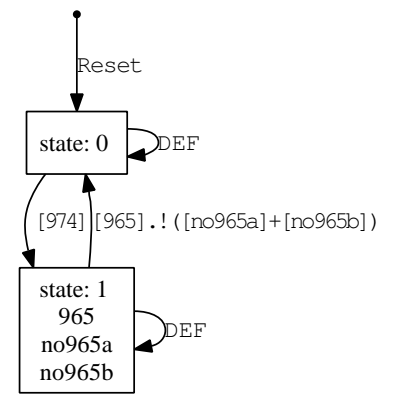

Figure 7.47: FSM Force965

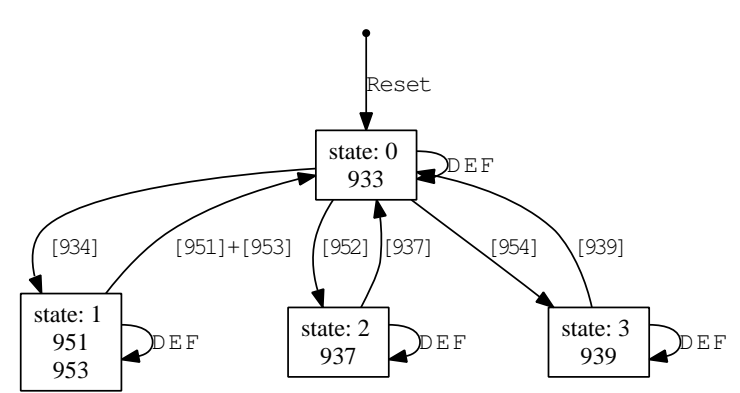

Figure 7.48: FSM B4

The next translation we examine is for B4, and the FSM are shown in Figure 7.48. Note at state 0 , we have a transition to state 1 with condition '[934]'. Strictly 
speaking this should be '[934] $![952] \cdot ![954]$ '. However, after examining the plant and other supervisors, we know that these three events can never occur in the same clock period. We can thus simplify this to '[934]' to keep our diagram simple. Similar for the '[952]' and '[954]' transitions. A similar example is at state 1. Here we have transition condition '[951] $+[953]$ '. Strictly speaking, this should be '[951] •![953]+![951] • [953]' but we know from the plant that these events can't occur in the same clock period, so we can simplify things.

The translation of the remaining FSM are straightforward so we do not need to discuss them individually. The translations for supervisors B6, B7, B8, LathePick, TakeB2, B4Path, Force961, handleSystDown, TakeB4PutB6, TakeB4PutB7, and AMChooser are shown in Figures 7.49 - 7.59,
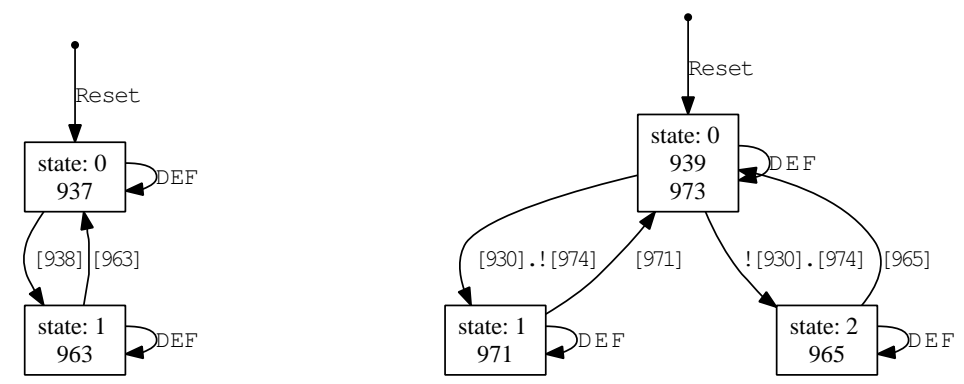

Figure 7.49: FSM B6

Figure 7.50: FSM B7 


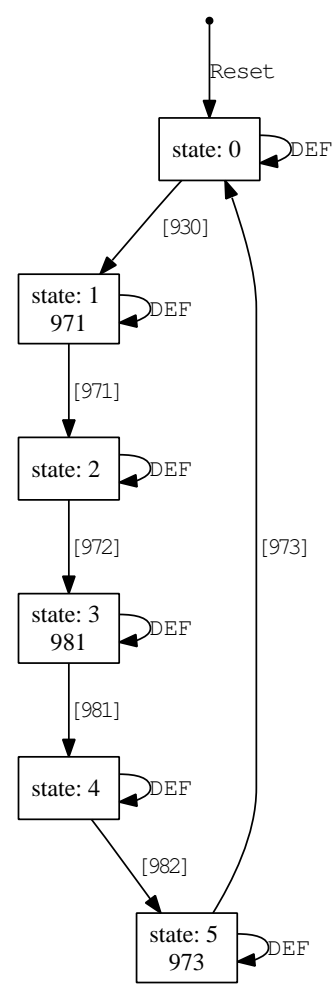

Figure 7.51: FSM B8

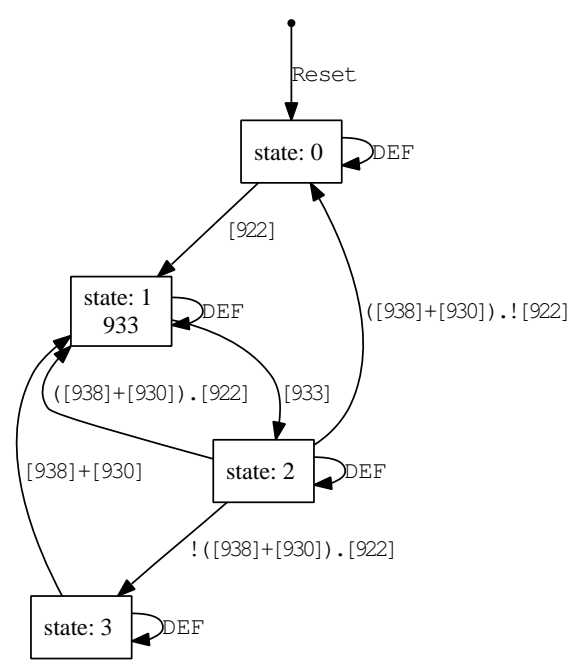

Figure 7.53: FSM TakeB2

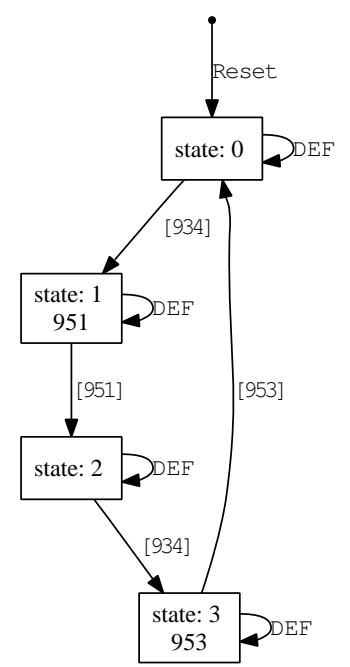

Figure 7.52: FSM LathePick

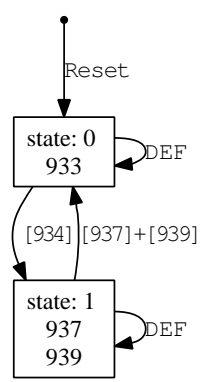

Figure 7.54: FSM B4Path 


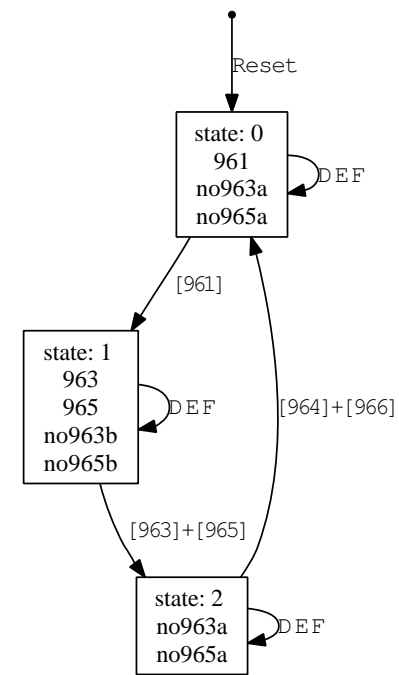

Figure 7.55: FSM Force961 Figure 7.56: FSM handleSystDown
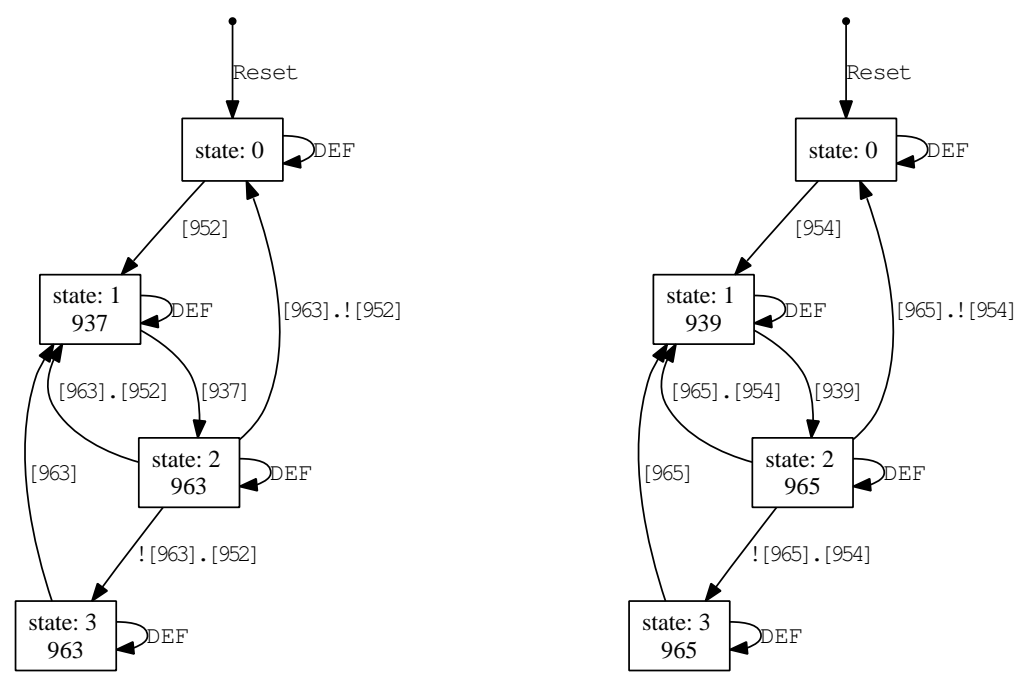

Figure 7.57: FSM TakeB4PutB6 Figure 7.58: FSM TakeB4PutB7 


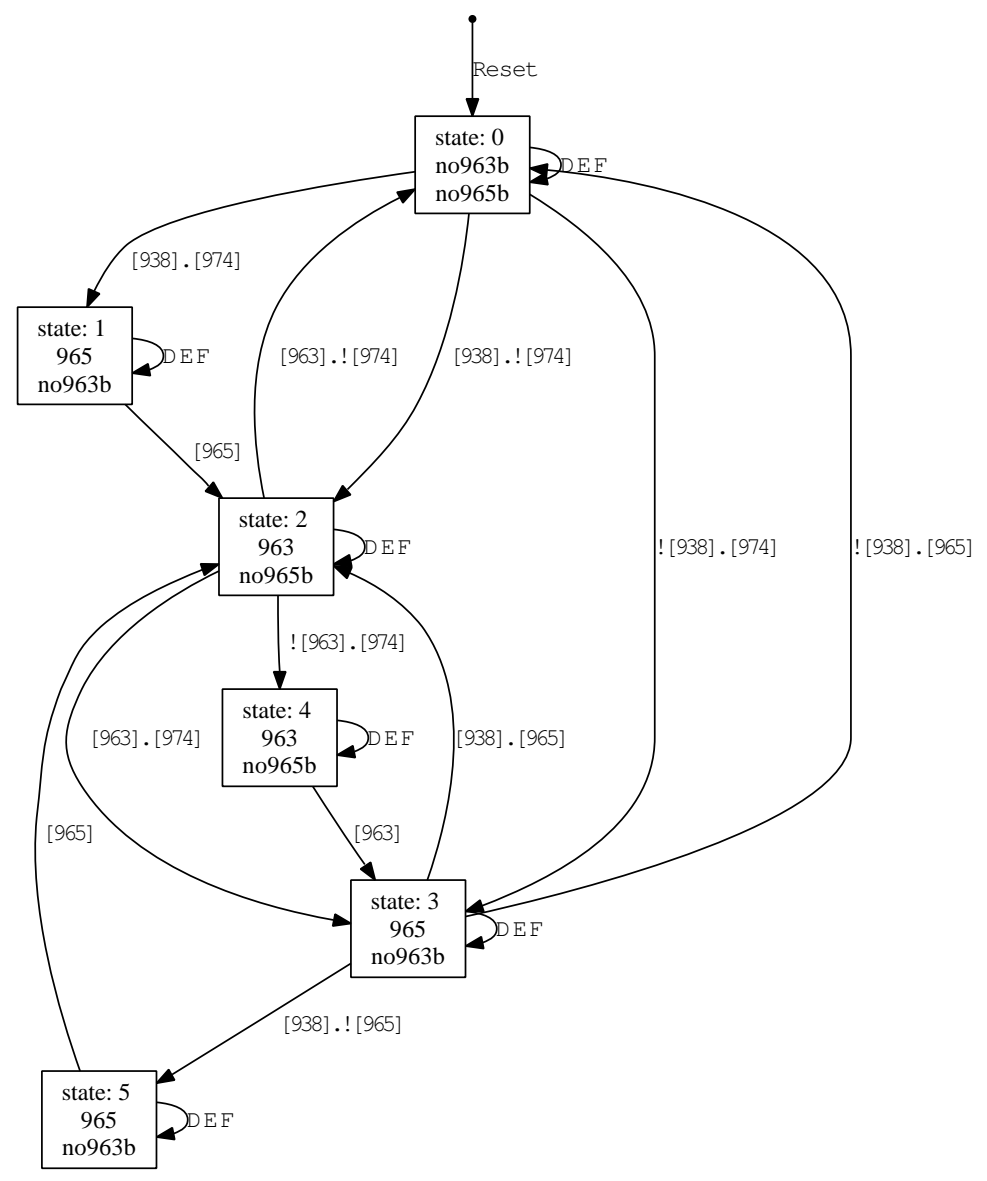

Figure 7.59: FSM AMChooser 


\section{Chapter 8}

\section{Conclusions}

This thesis focuses on issues related to implementing theoretical Discrete-Event Systems (DES) supervisors, and the concurrency and timing delay issues involved.

Sampled-data (SD) supervisory control deals with timed DES (TDES) systems where the supervisors will be implemented as SD controllers. An SD controller is driven by a periodic clock and sees the system as a series of inputs and outputs. On each clock edge (tick event), it samples its inputs, changes states, and updates its outputs. In our introduction, we identified several concurrency issues that are not covered by the standard controllability and nonblocking definitions.

In this thesis, we identify a set of existing TDES properties that will be useful to our work, but not sufficient. We require that our plant have proper time behavior, and is complete for our supervisor. We also require that our closed loop system is activity loop free and nonblocking. To these properties, we add two new conditions. First, we require that the plant have $\mathbf{S}$-singular prohibitable behavior, where $\mathbf{S}$ is our TDES supervisor. This condition restricts plant behavior such that prohibitable events can only occur at most once per clock cycle, but is only concerned with strings that are also accepted by our supervisor.

The main new condition we introduce is the SD controllability definition. This condition extends the standard TDES controllability definition by adding restrictions so that the TDES behavior is consistent with restrictions imposed by SD controllers, making it easier to translate a TDES into an SD controller. It includes conditions to ensure that the enablement and eligibility information is constant across a sampling 
period, and that when the controller forces an event, it will not occur when the plant model says it can't. It also ensures that when two strings that appear identical to an SD controller occur in the same sampling period, the strings have the same closed and marked future in the system's closed loop behavior. This means the SD controller will take the same control action for both, and either string will be sufficient to get us to a marked state. Finally, we require that only the empty string or a string ending in a tick can be marked. This ensures that marked strings will be observable to the controller.

We then establish a formal representation of an SD controller as a Moore Finite State Machine (FSM), and describe how to translate a TDES supervisor to a FSM. To be able to translate a given TDES into an FSM, we require that the TDES be CS deterministic. This new condition essentially says that if two concurrent strings can occur in the same clock cycle and they contain the same events (possibly in different order or number), then they must take us to the same state in the supervisor. This ensures our FSM is deterministic. We also discuss how to construct a single centralized controller, as well as a set of modular controllers and show that they will produce equivalent output. This is an important result, because we prefer a modularized design of controllers rather than a large, complex, centralized design.

Next, we capture the enablement and forcing action of a translated controller in the form of a TDES supervisory control map, and show that the closed-loop behavior of this map and the plant is the same as that of the plant and the original TDES supervisor. This is important as it shows that the behavior we expect from our TDES model is what we should actually get in the system, at least as far as enablement and forcing goes. As a controller chooses its next state based on which events occurred in the last clock period, this means the enablement and forcing actions the controller takes is irrespective to event ordering or number, but will have equivalent effect as that of our TDES supervisor. As we discuss at the end of Chapter 3, there are several time delay issues that we only partially address, leaving the remaining issues for future work.

We also show that our method is robust with respect to nonblocking and certain variations in the actual behavior of our physical system. Essentially, if there are two or more concurrent strings possible in a given clock cycle and they contain the same events (possibly in different order or number), we showed that as long as at 
least one of these strings is actually possible in the physical system, then the physical system and our SD controller will be nonblocking if our TDES closed loop system is nonblocking. This result is important as some implementations may be such that we actually get a subset of our expect behavior. This result says that as long as we get this minimal subset, we will remain nonblocking.

We also introduce a set of predicate-based algorithms to verify the SD controllability property, as well as the other conditions that we require. The algorithms are implemented on the top of the preceding code base of Raoguang Song and use binary decision diagrams (BDD). BDD is an efficient structure to store systems with large statespaces and to perform state set operations. The implemented software tool is able to verify a system whose synchronous product has more than 80,000 states, in less than 3 minutes. We expect that it will be able to handle quite large systems, but we did not have time to attempt this ourselves.

Finally to test our algorithms, we have produced a set of illustrative examples which fail the key conditions discussed in this thesis, as well as a successful application example based on a Flexible Manufacturing System (FMS). For all the supervisors in the FMS example, we also translated them into Moore FSM controllers using the translation method we created. Ideally, we would like to see an algorithm that converts these controllers into program source code in some computer language. This is left as future work and is beyond the scope of this thesis.

The source code of the software tool and the input files for the FSM example are included in the appendix. The software is single threaded, which limits its performance. A few choices for the next step for the software tool, are rewriting the code to be multithreaded, and/or implement a mechanism that can distribute the verification over multiple machines. We believe that our algorithms have good parallelizing potential. This is left as future work. 
8. Conclusions 


\section{Bibliography}

[1] D. S. Arnon, "Bibliography of quantifier elimination for real closed fields," J. Symb. Comput., vol. 5, no. 1-2, pp. 267-274, 1988. 6.2.2

[2] S. Balemi, "Input/output discrete event processes and communication delays," Discrete Event Dynamical Systems: Theory and Applications, pp. 41-85, 1994. 1.1, 1.2, 2.2 .3

[3] F. Basile and P. Chiacchio, "On the implementation of supervised control of discrete event systems," Control Systems Technology, IEEE Transactions on, vol. 15, no. 4, pp. 725-739, 2007. 1.2

[4] W. Bolton, Programmable Logic Controllers. Elsevier, 4th ed., 2006. 1

[5] B. Brandin and W. Wonham, "Supervisory control of timed discrete-event systems," IEEE Transactions on Automatic Control, vol. 39, pp. 329-342, Feb 1994. $1.1,1.2,2.3$

[6] B. A. Brandin, Real-time supervisory control of automated manufacturing systems. PhD thesis, University of Toronto. Graduate Department of Electrical and Computer Engineering, 1992. 1.1, 1.2, 2.3, 5.1

[7] S. Brown and Z. Vranesic, Fundamentals of Digital Logic with VHDL Design. McGraw Hill Higher Education, 3rd ed., 7 2008. 1, 3.1

[8] A. E. Bryant, "Symbolic boolean manipulation with ordered binary-decision diagrams," ACM Computing Surveys, vol. 24, pp. 293-318, 1992. 1.1, 1.2, 6, 6.2.2

[9] C. Dragert, J. Dingel, and K. Rudie, "Generation of concurrency control code using discrete-event systems theory," in SIGSOFT '08/FSE-16: Proceedings of 
the 16th ACM SIGSOFT International Symposium on Foundations of software engineering, (Atlanta, Georgia), pp. 146-157, ACM, 2008. 1.2

[10] A. Giua and C. Seatzu, "Modeling and supervisory control of railway networks using petri nets," IEEE Transactions on Automation Science and Engineering, vol. 5, no. 3, pp. 431-445, July 2008. 1.2

[11] R. C. Hill, Modular Verification and Supervisory Controller Design for DiscreteEvent Systems Using Abstraction and Incremental Construction. PhD thesis, Department of Mechanical Engineering, University of Michigan, 2008. 1.1, 7, $7.2,7.2 .2$

[12] R. J. Leduc, "PLC implementation of a DES supervisor for a manufacturing testbed: An implementation perspective," Master's thesis, Graduate Department of Computer and Electrical Engineering, University of Toronto, 1996. 1.2

[13] J. Lind-Nielsen, BuDDy: Binary Decision Diagram Package. IT-University of Copenhagen, 11 2002. 6]

[14] C. Ma, Nonblocking supervisory control of state tree structures. PhD thesis, Department of Electrical and Computer Engineering, University of Toronto, 2004. Adviser-W. M. Wonham. 1.2, 6.2, 6.3

[15] P. Malik, From Supervisory Control to Nonblocking Controllers for Discrete Event Systems. PhD thesis, Dept. of Computer Science, University of Kaiserslautern, Kaiserslautern, 2003. 1.2

[16] J. O. Moody and P. J. Antsaklis, Supervisory Control of Discrete Event Systems using Petri Nets. Kluwer Academic Publishers, 1998. 1.2

[17] E. Moore, "Gedanken-experiments on sequential machines," in Automata Studies (C. Shannon and J. McCarthy, eds.), pp. 129-153, Princeton, NJ: Princeton University Press, 1956. 1.1, 4

[18] M. Nourelfath and E. Niel, "Modular supervisory control of an experimental automated manufacturing system," Control Engineering Practice, vol. 12, no. 2, pp. 205-216, Feb. 2004. 1.2 
[19] J. S. Ostroff, "Deciding properties of timed transition models," IEEE Trans. Parallel Distrib. Syst., vol. 1, no. 2, pp. 170-183, 1990. 1.2

[20] J. S. Ostroff, Temporal logic for real time systems. New York, NY, USA: John Wiley \& Sons, Inc., 1989. 1.2

[21] J. Ostroff and W. Wonham, "A framework for real-time discrete event control," IEEE Transactions on Automatic Control, vol. 35(4), pp. 386-397, April 1990. 1.2

[22] S. Perk, T. Moor, and K. Schmidt, "Controller synthesis for an I/O-based hierarchical system architecture," Discrete Event Systems, 2008. WODES 2008. 9th International Workshop on, pp. 474-479, May 2008. 1.2

[23] P. J. Ramadge and W. M. Wonham, "Supervisory control of a class of discrete event processes," SIAM J. Control Optim., vol. 25, no. 1, pp. 206-230, 1987. 1

[24] A. Saadatpoor, "State-based control of timed discrete-event systems using binary decision diagrams," Master's thesis, Department of Electrical and Computer Engineering, University of Toronto, 2004. 1.2

[25] K. Schmidt and E. Schmidt, "Communication of distributed discrete-event supervisors on a switched network," Discrete Event Systems, 2008. WODES 2008. 9th International Workshop on, pp. 419-424, May 2008. 1.2

[26] R. Song, "Symbolic synthesis and verification of hierarchical interface-based supervisory control," Master's thesis, Dept. of Computing and Software, McMaster University, 2006. 1.1, 1.2, 6, 6.1.2, 6.2, 6.3, 6.3.2, 6.3.2, 6.4.1

[27] A. Vahidi, B. Lennartson, and M. Fabian, "Efficient analysis of large discreteevent systems with binary decision diagrams," in Proc. of the 44th IEEE Conf. Decision Contr. and and 2005 European Contr. Conf., (Seville, Spain), pp. 27512756, 2005. 1.2

[28] K. C. Wong and W. M. Wonham, "Hierarchical control of timed discrete-event systems," Discrete Event Dynamic Systems, vol. 6, pp. Pages 275 - 306, July 1996. 2.3 .2 
[29] W. M. Wonham, Supervisory Control of Discrete-Event Systems. Department of Electrical Engineering, University of Toronto, 2005. 1, 2.

[30] W. M. Wonham and P. J. Ramadge, "On the supermal controllable sublanguage of a given language," SIAM J. Control Optim., vol. 25, no. 3, pp. 637-659, 1987. 1

[31] S. Xu and R. Kumar, "Asynchronous implementation of synchronous discrete event control," Discrete Event Systems, 2008. WODES 2008. 9th International Workshop on, pp. 181-186, May 2008. [1.2

[32] Z. Zhang and W. M. Wonham, "STCT: an efficient algorithm for supervisory control design," in Proc. of SCODES 2001, (INRIA, Paris), pp. 82-93, July 2001. 1.2

[33] M. Zhou and F. DiCesare, Petri Net Synthesis for Discrete Event Control of Manufacturing Systems. Kluwer Academic Publishers, 1993. 1.2 


\section{Appendix A}

\section{SD Software Program}

\section{A.1 FMS Example Input Files}

The input files below are all for the FMS example that we presented in Section 7.2 .

\section{A.1.1 FMS Plants}

Listing A.1: Con2

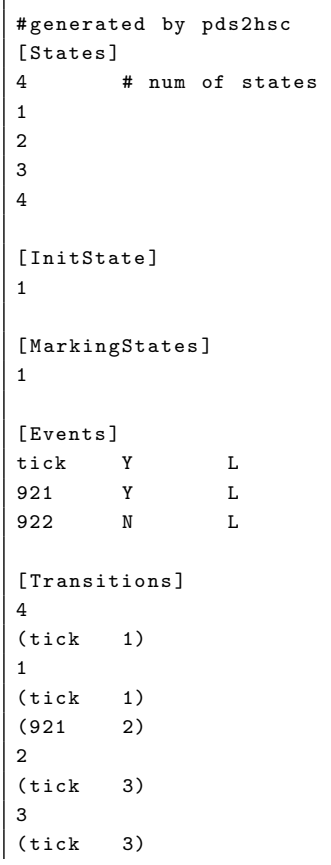


Listing A.2: Robot

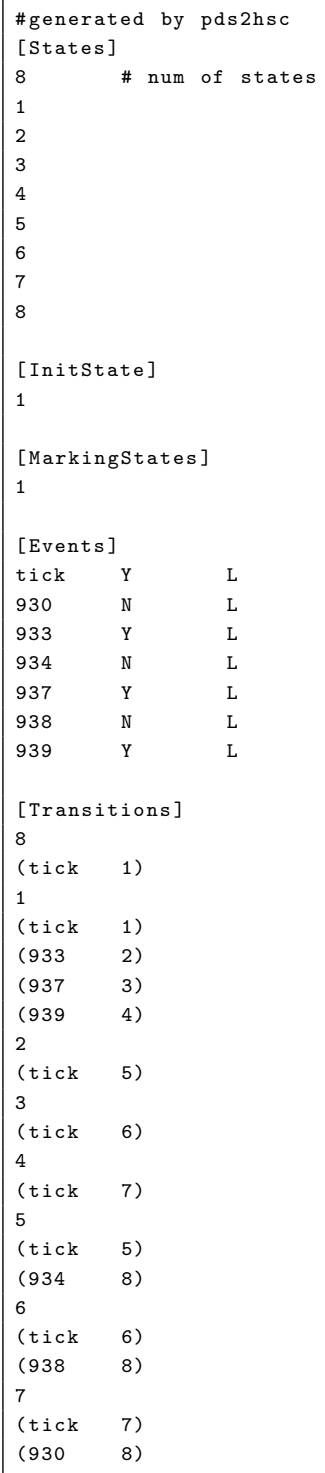

Listing A.3: Lathe

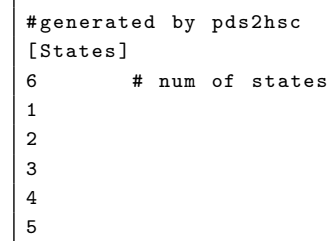




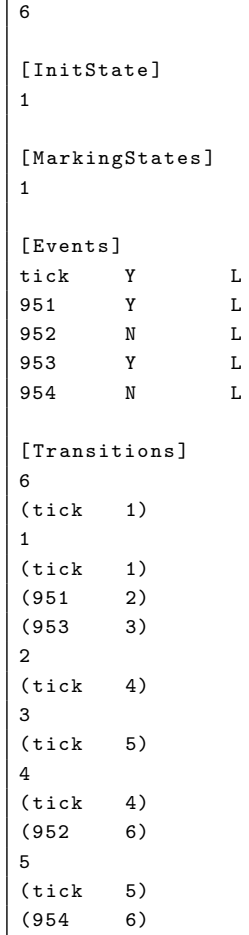

Listing A.4: AM

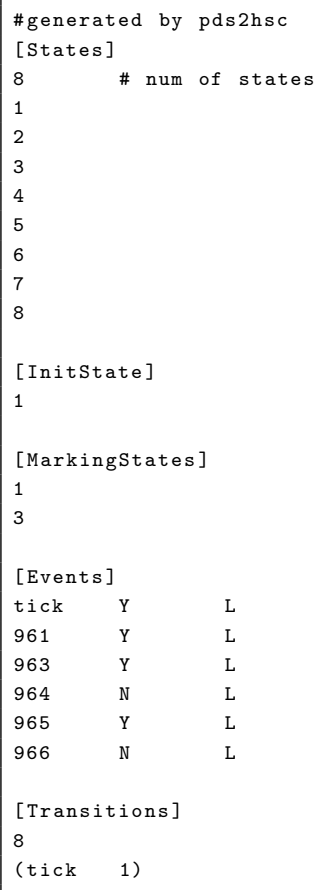




$\mid \begin{array}{ll}\text { (tick } & 1) \\ (961 & 2) \\ 2 & \\ (\text { tick } & 3) \\ 3 & \\ (\text { tick } & 3) \\ (963 & 4) \\ (965 & 5) \\ 4 & \\ (\text { tick } & 6) \\ 5 & \\ (\text { tick } & 7) \\ 6 & \\ (\text { tick } & 6) \\ (964 & 8) \\ 7 & \\ (\text { tick } & 7) \\ (966 & 8)\end{array}$

Listing A.5: Con3

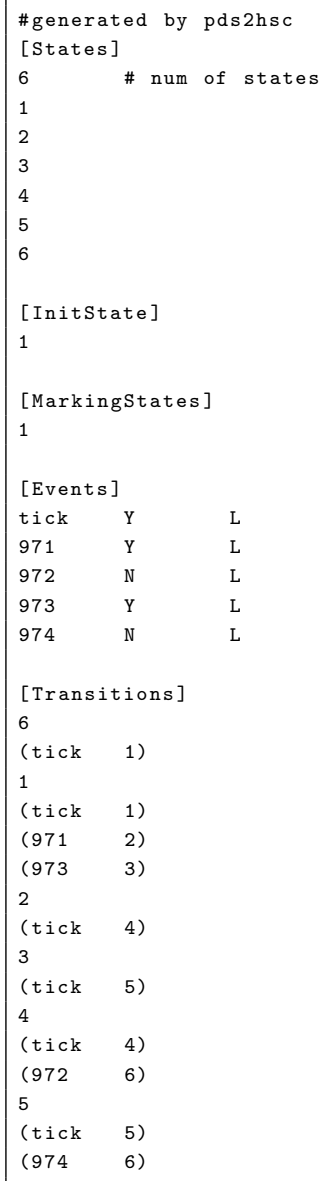

Listing A.6: PM

\#generated by pds2hsc 


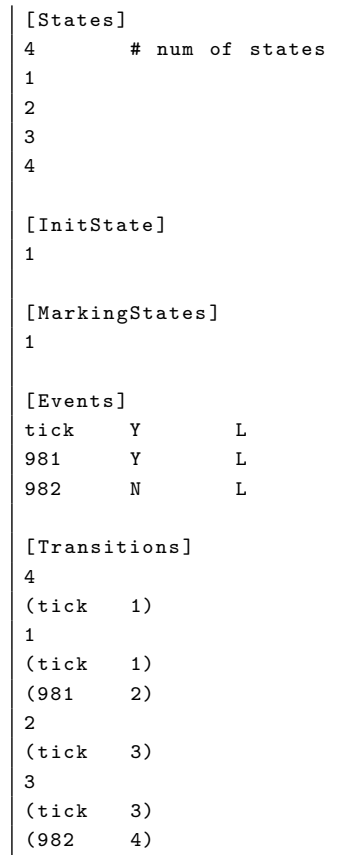

\section{A.1.2 Helper Plants}

Listing A.7: AddNo921

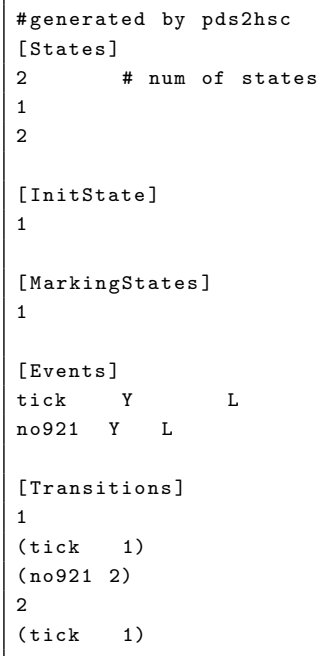

Listing A.8: AddNo963 


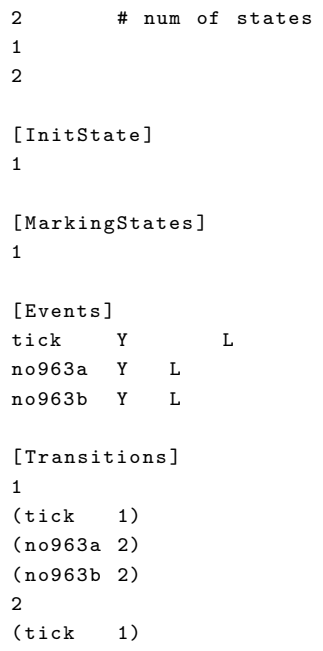

Listing A.9: AddNo965

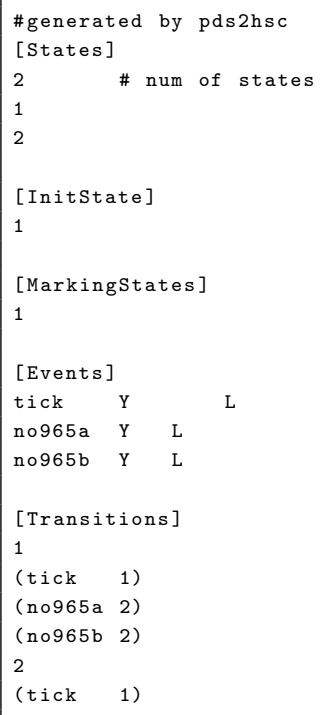

Listing A.10: SystDownNup

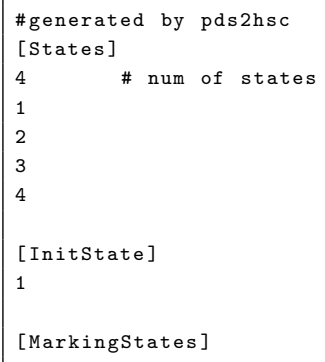




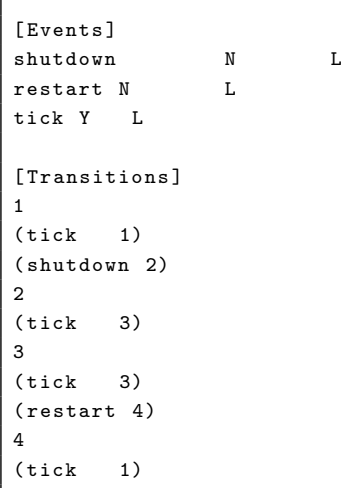

\section{A.1.3 Buffer Supervisors}

Listing A.11: B2

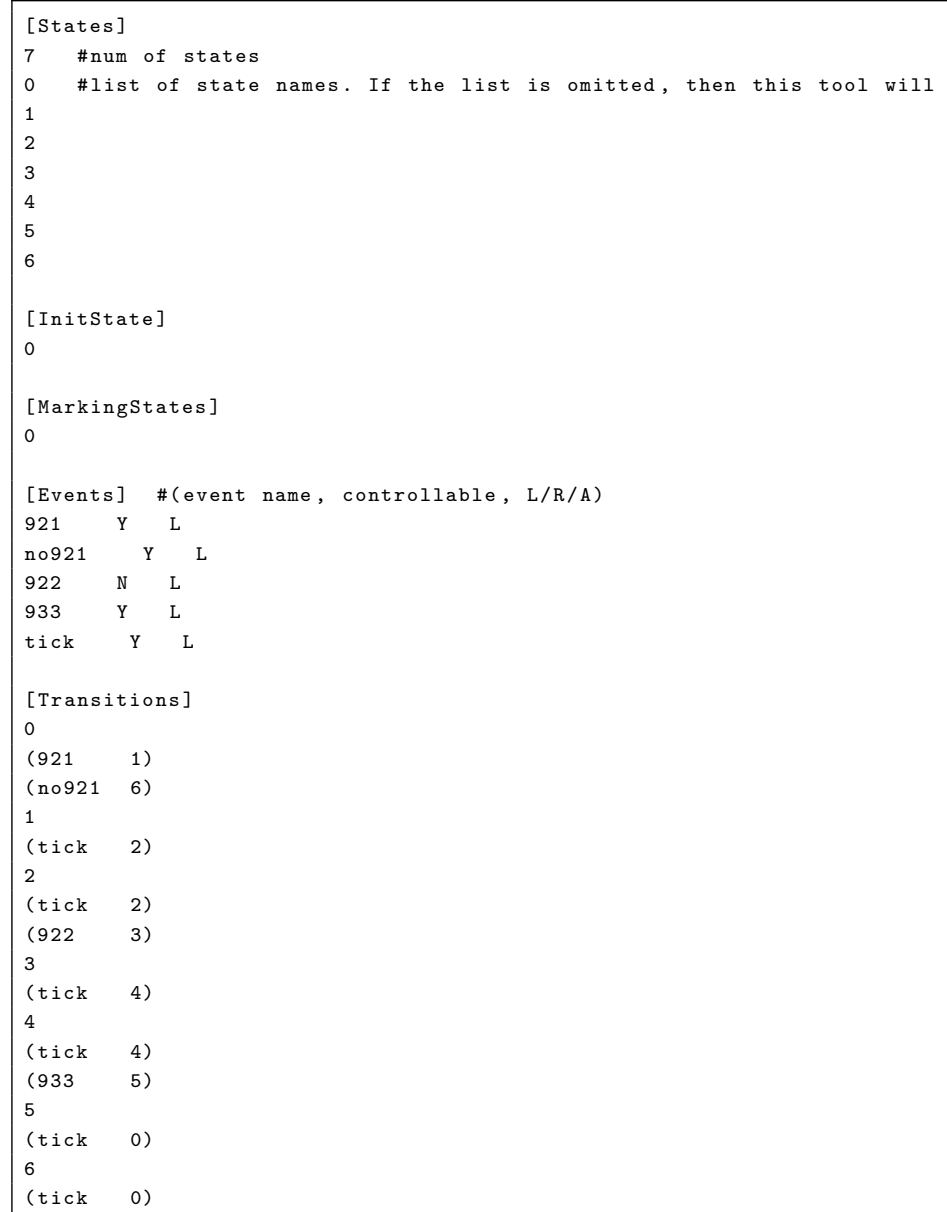




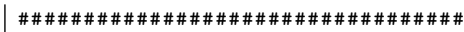

Listing A.12: B4

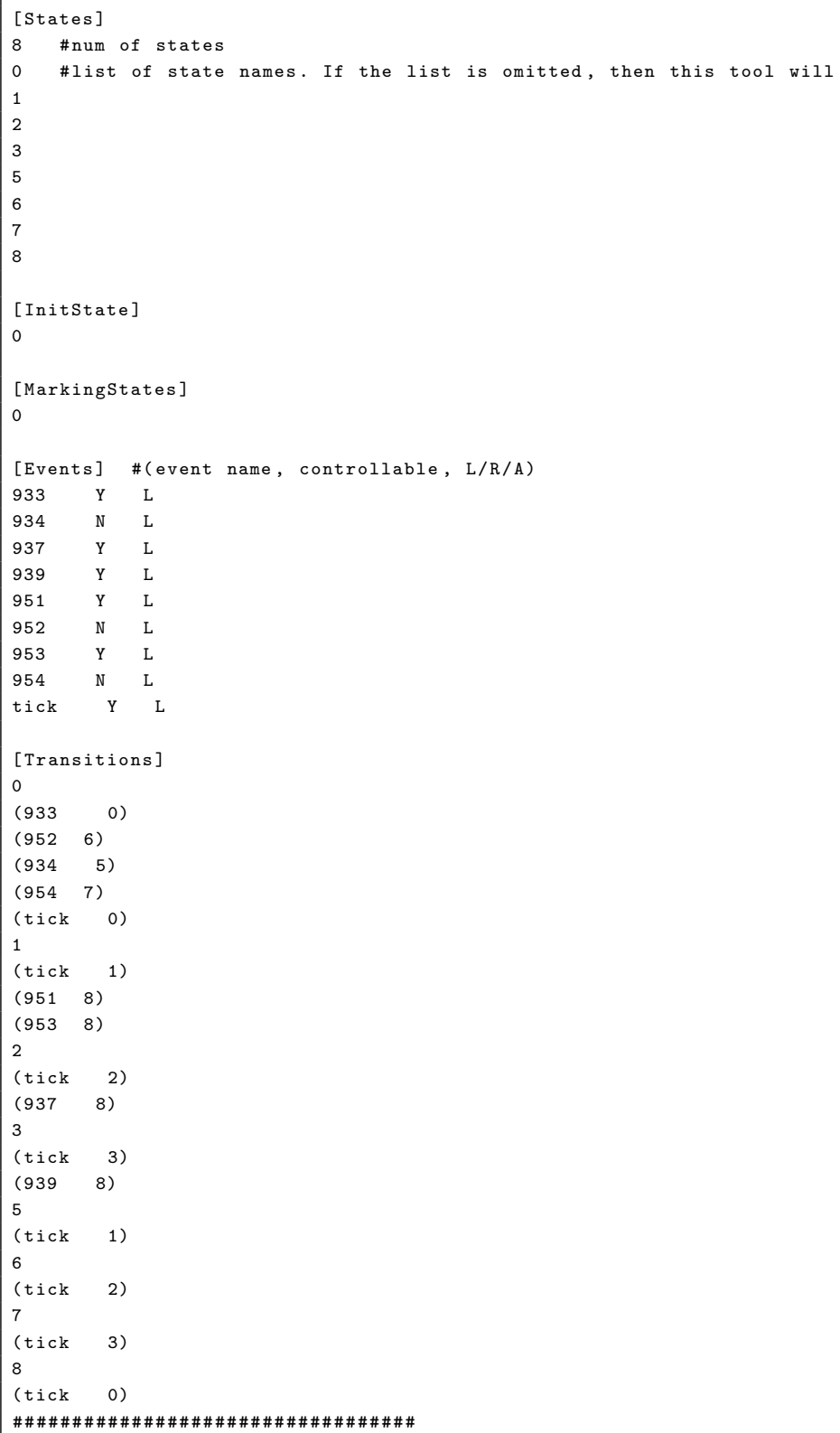

Listing A.13: B6

[States]

4 \#num of states

0 \#list of state names. If the list is omitted, then this tool will 


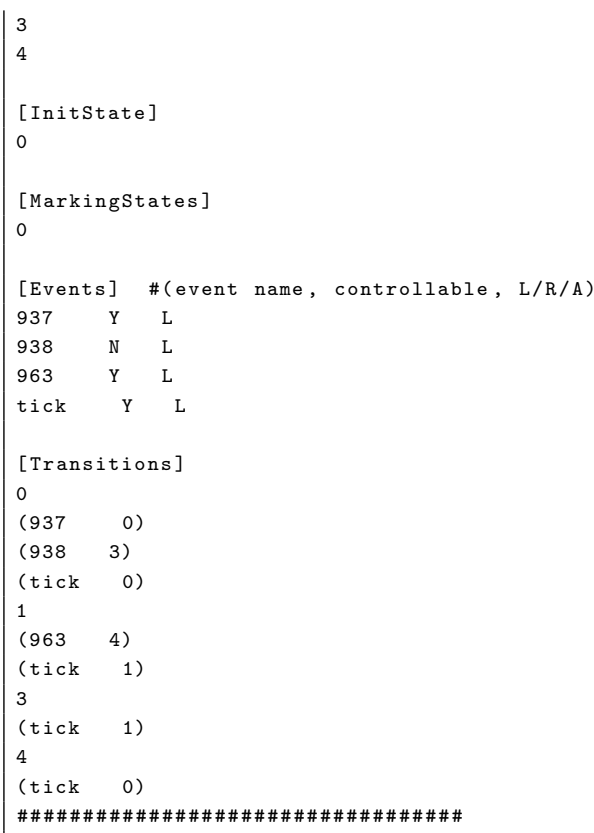

Listing A.14: B7

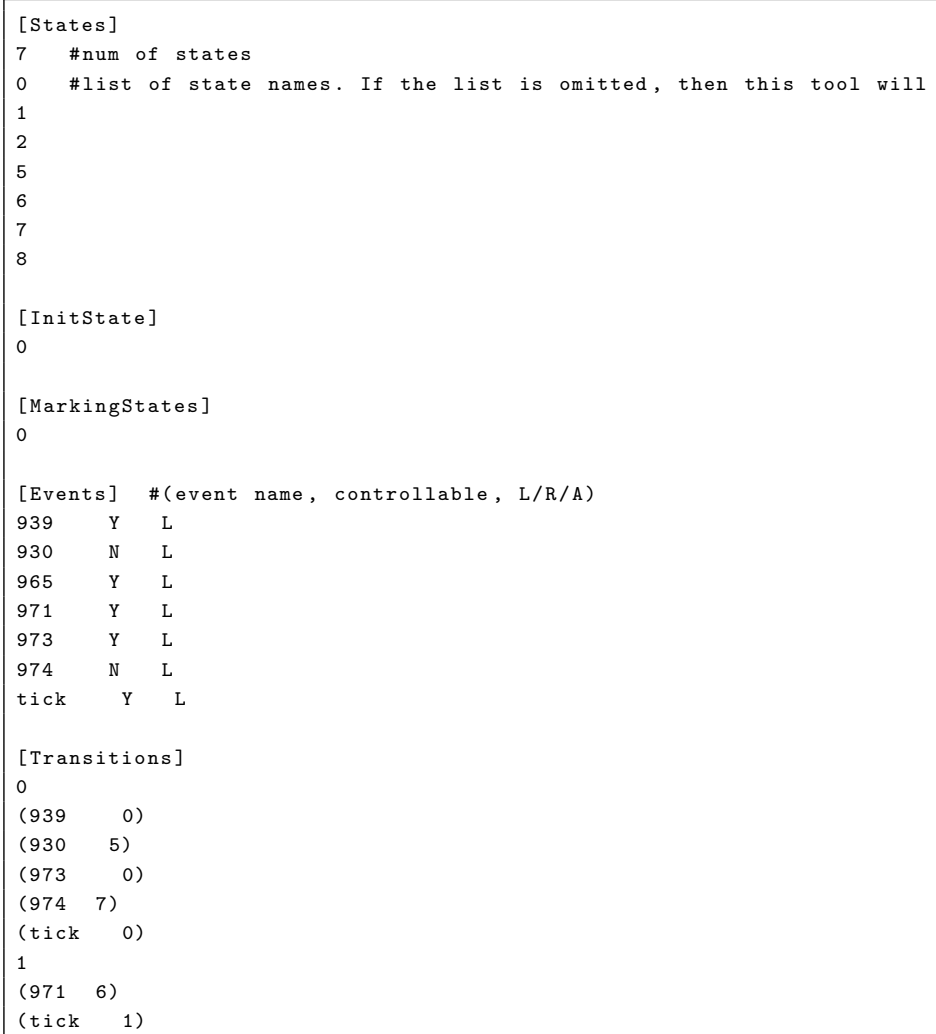




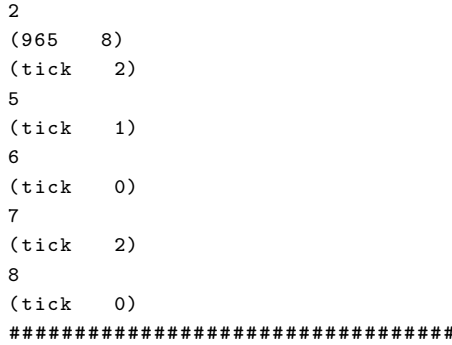

Listing A.15: B8

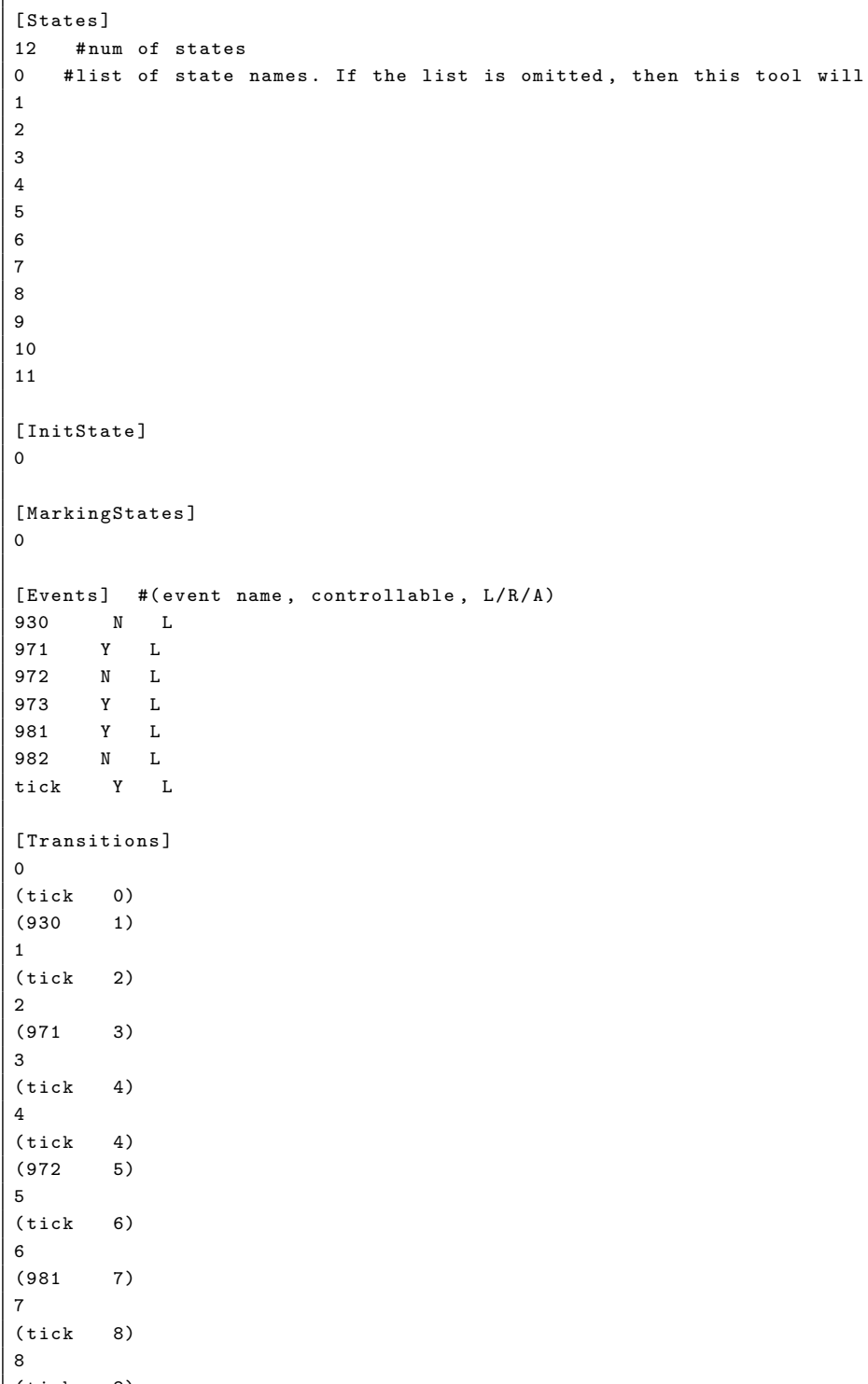




\section{A.1.4 Additional Supervisors}

Listing A.16: AMChooser

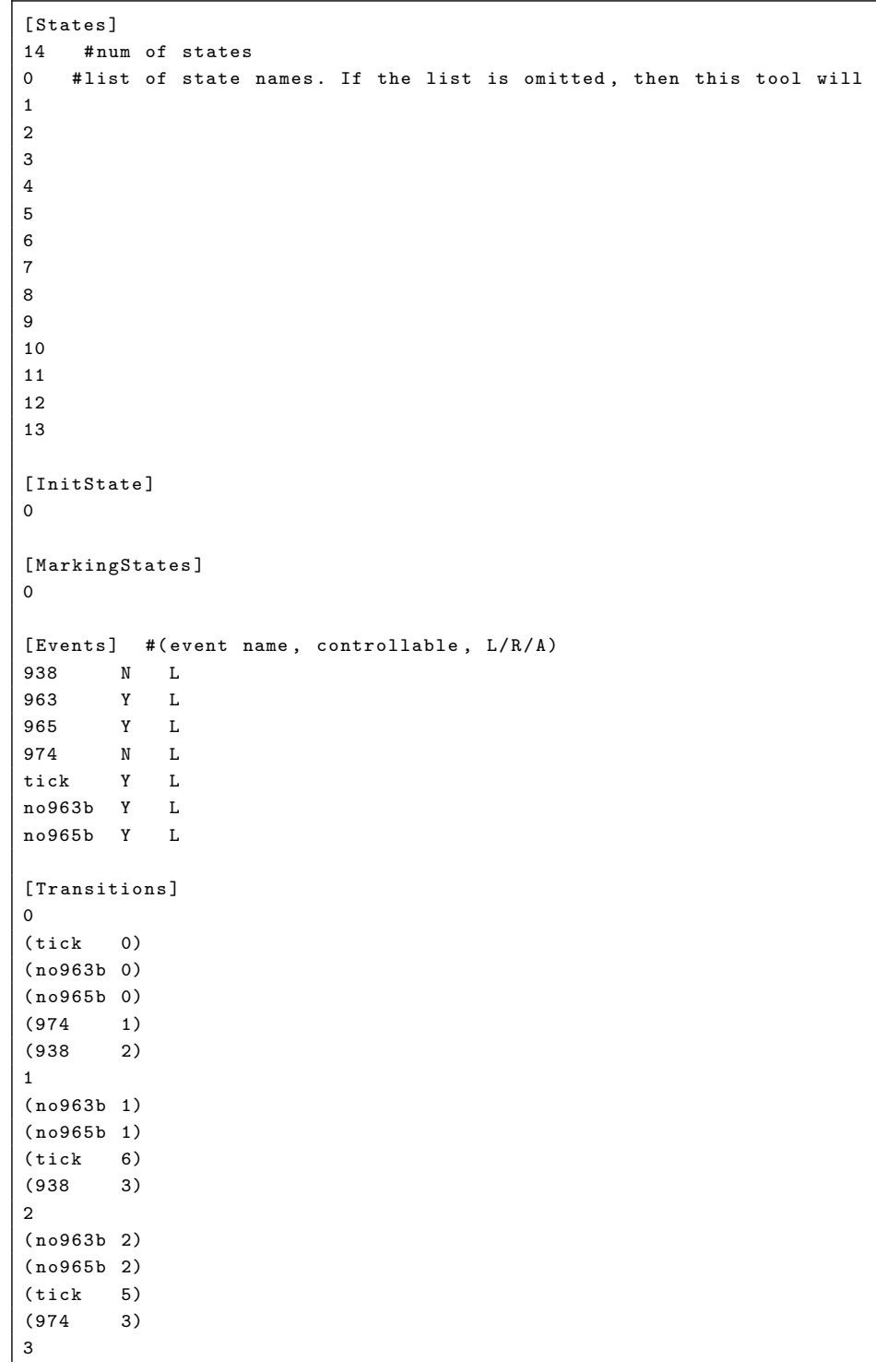




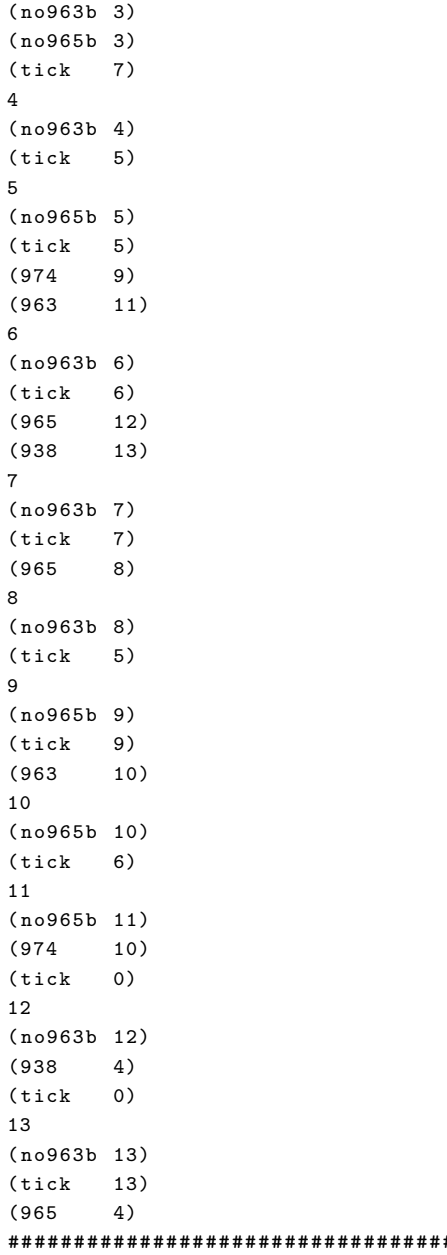

Listing A.17: B4Path

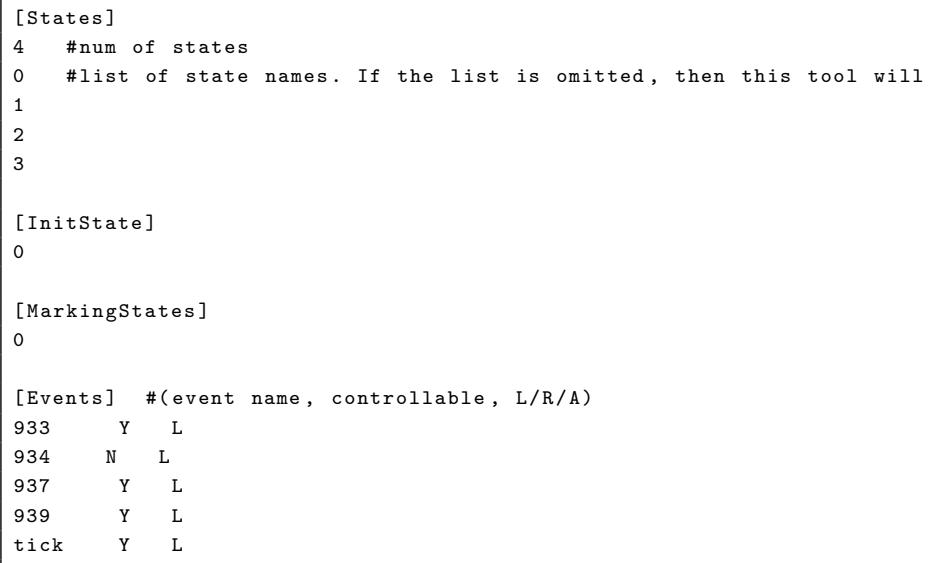




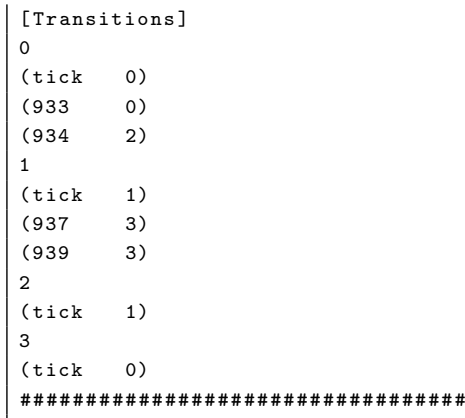

Listing A.18: Force961

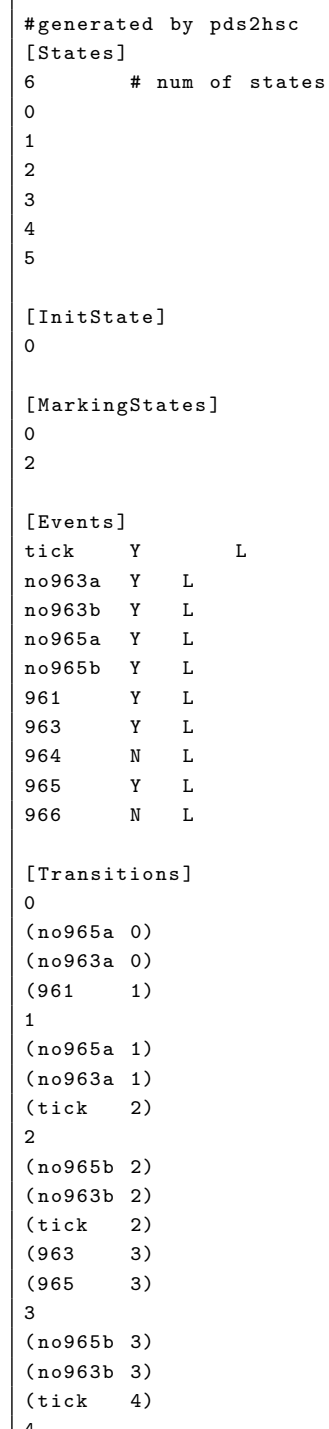




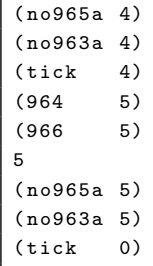

Listing A.19: Force963

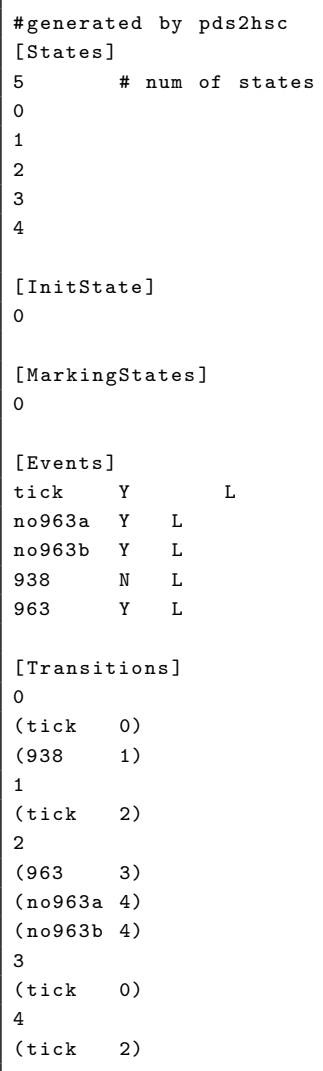

Listing A.20: Force965

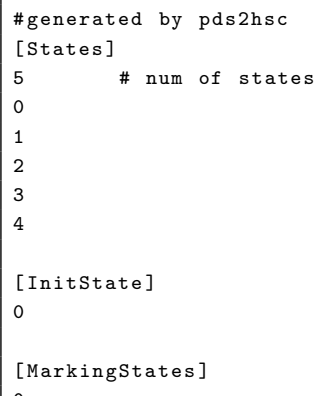




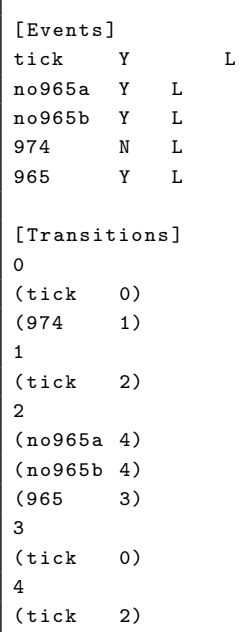

Listing A.21: LathePick

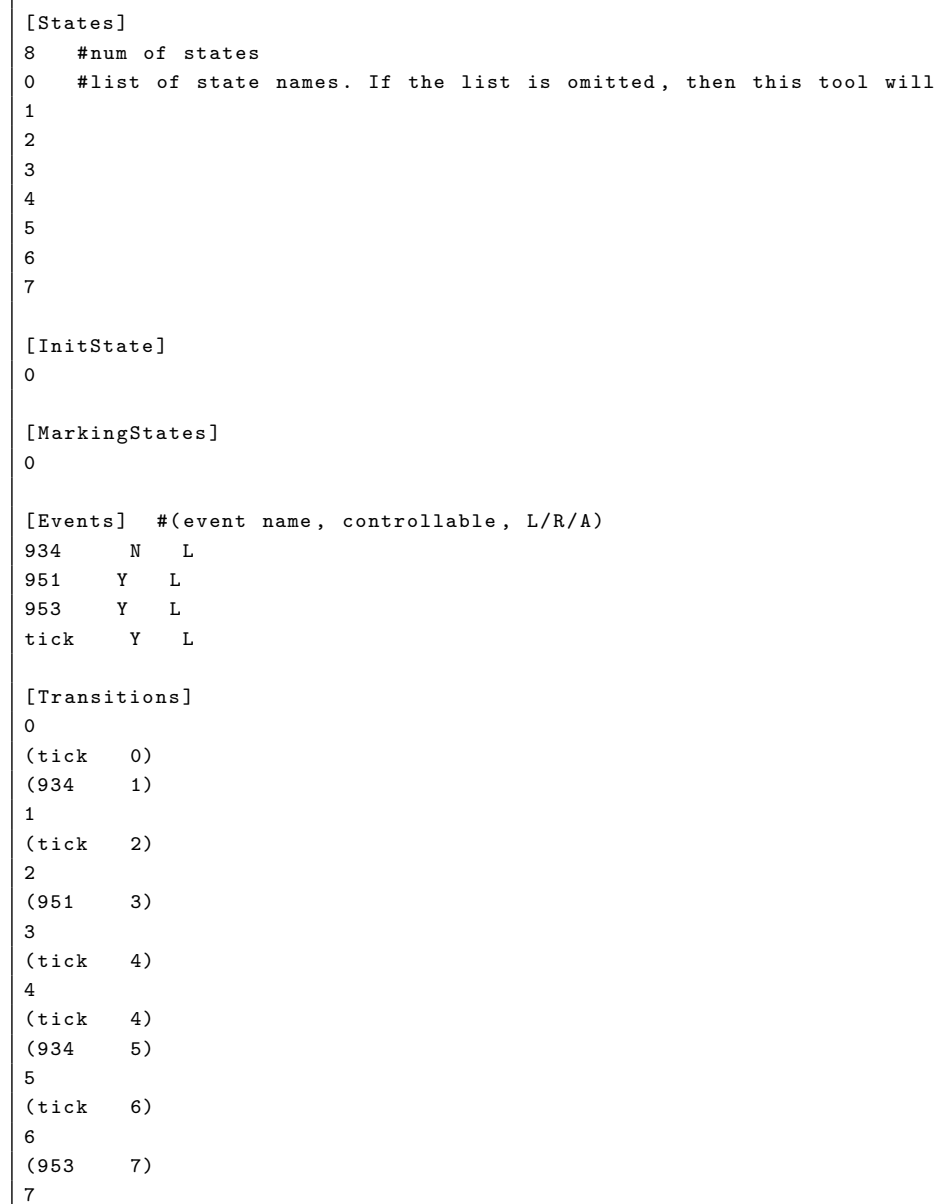


$\left(\begin{array}{ll}\text { tick } & 0\end{array}\right)$

Listing A.22: TakeB2

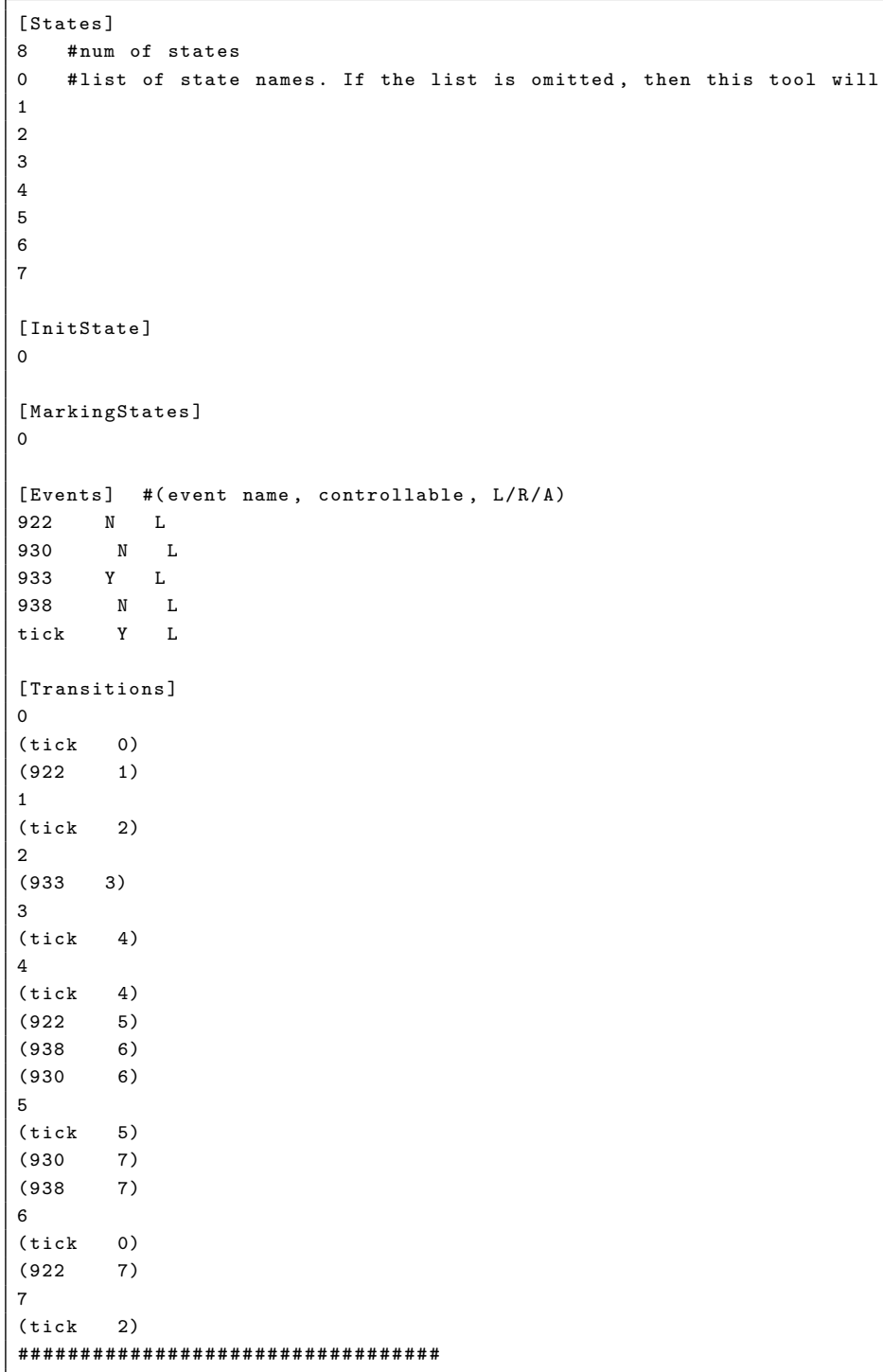

Listing A.23: TakeB4PutB6

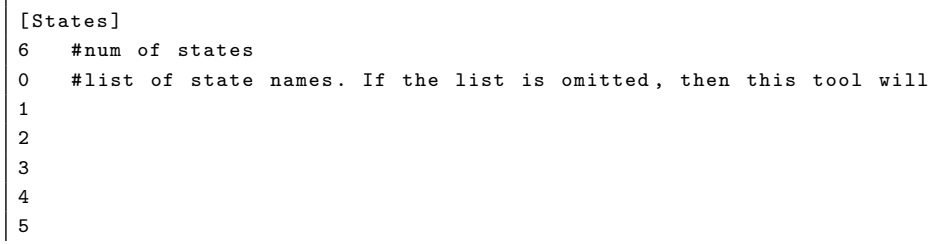




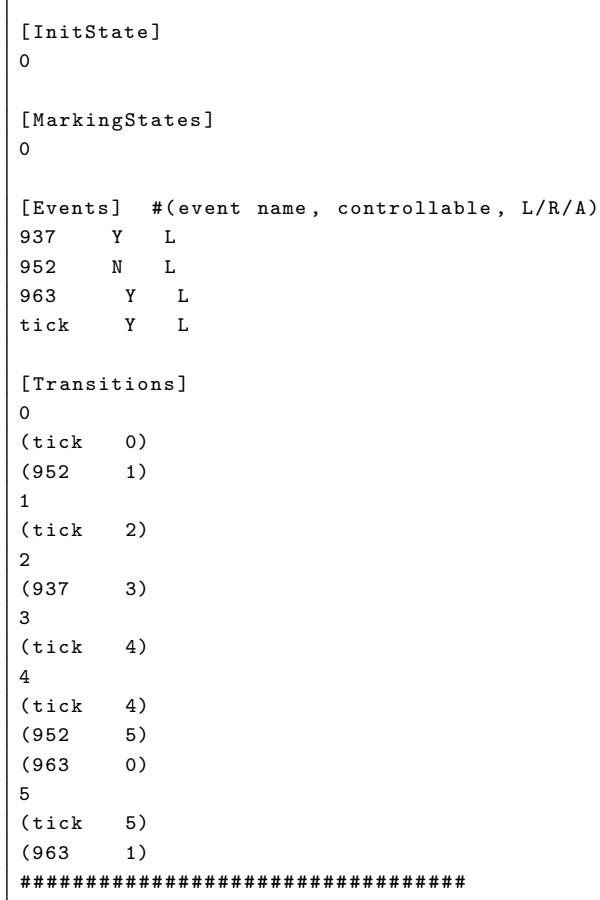

Listing A.24: TakeB4PutB7

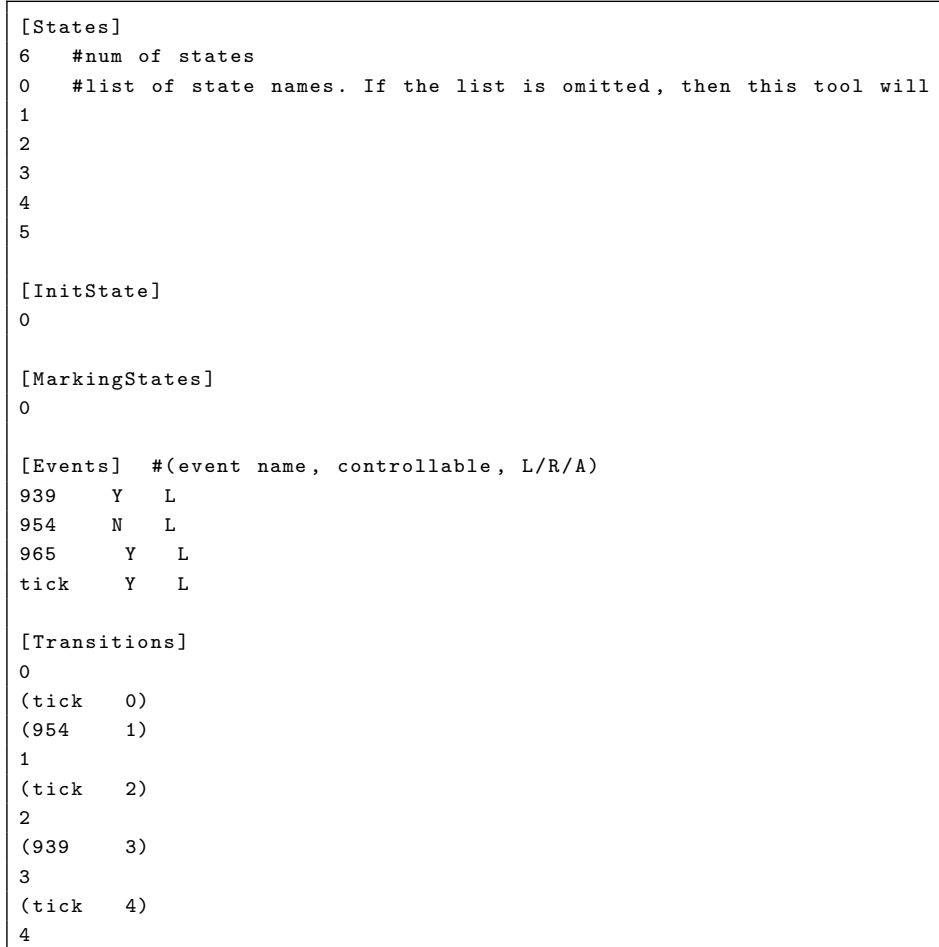


Listing A.25: handleSystDown

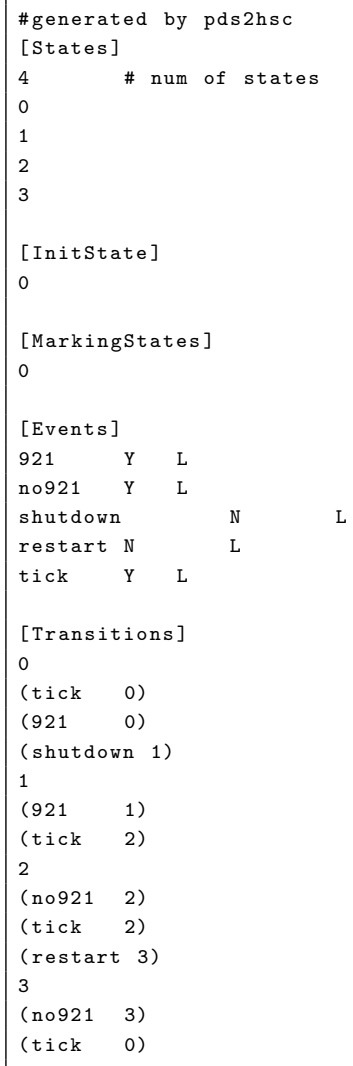

\section{A.2 Source code}

The source code files are to be compiled using gcc 4.3.2 or higher version. Optimization -0 is suggested for better performance. 


\section{A.2.1 Main}

\section{main.cpp}

001\{

002 bool bPrjLoaded = false;

003 char $\mathrm{ch}=$ ' $\backslash 0$ ';

004 char prjfile[MAX_PATH];

005 string errmsg;

006 prjfile [0] $={ }^{\prime} \backslash 0^{\prime}$;

007 int iret $=0$;

008 char prjoutputfile[MAX_PATH];

009

010 char savepath [MAX_PATH];

011 savepath [0] = ' $\backslash 0$ ';

012 HISC_SUPERINFO superinfo;

013

014 HISC_TRACETYPE tracetype;

015 int computetime $=0$;

016

017 while (ch != 'q' \&\& ch != 'Q')

018\{

021

022

023

\{

024

025

026

027

028

029

030

031

$$
\text { case ' } q \text { ': }
$$

case ' $Q$ ':

032

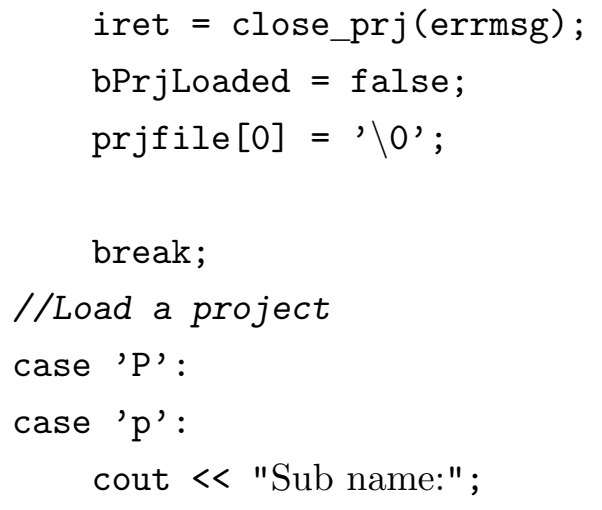


033

034

035

036

037

038

039

039

040

041

041

042

043

044

045

046

047

048

049

050

051

052

053

054

055

056

057

058

059

060

061

062

063

064

065 cin.getline(prjfile, MAX_PATH);

iret = load_prj(prjfile, errmsg);

if $($ iret $<0)$

\{

if (iret > -10000) //error bPrjLoaded = false;

else $\mathrm{bPrjLoaded}=$ true $; / /$ waring

\}

else

$\mathrm{bPrjLoaded}=$ true;

break;

//close the current project

case ' $\mathrm{C}$ ':

case ' $\mathrm{C}$ ':

iret $=$ close_prj (errmsg);

bPrjLoaded = false;

prjfile $[0]=' \backslash 0^{\prime}$;

break;

//File the current project

case 'f':

case 'F':

cout $<<$ "file name:" ;

cin.getline(prjoutputfile, MAX_PATH);

iret $=$ print_prj(prjoutputfile, errmsg);

break;

//Low Level verification

case 'l':

case 'L':

cout << "Show the blocking type(may take long time)(Y/N)?";

tracetype $=$ (HISC_TRACETYPE) getchoice_tracetype () ;

char verbosechoices [3] $=\left\{{ }^{\prime} 0^{\prime},{ }^{\prime}{ }^{\prime},{ }^{\prime}{ }^{\prime}\right.$ ' $\}$;

cout << "Verbose level (0 - disable, 1 - brief, 2 - full)?";

const char choice[2] $=\{$ getkeystroke(verbosechoices, 


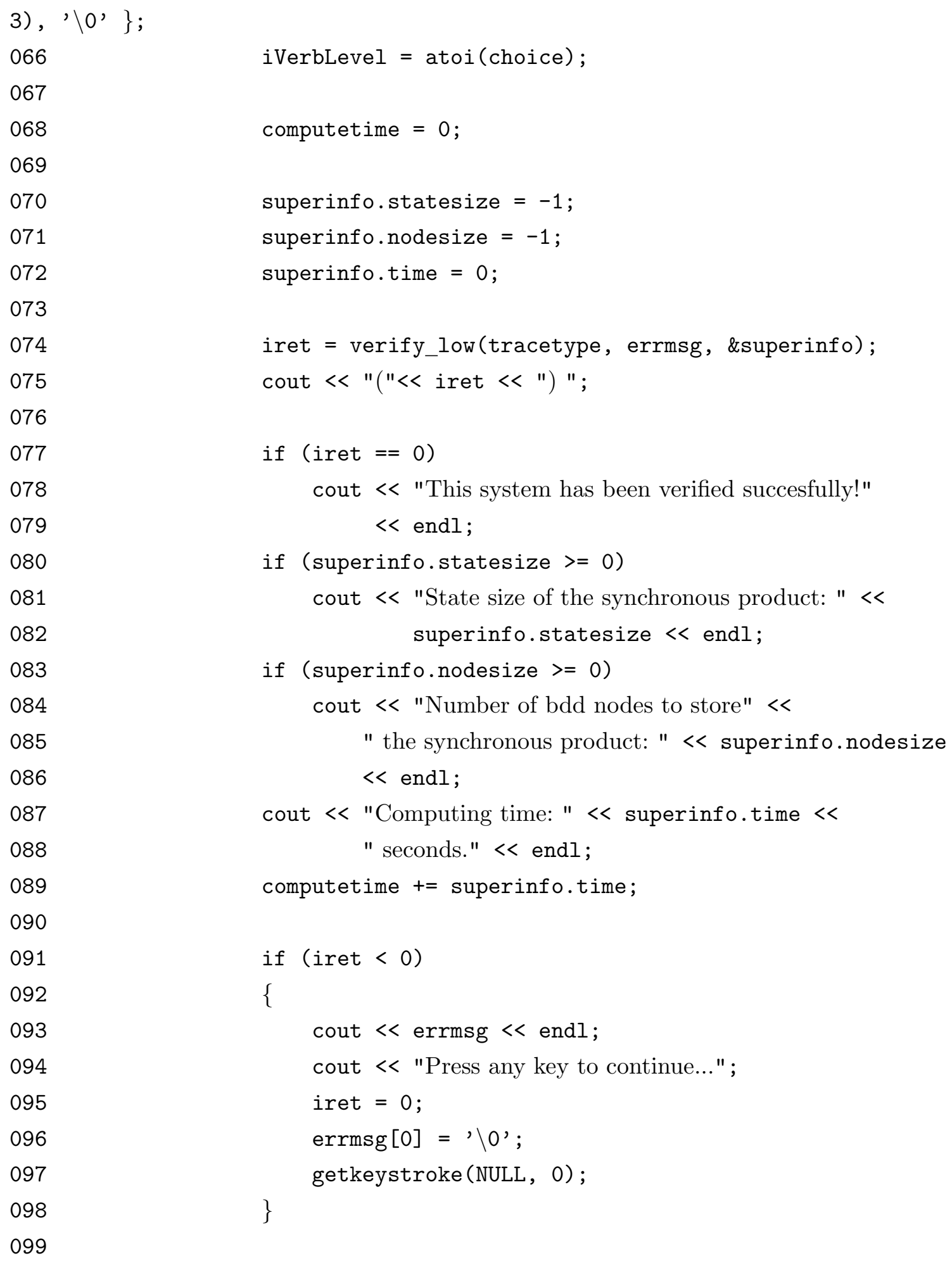


100 cout $<<$ "Total computing time:" < computetime $<$ "

seconds."

101 $<$ endl;

102

103

104

105

106

107

108

109

110

111

112

113

114

115

close_hisc();

116

117 return 0 ;

$118\}$

119

120 int getchoice(bool bPrjLoaded, const char $*$ prjfile)

121\{

122 char allowed_choice[50];

123 int numofchoice $=0$;

124

125 cout $<$ endl $<$ endl $<$ endl $<$ endl $<$ endl;

126 cout $<<" * * * * * * * * * * * * * * * * * * * * * * * * * * * * * * * * * * * * * * * * * * * * * * * * * 4 "<$ endl;

127 cout $<$ " Bdd-based HISC Synthesis and Verification Tool " $<$ endl;

128 cout $<" * * * * * * * * * * * * * * * * * * * * * * * * * * * * * * * * * * * * * * * * * * * * * * * * * * "<$ endl;

129 if (! bPrjLoaded)

130\{

131

132

133

allowed_choice [0] = 'p';

allowed_choice [1] = ' $\mathrm{P}$ ';

allowed_choice[2] = ' $q$ '; 


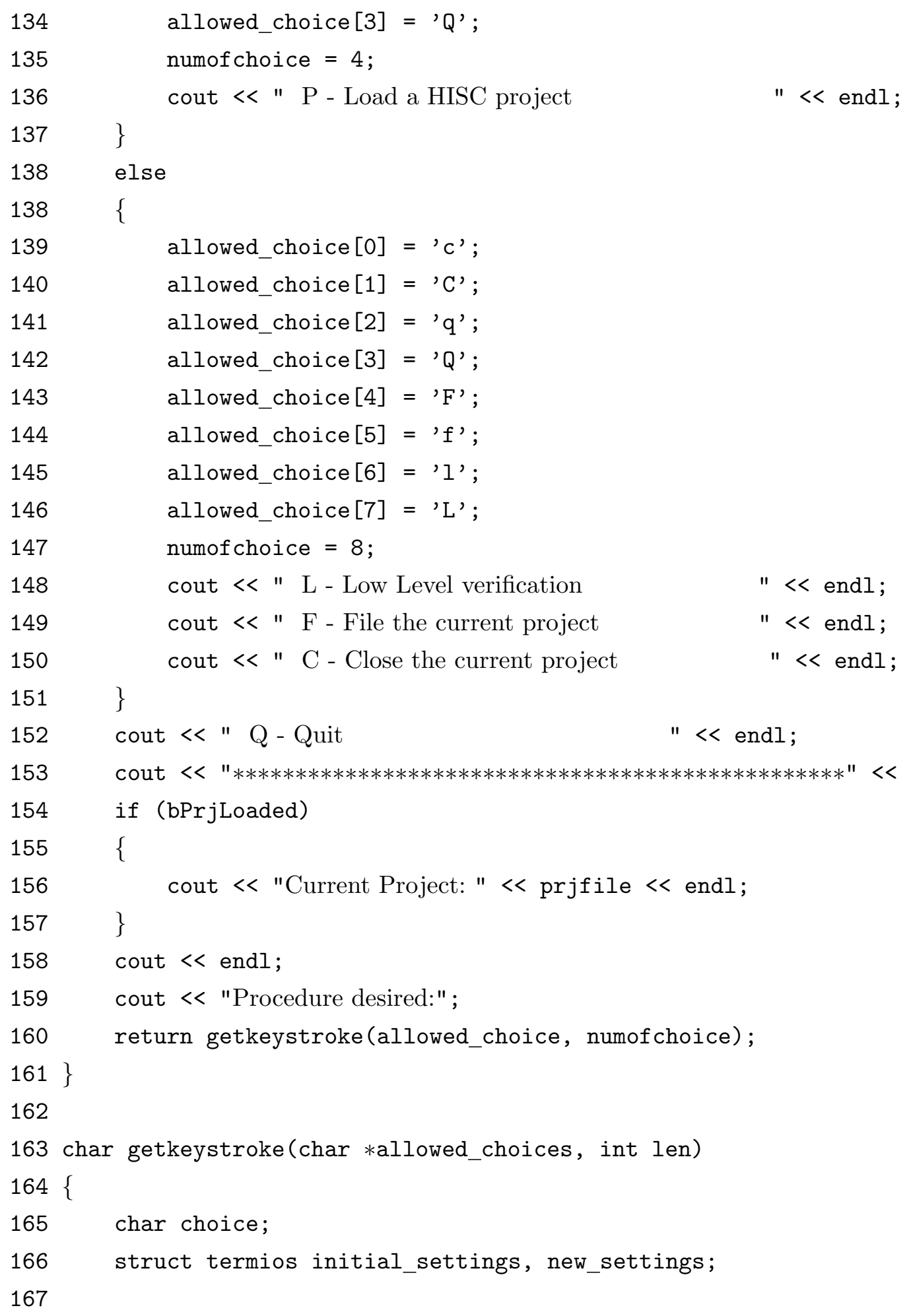




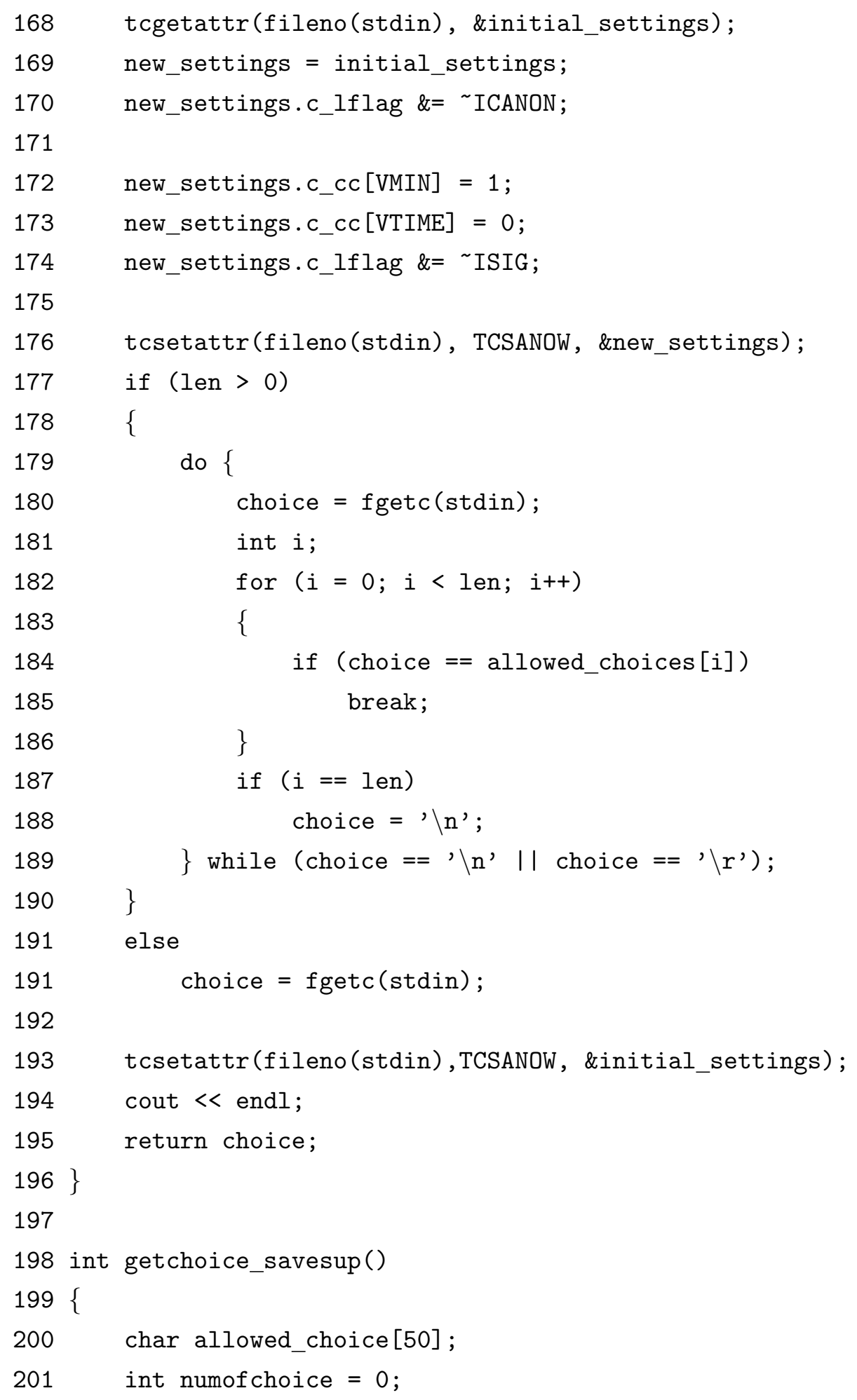




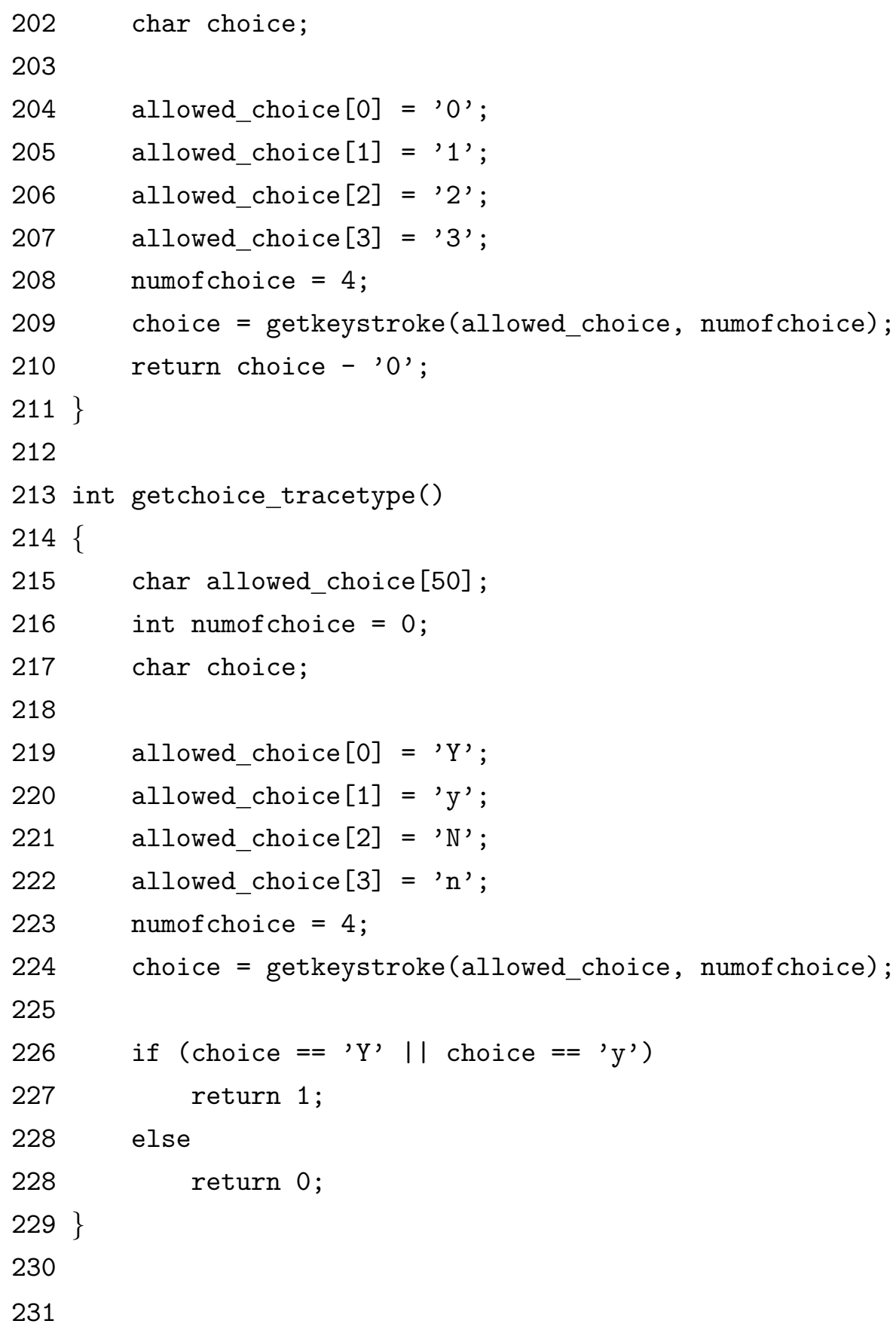




\section{A.2.2 Global Functions, Typedefs, Variables, Preprocessors symbols}

type.h

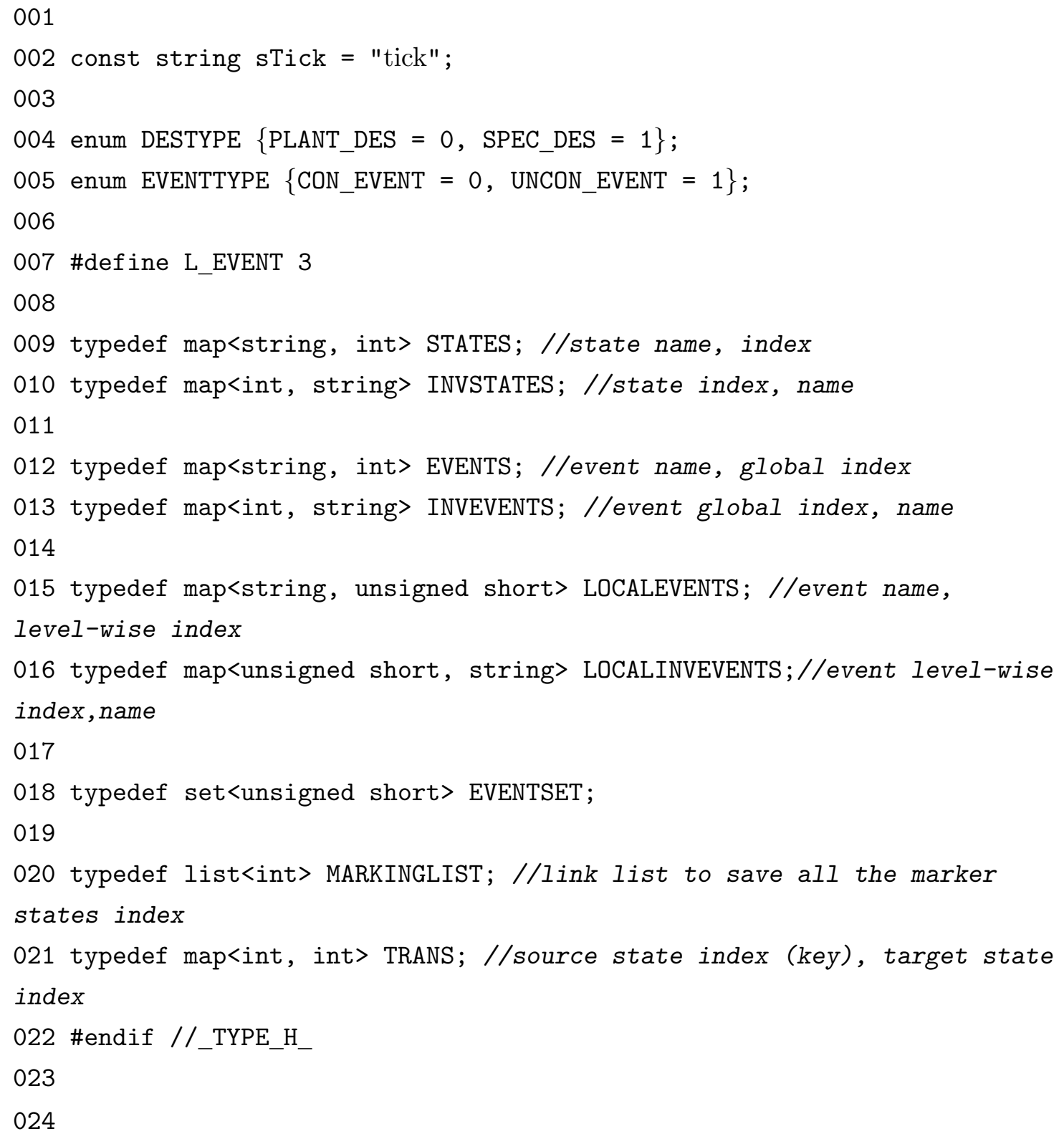




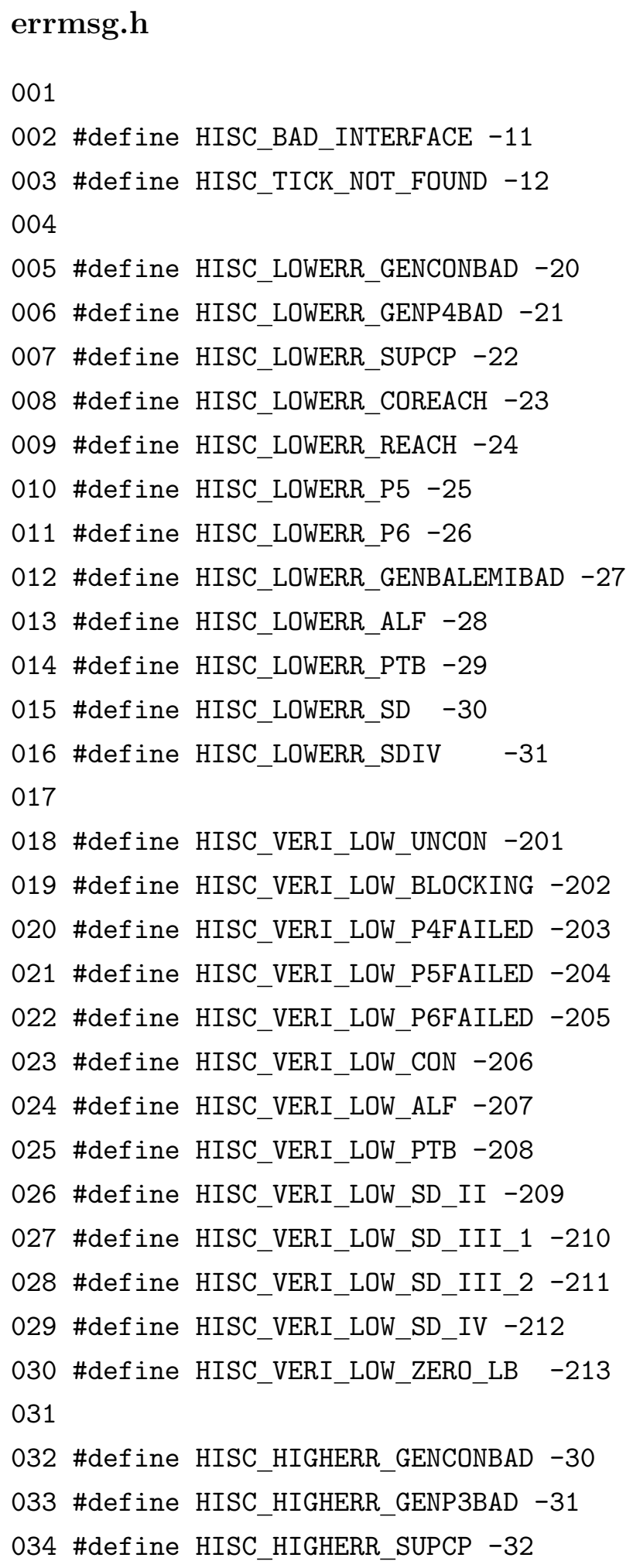




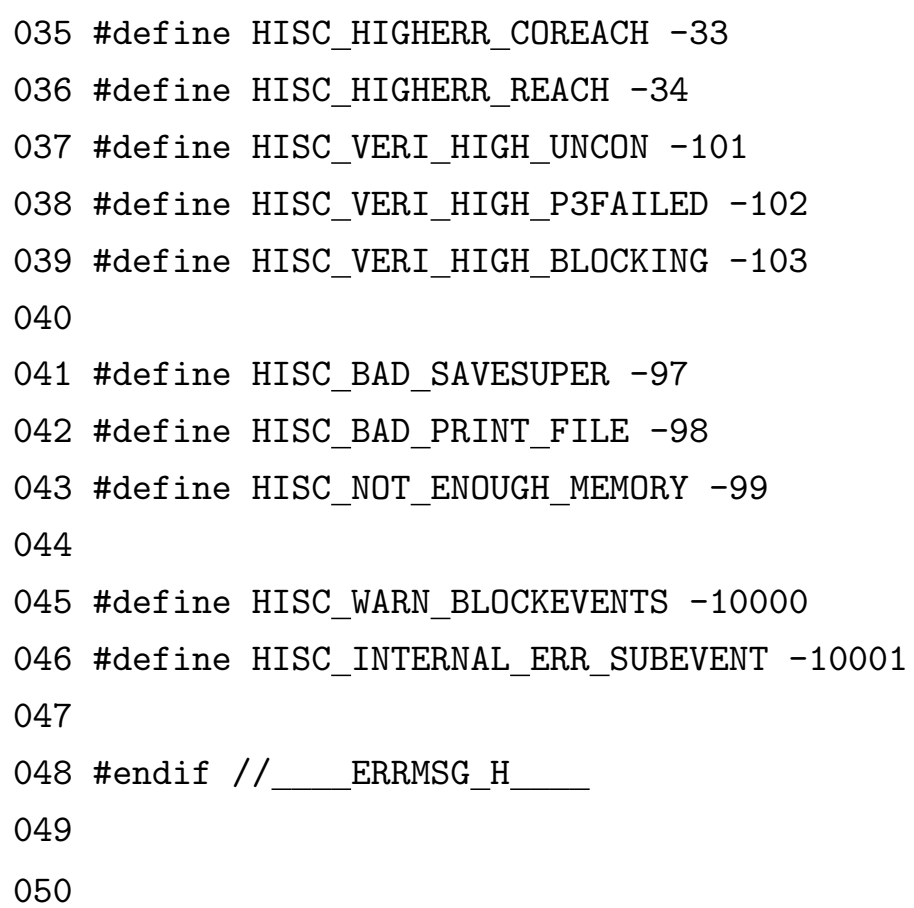

\section{pubfunc.h}

001 extern string str_upper(const string \&str);

002 extern string str_lower(const string \&str);

003 extern string str_itos(int ilnt);

004 extern string str_ltos(long long lLong);

005

006 extern string str_nocomment(const string \& str);

007 extern int scp_err(const string \& sErr, const int iErrCode);

008

009 extern string GetNameFromFile(const string \& vsFile);

010

011 extern int IsInteger(const string \&str);

012 extern int CompareInt (const void* pa, const void* pb);

013

014 extern void bddPrintStats (const bddStat \&stat);

015 extern void SetBddPairs(bddPair *pPair, const bdd \& bddOld, const bdd \& bddNew); 
016 extern int NumofSharedEvents (const int $*$ pEventsArr_a, const int viNumofEvents_a,

017 const int $*$ pEventsArr_b, const int viNumofEvents_b);

018 extern void my_bdd_gbchandler(int pre, bddGbcStat $*$ s);

019

020 \#endif //__ PUBFUNC_H__

021

022

\section{pubfunc.cpp}

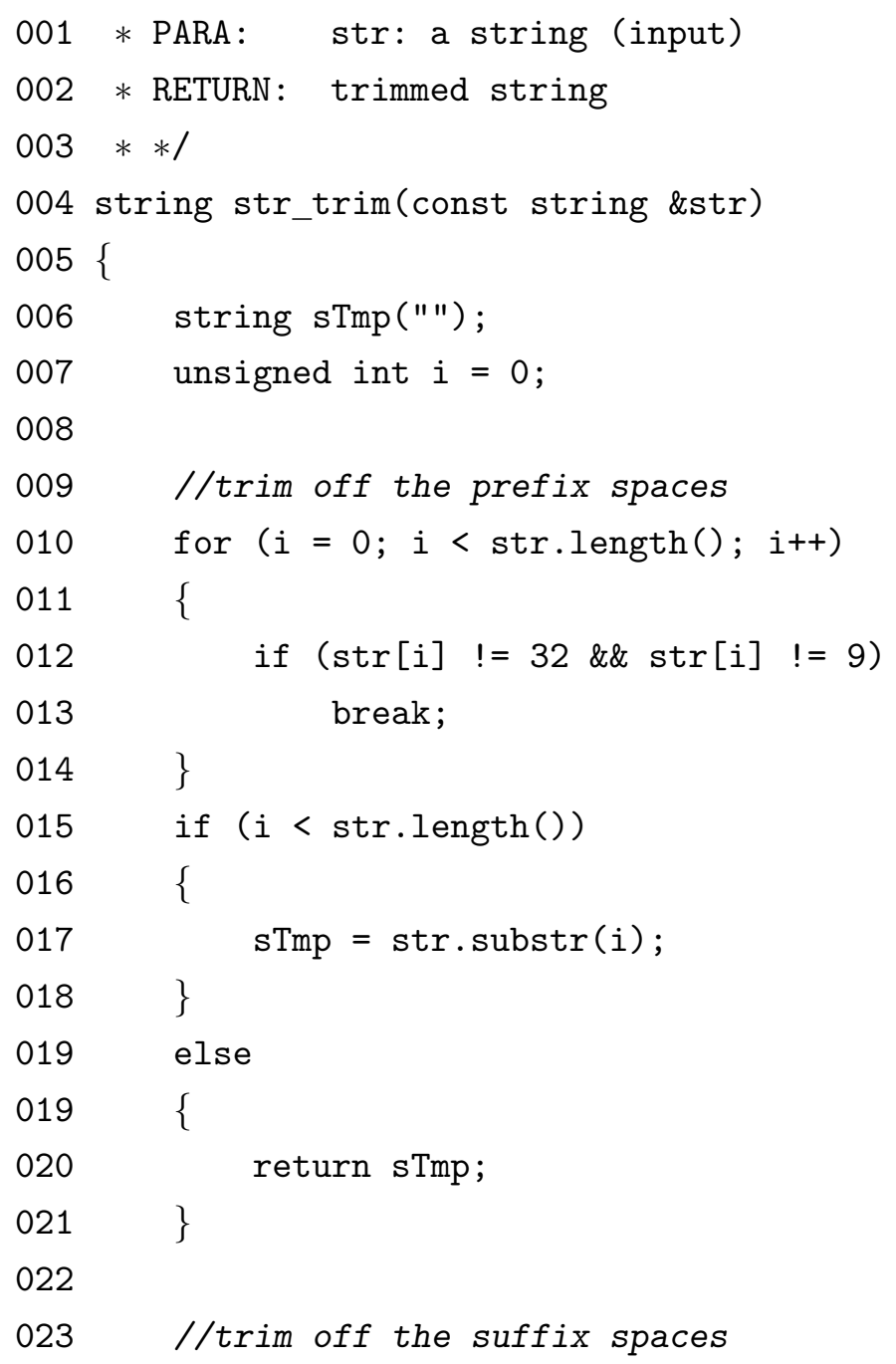




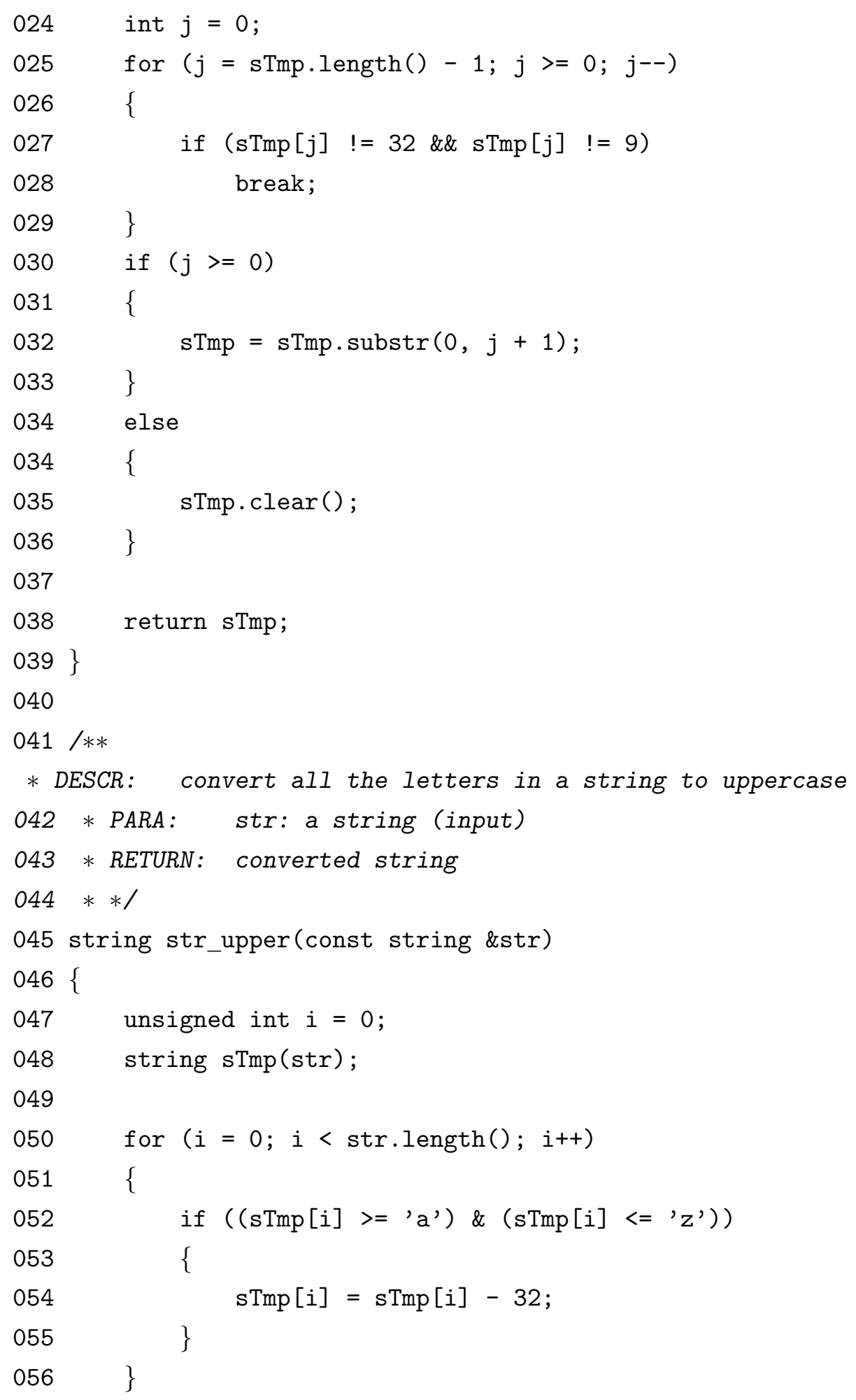




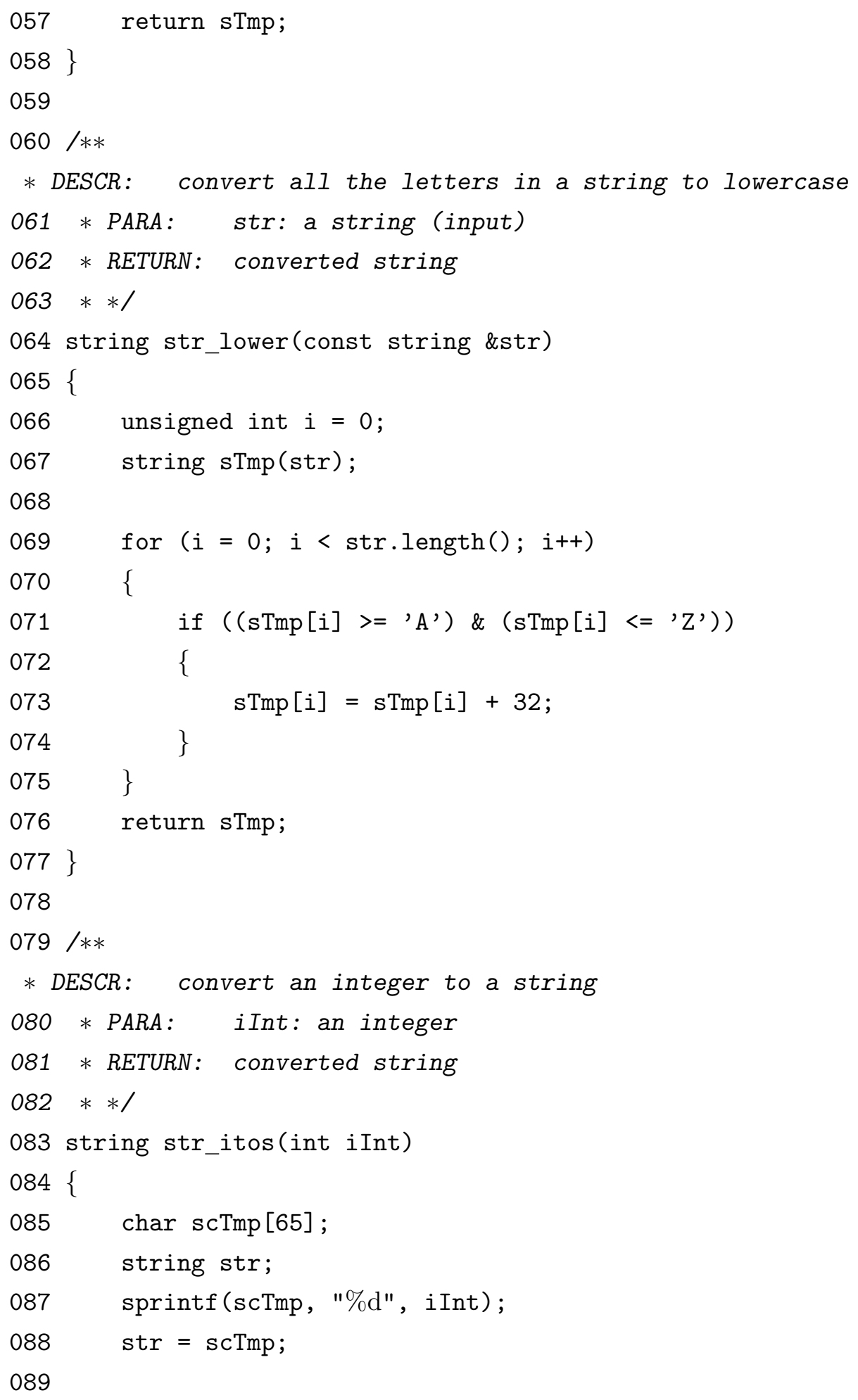




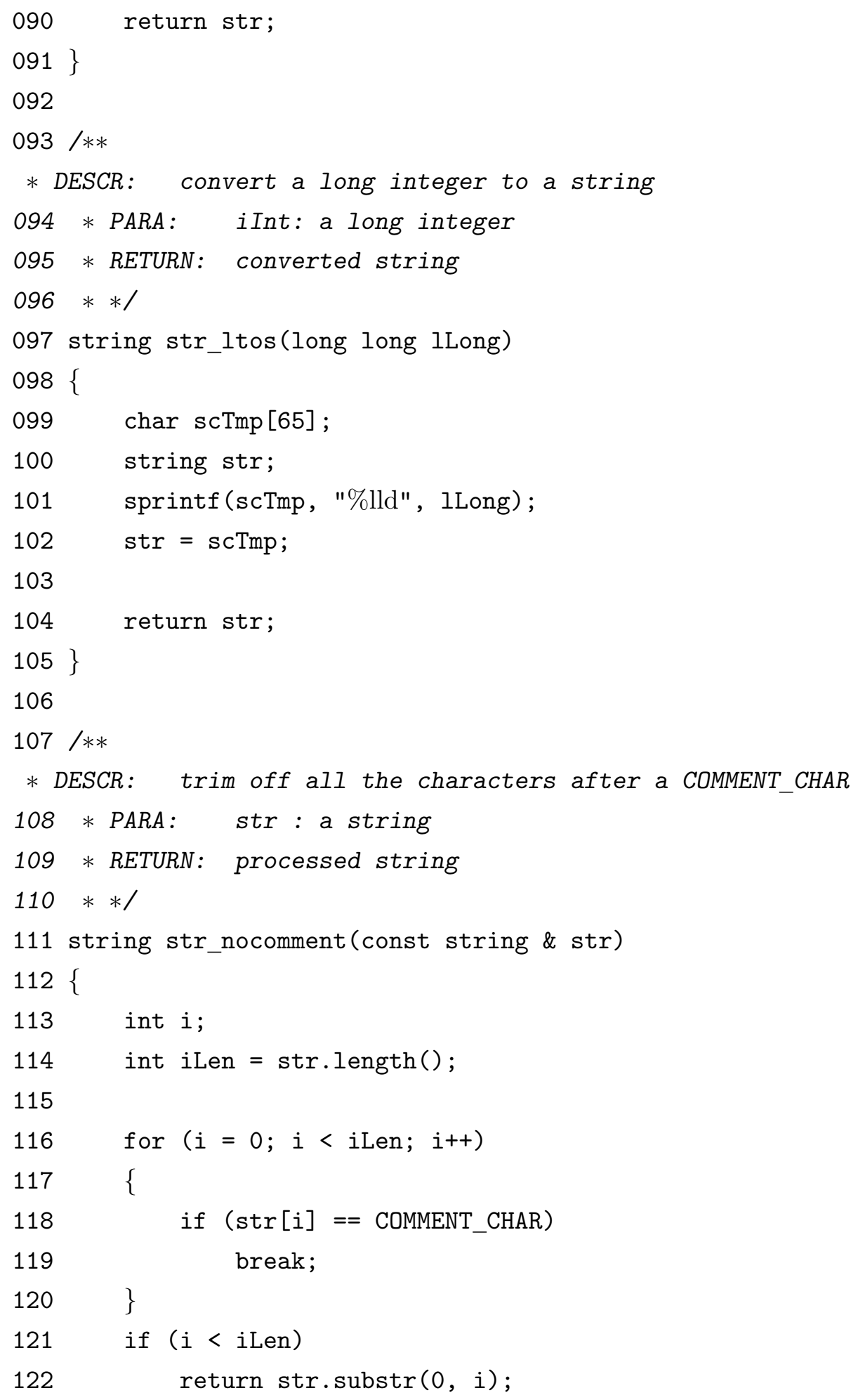




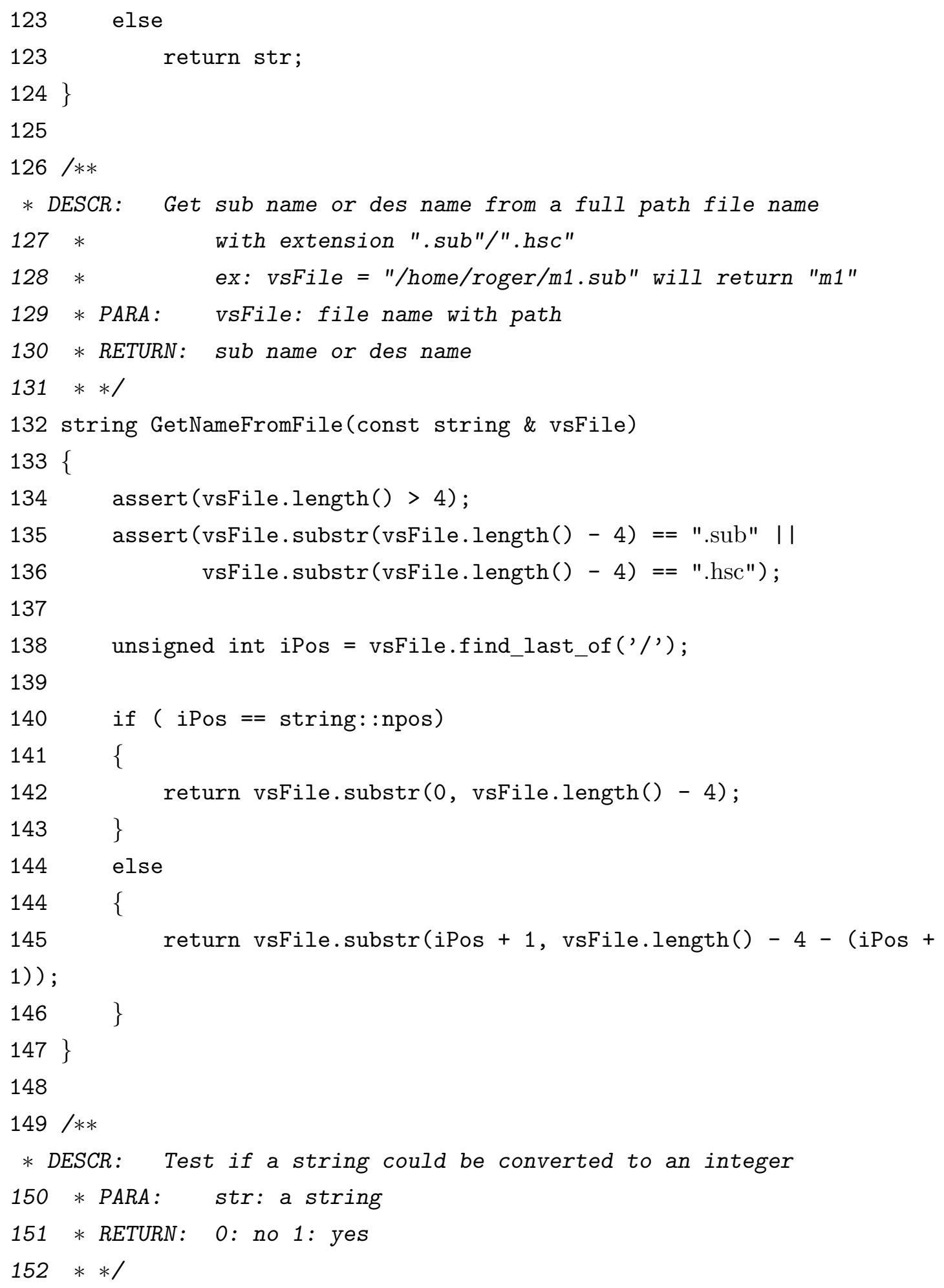


153 int IsInteger (const string \&str)

154\{

155 if (str.length ()$==0)$

156 return 0 ;

157 for (unsigned int $i=0 ; i<\operatorname{str}$. length(); $i++$ )

$158\{$ \{

159

160

if $(\operatorname{str}[i]>=$ '0' \&\& $\operatorname{str}[i]<=$ '9')

161

continue;

else

161 return 0 ;

$162\}$

163

164 return 1 ;

$165\}$

166

$167 / * *$

* DESCR: Compare two integers which are provided by two general

pointers.

$168 * \quad$ qsort, bsearch will use this function

169 * PARA: pa, pb: general pointers pointing to two integers

$170 *$ RETURN: $1: a>b$

$171 * \quad 0: a=b$

$172 * \quad-1: a<b$

$173 * * 1$

174 int CompareInt (const void* pa, const void* pb)

175\{

176 int $\mathrm{a}=*(($ int $*)$ pa $)$;

177 int $\mathrm{b}=*(($ int $*) \mathrm{pb})$;

178

179 if $(\mathrm{a}>\mathrm{b})$

180 return 1 ;

181 else if $(a<b)$

182 return -1 ;

183 else

183

return 0 ; 


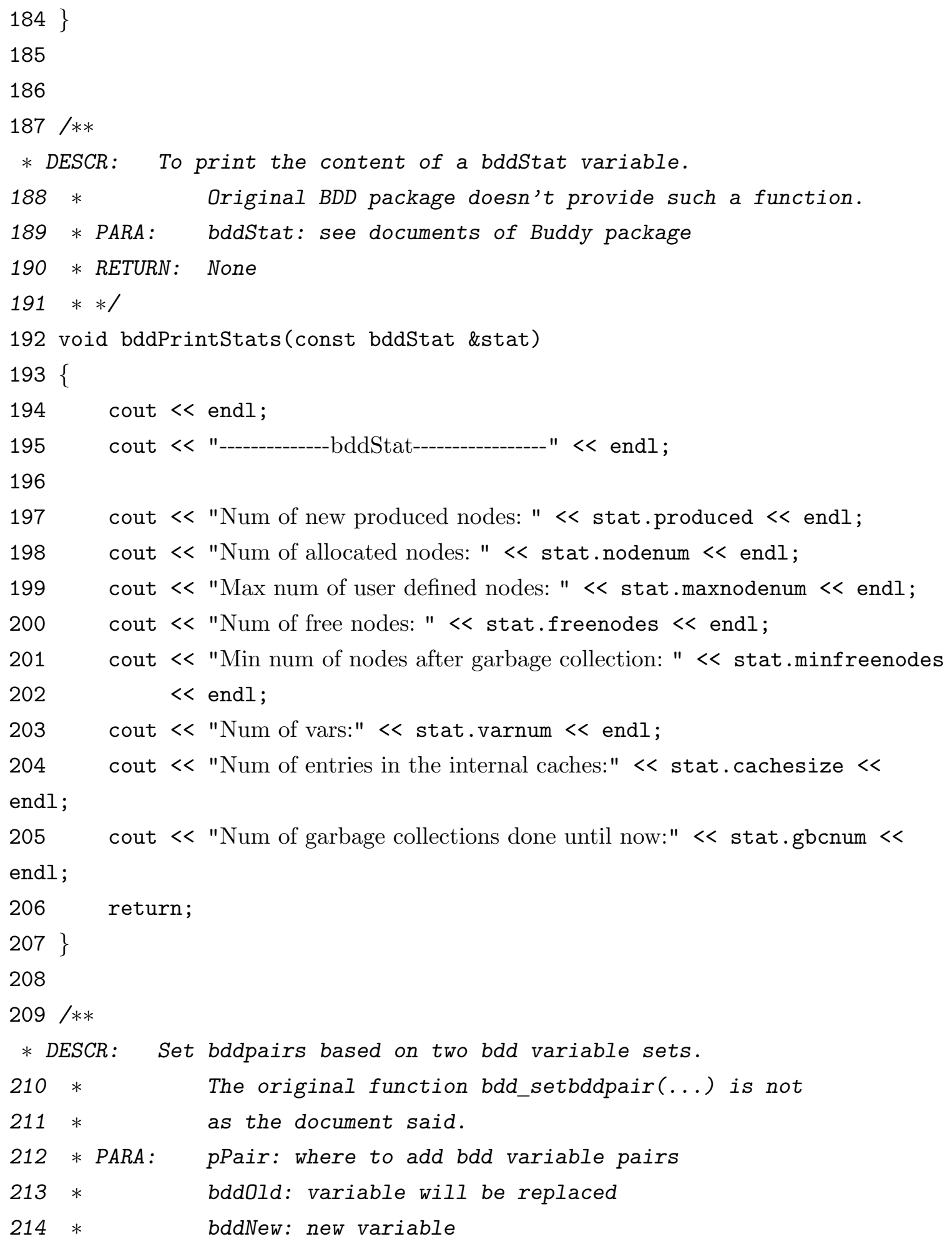




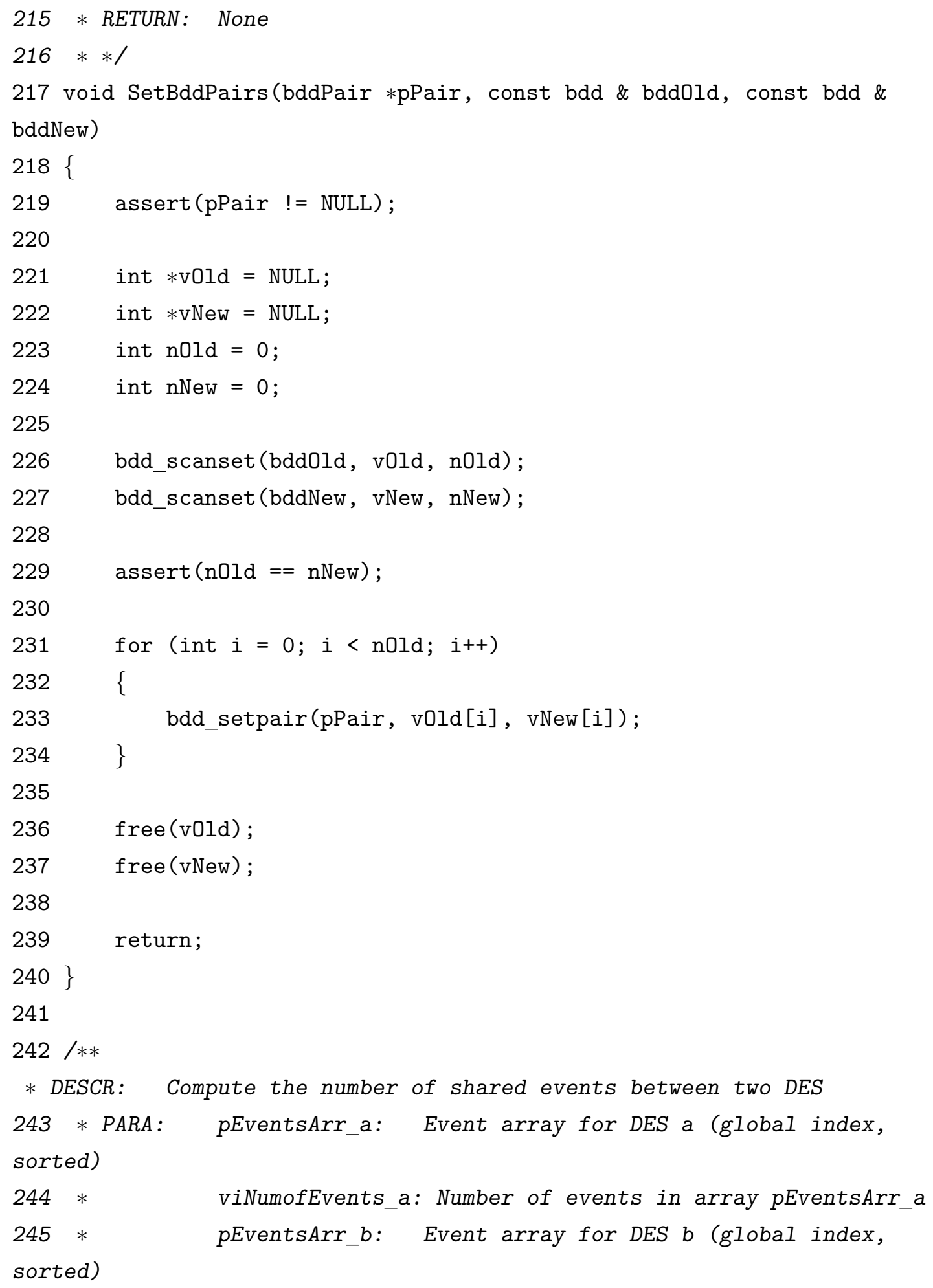




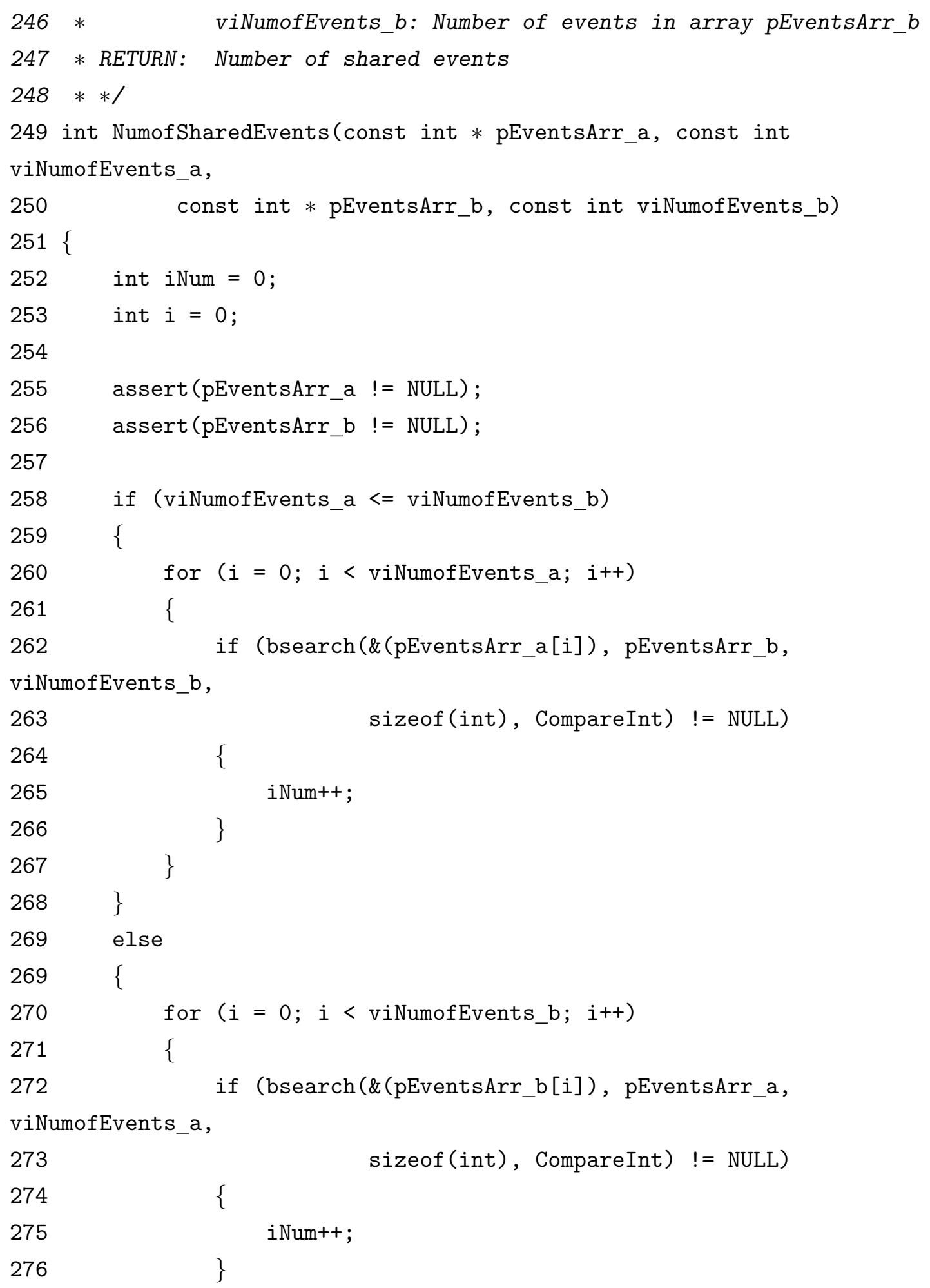




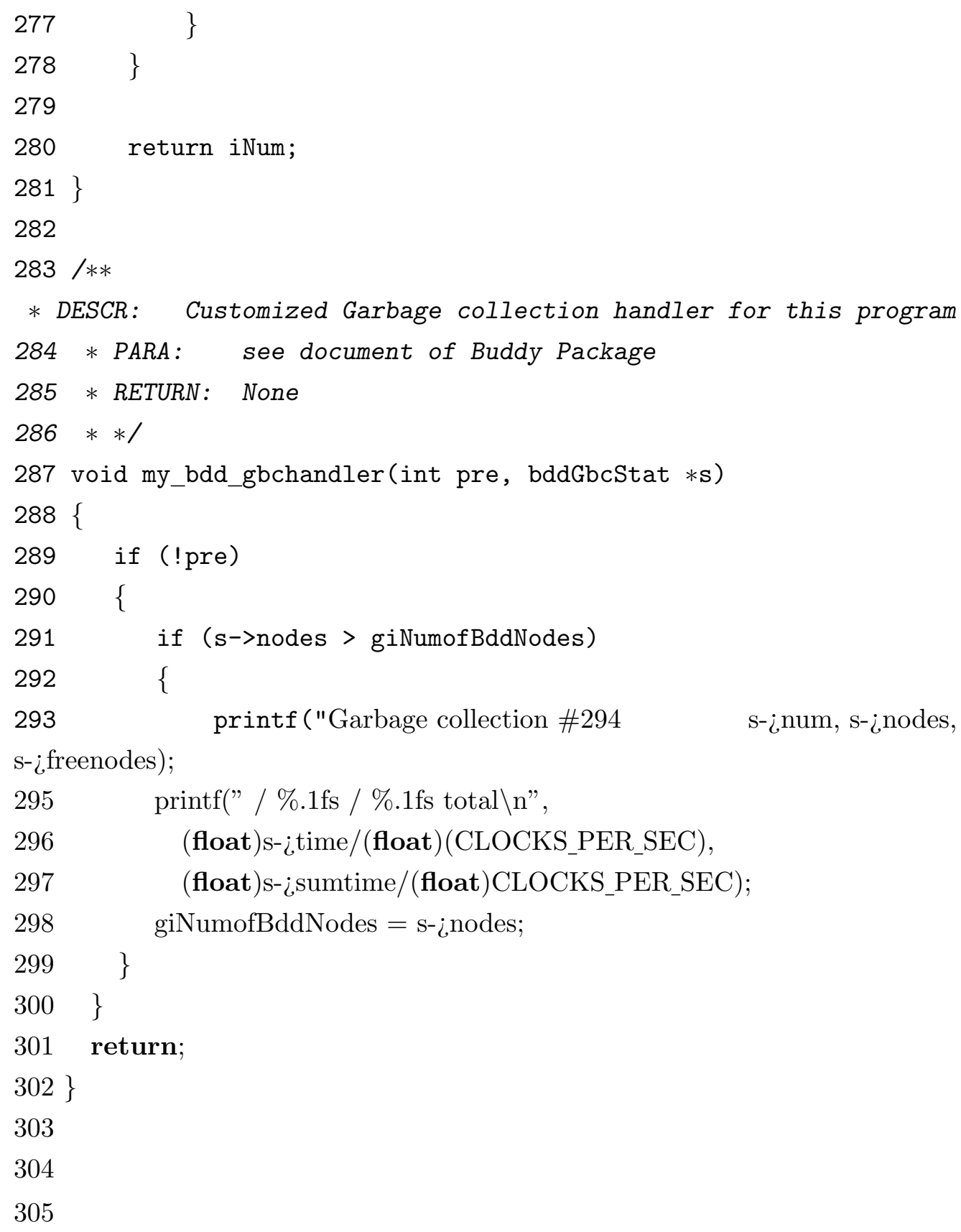

\section{BddSd.h}

001 int load_prj(const char *prjfile, std: :string \& errmsg); 002 


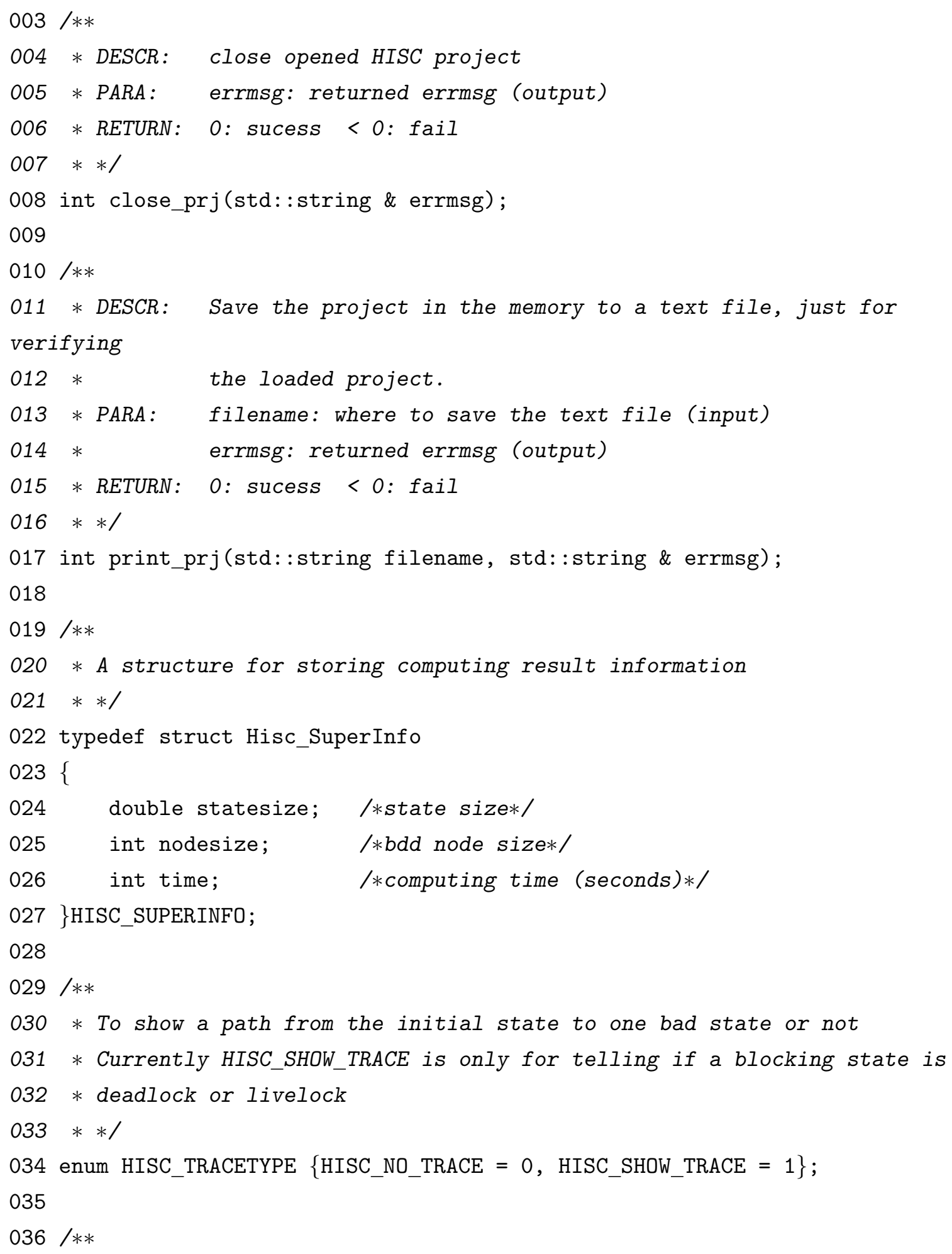




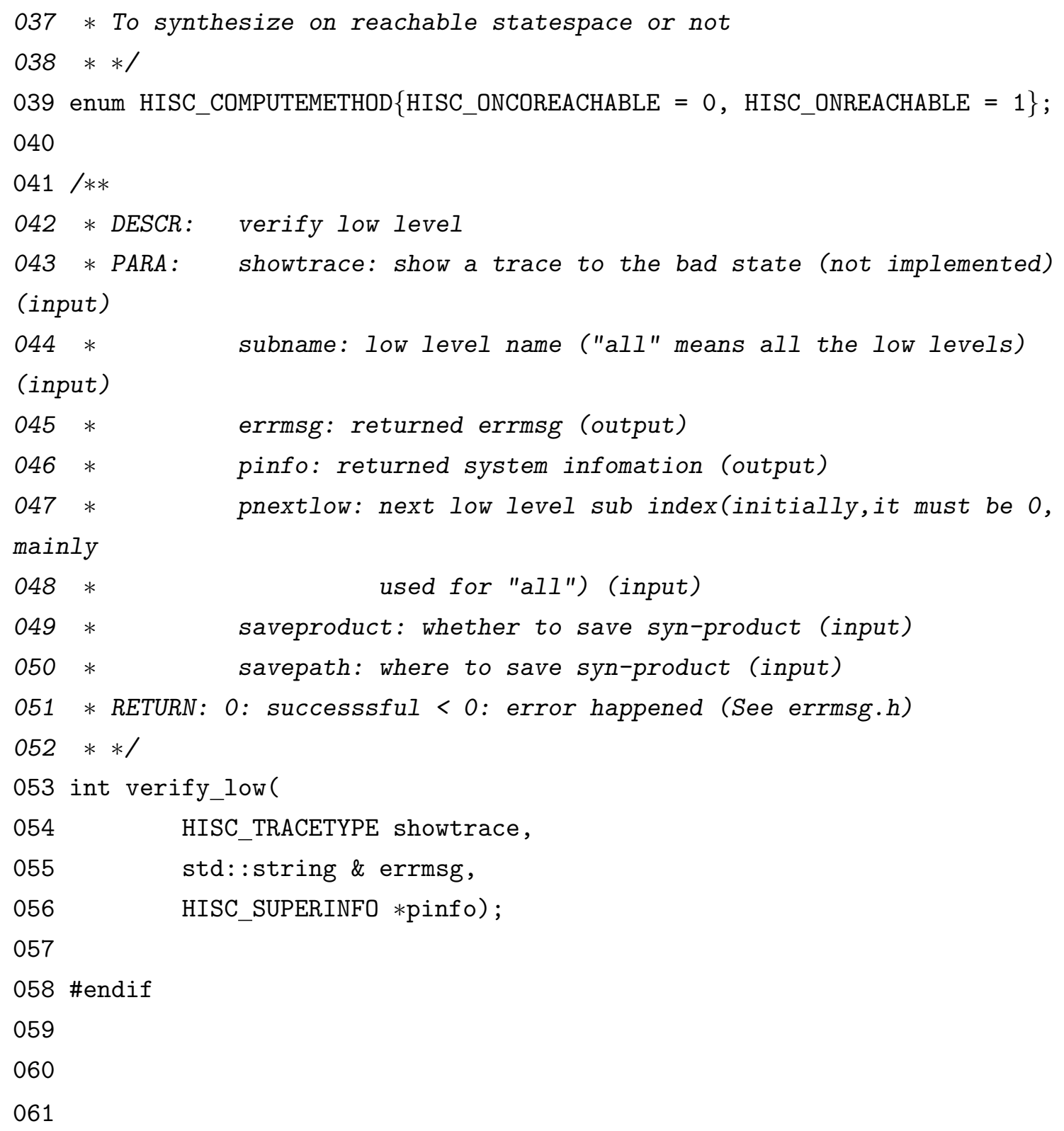

\section{BddSd.cpp}

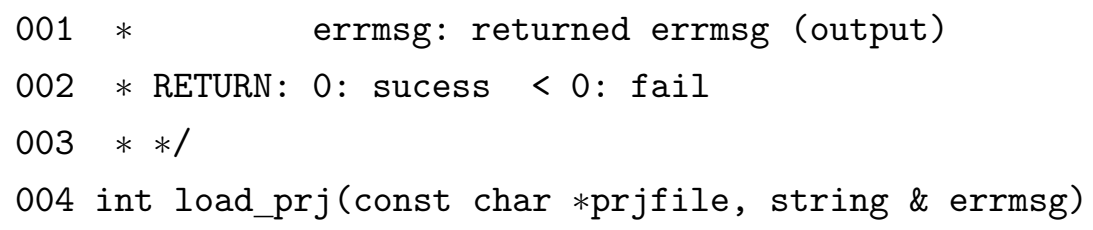




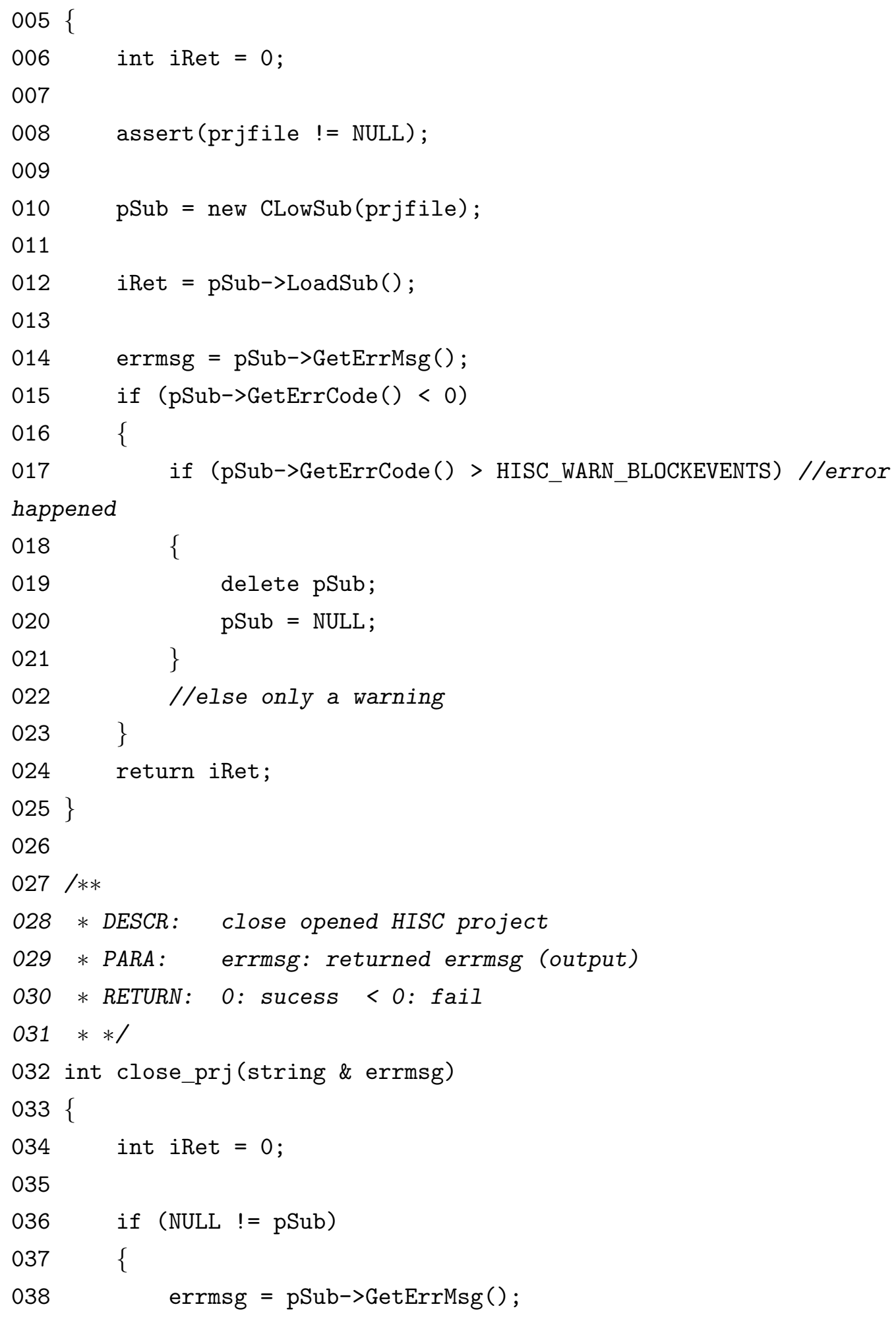




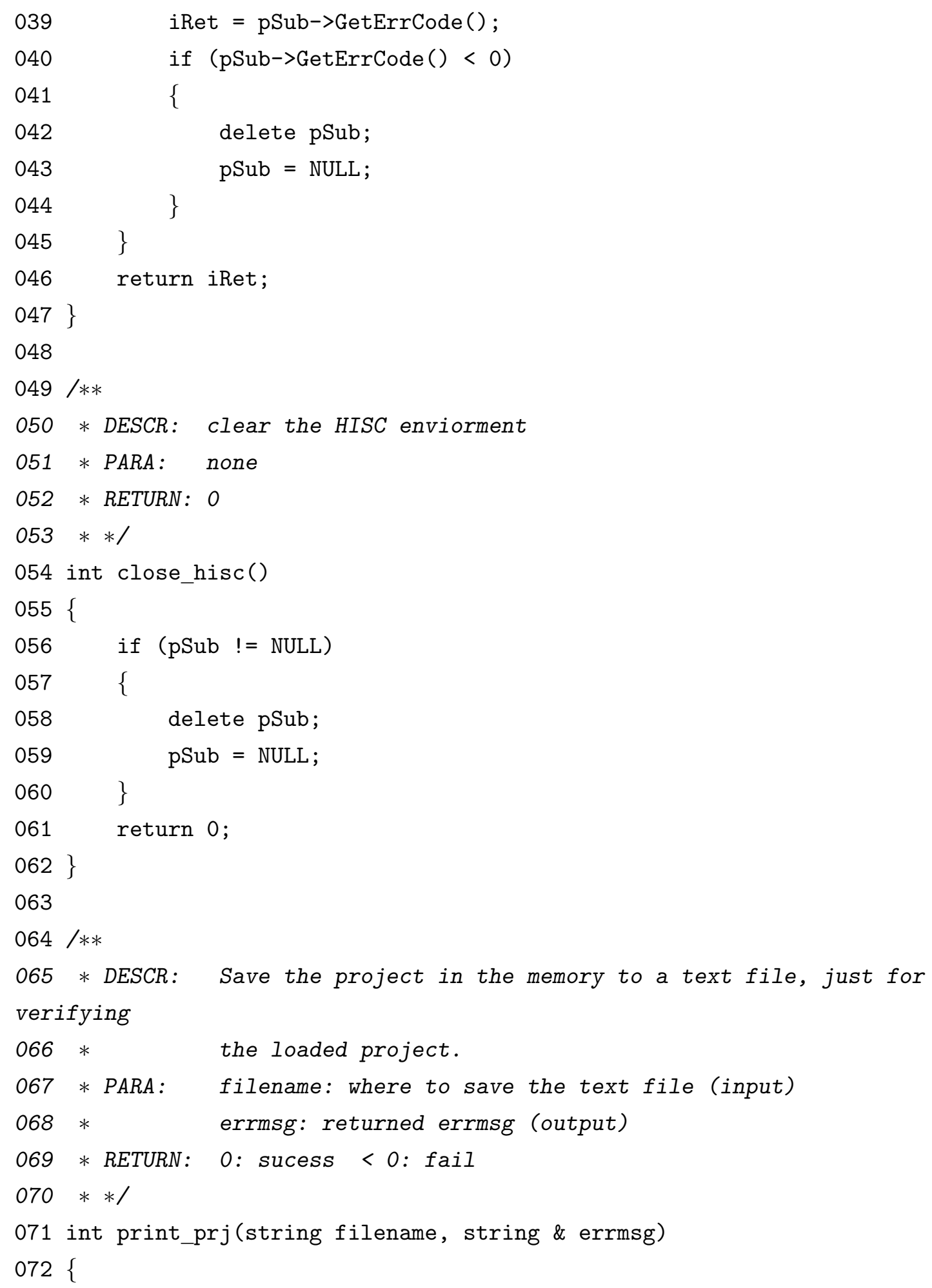




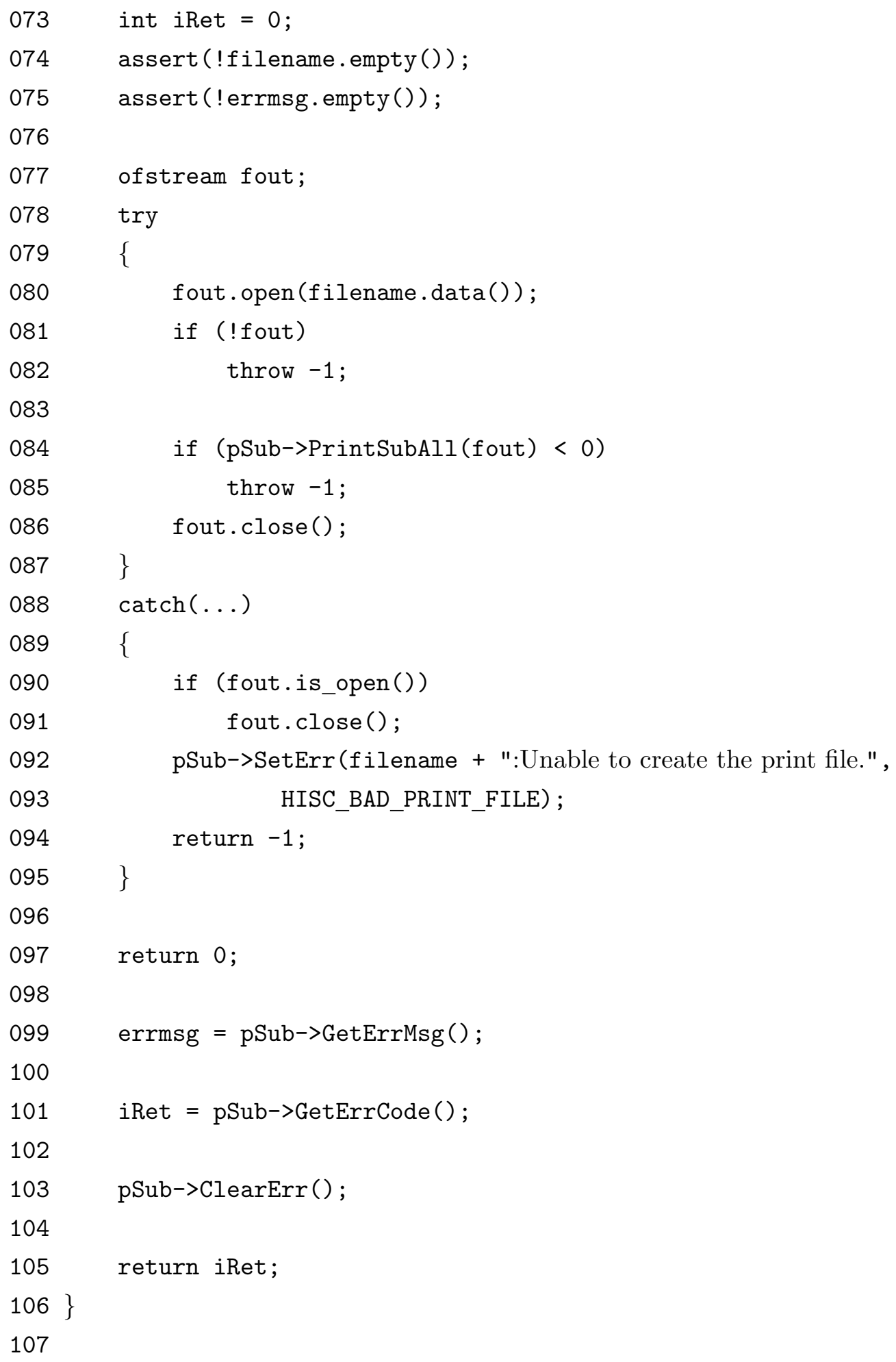




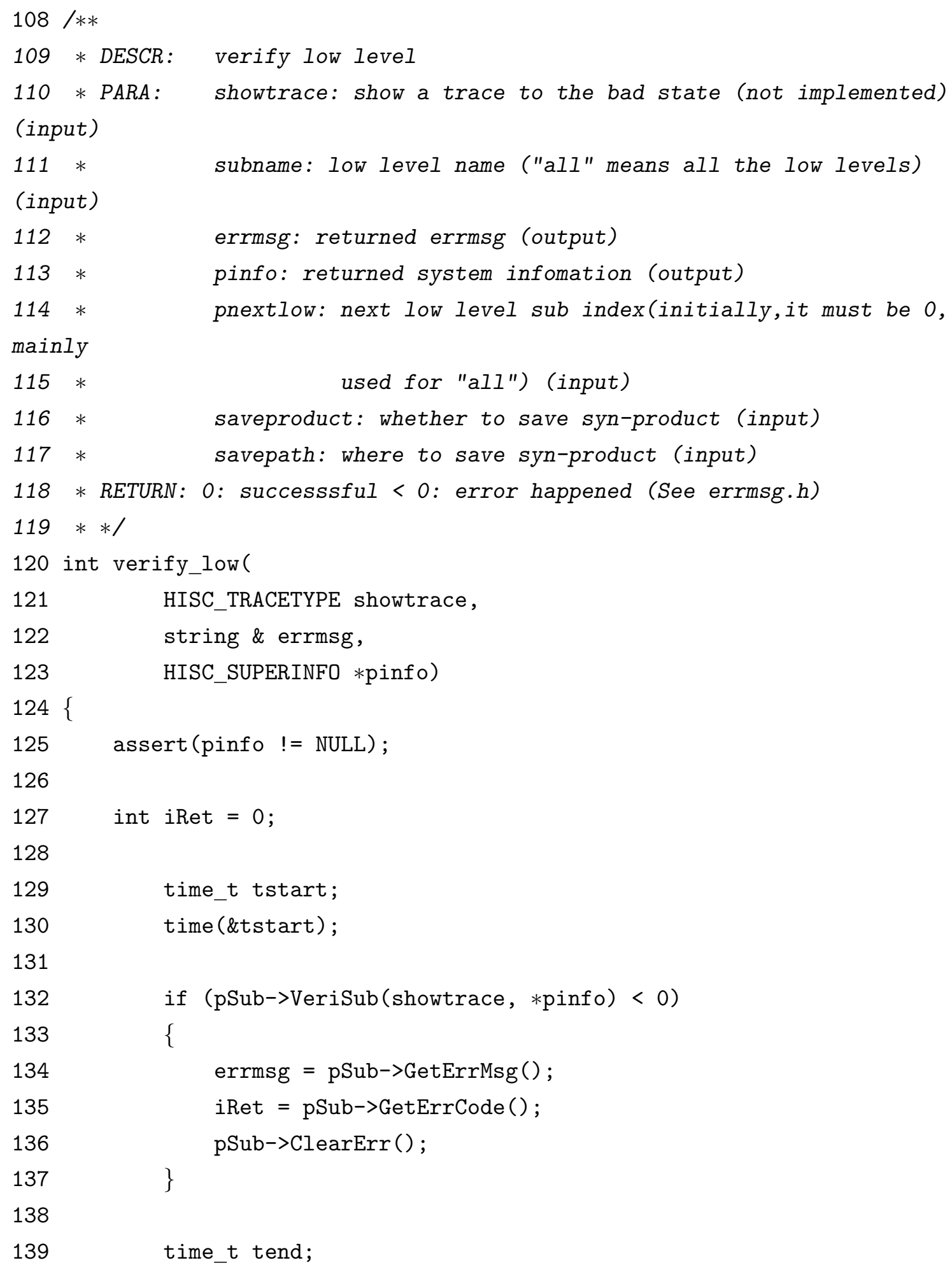




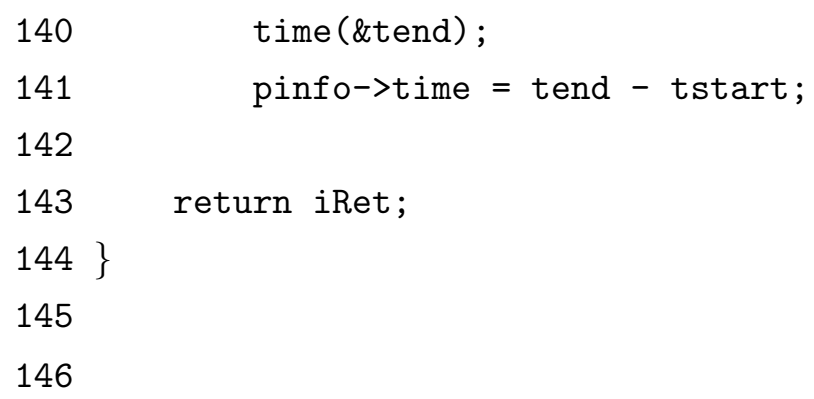

\section{A.2.3 DES Class}

\section{DES.h}

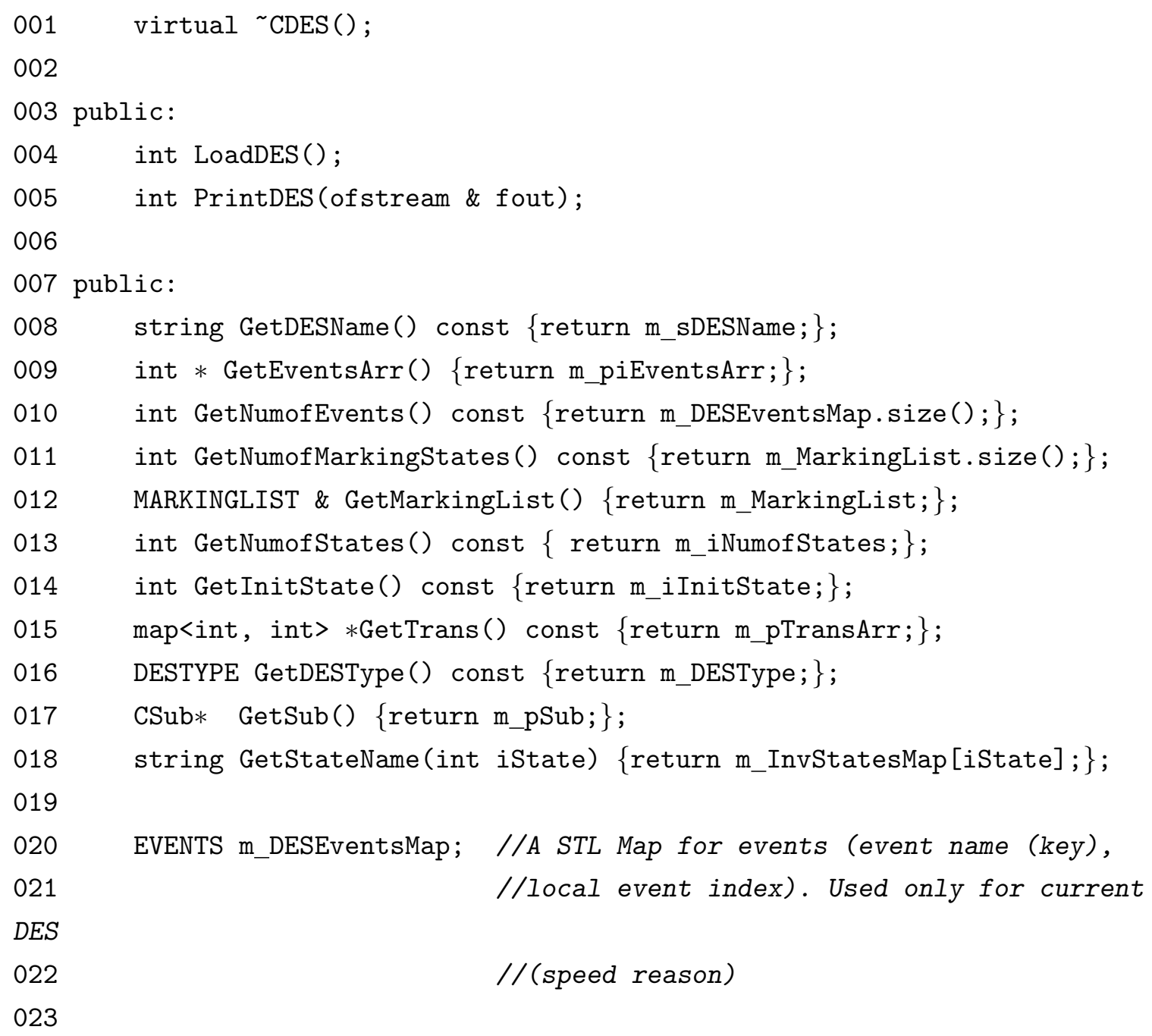


024 private: //data memeber

025 string m_sDESFile; //DES file name with path

026 string m_sDESName; //DES name without path and file extension

027 DESTYPE m_DESType; //DES type

028

029 int m_iNumofStates; //Number of States

030 int m_iInitState; //Initial state

031

032 MARKINGLIST m_MarkingList; //Link list containing all marking states

033

034 STATES m_StatesMap; //A STL Map for states (state name (key), state index)

035 INVSTATES m_InvStatesMap; //A STL Map for states (state index (key),

036 //state name)(for printing)

037

038 INVEVENTS m_InvDESEventsMap; //A STL Map for events (localindex (key),

039

//event name). Used only for current

$D E S$

040 $/ /$ (for printing)

041 EVENTS m_UnusedEvents; //A STL Map for blocked events (name: key, index)

042

043

int $*$ m_piEventsArr; //Save all the event indices ascendingly.

044 //used for find shared events between two

DESes.

045

046 TRANS *m_pTransArr; //Transiton Map array, indexed by event indices.

047

048 //TRANSMAP: first int: source state index

049 CSub *m_pSub; //which subsystem this DES belongs to 050 private: //internal function members 


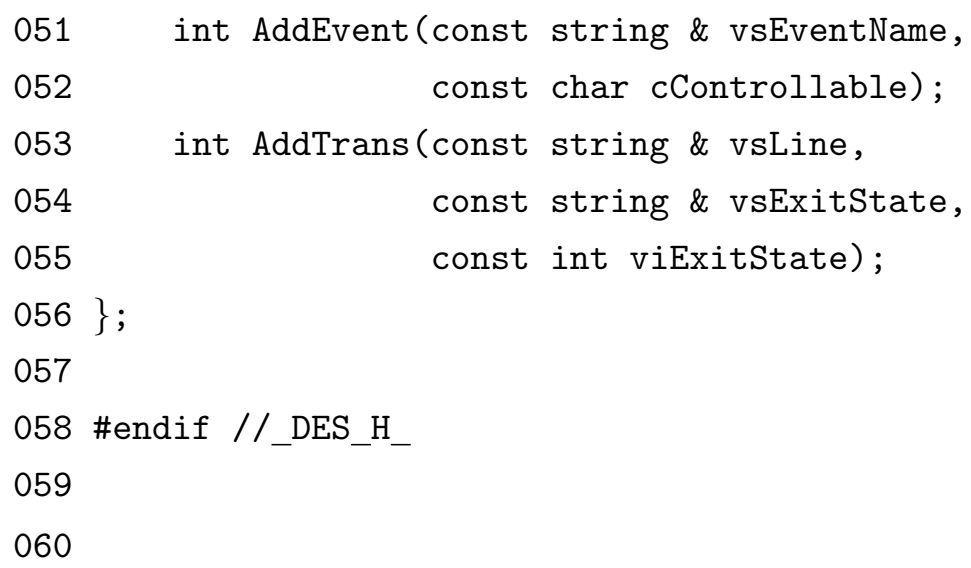

\section{DES.cpp}

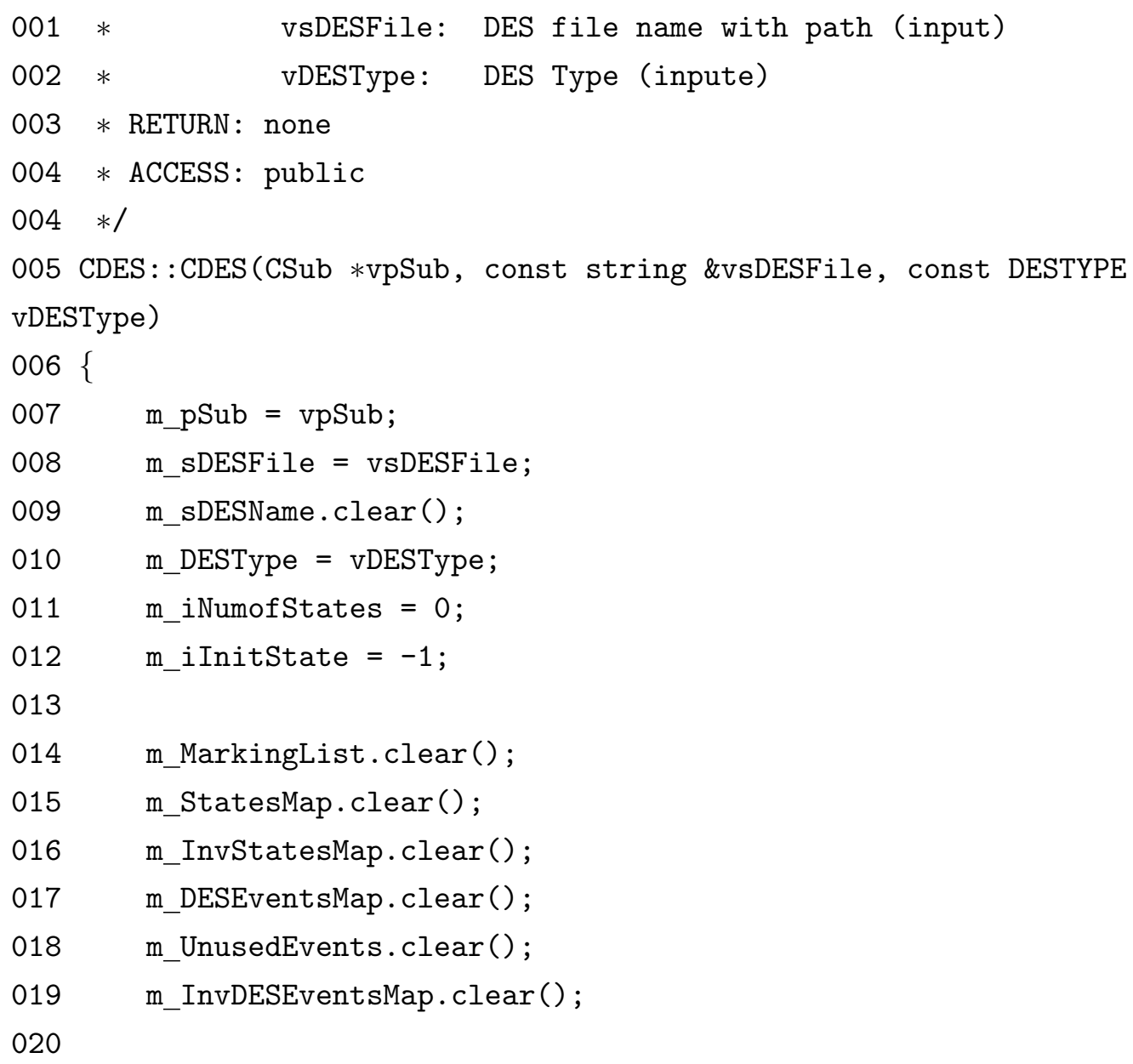




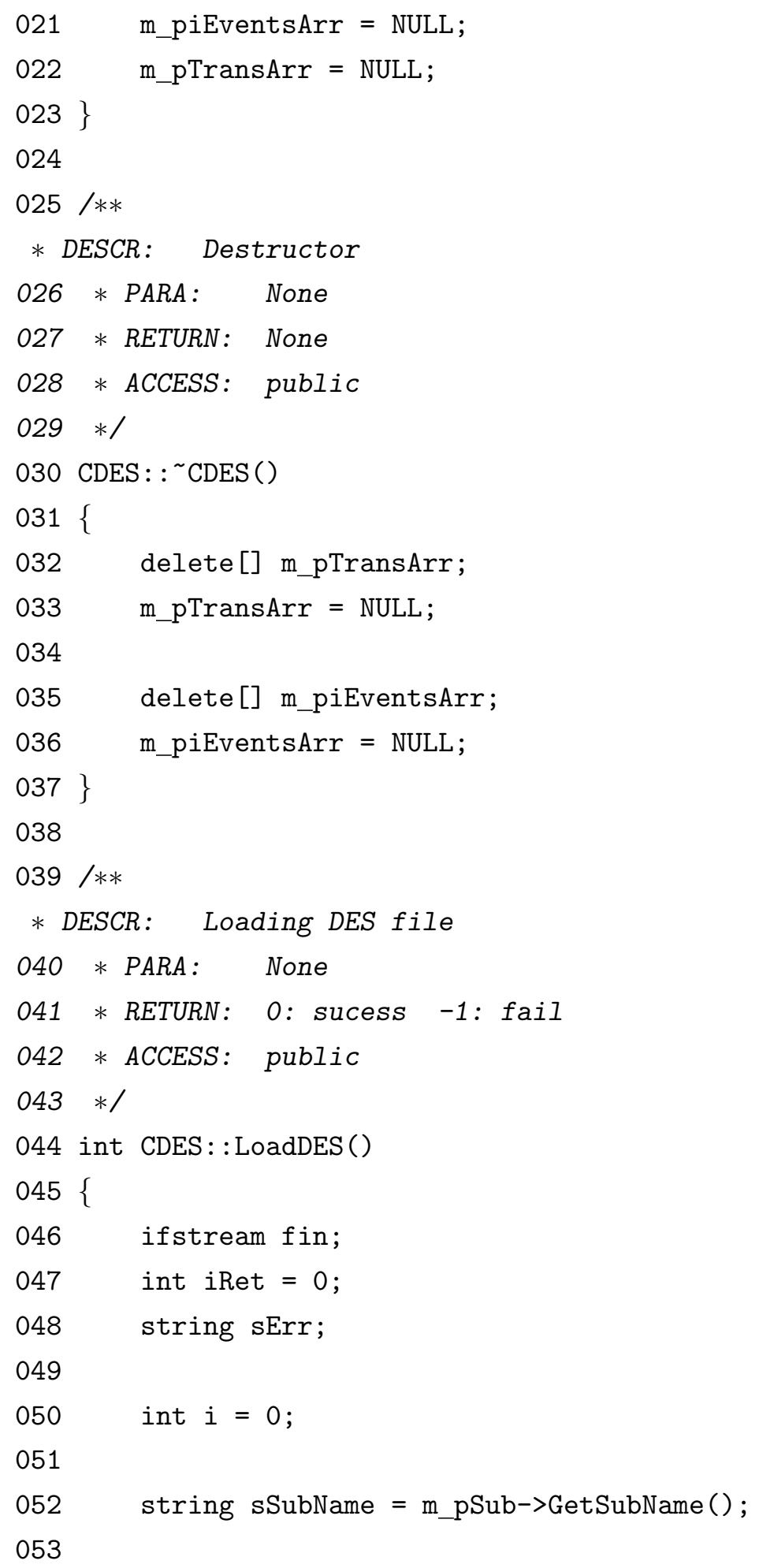




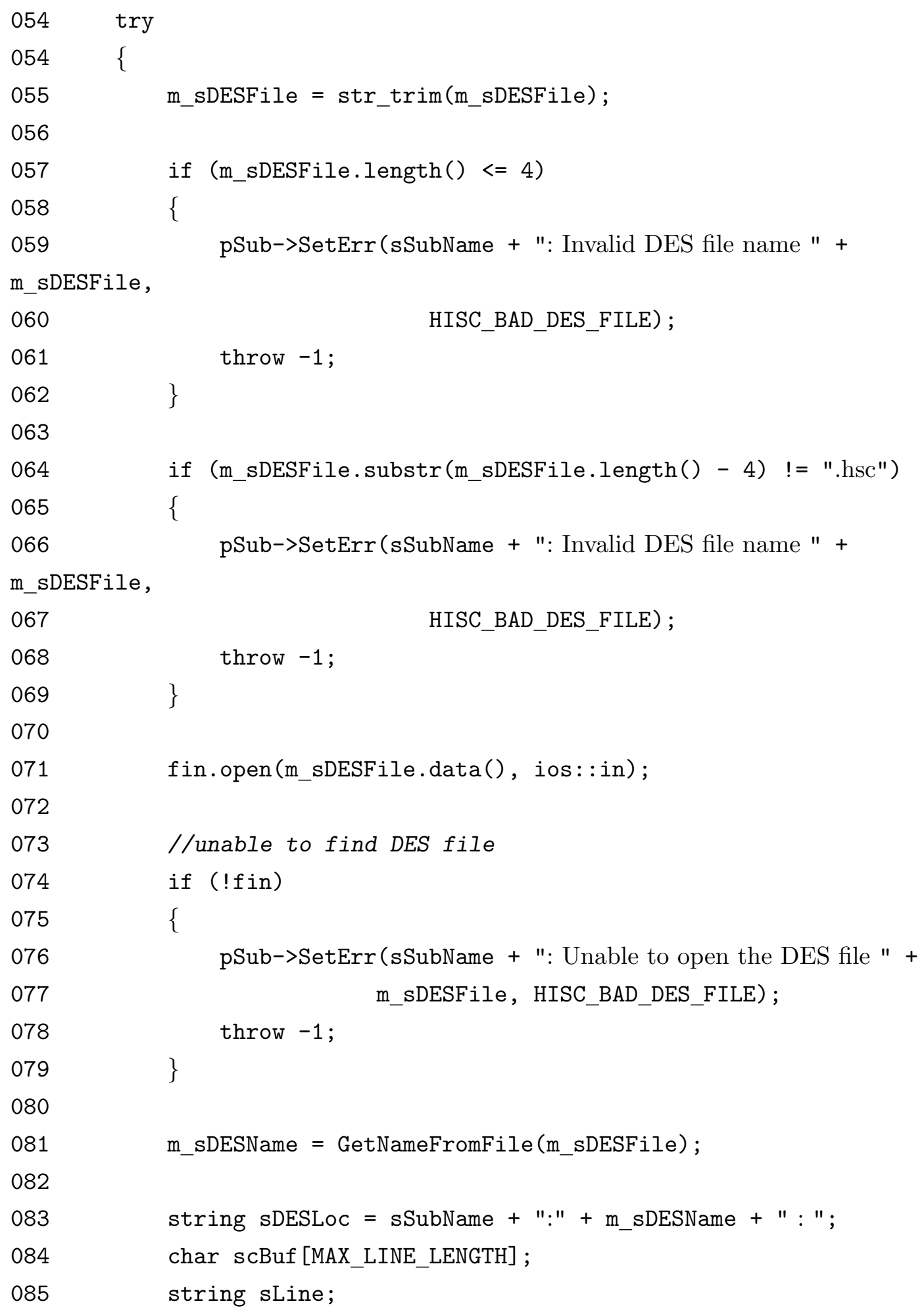


086 int iField $=-1 ; / / 0$ : States 1: InitState 2: MarkingStates

087 //3: Events 4: Transitions

088 char $*$ scFieldArr []$=\{$ "STATES", "INITSTATE", "MARKINGSTATES",

089 "EVENTS", "TRANSITIONS" \};

090 int iStatesFieldFlag $=0 ; / / 1$ : just finised reading the

[States] line,

091

// so next line should be the num

of states

092

//0: otherwise

093

int iTmpStateIndex $=0$;

094

int iTmpEventIndex $=0$;

095

char cEventSub $=$ ' $\backslash 0^{\prime}$;

096

char cControllable $={ }^{\prime} \backslash 0^{\prime}$;

097

098

string sExitState;

099

int iExitState $=-1$;

100

101

102

103

104

105

106

107

108

109

110

111

112

113

114

while (fin.getline(scBuf, MAX_LINE_LENGTH))

2

4

\{

sLine $=$ str_nocomment $($ scBuf $) ;$

sLine $=$ str_trim(sLine);

if (sLine.empty ())

continue;

115

116

//Field line

if (sLine $[0]==$ ' [' \&\& sLine $[$ sLine. $\operatorname{length}()-1]==$ '] ')

117

sLine $=\operatorname{sLine} \cdot \operatorname{substr}(1, \operatorname{sLine} \cdot$ length ()$-1) ;$

sLine $=\operatorname{sLine} . \operatorname{substr}(0$, sLine.length ()$-1) ;$

sLine = str_upper (str_trim(sLine) $)$;

iField++; 


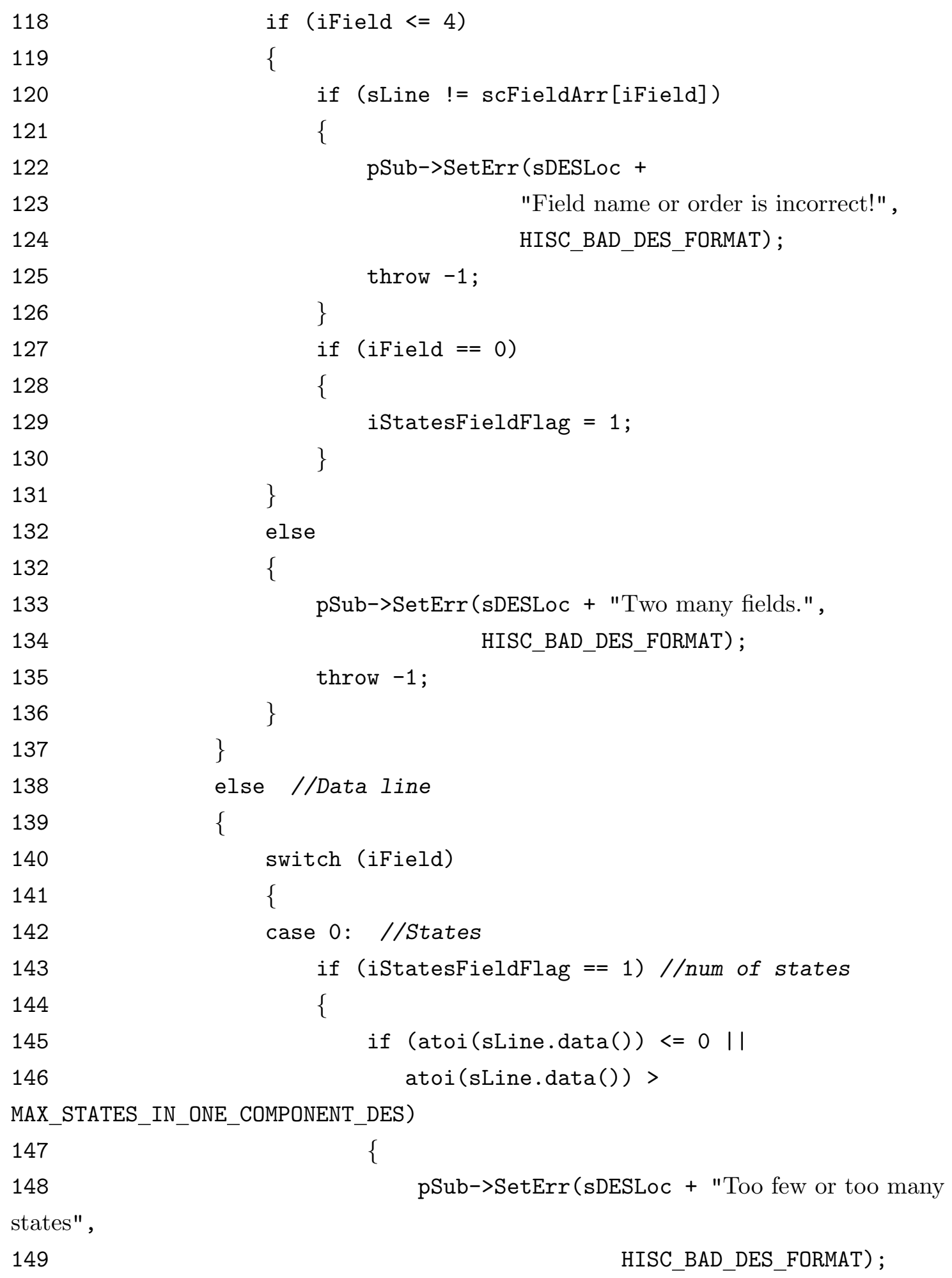


150

151

152

153

154

155

156

157

158

159

160

161

161

162

m_StatesMap.end ())

163

164

names--" +

165

HISC_BAD_DES_FORMAT) ;

166

167

168

169

170

171

172

173

174

175

175

176

1

177

sLine; throw -1 ;

\}

//initialize the number of states

m_iNumofStates $=$ atoi $($ sLine.data ()$)$;

//initialize the transition arrary

$\mathrm{m} \_\mathrm{pTransArr}=$ new TRANS[m_iNumofStates];

iStatesFieldFlag $=0$;

\}

else

\{

if (m_StatesMap.find(sLine) !=

\{ pSub->SetErr (sDESLoc + "Duplicate state sLine, throw -1 ;

\}

else if (sLine [0] $=={ }^{\prime}\left({ }^{\prime}\right)$

\{ pSub->SetErr (sDESLoc +

"The first letter of state names can not be (", HISC_BAD_DES_FORMAT) ;

throw -1 ;

\}

else

\{

m_StatesMap[sLine] = m_StatesMap.size ()$-$

m_InvStatesMap[m_StatesMap.size ()$-1]=$ 


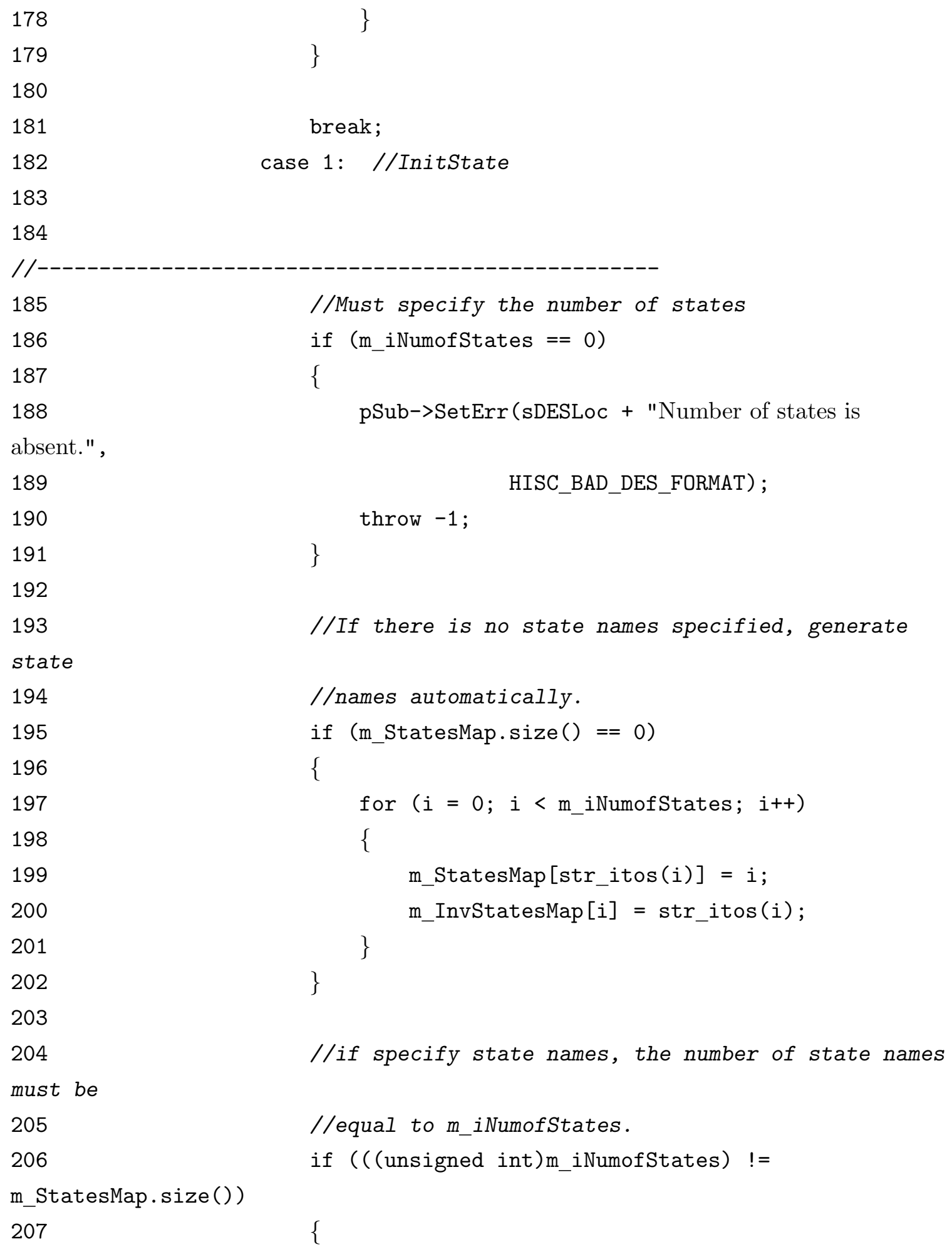

183

86

//Must specify the number of states 


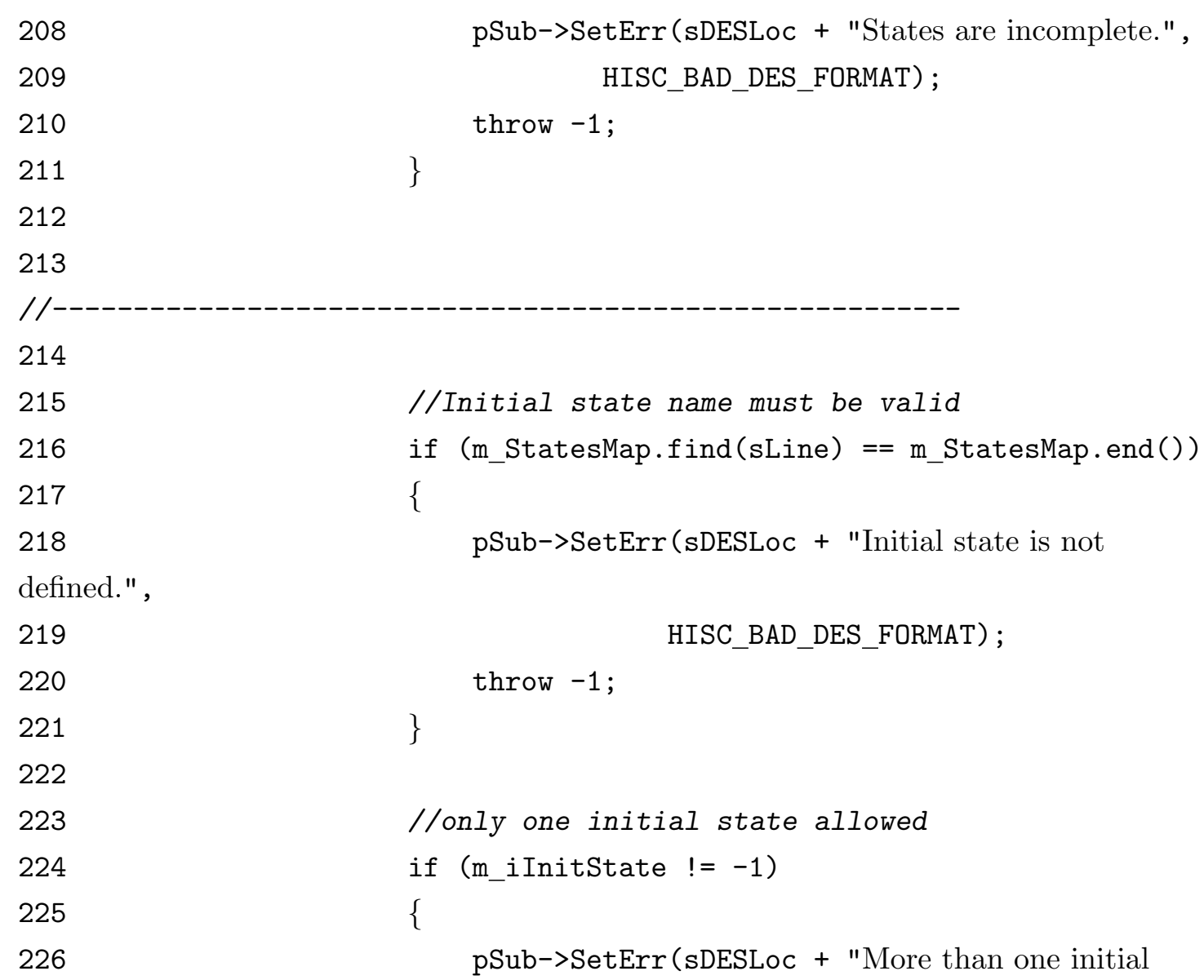




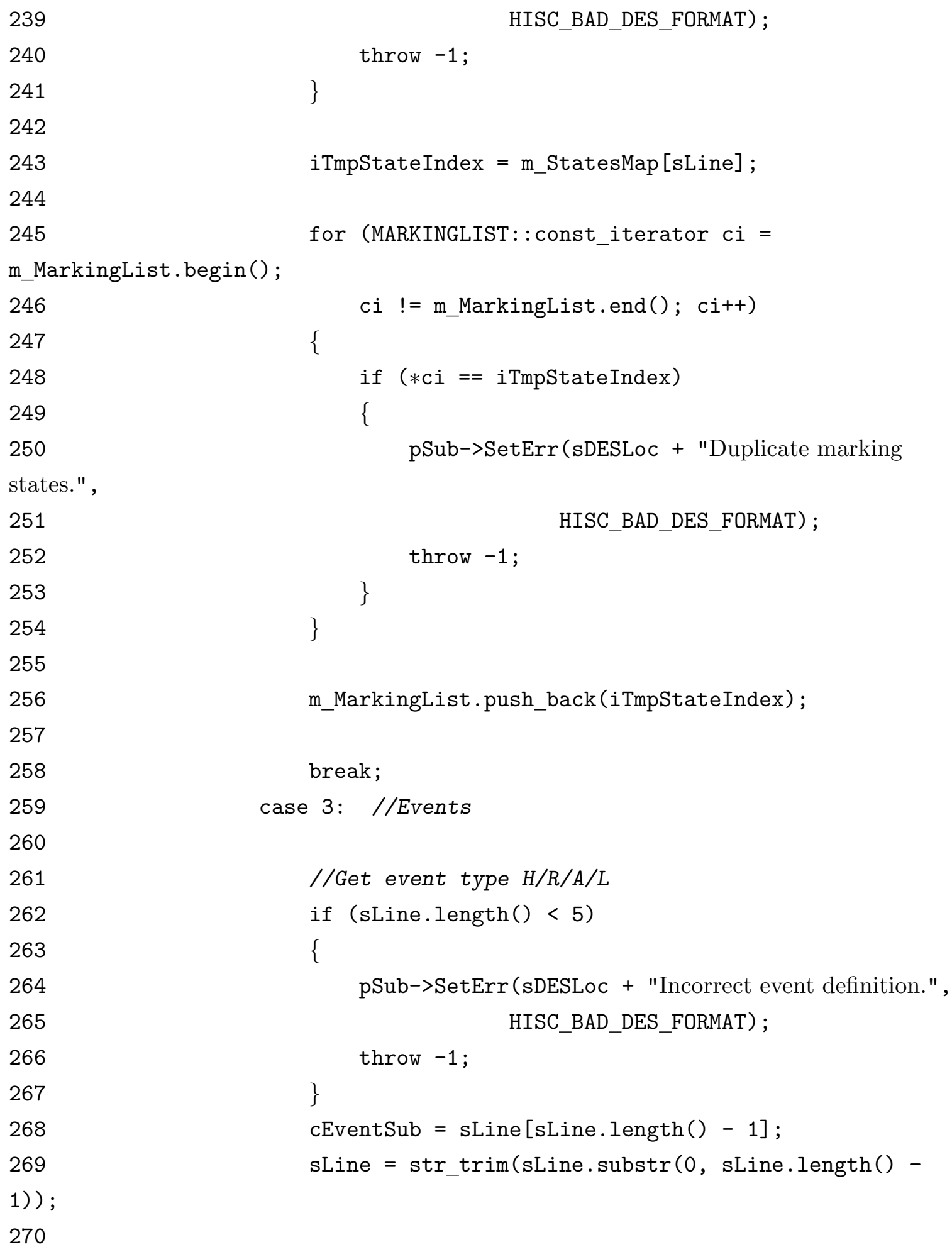


271

272

273

274

275

276

277

278

279

1)) ;

280

281

282

283

284

285

286

287

288

289

290

291

292

293

294

295

296

297

298

299

300

301

302

303

304
//Get controllable or not

if (sLine.length ()$<3$ )

\{ pSub->SetErr (sDESLoc + "Incorrect event definition.", HISC_BAD_DES_FORMAT);

throw -1 ;

\}

cControllable $=$ sLine $[$ sLine.length ()$-1]$;

sLine $=$ str_trim(sLine. $\operatorname{substr}(0$, sLine.length ()$-$

//Get event name

if (sLine.empty ())

\{

pSub->SetErr (sDESLoc + "Incorrect event definition.", HISC_BAD_DES_FORMAT);

throw -1 ;

\}

if (cEventSub $>=$ ' $a$ ') cEventSub $-=32$;

if (cControllable $>=$ ' $\mathrm{a}$ ')

cControllable $-=32$;

iTmpEventIndex $=$ AddEvent (sLine, cControllable);

if (iTmpEventIndex $<0$ ) throw -1 ; //Errmsg generated by AddEvent

m_DESEventsMap[sLine $]=i \operatorname{TmpEventIndex;~}$

m_UnusedEvents[sLine] = iTmpEventIndex;

m_InvDESEventsMap [iTmpEventIndex $]=$ sLine;

break;

case 4: //Transitions

//check exiting state 


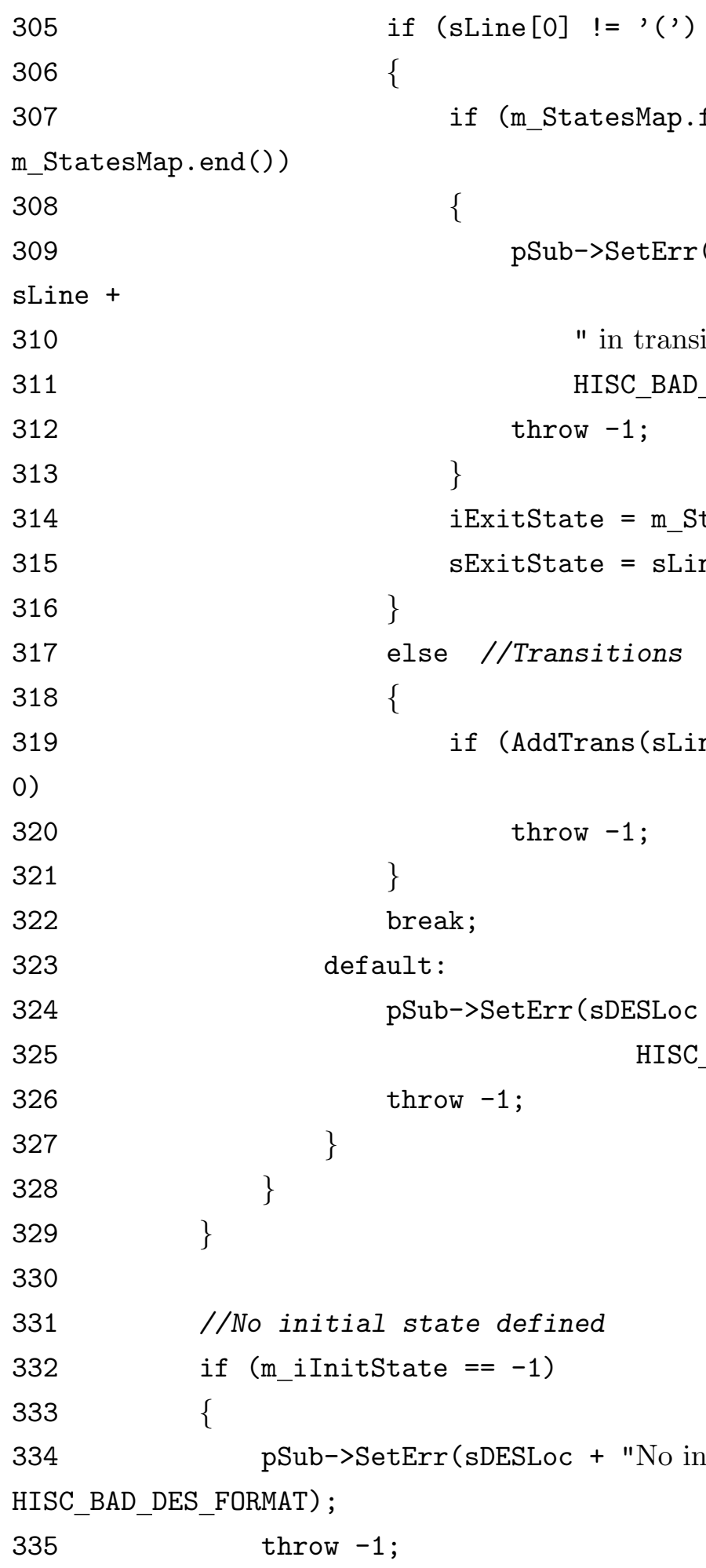




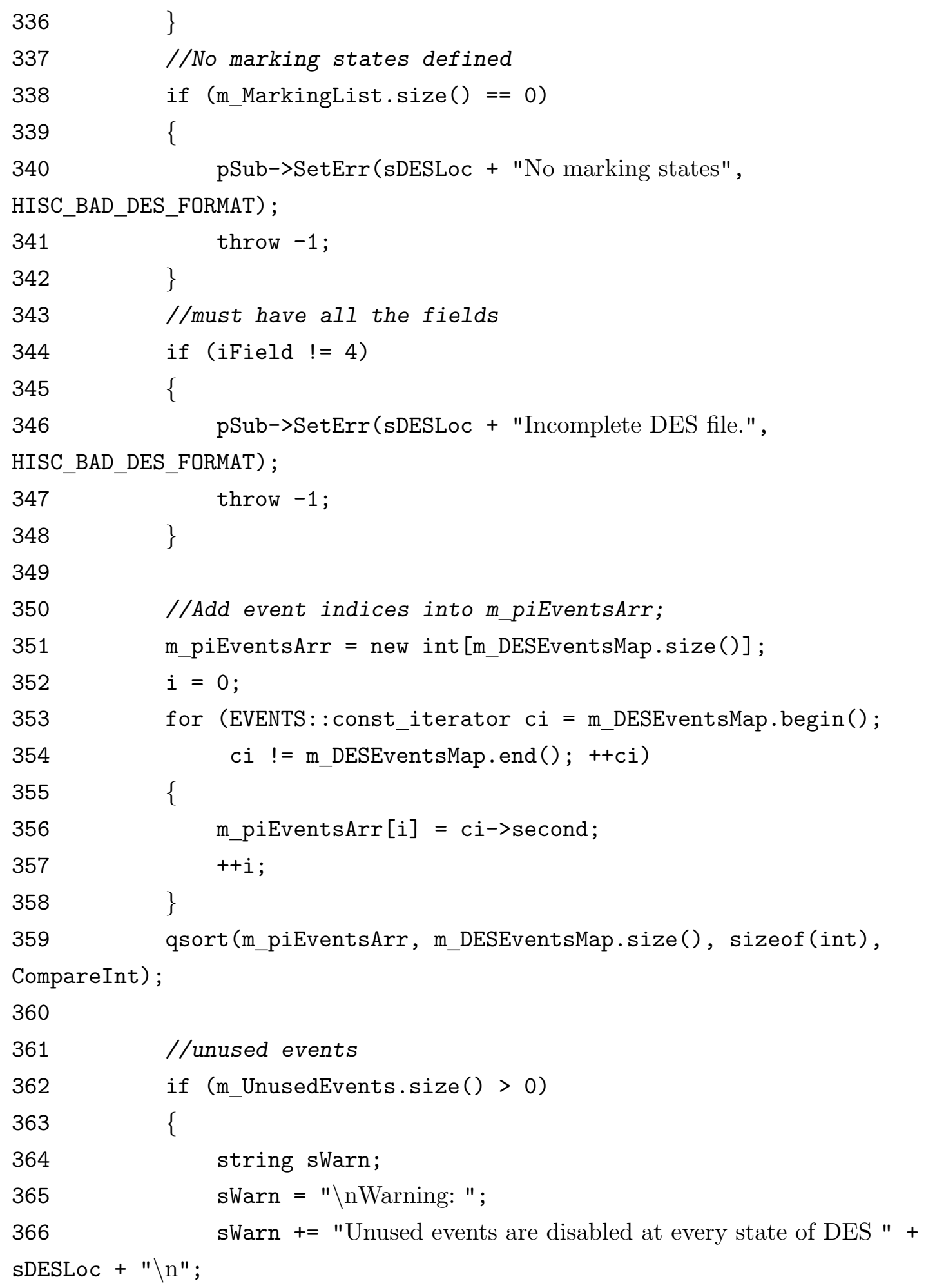




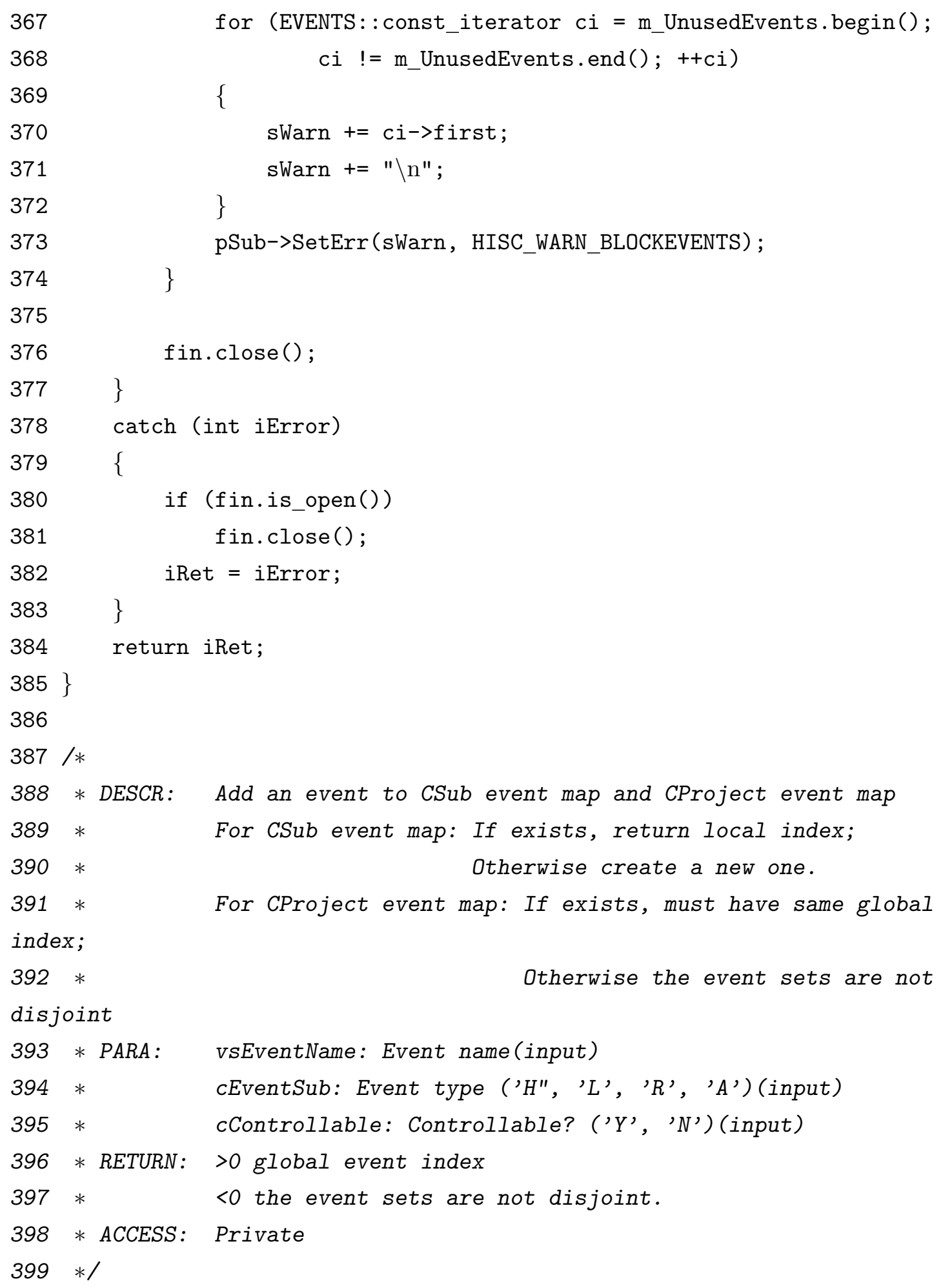


400 int CDES : :AddEvent (const string \& vsEventName, const char cControllable)

401\{

402 string sErr;

403

404 int $i$ TmpEventIndex $=0$;

405 int iTmpLocalEventIndex $=0$;

406

407 string sDESLoc $=$ m_pSub->GetSubName ()$+": "+$ m_sDESName + ": ";

408

409 //Controllable or uncontrollable

410 if (cControllable != 'Y' \&\& cControllable != 'N')

411\{

412 pSub->SetErr (sDESLoc + "Unknown event controllable type--" +vsEventName,

413

HISC_BAD_DES_FORMAT);

414 return -1 ;

$415 \quad\}$

416

417 //already defined in current DES

418 if (m_DESEventsMap.find (vsEventName) != m_DESEventsMap.end ())

419\{

420

421

422

423

424

425

//Compute local event index

426

427

iTmpLocalEventIndex $=\mathrm{m} \_$pSub->AddSubEvent (vsEventName,

428

429

(cControllable $==$ 'Y') ? CON_EVENT:UNCON_EVENT);

if ((cControllable $==$ ' $Y$ ' \&\& iTmpLocalEventIndex $\% 2==0)$ | |

430

431

432

pSub->SetErr (sDESLoc + "Duplicate events definition--" + vsEventName, HISC_BAD_DES_FORMAT);

return -1 ;

\}

$$
\text { (cControllable == 'N' \&\& iTmpLocalEventIndex } \% 2==1 \text { )) }
$$

\{

$$
\text { pSub->SetErr (sDESLoc + "Event " + vsEventName + }
$$




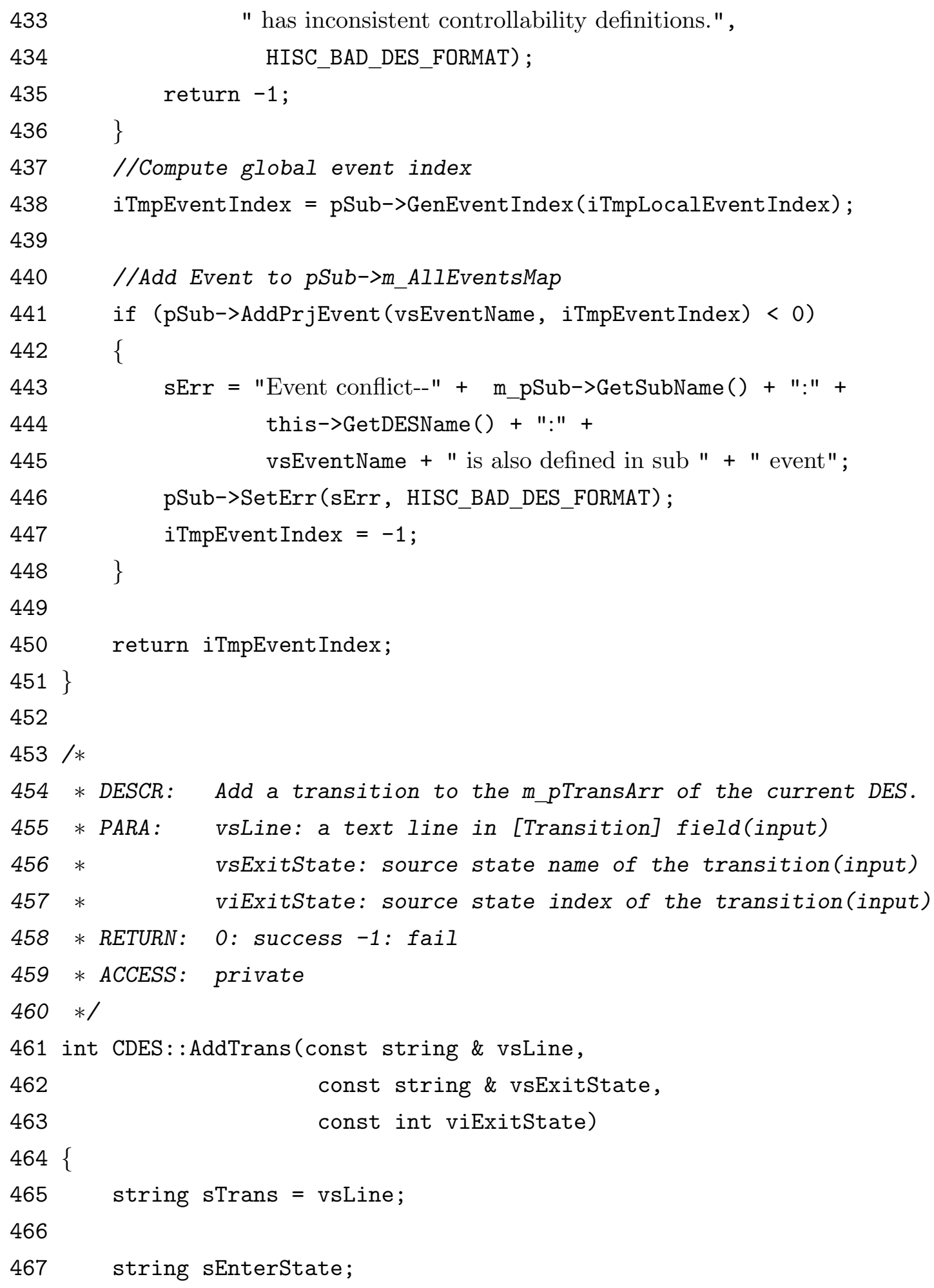




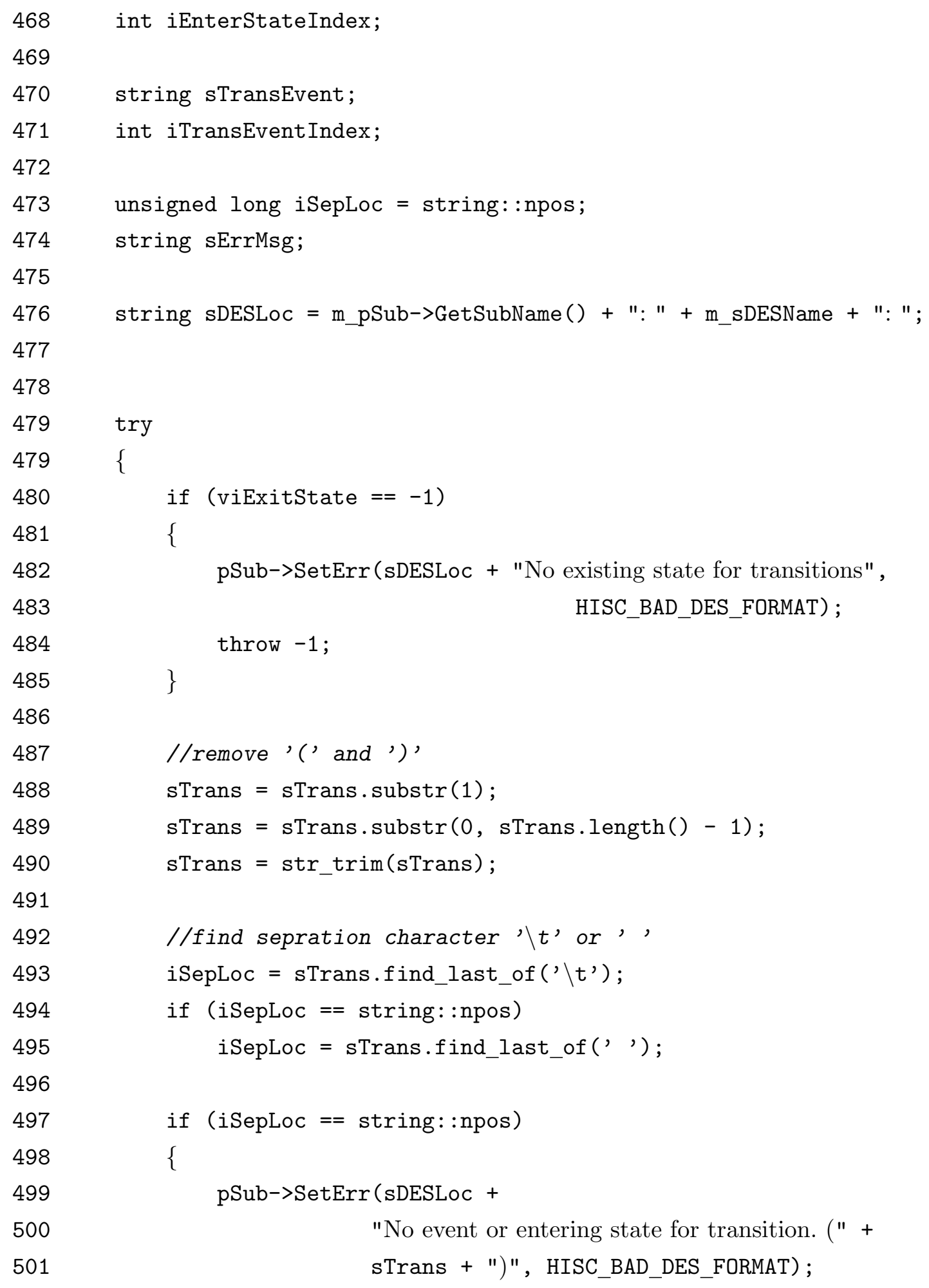




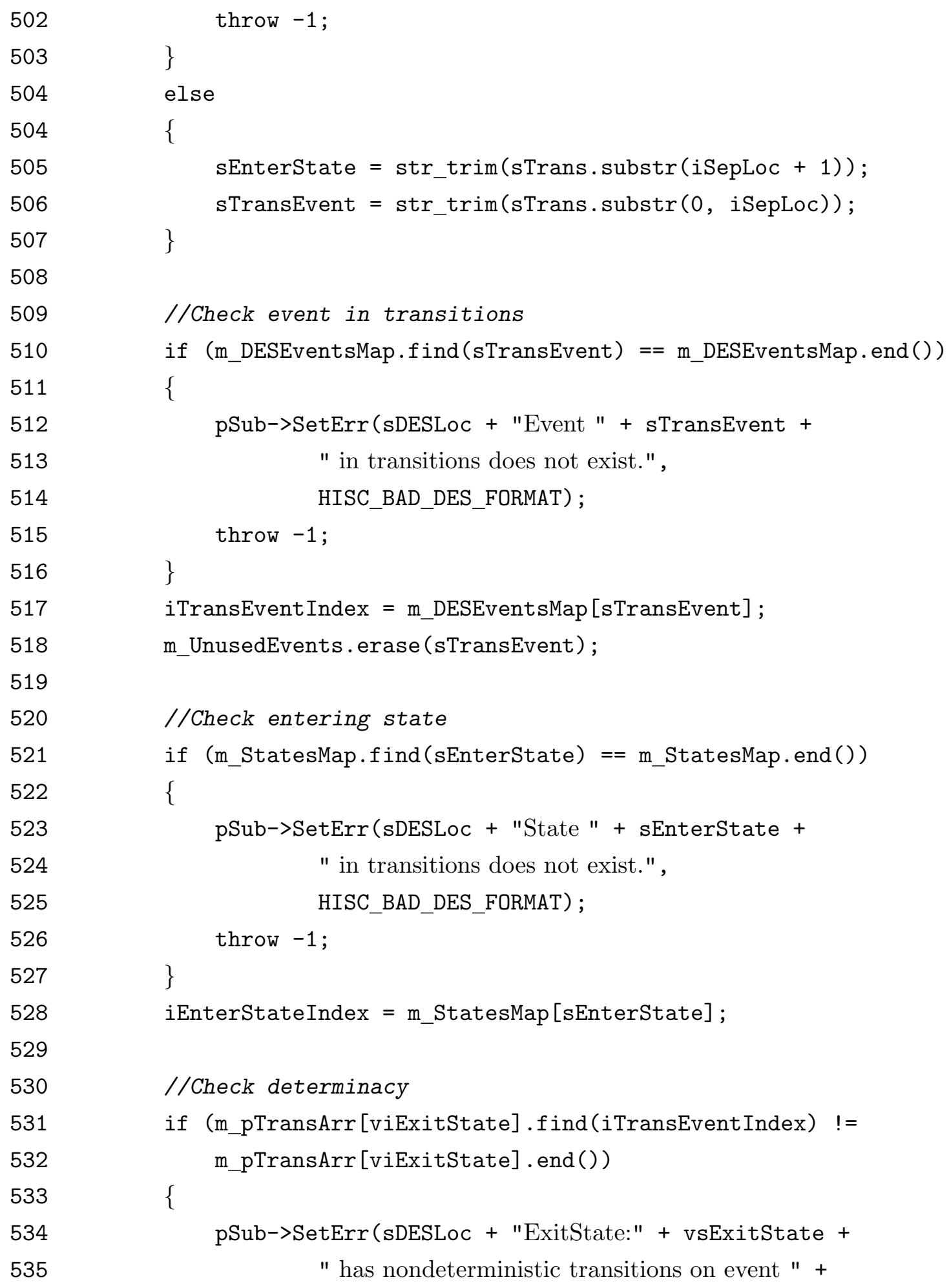


sTransEvent,

536

537

throw -1 ;

538 \}

539 m_pTransArr[viExitState] [iTransEventIndex] = iEnterStateIndex;

$540 \quad\}$

$541 \quad \operatorname{catch}($ int $)$

542\{

543 return -1 ;

$544 \quad\}$

545

546 return 0 ;

$547\}$

548

$549 / *$

550 * DESCR: Print this DES in memory to a file (for checking)

551 * PARA: fout: file stream(input)

$552 *$ RETURN: 0: success -1: fail

$553 *$ ACCESS: public

$554 * /$

555 int CDES: :PrintDES (ofstream \& fout)

556\{

557 try

557\{

558 int $i=0$;

559

560

fout $\ll$ endl $\ll$ "\#-DES: " $<$ m_sDESName $<"$ ---------" $<$

endl;

561

fout $<<$ "[States]" $<<$ endl;

562

fout $<$ m_iNumofStates $<$ endl;

563

564

565

for (INVSTATES: : const_iterator $\mathrm{ci}=\mathrm{m}_{-}$InvStatesMap.begin $($) ;

566

567 ci ! = m_InvStatesMap.end (); ++ci) 


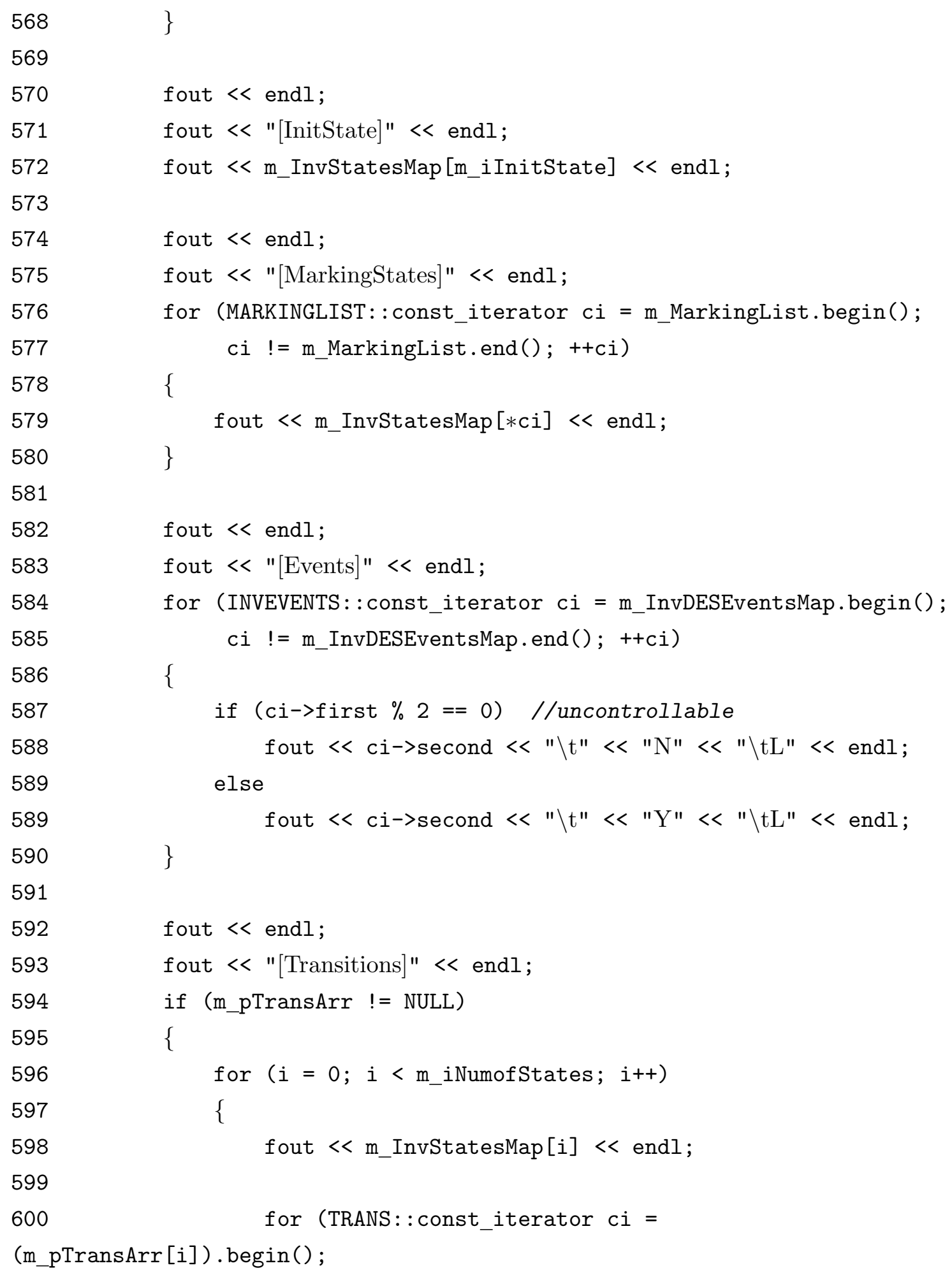




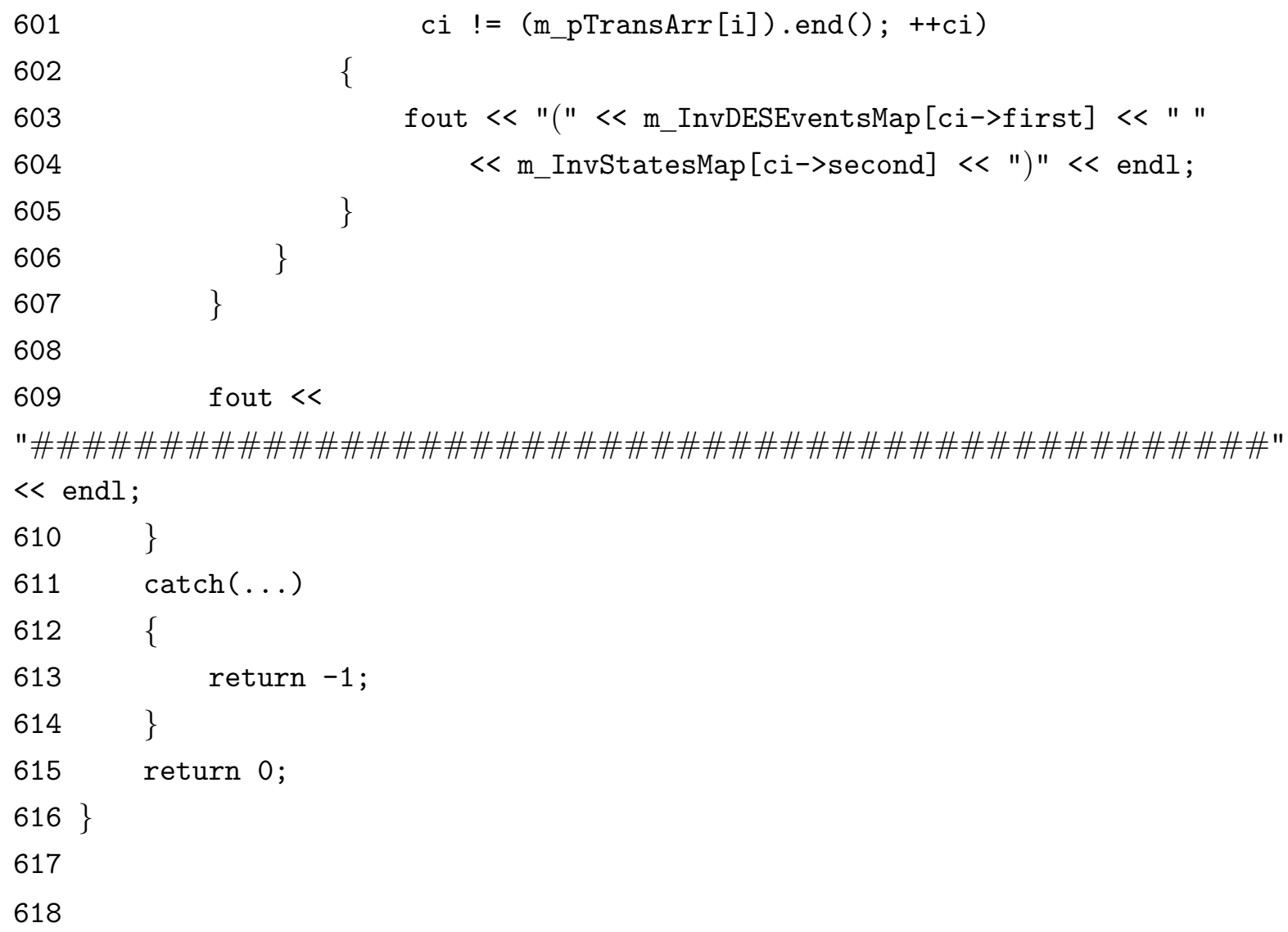

618

\section{A.2.4 Sub Class}

\section{Sub.h}

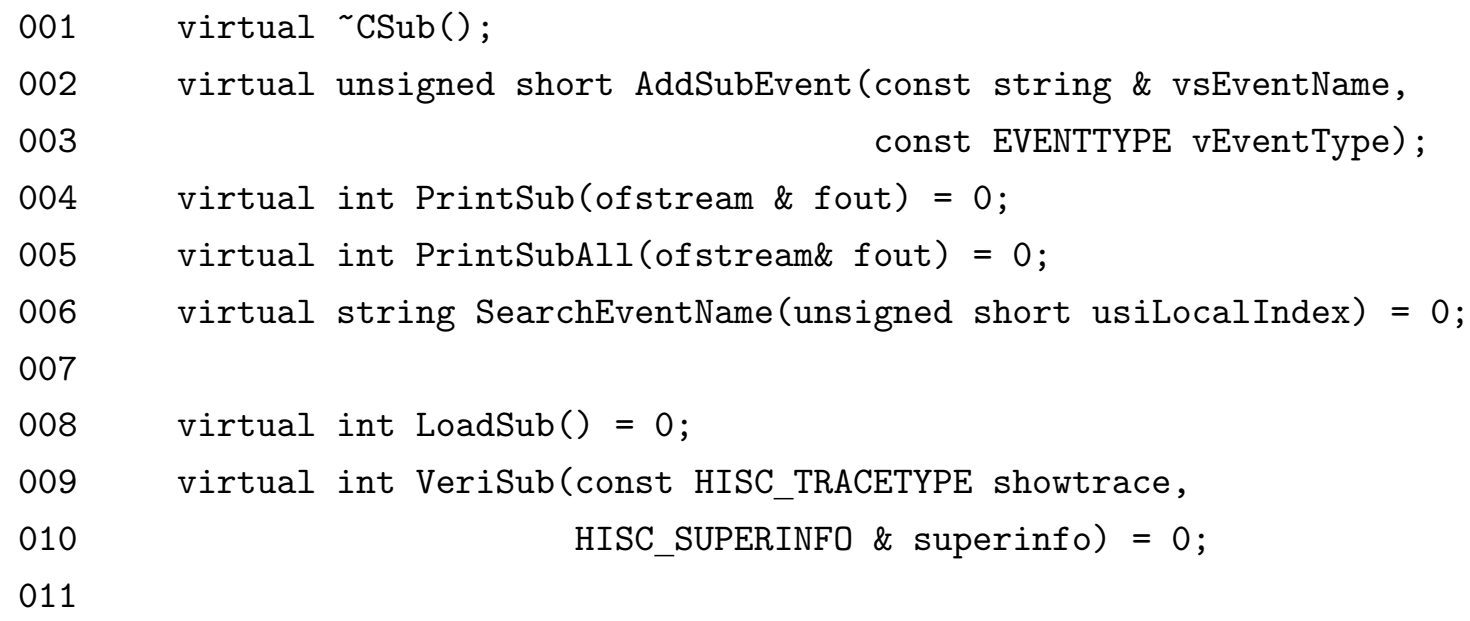




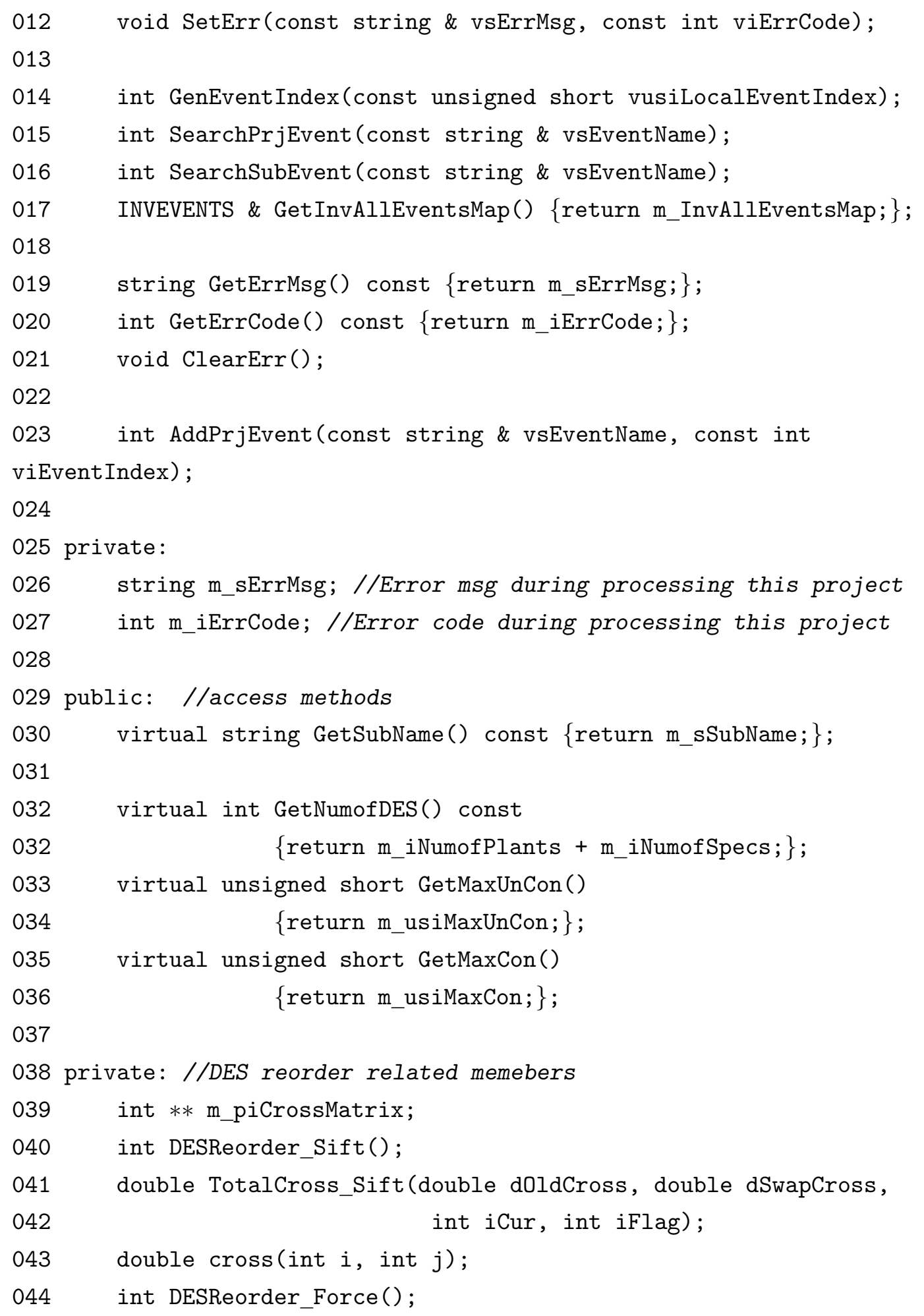




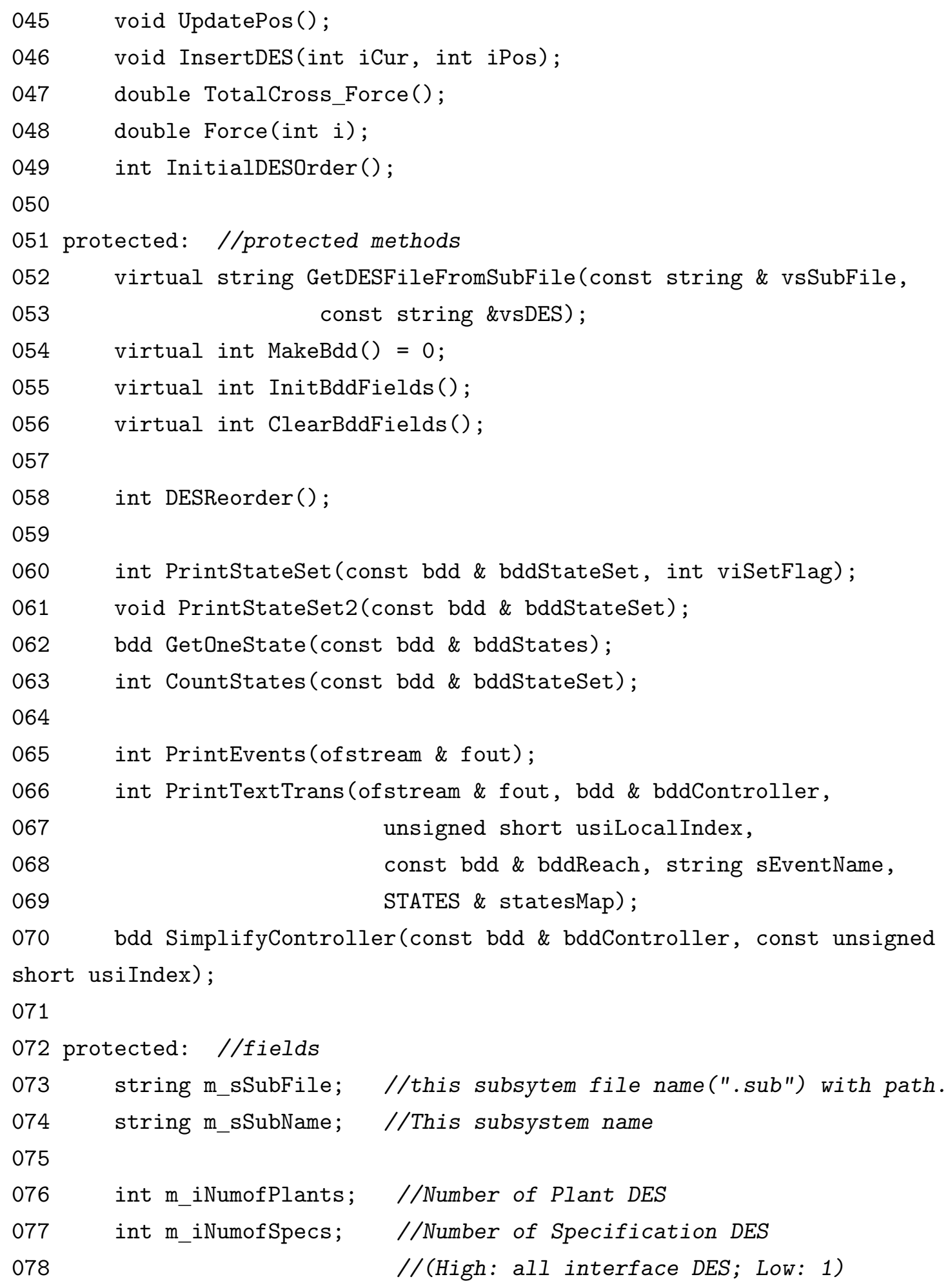




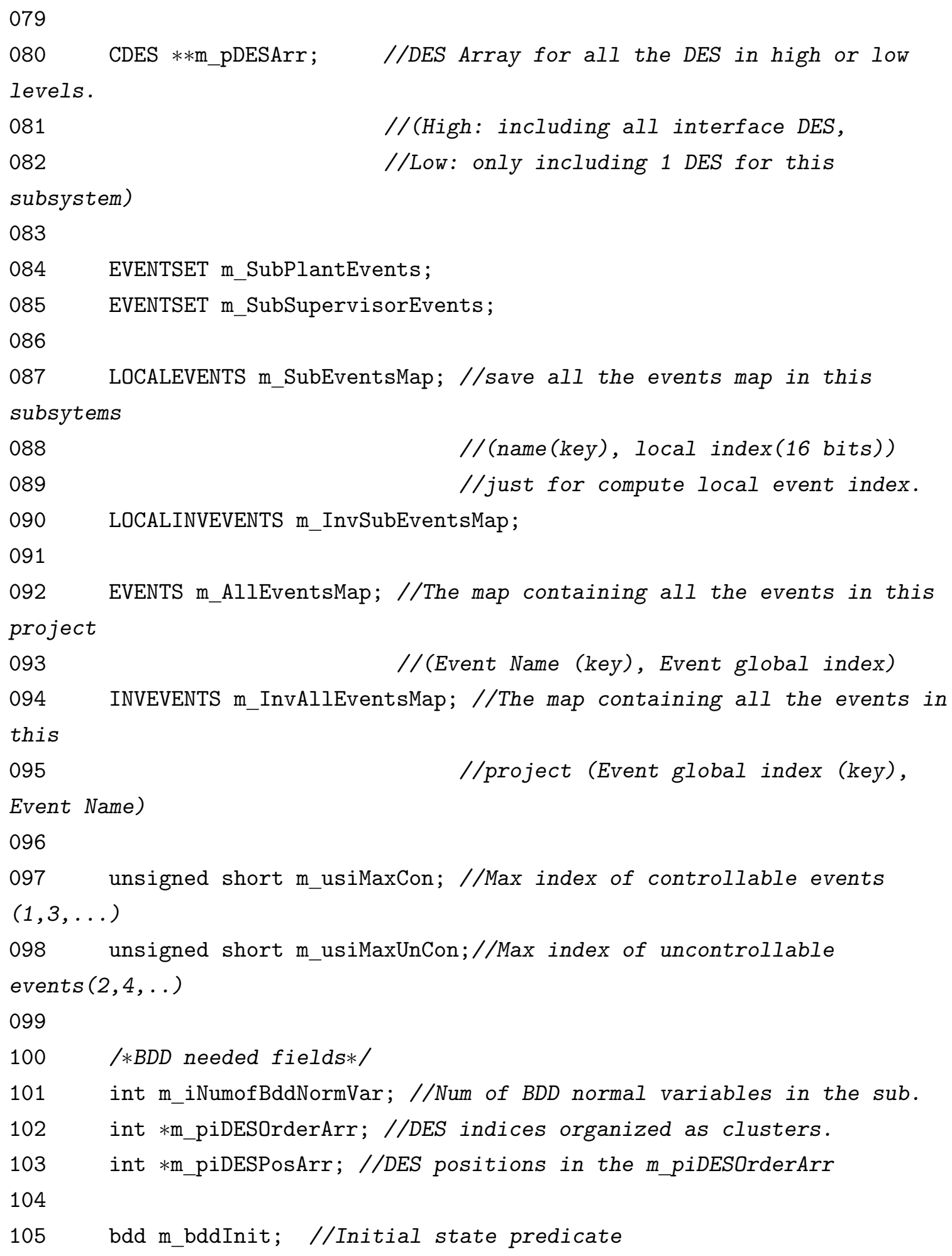


106 bdd m_bddMarking; //Marking states predicate

107 bdd m_bddSuper; //The generated supervisor

108

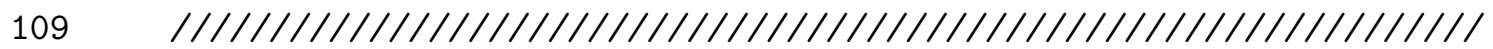

110 //Transition predicates and its variable sets, variable pairs.

111 //0: High level events

$112 \quad / / 1$ : Request events

$113 \quad / / 2$ : Answer events

$114 \quad / / 3$ : Low level events

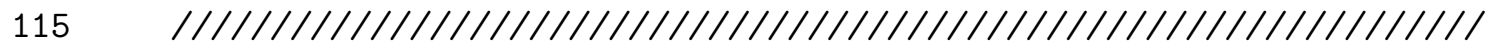

$116 \quad / / T r a n s i t i o n$ predicates

117 bdd $*$ m_pbdd_ConTrans;

118 bdd $*$ m_pbdd_ConPlantTrans;

119 bdd *m_pbdd_ConSupTrans;

120 bdd $*$ m_pbdd_UnConTrans;

121 bdd *m_pbdd_UnConPlantTrans;

122 bdd *m_pbdd_UnConSupTrans;

123

124 //variable(DES index) set for transition predicates

125 bdd $*$ m_pbdd_ConVar;

126 bdd *m_pbdd_ConVarPrim;

127 bdd $*$ m_pbdd_UnConVar;

128 bdd $*$ m_pbdd_UnConVarPrim;

$129 \quad / / p l a n t$ part variables

130 bdd $*$ m_pbdd_UnConPlantVar;

131 bdd $*$ m_pbdd_UnConPlantVarPrim;

132 bdd *m_pbdd_ConPhysicVar; //for simplifying controller (note:

physical)

133 bdd *m_pbdd_ConPhysicVarPrim;//for simplifying controller (note:physical)

134 //supervisor part variables

135 bdd $*$ m_pbdd_UnConSupVar;

136 bdd $*$ m_pbdd_UnConSupVarPrim;

137 bdd $*$ m_pbdd_ConSupVar; //for simplifying controller (note:

physical) 


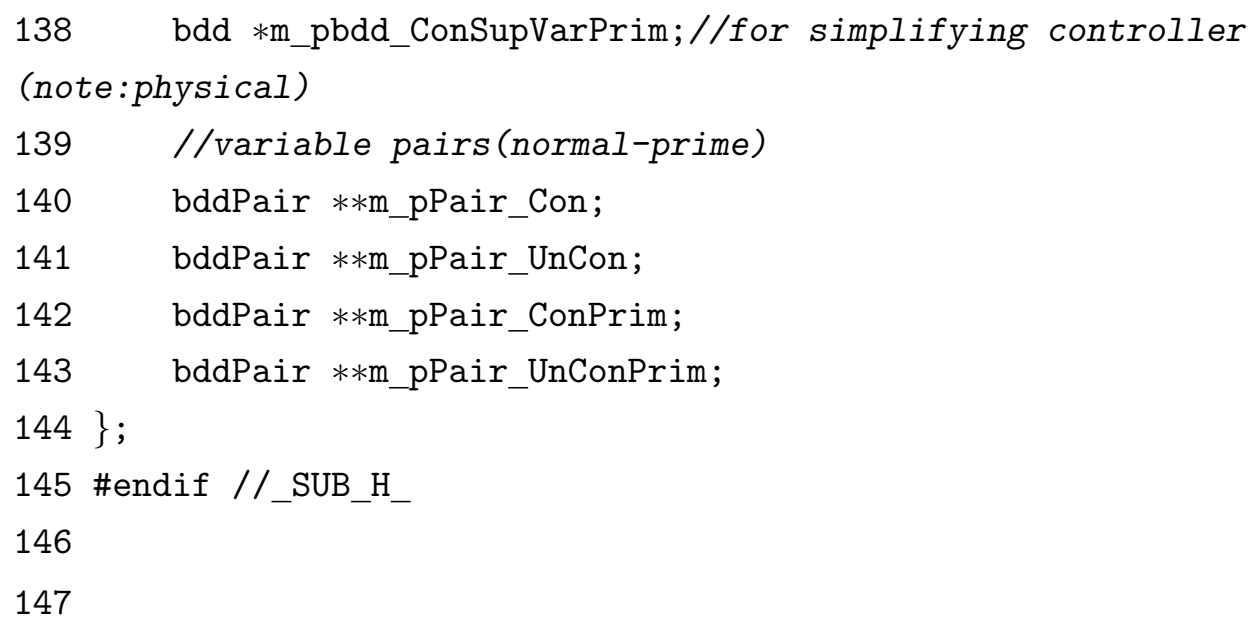

\section{Sub.cpp}

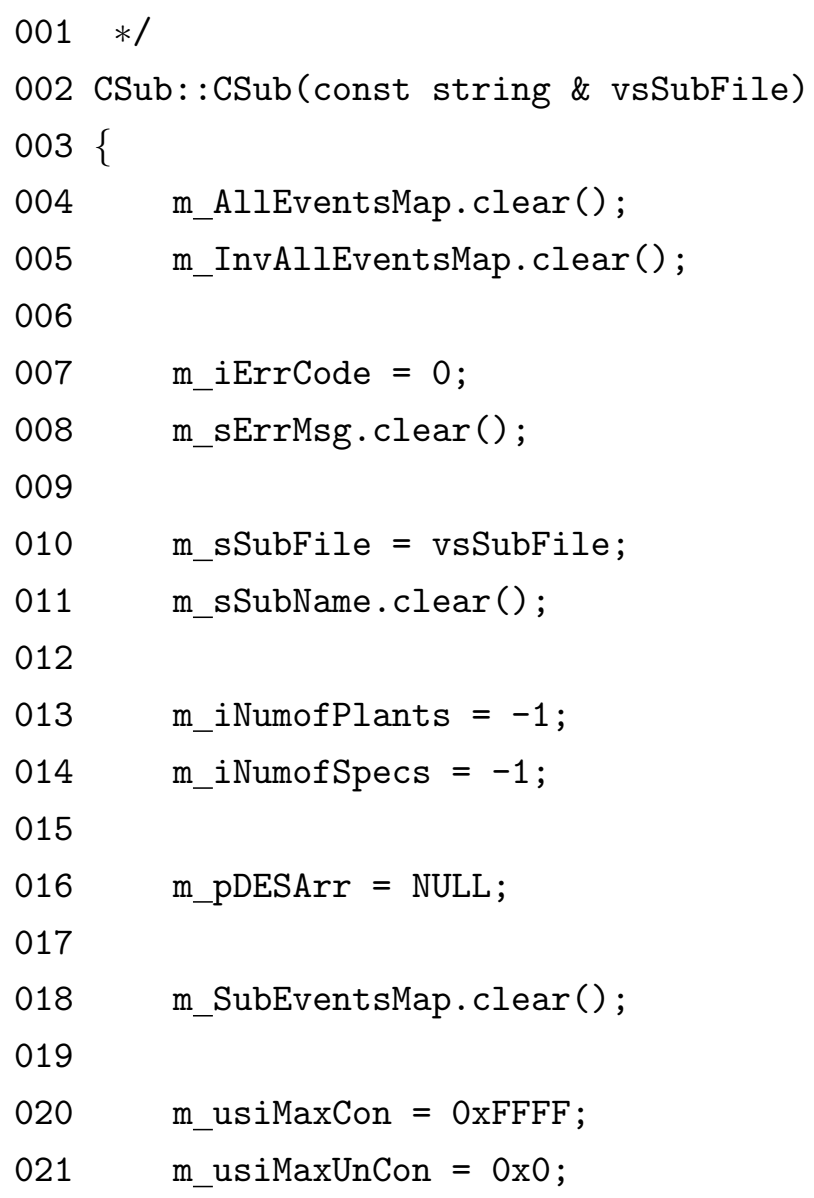




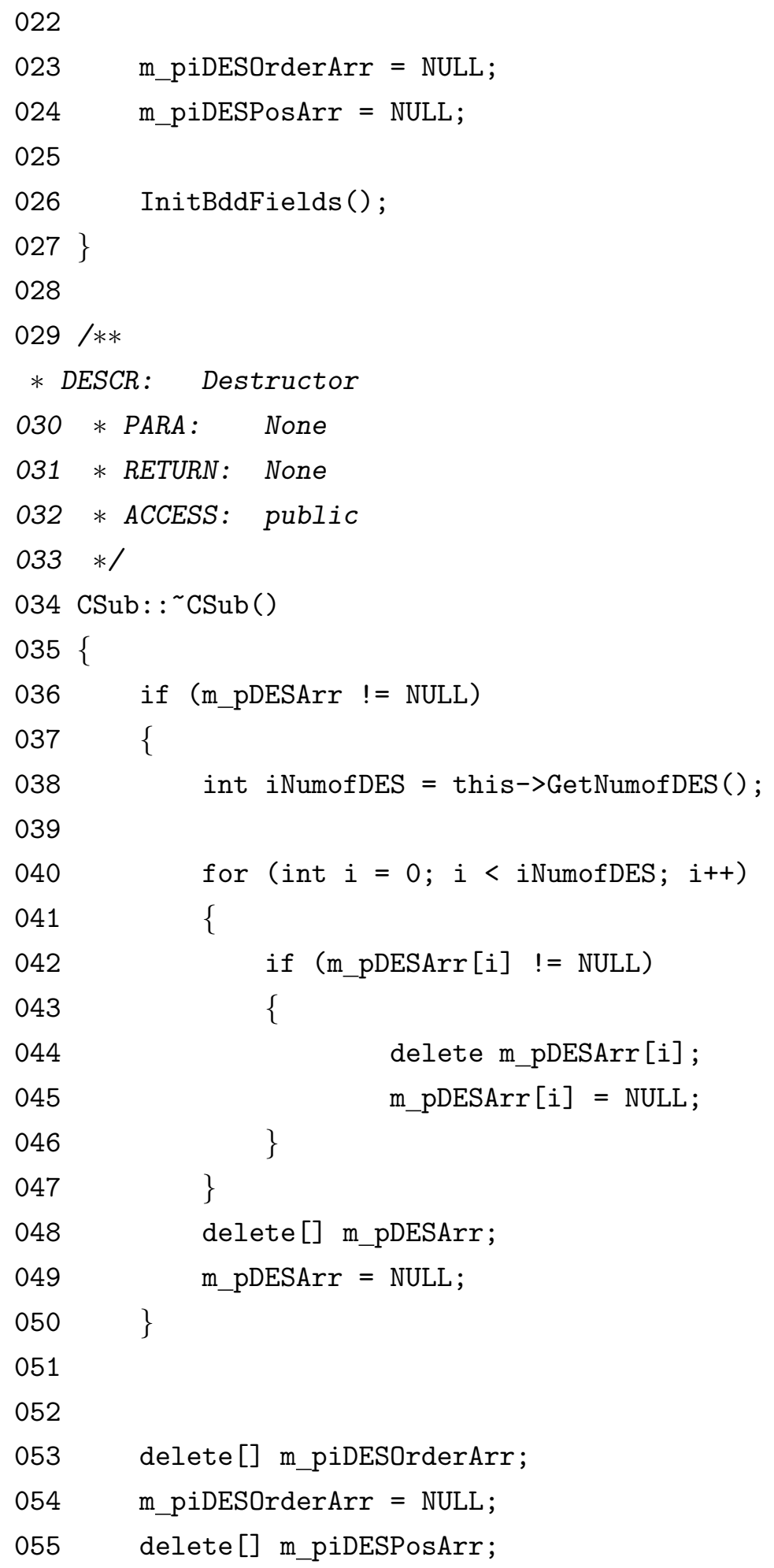




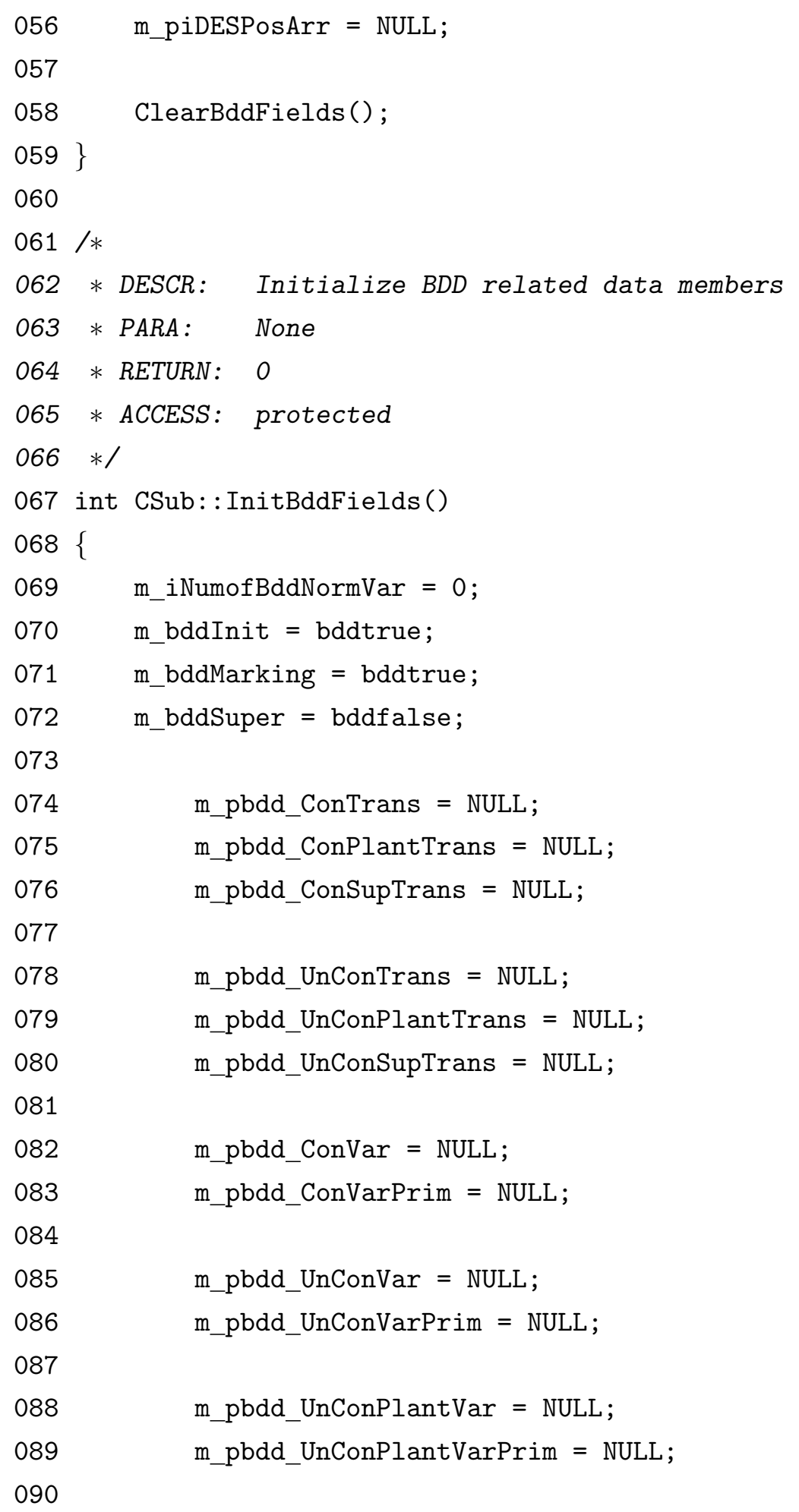




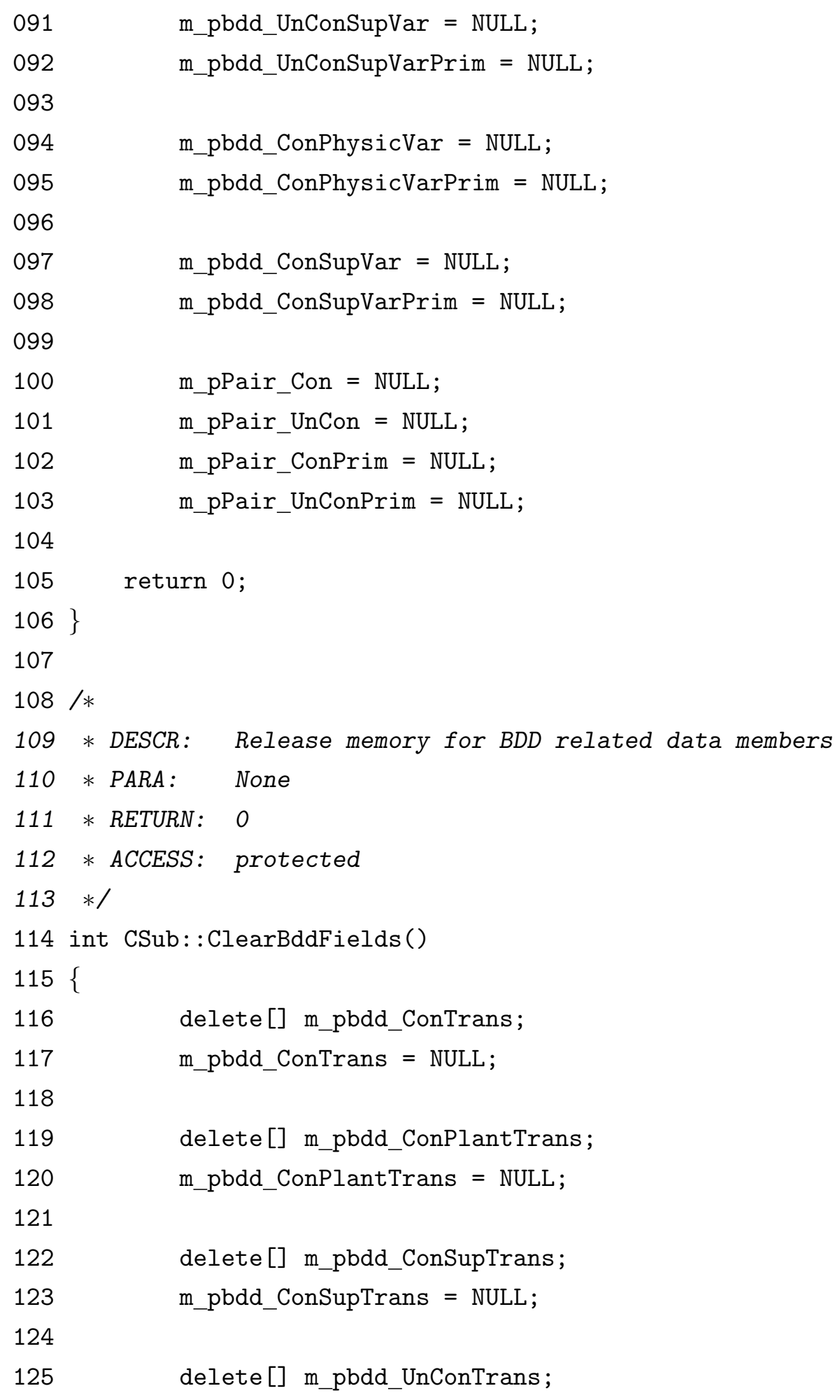


m_pbdd_UnConTrans $=$ NULL;

127

128

129

delete [] m_pbdd_UnConPlantTrans;

130

131

m_pbdd_UnConPlantTrans = NULL;

132

delete [] m_pbdd_UnConSupTrans;

133

134

m_pbdd_UnConSupTrans = NULL;

135

delete[] m_pbdd_ConVar;

136

m_pbdd_ConVar $=$ NULL;

137

delete[] m_pbdd_UnConVar;

138

139

$\mathrm{m} \_$pbdd_UnConVar $=$NULL;

140

141

142

delete[] m_pbdd_ConVarPrim;

m_pbdd_ConVarPrim = NULL;

143

144

145

delete [] m_pbdd_UnConVarPrim;

m_pbdd_UnConVarPrim = NULL;

146

147

delete [] m_pbdd_UnConPlantVar;

148

149

150

m_pbdd_UnConPlantVar = NULL;

151

delete[] m_pbdd_UnConPlantVarPrim;

152

m_pbdd_UnConPlantVarPrim = NULL;

153

154

155

delete [] m_pbdd_UnConSupVar;

m_pbdd_UnConSupVar = NULL;

delete[] m_pbdd_UnConSupVarPrim;

m_pbdd_UnConSupVarPrim = NULL;

156

157

158

159

delete[] m_pbdd_ConPhysicVar;

m_pbdd_ConPhysicVar = NULL;

delete[] m_pbdd_ConPhysicVarPrim;

160

m_pbdd_ConPhysicVarPrim = NULL;

delete [] m_pbdd_ConSupVar;

m_pbdd_ConSupVar = NULL; 


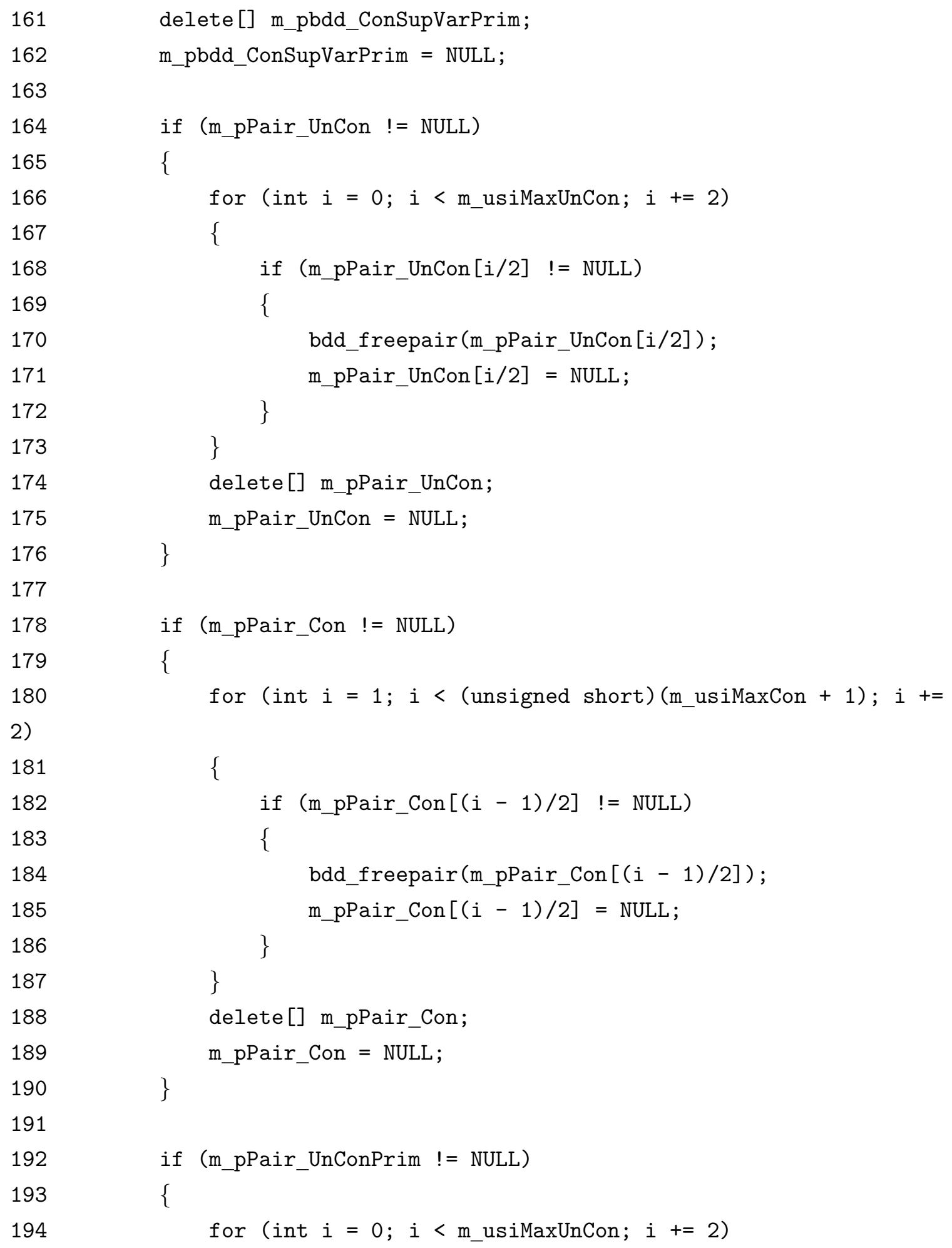




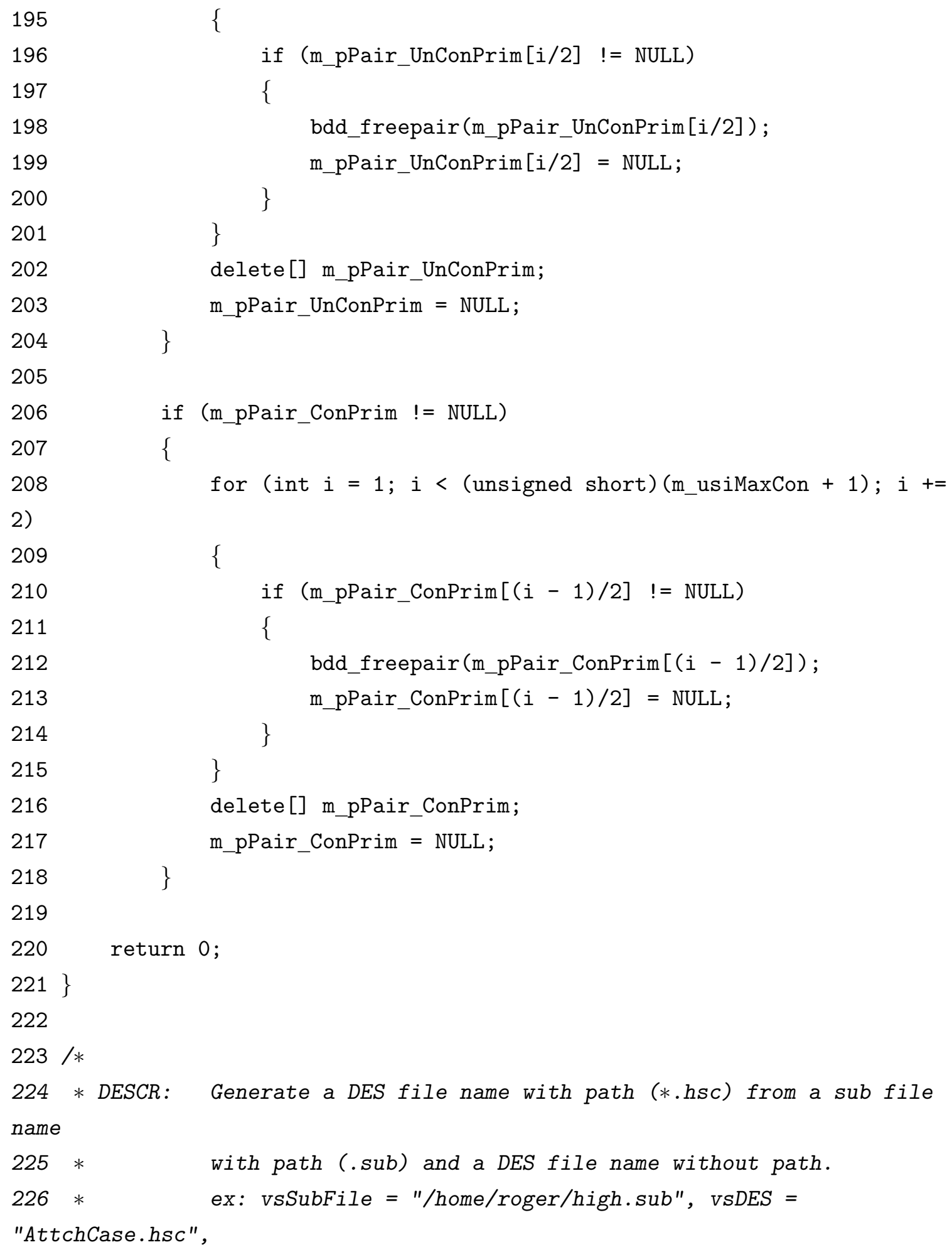




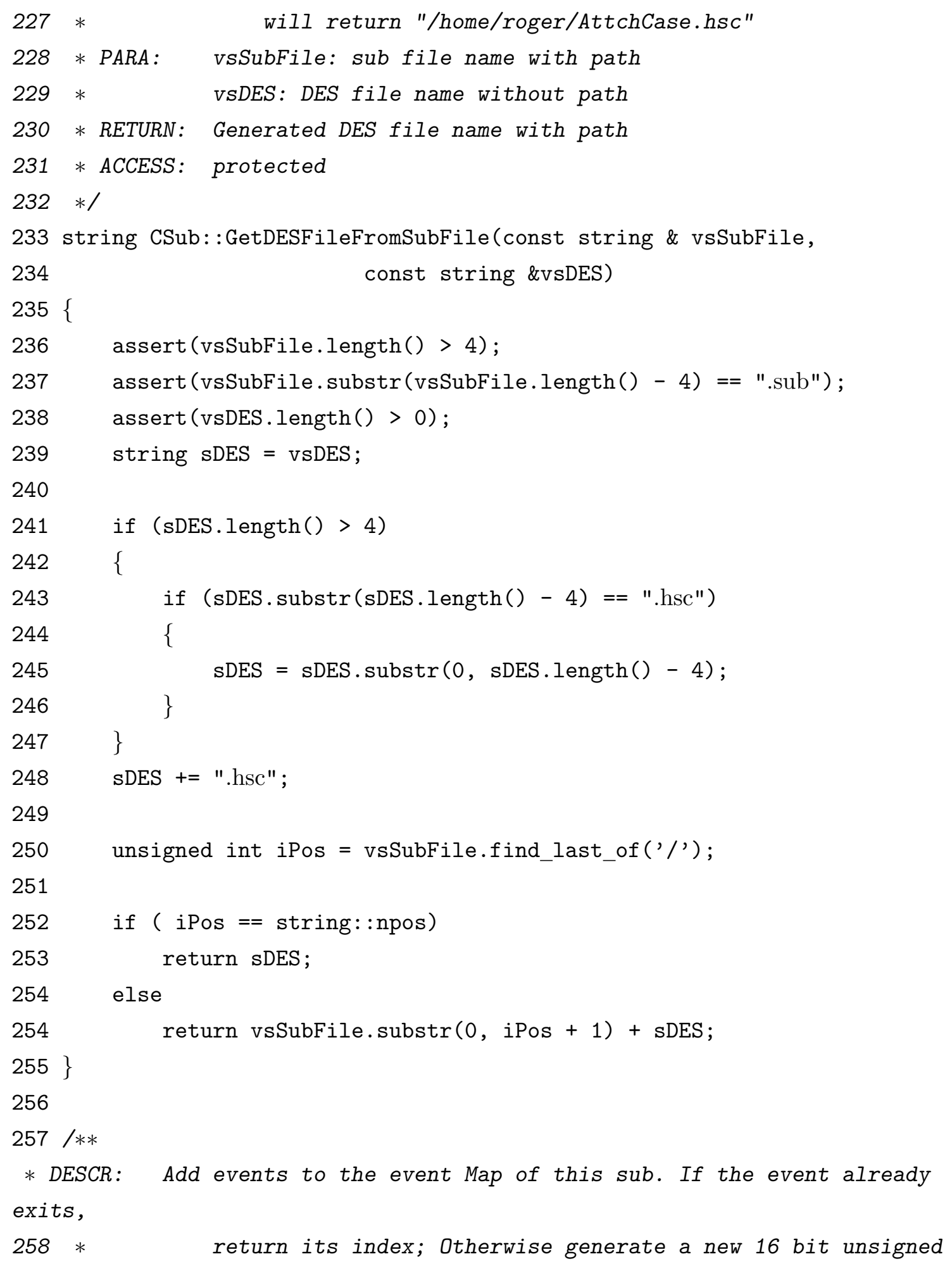




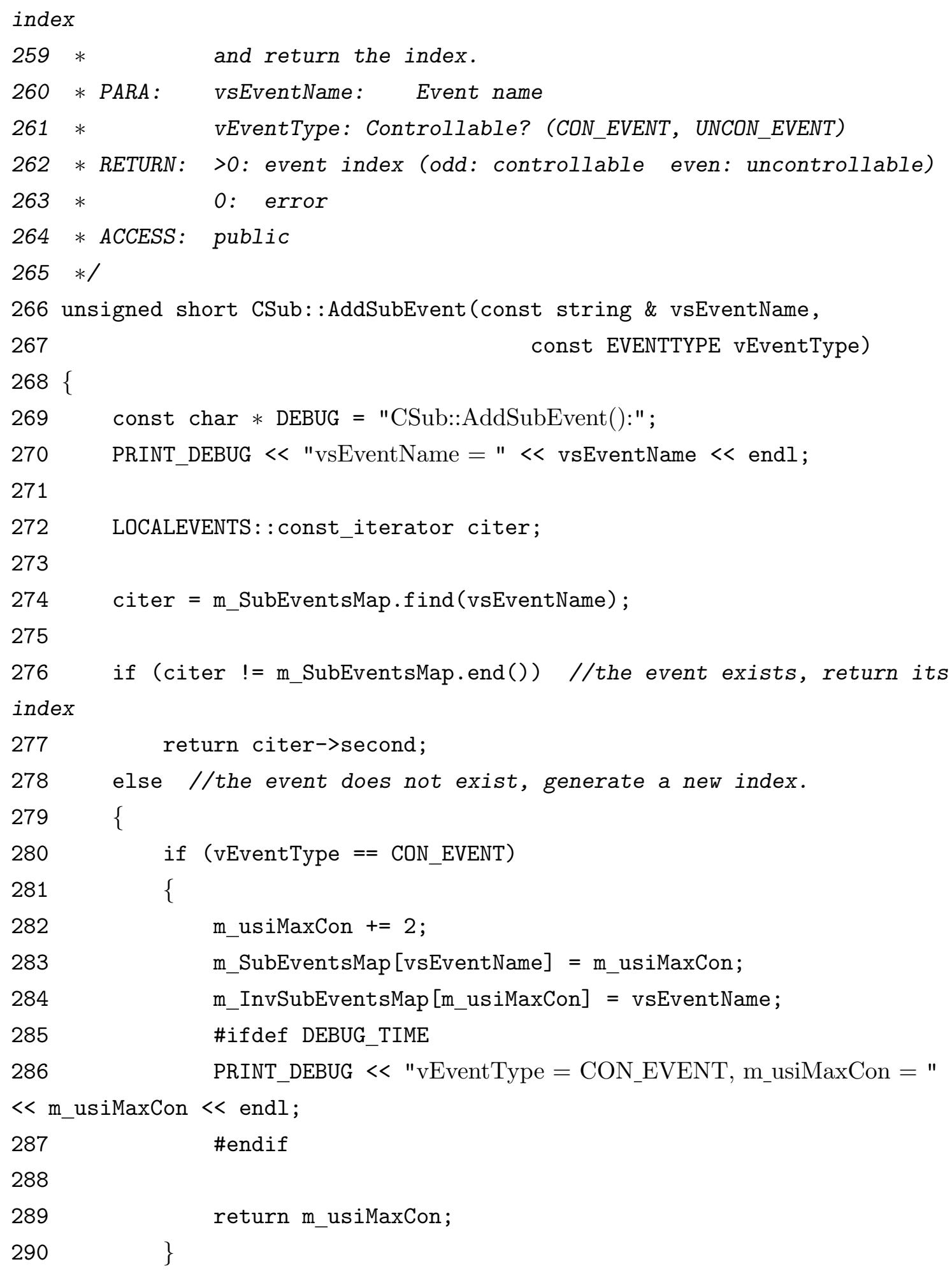




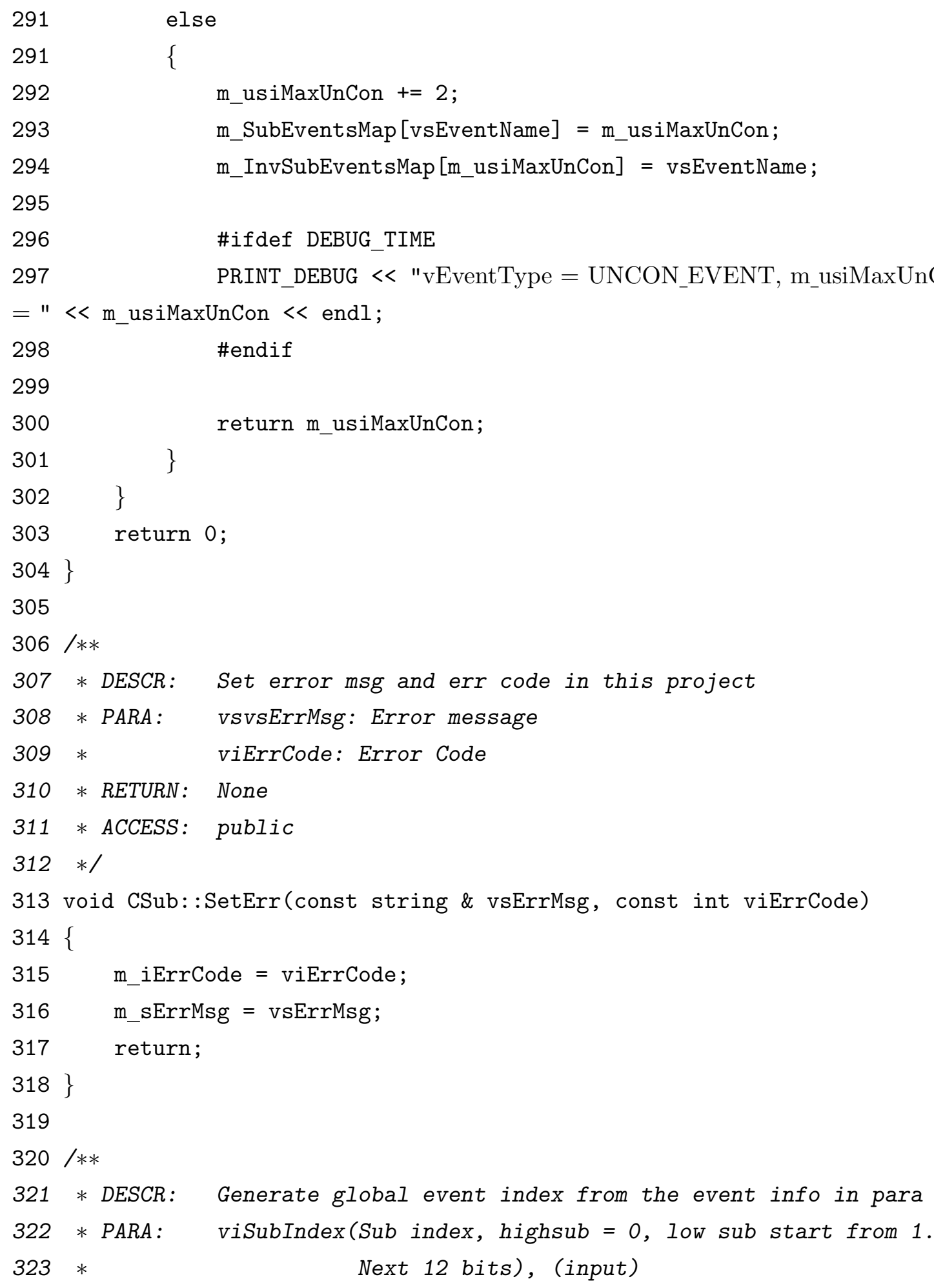




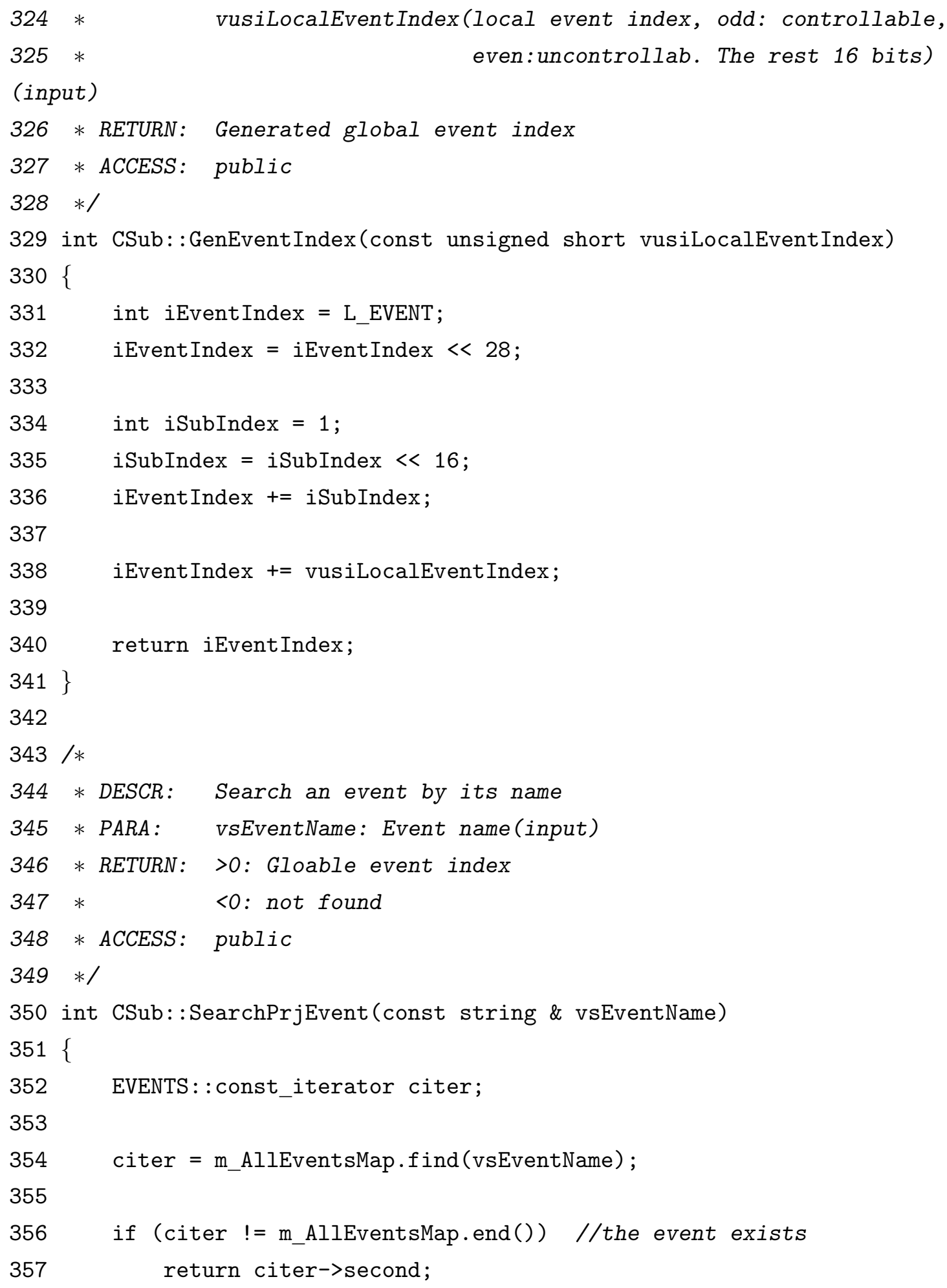




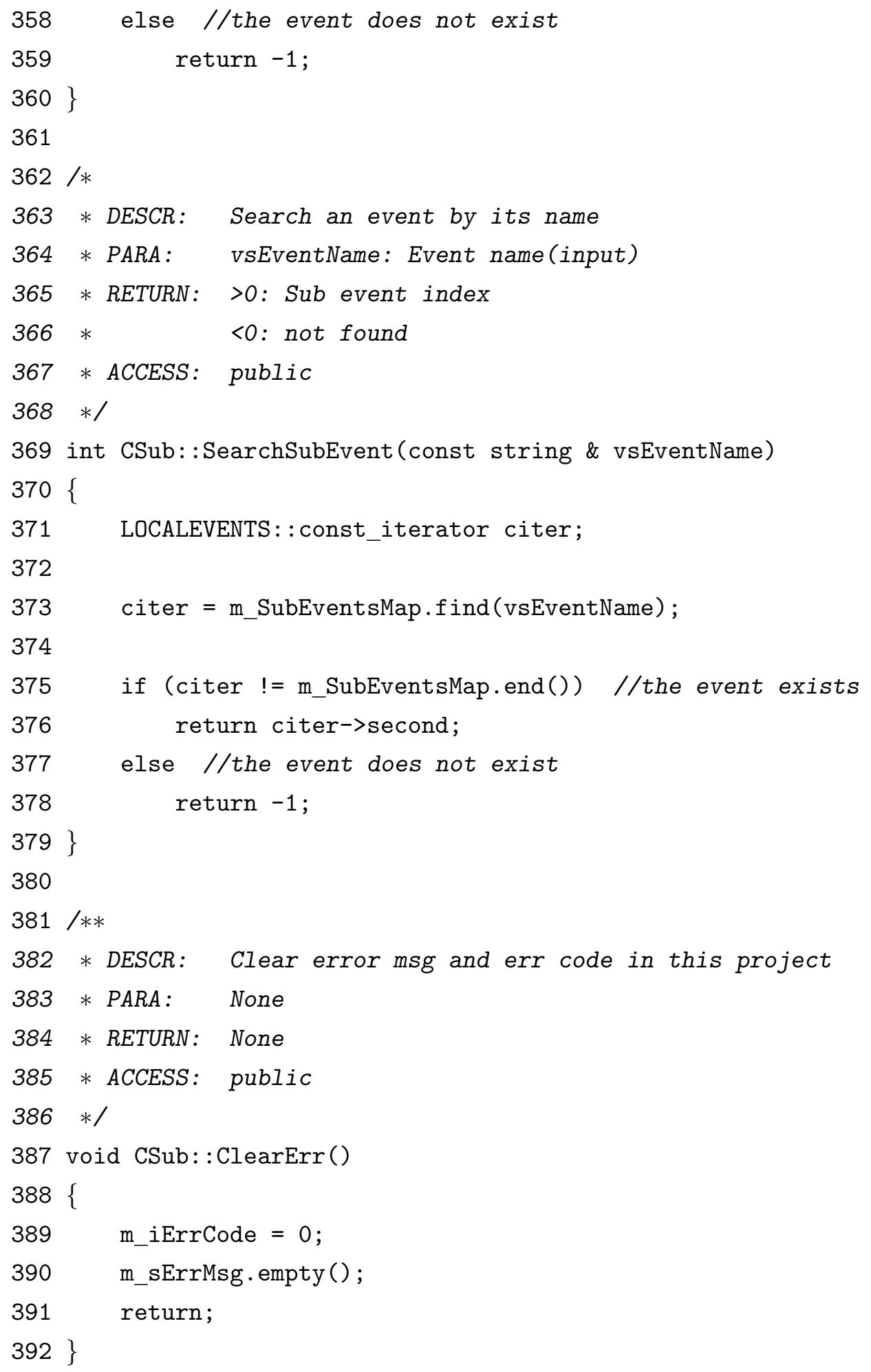




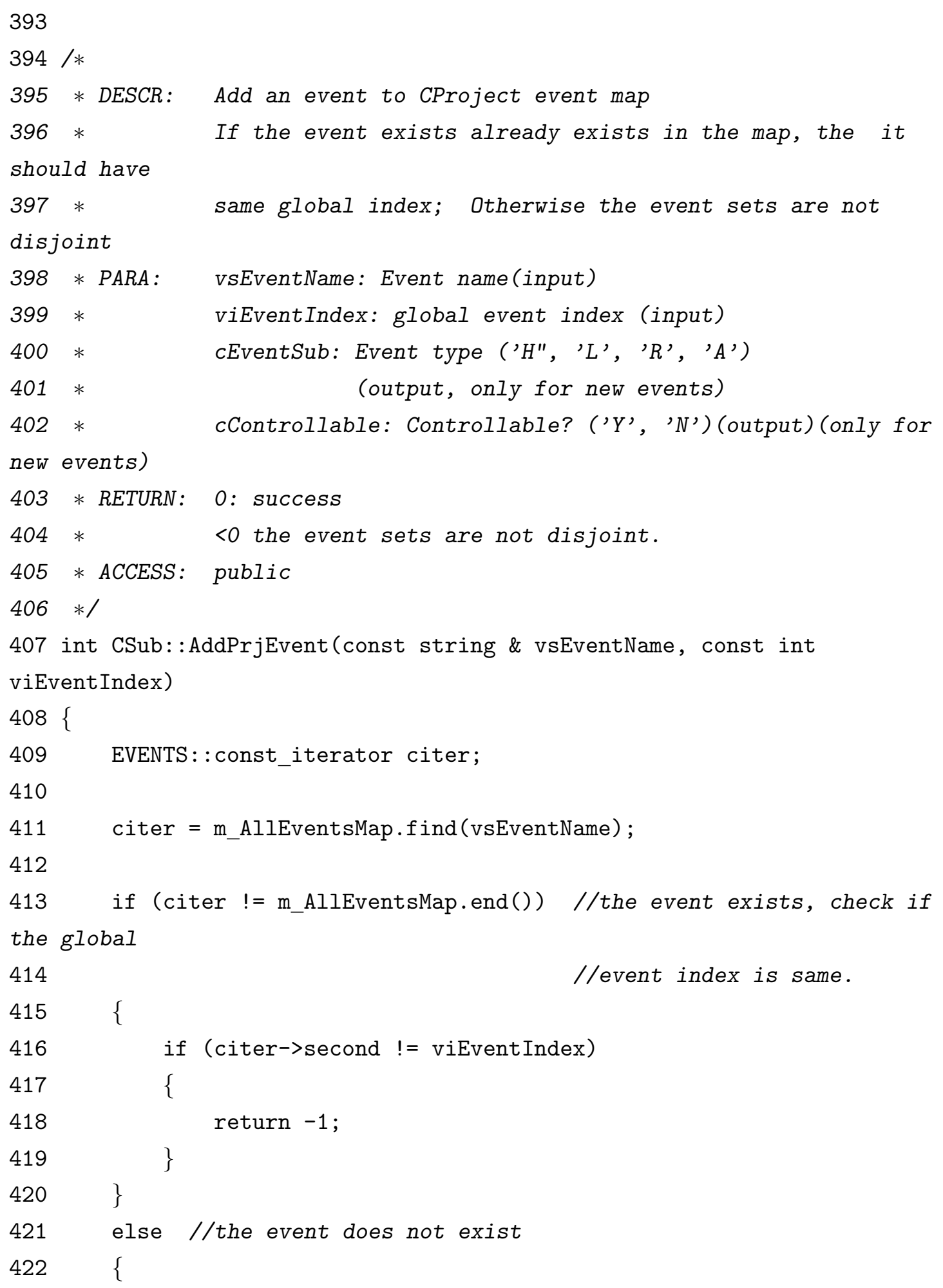




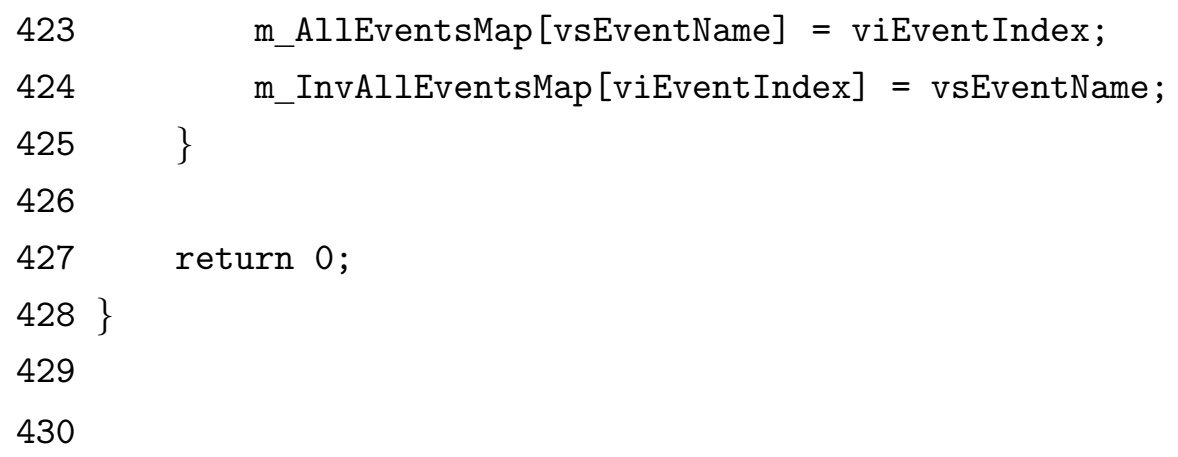

\section{Sub1.cpp}

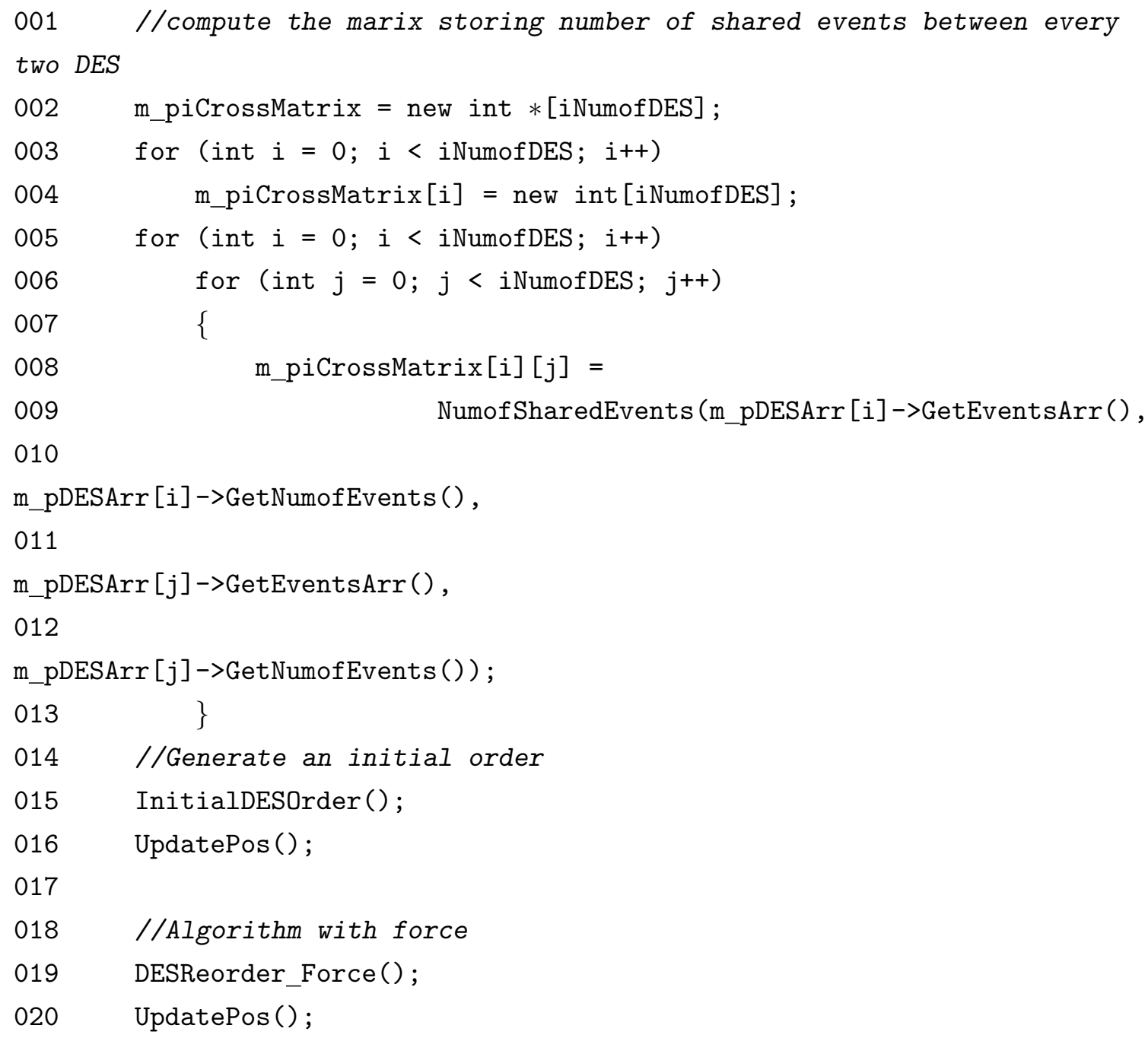




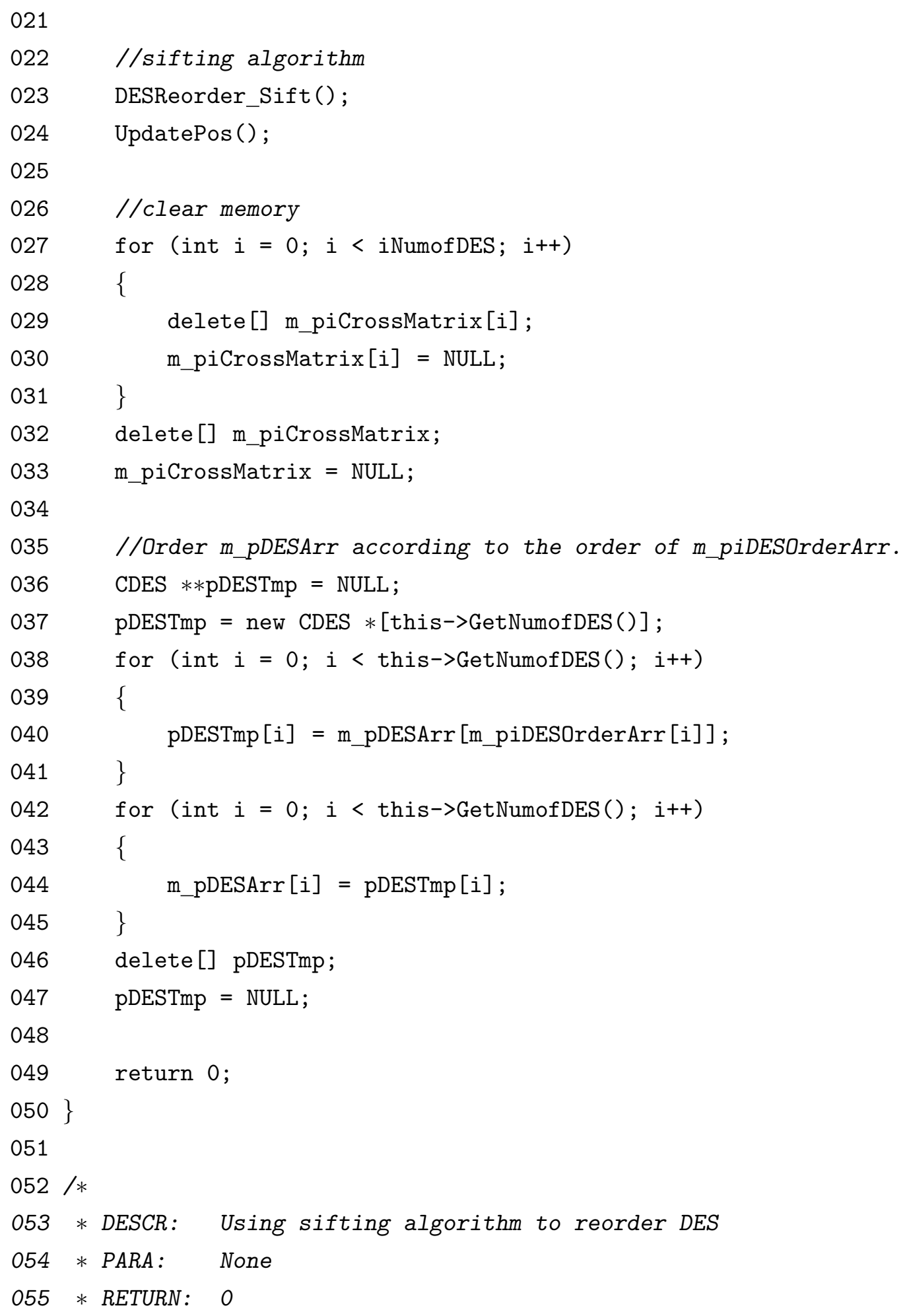




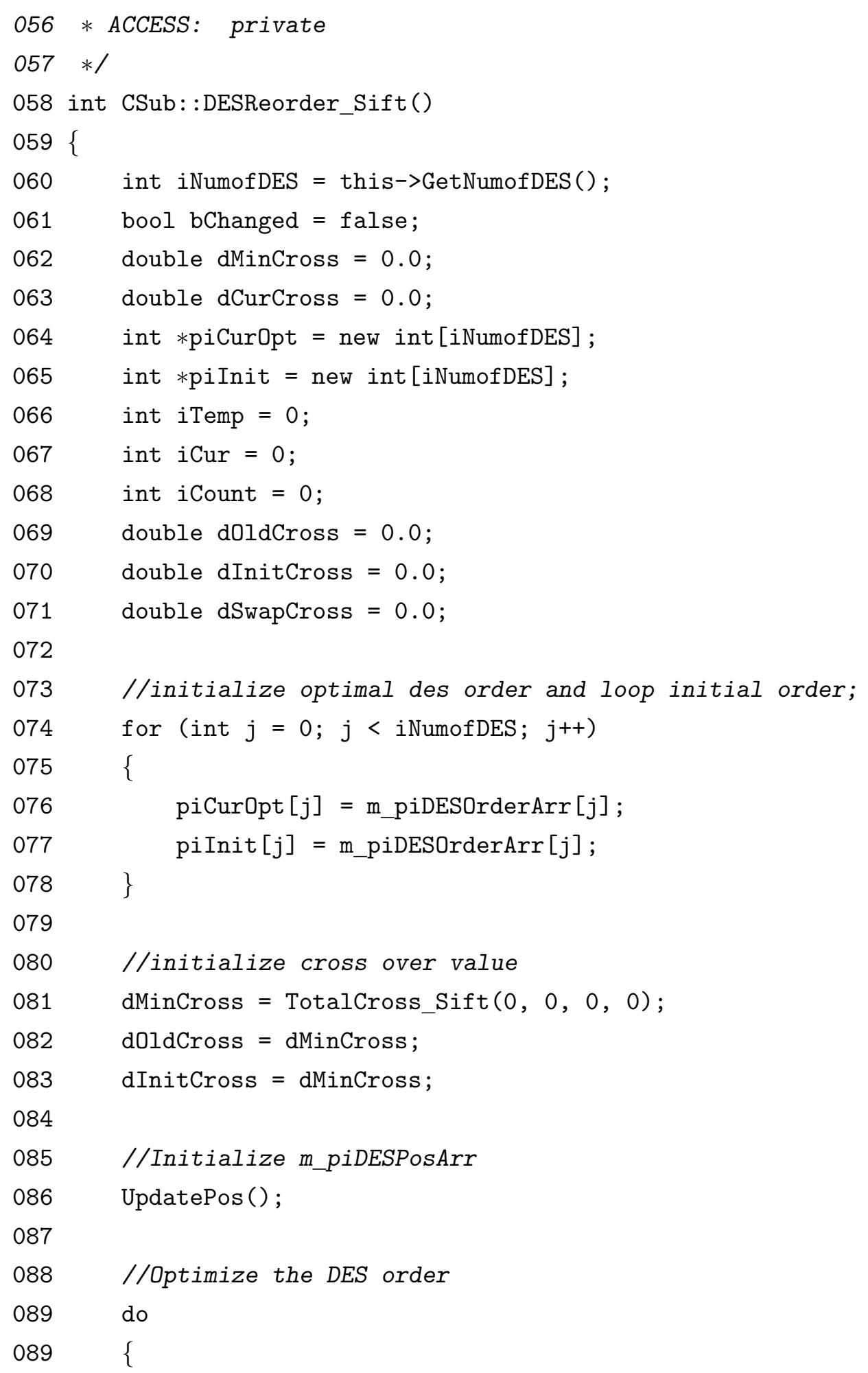




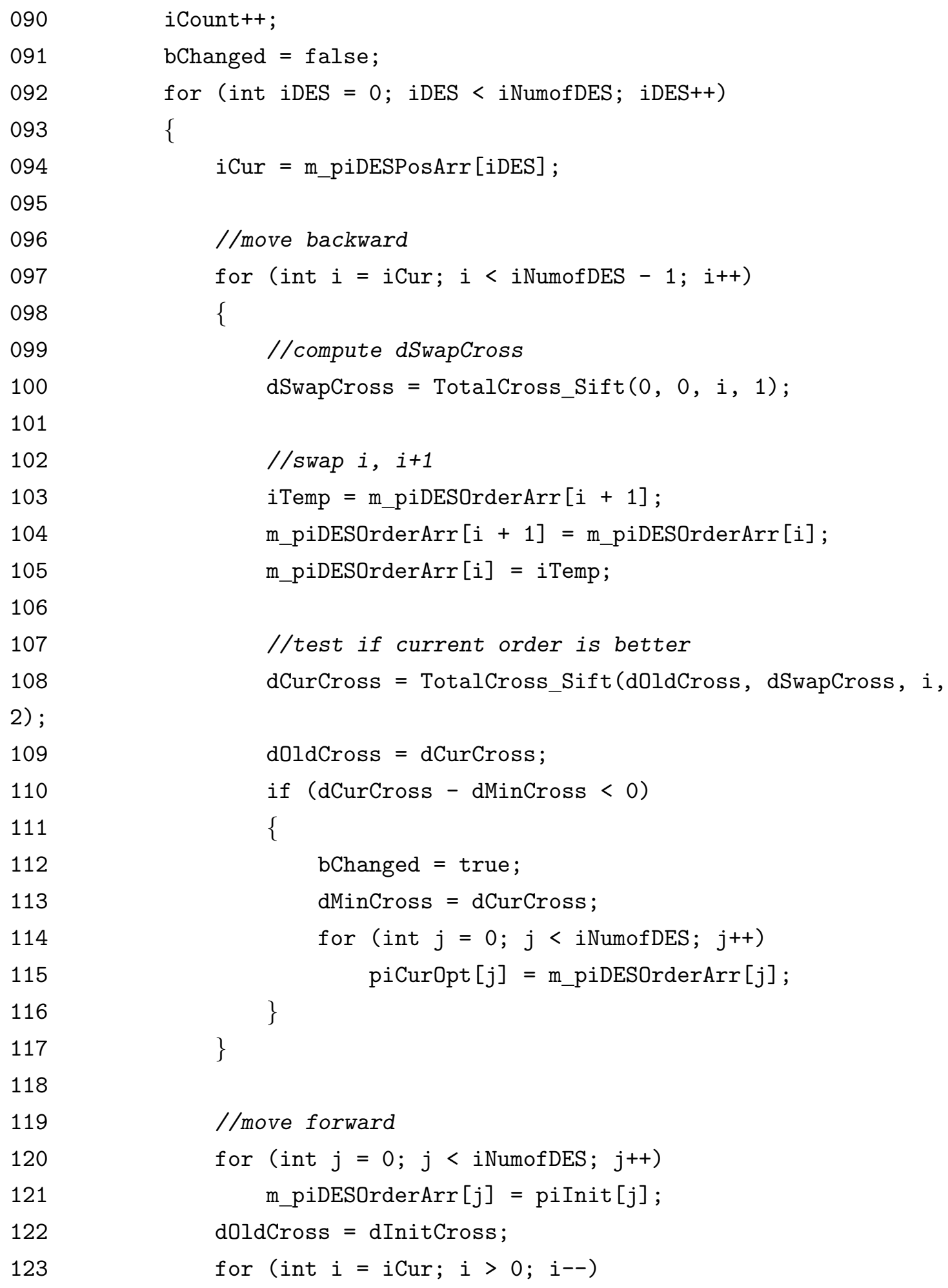


124

125

126

127

128

129

130

131

132

133

134

1,2);

135

136

137

138

139

140

141

142

143

144

145

146

147

148

149

150

151

152

153

154

155

155

156

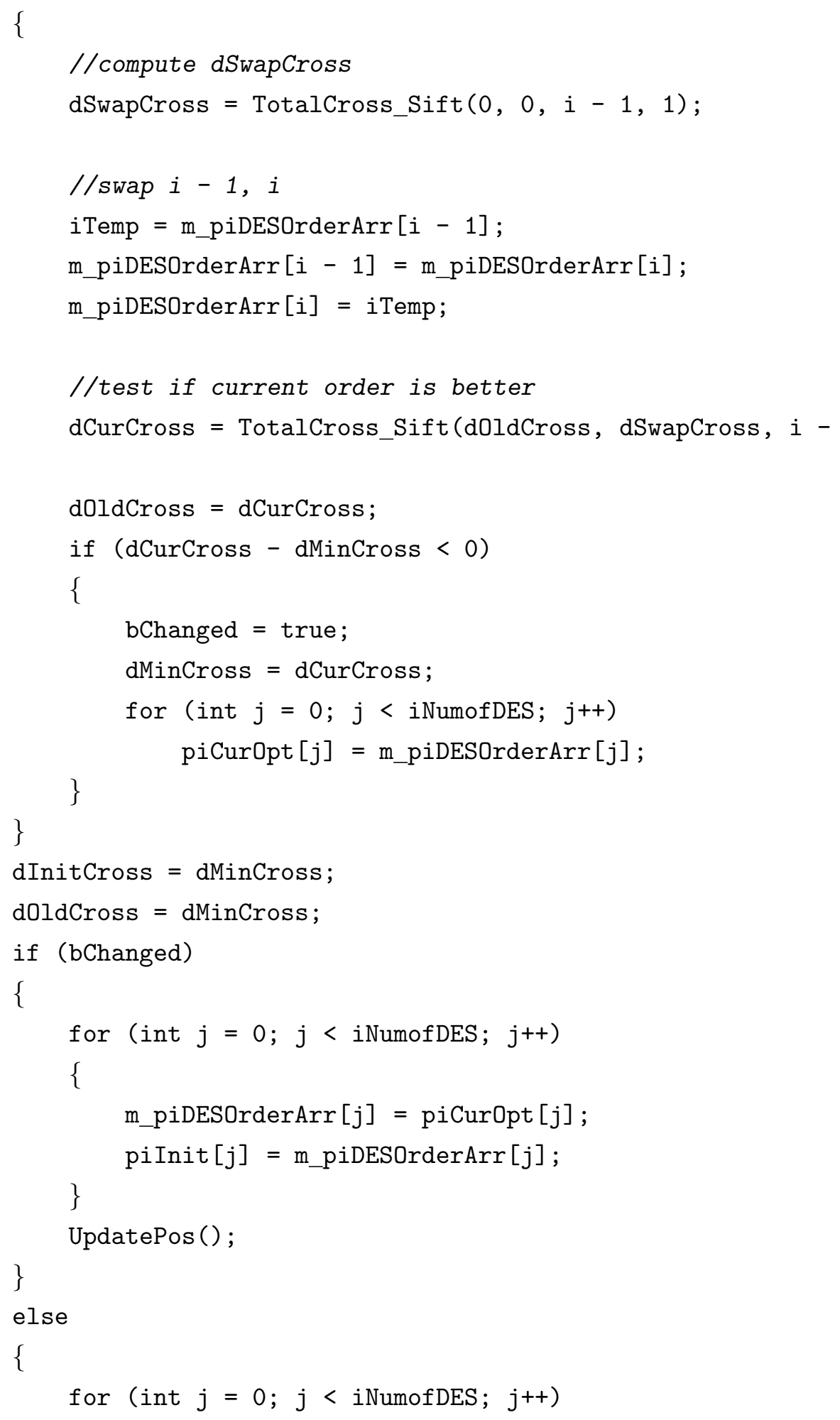




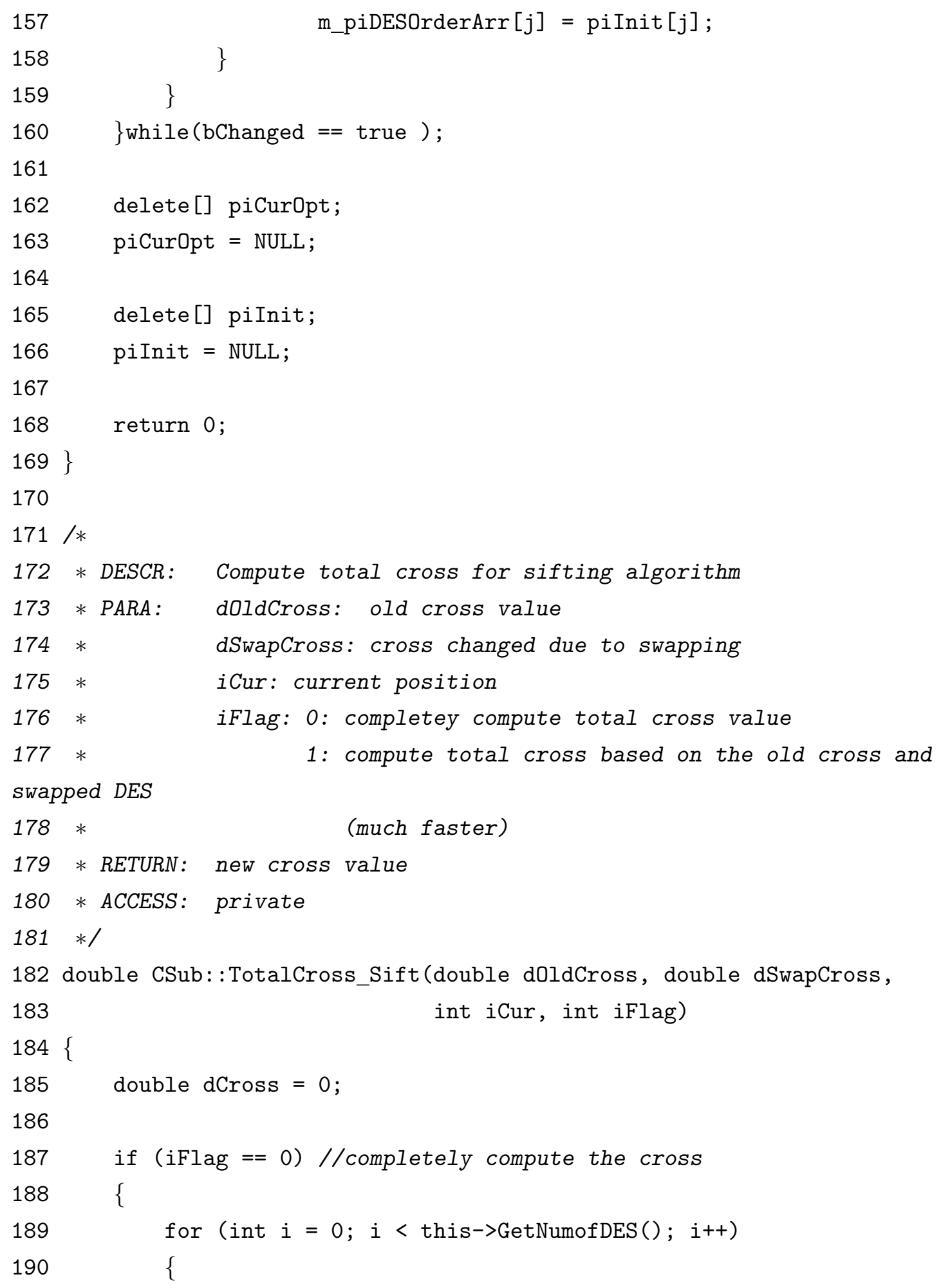




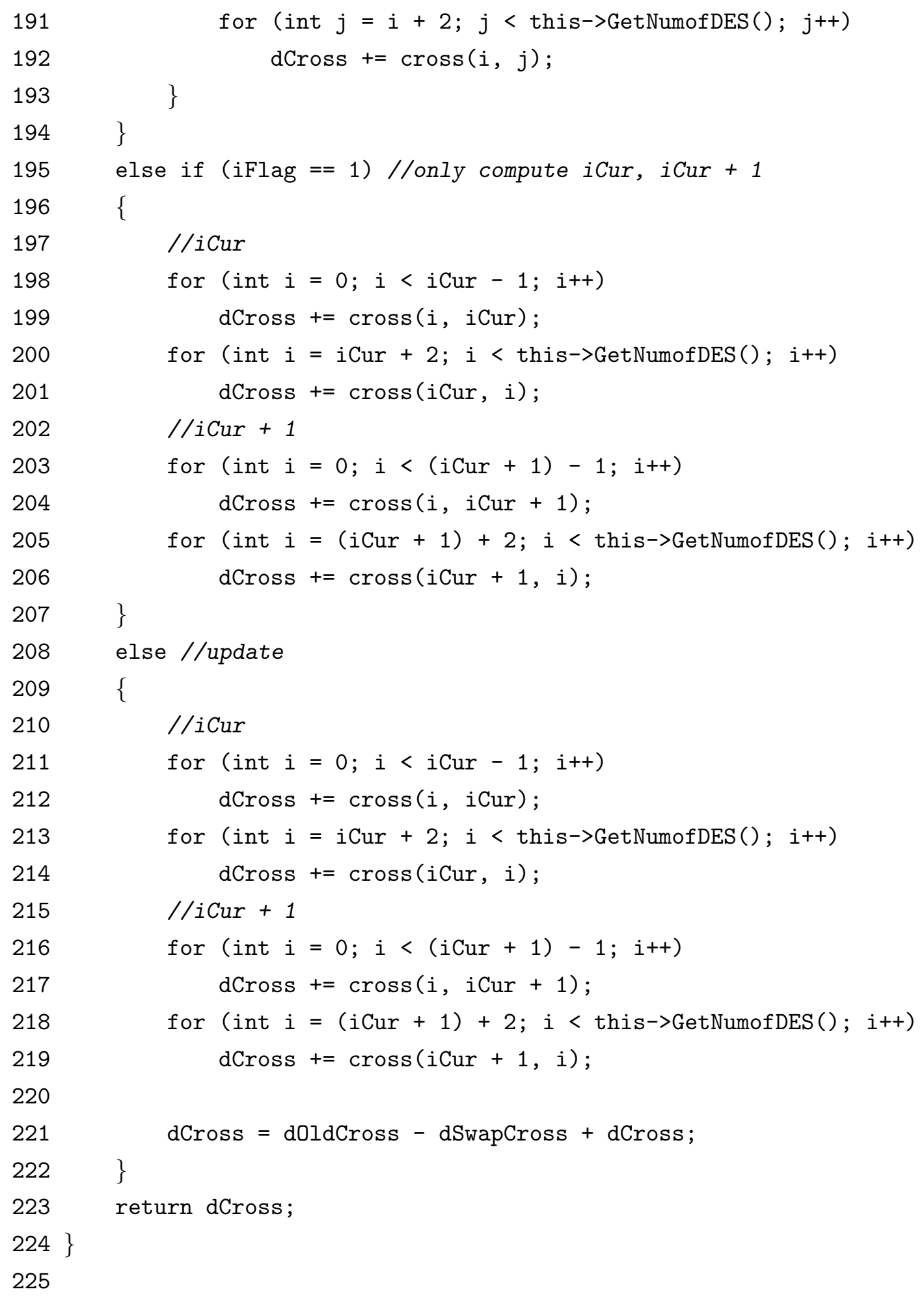




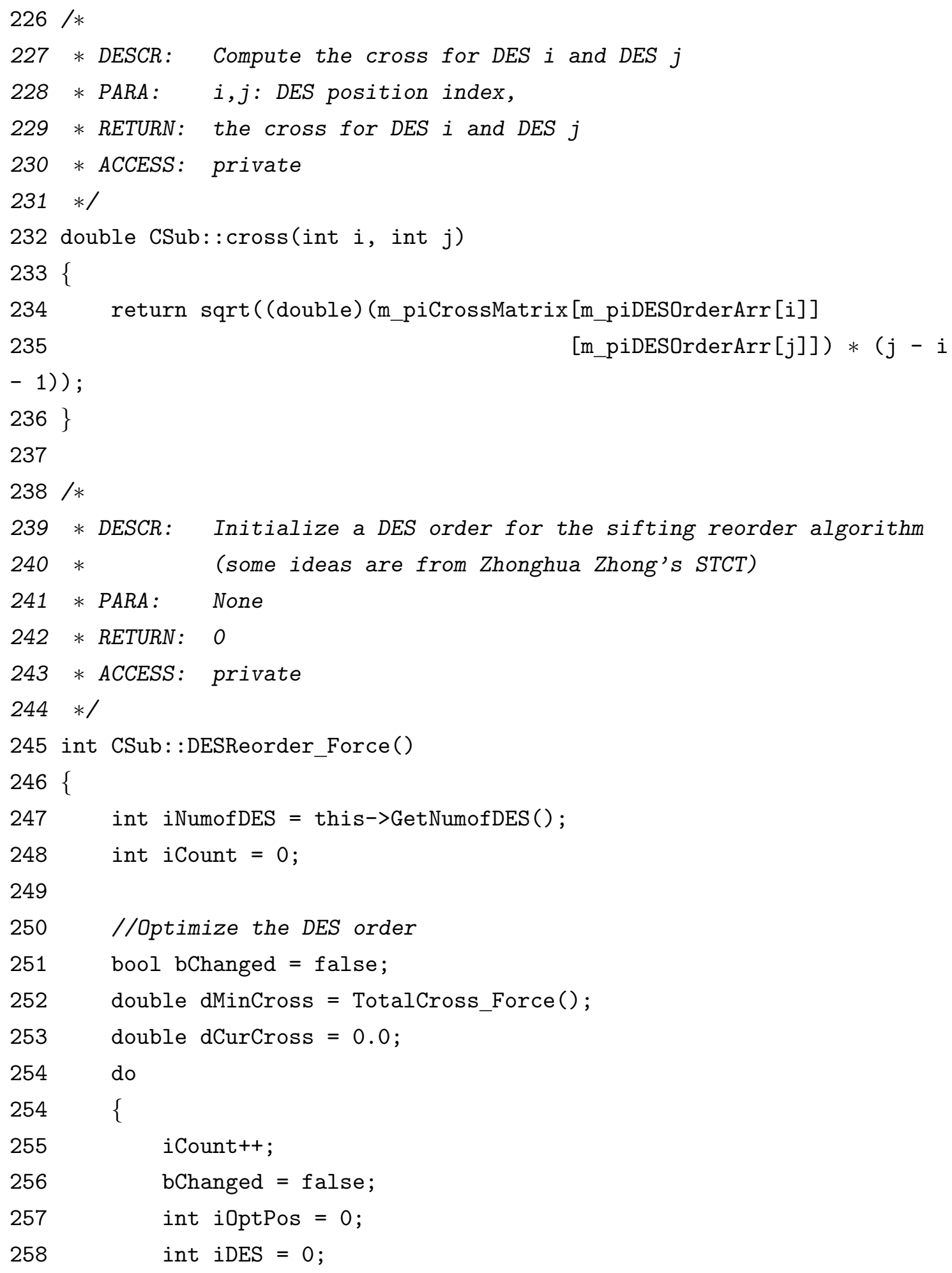




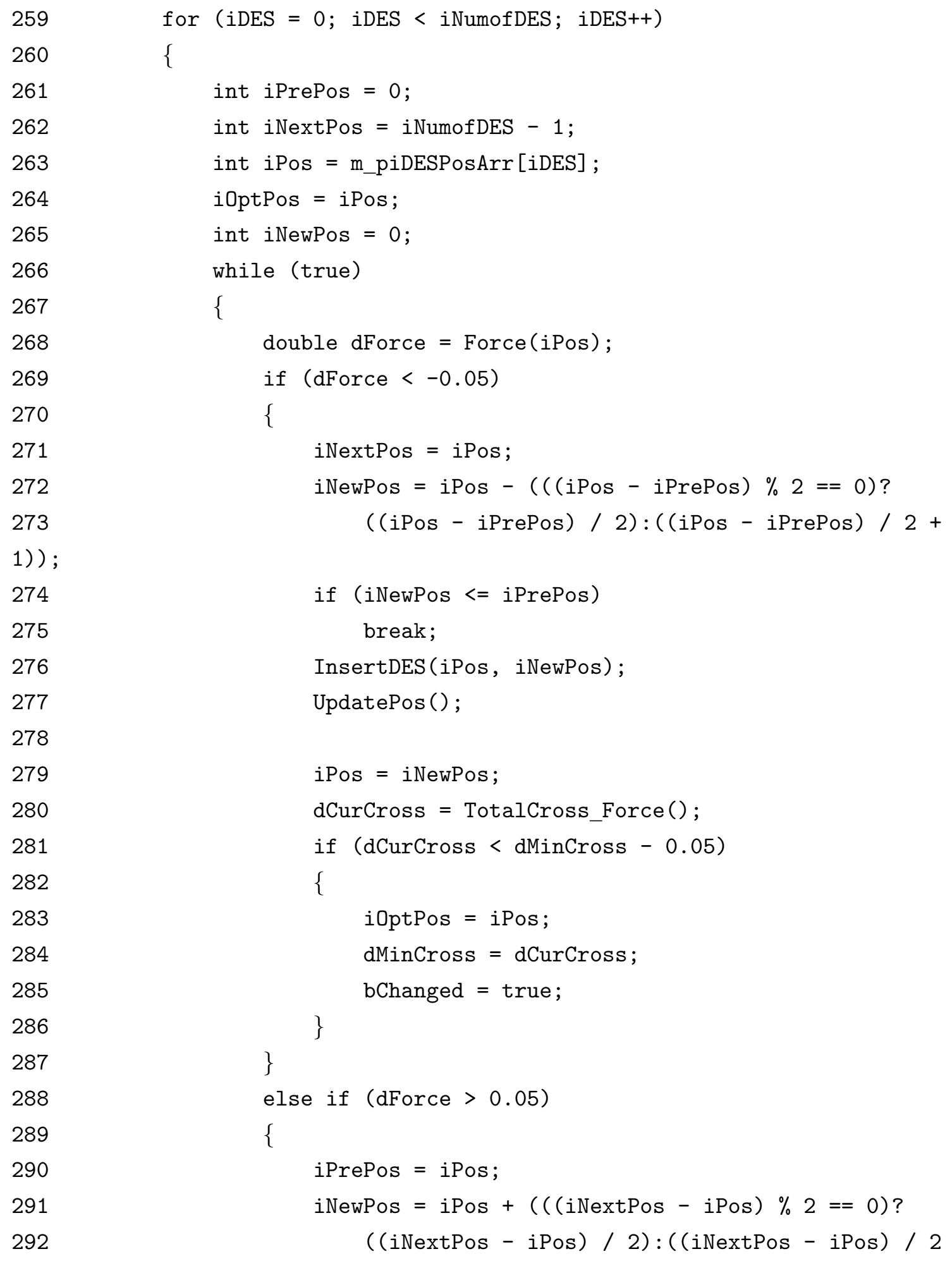


+ 1)); 293

294

295

296

297

298

299

300

301

302

303

304

305

306

307

307

308

309

310

311

312

313

314

$315\}$

316

$317 / *$

318 * DESCR: Update DES position in array m_piDESPosArr according the new order

319 * PARA: None

$320 *$ RETURN: None

$321 *$ ACCESS: private

$322 * /$

323 void CSub: :UpdatePos()

324\{ if (iNextPos <= iNewPos) break;

UpdatePos();

iPos = iNewPos;

dCurCross = TotalCross_Force () ;

if (dCurCross < dMinCross - 0.05)

\{

iOptPos = iPos;

dMinCross = dCurCross;

bChanged = true;

\}

\}

else

break;

\}

InsertDES (m_piDESPosArr [iDES], iOptPos);

UpdatePos ();

\}while (bChanged == true);

return 0 ;
InsertDES (iPos, iNewPos); 


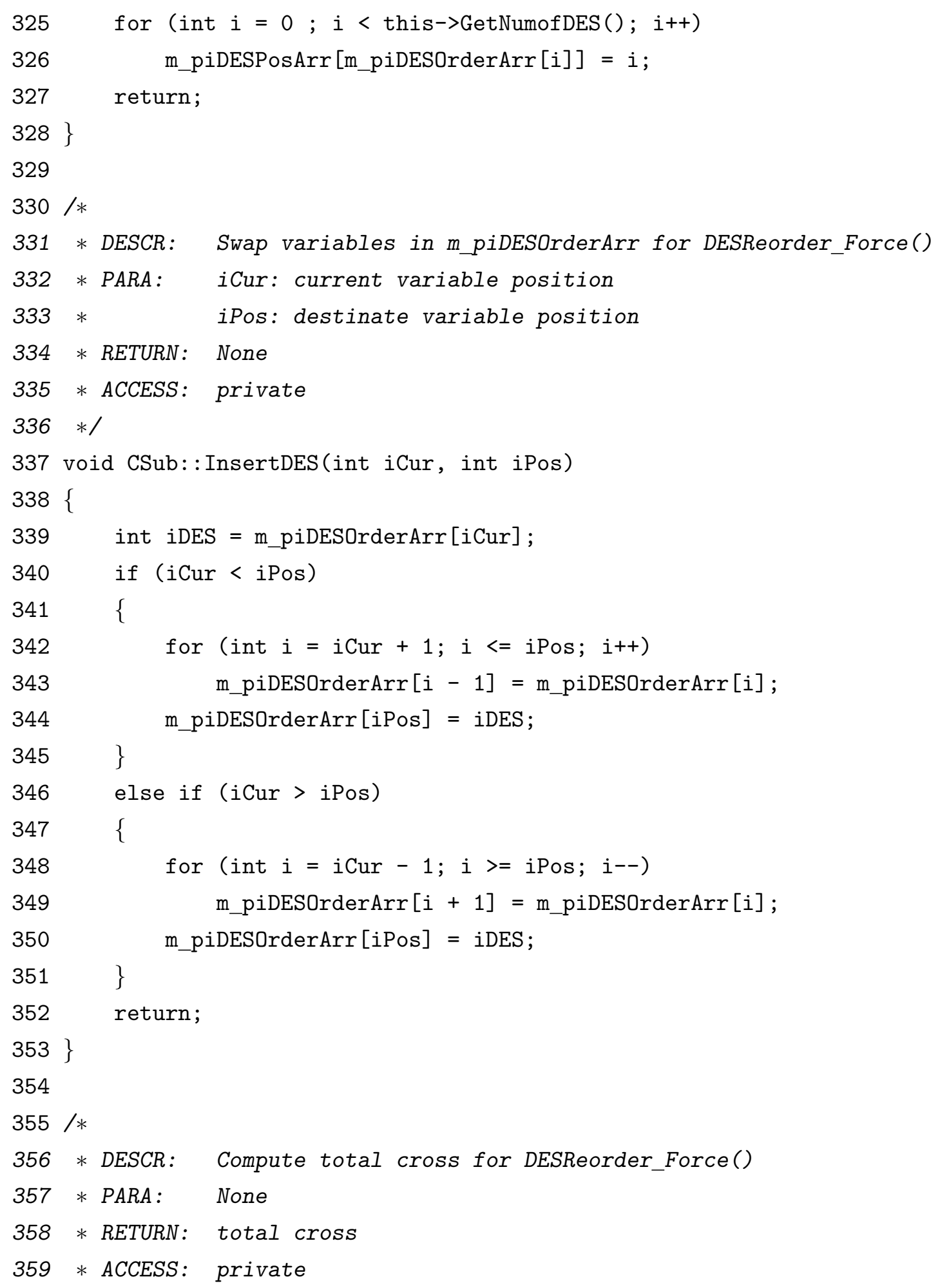




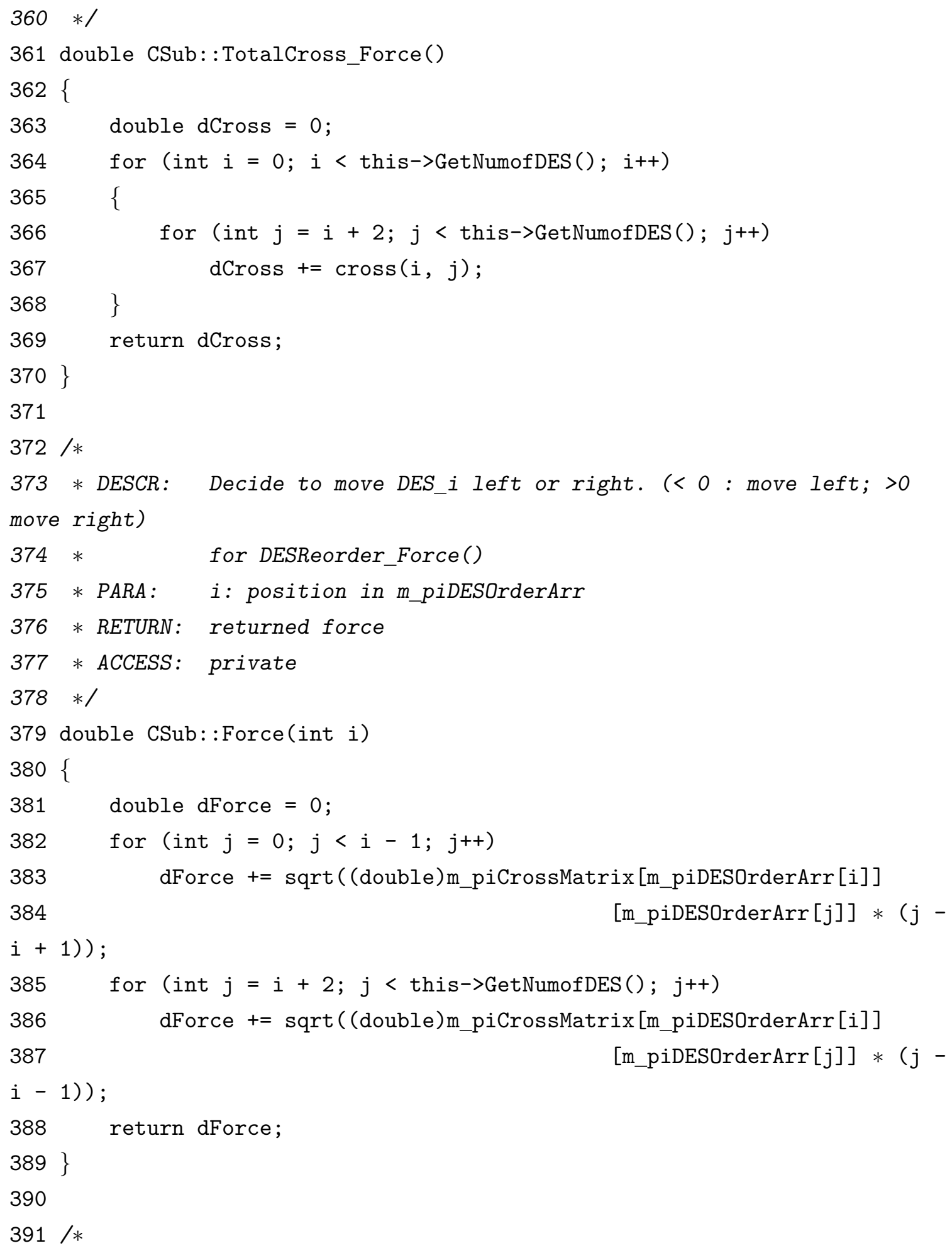




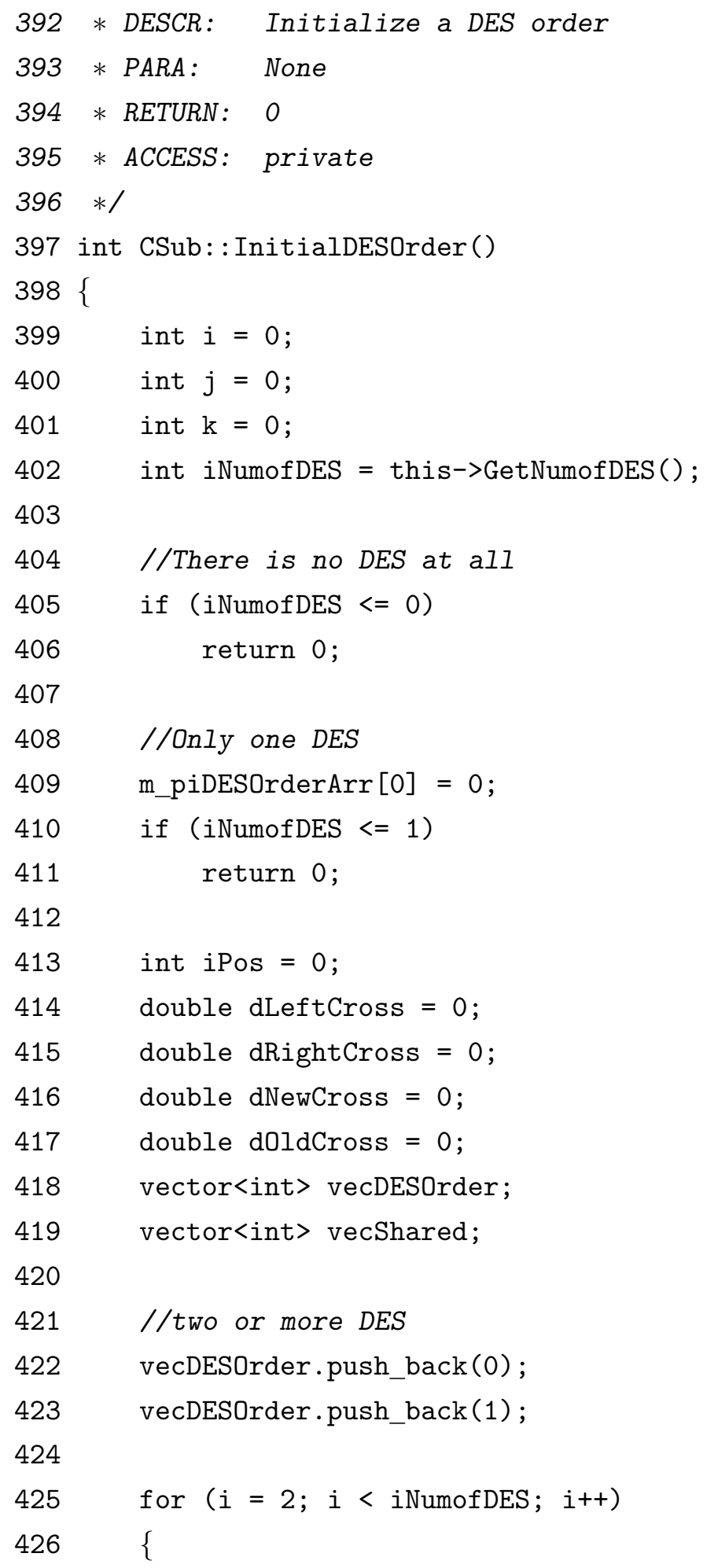




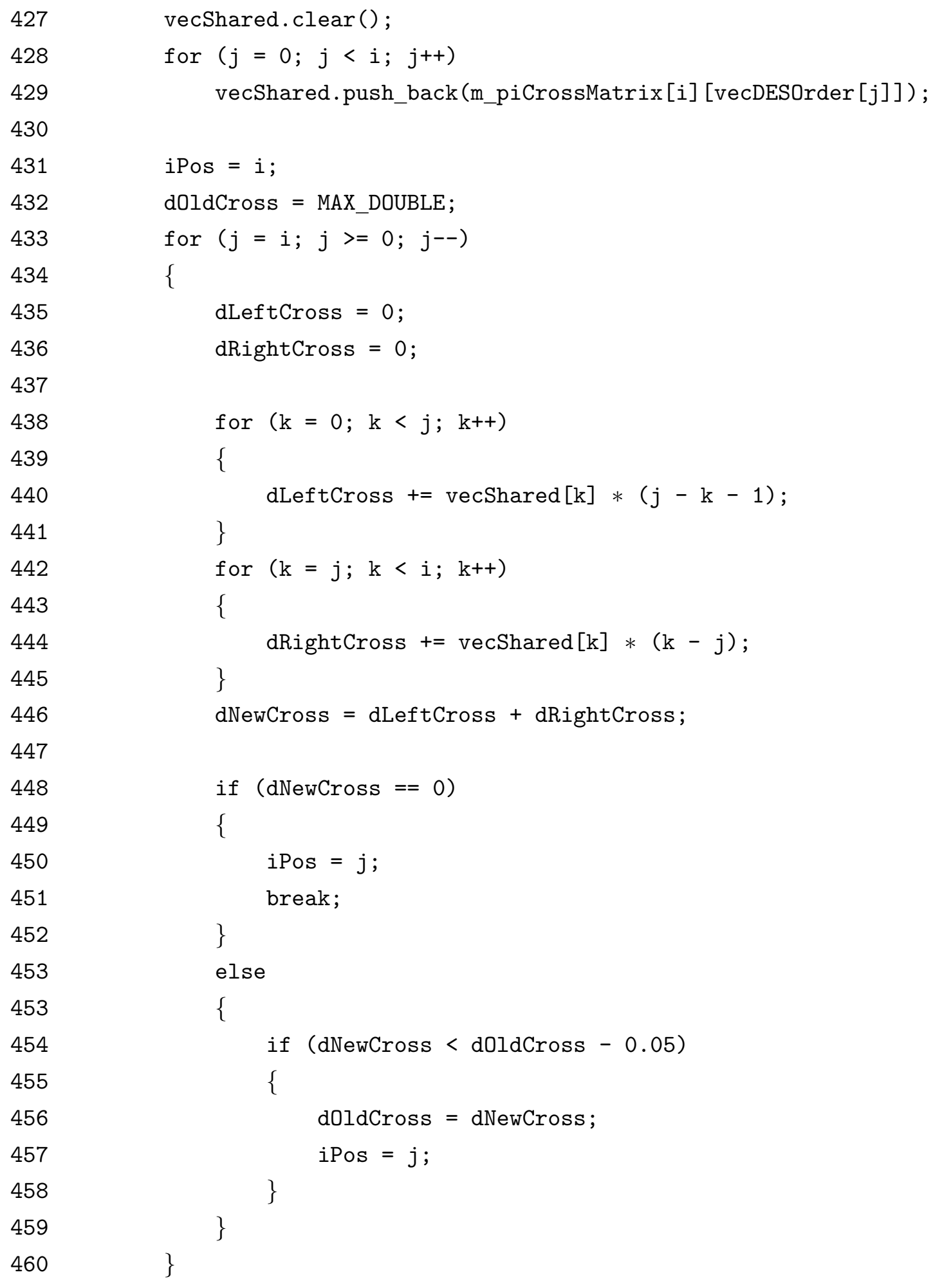


461

462

463

464

465

466

466

467

468

469

470

471

472

473

if $($ iPos $==0)$ vecDESOrder .insert (vecDESOrder.begin(), i);

else if (iPos $==$ i) vecDESOrder.push_back(i);

else

\{

vector<int>: : iterator itr $=$ vecDESOrder.begin () ;

itr $+=$ iPos;

vecDESOrder.insert(itr, i);

474

475 for $(i=0 ; i<$ (int)vecDESOrder.size ()$; i++)$

476\{

477 m_piDESOrderArr [i] = vecDESOrder [i];

$478 \quad\}$

479 return 0 ;

$480\}$

481

482

\section{Sub2.cpp}

001 bdd bddStates = bddStateSet;

002 int $*$ piStateSet $=$ fdd_scanallvar (bddStates);

003

004 int count $=0$;

005

006 while (piStateSet $!=$ NULL \&\& count $<3$ )

$007 \quad\{$

008 bdd bddVisitedState = bddtrue;

009 


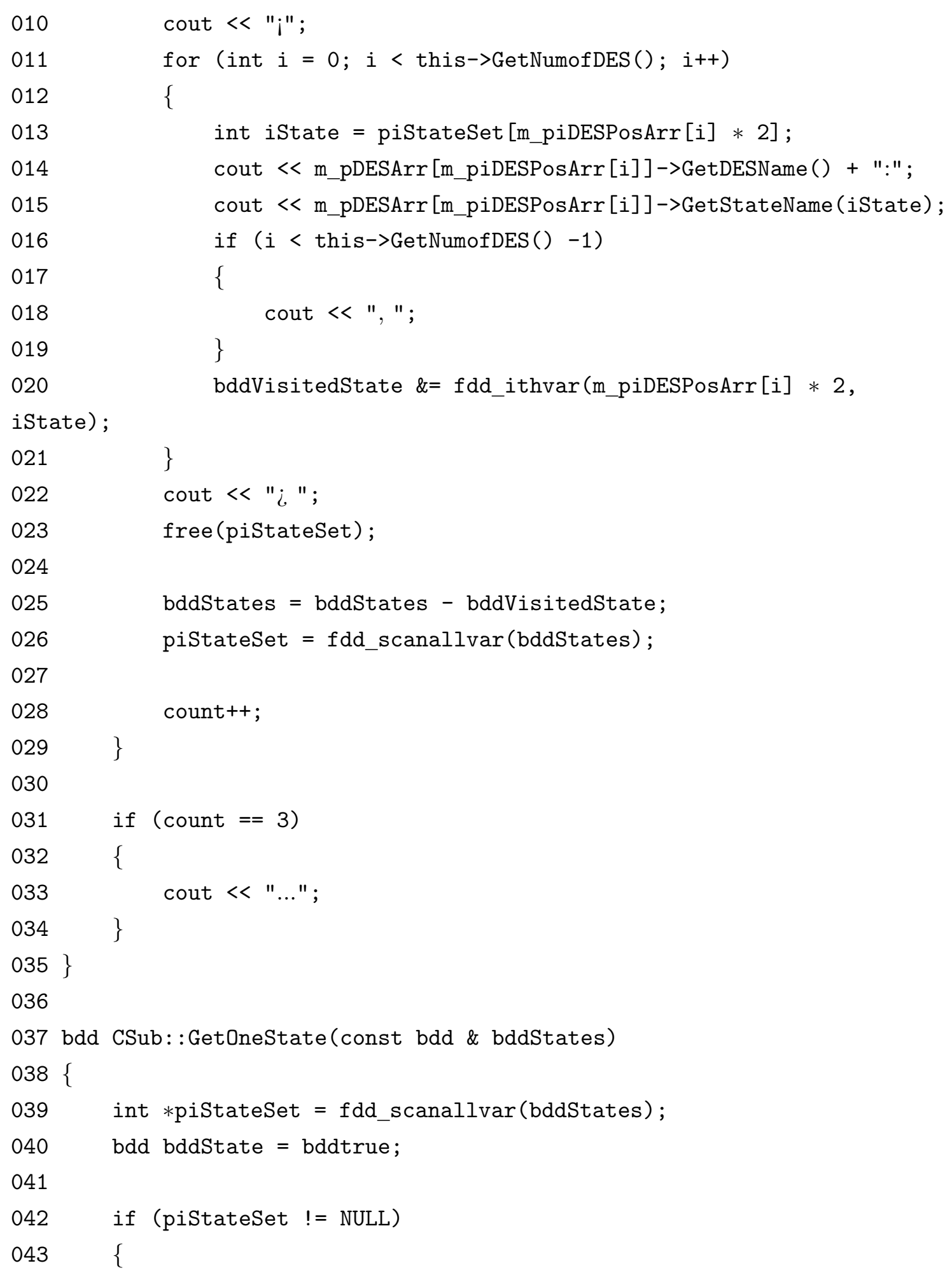




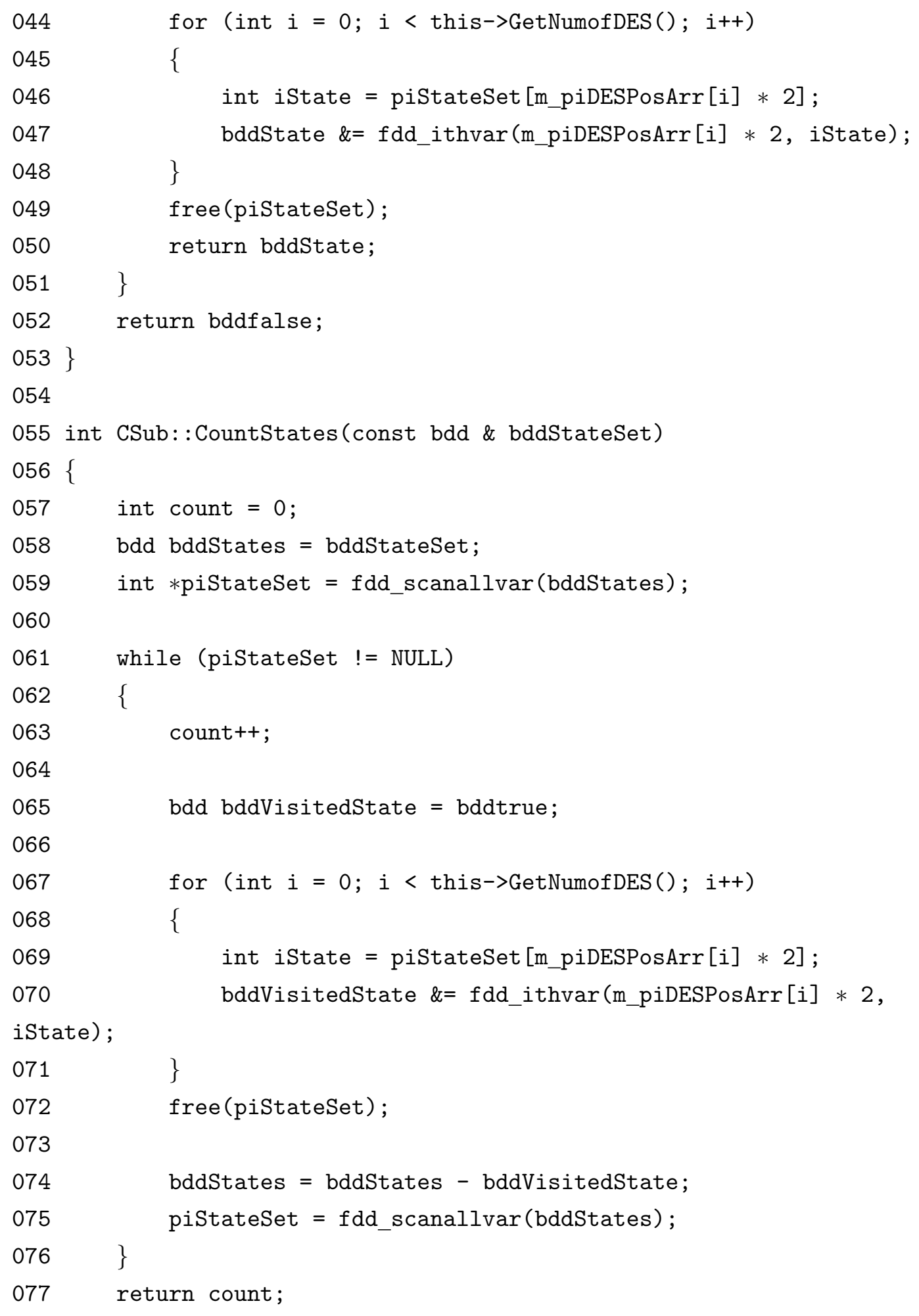




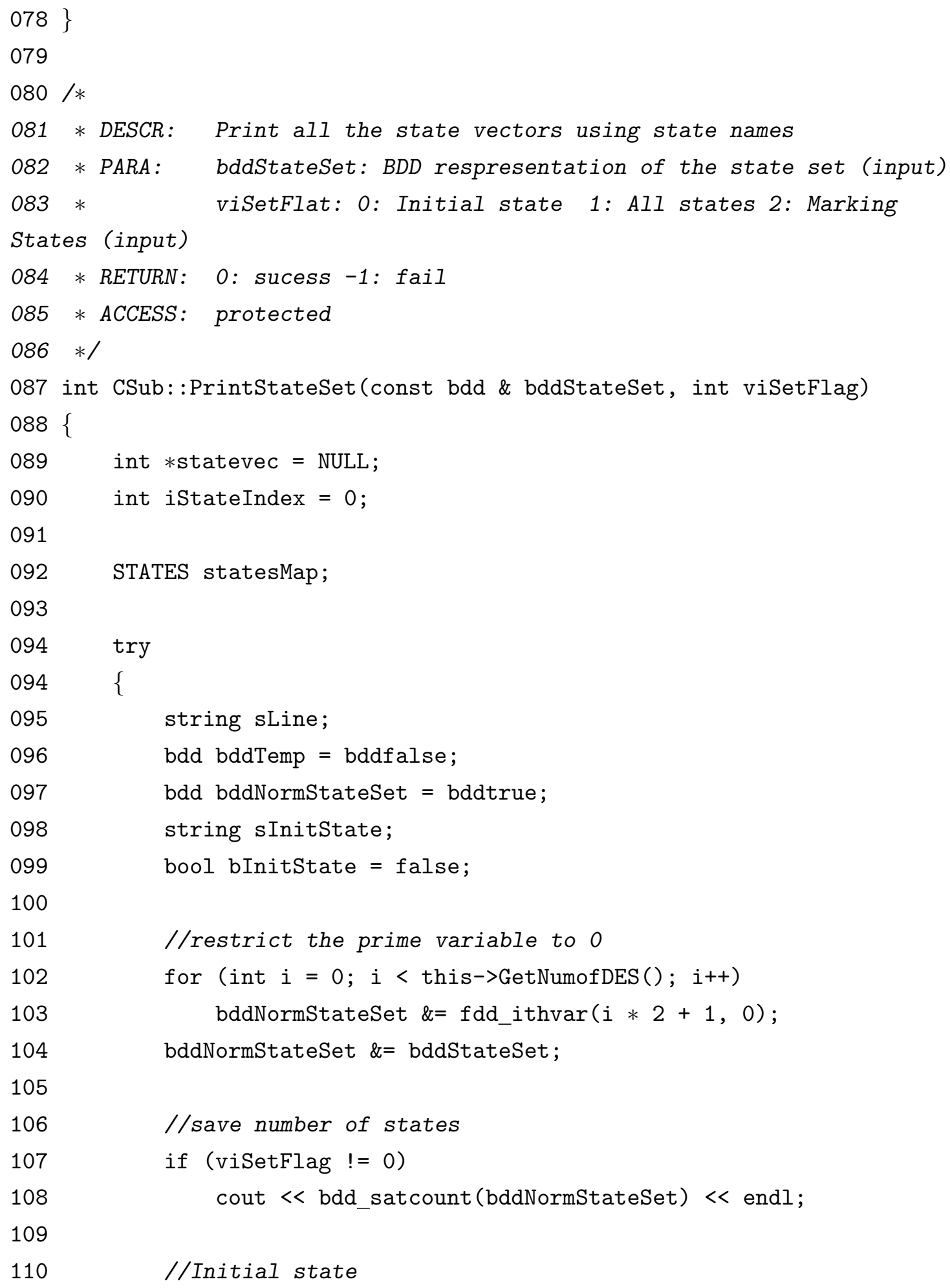




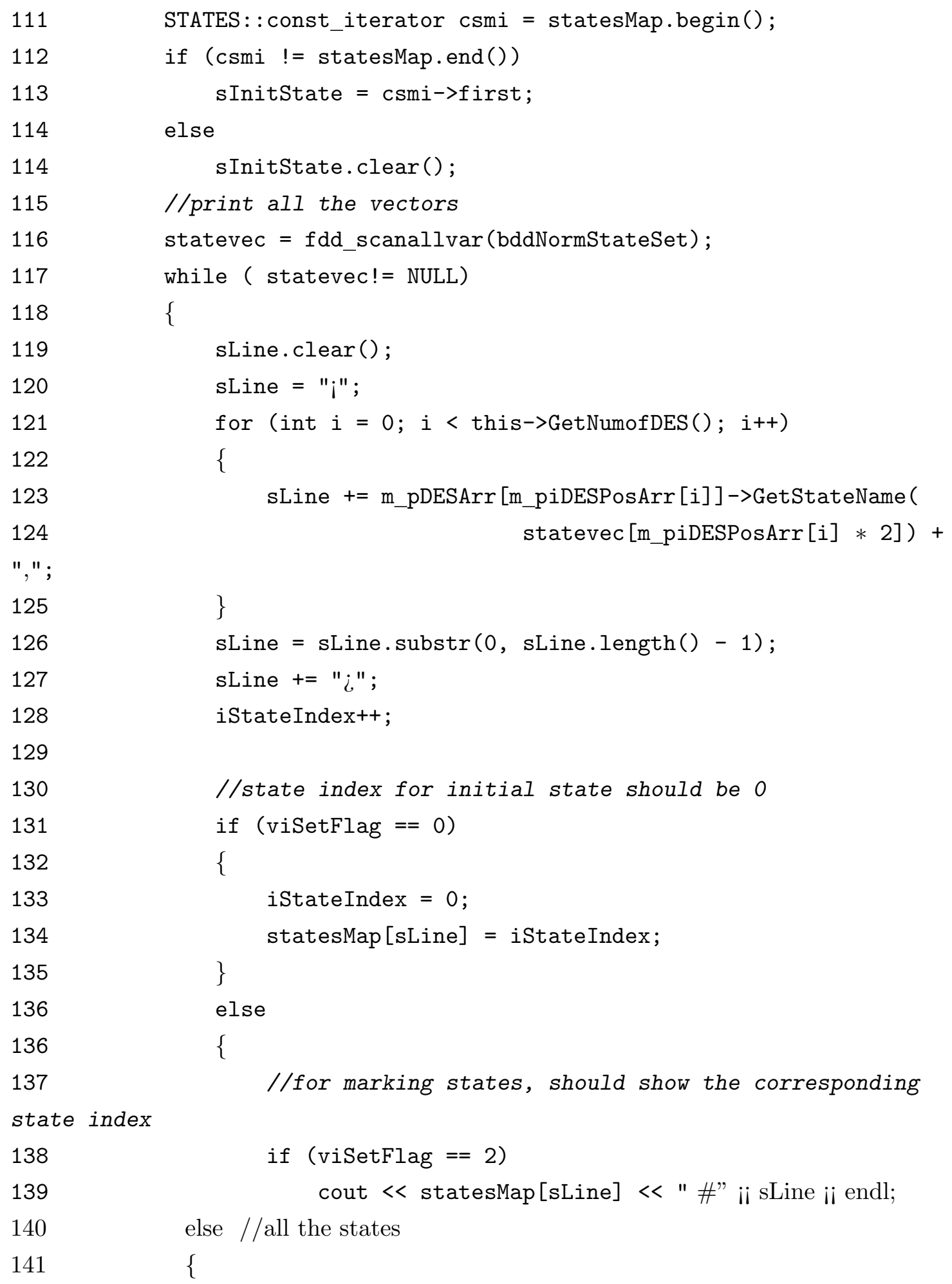




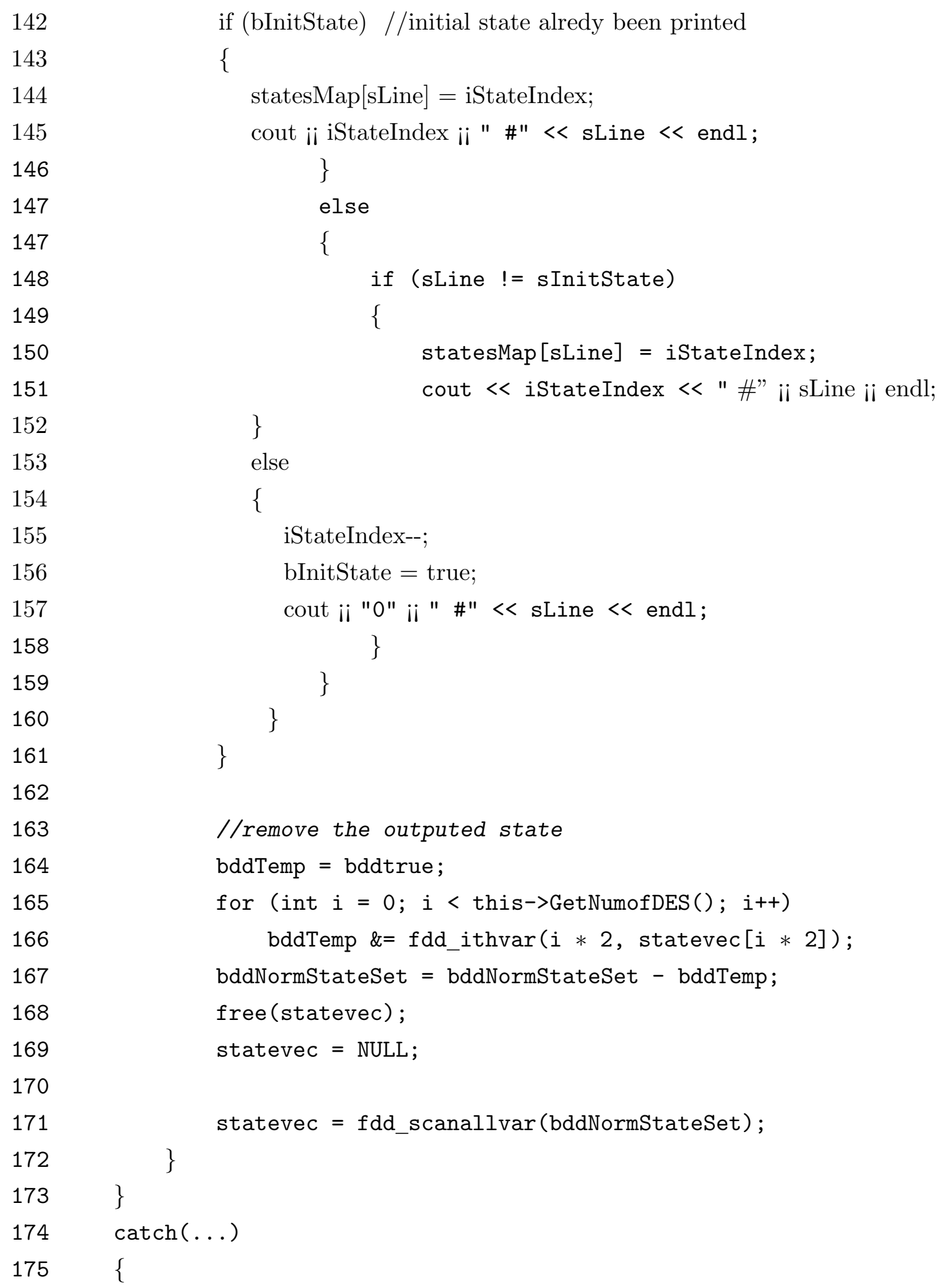

142

143

144

145

146

147

147

148

149

150

151

152

153

154

155

156

157

158

159

160

161

162

163

164

165

166

167

168

169

170

171

172

$173\}$

$174 \operatorname{catch}(\ldots)$

175

if (bInitState) //initial state alredy been printed

\{

statesMap[sLine $]=$ iStateIndex;

cout ii iStateIndex ii " \#" $<$ sLine $<$ endl;

\}

else

\{

if (sLine $!=$ sInitState)

\{

statesMap [sLine] = iStateIndex;

cout $<$ iStateIndex $<$ " \#" ii sLine ii endl;

\}

else

\{

iStateIndex--;

bInitState $=$ true;

cout ii "O" ii "\#" $<$ sLine $<$ endl;

\}

\}

\}

\}

//remove the outputed state

bddTemp = bddtrue;

for (int $i=0 ; i<$ this->GetNumofDES(); $i++$ )

bddTemp \&= fdd_ithvar $(i * 2$, statevec $[i * 2])$;

bddNormStateSet $=$ bddNormStateSet - bddTemp;

free (statevec);

statevec = NULL;

statevec $=$ fdd_scanallvar(bddNormStateSet); 


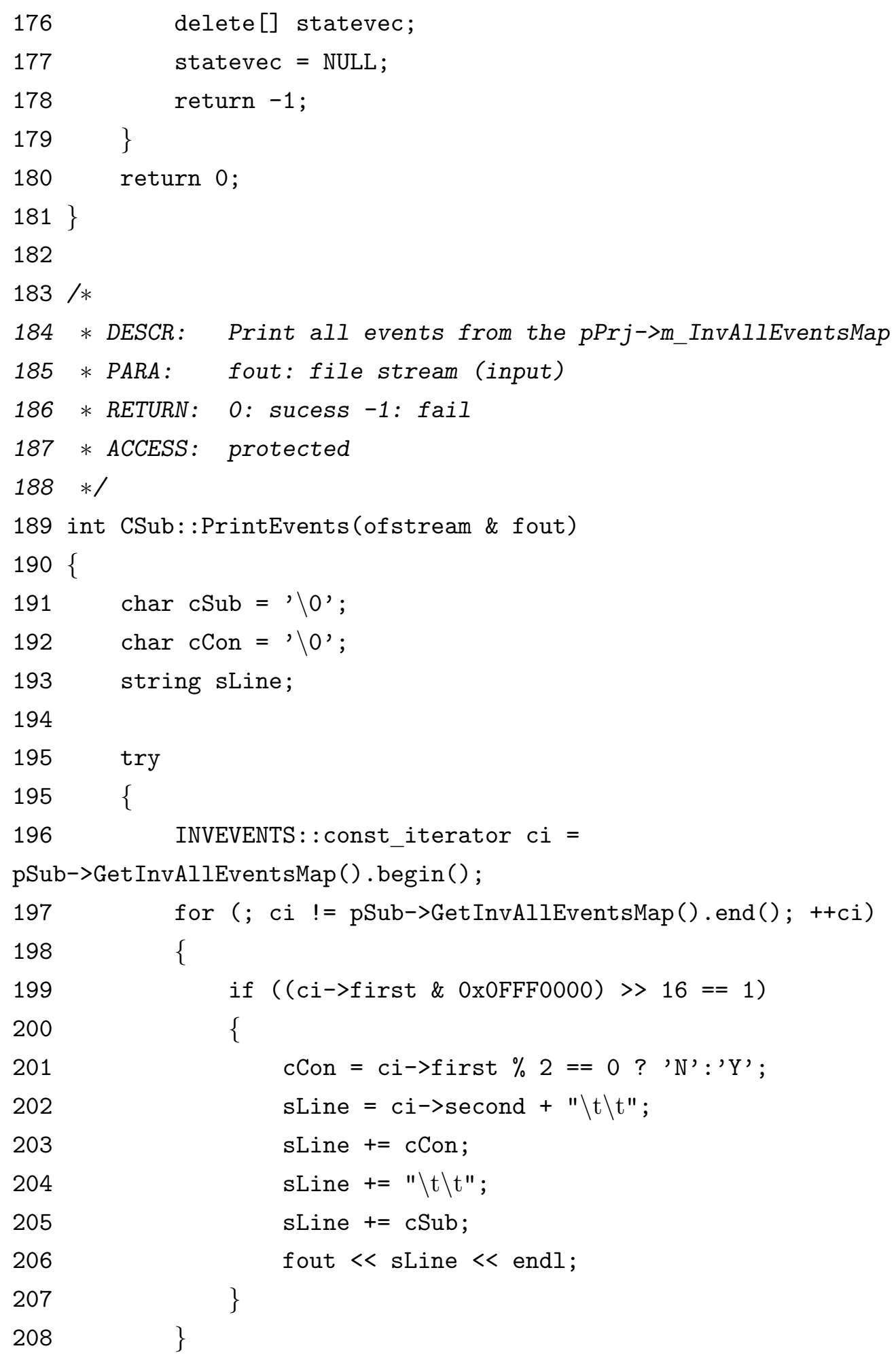




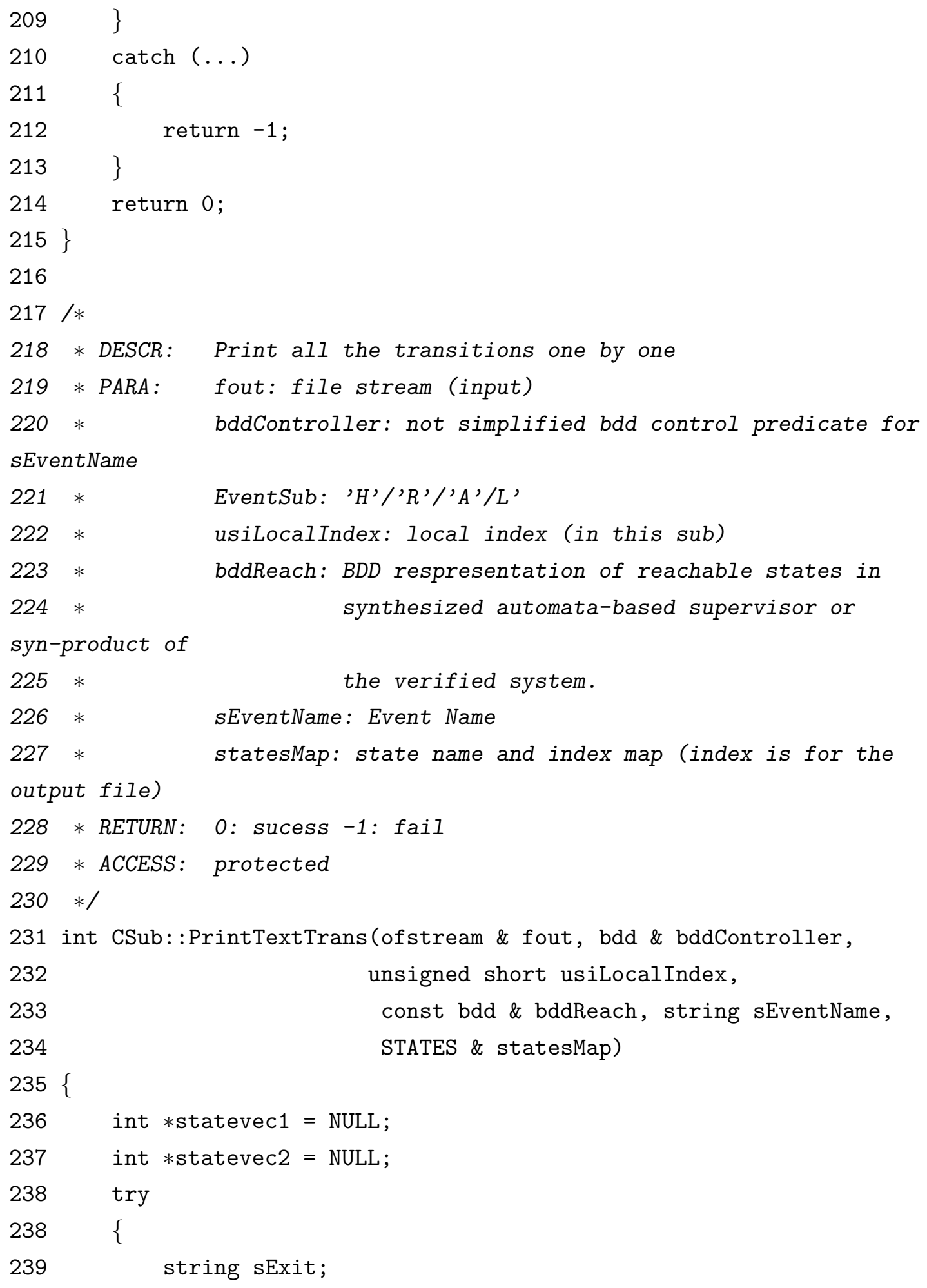




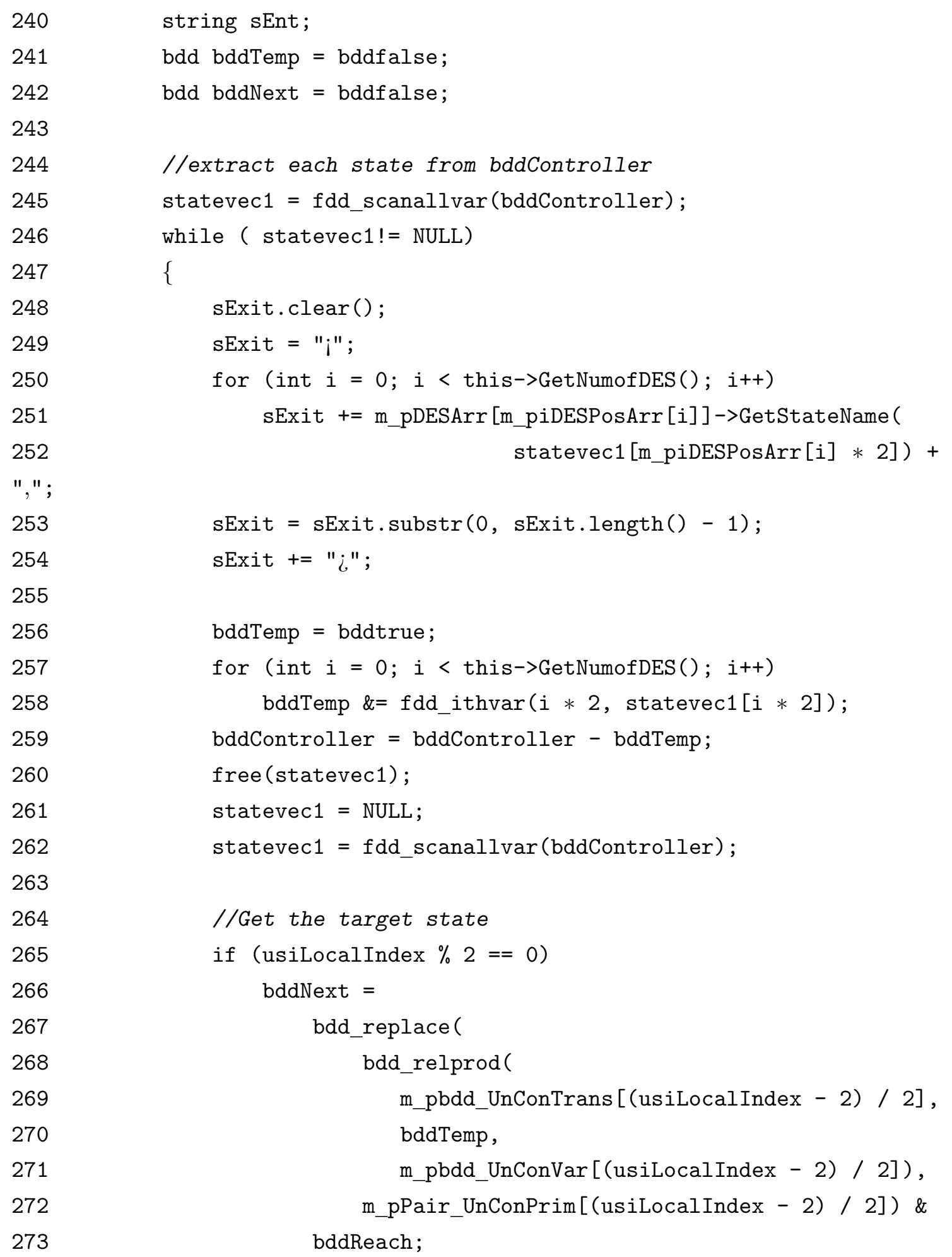




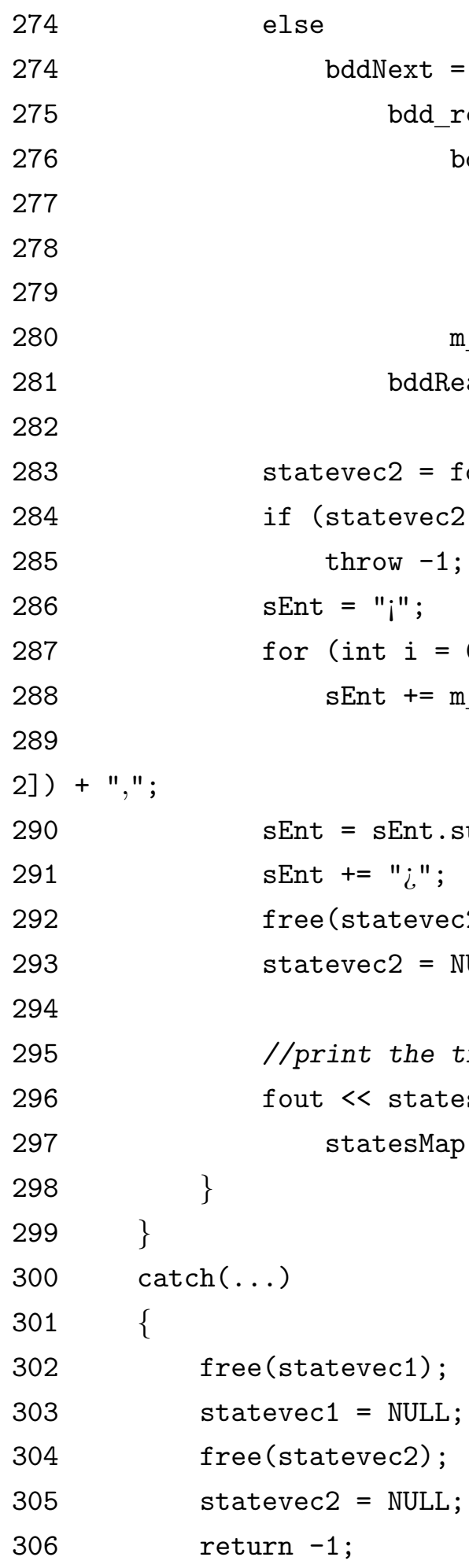




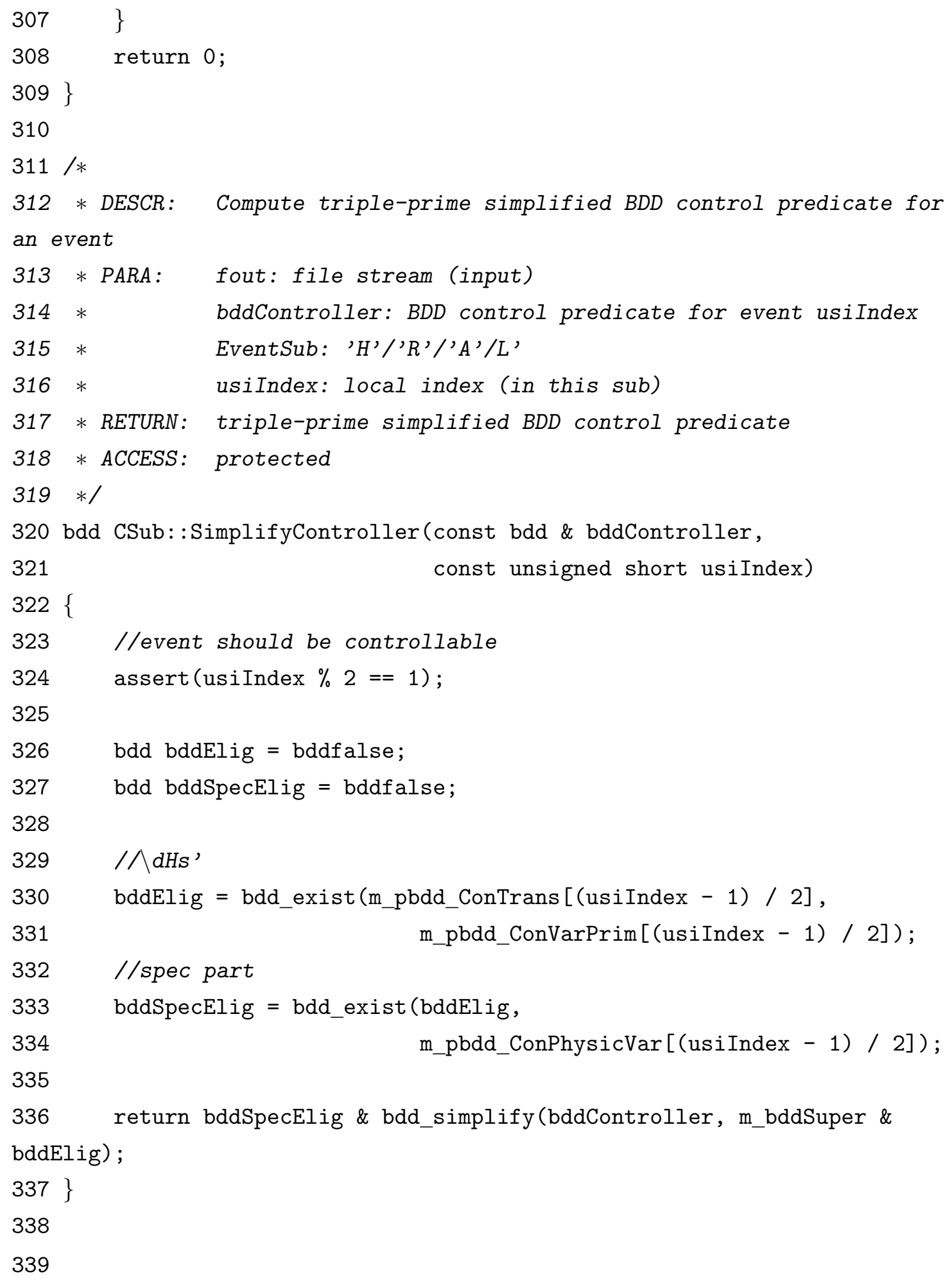




\section{A.2.5 LowSub Class}

\section{LowSub.h}

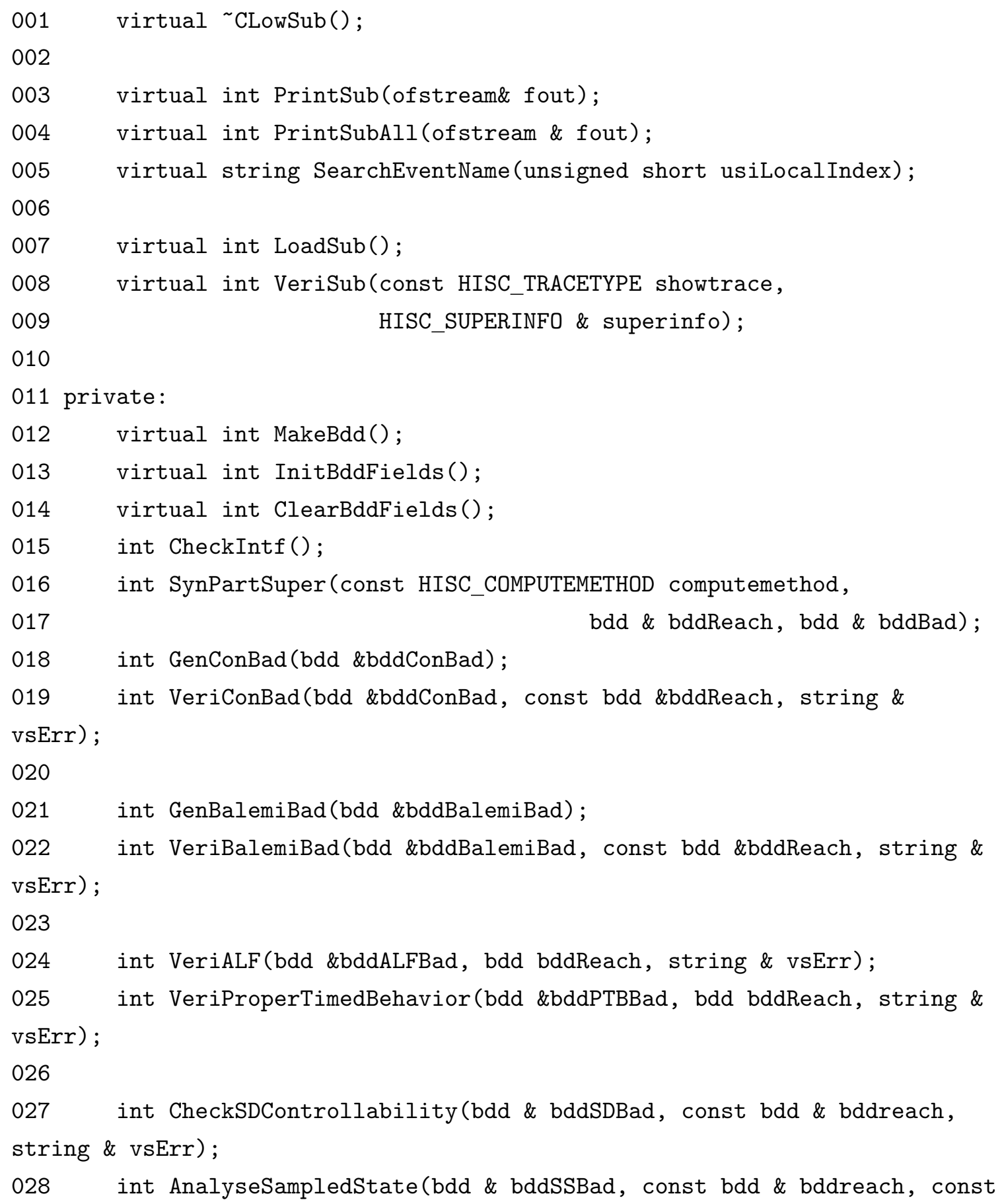


bdd \& bddSS,

029 list $<$ list<bdd $>>$ \& list_NerFail, bdd \& bddSF, stack<bdd $>$ \&

stack_bddSP, string \& vsErr);

030

031 int CheckTimedControllability(const EVENTSET \& eventsDis, const

EVENTSET \& eventsPoss);

032 int CheckTimedControllability(bdd \& bddTCBad, const bdd \& bddreach);

033

034 bool RecheckNerodeCells(bdd \& bddNCBad, const bdd \& bddreach, list< list<bdd > > \& list_NerFail);

035 bool RecheckNerodeCell (bdd \& bddNCBad, const bdd \& bddreach, const

list<bdd $>$ \& Zeqv, list< pair<bdd, bdd $>>$ \& listVisited);

036

037 int DetermineNextState(bdd \& bddLBBad, const EVENTSET \& eventsPoss, const bdd \& bddZ, const bdd \& bddreach, 038 const int \& intB, int \& intNextFreeLabel, map<int, bdd $>$ \&

B_map, stack<int> \& B_p,

039 bdd \& bddSF, stack<bdd> \& stack_bddSP, 040 map<int, EVENTSET> \& B_occu, map<int, bdd> \& B_conc, string \& vsErr);

041

042 void CheckNerodeCells (map<int, bdd> \& B_conc, map<int, EVENTSET> \& B_occu,

$043 \quad$ list< list<bdd $>>$ \& list_NerFail);

044

045 int CheckSDiv(bdd \& bddSDivBad, const bdd \& bddReach);

046

047 EVENTSET GetTransitionEvents (const bdd \& bddleave, const bdd \& bddenter);

048

049 int GenP4Bad (bdd \&bddP4Bad);

050 int VeriP4Bad (bdd \&bddP4Bad, const bdd \&bddReach, string \&vsErr);

051 int supcp (bdd \& bddP);

052 bdd cr (const bdd \& bddPStart, const bdd \& bddP, int \& iErr); 


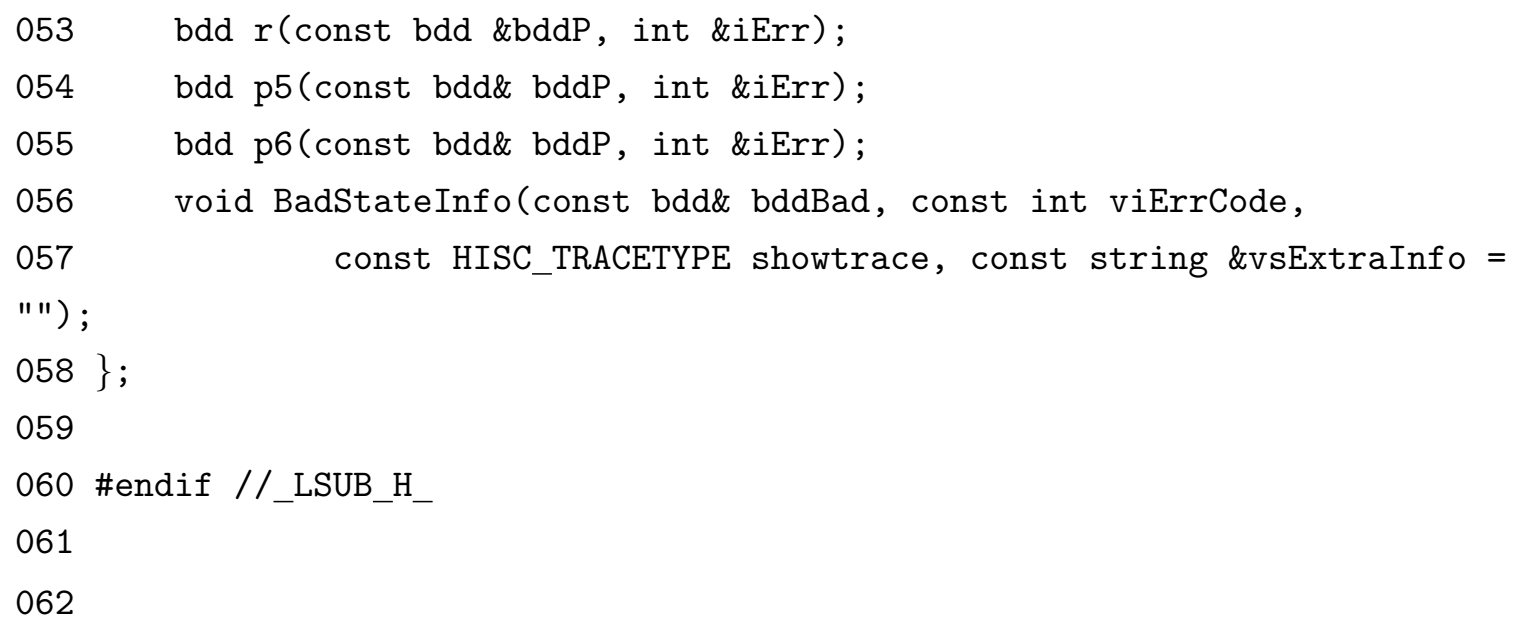

\section{LowSub.cpp}

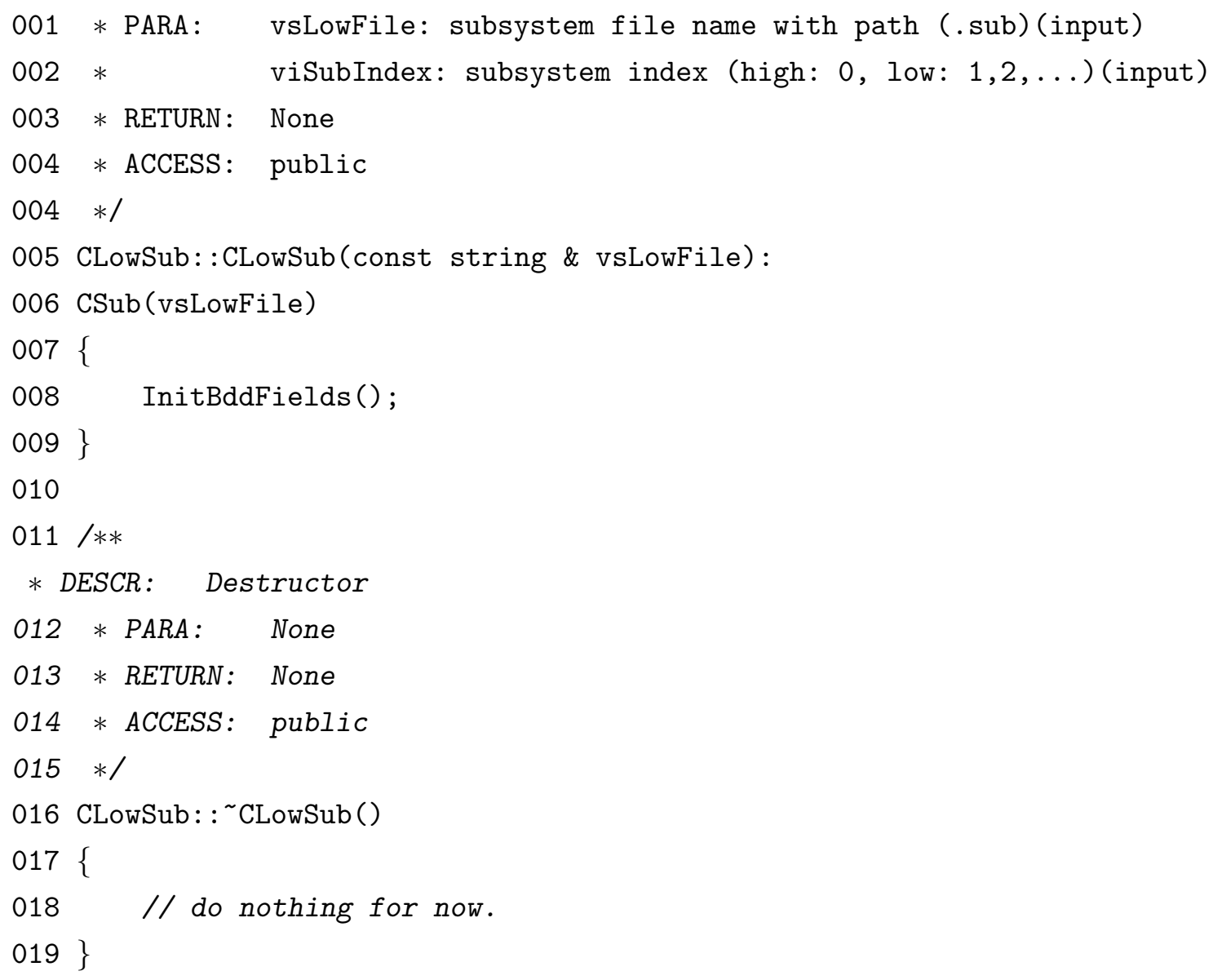




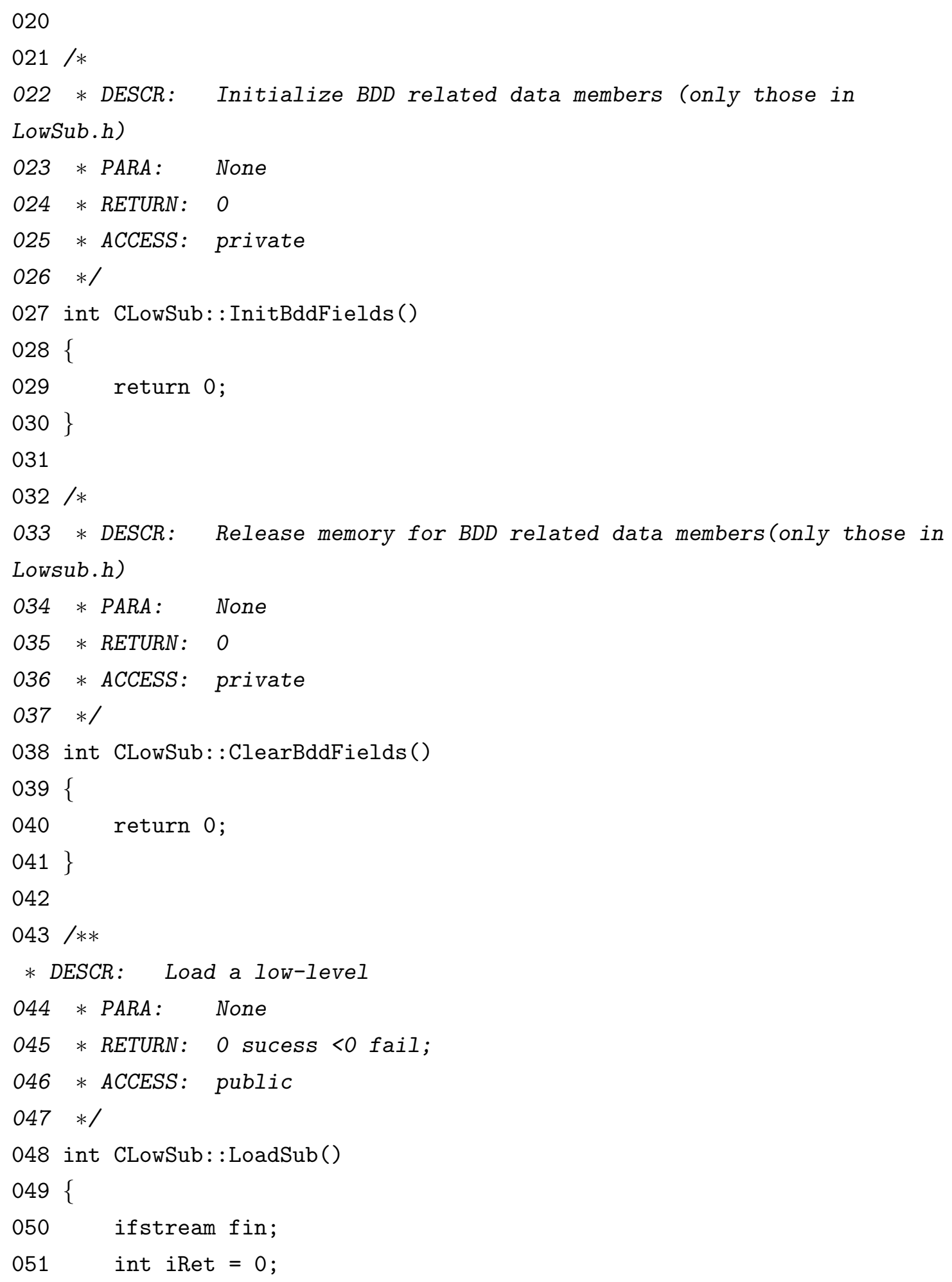




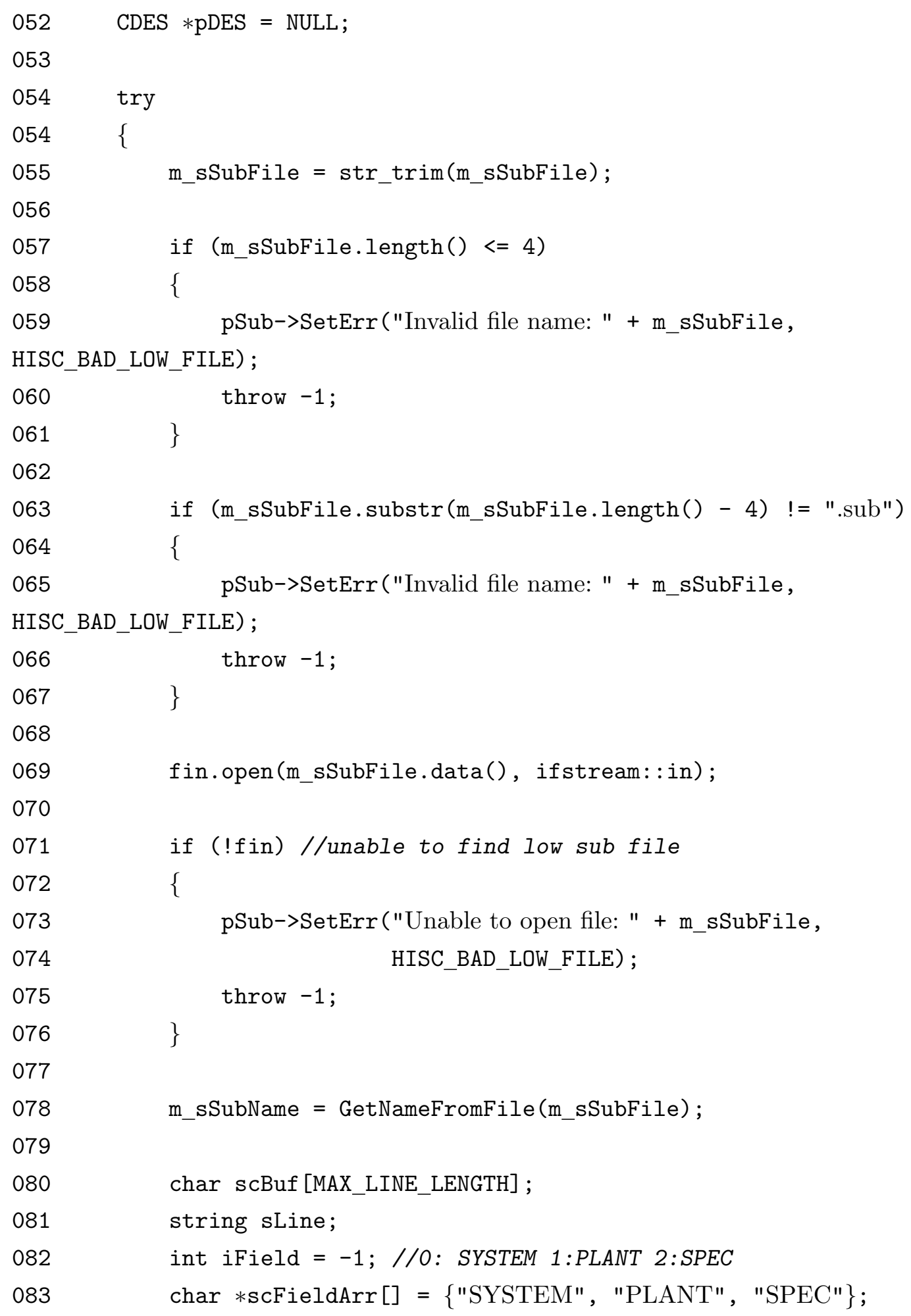




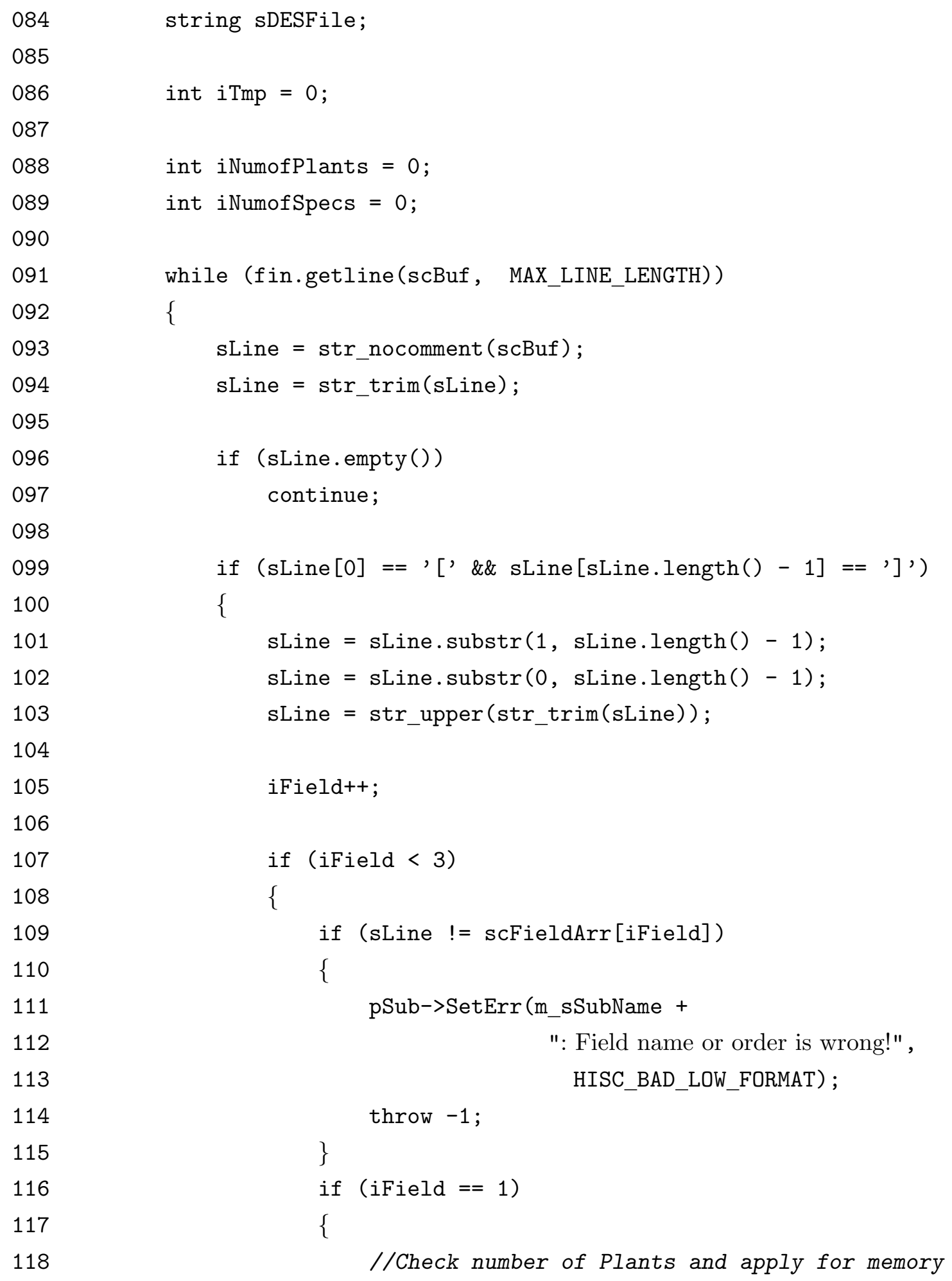




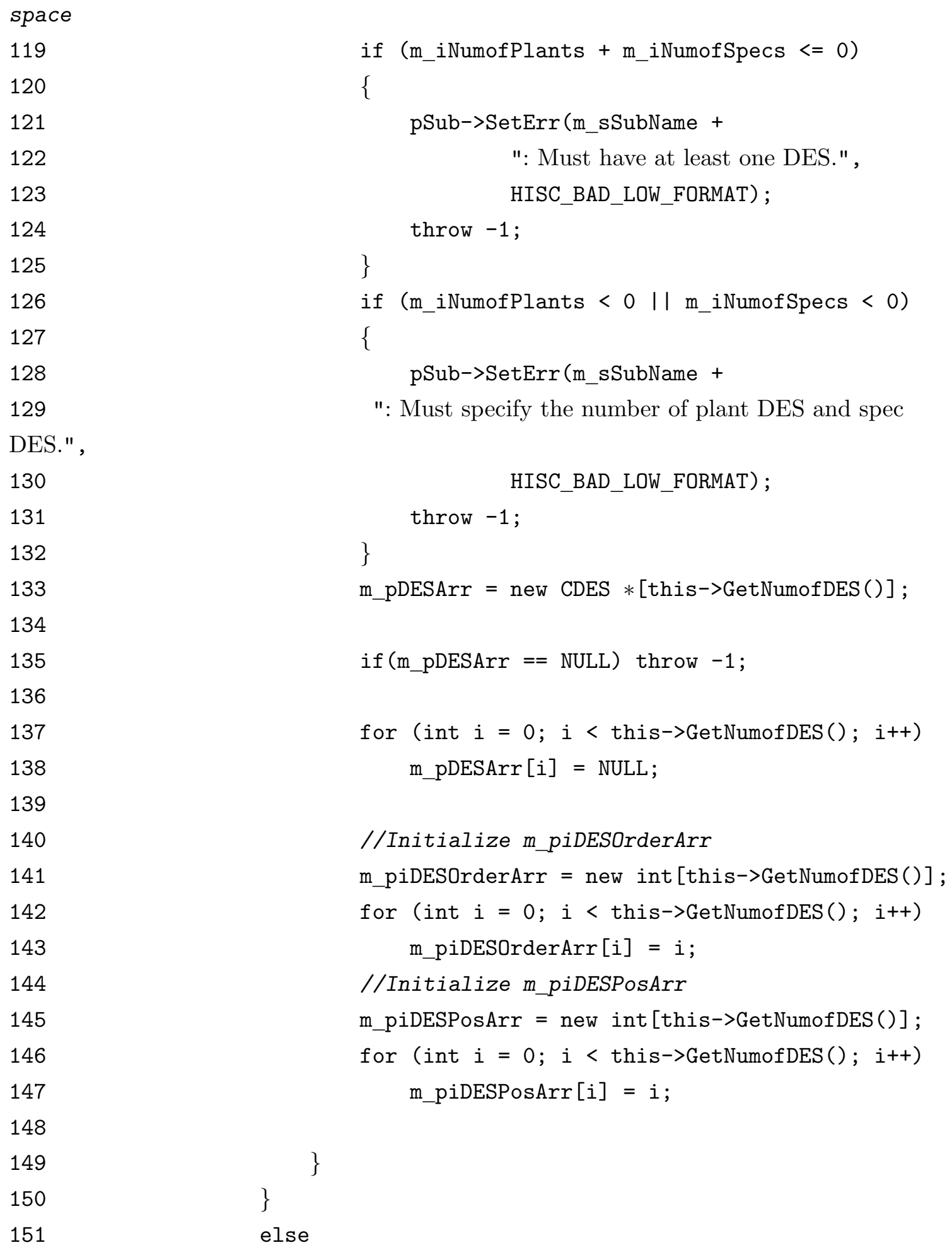


151

152

153

154

155

156

157

157

158

159

160

161

162

163

absent!",

164

165

166

167

168

169

170

171

172

173

174

175

176

177

178

179

179

180

181

182
\{ pSub->SetErr (m_sSubName + ": Too many fields!", HISC_BAD_LOW_FORMAT); throw -1 ;

\}

\}

else

\{

\{

case $0: \quad / /$ [SYSTEM]

if (! IsInteger(sLine))

\{ pSub->SetErr (m_sSubName + ": Number of DES is

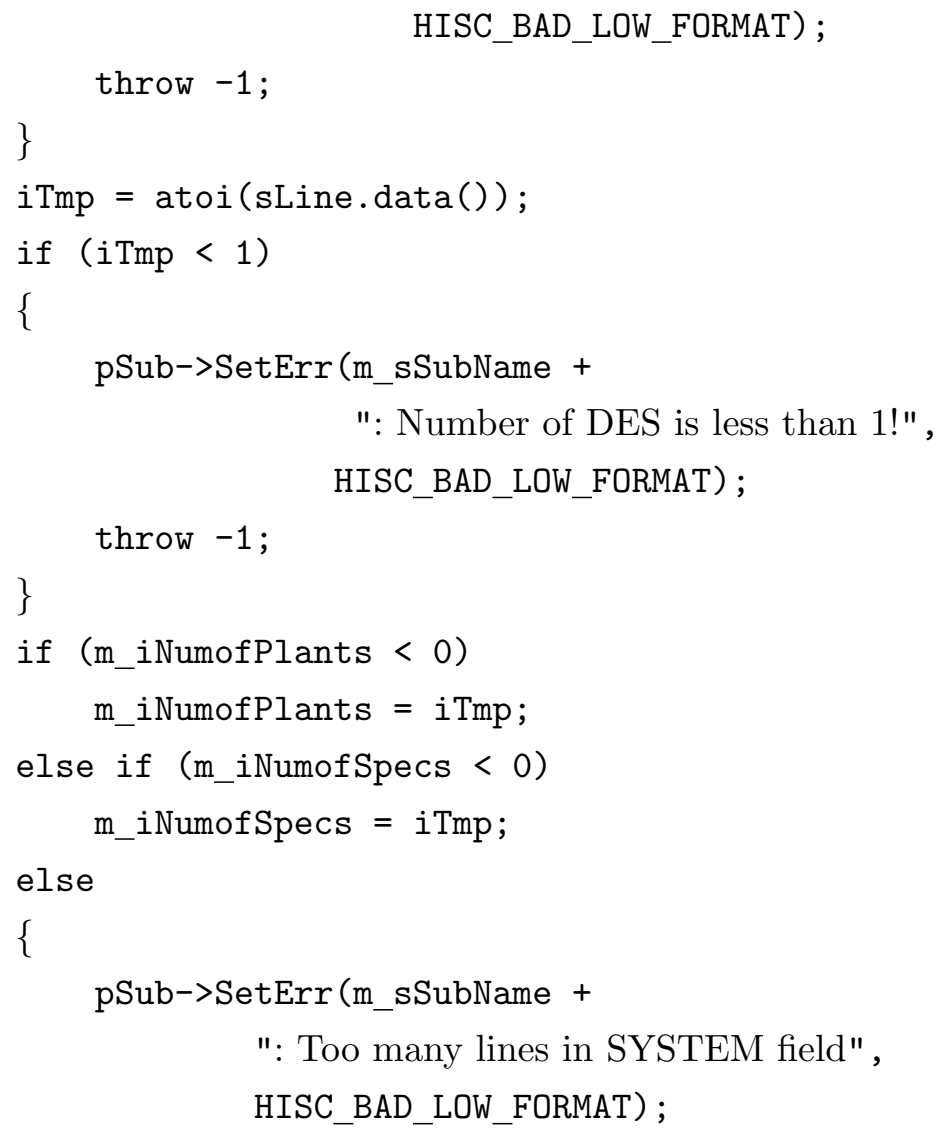




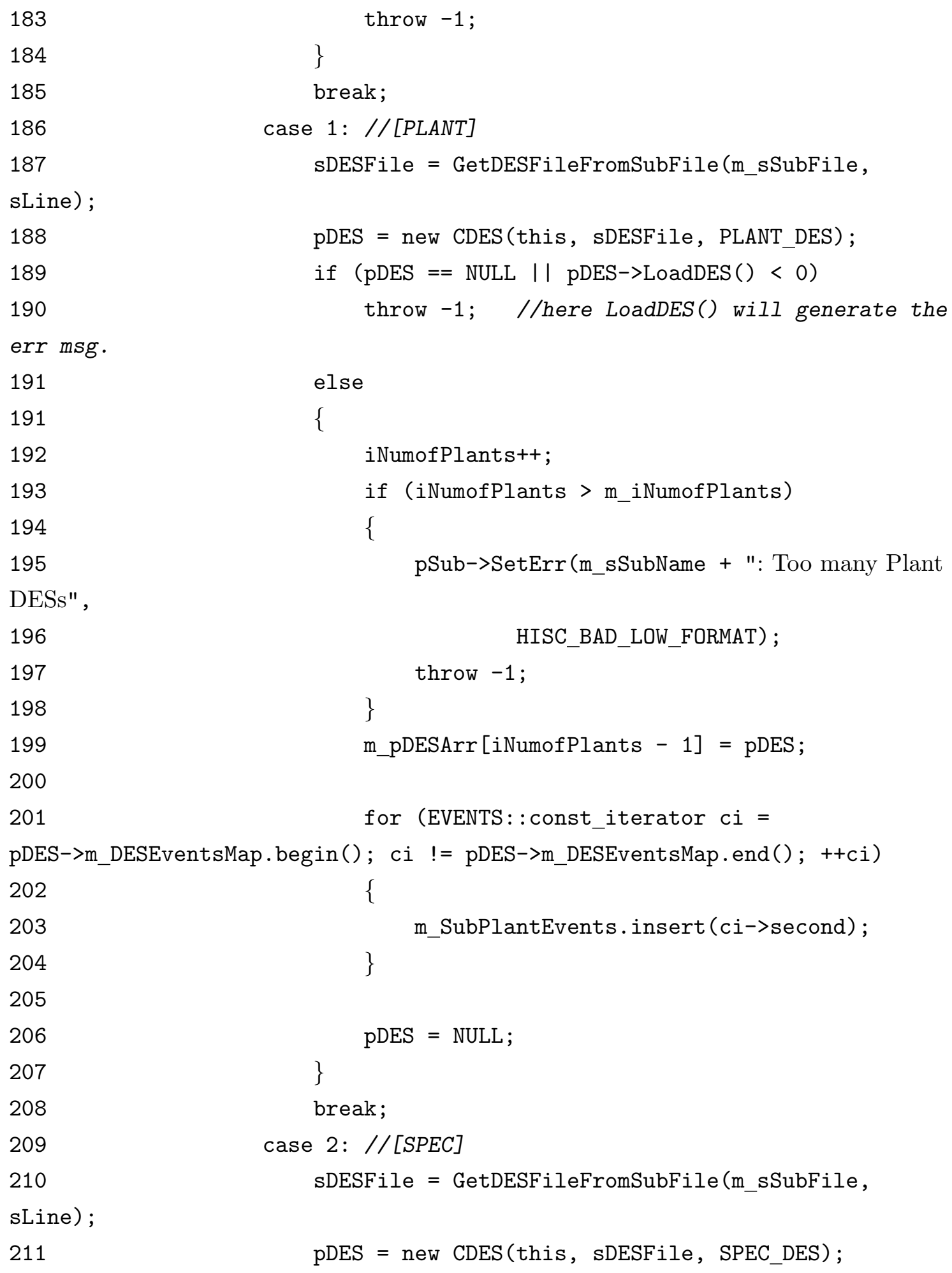




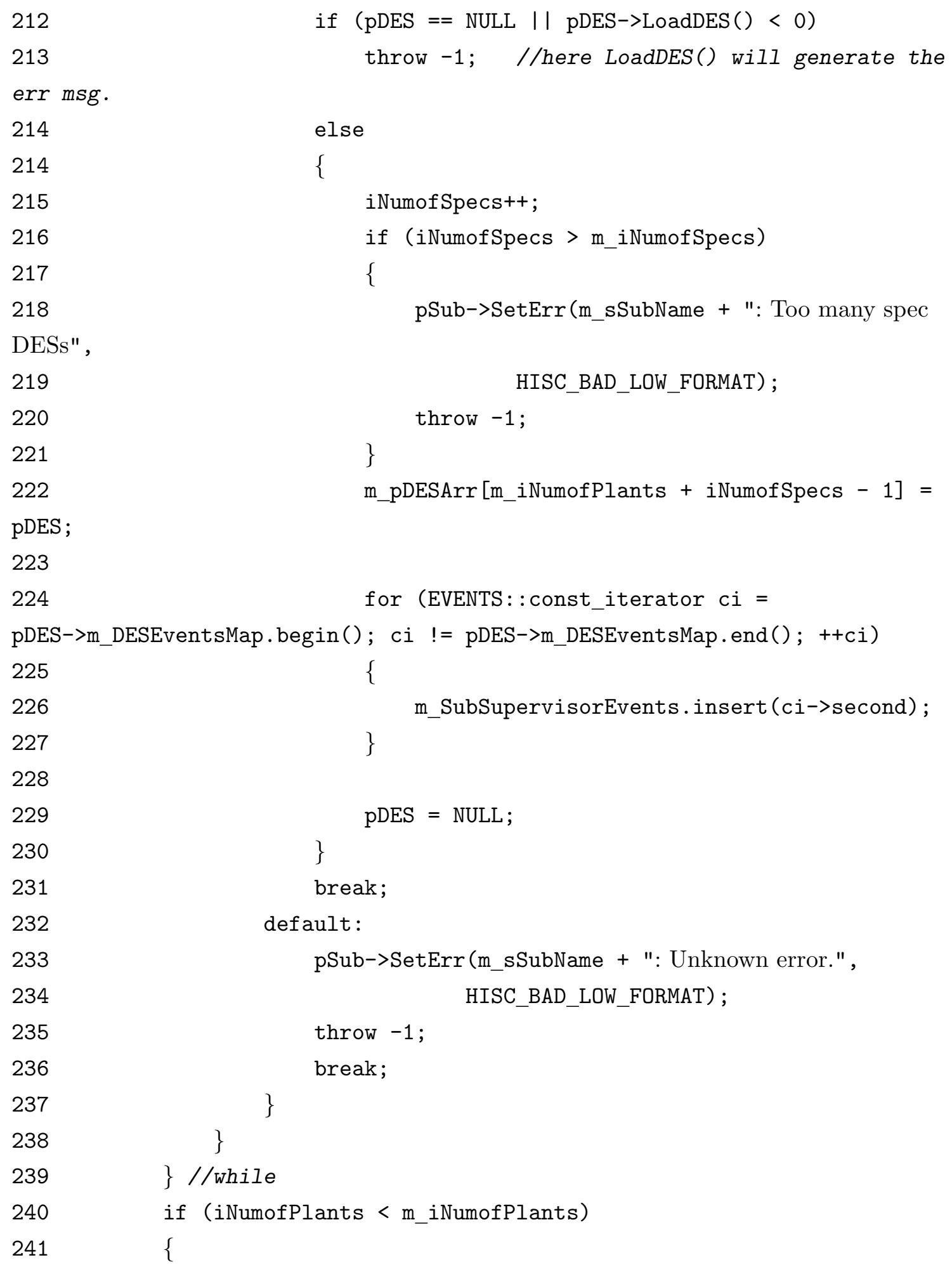




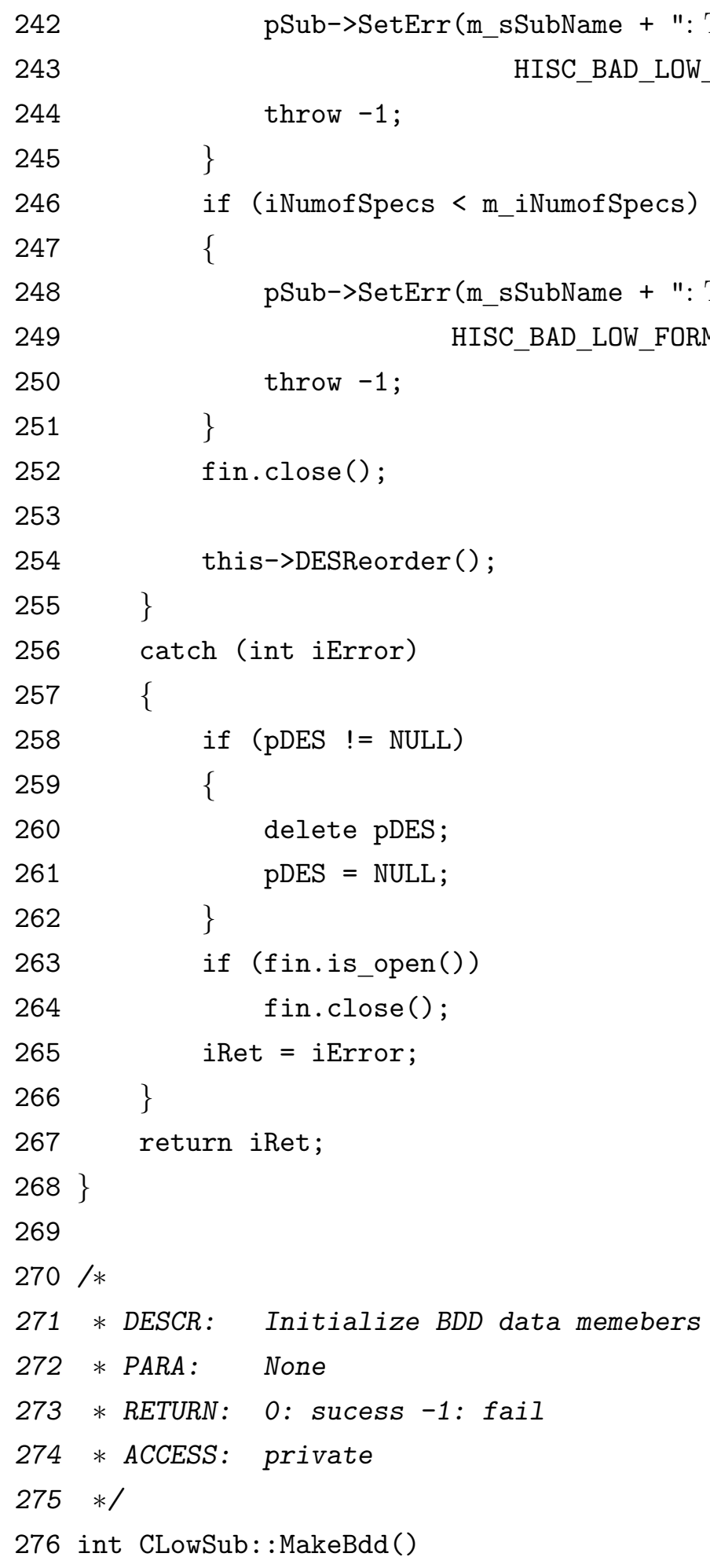


277\{

278 const char $*$ DEBUG $=$ "CLowSub::MakeBdd ()$: "$;

279

280 try

280\{

281

//Initialize the bdd node table and cache size.

282

long long lNumofStates $=1$;

283

284

285

286

287

288

289

290

291

292

293

294

295

296

296

297

298

299

300

301

302

for (int $i=0 ; i<$ this->GetNumofDES(); $i++$ )

\{

lNumofStates $*=$ m_pDESArr [i]->GetNumofStates ();

if (INumofStates >= MAX_INT)

break;

\}

if (lNumofStates < = 10000)

bdd_init (1000, 100);

else if (INumofStates <= 1000000)

bdd_init(10000, 1000);

else if (lNumofStates < $=10000000$ )

bdd_init (100000, 10000);

else

\{

bdd_init (2000000, 1000000);

bdd_setmaxincrease (1000000);

\}

303

304

305

306

307

308

giNumofBddNodes $=0$;

bdd_gbc_hook(my_bdd_gbchandler);

//define domain variables

int $*$ piDomainArr $=$ new int [2] ;

for (int $i=0 ; i<2 *$ this $->\operatorname{GetNumofDES}() ; i+=2$ )

m_pDESArr $[i / 2]->\operatorname{GetDESName}()<$ endl; $\}$ 


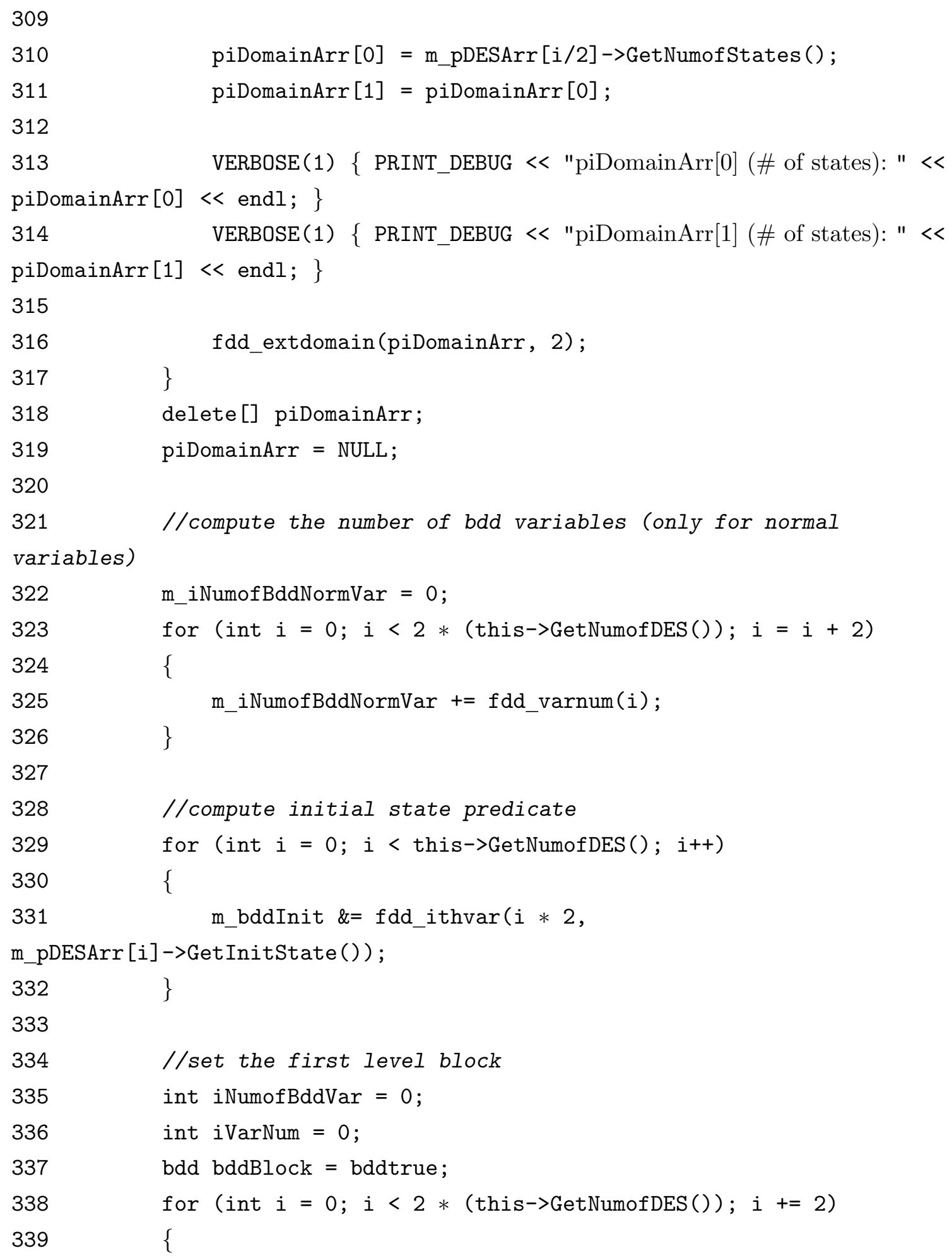




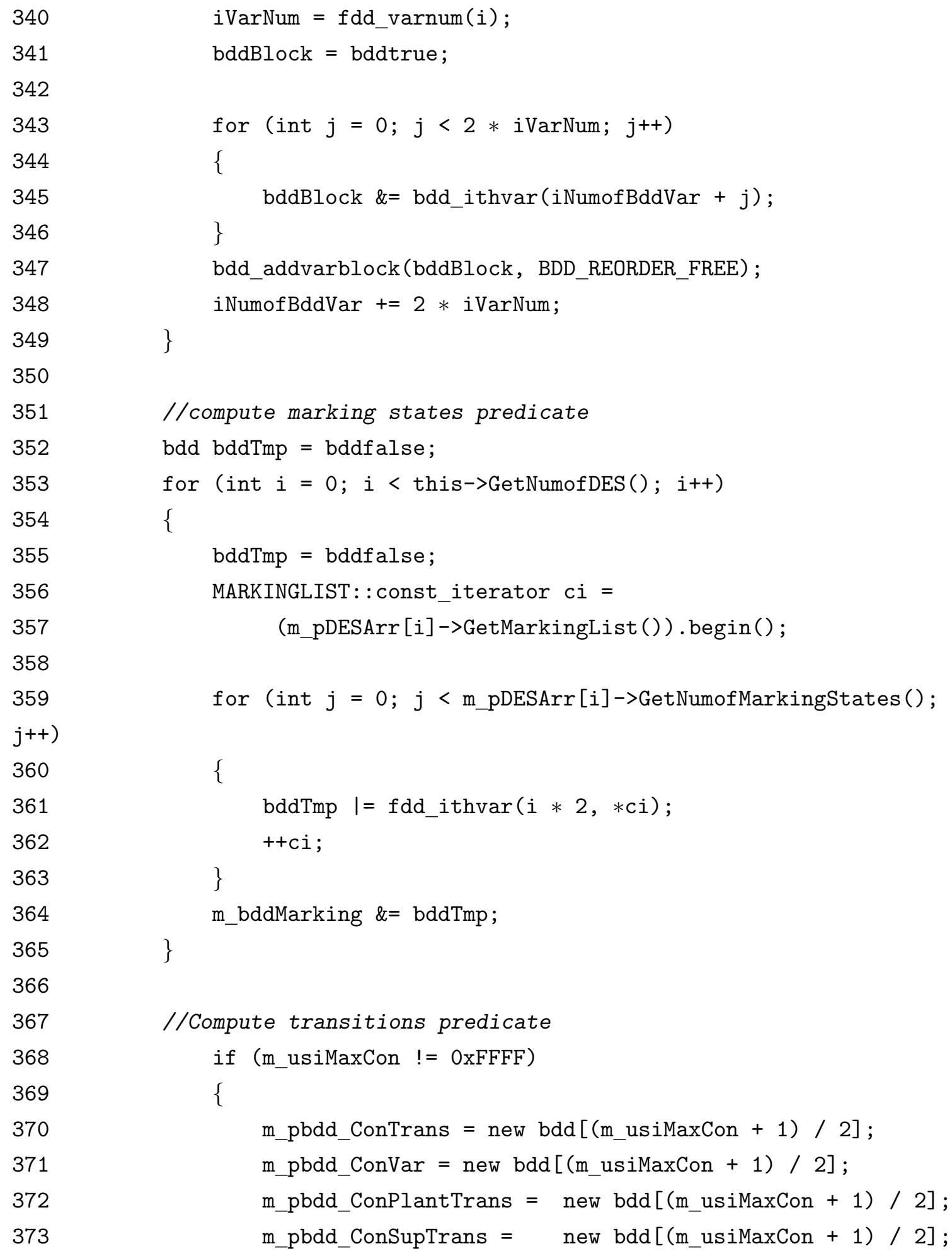




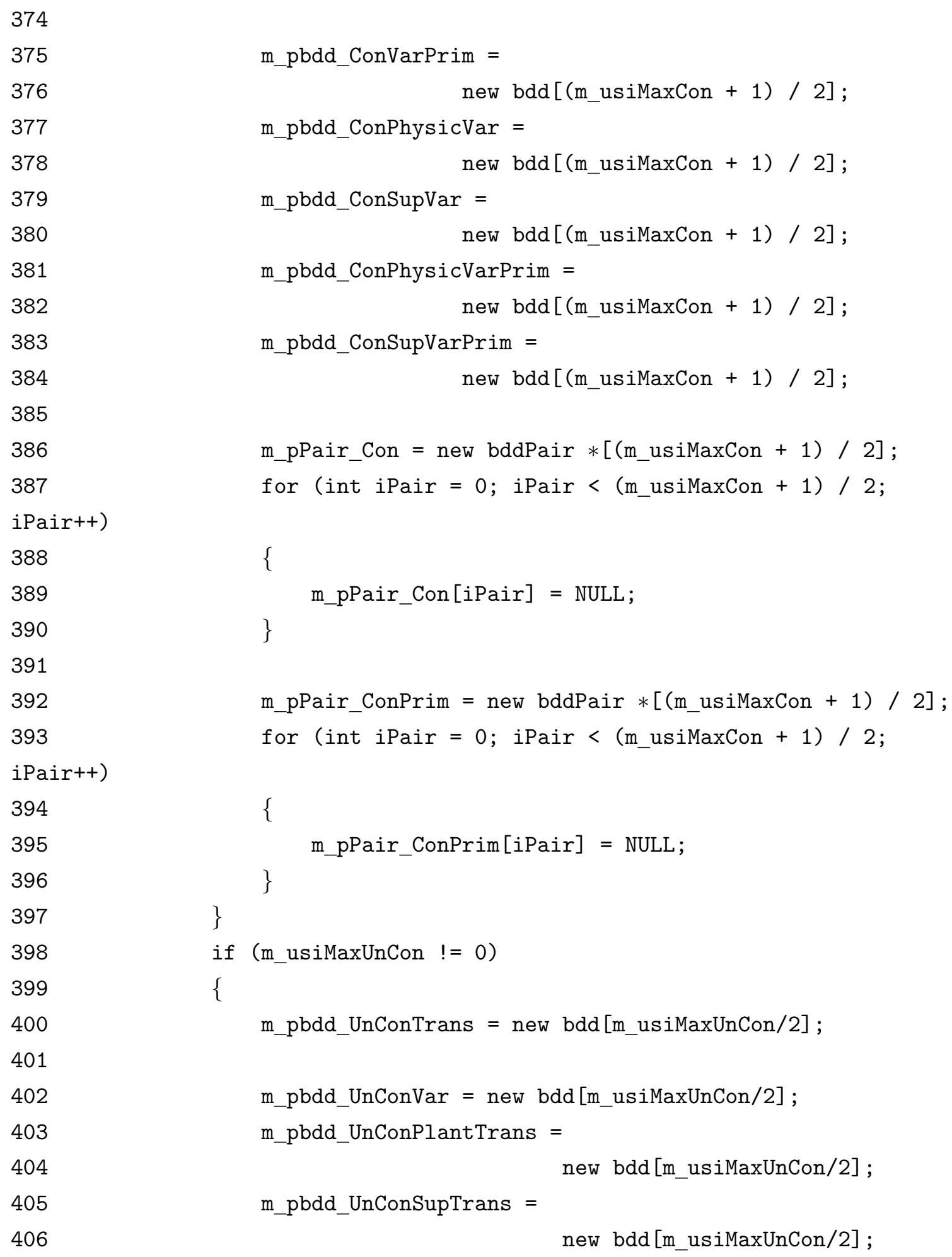

375

376

377

378

379

380

381

382

383

384

385

386

387

iPair++)

388

389

390

391

392

393

iPair++)

394

395

396

397

398

399

400

401

402

403

404

405

406 
407

408

409

410

411

412

413

414

415

416

417

418

419

420

421

422

423

424

425

426

427

428

429

430

431

432

it.

433

434

435

436

bddfalse;

437

438

439

m_pbdd_UnConVarPrim = new bdd[m_usiMaxUnCon/2];

m_pbdd_UnConPlantVar = new bdd[m_usiMaxUnCon/2];

m_pbdd_UnConSupVar $=$ new bdd[m_usiMaxUnCon/2];

m_pbdd_UnConPlantVarPrim = new bdd[m_usiMaxUnCon/2];

m_pbdd_UnConSupVarPrim $=$ new bdd[m_usiMaxUnCon/2];

m_pPair_UnCon $=$ new bddPair $*\left[\mathrm{~m} \_\right.$usiMaxUnCon/2];

for (int iPair $=0$; iPair < m_usiMaxUnCon/2; iPair++)

\{

m_pPair_UnCon[iPair $]=$ NULL;

\}

m_pPair_UnConPrim = new bddPair $*$ [m_usiMaxUnCon/2];

for (int iPair = 0; iPair < m_usiMaxUnCon/2; iPair++)

\{

m_pPair_UnConPrim[iPair] = NULL;

\}

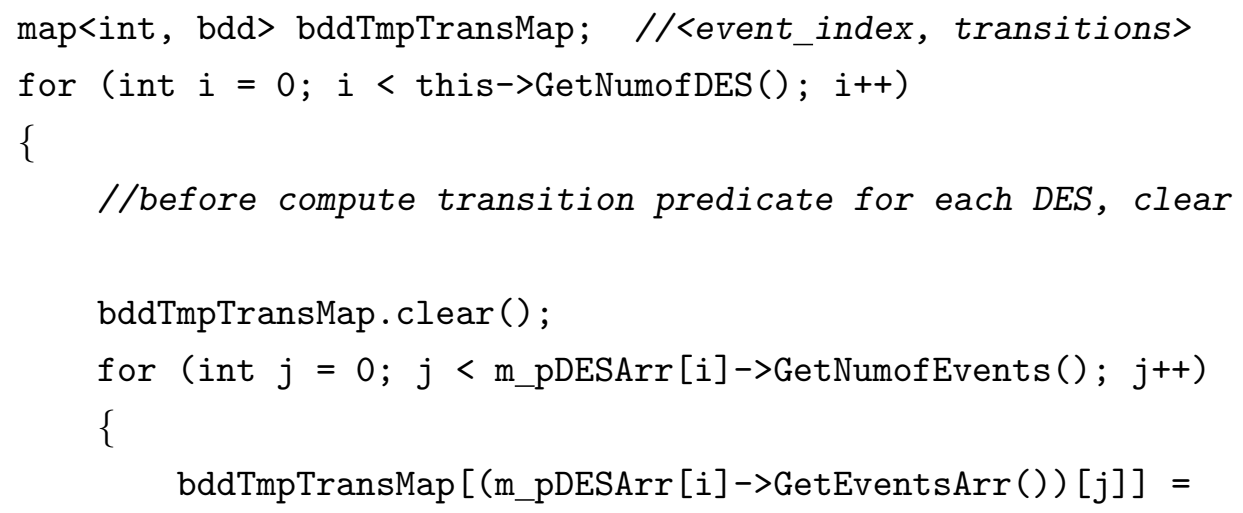


440

441

442

443

j)). begin();

444

$++c i)$

445

446

447

448

449

450

451

452

453 for (int $j=0 ; j<m \_p D E S A r r[i]->\operatorname{GetNumofStates}() ; j++$ )

$$
\{
$$

TRANS : : const_iterator $\mathrm{ci}=$

$$
\left(* \left(m \_p D E S A r r[i]->\operatorname{GetTrans}()+\right.\right.
$$

for $\left(;\right.$ ci $!=\left(*\left(m \_p D E S A r r[i]->\operatorname{Get} \operatorname{Trans}()+j\right)\right)$.end () ;

\{ bddTmpTransMap[ci->first] I=fdd_ithvar $(i * 2, j) \&$ fdd_ithvar( $i * 2+1$, ci->second);

\}

//combine the current DES transition predicate to //subsystem transition predicate map<int, bdd $>$ : : const_iterator $\operatorname{ciTmp}=$

bddTmpTransMap.begin();

454

455

456

for (; ciTmp != bddTmpTransMap.end (); ++ciTmp)

\{

if (ciTmp->first \% $2==0$ ) //uncontrollable, start

from 2

457

458

int index $=($ ciTmp->first \& 0x0000FFFF $) / 2-1$;

459

460

461

462

463

464

465

466

467

468

469

1); if (m_pbdd_UnConVar[iIndex] == bddfalse)

\{

m_pbdd_UnConTrans[iIndex] = bddtrue;

$\mathrm{m} \_$pbdd_UnConVar[iIndex] = bddtrue;

m_pbdd_UnConVarPrim[iIndex] = bddtrue;

\}

m_pbdd_UnConTrans[iIndex] \&= ciTmp->second;

m_pbdd_UnConVar[iIndex] $\&=$ fdd_ithset $(i * 2)$;

m_pbdd_UnConVarPrim[iIndex] \&= fdd_ithset $(i * 2+$ 
470

471

472

473

474

475

476

477

478

479

480

481

ciTmp->second;

482

2) ;

483

fdd_ithset $(i * 2+1)$;

484

485

486

487

488

489

490

491

492

493

494

495

2) ;

496

* $2+1$ );

497

498

499 //compute uncontrollable plant vars and varprimes if (m_pDESArr[i]->GetDESType $($ ) == PLANT_DES)

\{

$$
\text { if (m_pbdd_UnConPlantVar[iIndex] == bddfalse) }
$$

\{ m_pbdd_UnConPlantTrans[iIndex] = bddtrue; m_pbdd_UnConPlantVar[iIndex] = bddtrue; m_pbdd_UnConPlantVarPrim[iIndex] = bddtrue;

\}

m_pbdd_UnConPlantTrans[iIndex] \&= m_pbdd_UnConPlantVar[iIndex $] \quad \&=$ fdd_ithset $(i *$ m_pbdd_UnConPlantVarPrim[iIndex] \&= \} else if (m_pDESArr[i]->GetDESType ()$==$ SPEC_DES) \{ if (m_pbdd_UnConSupVar[iIndex] == bddfalse) \{ m_pbdd_UnConSupTrans[iIndex] = bddtrue; m_pbdd_UnConSupVar [iIndex] = bddtrue; m_pbdd_UnConSupVarPrim[iIndex] = bddtrue;

\}

m_pbdd_UnConSupTrans[iIndex] \&=ciTmp->second; m_pbdd_UnConSupVar[iIndex] \&= fdd_ithset $(i *$ m_pbdd_UnConSupVarPrim[iIndex] \&=fdd_ithset $(i$ 


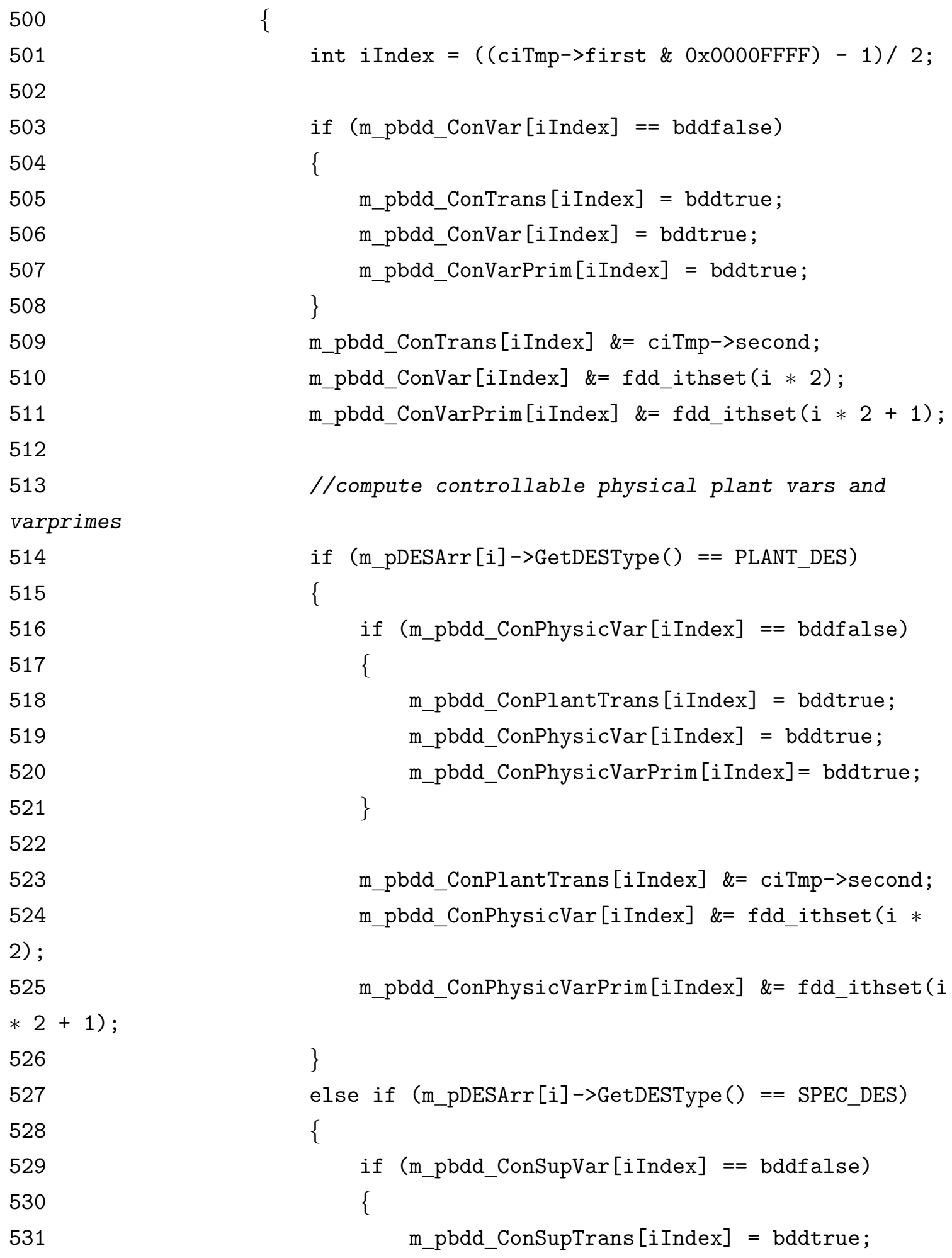


532

533

534

535

536

537

538

$2+1)$;

539

540

541

542

543

544

if the event

545

(plants).

546

547

548

549

550
m_pbdd_ConSupVar[iIndex $]=$ bddtrue;

m_pbdd_ConSupVarPrim[iIndex]= bddtrue;

\}

m_pbdd_ConSupTrans [iIndex] \&= ciTmp->second;

m_pbdd_ConSupVar[iIndex] \&= fdd_ithset $(i * 2)$;

m_pbdd_ConSupVarPrim[iIndex] \&= fdd_ithset $(i *$

m_SubSupervisorEvents.end ())

551 \&\& (m_SubPlantEvents.find(sig) !=

m_SubPlantEvents.end ()))

552

553

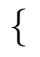

554

555

else if ((m_SubSupervisorEvents.find(sig) !=

m_SubSupervisorEvents.end ( ) )

556 \&\& (m_SubPlantEvents.find (sig) ==

m_SubPlantEvents.end ()))

557

558

559

m_pbdd_ConPlantTrans [iIndex] = bddtrue; 


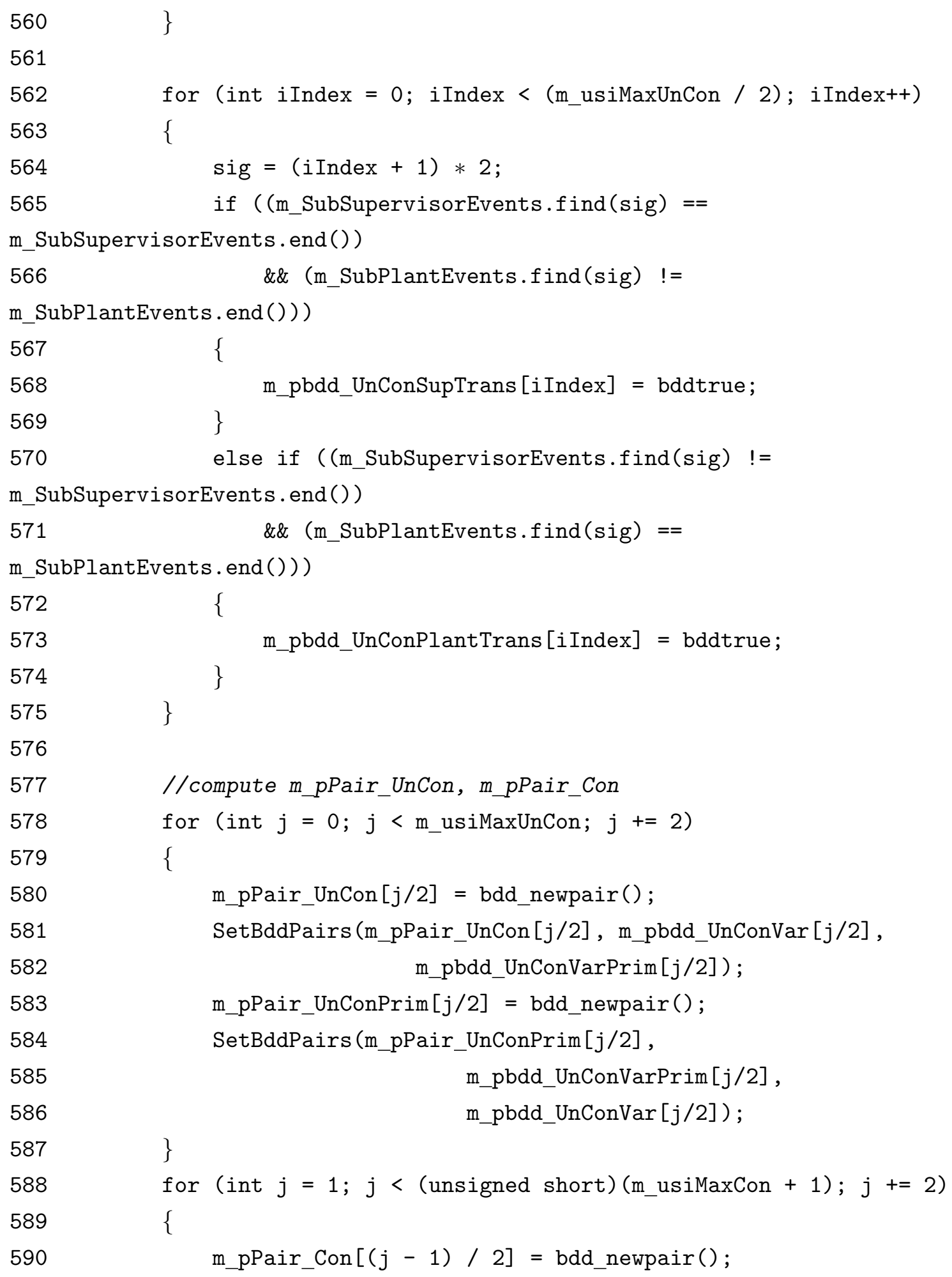




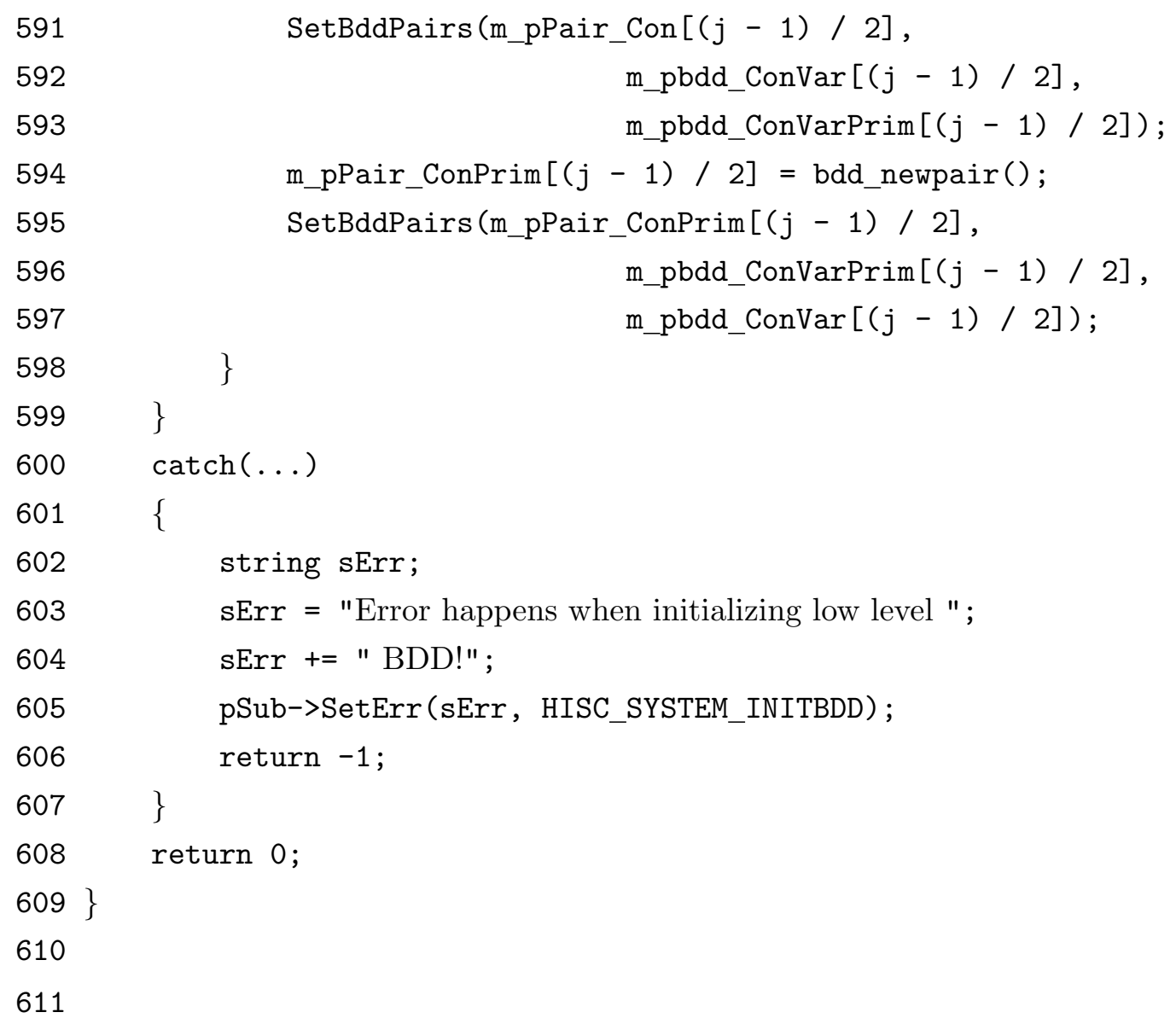

\section{LowSub1.cpp}

001 * DESCR: Save DES list of low-levels in memory to a file (for checking) 


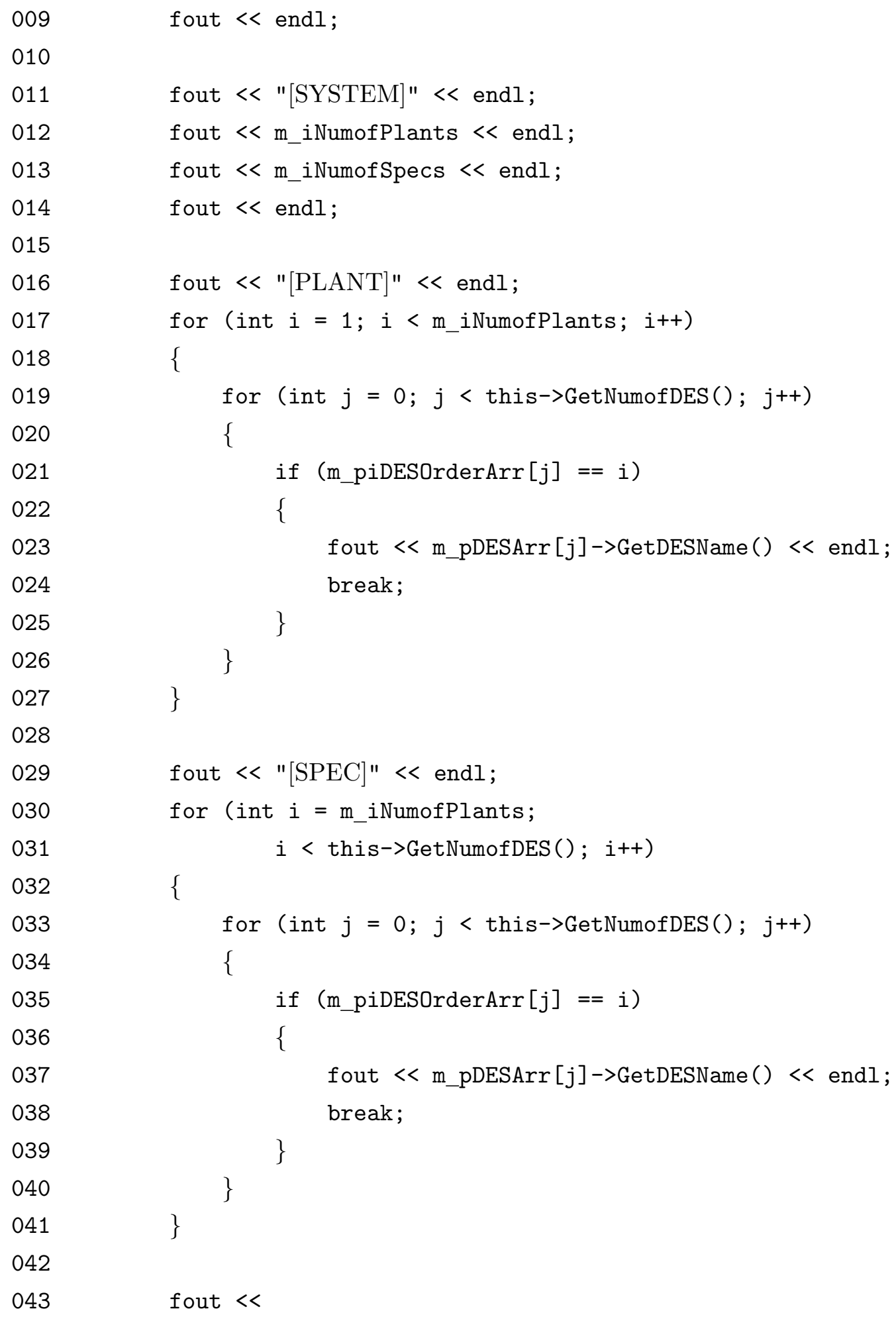




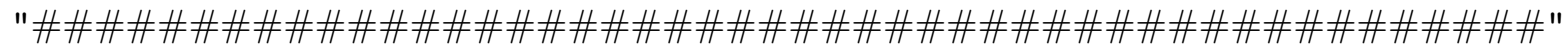
$<$ endl;

$044\}$

$045 \quad \operatorname{catch}(\ldots)$

046\{

047 return -1 ;

$048 \quad\}$

049 return 0 ;

$050\}$

051

$052 / * *$

* DESCR: Save all the DES in low-levels to a text file for checking 053 * PARA: fout: output file stream

$054 *$ RETURN: 0 : sucess -1 : fail

$055 *$ ACCESS: public

$056 * 1$

057 int CLowSub: :PrintSubAll (of stream \& fout)

058\{

059 try

059\{

060

061

if (PrintSub (fout) $<0)$

062

063

064

065

066

throw -1 ;

$067 \quad\}$

$068\}$

$069 \operatorname{catch}(\ldots)$

$070 \quad$ \{

071 return -1 ;

$072\}$

073 return 0 ;

$074\}$ 


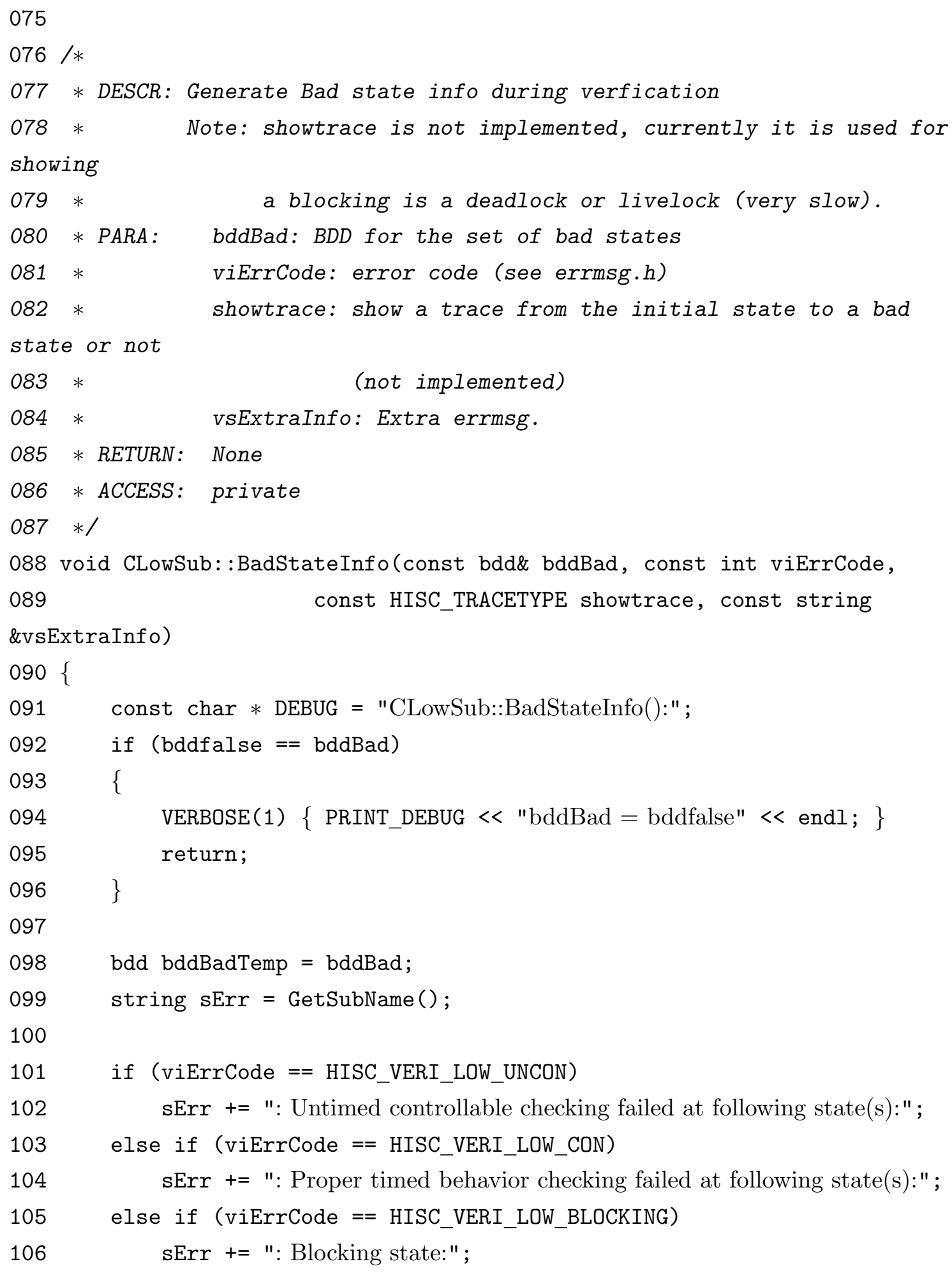


107 else if (viErrCode == HISC_VERI_LOW_P4FAILED)

108 sErr $+=$ ": Interface consistent conditions Point 4 checking failed state:" ;

109 else if (viErrCode == HISC_VERI_LOW_P5FAILED)

$110 \quad$ sErr += ": Interface consistent conditions Point 5 checking failed state:" ;

111 else if (viErrCode == HISC_VERI_LOW_P6FAILED)

112 sErr += ": Interface consistent conditions Point 6 checking failed state:" ;

113 else if (viErrCode == HISC_VERI_LOW_ALF)

114 sErr += ": ALF checking failed state:";

115 else if (viErrCode == HISC_VERI_LOW_PTB)

116 sErr += ": Not proper timed behavior at state:" ;

117 else if (viErrCode == HISC_VERI_LOW_SD_II)

118 sErr += ": Failed SD Controllability condition II at state:" ;

119 else if (viErrCode == HISC_VERI_LOW_SD_III_1)

120 sErr += ": Failed SD Controllability condition III.1 at state:" ;

121 else if (viErrCode == HISC_VERI_LOW_SD_III_2)

122 sErr += ": Failed SD Controllability condition III.2 at state:";

123 else if (viErrCode == HISC_VERI_LOW_SD_IV)

124 sErr += ": Failed SD Controllability condition IV at state:" ;

125 else if (viErrCode == HISC_VERI_LOW_ZERO_LB)

126 sErr += ": There is some event has a lower bound less than 1 tick:" ;

127

128

129

130

131

132

133

134

135

136

sErr += "\n";

137

138

139

140

int count $=0$;

while (bddfalse != bddBadTemp \&\& count < 10)

\{

141

$$
\begin{aligned}
& \text { bdd bddstate = GetOneState (bddBadTemp); } \\
& \text { bddBadTemp -= bddstate; }
\end{aligned}
$$

int $*$ piBad $=$ fdd_scanallvar (bddstate $)$;

if $($ NULL $==$ piBad) break;

//for blocking state, try to find the deadlock state

//if there is no deadlock state, only show one of the live lock 


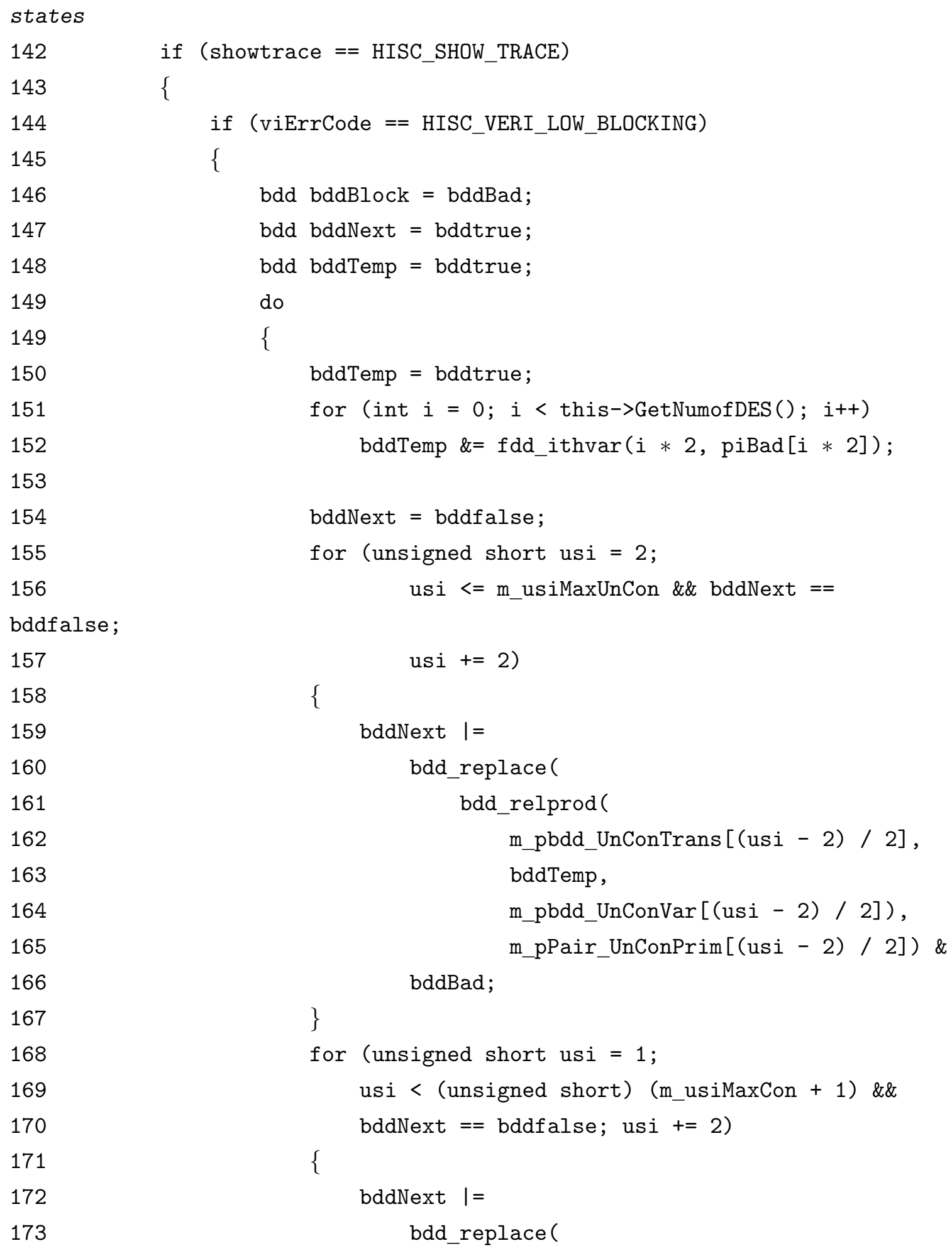


174

175

176

177

178

179

180

181

182

state

183

184

185

186

187

188

189

190

191

192

193

194

195

196

197

198

199

200

201

202

203

204

205

206

207 bdd_relprod(

m_pbdd_ConTrans [(usi - 1) / 2], bddTemp, m_pbdd_ConVar[(usi - 1) / 2]), m_pPair_ConPrim[(usi - 1) / 2]) \& bddBad;

\}

if (bddNext $==$ bddfalse) //this is a deadlock

\{ sErr += "[DeadLock $] "$; break;

\} else //not a deadlock state \{ bddBlock = bddBlock - bddTemp; free (piBad); piBad = NULL; piBad = fdd_scanallvar(bddBlock);

\}

count++;

\} while (piBad != NULL);

if ( $\mathrm{piBad}==$ NULL) //live lock

\{

sErr $+=$ "[LiveLock $] "$;

piBad = fdd_scanallvar (bddBad);

\}

\}

\}

sErr $+=" \backslash t_{i} " ;$ 


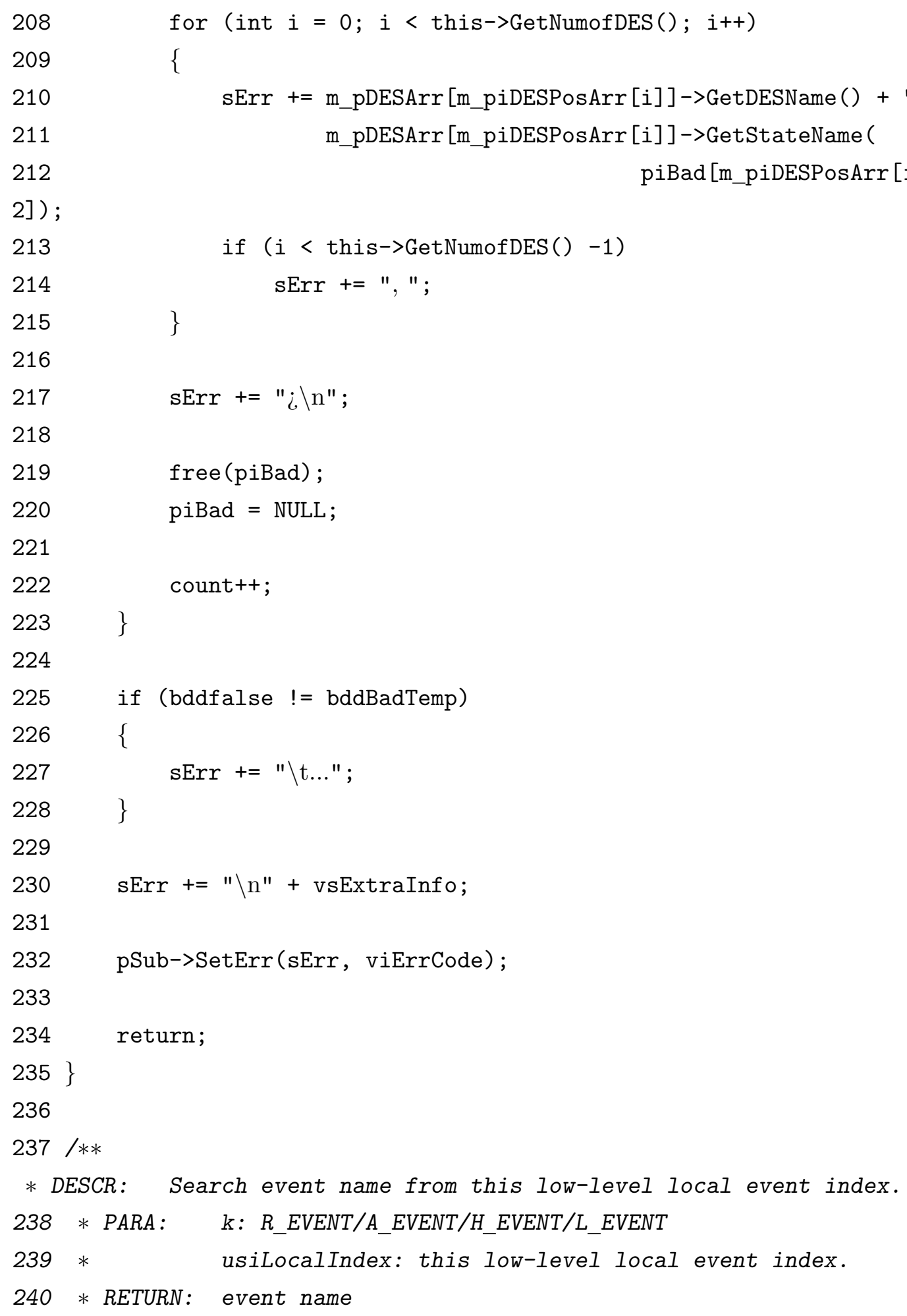


$241 *$ ACCESS: public

$242 * 1$

243 string CLowSub: :SearchEventName(unsigned short usiLocalIndex)

244\{

245 int iEventIndex $=0$;

246 iEventIndex $=$ pSub->GenEventIndex (usiLocalIndex);

247 return (pSub->GetInvAllEventsMap ()) [iEventIndex];

$248\}$

249

250

\section{LowSub3.cpp}

001 int CLowSub::VeriSub(const HISC_TRACETYPE showtrace, HISC_SUPERINFO \& superinfo)

002\{

003 int iRet $=0$;

004 int iErr $=0$;

005 //Initialize the BDD data memebers

006 CSub: :InitBddFields();

007 InitBddFields ();

008 bdd bddReach = bddfalse;

009 string sErr;

010

011 \#ifdef DEBUG_TIME

012 timeval tv1, tv2;

013 \#endif

014

015 try

015\{

016 //Make transition bdds

017 if (MakeBdd ()$<0)$

018 throw -1 ;

019

020 bdd bddConBad = bddfalse; 


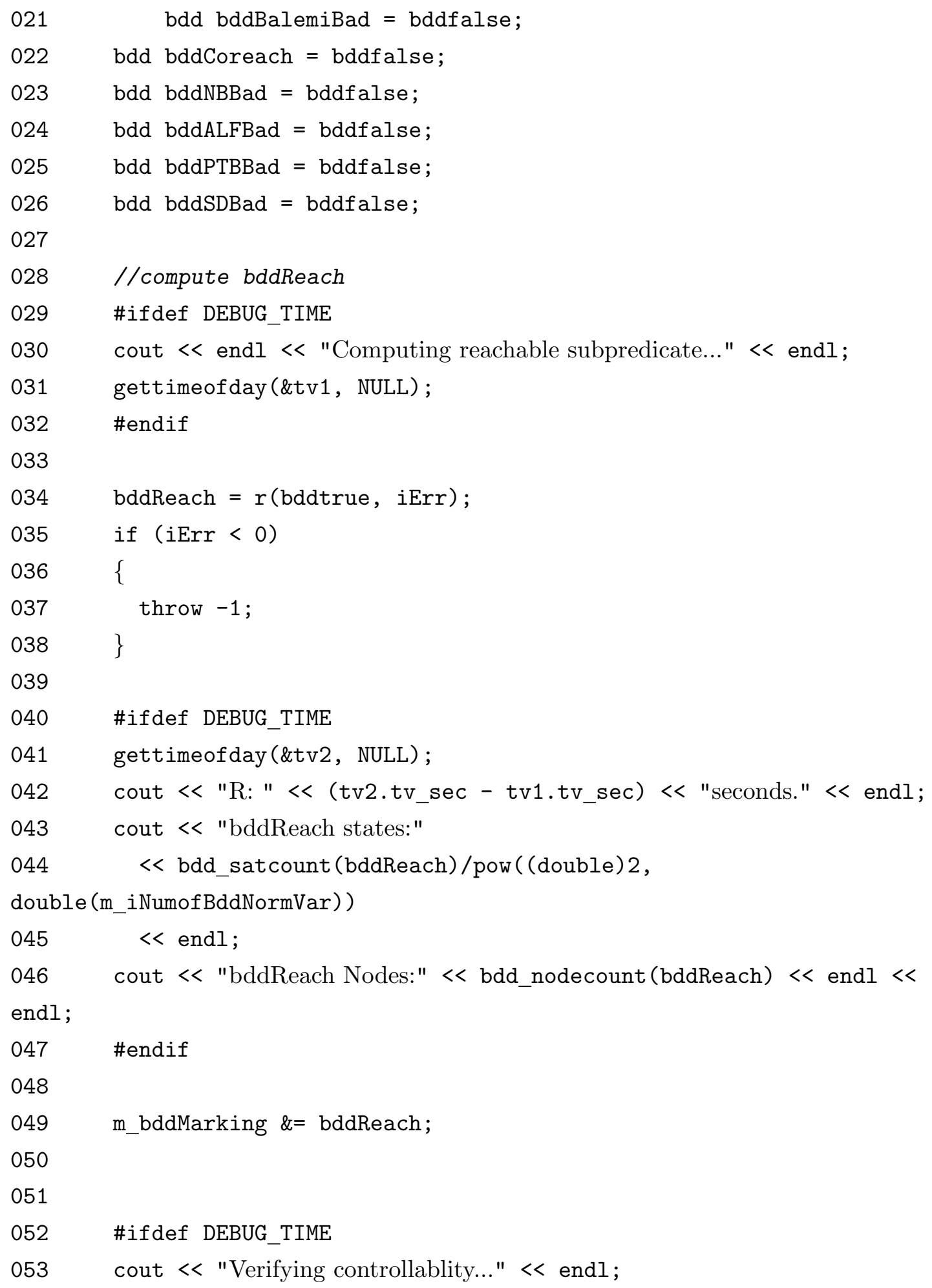




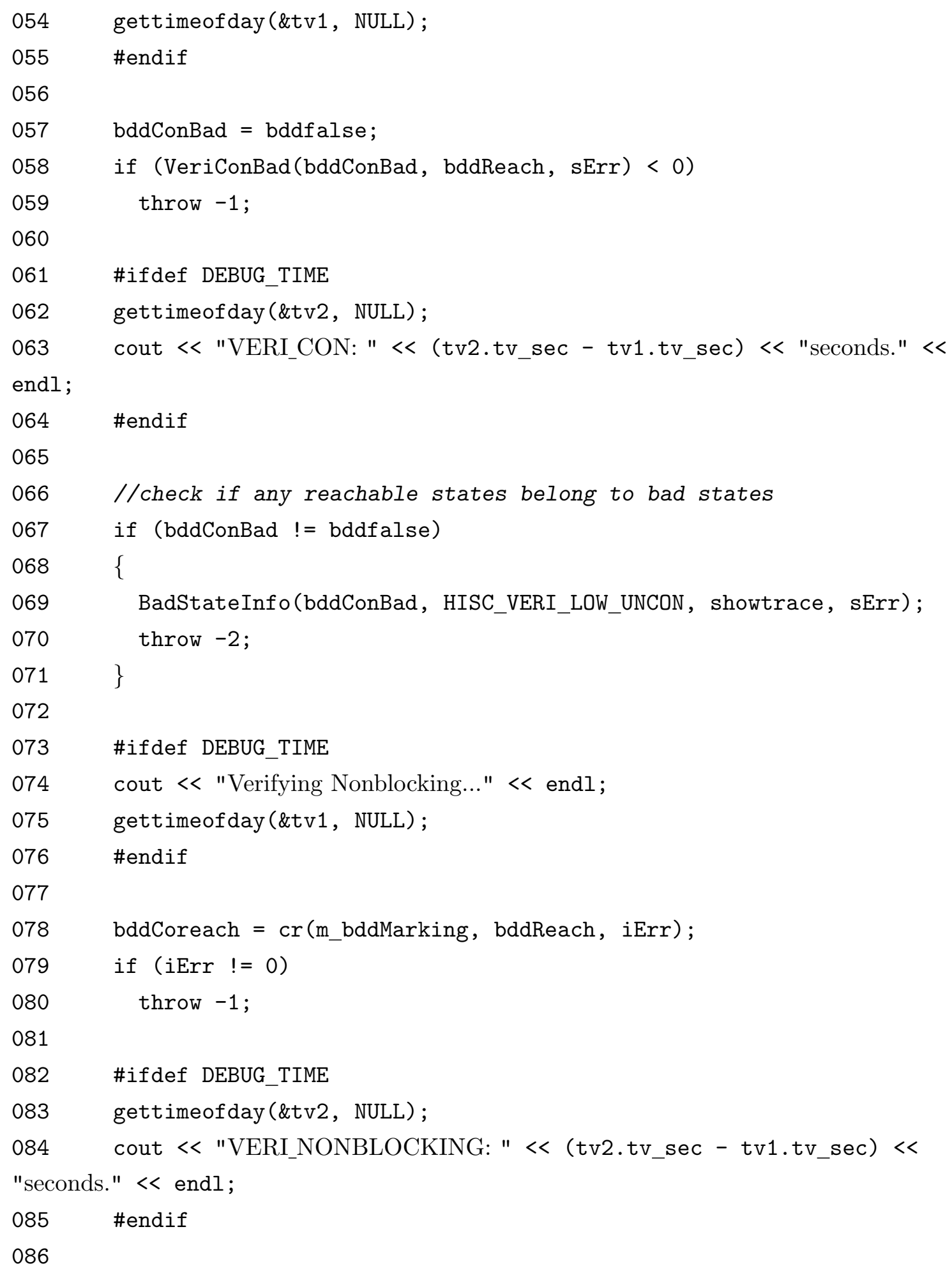




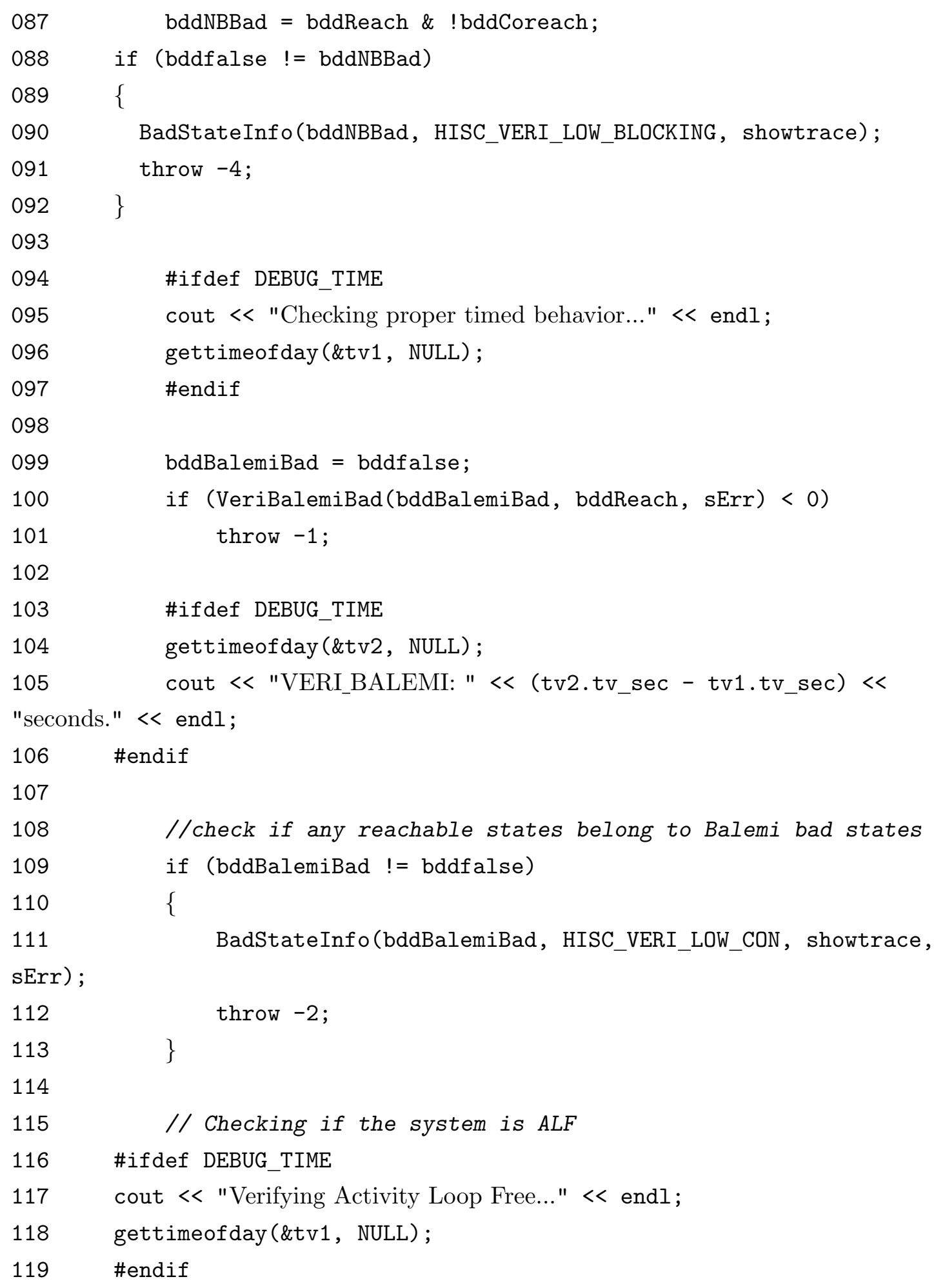


120

121

122

123

124

125

126

127

$<<$ endl;

128 \#endif

129

130 if (bddALFBad != bddfalse)

131\{

132

bddALFBad = bddfalse;

if (VeriALF (bddALFBad, bddReach, sErr) < 0)

throw -1 ;

sErr);

133

\#ifdef DEBUG_TIME

gettimeofday (\&tv2, NULL);

cout $\ll$ "VERI_ALF: " $<$ (tv2.tv_sec - tv1.tv_sec) $<$ "seconds."

$134\}$

135

136

137

138

139

140

141

142

143

144

145

146

147

148

BadStateInfo(bddALFBad, HISC_VERI_LOW_ALF, showtrace,

$$
\text { throw }-2 \text {; }
$$

\}

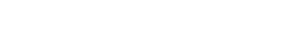

151

if (bddPTBBad $!=$ bddfalse) 


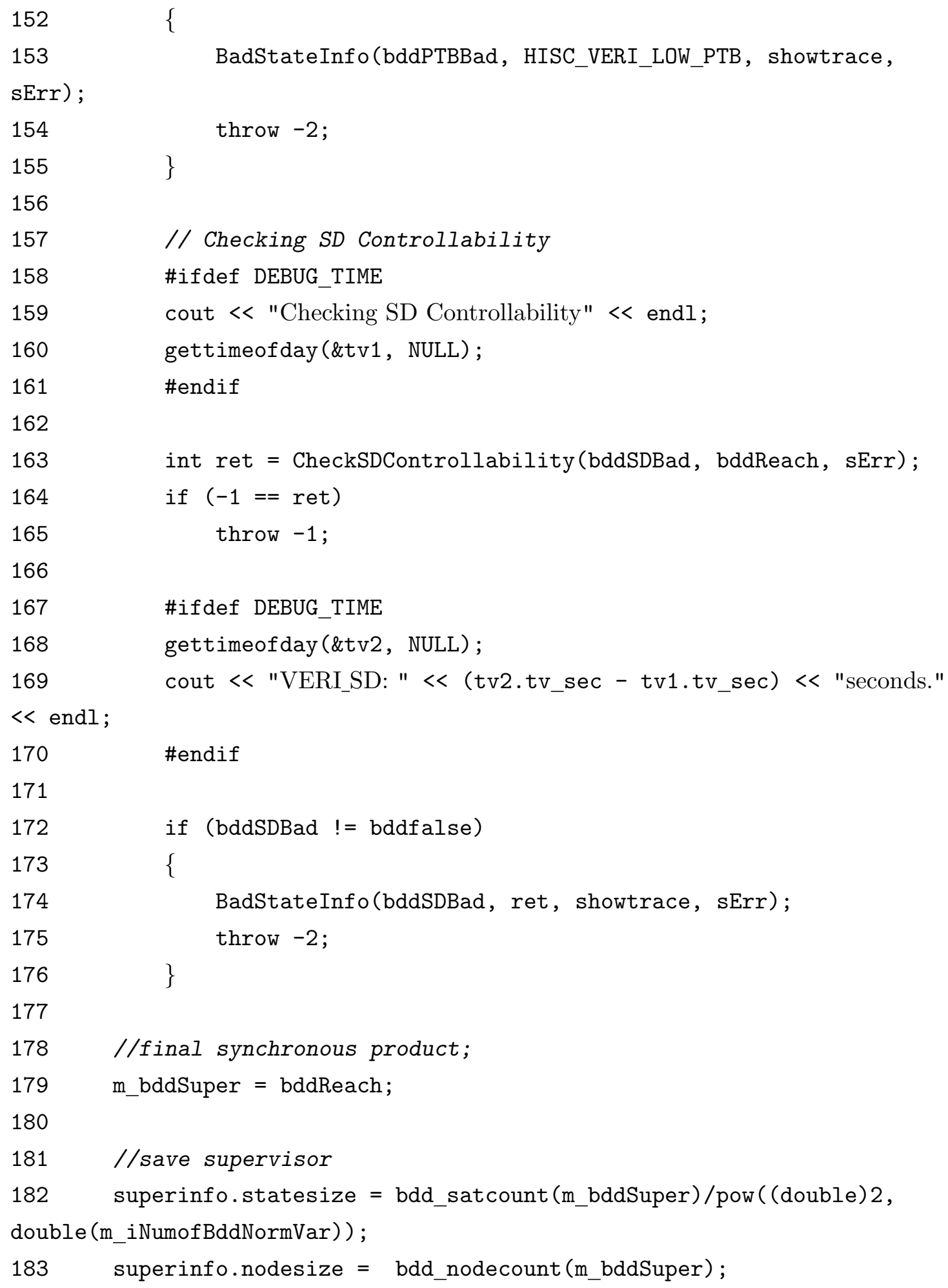




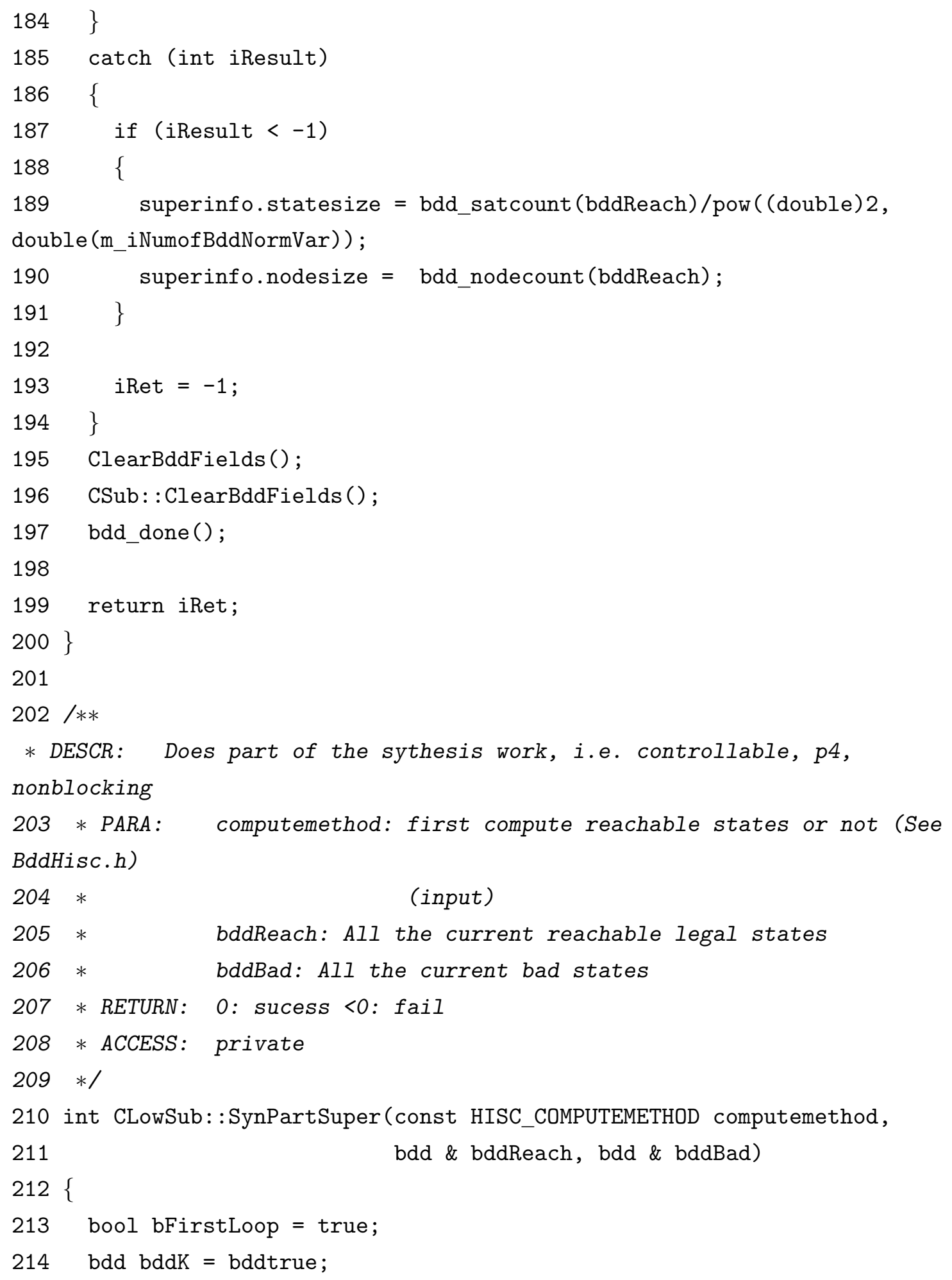




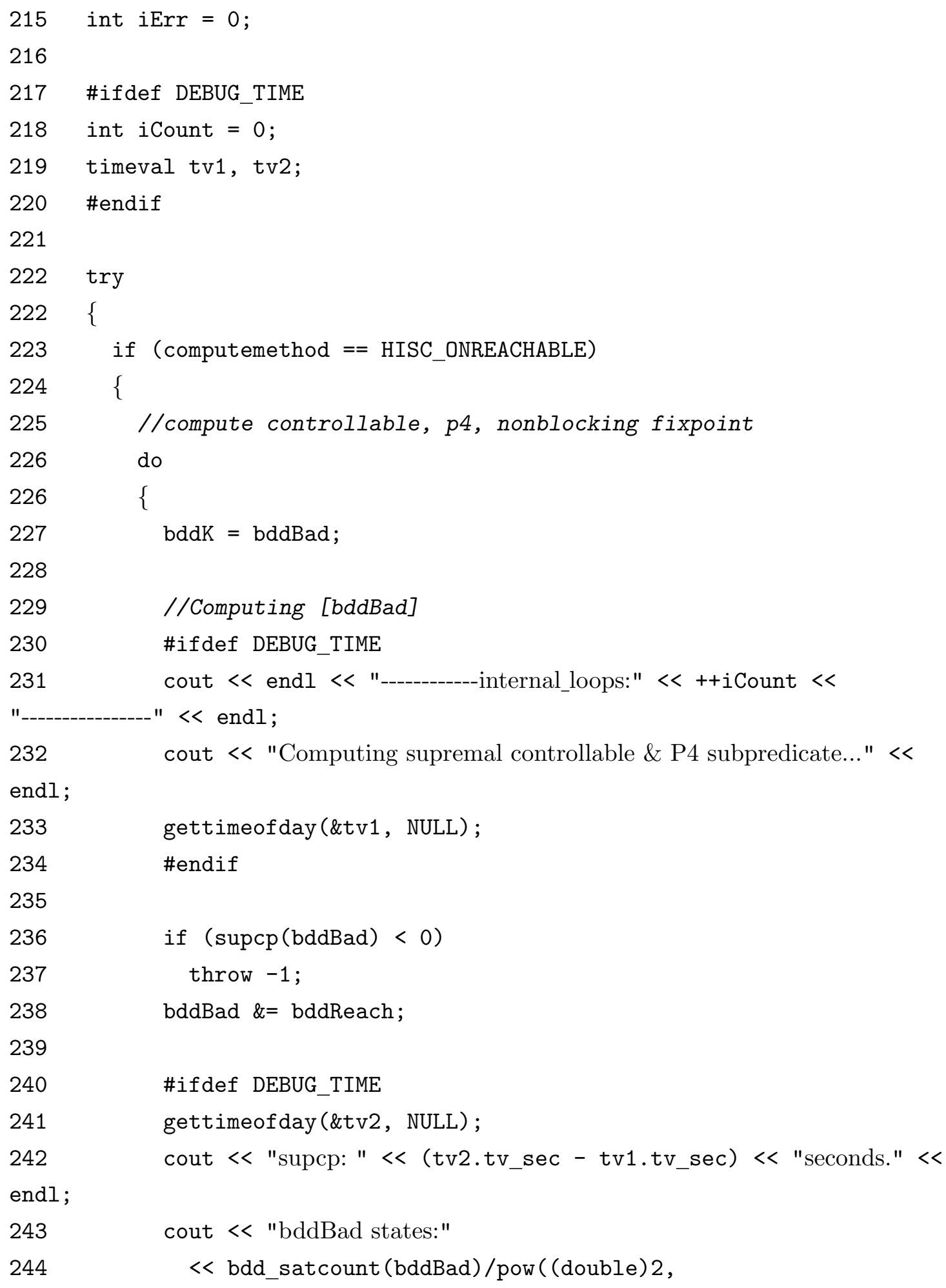


double(m_iNumofBddNormVar))

245

$<$ endl;

246

cout $<<$ "bddBad Nodes:" $<<$ bdd_nodecount(bddBad) $<$ endl;

247

\#endif

248

249

if $(\mathrm{bddK}==$ bddBad \&\& bFirstLoop $==$ false)

250

break;

251

252

//Computing CR(not(bddBad))

253

bdd bddTemp = bddReach - bddBad;

254

255

\#ifdef DEBUG_TIME

256

cout $<$ endl $<$ "bddGood states:"

257

$<$ bdd_satcount (bddTemp)/pow ((double)2,

double (m_iNumofBddNormVar))

258

$<<$ endl;

259

cout $<<$ "bddGood Nodes:" $<<$ bdd_nodecount (bddTemp) $<<$ endl;

260 cout $<<$ endl $<<$ "Computing coreachable subpredicate..." $<<$ endl;

261

262 gettimeofday (\&tv1, NULL);

263

264

\#endif

265

bddBad $=$ bdd_not $\left(\mathrm{cr}\left(\mathrm{m} \_\right.\right.$bddMarking, bddTemp, iErr $\left.)\right)$;

266

if (iErr !=0)

267

throw -1 ;

268

bddBad \&= bddReach;

269

270

bFirstLoop = false;

271

\#ifdef DEBUG_TIME

272

gettimeofday (\&tv2, NULL);

272

endl;

273

cout $\ll$ "cr: " $<$ (tv2.tv_sec - tv1.tv_sec) $<<$ "seconds." $<<$

274

cout $<<$ "bddBad states:"

double (m_iNumofBddNormVar))

275

$<$ endl; 


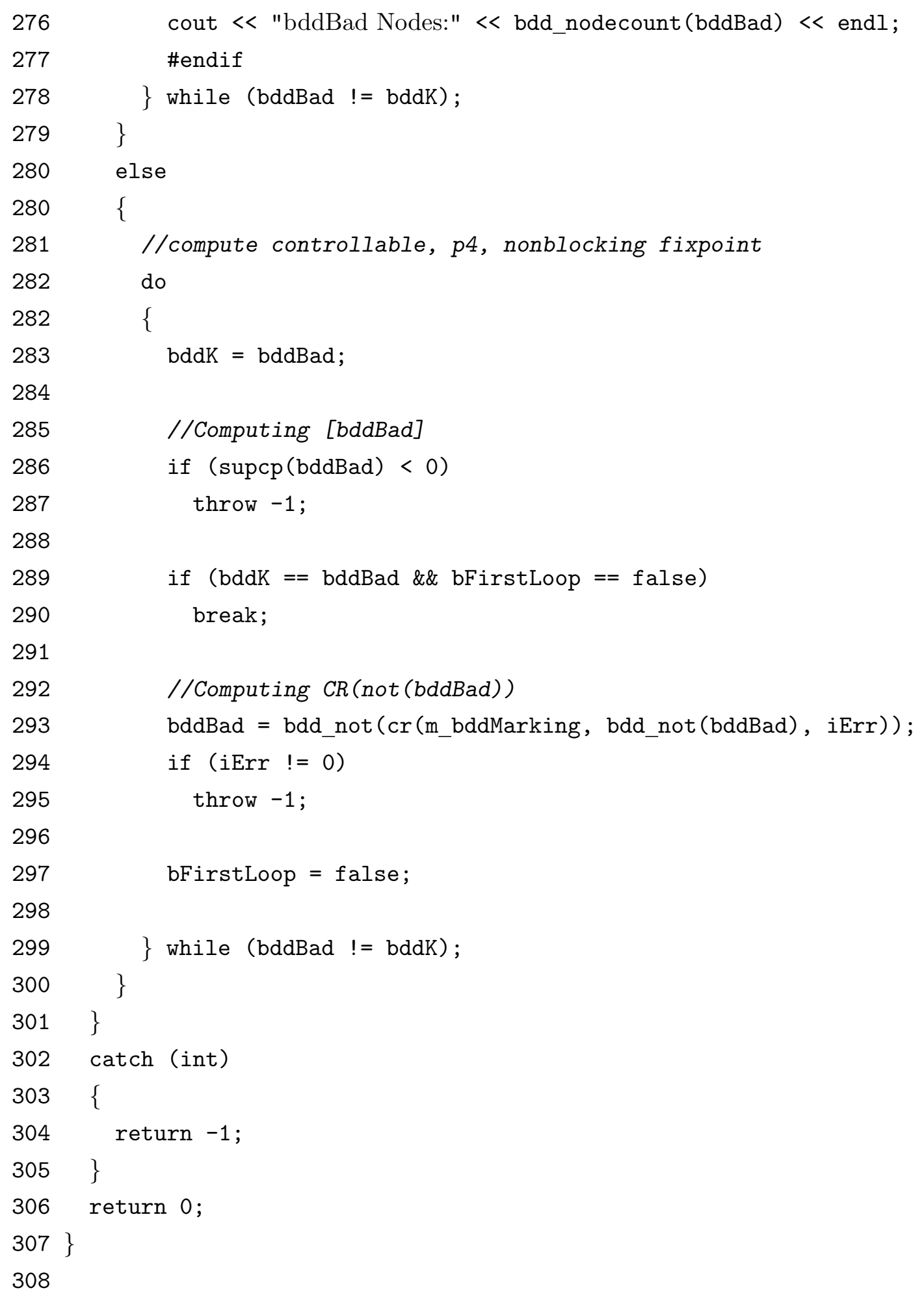




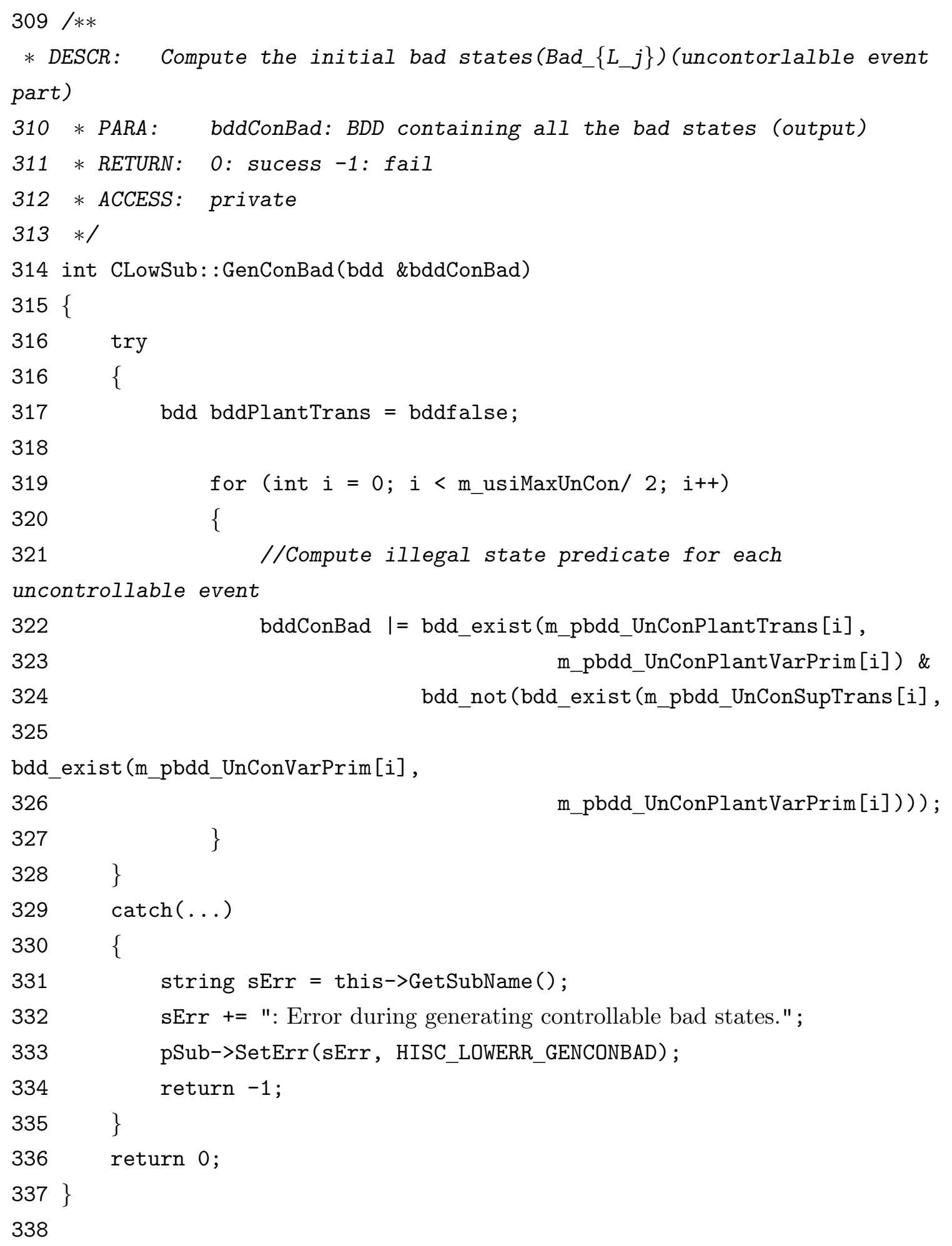




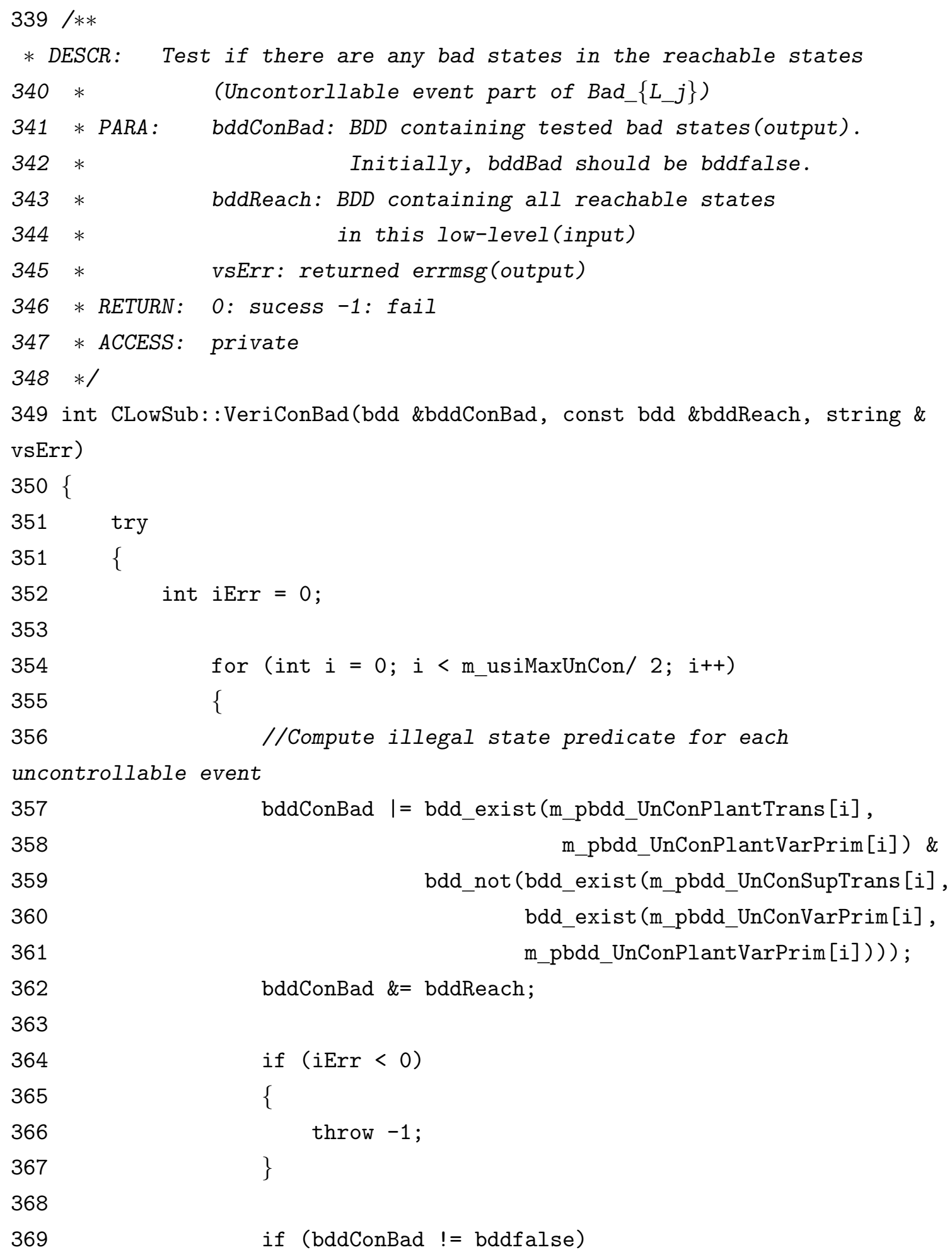




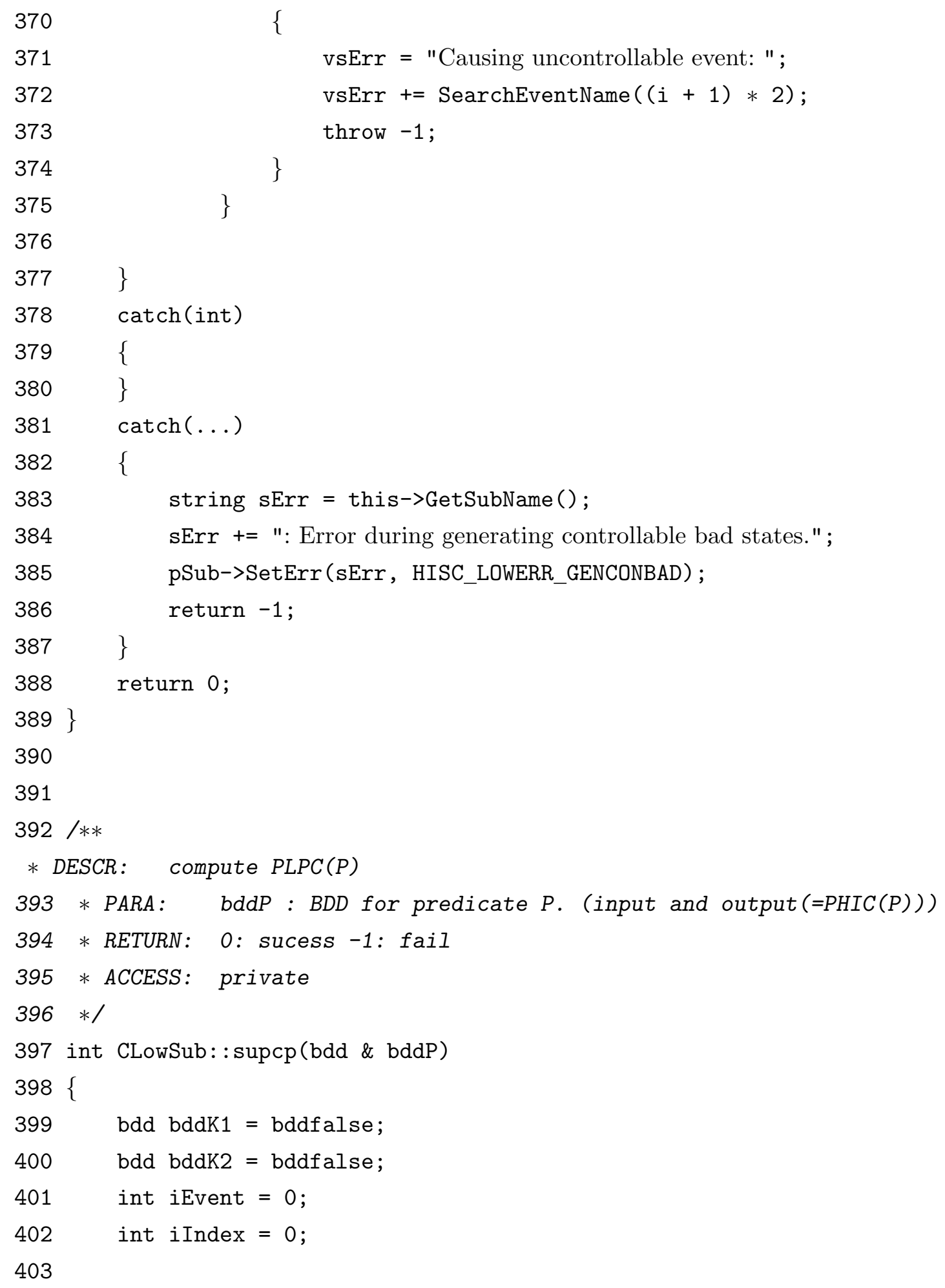




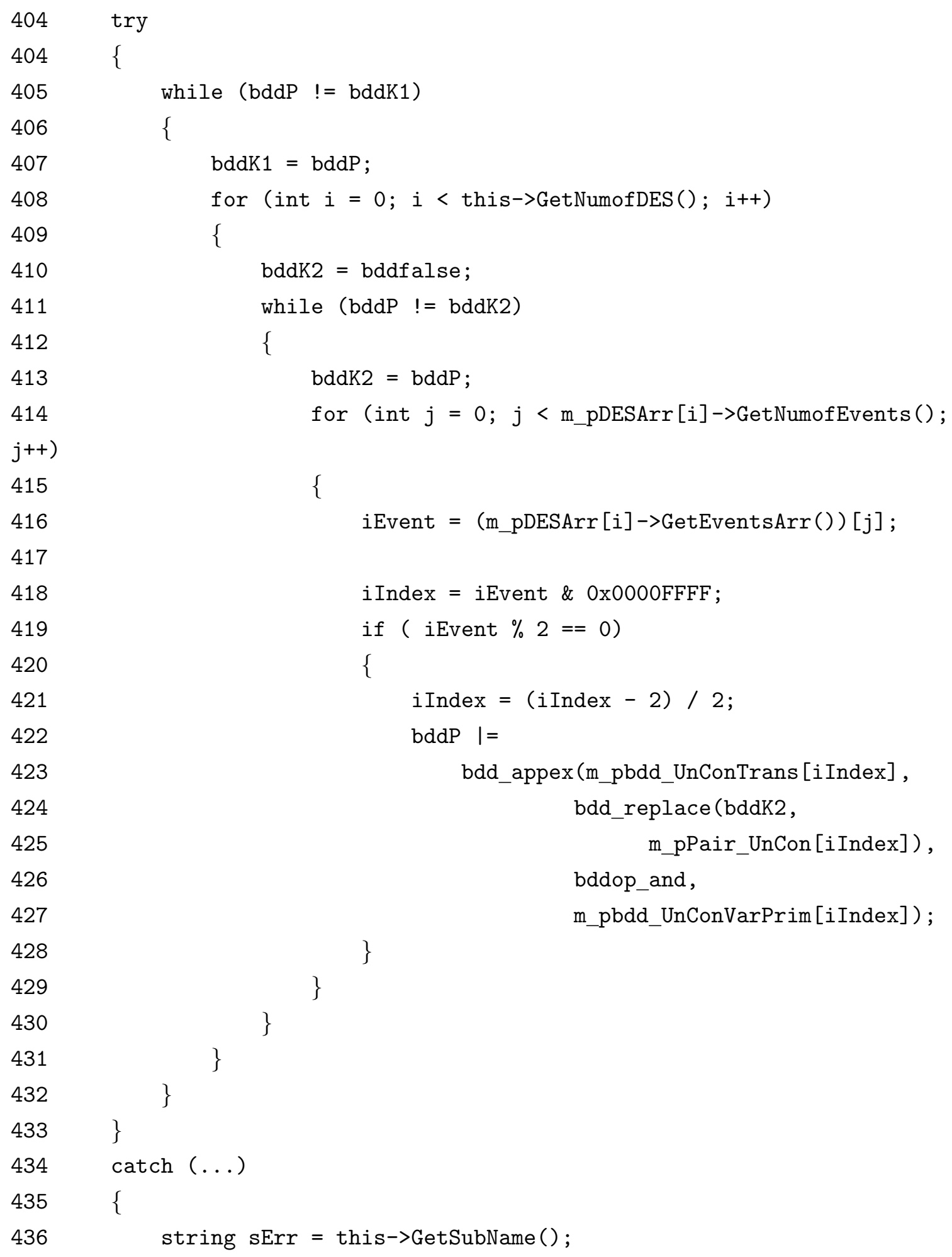




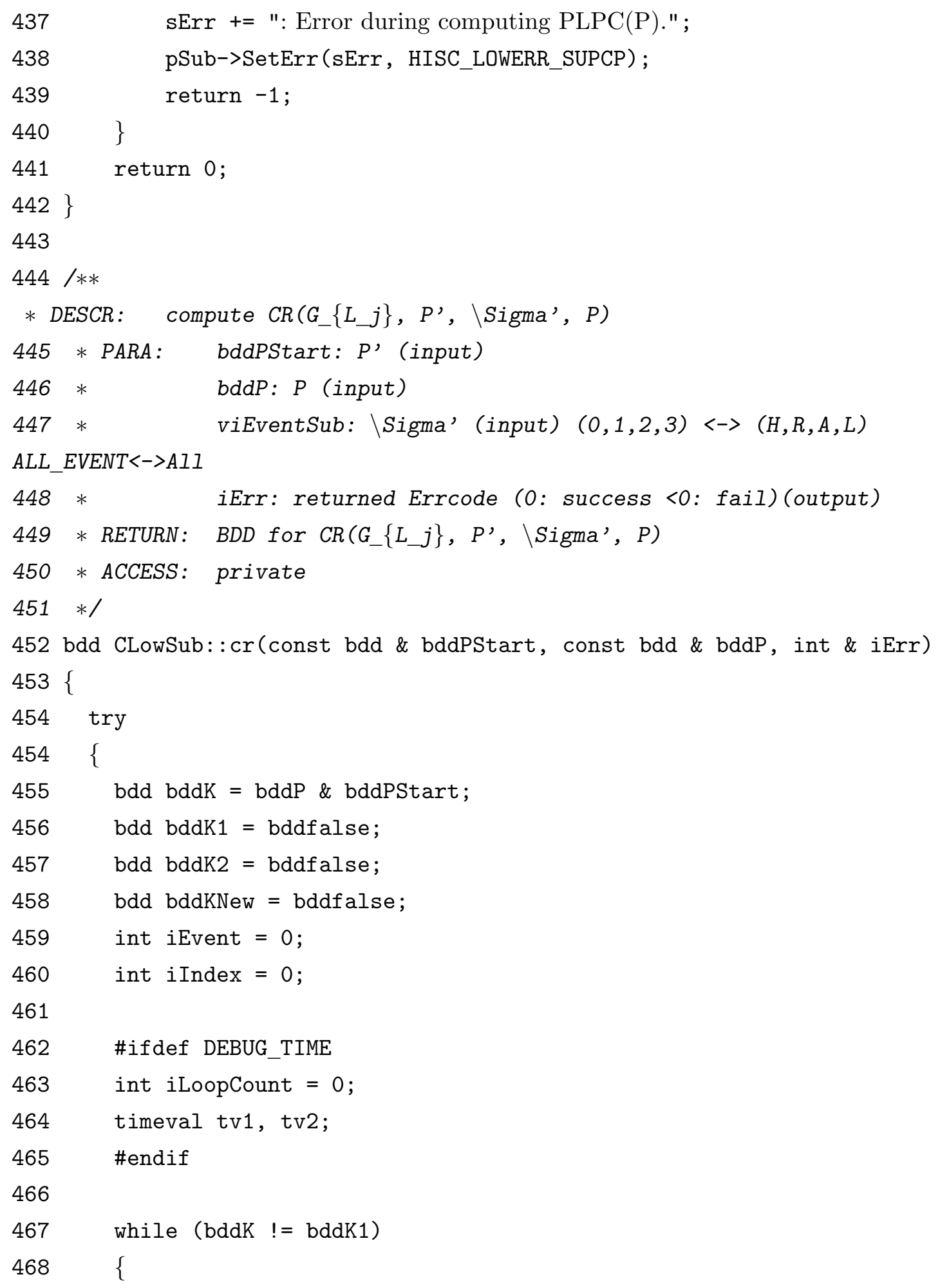




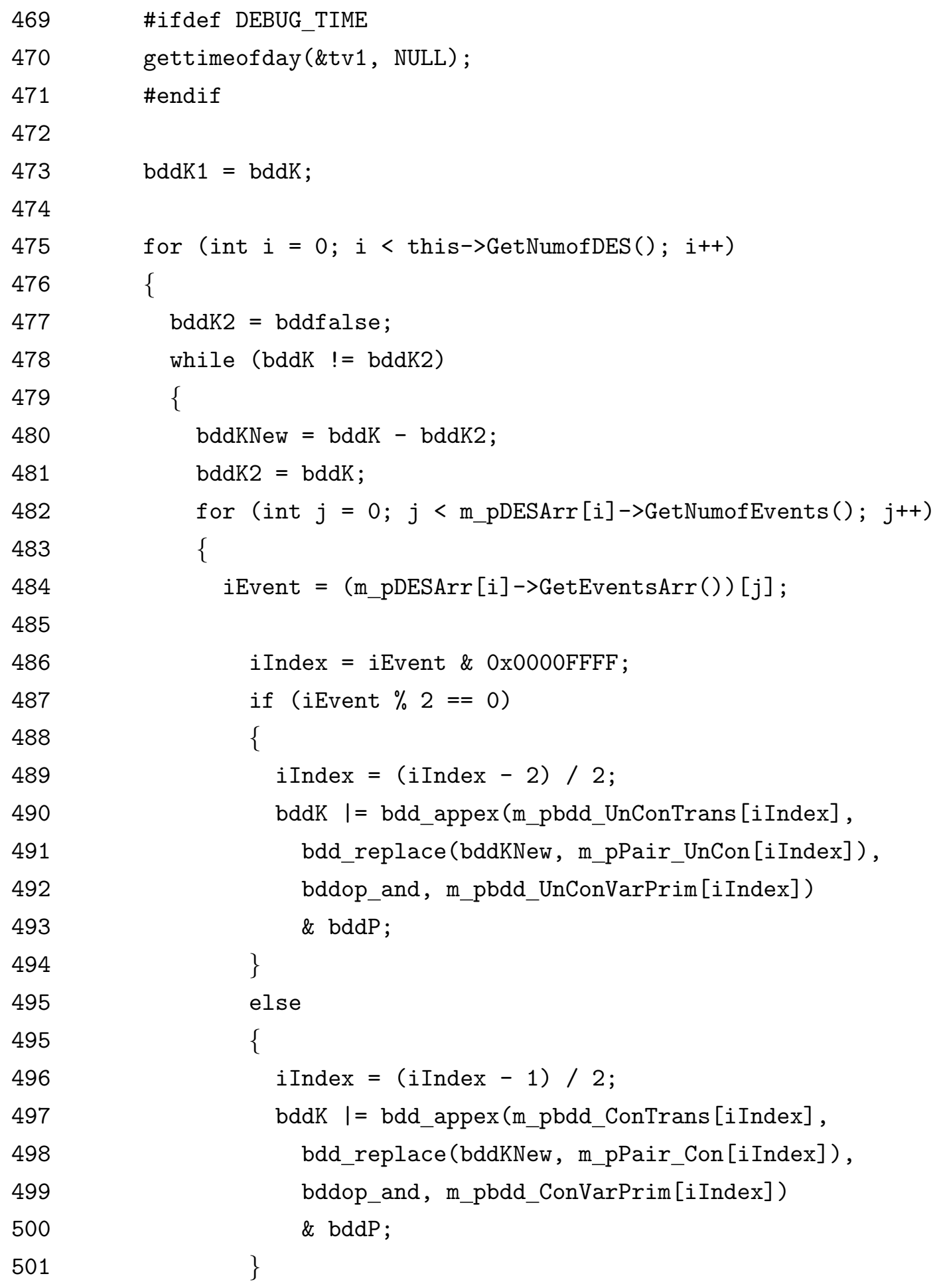




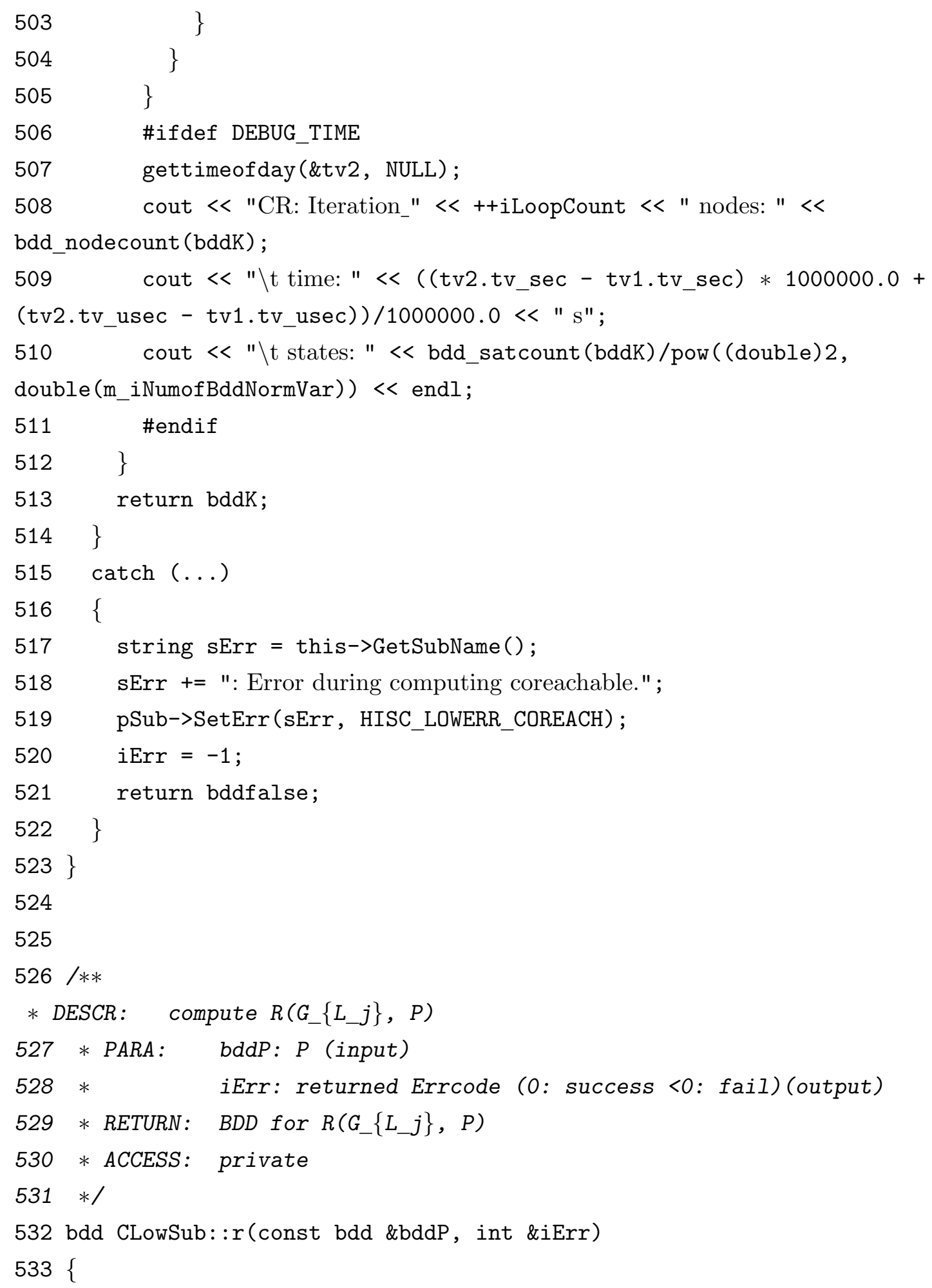




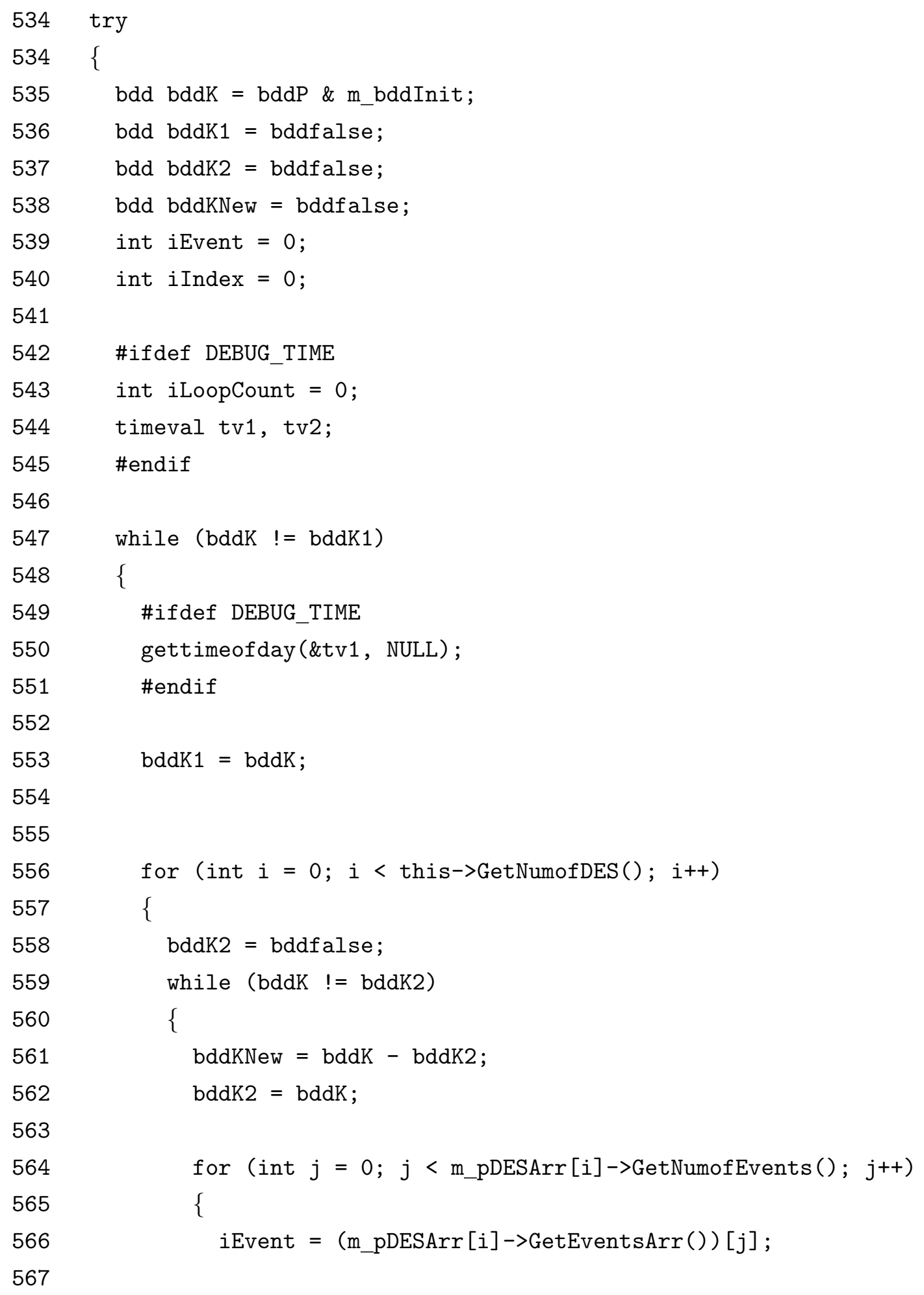




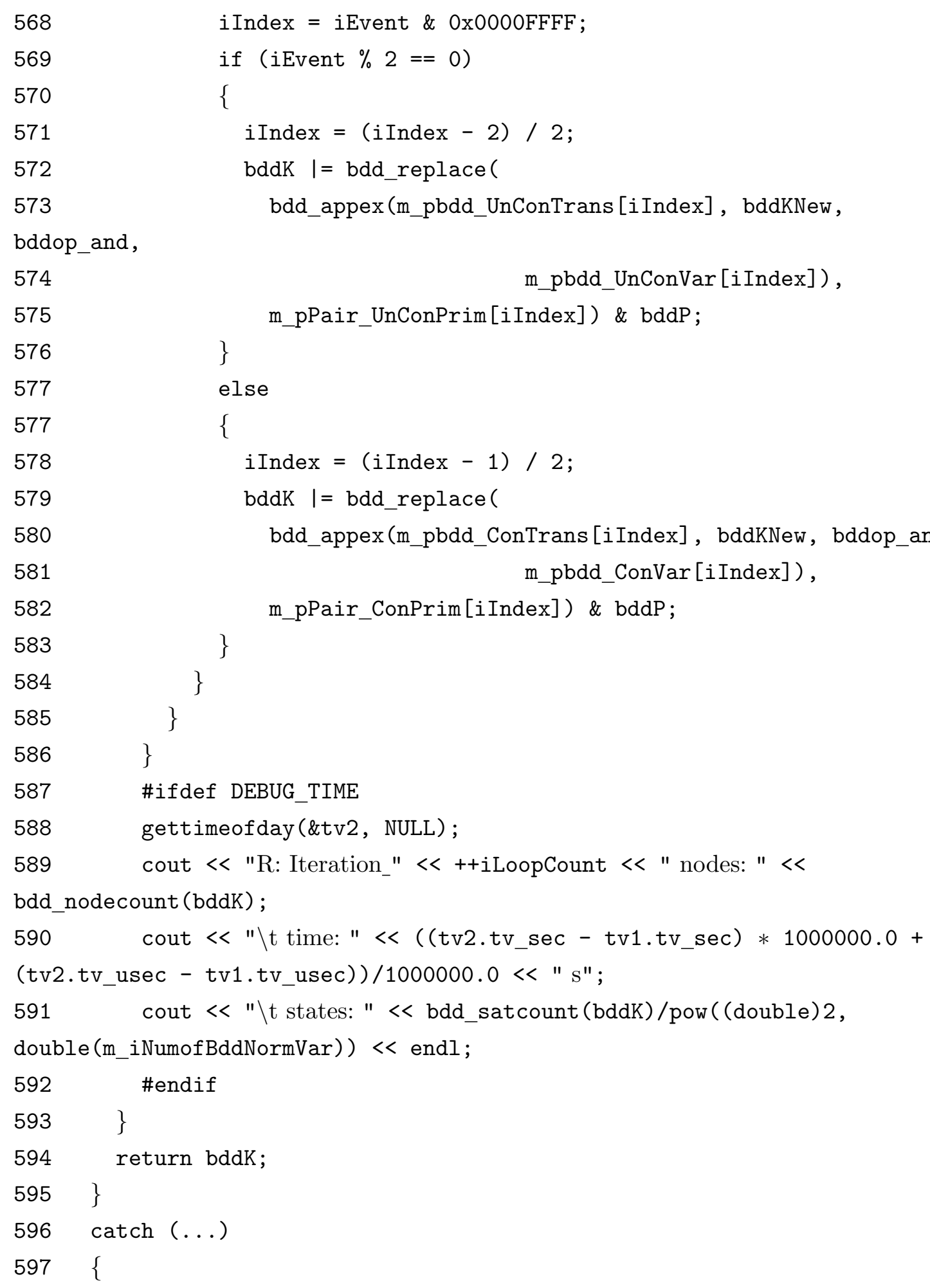




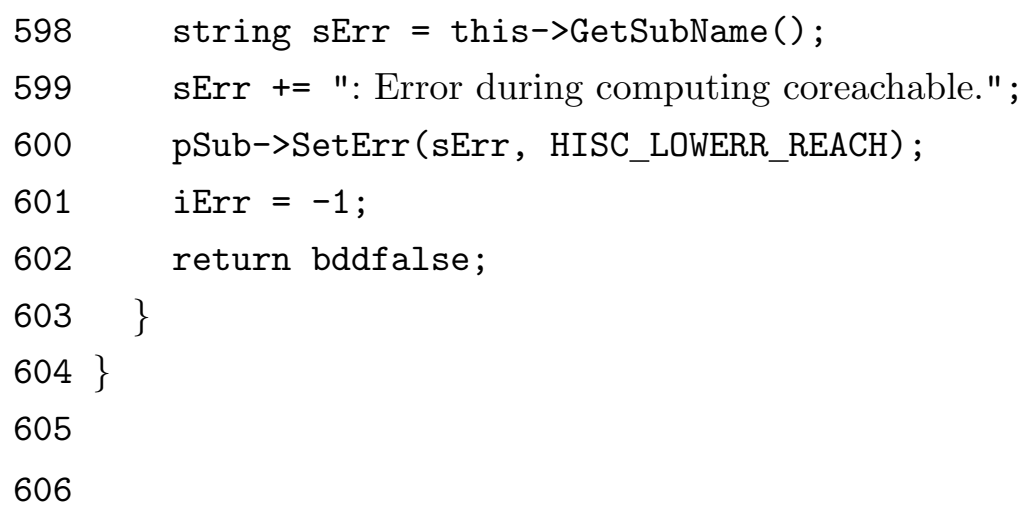

\section{LowSub4.cpp}

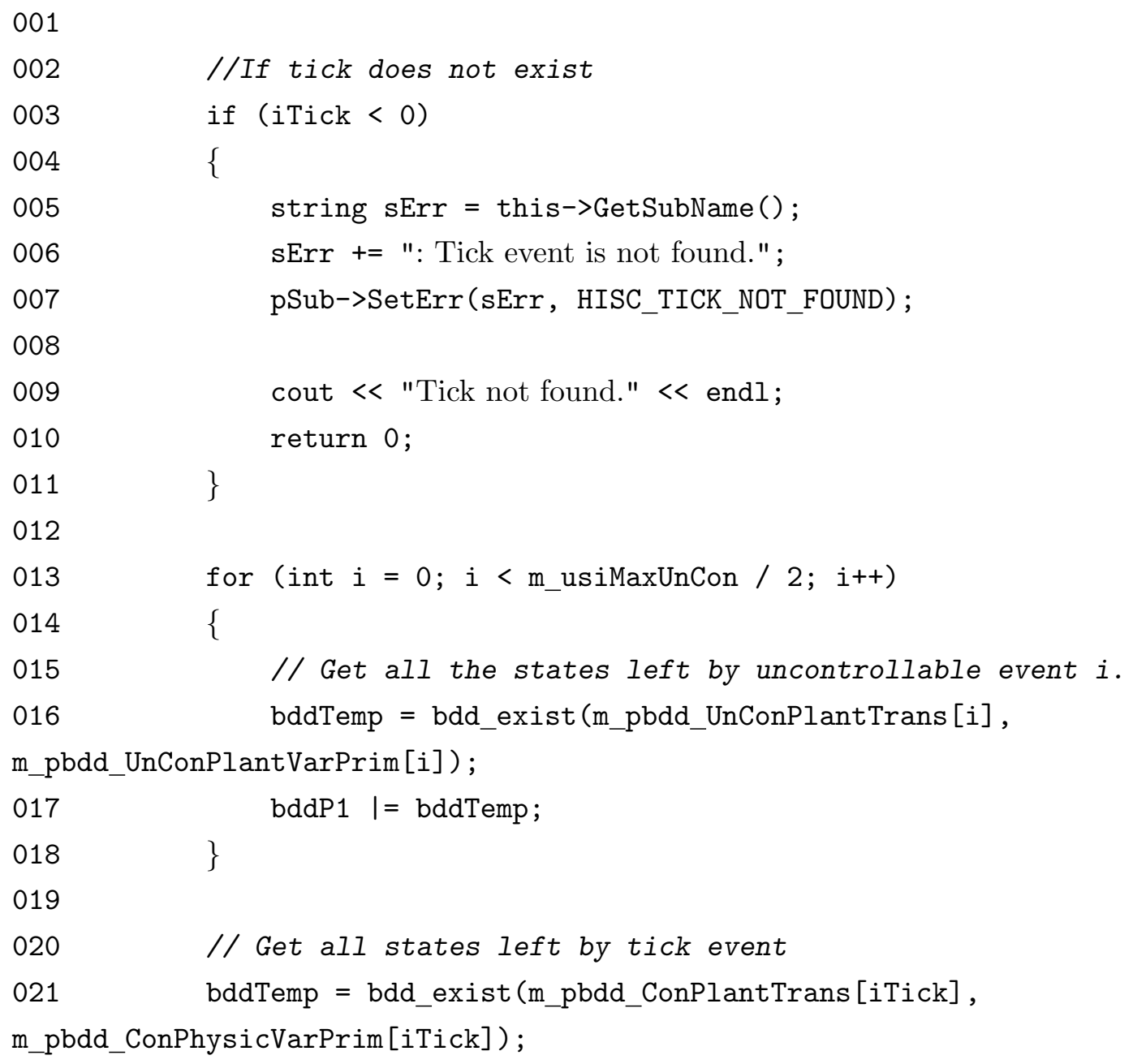


022

023

bddP1 | = bddTemp;

024

025

026

027

028

029

030

031

032

bddPTBBad $=$ bddReach - bddP 1 ;

033

034

035

036

037

038

039

040

041

042

043

044

VERBOSE (2)

\{

PRINT_DEBUG << "bddReach: ";

PrintStateSet2 (bddReach);

cout $<<$ endl;

\}

045

$046 \quad\}$

047 catch(int)

$048 \quad\{$

$049\}$

$050 \quad \operatorname{catch}(\ldots)$

051\{

052

if (bddPTBBad $!=$ bddfalse)

\{

VERBOSE (2)

\{

PRINT_DEBUG 《< "bddPTBBad: " ;

PrintStateSet2 (bddPTBBad);

cout $<<$ endl;

\}

vsErr = "Not proper timed behavior." ;

throw -1 ;

053

054

055

\}

\}

\}

\{

string sErr $=$ this $->$ GetSubName () ;

sErr += ": Error when checking proper timed behavior.";

pSub->SetErr (sErr, HISC_LOWERR_PTB);

$056\}$

return -1 ; 


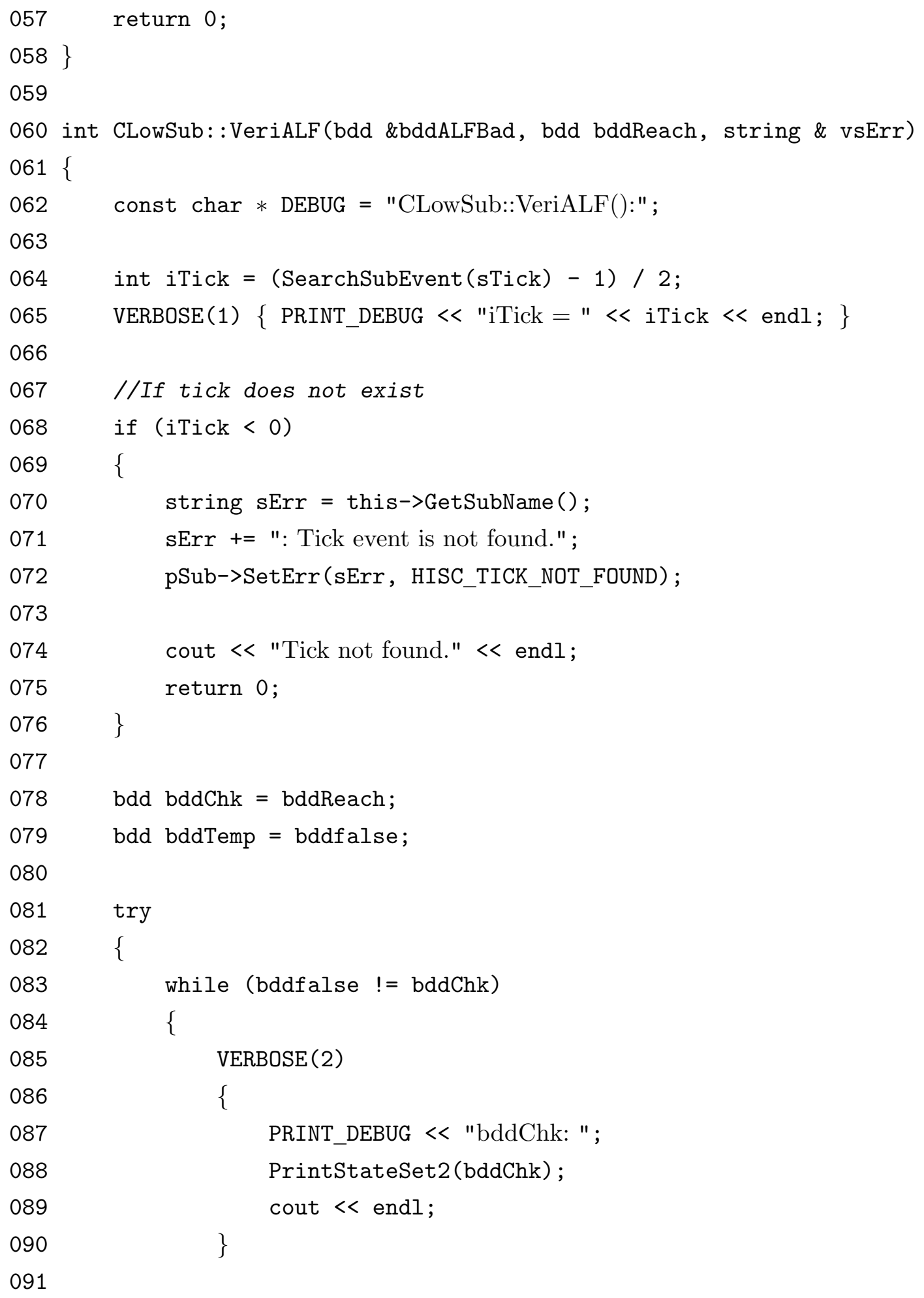




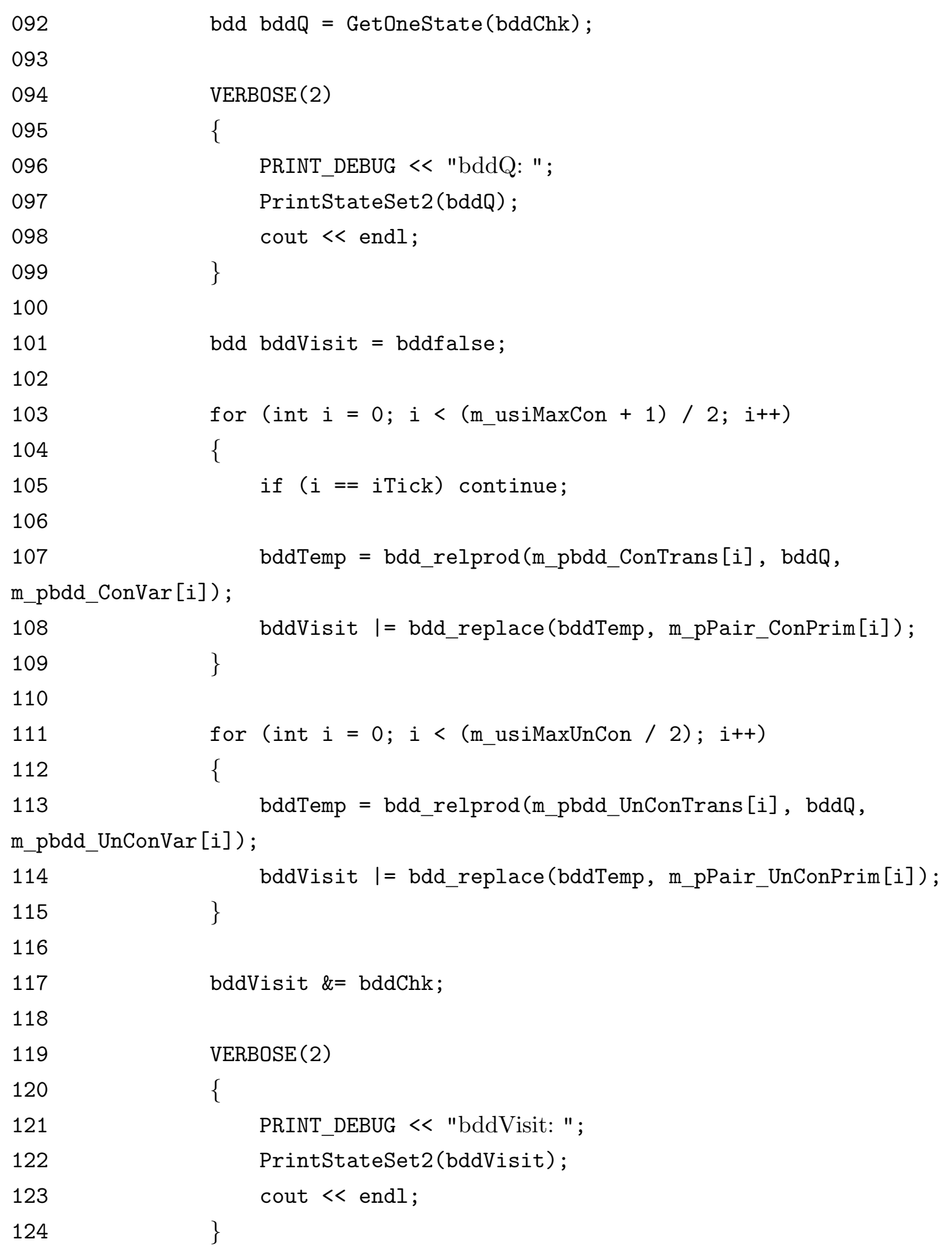




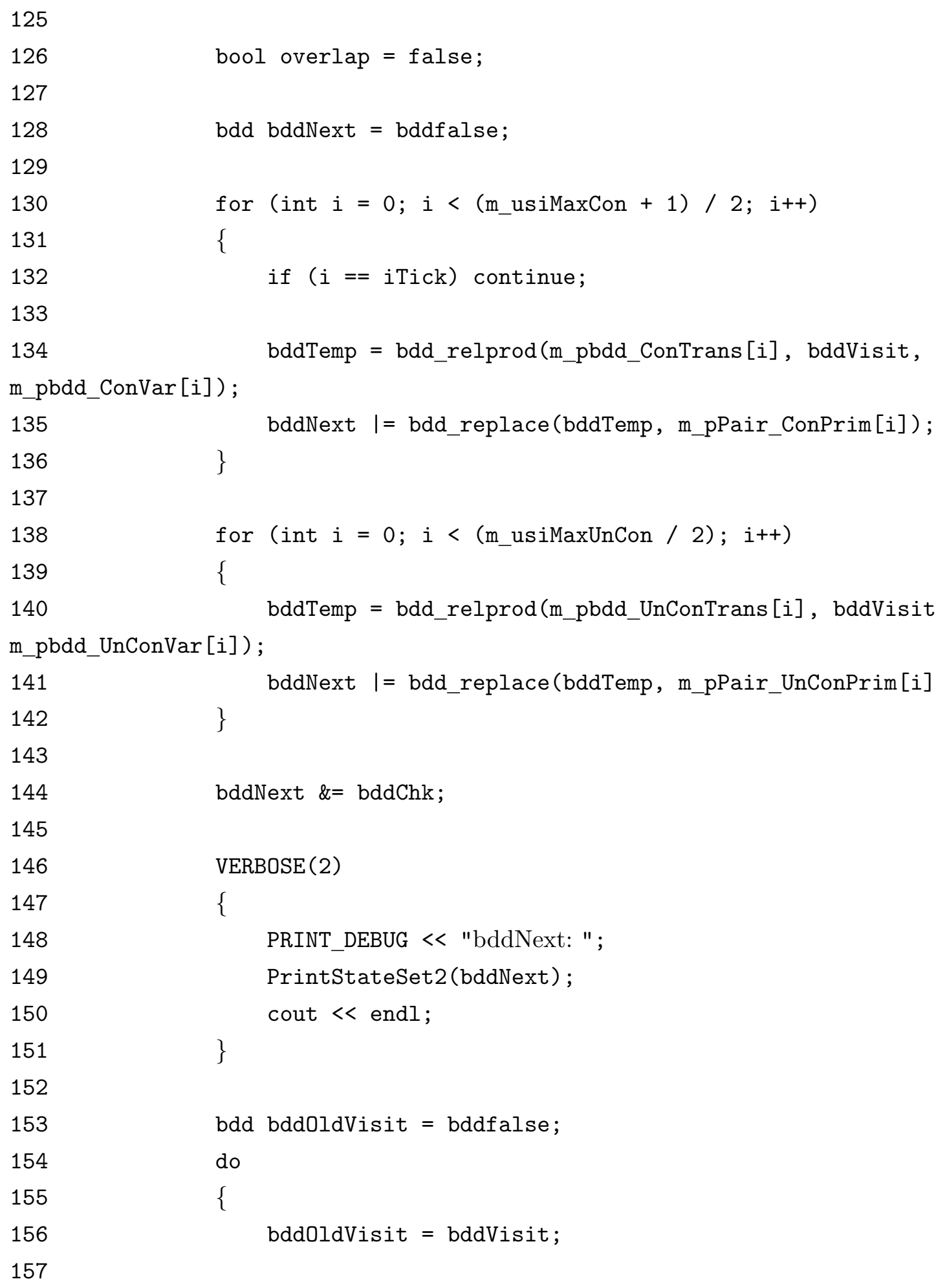




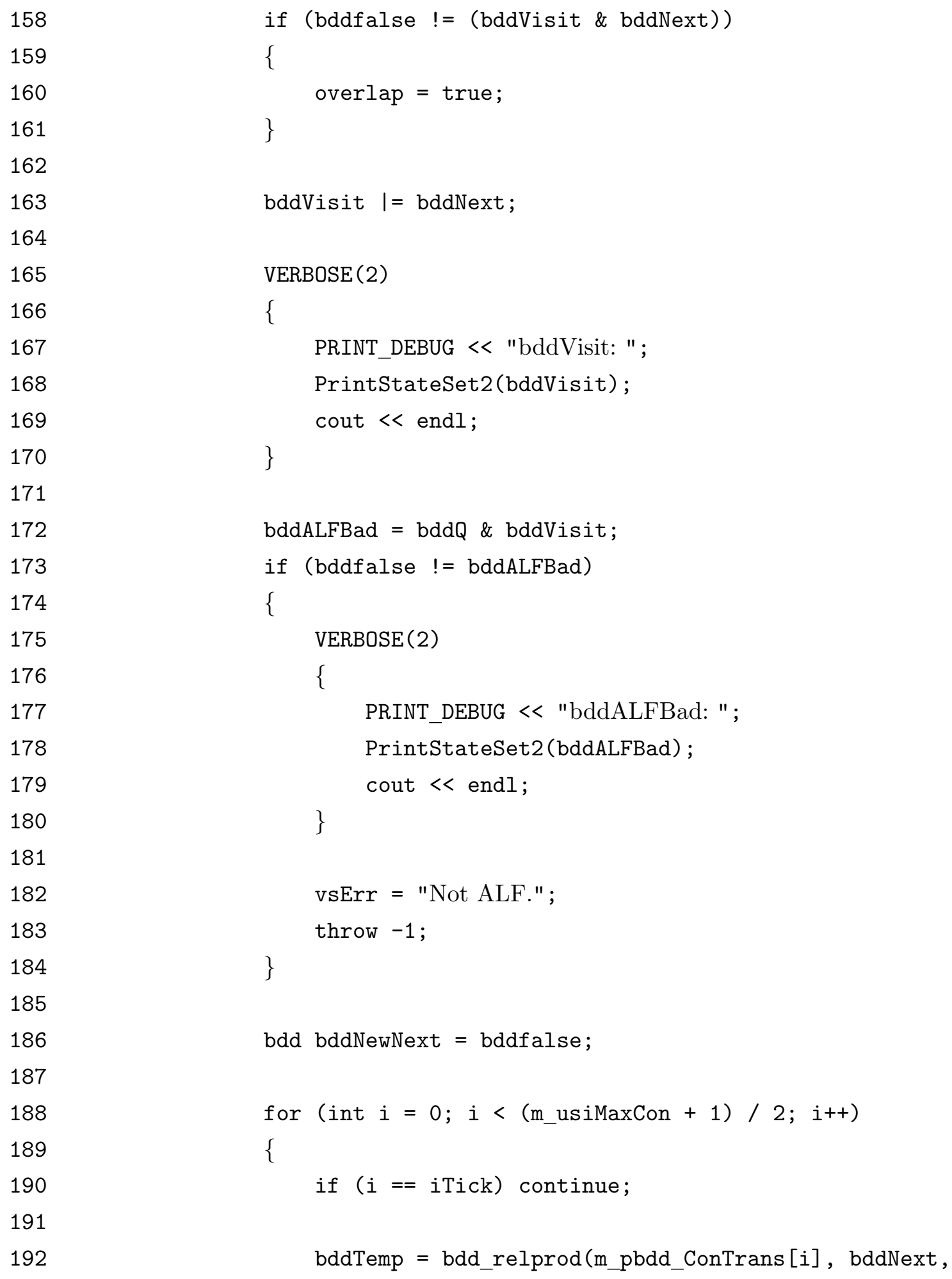




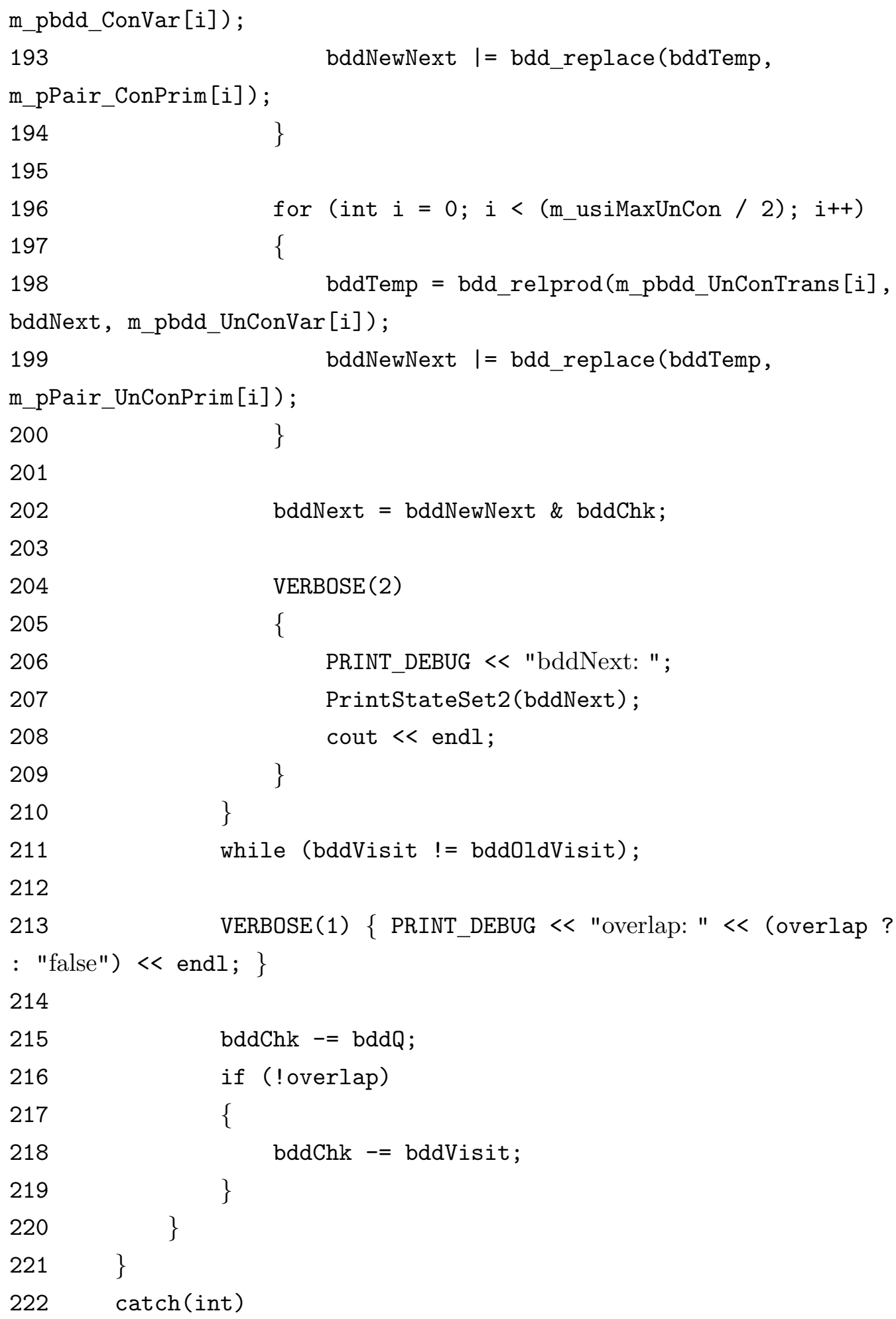

210

211

212

213

\{ 


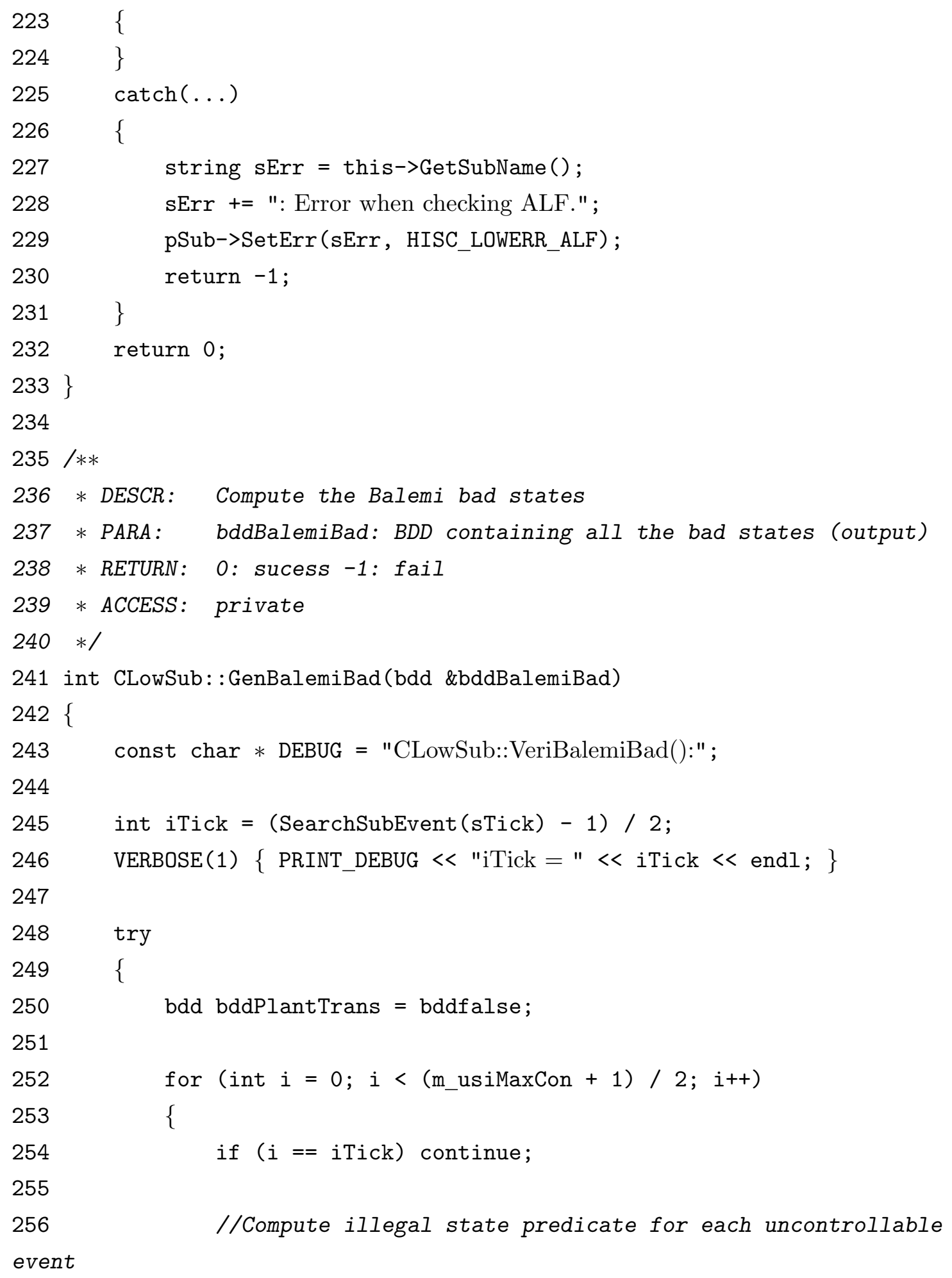




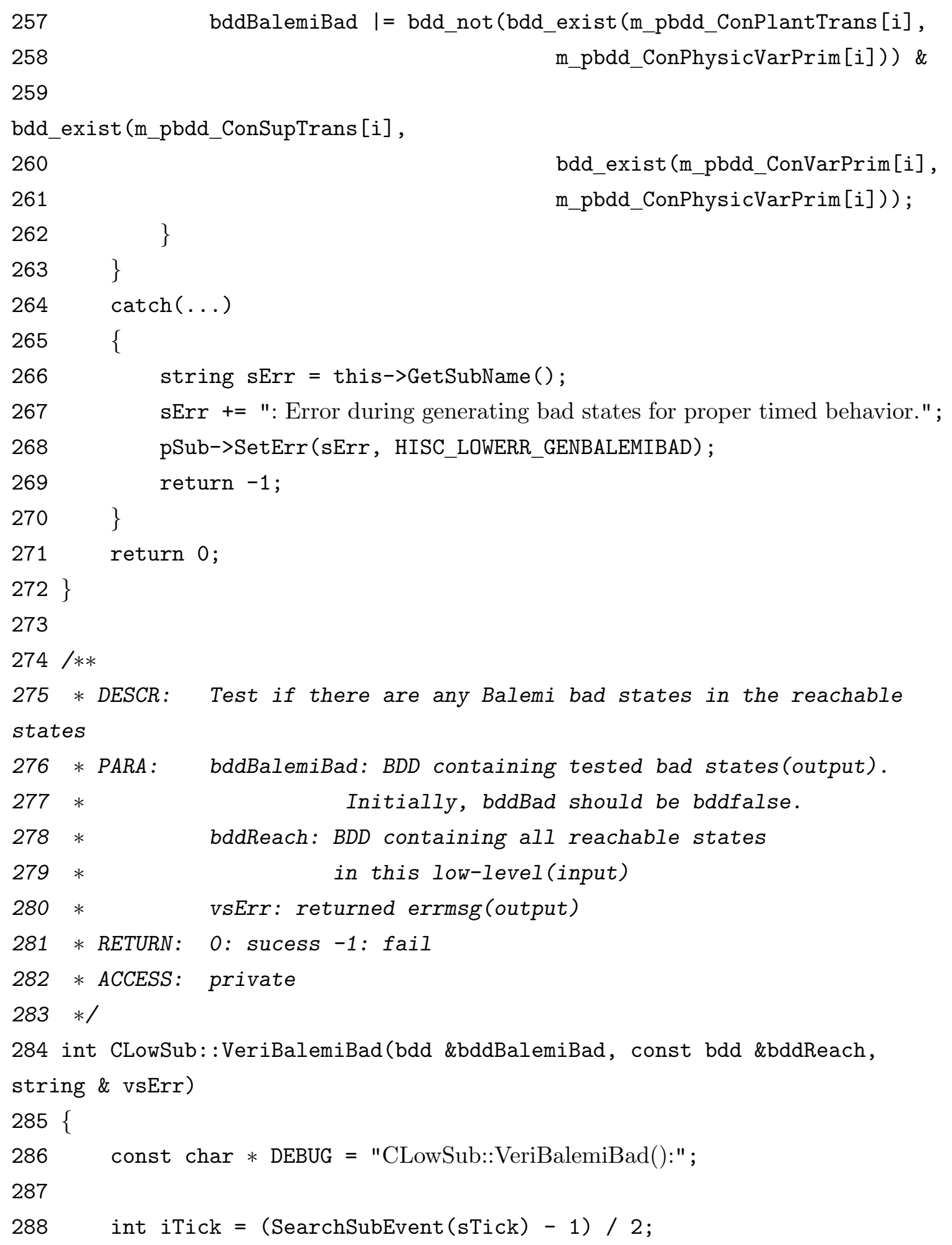




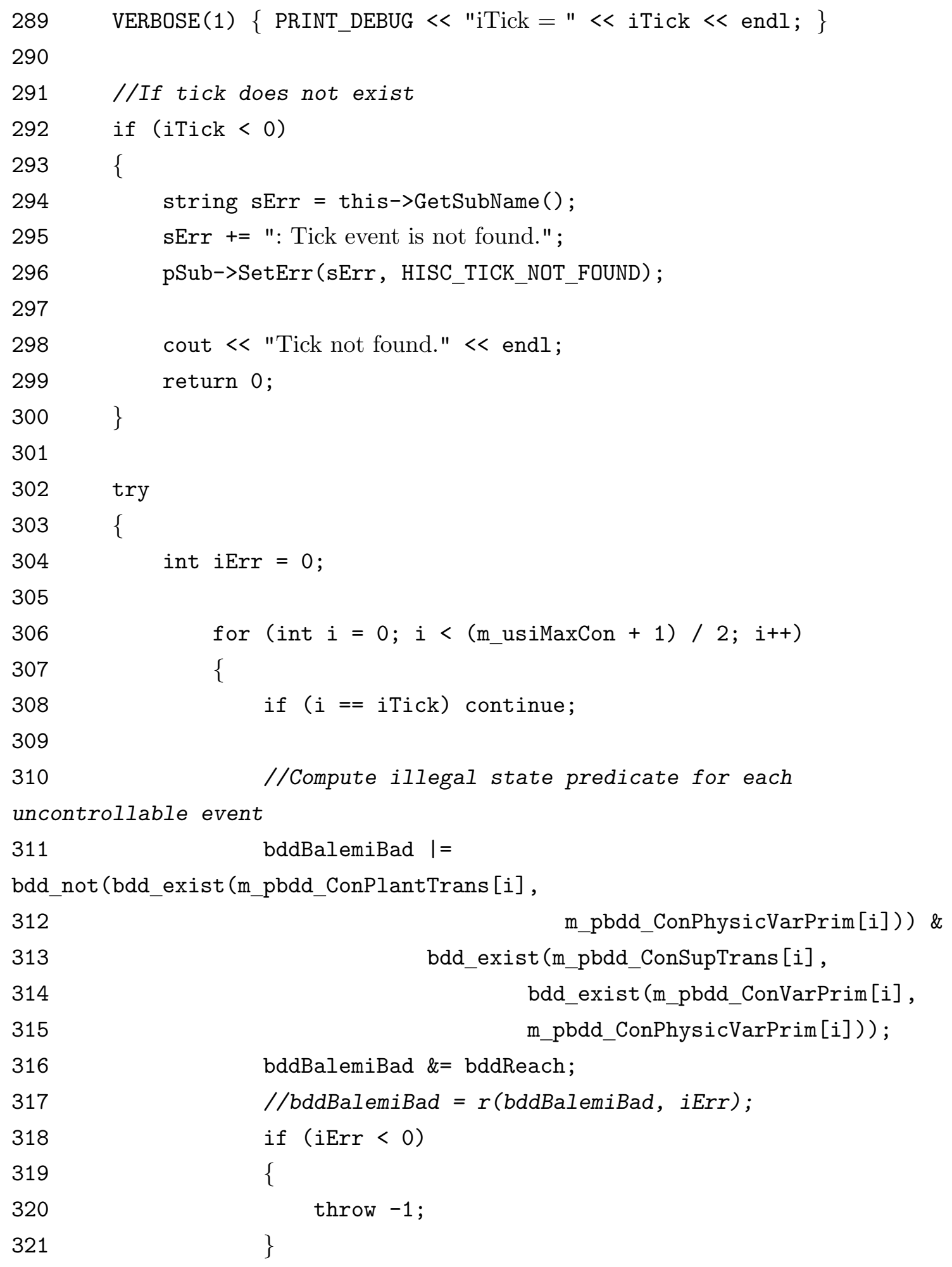




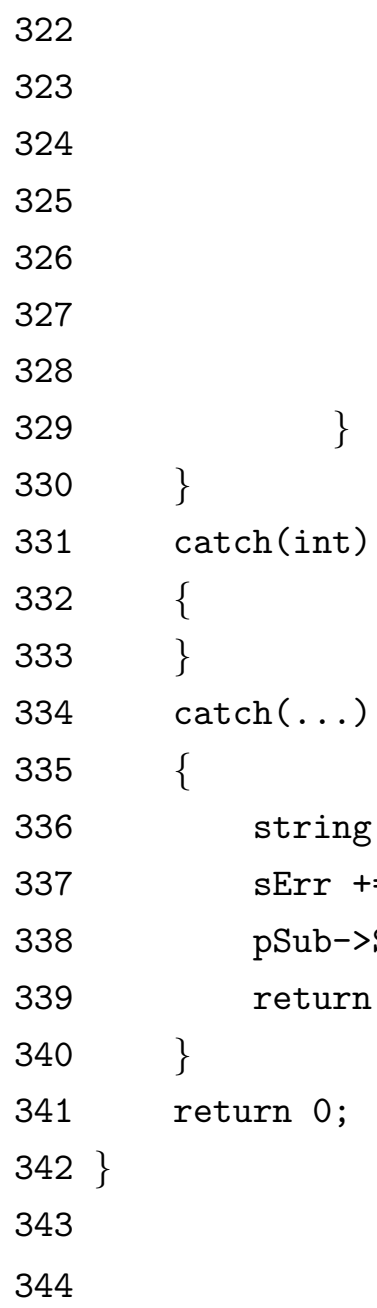

\section{LowSub5.cpp}

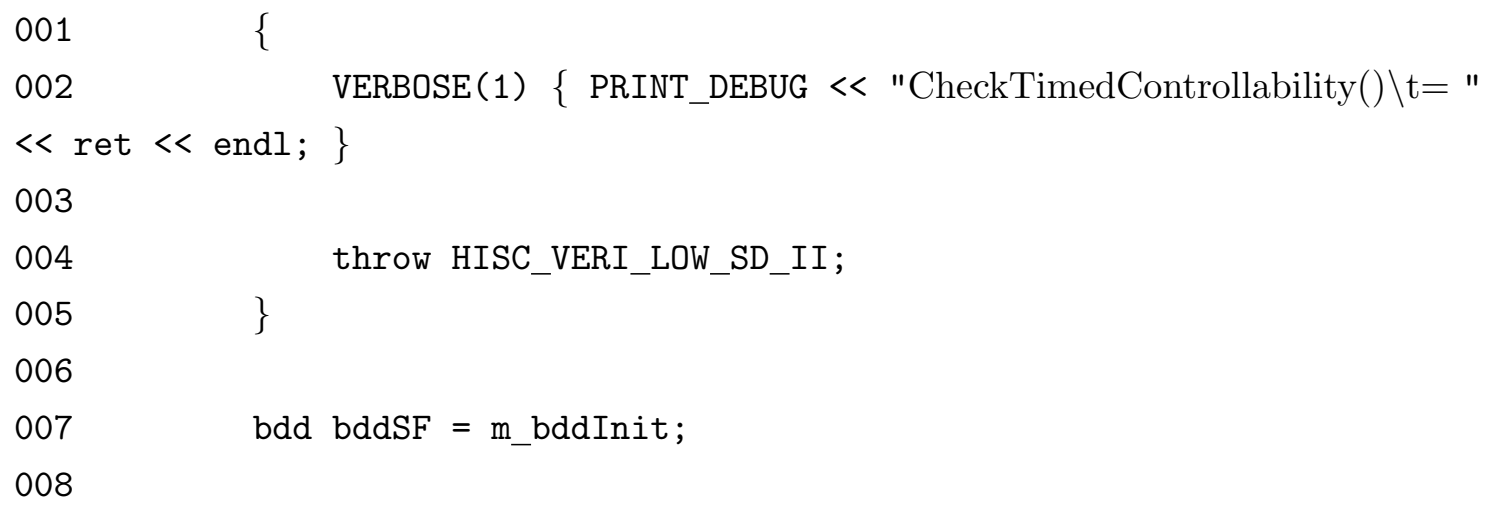




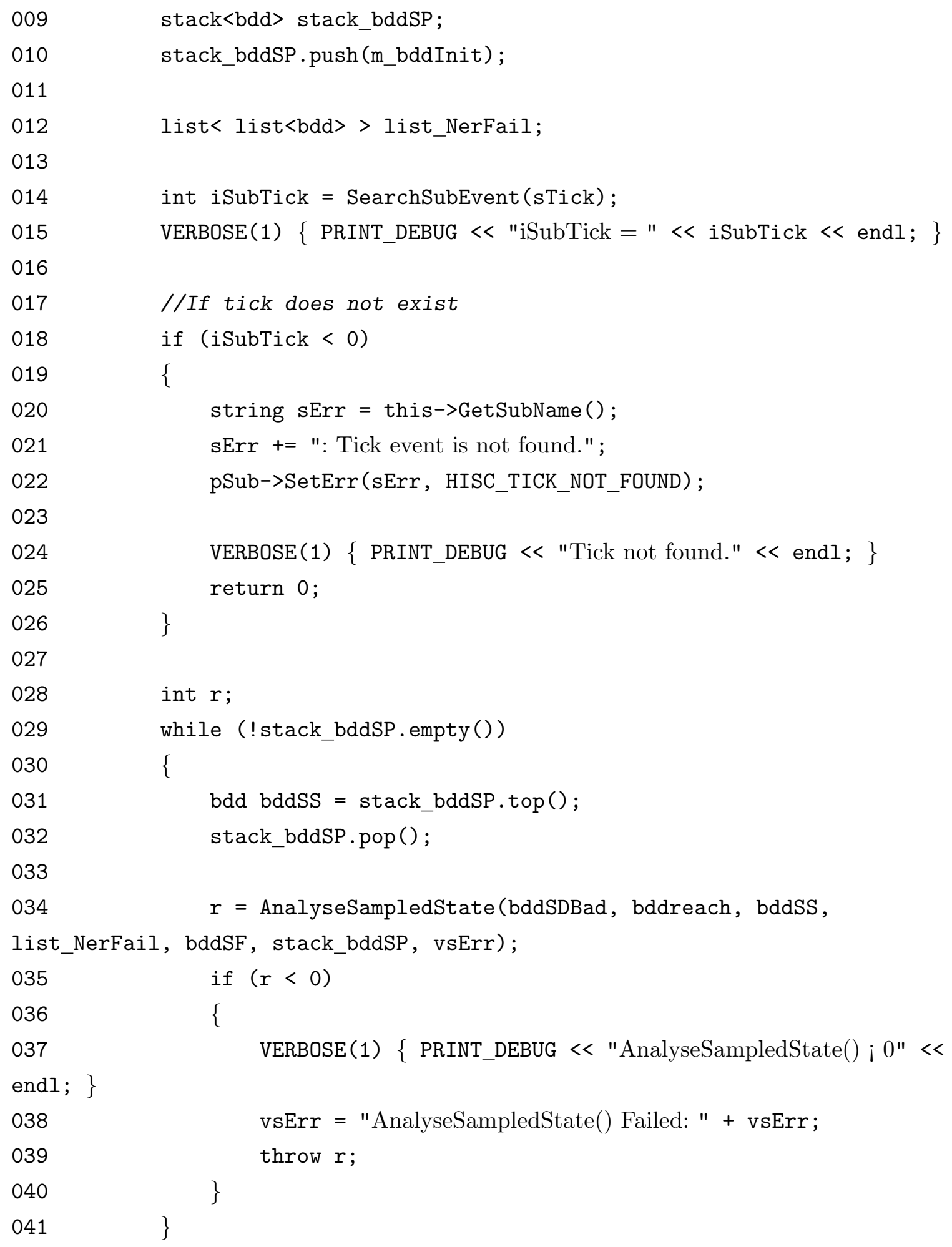




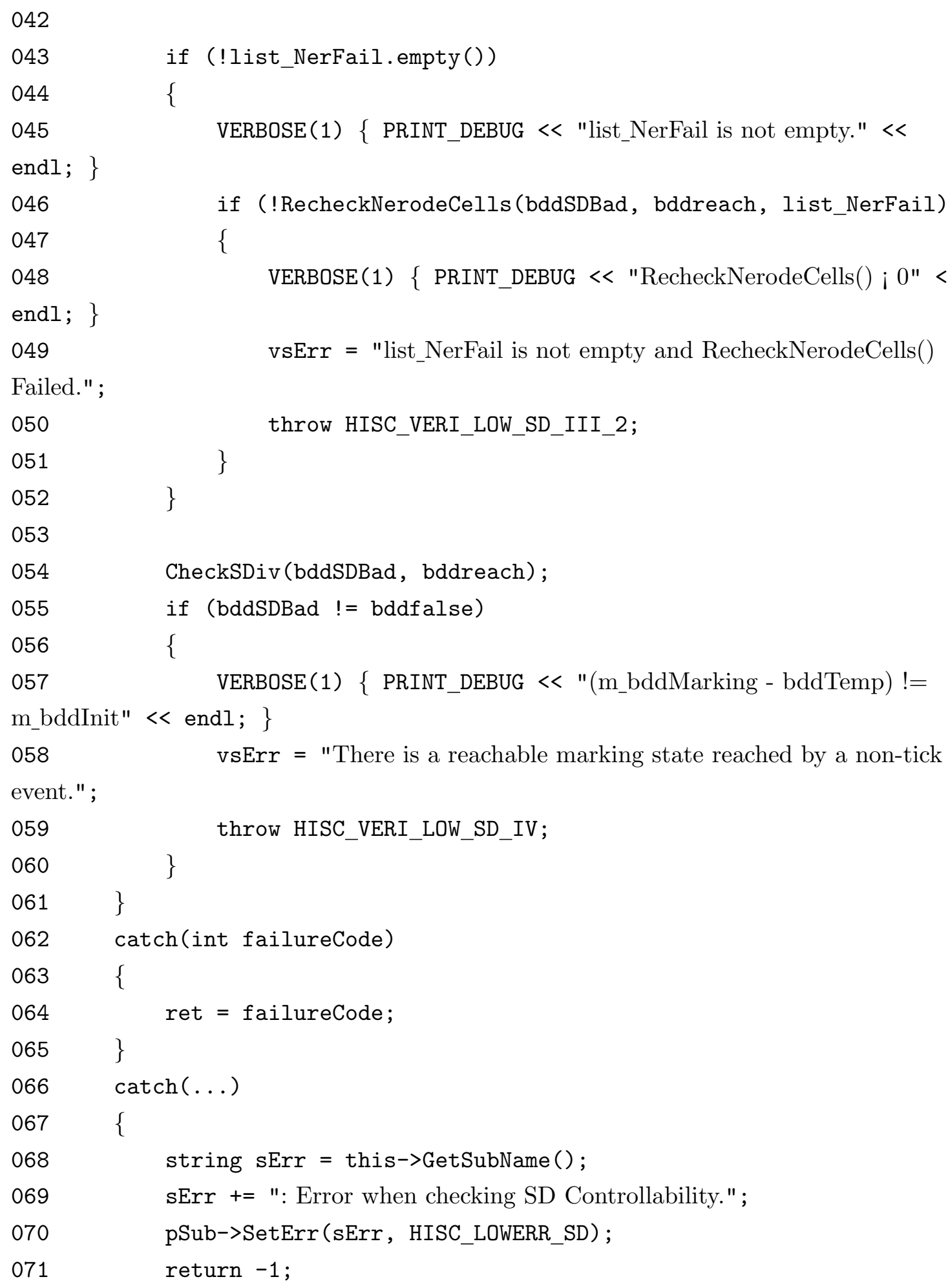




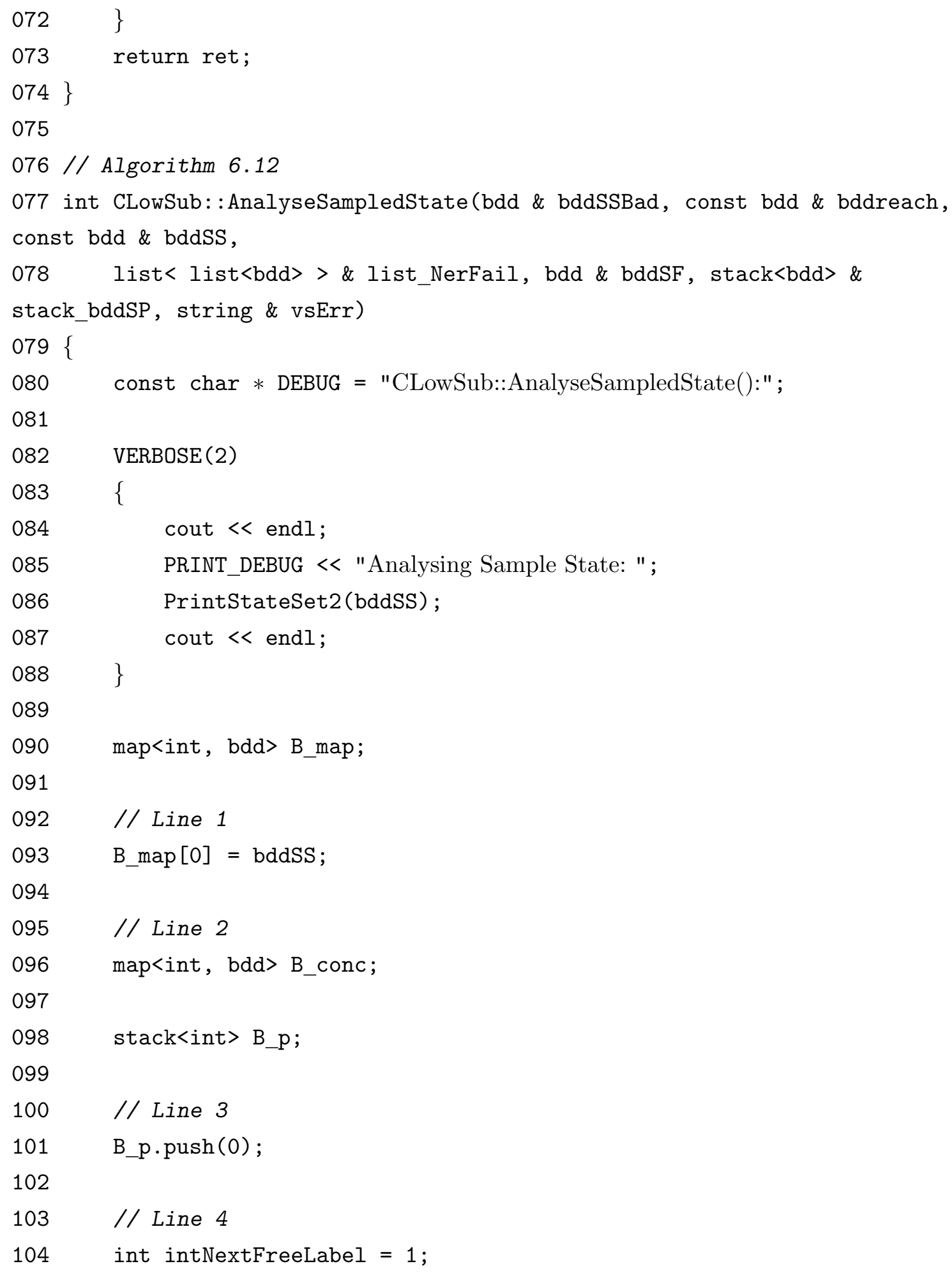




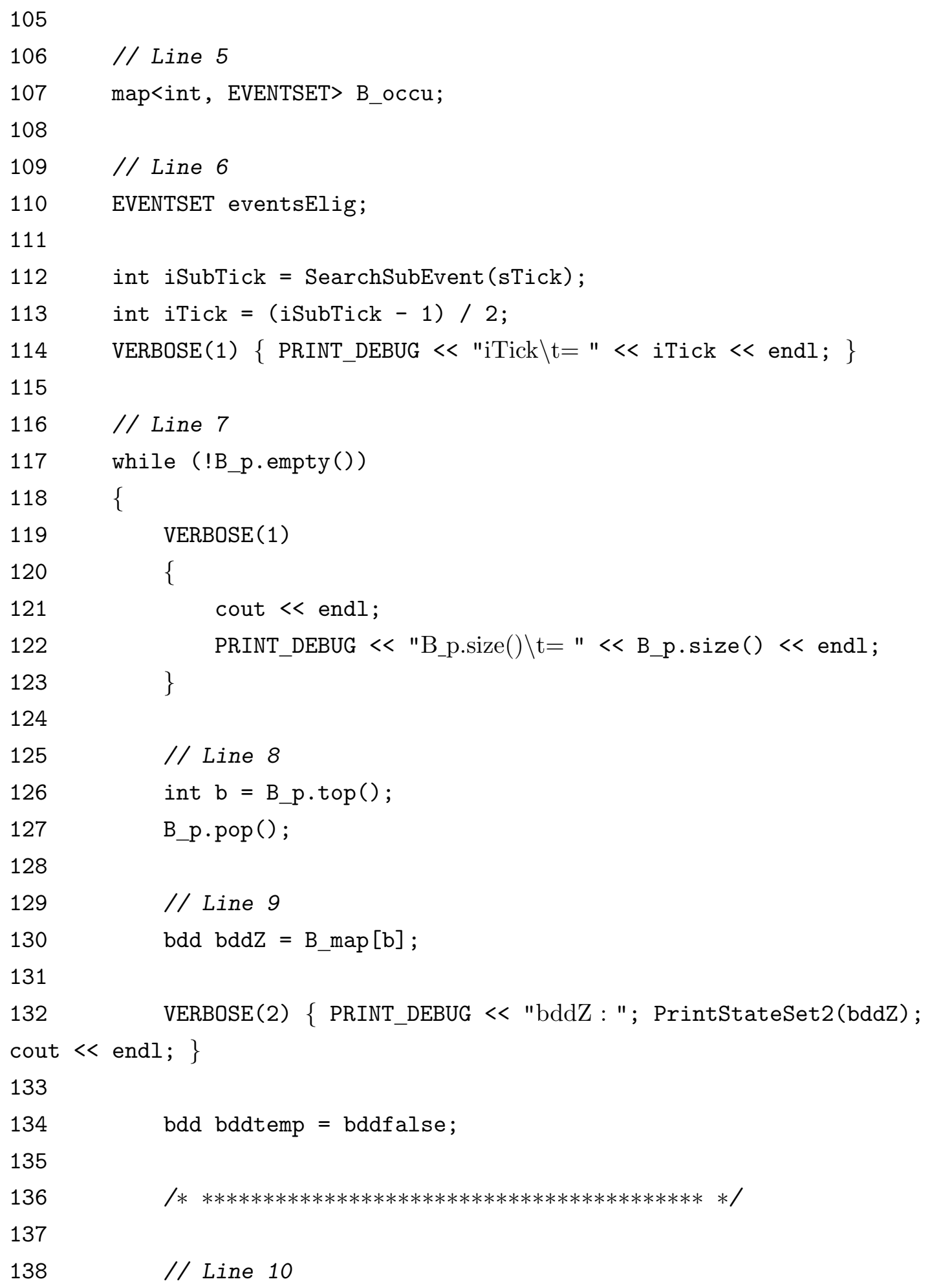


139 EVENTSET eventsA;

140

141 VERBOSE (1) \{ PRINT_DEBUG $\ll$ "FOR-LOOP START :

m_SubPlantEvents" < endl; \}

142

143

// Line 11

144

for (EVENTSET: : iterator $i=m_{-}$SubPlantEvents.begin () ; $i \quad !=$

m_SubPlantEvents.end (); i++)

145

146

int iIndex, event $=* i$;

147

if (event $<1$ )

148

149

\{

event index lower than $1 "<$ endl; $\}$

150

return HISC_INTERNAL_ERR_SUBEVENT;

151

152

153

\}

m_InvSubEventsMap [event] $\ll$ endl; $\}$

154

155

156

if $(1==$ event $\% 2) / /$ Controllable

157

iIndex $=($ event -1$) / 2$;

bddZ, m_pbdd_ConVar[iIndex]);

$158 \quad$ bddtemp = bdd_replace (bddtemp,

m_pPair_ConPrim[iIndex]);

159

160

\}

161

162

else //Uncontrollable

162

\{

163

iIndex $=($ event $/ 2)-1$;

bddZ, m_pbdd_UnConVar[iIndex]);

164

bddtemp = bdd_replace (bddtemp,

m_pPair_UnConPrim[iIndex]);

165 
166

167 bddtemp \&= bddreach;

$168 \quad \operatorname{VERBOSE}(2)\{$ PRINT_DEBUG $<$ "bddtemp $\backslash \mathrm{t}=$ ";

PrintStateSet2 (bddtemp); cout $\ll$ endl; $\}$

169

170

// Line 12

171

if (bddtemp != bddfalse)

172

173

VERBOSE (1) \{ PRINT_DEBUG $<$ "bddtemp != bddfalse" $<<$

endl; $\}$

174

175

// Line 13

176 eventsA.insert (event);

177 $\operatorname{VERBOSE}(1) \quad\{$ PRINT_DEBUG $<$ "eventsA.size ()$\backslash \mathrm{t}="<<$

eventsA.size ()$<$ endl; $\}$

178

179

// Line 14

180

181

182

// Line 15

183

184

\}

$<$ endl; \}

185

186 // Line 16

187 EVENTSET eventsD;

188

189 VERBOSE (1) \{ PRINT_DEBUG $<$ "FOR-LOOP START :

m_SubSupervisorEvents" $<$ endl; \}

190

$191 \quad / /$ Line 17

192 for (EVENTSET: :iterator $i=$ m_SubSupervisorEvents.begin(); i != m_SubSupervisorEvents.end (); i++)

193

194

bdd bddSupervisorTrans = bddfalse; 


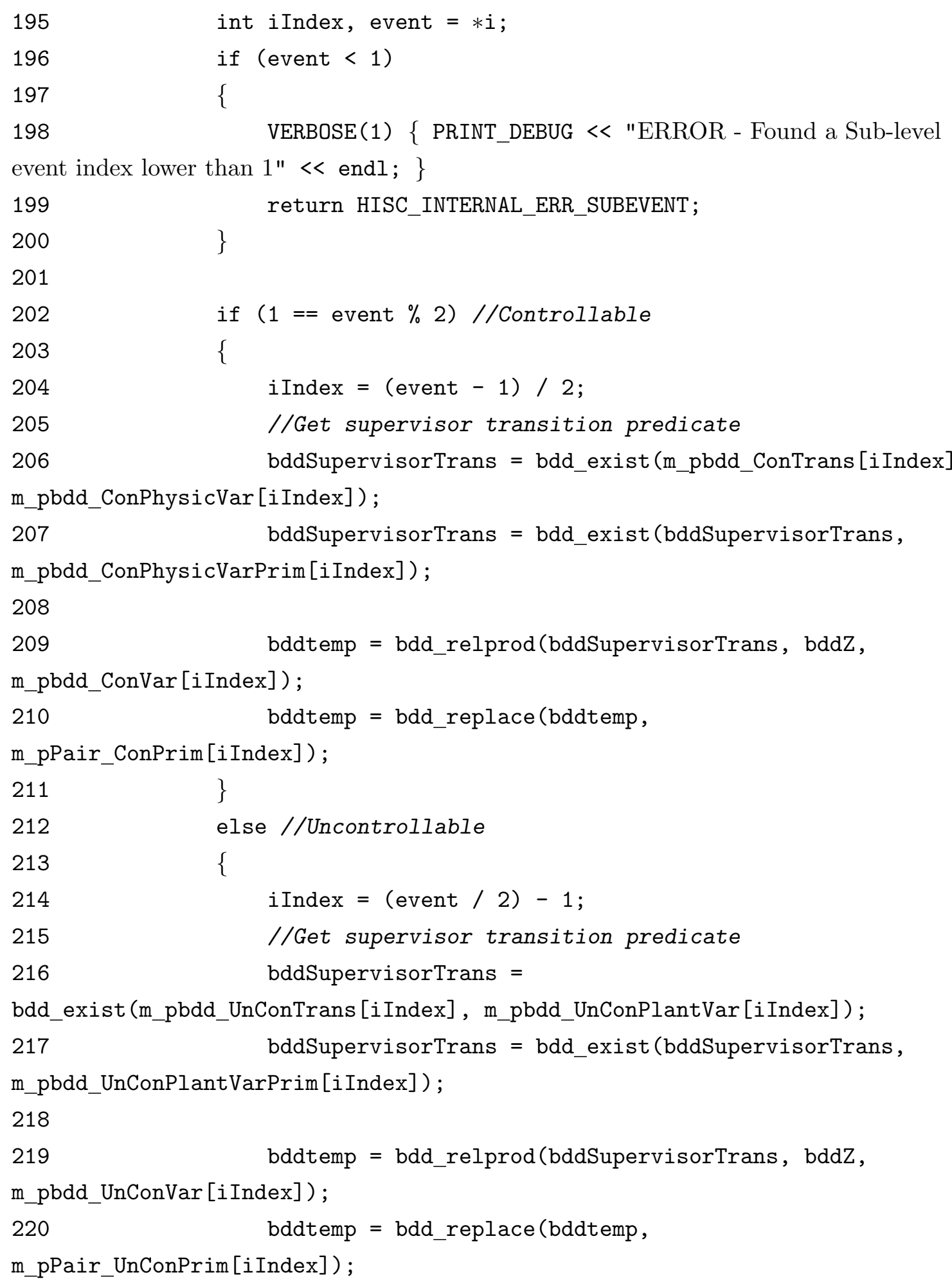




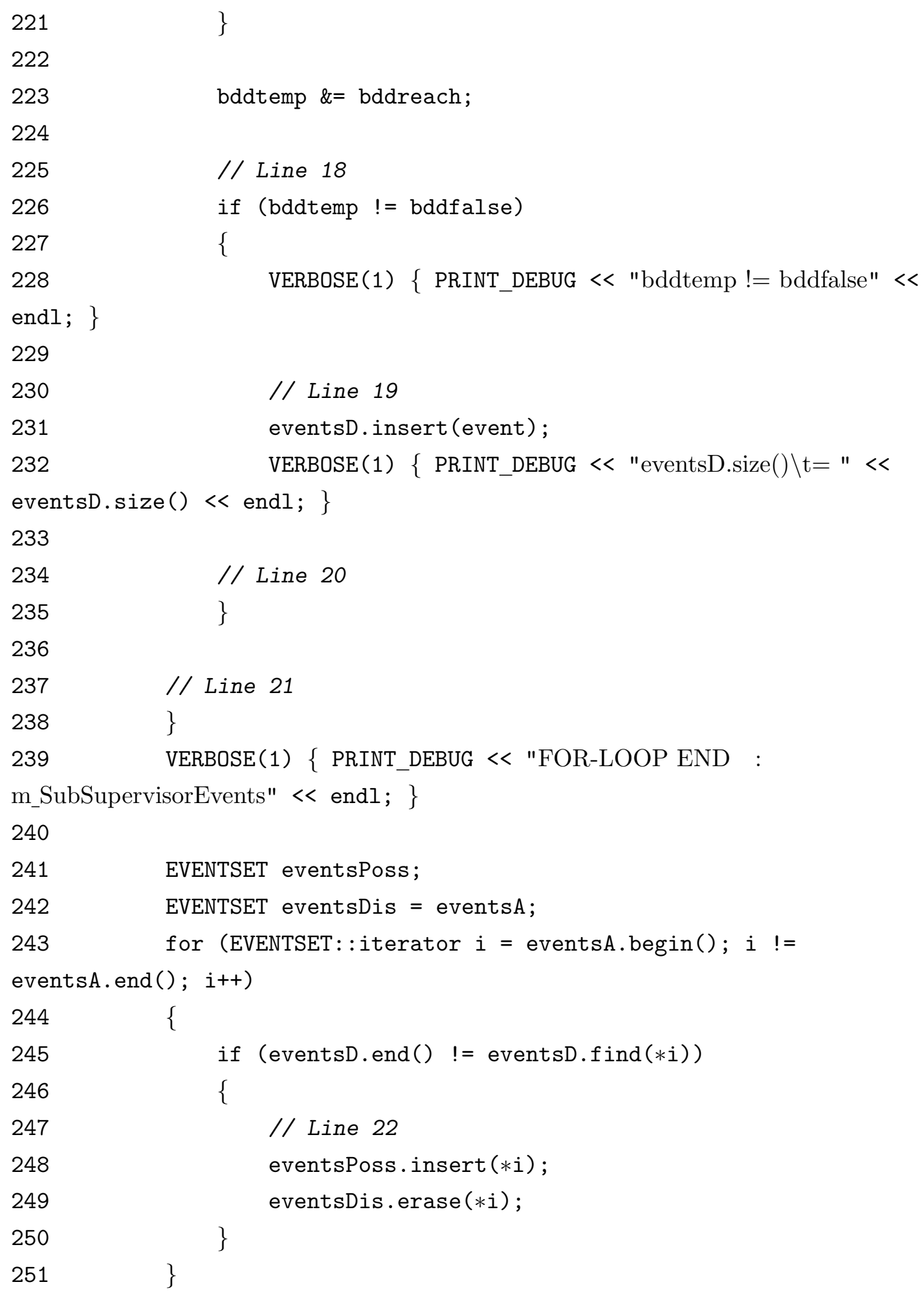


252

$253 / * * * * * * * * * * * * * * * * * * * * * * * * * * * * * * * * * * * * * * * * * * \quad * /$

254

255 VERBOSE (1) \{ PRINT_DEBUG $<<$ "FOR-LOOP START : eventsPoss" $<<$ endl; \}

256 for (EVENTSET: :iterator $i=$ eventsPoss.begin(); $i$ != eventsPoss.end (); i++)

257

258

if $((* i)<1)$

259

260

\{

event index lower than $1 "<$ endl; \}

261

return HISC_INTERNAL_ERR_SUBEVENT;

262

263

\}

m_InvSubEventsMap $[(* i)] \ll$ endl $;\}$

$264 \quad\}$

265

VERBOSE (1) $\{$ PRINT_DEBUG $\ll$ "FOR-LOOP END : eventsPoss" $<$

endl; $\}$

266

267 VERBOSE (1) $\{$ PRINT_DEBUG $\ll$ "FOR-LOOP START : eventsDis" $<<$ endl; $\}$

268 for (EVENTSET: :iterator $i=$ eventsDis.begin(); $i \quad !=$ eventsDis.end (); $i++)$

269

270

271

272

273

\{

event index lower than $1 "<$ endl; $\}$

274

return HISC_INTERNAL_ERR_SUBEVENT;

275

276

if $((* i)<1)$

\{

VERBOSE (1) \{ PRINT_DEBUG $\ll$ "ERROR - Found a Sub-level

m_InvSubEventsMap $[(* i)]<$ endl; $\}$

277

\}

$\operatorname{VERBOSE}(1)\{$ PRINT_DEBUG $\ll$ "eventsDis : " $<$

\} 


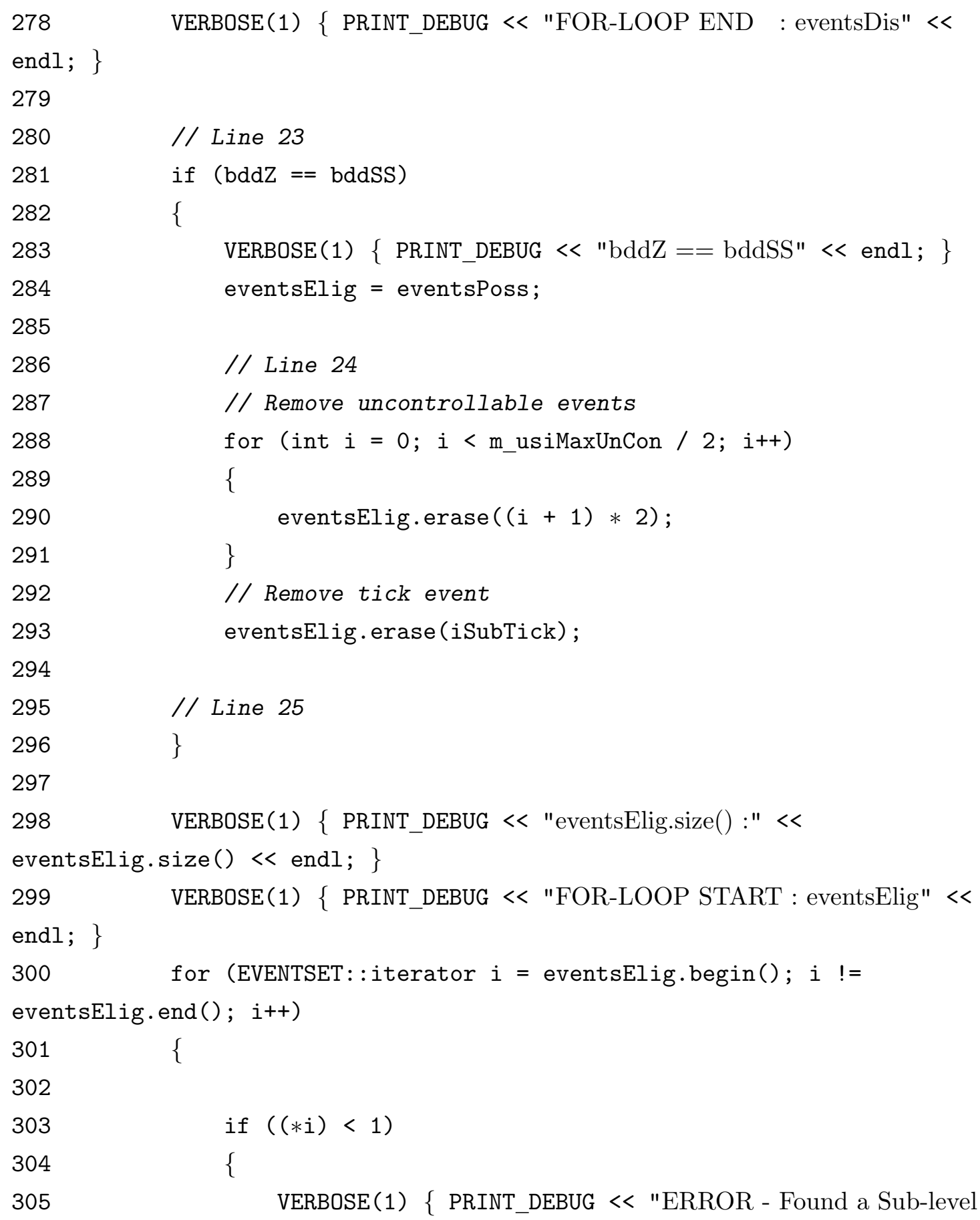




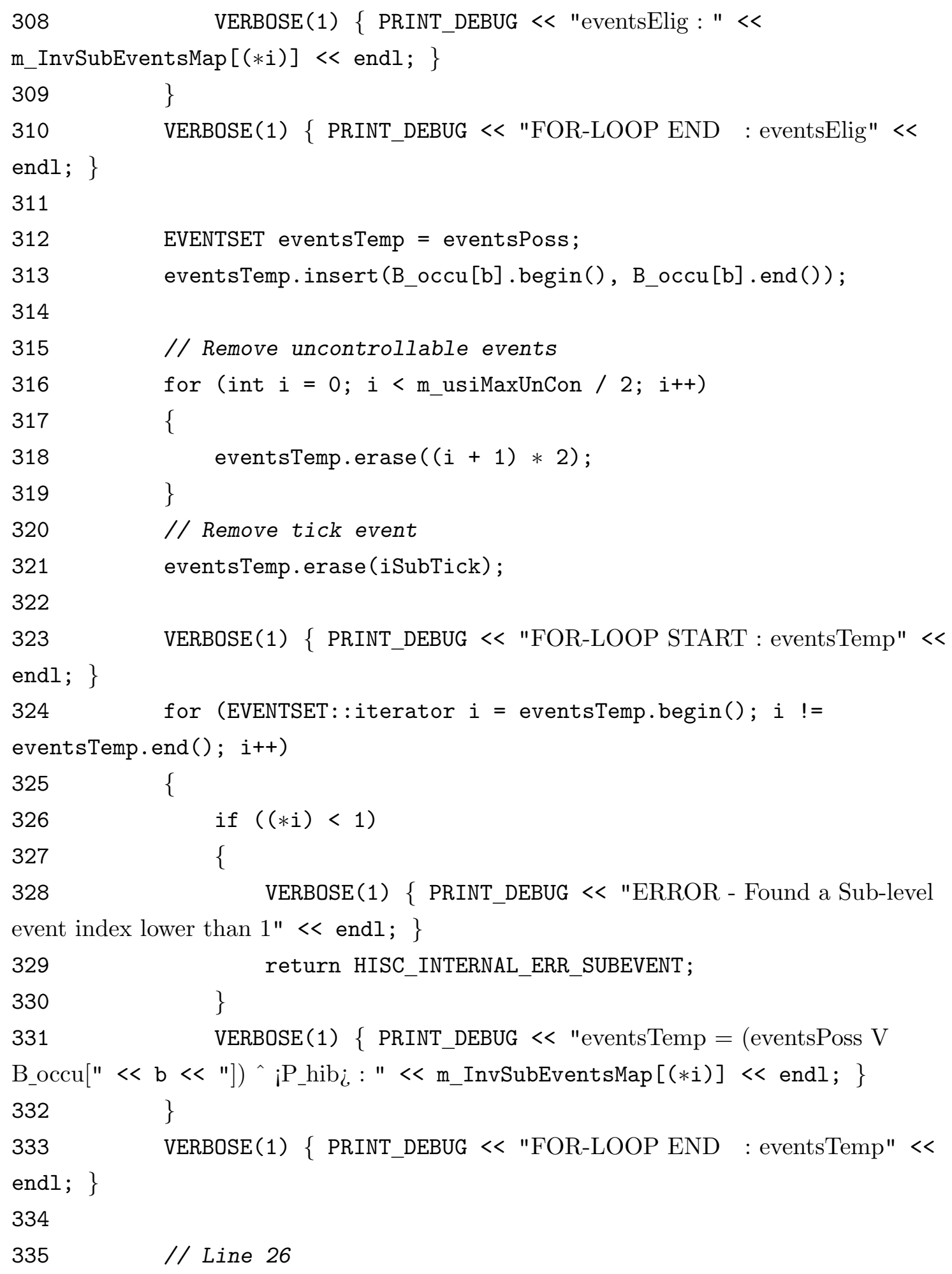




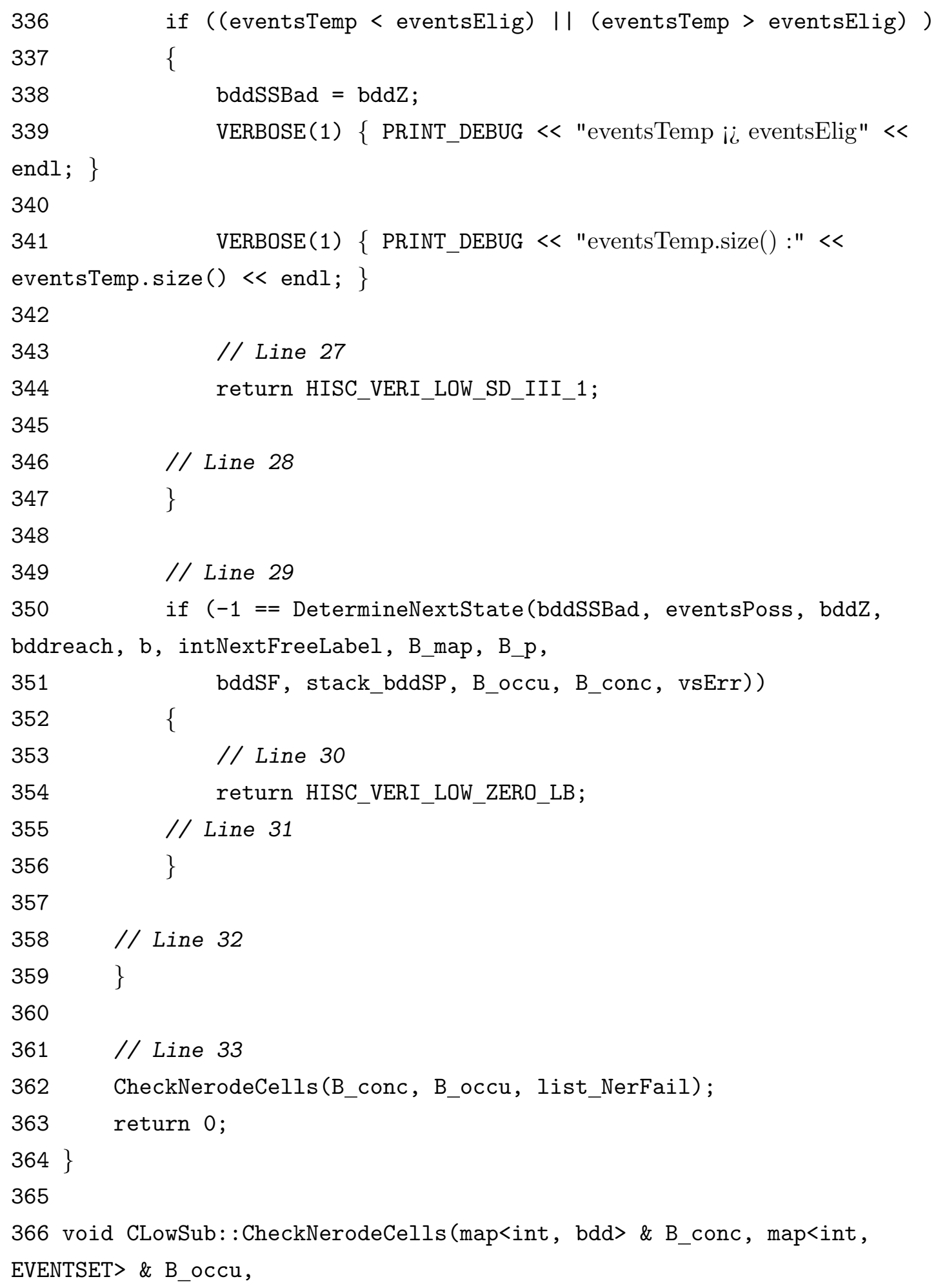




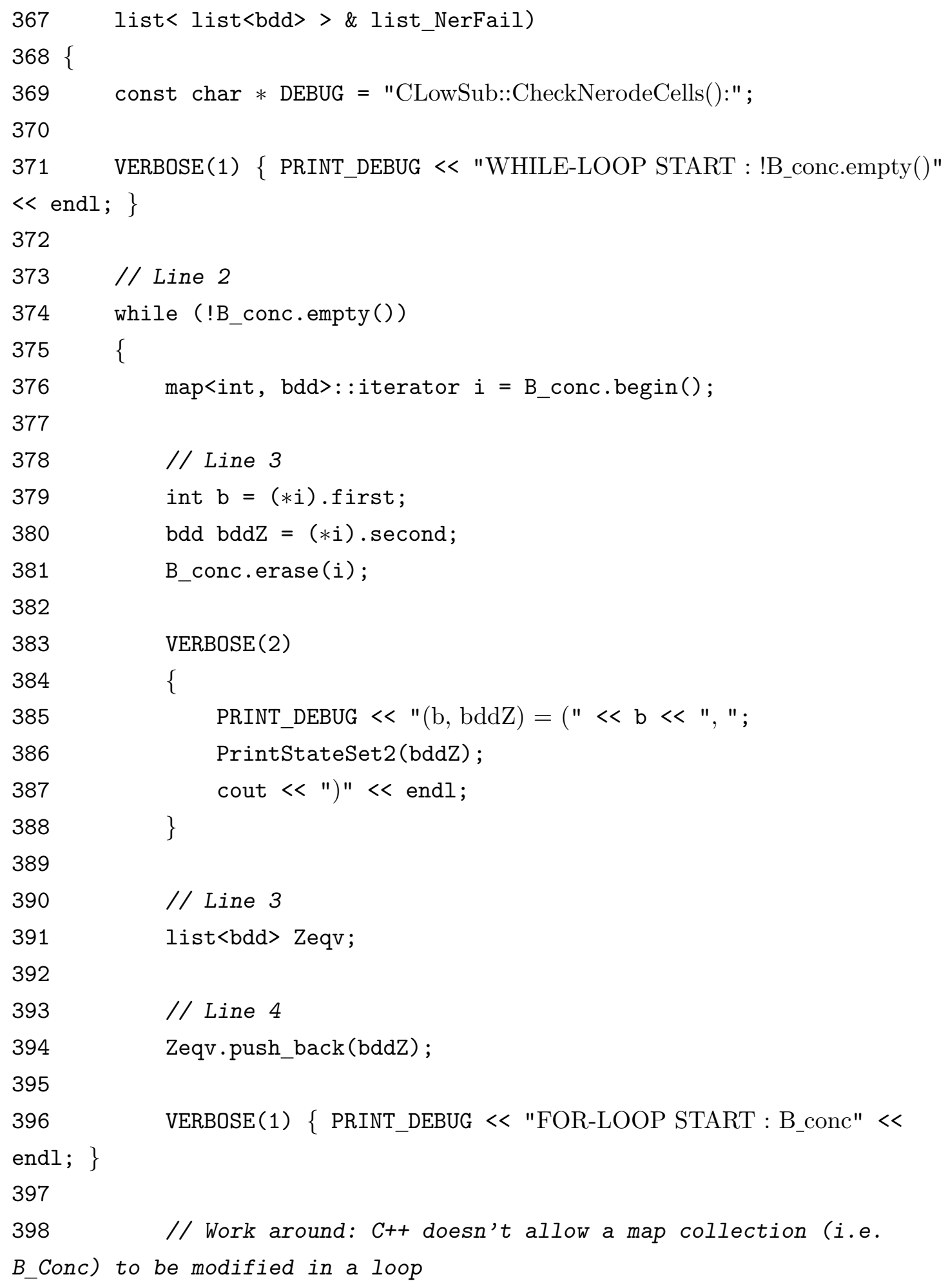




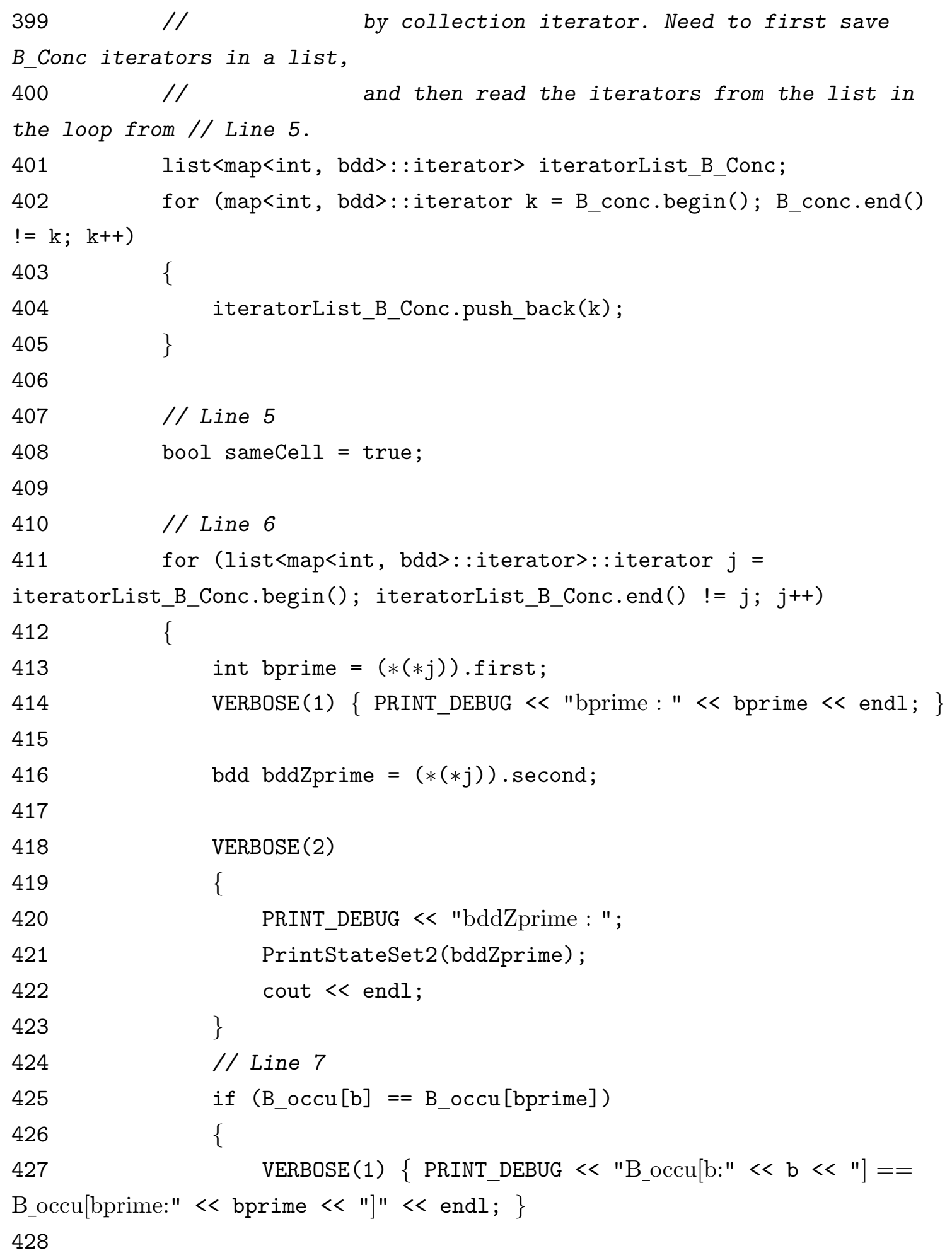




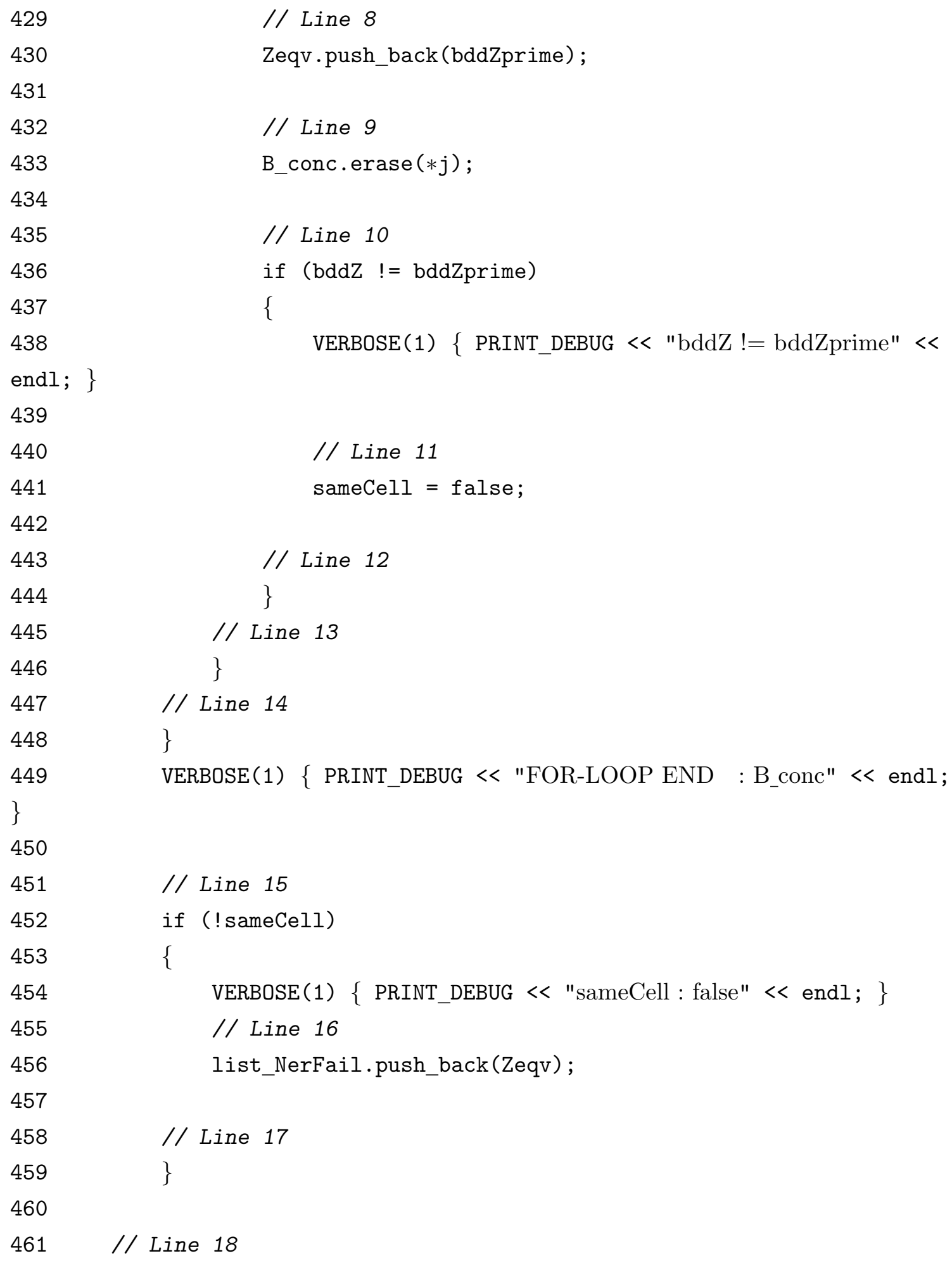

50




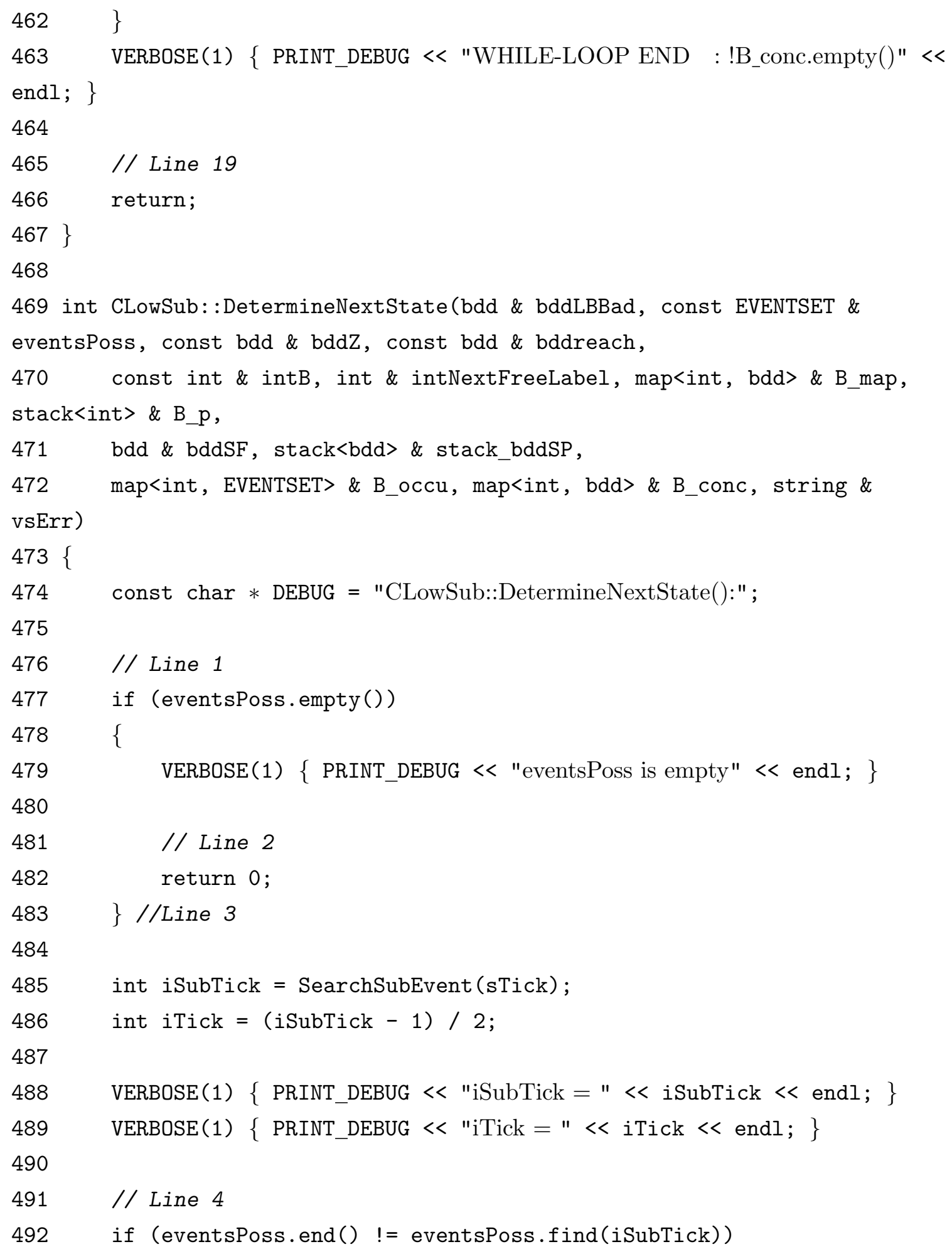




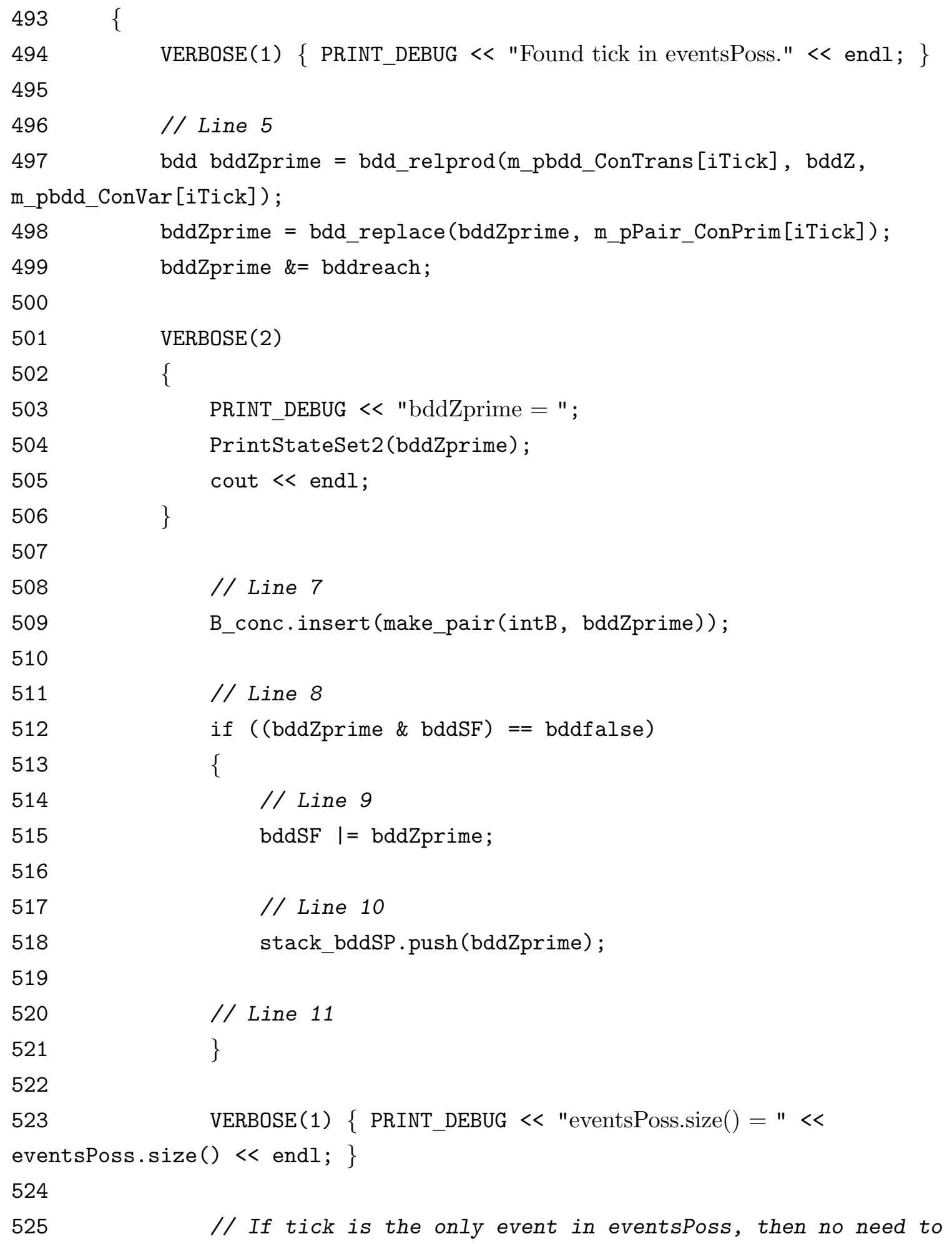




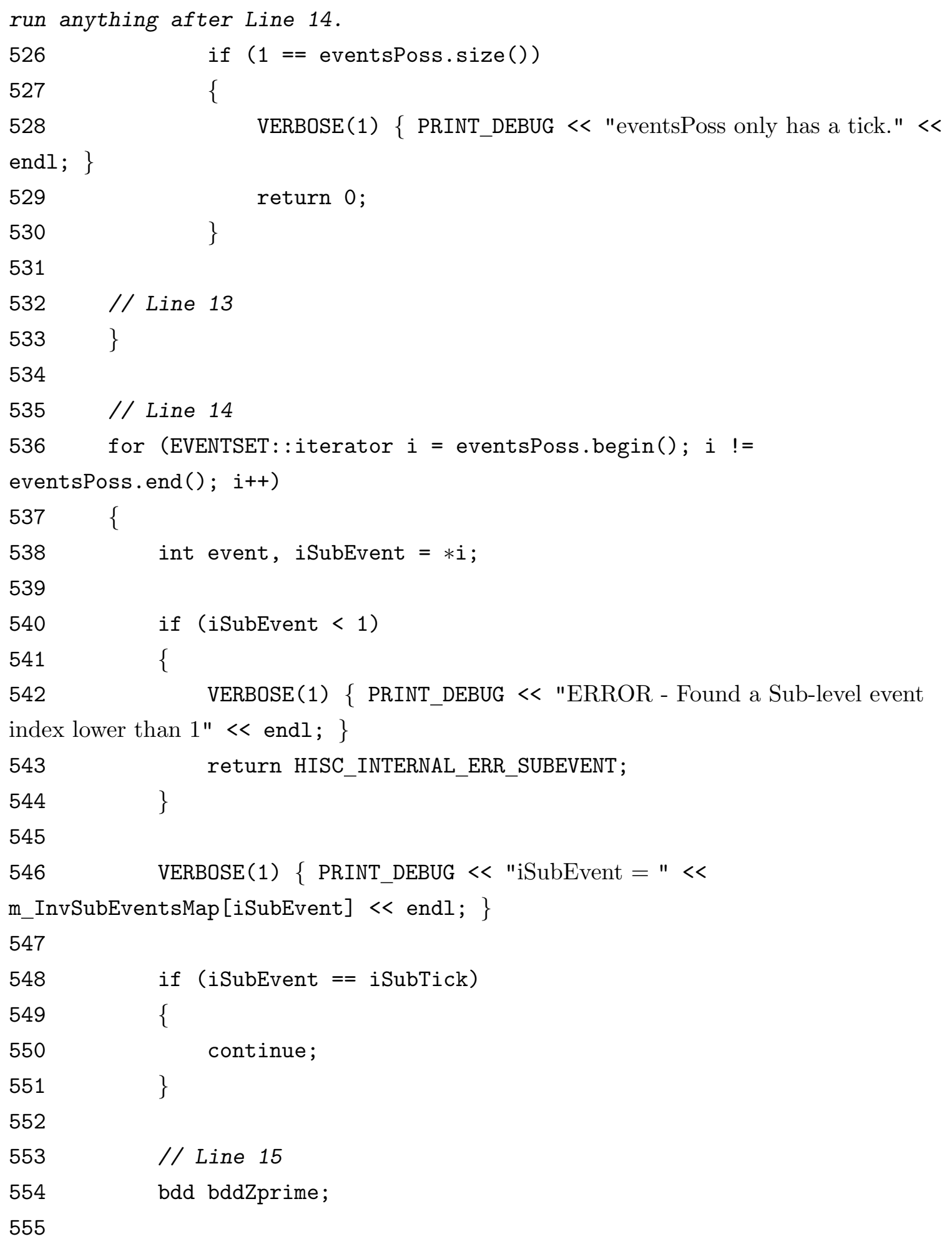




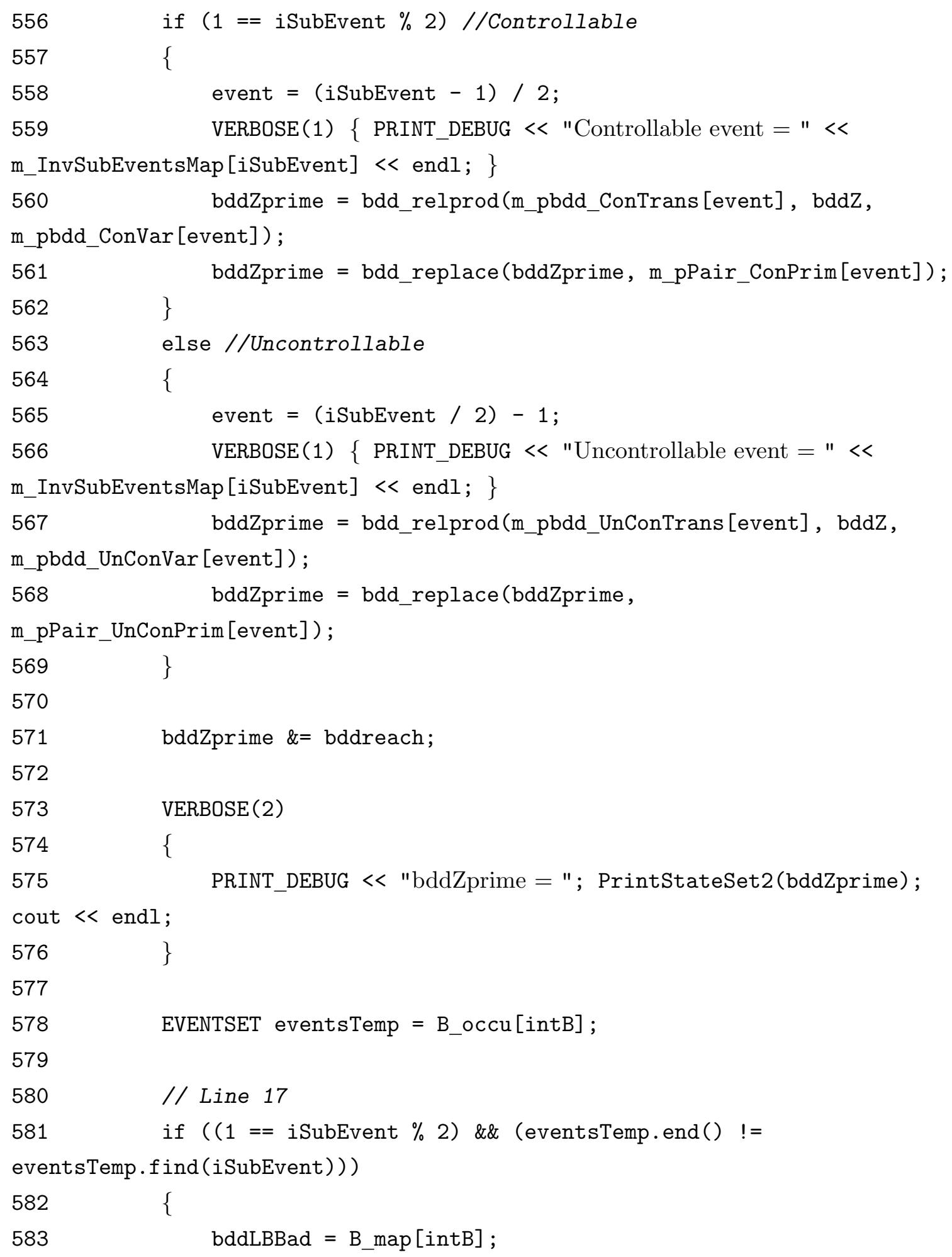




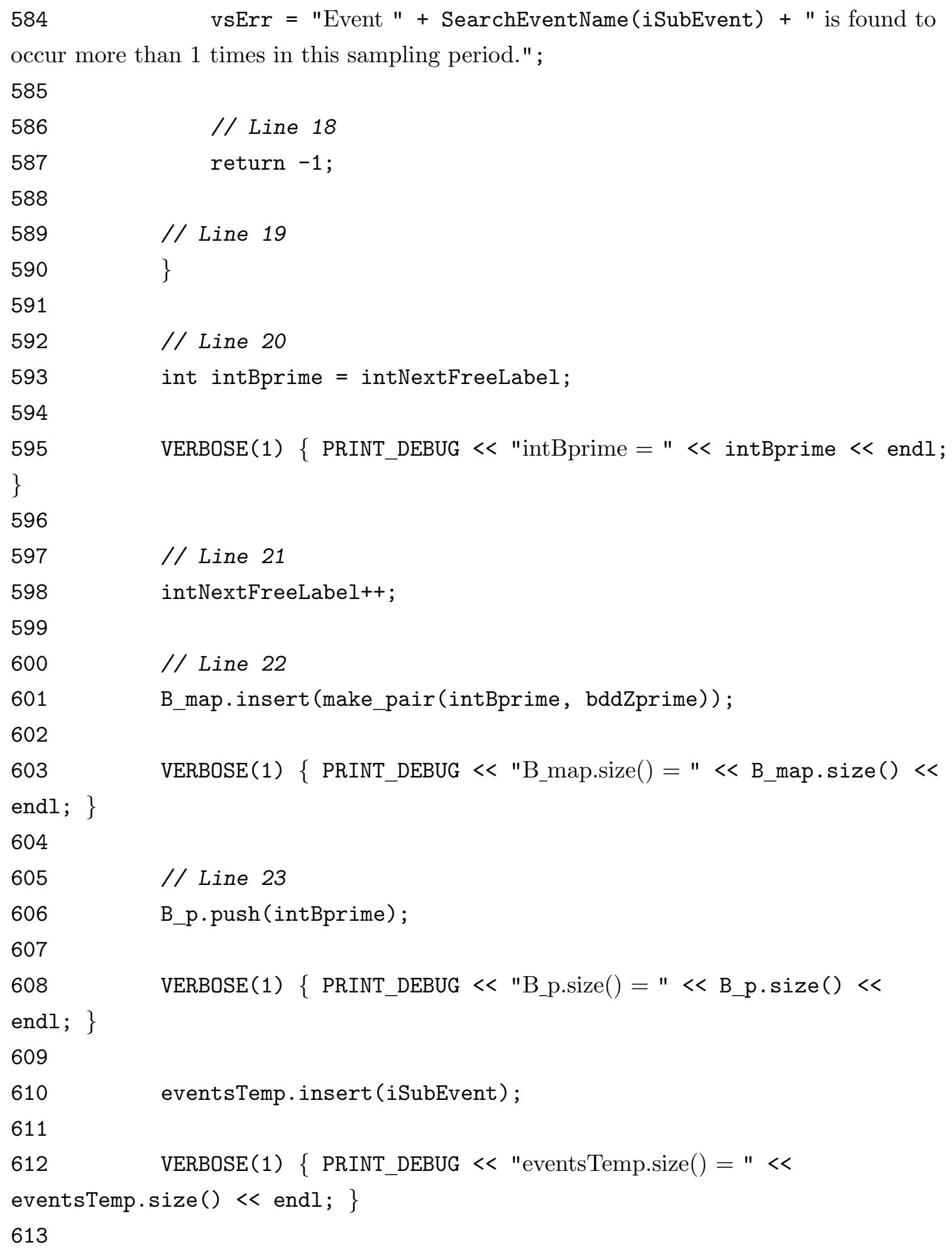




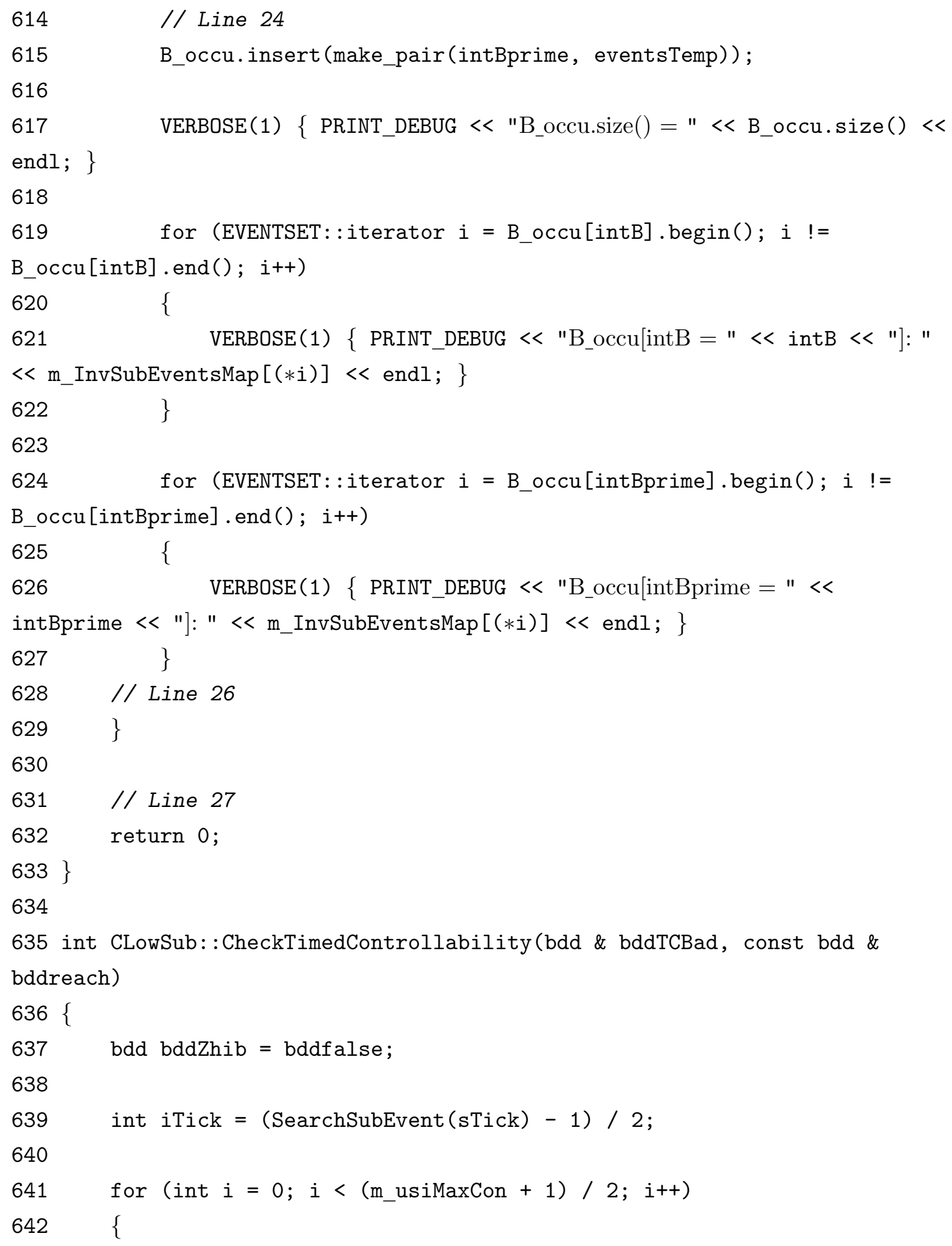




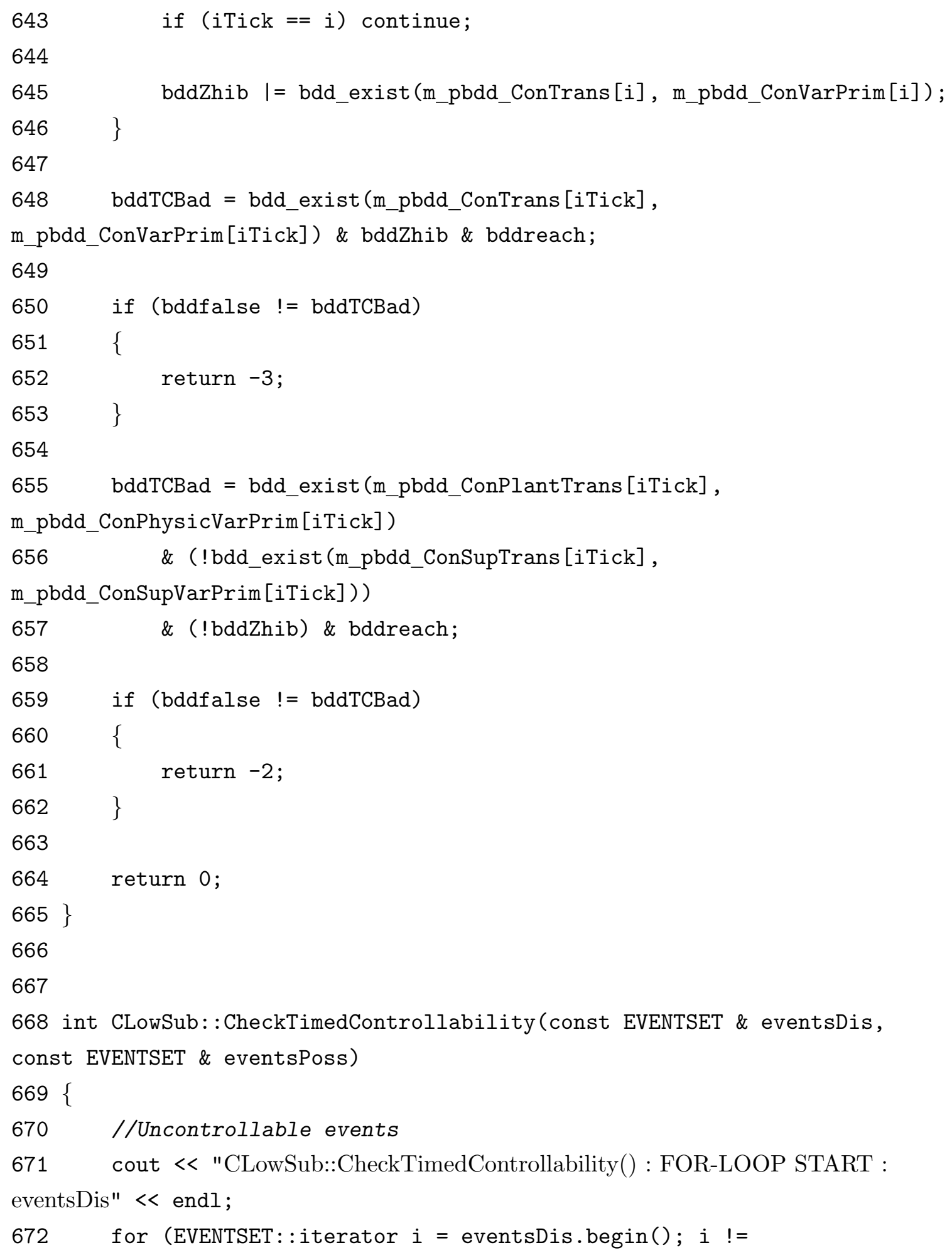




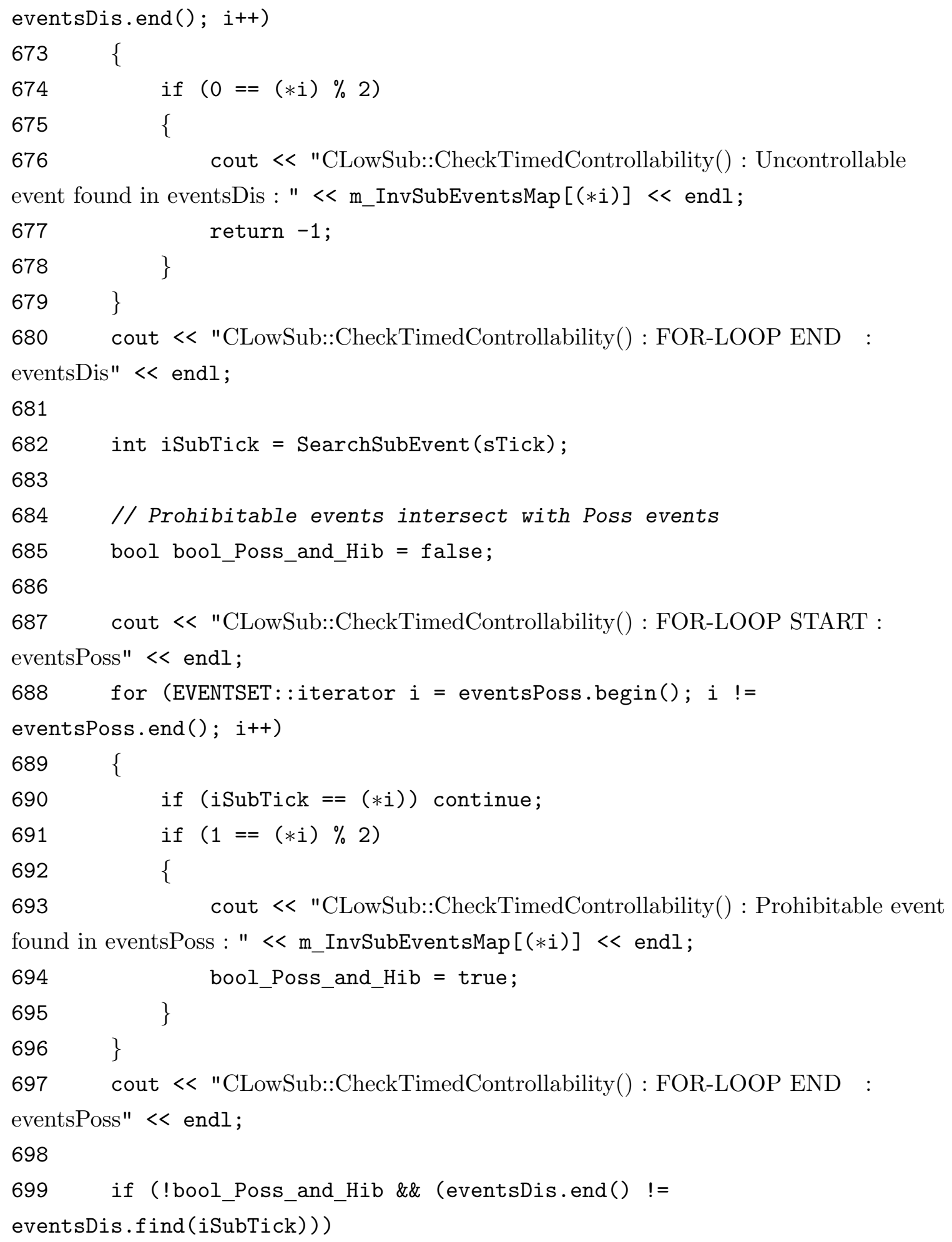




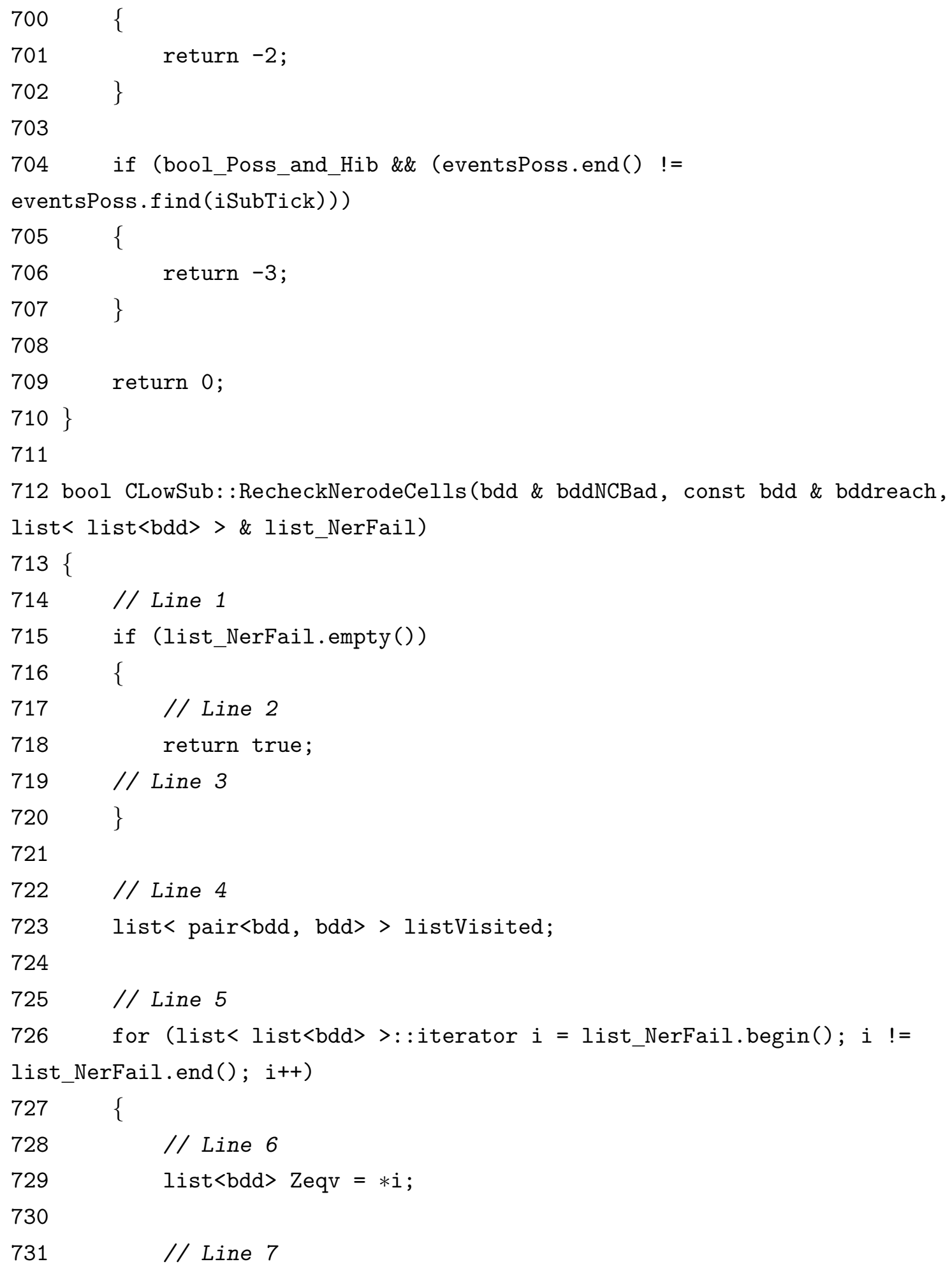




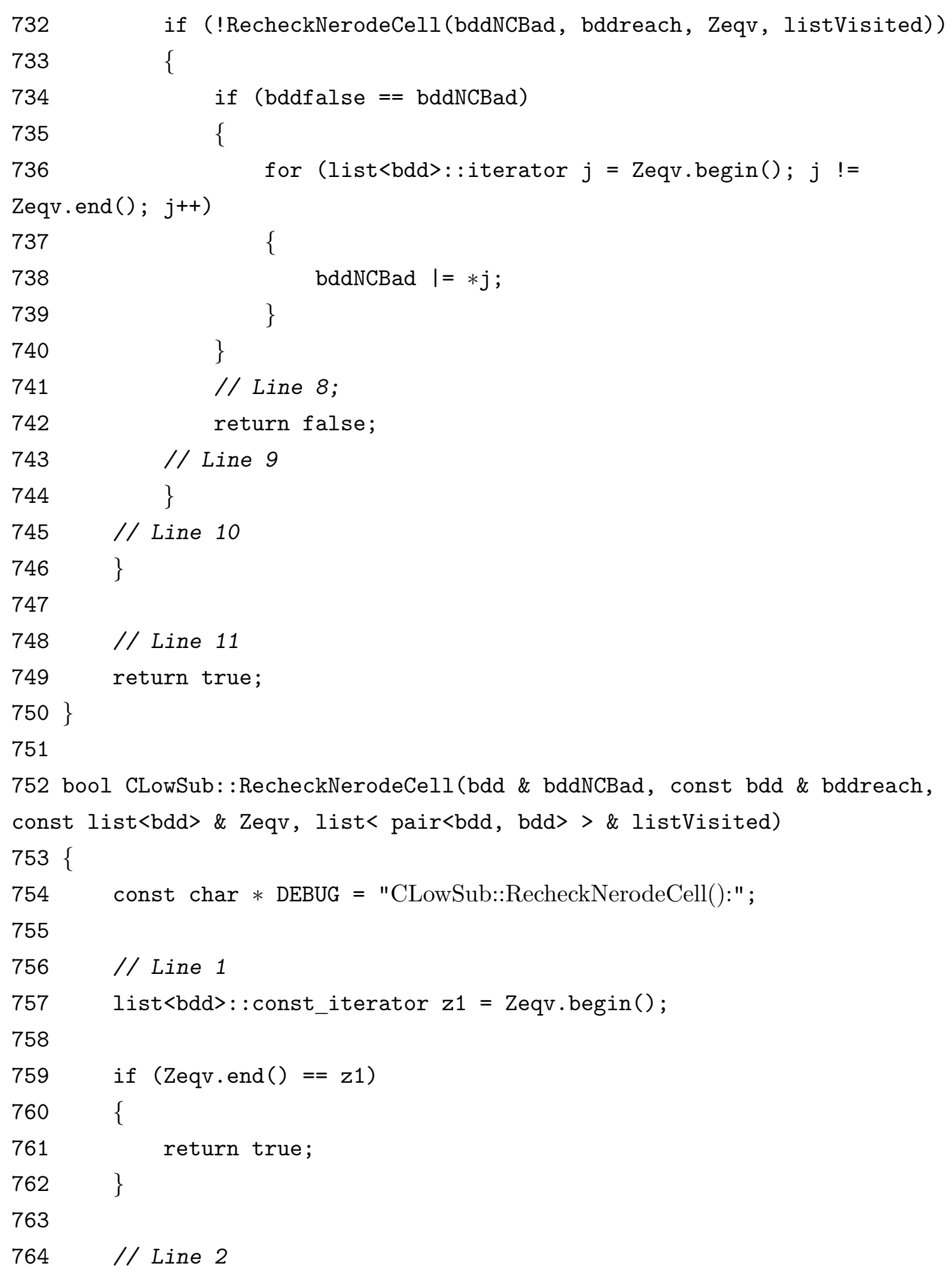




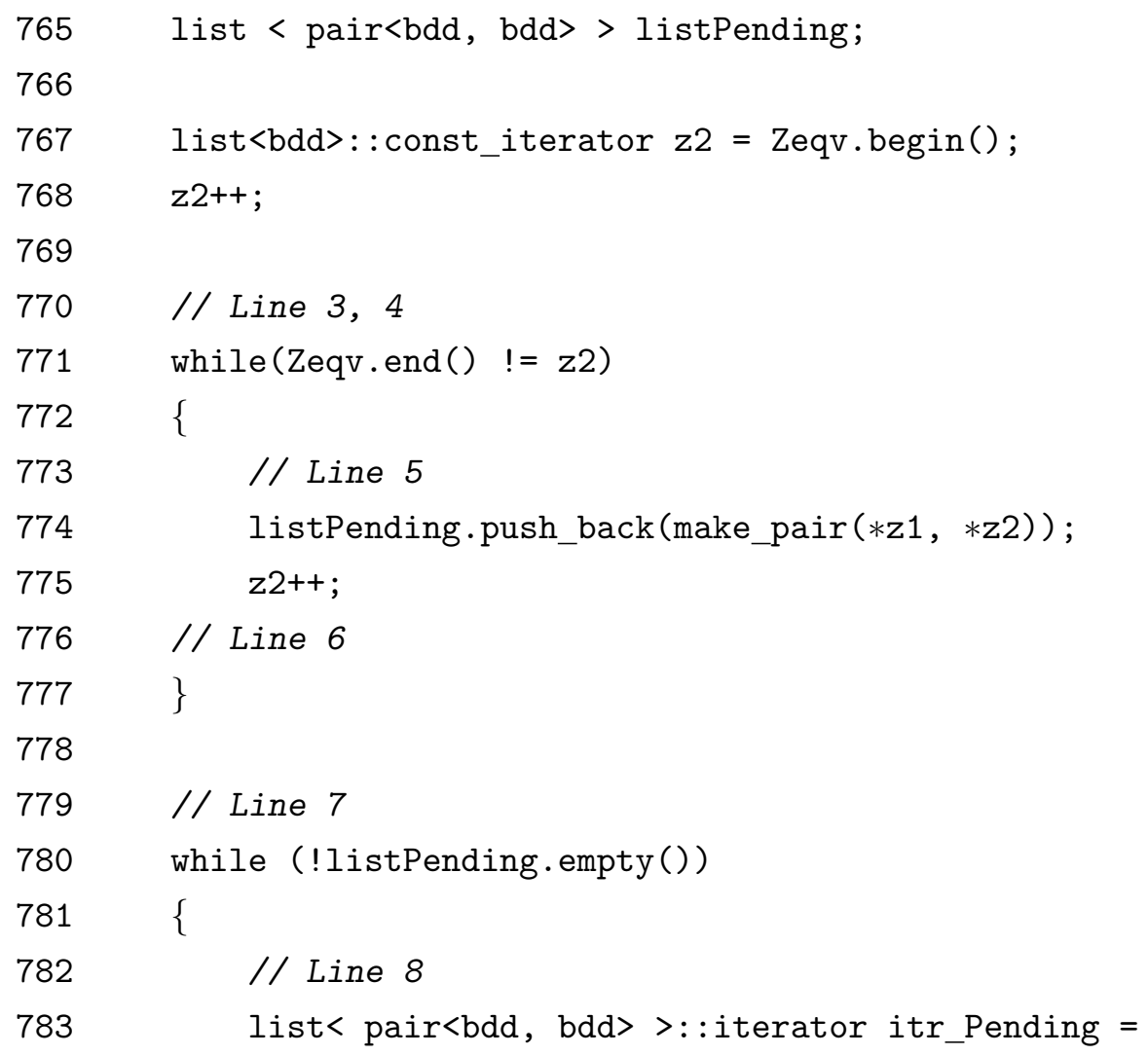

listPending. $\operatorname{begin}()$;

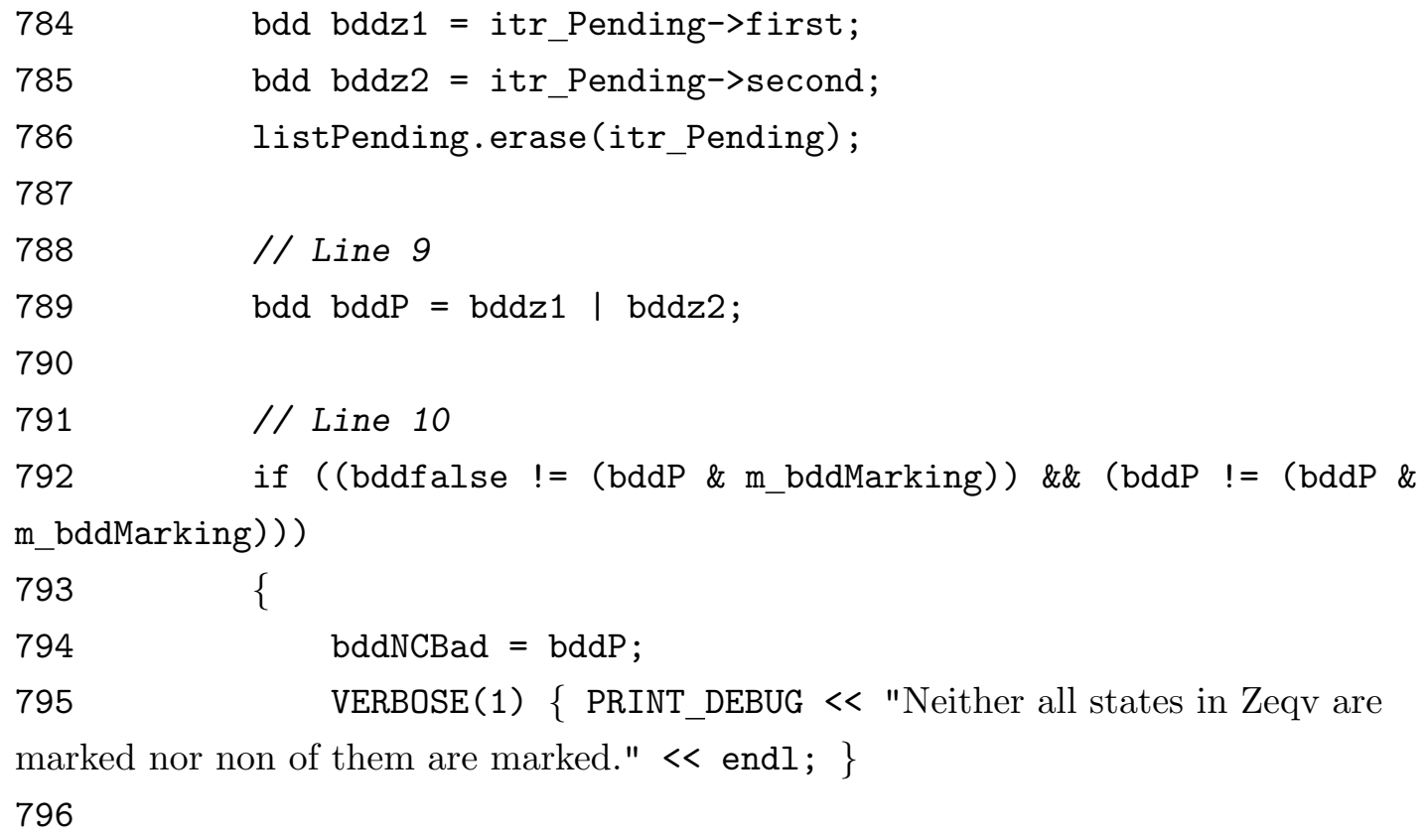




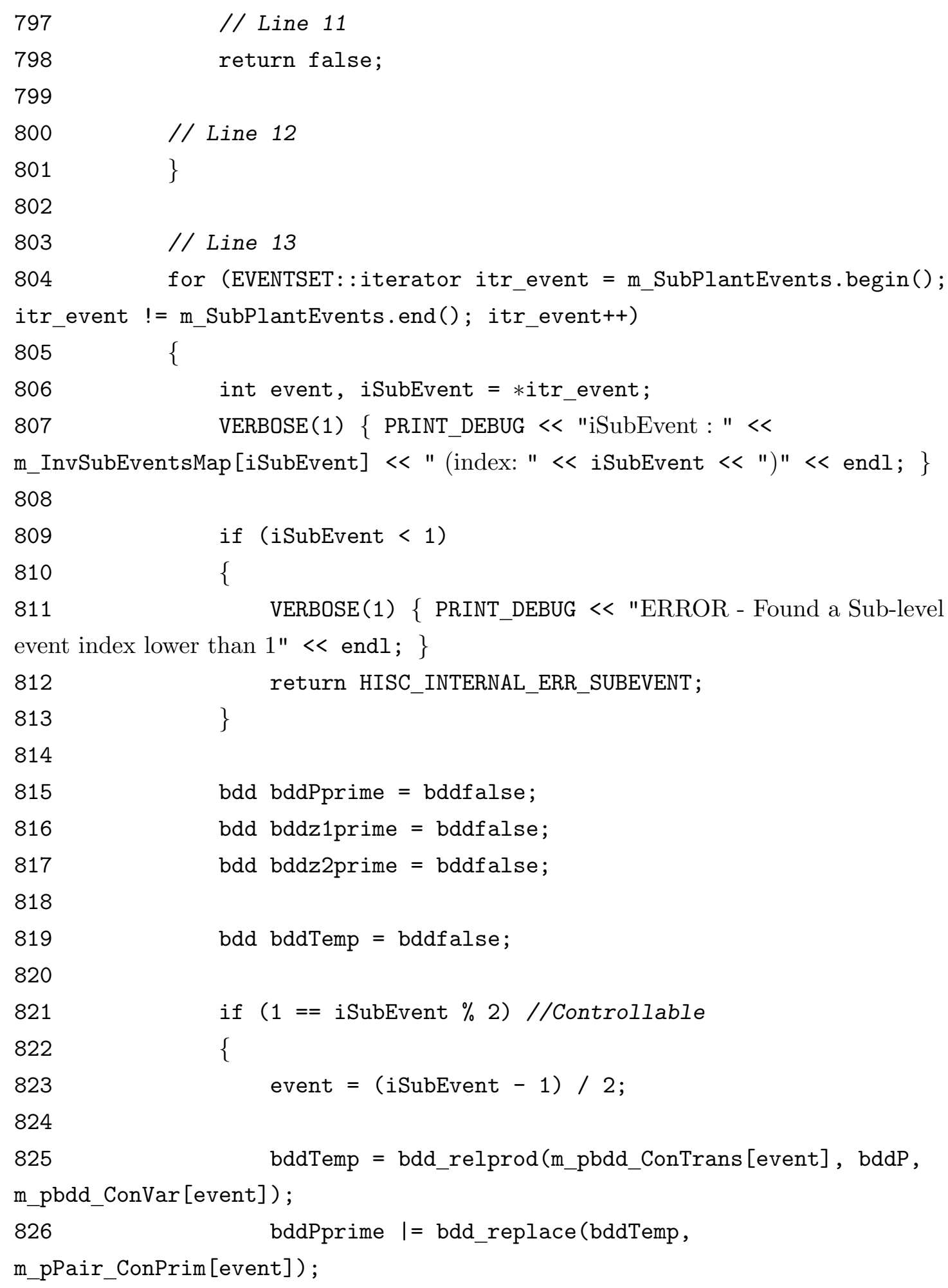




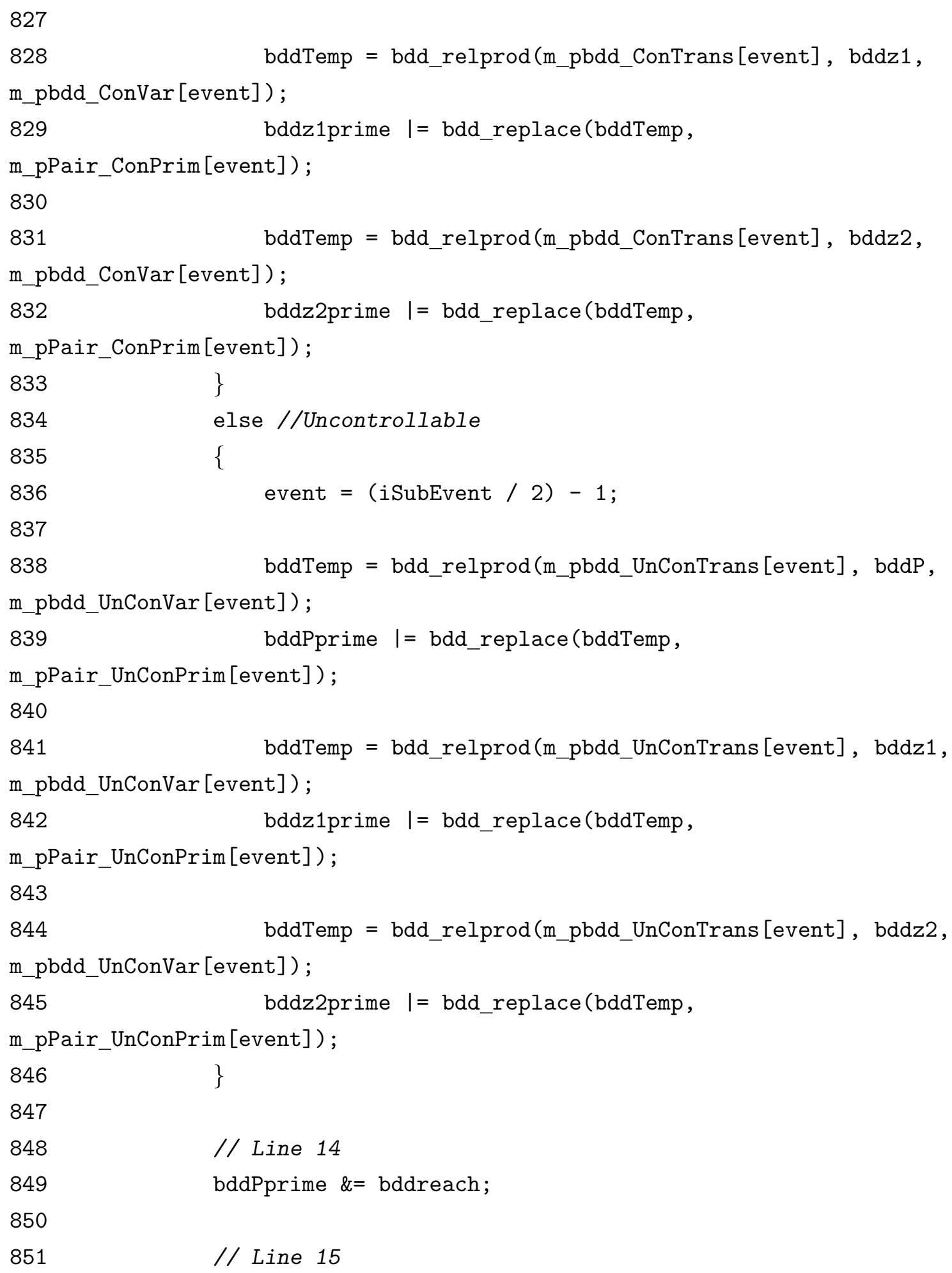




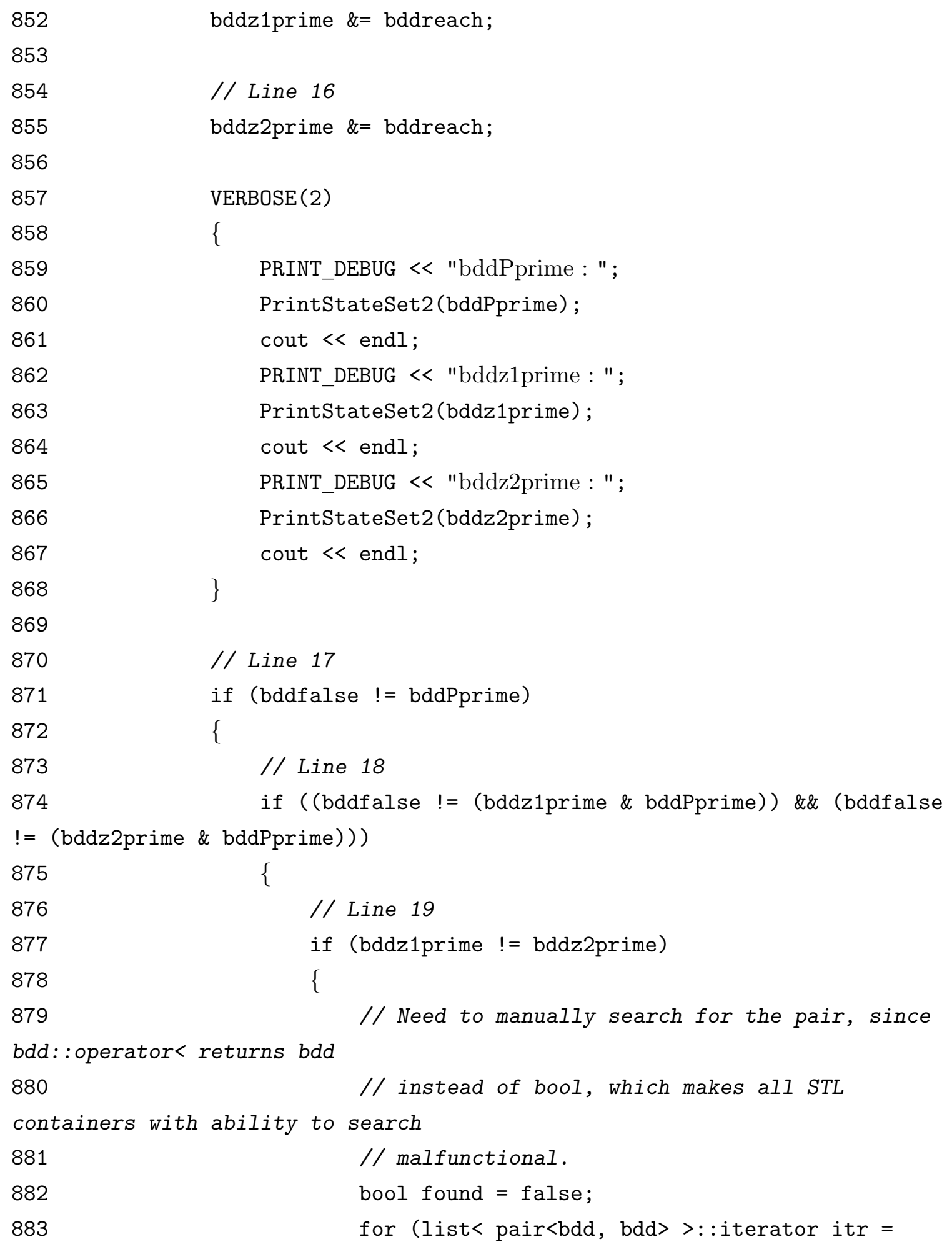




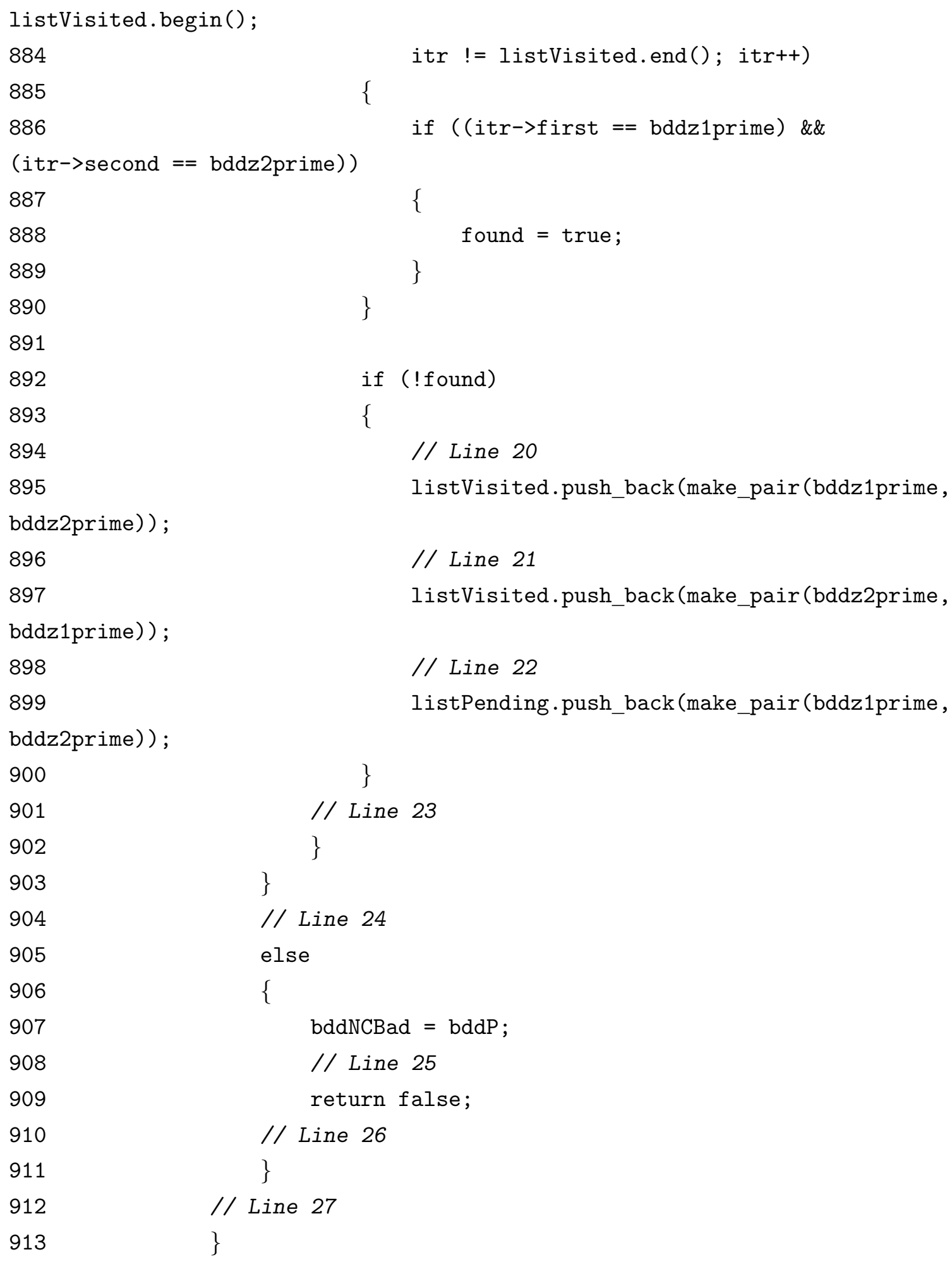




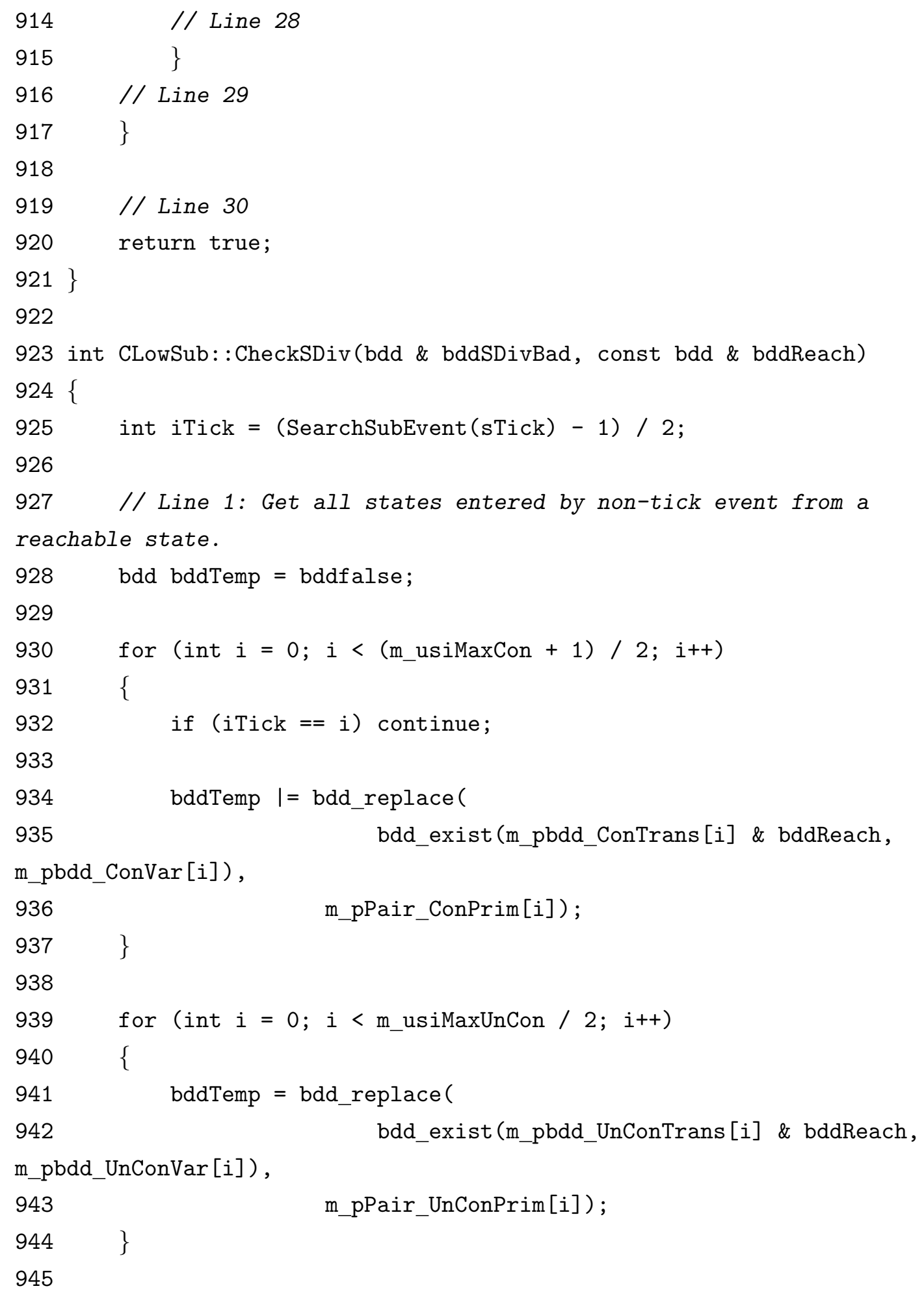


946 // Line 2 - 4: Each reachable marking states must not be reached by a non-tick event from a reachable state.

947 bddSDivBad = (m_bddMarking \& bddReach) \& bddTemp;

948

949 return 0 ;

$950\}$

951

952 EVENTSET CLowSub: :GetTransitionEvents (const bdd \& bddleave, const bdd \& bddenter)

953\{

954 EVENTSET events;

955 events.clear();

956

957 if ((bddleave $==$ bddfalse) || (bddenter $==$ bddfalse) $)$

958\{

959 cout $<<$ "CLowSub::GetTransitionEvents() : bddleave is empty or bddfalse is empty." $<<$ endl;

960 return events;

$961\}$

962

963 //Controllable events

964 for (int $i=0$; $i<$ (m_usiMaxCon +1 ) / 2 ; $i++$ )

965\{

966 bdd bddtrans = bddleave \& bdd_replace(bddenter,

m_pPair_Con[i]);

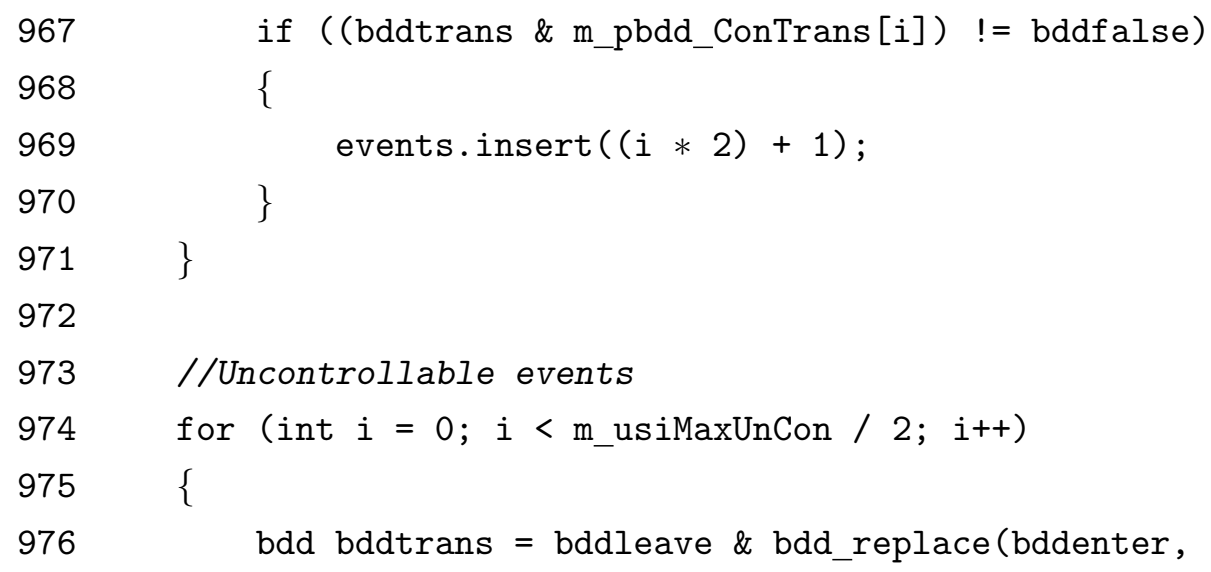


m_pPair_UnCon[i]);

977 if ((bddtrans \& m_pbdd_UnConTrans[i]) != bddfalse)

978

979 \{

980

$981\}$

982

983 return events;

$984\}$

985

986 